\title{
Collective habits and social norms in labour supply : from micromotives to macrobehaviour
}

Citation for published version (APA):

Vendrik, M. C. M. (1993). Collective habits and social norms in labour supply : from micromotives to macrobehaviour. [Doctoral Thesis, Maastricht University]. Datawyse / Universitaire Pers Maastricht. https://doi.org/10.26481/dis.19931022mv

Document status and date:

Published: 01/01/1993

DOI:

10.26481/dis.19931022mv

Document Version:

Publisher's PDF, also known as Version of record

\section{Please check the document version of this publication:}

- A submitted manuscript is the version of the article upon submission and before peer-review. There can be important differences between the submitted version and the official published version of record.

People interested in the research are advised to contact the author for the final version of the publication, or visit the DOI to the publisher's website.

- The final author version and the galley proof are versions of the publication after peer review.

- The final published version features the final layout of the paper including the volume, issue and page numbers.

Link to publication

\footnotetext{
General rights rights.

- You may freely distribute the URL identifying the publication in the public portal. please follow below link for the End User Agreement:

www.umlib.nl/taverne-license

Take down policy

If you believe that this document breaches copyright please contact us at:

repository@maastrichtuniversity.nl

providing details and we will investigate your claim.
}

Copyright and moral rights for the publications made accessible in the public portal are retained by the authors and/or other copyright owners and it is a condition of accessing publications that users recognise and abide by the legal requirements associated with these

- Users may download and print one copy of any publication from the public portal for the purpose of private study or research.

- You may not further distribute the material or use it for any profit-making activity or commercial gain

If the publication is distributed under the terms of Article $25 \mathrm{fa}$ of the Dutch Copyright Act, indicated by the "Taverne" license above, 
COLLECTIVE HABITS AND SOCIAL NORMS IN LABOUR SUPPLY 



\title{
COLLECTIVE HABITS AND SOCIAL NORMS IN LABOUR SUPPLY
}

\author{
From Micromotives to Macrobehaviour
}

\section{PROEFSCHRIFT}

ter verkrijging van de graad van doctor aan de Rijksuniversiteit Limburg te Maastricht, op gezag van de Pro-Rector, Prof. dr. L. Boon, volgens het besluit van het College van Dekanen, in het openbaar te verdedigen op vrijdag 22 oktober 1993 om 16.00 uur door

Martinus Cornelis Maria Vendrik

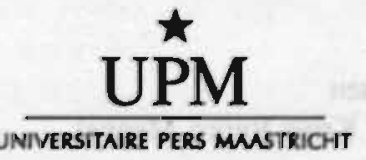


Promotores:

Prof. dr. J. Muysken

Prof. dr. C.R.J. de Neubourg

Beoordelingscommissie: Prof. dr. J.A.H. Maks (voorzitter)

Prof. dr. A. Kapteyn (Katholieke Universiteit Brabant)

Prof. dr. H.J.M. Peters

CIP-DATA KONINKLIJKE BIBLIOTHEEK, DEN HAAG

Vendrik, Martinus Cornelis Maria

Collective habits and social norms in labour supply : from micromotives to macrobehaviour / Martinus Comelis Maria Vendrik. - Maastricht : Universitaire Pers Maastricht. III., fig., tab.

Thesis Maastricht. - With ref. - With summary in Dutch. ISBN 90-5278-093-5

Subject headings: labour supply : preference formation ; social norms. 
"The strongest force is the force of habit."

Jan Tinbergen

To my parents and Hilda 


\section{Contents}

Preface

CHAPTER 1 Introduction

1.1 Individual habit formation 3

1.2 Behavioural preference interdependence 5

$\begin{array}{lll}1.3 & \text { Collective habit formation } & 6\end{array}$

1.4 Dynamics of social norms 8

1.5 Dynamics of female participation 9

1.6 Approach 10

CHAPTER 2 Microeconomic Theory of Labour Supply 13

2.1 The standard model 13

2.2 Extensions 19

$\begin{array}{lll}2.2 .1 & \text { Scope } & 19\end{array}$

$\begin{array}{ll}2.2 .2 & \text { Imperfect information }\end{array}$

$\begin{array}{ll}2.2 .3 & \text { Taxes and transfers }\end{array}$

2.2.4 Rationings and endogenous wages 24

$\begin{array}{lll}2.2 .5 & \text { Psychic income of labour time } & 27\end{array}$

2.2.6 Preference variations and dynamics of state variables 33

2.2.7 Family labour supply 35

2.3 The short-run models in this study 37

CHAPTER 3 Individual Habit Formation: General Model

3.1 General model $\quad 42$

3.2 Multiple equilibria and hysteresis 44

$\begin{array}{lll}3.3 & \text { Stability conditions } & 47\end{array}$

3.4 Short and long-run wage elasticities $\quad 50$

3.5 Catastrophes, hysteresis and quasi-hysteresis 52

3.6 Discontinuous short-run labour supply schedules and hysteresis 56

3.7 Concluding remarks $\quad 58$

Appendix 3A Formal equilibrium and stability analysis 59

Appendix 3B Derivation of relations between short and long-run wage effects

CHAPTER 4 Individual Habit Formation:

Additive Quadratic Model and Aggregation 61

4.1 Short-run labour supply 62

4.2 Catastrophes and hysteresis 65

4.3 Always stable long-run case 68 
$4.4^{*} \quad$ Unstable long-run cases $\quad 74$

$\begin{array}{lll}4.5^{*} & \text { Workaholism } & 83\end{array}$

4.6 Long-run utility functions $\quad 89$

4.7 Aggregation: short run $\quad 92$

4.8 Aggregation: long run $\quad 95$

4.9 Concluding remarks 103

Appendix 4A Derivation of results for Case 1 in Section 4.3 103

Appendix 4B Derivation of results for Case 2 in Section $4.4 \quad 104$

Appendix 4C Derivation of results for Cases 5 and 6 in Section 4.4105

Appendix 4D Derivation of exit curve for $c^{*} \leq 0 \quad 106$

Appendix 4E Derivation of adjustment paths of the

average habit stock in Figure 4.12

$\begin{array}{lll}\text { CHAPTER } 5 & \text { Behavioural Preference Interdependence } & 108\end{array}$

$5.1 \quad$ Classification of social interactions 109

5.1.1 General notions 109

$\begin{array}{lll}5.1 .2 & \text { Sources of BPI } & \$ 11\end{array}$

$\begin{array}{ll}5.1 .3 & \text { Endogenous dynamics of BPI } \\ 5.115\end{array}$

5.1.4 Other forms of preference (inter)dependence 116

$\begin{array}{lll}5.2 & \text { General model } & 117\end{array}$

5.3 Multiple equilibria, hysteresis and stability conditions $\quad 123$

$5.4 \quad$ Wage elasticities, catastrophes and hysteresis $\quad 127$

$\begin{array}{lll}5.5 & \text { Additive quadratic model } & 130\end{array}$

$\begin{array}{lll}5.5 .1 & \text { Short-run labour supply } & 130\end{array}$

$\begin{array}{ll}5.5 .2 \quad \text { Long-run labour supply } & 131\end{array}$

5.5.3* Conditions for catastrophes and hysteresis $\quad 135$

$\begin{array}{lll}5.6 & \text { Concluding remarks } & 141 .\end{array}$

CHAPTER 6 Collective Habit Formation: BPI and Stable IHF 143

$\begin{array}{lll}6.1 & \text { Stability conditions } & 144\end{array}$

6.1.1 General analysis $\quad 144$

6.1.2 Case of one uniform habit slope $\quad 145$

6.1.3 Case of two uniform habit slopes 149

6.2 Additive quadratic model 151

6.2.1 Short-run labour supply 151

6.2.2 Case of one uniform habit slope 153

6.2.3* Case of two uniform habit slopes 154

6.2.4* Conditions for collective catastrophes and hysteresis 157

6.3 BPI and stable IHF in consumption 163

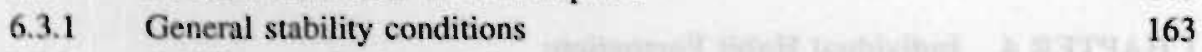

$\begin{array}{ll}6.3 .2 & \text { Additive quadratic model } \\ 6.464\end{array}$

6.4 BPI and stable IHF in corporate time $\quad 165$

6.5 Concluding remarks 166

$\begin{array}{ll}\text { Appendix } 6 \text { A Derivation of stability condition (6.6) } & 167\end{array}$ 
CHAPTER 7 Collective Habit Formation: BPI and Unstable IHF

7.1 General analysis

7.2 Case A: variation in household time taste and IHF strength 174

7.3 Case B: variation in susceptibility to social influence 180

$\begin{array}{lll}7.4 & \text { Conditions and configurations of equilibria } & 184\end{array}$

$\begin{array}{lll}7.5 & \mathrm{BPI} \text { and unstable IHF in consumption } & 188\end{array}$

7.6 BPI and unstable IHF in corporate time $\quad 190$

$\begin{array}{lll}7.7 & \text { Concluding remarks } & 192\end{array}$

Appendix 7A Derivation of (7.3) 195

Appendix 7B Derivation of equality of surface areas

$S_{1}$ and $S_{2}$ in Figure 7.2

\section{CHAPTER 8 Dynamics of Social Norms}

8.1 Theory of social custom

8.2 Social norrns in male labour supply

8.3 Social norms in female labour supply

8.4 Household norm and collective habit

8.5 Dynamics of social norm beliefs

8.6 Collective catastrophes and hysteresis

8.7 Comparison with social custom literature

8.8 Financial independence norm: Akerlof specification

8.9 Financial independence norm: AQM

8.10 Household norm and financial independence norm 227

8.11 Concluding remarks

9.1 Female labour force participation; the Dutch case

9.2 Impacts of social norms and collective habits

9.3 General participation equation for one social group

9.4 Logit participation equation for one social group

9.5 Aggregation and BPI between social groups

9.6 Social norm proxies and econometric evidence 256

$\begin{array}{ll}9.7 & \text { Endogenous dynamics of social norms }\end{array}$

$\begin{array}{lll}9.8 \text { Long-run elasticities } 261 & \end{array}$

9.9 Paricipation rate dynamics and hysteresis 267

$\begin{array}{lll}9.10 & \text { Collective catastrophes } & 272\end{array}$

$\begin{array}{lll}9.11 & \text { Collective satiation } & 278\end{array}$

9.12 Concluding remarks 282

Appendix 9A Derivations of (9.5) and (9.7) 283 
References

Nederlandse Samenvatting / Summary in Dutch

Curriculum Vitae

Sections and subsections which are marked by an ", are the most technical ones (see Sec. 1.6).

An abridged version of Chapter 3 has been published as Vendrik, Maarten C.M. (1993), "Habits, Hysteresis and Catastrophes in Labor Supply", Journal of Economic Behavior and Organization 20: 353-372.

Parts of Chapters 8 and 9 have appeared as De Neubourg and Vendrik (1989) and are forthcoming in the Journal of Economic Psychology. 


\section{Preface}

This study tries to incorporate some psychological and sociological insights into microeconomic analysis. While I have long been interested in psychology and sociology, my concern with the specific subject of this book has its main origins in certain experiences during my econometrics studies at the Erasmus University of Rotterdam. In particular, I remember a discussion on the desirability of economic growth in a seminat on theoretical economics, in which I disagreed with the teacher Prof. C. van Eijk. One of his arguments in favour of economic growth in developed countries was that there are still so many needs to be fulfilled in these countries. Implicit in this argument, however, was the assumption that needs are exogenous to the economic system. It was precisely this crucial assumption and, more generally, the desirability of ever continuing economic growth that I came increasingly to question. In order to investigate these issues further I joined a group of Dutch economists who had the same doubts and who, following Bob Goudzwaard and Harry de Lange, advocate an "Economics of Enough". Through my experiences in this group and by attending many lectures and seminars in social and economic philosophy and sociology, I learned to place the problems mentioned in a wider social-scientific context.

In addition, I became increasingly fascinated by the immense significance which paid work has for people in our Western societies. When I thought about it, I realized that this must have something to do with so many needs being satisfied (as well as dissatisfied) in and by means of a job. Indeed, one can say that almost the entire needs hierarchy of Maslow is involved (exceptions are the needs for sleep and sex, but even these needs are fulfilled in certain jobs). Nevertheless, the resulting strong want for paid work (which may be referred to as "job libido") seems again not exogenous to the economic system, but rather subject to endogenous change. For example, nowadays married women in OECD countries have, on average, a much stronger want for paid work than they had some decades ago, and this change does not seem exogenous to developments in the economy. Traditionally, economists have attributed the ensuing increases in female labour force participation to alterations in budget and other kinds of constraints, but historical and econometric evidence strongly suggests that shifts in preferences are also important causes.

Against this background, I was very happy when, after graduating in econometrics, I got the opportunity to conduct doctoral research at the economics department of the University of Limburg on the possible role of psychological and sociological factors in an economic analysis of labour supply. I began by constructing a general utility-maximizing framework, in which I integrated economic as well as some psychological and sociological motivation theories. However, although successful in terms of my own research objectives, this broad approach turned out not to fit in very well with the rather restrictive research cuiture of mainstream economists. Therefore, after two years I decided to shift the emphasis to an analysis of a non-linear dynamics of preference formation in labotir supply. This had the advantage that I could use my skills and experience in non-linear dynannics and, more specifically, catastrophe theory, which I had acquired during studies of theoretical physics 
prior to my econometrics studies. Moreover, I found it fascinating to see how collective phenomena in human behaviour resemble those in the behaviour of atoms and molecules. On the other hand, my ability to master the complexities of the non-linear dynamics of preference formation contributed to making the research extensive as well as fairly abstract and technical. Therefore, I was glad to be aided by my supervisors, Joan Muysken and Chris de Neubourg, in learning to write papers and chapters of this book which were not too long and not too technical. Yet, I feel I have not completely succeeded in that.

More generally, Joan and Chris helped me to adapt to the economic research culture. Moreover, they urged me to restrict myself so as to finish the project within reasonable time limits. Still, I was stubborn enough to exceed time schemes repeatedly, since I felt the project as well as a part of myself needed that. This brought me in continual conflict with other parts of myself and it made necessary frequent appeal to the patience of my supervisors. I thank them for their help as well as their patience. Furthermore, I am indebted to Joan and Chris for bringing me in contact with the work of George Akerlof. This work has been a constant source of inspiration for me, since I feel very much at home with his broad, interdisciplinary, and yet formal approach. Another source of inspiration has been the work of Arie Kapteyn, Bernard van Praag and their fellow workers, and I am indebted to Arie for helpful comments on chapters of this book. At Maastricht my most valuable and pleasant discussion partner has been Lex Borghans, with whom I could discuss all kinds of aspects of my research. I thank him for that. Furthermore, my gratitude goes to John Spangenberg, Geert Woltjer and Sabine van de Zande for their moral and other kinds of support, to Arjen van Witteloostuijn for helpful advice, and to Stephen Lea, Siegwart Lindenberg. Joan Muysken. Chris de Neubourg, Erik de Regt, Jaeques Siegers and Danicl Weiserbs for useful discussions and/or comments. I am indebted to Hans Maks and Hans Peters for their comments on this thesis as members of the evaluation committee, especially the latter for his detailed remarks. I thank Sorin Ducaru, Erik van Ballegoij and Marce! Jansen for drawing the figures and most tables, Aad van Mourik and Ruud Leliveld for advice on the lay-out of this thesis, and the latter also for his contribution to the design of the cover of this book. Moreover. I am indebted to Bob Wilkinson for his advice on the English of this book, to Huub van Kan and Maud Soudant for printing support, and to Jan van Ours for kindly sending me data on labour force participation rates.

Last but not least, my sincere gratitude goes to my parents for giving me the cultural baggage and inspiration which is needed for this kind of research, and for their strong and continuous trust in my risky undertakings. I am also very grateful to my wife Hilda for her moral support and for having so much patience with me having so little energy left for her and the housekeeping. Finally, I thank my little daughter Kanuna for making me feel more and think less. 


\section{CHAPTER 1}

\section{Introduction}

A remarkable phenomenon in economic science is that the assumption of stable preferences or tastes is still a point of departure of many of its models, despite the fact that there is growing evidence to the contrary. One striking example of preference shifts in the field of labour supply is given by the changes in attitude of "emancipating"1 married women towards their participation in the labour market. Since World War II female participation in the labour force has strongly increased in many OECD countries. Traditionally, economists have been trying to explain these developments as the result of changes in pecuniary variables like the women's own wage rate, the income of the husband and the tax system, and of changes in demographic and socio-economic variables like the number of children and the level of education of the women. Although such variables can account for a substantial part of the increases in female labour force participation, they seem only a part of the truth. Historical evidence as well as casual observation strongly suggest that changes in habits and social norms and social influence processes have also played important roles in the female participation increases. Such changes and processes directly affect the preferences of the women involved, implying that the assumption of stable preferences should be relaxed.

Allowing unstable preferences makes most economists feel uneasy, since they are not used to it, since it tends to make their models much more complex and since it urges them to think about psychological and sociological issues. Historically, the division of labour in social science has relegated the study of preference formation to the realms of psychology and sociology. Nevertheless, in contrast to this division of labour, social reality is not separated in disjunctive fragments. In particular, this holds for the reality of the rises in female labour force participation, such that the present state of separate research on this subject by economists, sociologists and psychologists is unsatisfactory. It seems highly desirable to integrate sociological and psychological insights into the economic models of female labour force participation. The present study aims to contribute to that.

Although the importance of preference formation was recognized by early economists (e.g., Marshall, 1920, App. H, Sec. 3), attempts to incorporate preference formation into

${ }^{1}$ In American Engiish the terms "emancipating" and "emancipation" seem to have a stronger and more ideology-laden meaning than in British English. Therefore, such terms are put between quotation marks in this study. 
mainstream economic theory seem to be relatively recent. Duesenberry (1949) was among the first to give a systematic treatment of (endogenous) preference formation in the context of saving and consumption behaviour. ${ }^{2}$ In the wake of this pioneering work two major theories of preference formation have been developed, namely the theory of individual habit formation and the theory of (behavioural) preference interdependence. Individual habit formation is the phenomenon that an individual gets used to a certain behaviour and that this reinforces her/his preferences in favour of that behaviour. Behavioural preference interdependence means that relevant others displaying a certain behaviour affect an individual's preferences with respect to that behaviour. ${ }^{3}$ Another, but related theory of preference formation has been introduced by Akerlof (1980). His theory of social custom models the impacts of beliefs in social norms on economic behaviour as well as the feedback of that behaviour on the social norm beliefs. Finally, the counterpart of individual habit formation is individual long-run satiation (e.g., Iannacone, 1986). This is the phenomenon that an individual gets increasingly satiated with a certain behaviour, thus weakening her/his preferences in favour of that behaviour in the long run. It is usually considered as a part of the theory of individual habit formation.

All three theories of preference formation, including individual long-run satiation, seem highly relevant for the development of the female labour force participation and, more generally, for the labour supply behaviour of women as well as men (think of work ethic norms). ${ }^{4}$ On the other hand, research on this subject is rare (see the next section). Therefore, the present study will apply the three preference formation theories to labour supply by incorporating them into the microeconomic theory of labour supply and by deriving implications of this incorporation. In doing so, the three preference formation theories will be extended and integrated. Moreover, the focus will be on the effects of nonlinear forms of dynamics of the preference formations in combination with aggregation from the microeconomic to the macroeconomic behaviour.

Sections 1.1-1.5 of this introductory chapter give more detailed accounts of the contributions of the successive chapters of this study to the state of the art of the three preference formation theories. Section 1.6 provides a motivation of the theoretical and deductive approach of this study and gives some directions to the reader.

2 In principle, preference fonmation can also be exogenous to the socio-economic system as, for example, expressed by a time trend on some parimeters of consumption demand or labour supply equations. Usually, however, a certain degree of endogeneity of preferences to the socio-economic system seems likely, so exogenous preference formation did not receive much attention in the literature.

${ }^{3}$ In the literature this is usually referred to as preference interdependence. but $I$ add the adjective "behavioural", since there are also other kinds of preference interdependence (see Sec. 5.1).

"Two other important types of endogenous preferences are those influenced by advertising (see. e.g. Galbraith, 1958, and Schmalensee, 1972) or by prices (see. e.g. Pollak. 1977). However, in the context of labour supply such endogenous preferences seem less important than those mentioned above. 


\subsection{Individual habit formation}

Whereas individual habit formation in consumption has been the subject of a large number of theoretical and empirical investigations (see Pollak, 1978, and Muellbauer, 1988, for surveys), individual habit formation in labour supply has received much less attention. Only a few empirical studies have been conducted. Phlips (1978) gives an empirical analysis of individual habit formation with respect to consumption categories, leisure and transaction money in a linear expenditure system. Using household data, Johnson and Pencavel (1984) investigate a similar linear expenditure model for individual habit formation with respect to total family consumption and leisure times of husband and wife. Hotz et al. (1988) estimate a more sophisticated specification for individual habit formation with respect to leisure. Finally, Kapteyn and Woittiez (1990) develop and estimate a detailed model of both individual habit formation and behavioural preference interdependence with respect to leisure or labour times of husband and wife (see also Secs. 1.2 and 1.3).

While the studies of Phlips, Johnson and Pencavel, and of Kapteyn and Woittiez assume myopic (individual) habit formation, Hotz et al. extend this to rational habit formation. Myopic habit formation means that the individual does not take into account the feedback of her (or his) present decision on her future preferences. In contrast, in rational habit formation models the individual has perfect foresight with respect to this feedback and takes fully into account the ensuing future utility costs or benefits of his present decision. However, as Deaton and Muellbauer (1980a, p. 374) put it, "by making no concession to imperfect knowledge or to questions of calculation", rational habit formation models "are extremely implausible as descriptions of observed behaviour" (see also Akerlof, 1991). ${ }^{5}$ Moreover, Muellbauer (1988) and Winder (1988) report that empirical evidence on life cycle consumption appears to favour myopic rather than rational habit formation. On the other hand, Pashardes (1986) obtains the opposite result. For labour supply there is no empirical evidence, but in that case myopic habit formation may be a more plausible approximation than in the case of consumption behaviour, since labour supply decisions tend to be less frequent than consumption decisions and hence may induce less awareness of habit formation.

Anyway, all four labour supply studies mentioned above suggest that, especially with respect to leisure, habit formation is rather strong. Still, the habit formation is shown to be mostly stable in the sense that the stability conditions for the long-run equilibrium are fulfilled. Such a stable long-run equilibrium is the result of a long-run multiplier process in which, for example, a person who has been induced to take a part-time job, gets used to the smaller amount of leisure, which makes her prefer even less leisure and take a job of more hours, which she also gets used to, which makes her prefer even less leisure and take a job of even more hours, etcetera until a long-run equilibrium is reached. A simple extension of such a model of stable habit formation can explain how after taking a part-time job a person can develop a strong orientation towards paid work and may even develop into a "workaholic". On the other hand, such a model cannot explain why some long-term unemployed,

5 On the other hand, rational habit formation models may be very important in a nomative welfare-theoretical context (see Pollak, 1976b, p. 296). 
although having lost their old job involuntarily, become little motivated to get a new job. Similarly, such a stable habit formation model can give an explanation of the strong orientation of many housewives to household work, but it may not adequately explain how in a process of "emancipation" the same women can develop a strong orientation towards paid work.

The present study models such profound changes in preferences and behaviour as the outcomes of unstable habit formation as in the addictive consumption model of Becker and Murphy (1988). The unstable habit formation is described by an unstable long-run equilibrium which is located between two stable long-run equilibria. This leads to a polarization in behaviour and habit state: on the one hand, when the initial hours of work are above the unstable equilibrium value, the habit formation induces a rise in hours towards a stable equilibrium of relatively high hours and ensuing accustomization. Such an equilibrium may describe the behaviour and habits of an "emancipated" woman with a job or a workaholic (when the number of hours worked is very high). On the other hand, when the initial hours are below the unstable equilibrium value, the habit formation causes falls in hours towards a stable equilibrium of low or zero hours and similarly ensuing accustomization. The zero hours equilibrium may then correspond to a housewife or a "voluntarily long-term unemployed" person. Since the initial hours depend on the time paths of exogenous variables like the wage rate or rationings in the past, these time paths determine which of the two stable equilibria is actually approached. This I call individual hysteresis. ${ }^{6}$ At certain values of exogenous variables the stable equilibrium which is approached may become unstable and subsequently disappear (this is not recognized by Becker and Murphy). As a kind of "snowball effect" this induces an unstable habit formation process towards the other stable equilibrium. Such a transition is referred to as an individual catastrophe in the sense of Thom (1975). Individual catastrophes in behaviour and habits may have occurred (and still occur) in the "emancipation" process of individual married women. In contrast to Becker and Murphy, 1 assume that the habit formation is myopic, since this seems a plausible approximation for labour supply (see above). Moreover, it simplifies the analysis and facilitates the introduction of more than one habit state variable for consumption, household time (or leisure, see Sec. 2.3) and corporate time (or labour time).

Chapter 3 of the present study analyses this non-linear dynamics of individual habit formation in labour supply for a general (additive) utility function. In particular, necessary and sufficient (in)stability conditions are derived and in the case of instability schedules of individual long-run labour supply as a function of the wage rate are obtained, displaying individual hysteresis and catastrophes. At the end of the chapter the unstable individual habit formation model is complemented by individual long-run satiation, and cases of discontinuous short-run labour supply schedules are considered. Chapter 4 derives necessary and

${ }^{6}$ See Cross and Allen (1988) and Franz (1990) for the origins of the concept of hysteresis in the natural sciences and for surveys of research on hysteresis in economics. See Goodwin (1977) and Heiner (1983, p. 582) for hysteresis in relation to habits and behavioural rules.

${ }^{7}$ There is a huge literature on applications of catastrophe theory in various disciplines, ranging from physics and biology to linguistics and history (see, e.g.. Zeeman, 1977). One interesting application in economics is the generalization of the Kaldor model of a business cycle by Varian (1979) and George (1981). One application in physics is Vendrik (1979a, 1979b). 
sufficient conditions for individual hysteresis and catastrophes for an additive quadratic specification of the utility function, which is similar to the specifications used by Houthakker and Taylor (1970) and Becker and Murphy (1988) for the case of consumption. Moreover, this specification is used to trace the macroeconomic implications of the derived schedules of individual long-run labour supply by aggregating them to schedules of average (i.e. per capita) long-run labour supply under various assumptions on the individual variation in utility function parameters, exogenous variables and habit states.

\subsection{Behavioural preference interdependence}

In contrast to individual habit formation in consumption, (behavioural) preference interdependence in consumption has only been investigated in a limited number of studies (e.g., Veblen, 1899, Leibenstein, 1950, Gaertner, 1974, Pollak, 1976a, and Kapteyn et al., 1989). Leibenstein makes an important distinction between "bandwagon effects" and "reverse bandwagon effects" (or "snob effects"). A bandwagon effect means that relevant others displaying a certain behaviour reinforce an individual's preferences with respect to that behaviour. On the other hand, a reverse bandwagon effect is said to occur when relevant others displaying a certain behaviour diminish an individual's preferences with respect to that behaviour (cf. the distinction between the reinforcing and weakening effects of individual habit formation and individual long-run satiation, respectively). The literature mainly focuses on bandwagon effects. A fortiori this holds for preference interdependence in labour supply, on which there is only one publication I know, namely that of Kapteyn and Woittiez (1990). They model both preference interdependence and individual habit formation with respect to leisure or labour times of husband and wife, and in an estimation for Dutch cross-section data they find a significant contribution of preference interdependence. On the other hand, this contribution is surprisingly small, considering empirical results for preference interdependence in consumption.

Just like practically all models of preference interdependence in consumption, the estimated model of Kapteyn and Woittiez implies a unique stable long-run equilibrium for preference interdependence (at given long-run equilibrium values of the habit states). Such a stable long-run equilibrium is the result of a long-run multiplier process in which, for example, some members of a social group who have been induced to take a job, induce other members also to take a job or to take a job of more hours, which again induces other members to take a job too or to take a job of more hours, etcetera until a long-run equilibrium is reached. However, analogously to a stable individual habit formation model, such a model of stable preference interdependence cannot explain why members of certain subcultures of long-term unemployed persons, although having lost their old job involuntarily, become little motivated to get a new job. Similarly, such a stable preference interdependence model may not adequately explain how in a collective process of "emancipation" social groups of former housewives can develop a strong orientation towards paid work.

Again, the present study models such profound changes in preferences and behaviour as the outcomes of unstable (bandwagon) preference interdependence as described by an unstable long-run equilibrium between two stable long-run equilibria. In one of the latter 
stable equilibria a majority of the social group may not participate in the labour market. In the other stable equilibrium a majority of the same social group may have a job. Which of the two equilibria is actually approached at a certain moment depends on the past history of the social group. This I call collective hysteresis. Moreover, under certain conditions the social group may, as a kind of "snowball effect", collectively enter or leave the labour market in an unstable preference interdependence process from one towards the other equilibrium. This is referred to as a collective catastrophe. Such collective transitions within social groups may have played (and still play) a role in the strong rise of the labour force participation of married women in many OECD countries since World War II.

Chapter 5 of the present study analyses this non-linear dynamics of behavioural preference interdependence in labour supply. First, a classification is given of the various kinds of social interaction underlying behavioural preference interdependence and other forms of preference interdependence in labour supply. Next, the social interactions underlying (bandwagon) behavioural preference interdependence in labour supply are summarized in a general model of (behavioural) preference interdependence with respect to the average consumption, the average household time and the average corporate time in a social group (as formulated by Iannacone (1989) for consumption only). An attractive feature of this preference interdependence model is that its structure is analogous to the structure of the general individual habit formation model of Chapter 3 (see also Deaton and Muellbauer, 1980, pp. 374-375). This analogy is employed in deriving necessary and sufficient (in)stability conditions as well as schedules of average long-run labour supply as a function of the average wage rate, displaying collective hysteresis and catastrophes. Note, however, that, whereas the individual habit formation model holds for individual behaviour, the preference interdependence model is formulated on the level of average behaviour in a social group. This implies an additional complication in the preference interdependence model as compared to the individual habit formation model, namely that of individual variation within the social group. The stabilizing effects of this individual variation are illustrated for an additive quadratic specification of the utility function. Then, for this specification necessary and sufficient conditions for collective catastrophes and hysteresis can be derived. Finally, a comparison is made between the different effects on average labour supply of unstable preference interdependence and unstable individual habit formation.

\subsection{Collective habit formation}

A subsequent question is what a combination of the effects on average labour supply of (unstable) preference interdependence and of (unstable) individual habit formation may look like. For consumption demand, integrations of a (bandwagon) preference interdependence model and an individual habit formation model have been made by Pollak (1976a) and Alessie and Kapteyn (1991). For labour supply, such an integration has only been carried out by Kapteyn and Woittiez (1990) (see the previous section). For the sake of brevity, we refer 
to these integrated models as models of collective habit formation, ${ }^{8}$ Again, all the collective habit formation models mentioned imply or are estimated to have a unique stable long-run equilibrium for the combined individual habit formation/preference interdependence process. Just as the separate stable individual habit formation or stable preference interdependence models, such a stable collective habit formation model cannot or may not adequately explain profound changes in labour supply preferences like those mentioned in the previous two sections. To model such phenomena in a more complete way we integrate the individual habit formation models of Chapters 3 and 4 with the preference interdependence models of Chapter 5 into (unstable) collective habit formation models.

First, for two moderately general subcases of collective habit formation with respect to household time only, simple necessary and sufficient (in)stability conditions are derived in Section 6.1. However, these conditions tum out not to be the relevant (in)stability conditions in the case of preference interdependence and separately unstable individual habit formation. For that case a modified relevant (in)stability condition is derived in Chapter 7. Accordingly, Section 6.2 and subsequent sections of Chapter 6 are restricted to the case of preference interdependence and separately stable individual habit formation, whereas Chapter 7 analyses the case of preference interdependence and separately unstable individual habit formation. In order to investigate the labour supply dynamics implied by the conditions in Section 6.1, Chapter 6 considers an additive quadratic specification of the collective habit formation model. It is shown that, in the presence of exogenous individual variation, the individual habit formation does not only reinforce the effect of preference interdependence, but also counteracts it by amplifying the individual variation (a point not recognized by Kapteyn and Woittiez). Moreover, as a generalization of the case of unstable preference interdependence and no individual habit formation as treated in Chapter 5, necessary and sufficient conditions for collective catastrophes and hysteresis to occur for changing values of the wage rate are formulated. Finally, preference interdependence and separately stable individual habit formation with respect to consumption and corporate time are added to the models.

The case of preference interdependence and (separately) unstable individual habit formation is analysed for the additive quadratic specification in Chapter 7 . Then collective catastrophes and hysteresis can arise as results of a sort of "two-slage rocket". In the first stage the unstable individual habit formation induces individual catastrophes of some group members from zero to maximal corporate hours or the reverse. The second stage fires when, due to a sufficiently strong preference interdependence, these individual catastrophes trigger subsequent individual catastrophes of other group members to such an extent that collective catastrophes arise. Then, for instance, a rocketing number of group members start to work a rocketing number of corporate hours. Such collective catastrophes are described by the modified (in)stability condition mentioned above. The implied labour supply dynamics is analysed by considering the case of unstable individual habit formation and preference interdependence as an extension of the aggregate additive quadratic case of only unstable individual habit formation and no preference interdependence as treated in Chapter 4 . First considering unstable individual habit formation and preference interdependence with respect

${ }^{8}$ This term has been inspired by the connection between individual habit formation and preference interdependence as shown by Deaton and Muellbauer (1980, pp. 374-375). 
to household time only, two subcases are distinguished, namely Case A of individual variation only in household time taste and individual habit formation strength and Case B of individual variation only in susceptibility to social influence. For both subcases (necessary and) sufficient conditions for collective catastrophes are derived as well as interesting longrun average labour supply schedules with two domains of catastrophic behaviour alongside a domain of persistent and hysteretic behaviour. Finally, unstable individual habit formation and preference interdependence with respect to consumption and corporate time are added to the models.

\subsection{Dynamics of social norms}

Another, but related theory of preference formation is the theory of social custom (TSC) of Akerlof (1980). This theory models the impacts of beliefs in social norms on economic behaviour as well as a long-run feedback of that behaviour on the social norm beliefs. The former impacts represent the effects of internalized social norms and social control as are well known in sociology. The latter feedback describes processes of internalization and "externalization" of a social norm, by which beliefs in that social norm change.

In Chapter 8 a variant of the TSC along the lines of Romer (1984) is applied to labour supply. First, an overview is given of possible social norms in male and female labour supply. Only the implications of social norms in female labour supply are elaborated, but the implications of social norms in male labour supply are similar. The application of the theory of social custom to a so-called household norm is shown to be analogous in structure to a limiting case of Case B of the additive quadratic model of collective habit formation in Chapter 7 (see the previous section). This is with the proviso that the intergenerational process by which social nom beliefs change in the conception of Akerlof is replaced by an intrapersonal belief changing process. On a conceptual level, this analogy relativizes the distinction between social norms and habits as made by Elster (1989, p. 100). If we envision the concept of habit in a broader sense as general attitude or habitus (cf. Bourdieu and Passeron, 1971) and with the qualification just mentioned, the effects and dynamics of social norms in the theory of social custom can then be interpreted as one of the possible "channels" through which unstable individual habit formation and preference interdependence may proceed. Moreover, it is argued that the parameter restrictions on the additive quadratic model which correspond to the labour supply application of the theory of social custom, are implausible. This is important, since, among other points, the restrictions automatically imply unstable individual habit formation, and hence individual catastrophes and individual and collective hysteresis as well as very weak conditions for collective catastrophes, whereas in the additive quadratic model stable individual habit formation and absence of catastrophes and hysteresis are certainly possible (see also the literature in the previous section). Chapter 8 continues with a comparison with other applications of the theory of social custom by Akerlof (1980), Romer (1984), Booth (1985) and Naylor (1989). This comparison makes clear that the present application adds two pairs of essential elements to the overall picture, namely the pair of individual and collective catastrophes and the pair of individual and collective hysteresis. Finally, the female labour supply model is further extended by introducing the effects of a social norm of financial independence. 


\subsection{Dynamics of female participation}

Chapter 9 of this study investigates the empirical relevance of the theoretical models which are set forth in the preceding chapters, by applying them to the developments of the labour force participation of Dutch and American married women since World War II. Whereas the participation rate of Dutch married women was remarkably low and stable till 1960, it grew strongly after 1960. Econometric time-series analyses of this development have been given by Mol et al. (1988) for the period 1889-1985 and by Romme $(1987,1990)$ for the shorter period 1971-1985. Although the empirical results of the former study seem plausible, the study suffers from the drawback of not explicitly taking into account the probably important impacts of collective habit formation and social norms. In contrast, this is done by Romme, who finds consistently significant, stable and important impacts of preference interdependence and social norms (in line with Kapteyn and Woittiez). However, Romme's specifications of his participation equation are not systematically derived from an underlying microeconomic labour supply model. Moreover, he does not distinguish the female cohorts according to civil status (married or not) and number and age of children.

As an improvement on that, Chapter 9 of the present study derives general and logit participation equations for a social group, cohort and subpopulation of married women with school-going (or small) children on the basis of the theoretical models of the previous chapters. These participation equations suggest that the participation equations of Romme contain severe specification errors. Moreover, the equations derived by us have different specifications for entry and exit behaviour as a result of hysteretic persistence effects of individual habit formation. The modelling of such effects by Clark and Summers (1982) is shown to be incorrect due to a severe aggregation enror.

The endogenous dynamics of collective habit formation and social norm beliefs according to our theoretical models turn out to imply long-run elasticities of the participation rate with respect to the wage rate and other exogenous variables which vary with the level of the participation rate according to $\mathbf{a}$ hump-shaped profile. This is shown to imply serious doubts on the empirical simulation finding of Hartog and Theeuwes (1985) of a strong and dominant impact of the own wage rate on the development of the participation rate of Dutch married women since World War II. If we make a simplifying assumption, the hump-shaped participation rate profile of the long-run elasticities is demonstrated to lead, in a regime of constant relative change rates of the exogenous variables encouraging participation, to a sigmoid shape of the time profile of the participation rate which is considerably "sharper" than the sigmoid shape of a logistic curve implied by static labour supply models like that of Hartog and Theeuwes. We call the former curve super-exponential-logistic. More adequately than the logistic curve, such a curve can account for the empirical pattern of a remarkably persistent low participation rate of Dutch married women before 1960 and a strongly growing participation rate of these women after 1960. The persistence of the low participation rate before 1960 is then partially explained by low values of long-run elasticities of exogenous variables, whereas the strong rise of the participation rate after 1960 is partially explained by higher values of such elasticities. In close connection, the persistence of the participation rate of American married women just after World War II (Clark and Summers, 1982) is explained as the result of hysteresis effects of individual habit formation. 
Such hysteresis effects are also shown to explain differences in as well as convergence of participation rates among countries.

The strong rises in the participation rate of Dutch married women after 1960 and of American married women after 1950 may have been caused by collective catastrophes within social groups. Then the long-run elasticities of the participation rate of the social group become infinite as a result of unstable preference interdependence. A necessary and sufficient condition for such a collective catastrophe in the participation rate is derived. Just as in Chapter 7, we obtain a labour supply schedule with two catastrophic domains alongside a persistent and hysteretic domain. Aggregating such schedules to the level of cohorts or (sub)populations is shown to preserve the hysteresis properties, but to "smooth out" the collective catastrophes. Because of the scarcity of disaggregated data on the level of social groups, the latter property severely hinders the identification of collective catastrophes on that level. The hysteresis properties imply that the persistence of the participation rate of American married women just after World War II can be explained not only by individual habit formation, but also by unstable preference interdependence within social groups. Finally, it is argued that the development of the participation rate of Dutch married women after World War II may be conceived in a stylized way as the result of a cultural cycle superimposed on an upward labour demand trend. The cultural cycle is caused by a combination of collective habit (and norm belief) formation in a medium long run and collective satiation in a very long nun. Since the two mechanisms can be associated with conceptions of Marx and Maslow $(1943,1970)$ respectively, the cycle is referred to as the Marx-Maslow cycle.

\subsection{Approach}

The approach which has been chosen in this study is theoretical and deductive. It can be regarded as an exploration of theoretical possibilities on the basis of non-linear dynamic extensions of the microeconomic theory of labour supply. There are several arguments in favour of such an approach.

First, on the one hand, historical evidence, casual observation and some econometric evidence (c.g., Romme) suggest that the theoretical extensions which are analysed in this study may have empirical relevance. Chapter 9 tries to make this plausible. On the other hand, the econometric evidence based on essentially (log)linear collective habit formation (and social norm) models cannot say very much on the plausibility of certain theoretical assumptions of non-linear collective habit formation models. For instance, at first sight the existence of an unstable intermediate long-run equilibrium seems in contradiction with the fact that in the empirical studies by Phlips (1978), Johnson and Pencavel (1984), Hotz et al. (1988) and Kapteyn and Woittiez (1990) the stability conditions are mostly fulfilled. However, these conditions apply to averages of (other) stable equilibria over the persons to which the data employed refer, implying that on average the stability conditions are met without excluding the existence of an unstable intermediate equilibrium. More generally, empirical testing of non-linear dynamic phenomena seems very difficult and may require the development of new estimation methods and/or data. Furthermore, it is problematic to assess in advance which out of the wide range of non-linear dynamic extensions is more plausible 
than the others. For example, in Chapter 4 it is argued on the basis of the empirical studies mentioned above that a combination of (locally) unstable individual habit formation with respect to household time and stable individual habit formation with respect to consumption has a higher chance to occur than the opposite combination. However, this is only a conjecture, so it seems premature to rule out the latter combination. More generally, it seems a sensible research strategy to construct an overview of more or less plausible non-linear dynamic extensions of the microeconomic theory of labour supply within an integrated framework with certain limits (e.g., chaos is excluded by the assumed form of the differential equations). Such a strategy also seems sensible for the following additional reasons.

When one wants to obtain a deeper insight in a non-linear dynamics of labour supply, it turns out to be very useful to explore the possible scope of such a dynamics. Moreover, such a deeper insight can lead to an integration of partial theories. A striking example from the present study is the integration of the theory of social custom of Akerlof into collective habit formation theories. More generally, an exploration of the scope of possible extensions of the microeconomic theory of labour supply can clarify a deep controversy between neo-classical economists and their critics on the scope of microeconomic models. For instance, Bürgenmeier (1991) criticizes the microeconomic theory of labour supply for its inability to account for important institutional, psychological and social aspects. However, the development of labour supply research in the last two decades has shown that such aspects can be incorporated into the microeconomic theory of labour supply to a considerable extent. In particular, the present study demonstrates that even extreme labour supply phenomena like profound preference changes can be covered by extensions of the microeconomic theory. At least to the author, the way this is done seems to do sufficient justice to the fundamental nature of these preference changes in the present context, so it may meet some objections of the critics. On the other hand, there remains a fundamental divergence of views on the validity and scope of the one-utility maximizing model (see, e.g., Etzioni, 1988, Lutz and Lux, 1988, and Bürgenmeier, 1991). In my view this model can account for the multiple and even conflicting motives which are abundant in female labour supply and applies not only to conscious (or rational) choice behaviour, but also to non-choice behaviour. However, an argumentation of this view would lead us into the problematic and extensive discussion about the concept of rationality in economics and is beyond the scope of this study. Therefore, I confine myself here to the above brief statement of my view of the scope of the one-utility maximizing model, which underlies the present study. ${ }^{9}$

The present study thus remains within the microeconomic framework of one-utility maximizing. On the other hand, it fleshes out this framework in a much broader way than is usually done by neo-classical economists. Hopefully this contributes to broadening the views of such neo-classical economists. However, I am not too optimistic about the short-run possibilities to bring the positions of the neo-classicals and their critics nearer to each other, since the polarization between them seems to be reinforced by strong sociological and psychological interests as suggested by the theoretical models of the present study (according

${ }^{9}$ Detailed arguments for and against aspects of this view can be found in a discussion by letter between Mark Lutz and the author. Copies of this discussion are available from the author. 
to the theory of social custom, motives of reputation and reduction of cognitive dissonance lead to a polarization into two extreme comer equilibria).

Notwithstanding all these advantages of the theoretical approach of the study, the theoretical models developed remain to be tested empirically. Unfortunately, in connection with the difficulties involved, this is beyond the scope of the study, but it is intended to be a subject of further research. Another possible limitation of this study might be raised by sociologists and especially psychologists who find the behavioural assumptions of the theoretical models too crude. However, they should bear in mind that these assumptions about motives on a micro level are made in order to derive their implications for the labour supply behaviour on a macro level. To allow such derivations the assumptions should be sufficiently simple, while, on the other hand, making the assumptions more complex will not often imply an essential difference in macro dynamics. The latter robustness of the macro dynamics is suggested by the mathematical theories of non-linear dynamics, in particular catastrophe theory.

An implication of the theoretical and deductive approach of this study is that the analysis tends to be rather abstract and technical. I have tried to avoid more technicalities than necessary or to relegate them to appendices, but some sections and subsections are still quite technical. For readers who are interested in "concrete results" rather than in obtaining insights into the underlying structures, I have indicated the most technical sections and subsections by an asterisk. The readers concerned are advised to skip through these sections and to focus on the main propositions. Another point worth mentioning is that the analyses often switch between speaking in terms of "she" and "he". This depends on whether the model cases considered seem more relevant for female or male labour supply.

The next chapter gives a presentation of the standard microeconomic model of individual labour supply and an overview of some extensions of this model, which are relevant for the present study. At the end of the chapter the general structure and implications of the shortrun labour supply models to be used in this study are formulated. After that chapter the study follows the outline which has been sketched in the previous sections. 


\section{CHAPTER 2}

\section{Microeconomic Theory of Labour Supply}

This chapter gives a presentation of the standard microeconomic model of individual labour supply in Section 2.1 and an overview of some important extensions of this model in Section 2.2. The latter section discusses especially extensions which are relevant in the context of the present study. Finally, Section 2.3 formulates the general structure and comparative statics of the short-run labour supply models to be used in this study.

\subsection{The standard model}

In the standard microeconomic model of individual labour supply ${ }^{1}$ a person is viewed as selling from his (or her) total time endowment $T$ of twenty-four hours a day a part as labour time $H$ against an exogenous price equal to the hourly wage rate $w$ and as consuming the remaining time $L$ as leisure. The sale of $H$ results in a total income

$$
Y=w H+Y_{O} \text {. }
$$

where $Y_{O}$ is exogenous other than labour income, e.g. property income ${ }^{2}$. The total income $Y$ is planned to be spent on quantities of consumption goods $X_{1}, \ldots, X_{n}$ with exogenous prices $p_{1}, \ldots, p_{n}$, taking into account the income-budget constraint

$$
\sum_{i=1}^{n} p_{i} X_{i} \leq Y
$$

This constraint can be combined with (2.1) into the constraint

1 See, e.g., Deaton and Muellbauer (1980, Secs. 4.I and 11.1), Killingsworth (1983, Ch. 1). Pencavel (1986, Sec. 3.1) and Varian (1987, Ch. 10).

${ }^{2}$ This $Y_{O}$ may also contain (non-wage) transfer payments in as far as these are exogenous, so independent of other than transfer income. Most transfers, such as social security benefits, do depend on other income and require an extension of the standard model (see Subsec. 2.2.3). A possible exception is a base income provided by the govemment to everybody. 


$$
\sum_{i=1}^{n} p_{i} X_{i} \leq w H+Y_{O} .
$$

In planning his labour supply, the individual is assumed to maximize a utility function $U^{o}\left(X_{1}, \ldots, X_{n}, L\right)$ subject to the income-budget constraint (2.3) and a time-budget constraint

$$
H+L=T \text {. }
$$

The constraint (2.4) implies $H=T-L$, and substituting this into the constraint (2.3) we obtain one overall constraint in the $\left(X_{1}, \ldots, X_{n}, L\right)$ space, which can be written as

$$
\sum_{i=1}^{n} p_{i} X_{i}+w L \leq w T+Y_{O}
$$

This is an interesting equation. Interpreting leisure as a consumption good and its opportunify cost per time unit in terms of forgone wage income as its price, we have on the left-hand side of (2.5) the total "expenditures" on the consumption goods $X_{1}, \ldots, X_{n}$ and $L$, and on the right-hand side the maximum income attainable when all time is devoted to (paid) work. The latter income is sometimes called full income (Becker, 1965) or also implicit income (see Varian, 1987). It measures the market value of the consumer's endowments of own time and other income ${ }^{3}$. The terms on the left show that full income is spent in part directly on market goods $X_{1}, \ldots, X_{n}$ and in part indirectly on the time $L$ used to generate utility rather than earnings (Becker, 1981, p. 6).

An advantage of this approach is that it allows treating labour supply behaviour as similar to consumption behaviour. Just as in the latter case, it is convenient to reduce the utility maximization problem in terms of the $n+1$ choice variables $X_{1}, \ldots, X_{n}$ and $L$ to a problem with two choice variables, viz. $L$ on the one hand and an aggregate of all other goods $X_{1}, \ldots, X_{n}$ on the other hand. According to the composite commodity theorem of Hicks $(1936)^{4}$ this can be done in the following way. Assume that the prices $p_{1}, \ldots, p_{n}$ bear some fixed ratio $p$ to some base period prices $p_{01}, \ldots, p_{0 n}$, that is $p_{i}=p p_{0 i}, i=1, \ldots, n$, where $p$ may vary with time, but is common to all prices so that the relative prices $p_{i} / p_{j}, j \neq i$, remain fixed at $p_{0 i} / p_{0 j}$. We can then write the expenditures on $X_{1}, \ldots, X_{n}$ as

$$
\sum_{i=1}^{n} p_{i} X_{i}=p \sum_{i=1}^{n} p_{0 i} X_{i} \equiv p X
$$

where $X$ may be considered as a composite good quantity with price $p \cdot \cdot^{5}$ Moreover, the utility maximization problem can be split up in the following two steps. First, $U^{o}\left(X_{1}, \ldots, X_{n}, L\right)$ is maximized with respect to $X_{1}, \ldots, X_{n}$ under the constraint formed by the definition of $X$ in (2.6) for each relevant $X$ and $L$. This yields a maximal utility value for each relevant $X$

\footnotetext{
${ }^{3}$ This other income can be considered as the market value of an endowment of consumption goods (see Varian, 1987, p. 17I).

4 An essentially similar result was preved by Leontief (1936). See for medem expositions of the theorem Deaton and Muellbauer (1980, pp. 120-122), and Varian (1984, p. 147).

${ }^{5}$ Note from (2.6) that $p$ can be regarded as a Paasche price index.
} 
and $L$, and hence an indirect or "derived" utility function $U(X, L)$. Secondly, $U(X, L)$ is maximized under the remaining overall constraint in the $(X, L)$ space, which is obtained by substituting (2.6) into $(2.5)^{6}$ :

$$
p X+w L \leq w T+Y_{O} .
$$

For each possible combination of values of the exogenous variables, i.e. the price $p$, wage rate $w$ and other income $Y_{O}$, the constraint (2.7) defines a budget set in the $(X, L)$ space as indicated by, e.g., the quadrilaterals $O A B C, O A^{\prime} B C$ and $O A^{\prime \prime} B C$ in Figure 2.1. The upper frontiers of these budget sets, or budget lines $A B, A^{\prime} B$ and $A^{\prime \prime} B$ are defined by (2.7) with the equality sign $=$ instead of $\leq$. The maximum consumption level (e.g., $O A$, $O A^{\prime}$ or $O A^{\prime \prime}$ ) is attainable for $L=0$ (so that all available time is devoted to work) and is equal to the real full income $\left(\boldsymbol{w} T+Y_{O}\right) / p$. Furthermore, the consumption level $B C$, attainable for $L=T$, is equal to the real other income $Y_{O} / p$.

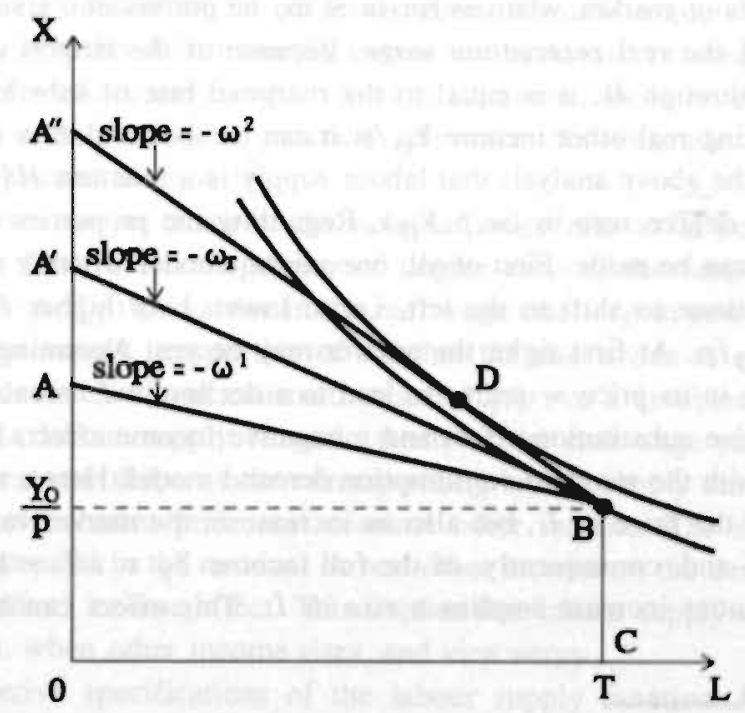

Figure 2.1 Determination of labour supply in the standard model. The bold lines are budget lines.

An optimal, i.e. utility maximizing, solution for $(X, L)$ is now obtained by confronting the budget set with indifference curves for the utility function $U(X, L)$. Usually, this utility function is assumed to be twice continuously differentiable and to describe positive marginal utilities $U_{X}$ and $U_{L}$ and positive and diminishing marginal rates of substitution $-d X / d L$ for all relevant $X$ and $L{ }^{7}$ These properties imply, successively, that the indifference curves for

${ }^{6}$ This two-stage utility maximization procedure should be distinguished carefully from the twostage utility maximization procedure in the context of two-stage budgeting (see, e.g., Deaton and Muellbauer, 1980, Secs. 5.1-5.2).

${ }^{7}$ See, e.g., Siegers (1985, Sec. II.3.3). 
$U(X, L)$ are "continuous" and even smooth (no kinks), represent increasing utility levels when moving outwards from the origin, and are strictly convex to the origin ${ }^{8}$.

As can easily be seen from Figure 2.1 for a case of strict convexity, the "increasing utility property" of the indifference curves implies that an optimal solution for $(X, L)$ is given by a point like $D$ where the budget line just touches an indifference curve. In addition, it follows from the combination of strict convexity to the origin of the indifference curves and concavity to the origin of the budget line ${ }^{9}$ that this optimal point is unique. Under these conditions two kinds of situations are possible. Both are pictured in Figure 2.1. For a sufficiently high real other income $Y_{Q} / p=B C$ and a sufficiently low real wage $w / p=\omega$, e.g. $\omega^{1}$, the optimal point is the corner solution $B$, so the person does not want to spend time on (paid) work. However, when, at constant $Y_{O} / p, \omega$ rises, the line $A B$ rotates upwards around $B$ and becomes tangent to the indifference curve through $B$ for $\omega=\omega_{r}$. When $\omega$ subsequently rises beyond $\omega_{r}$, the line becomes tangent to "higher" indifference curves at interior points left to $B$, for instance point $D$ for $\omega=\omega^{2}$. So, for $\omega>\omega_{F}$, the subject prefers to participate to the labour market, whereas for $\omega \leq \omega_{r}$, he prefers not. Usually, the "threshold wage" $\omega_{r}$ is called the real reservation wage. Because of the tangency of the line to the indifference curve through $B$, it is equal to the marginal rate of substitution $-d X / d L$ for $L=T$. For increasing real other income $Y_{O} / p$ it can be shown also to increase. ${ }^{10}$

It follows from the above analysis that labour supply is a function $H\left(w / p, Y_{0} / p\right)$, which is homogeneous of degree zero in $\left(w, p, Y_{O}\right)$. Regarding the properties of this function the following remarks can be made. First of all, one might wonder whether the interior solution for $(X, L)$ will continue to shift to the left, i.e. to lower $L$ or higher $H$, when $\omega$ further rises at constant $Y_{O} / p$. At first sight, the answer may be yes. Assuming that leisure $L$ is a normal good, a rise in its price $w$ seems to lead to a decline in $L$ because of the combined working of a negative substitution effect and a negative income effect. However, there is a crucial difference with the standard consumption demand model. Here a rise in $w$ means not only an increase of the price of $L$, but also an increase in the market value $w T$ of the total time endowment $T$ and, consequently, of the full income $Y_{F} \equiv w T+Y_{O}$. Leisure being a normal good, the latter increase implies a rise in $L$. This effect can be called a pasitive

8 In my interpretation this means that, for any pair of points on the same indifference curve, linear combinations of these points (on a straight line) in-between are lying at the other side of the indifference curve than the origin. In contrasi to what is asserted by Deaton and Muellbauer (1980, pp. 30 and 32), this includes, in my view, cases in which indifference curves join an axis of the diagram and stop there. See, for more on indifference curves, also Varian (1984, p. 113) or Maks (1981, Ch. 2, Sec. 3). The assumptions on the utility function presuppose its existence, which is based on the preference ordering axioms of reflexivity, completeness, transitivity or consistency, and continuity (see, e.g. Deaton and Muellbauer, 1980, pp. 26-28, and Varian, 1984, pp. 111-112).

${ }^{9}$ This means that intemediate linear combinations of any pair of points on the budget line are lying either on the budget line or at the same side of the budget line as the origin. Since the budget line is linear, the former situation applies. The literature usually speaks of convexity of the budget set. In the present usual case that all points between the budget line and the origin are part of the opportunity set, this is equivalent to concaviry to the origin of the budget line. I prefer to use the latter concept, since this better matches the concept of strict convexity to the origin of indifference curves.

${ }^{10}$ See Killingsworth (1983, p. 8, footnote 6). 
endowment income effect. ${ }^{11}$ The total effect of a change in $w$ on $L$ can then be decomposed according to the Slutsky equation

$$
\frac{\partial L}{\partial w}=\left.\frac{\partial L}{\partial w}\right|_{U}-\frac{\partial L}{\partial Y_{F}} L+\frac{\partial L}{\partial Y_{F}} T=\left.\frac{\partial L}{\partial w}\right|_{U}+\frac{\partial L}{\partial Y_{F}} H .
$$

From the ieft to the right, the terms in the second member of this equation represent the substitution effect (at constant utility level $U$ ), the normal income effect, and the endowment income effect. In the third member the income effect terms have been added up, yielding an always non-negative total income effect. When $H=0$, so $L=T$, this effect is zero, so an increase in $w$ will result in a pure negative substitution effect and thus a decrease in $L$ or an increase in $H$. This effect has already been found above. However, when $H$ becomes larger than zero, the total income effect becomes positive and may, for larger values of $H$, overrule the negative substitution effect. As easily seen, this is more likely to occur to the extent to which leisure is a luxury rather than a necessary good. It would imply a backward-bending labour supply curve. Whether labour supply curves are backward bending in practice and if so, for which kind of workers and in which kind of situations, are to be considered as major empirical questions and of obvious practical relevance, for example, for analysing the incentive effects of income taxation. ${ }^{12}$

The analysis above was in terms of the nominal wage rate $w$ at constant $p$ and $Y_{D}$. However, it follows from the homogeneity of degree zero of the labour supply function $H\left(w / p, Y_{O} / p\right)$ in $\left(w, p, Y_{O}\right)$ that a rise in the real wage rate $w / p$ at constant $Y_{O} / p$ has the same effect on labour supply irrespective of whether the rise is due to a rise in $w$ at constant $p$ and $Y_{O}$, a decline in $p$ at constant $w$ and $Y_{O} / p$, or a combination of these situations. ${ }^{13}$ Therefore, a labour supply curve in terms of the real wage rate at constant real other income has the same shape as the corresponding labour supply curve in terms of the nominal wage rate at constant price and nominal other income. The effect of a, ceteris paribus, increase (decrease) in the nominal or real other income on the demand for leisure is a pure positive (negative) income effect. This implies that labour supply curves will shift to the left and upwards, when other income rises, and vice versa.

If we want to derive specifications of the labour supply function $H\left(w / p, Y_{O} / p\right)$ for specific forms of the utility function $U(X, L)$, we can use, for $w / p$ larger than or equal to

${ }^{11}$ See, e.g., Deaton and Mucllbauer (1980, p. 91) and Varian (1987, p. 173).

12 See Deaton and Muellbauer (1980, p. 92). The possibility that work hours may be increased by a fall in wages and, hence, by a rise in income taxation is regarded with incredulity by some politicians, but is quite meaningful to many survey respondents (see Atkinson and Stiglitz, 1980, p. 34. footnote 5). Note that a shift of interior solutions to a comer solution with $L=0$ is implausible for most jobs.

${ }^{13}$ The total effect of a decline in $p$ at constant $w$ and $y_{O} / p$ on $L$ can be decomposed in a negative (cross) substitution effect and a positive normal income effect as well as a negative endowment income effect due to a decrease in $Y_{O}$ to keep $Y_{G} / p$ at a constant level. As easily shown, the two income effects add up to an always non-negative total income effect. Thus, we see that the total effect of a decline in $p$ at constant $w$ and $Y_{O} / p$ on $L$, and hence on $H$, has the same kind of components and has the same ambiguity of sign as a rise in $w$ at constant $p$ and $Y_{Q}$. 
the real reservation wage, the tangency condition, leading to the Second Law of Gossen for the case of labour supply

$$
\frac{U_{L}}{U_{X}}=-\frac{d X}{d L}=\frac{w}{p} .
$$

For each relevant combination of values of $w / p$ and $Y_{O} / p, L$, and hence $H$ (as well as $X$ ), can be solved from equation (2.9) in conjunction with the constraint equation (2.7). Note that a Cobb-Douglas utility function (with an elasticity of substitution equal to one) implies, for positive other income, a labour supply curve which merely slopes forward ${ }^{14}$, whereas a Leontief utility function describing perfect complementarity of consumption and leisure (a limiting case of the standard model with zero elasticity of substitution) leads, for not too large other income, to an only backward-sloping labour supply curve ${ }^{15}$. Hence, for modelling a backward-bending labour supply curve a more complex specification of the utility function is needed, e.g., a CES specification with a positive elasticity of substitution smaller than one (see Barzel and McDonald, 1973) or an additive quadratic specification as proposed by Gossen (1854) (see Sec. 4.1).

Finally, we remark that in the present situation of no full satiation, where the utility maximum is attained at the budget line, the "smaller or equal sign" $\leq$ in the income constraint (2.2) can be replaced by the equality sign $=$. From the resulting equation in combination with (2.6) it then follows that $X=Y / p$, i.e., the composite good quantity $X$ is equal to the real total income $Y / p$. This explains why total income often shows up as the other choice variable than leisure in more traditional presentations of the standard model of labour supply. ${ }^{16}$

From the exposition of the standard model of individual labour supply in the present section it may be concluded that, despite its simplicity, the model is rather powerful as well as flexible. In particular, it explains the existence of a positive reservation wage threshold to labour market participation and covers a broad range of possible shapes of the labour supply curve. Thereby the theoretical notion of substitution and income effects has proven to be a useful analytical construct. However, the model has, of course, also its limitations, and much recent work on labour supply has been aimed at modifying and extending the model. In the next section we will discuss some important extensions, especially those which are relevant in the context of the present study.

${ }^{14}$ See, e.g. Atkinson and Stiglitz (1980, p. 35). For zero other income the labour supply curve is vertical.

${ }^{15}$ For other income langer than or equal to a certain threshold value (which may differ from person to person), the labour supply curve is vertical at $\boldsymbol{H}=0$. The reservation wage can then be considered as infinitely high, whereas it is zero for other income below the threshold mentioned. These properties play a role in discussions on a basic income provided by the govemment for everybody.

${ }^{16}$ See, e.g., Atkinson and Stiglitz (1980, Sec. 2.2) and Siegers (1985, Sec. II.3.4.2). 


\subsection{Extensions}

\subsubsection{Scope}

The standard microeconomic model of individual labour supply can be modified or extended in several ways. In this context the distinction between utility maximizing models with a planning horizon of one period and those with a planning horizon of multiple periods is important. ${ }^{17}$ The standard model of the previous section is a one-period model. In such a model the subject can be considered as planning his labour supply for one period $t_{0}$ at the beginning of that period. However, the subject may simultaneousty, in the context of a career planning, plan a time path for his labour supply in future periods $t>t_{0}$. This could be modelled by a multiple-period or intertemporal utility maximizing model. Well-known examples of such a model are life cycle and human capital models. In these models the timing of behaviour plays an important role. ${ }^{18}$

Yet, in my view, a short-run or one-period labour supply decision tends to be taken not simultaneously with a long-run or multiple-period career planning, but after, and fitting as well as possible with such a planning. (Compare a tactic business decision within the framework of strategic planning.) This career plan can then be conceived as being given for a short-run labour supply decision. ${ }^{19}$ Thus, the short-run labour supply decisions may, to a large extent, determine actual labour supply behaviour, whereas the long-run career plans do so only through short-run decisions. As a consequence, these short-run decisions will take into account their possible impacts on future opportunities for realizing the career plan, e.g., in the case of an unemployed person, the possible negative "screening device" effects of not accepting a job offer now on future chances of getting a job ${ }^{20}$. Such anticipation in shortrun decisions on their expected long-run career effects can, to a certain extent, be incorporated into a one-period model via a job quality variable (see Subsec. 2.2.5). This study focuses on short-run labour supply decisions, and hence on corresponding one-period extensions of the standard model of labour supply. The next subsections give an overview of some of these extensions, namely those regarding imperfect information, taxes and transfers, rationings and endogenous wages, psychic income of labour time, preference variations and dynamics of state variables, and family labour supply.

17 This distinction corresponds to the distinction between static and dynamic labour supply models which is made by Killingsworth (1983). However, I do not think the latter terminology is very fortunate, for reasons to become clear in Subsection 2.2.6.

${ }^{18}$ See for surveys of multiple-period models, e.g. Deaton and Muellbauer (1980, Sec, 4.2 and Ch. 12), and, in the context of labour supply, Killingsworth (1983, Ch. 5). See for the timing aspect also Clark and Summers (1982).

${ }^{19}$ However, this does not exclude that, in the longer run, a career plan is adjusted, for instance in connection with experiences of frustration of short-run labour supply plans. In my view, as opposed to the usual practice of multiple-period utility maximizing models, long-run careeir plans tend to be not as detailed as short-run labour supply plans, since (among other reasons) otherwise the latter plans would be determined completely by the former, implying no room for a flexible response to new (labour demand) opportunities (unless long-run career plans are revised fastly).

${ }^{20}$ See, e.g., Deaton and Muellbauer (1980, p. 285), and De Neubourg (1985). 


\subsubsection{Imperfect information}

In one-period models it is usually assumed (implicitly) that the constraints which the subject takes into account, are identical to actual constraints, i.e., the subject has perfect information and foresight with respect to the (complex) combination of all these constraints. This assumption may be made more plausible by presupposing, by analogy to Marshall's "shor period" and in the spirit of Hicks, that the length of the period is such that the values of the exogenous variables are (in sufficiently good approximation) constant during the period. ${ }^{21}$ At the beginning of the next period the values of the exogenous variables are then assumed to have, in general, changed (discontinuously), implying that labour supply plans are revised.

In practice, however, the length of such a period could be very short (think of price changes!), whereas the "average period" after which labour supply plans are revised (e.g., about a year) may be much longer. ${ }^{22}$ The latter period seems the more relevant one and perfect foresight with respect to this period is, in many cases, implausible. ${ }^{23}$ Therefore, it appears appropriate to relax both the Marshallian presupposition and the assumption of perfect information and foresight. In the present theoretical context this is not a problem. We may assume that the subject plans her (average) labour supply for a period of certain length (e.g., a year) on the basis of more or less correct expectations on the existence and structure of constraints she will face and on the average (e.g., wages and prices) or total (e.g., other income) values of exogenous variables affecting these constraints. If, after the period, the constraints are perceived to have changed or expectations have turned out to be false, the expectations and, as a consequence, the short-run labour supply plan will be revised. Another reason for revising the labour supply plan may be that the person's own preferences have changed. More on these preference changes will be said in Subsection 2.2.6. In the following, when speaking about expected or perceived constraints or values of exogenous variables, I shall often, for the sake of brevity, omit the adjective "expected" or "perceived".

\subsubsection{Taxes and transfers}

A second extension of the standard model consists of incorporating taxes and transfers into the model. Taxes and transfers may affect the income constraints (2.3) and (2.7) as well as the corresponding utility functions. Here we will discuss only the effects on the incomebudget constraints. ${ }^{24}$ An important category of taxes is income taxation. Income taxes

${ }^{21}$ In the analysis of Marshall these variables are stock variables, and, in the contexi of the standard model, the real wage rate and real property income could be considered as indices of human capital and physical capital, respectively (see Killingsworth, 1983. p. 45). In general, however, we do not require that the exogenous variables are stock variables or could be interpreted as such.

${ }^{22}$ Reasons of this much longer period may be labour contracts, commitments, terms of notice and application, replanning costs and adjustment costs of behaviour.

23 This may even be the case for the Marshallian period because of imperfect information about current constraints, in particular about current values of exogenous variables affecting them. See, e.g., Deaton and Muellbauer (1980, pp. 337-338).

${ }^{24}$ See Killingsworth (1983, pp. 340-343) for a discussion of the effects of taxes and transfers on the utility function $U(X, L)$. 
impose a liability on the individual that is generally a function of earnings or gross labour income $Y_{G H}=w H$ and (different kinds of) gross other income. The latter income may contain property income as well as transfers from the government or relatives. However, these transfers are, in many cases, not exogenous, but negatively dependent on $Y_{G H}$ and exogenous kinds of gross other income $Y_{G O}$ such as property income. They can then be regarded as negative income taxes and, together with the positive income taxes, be described by a (net) tax function $t\left(Y_{G H}, Y_{G O}\right)$. This results in a total net income

$$
Y=w H+Y_{G O}-t\left(w H, Y_{G O}\right) .
$$

Substituting this expression and (2.6) into the income-budget constraint (2.2), we obtain the constraint

$$
p X \leq w H+Y_{G O}-t\left(w H, Y_{G O}\right) .
$$

For most current tax systems, the rate structure of the positive income taxes is progressive and the implicit tax rates of the negative income taxes are high and complex. The constraint (2.11) then defines a budget line which has a highly non-linear, winding shape, is nonsmooth, i.e., has kinks, and is often neither concave nor convex to the origin. This has various interesting implications ${ }^{25}$, but in the context of this study (see Sec. 2.3) it suffices to consider as an example the impacts of one special and fairly simple negative income tax, which is given by

$$
\begin{array}{ll}
t\left(w H, Y_{G O}\right)=-\left(Y_{M}-w H-Y_{G O}\right), & \text { if } w H+Y_{G O} \leq Y_{M}, \\
t\left(w H, Y_{G O}\right)=0, & \text { if } w H+Y_{G O} \geq Y_{M} .
\end{array}
$$

where $Y_{M}$ is a guaranteed minimum income and where (2.12a) implies an implicit tax rate of 100 percent. ${ }^{26}$ Assuming $Y_{G O}<Y_{M}$, both the transfer regime defined by (2.12a) and the no-transfer regime defined by $(2.12 \mathrm{~b})$ can occur for particular values of the wage rate $\boldsymbol{w}$. Substituting the tax function for these regimes into (2.10) leads to the non-linear constraint

$$
\begin{array}{ll}
p X \leq Y_{M}, & \text { if } w H+Y_{G O} \leq Y_{M} . \\
p X \leq w H+Y_{G O}, & \text { if } w H+Y_{G O} \geq Y_{M} .
\end{array}
$$

${ }^{25}$ See, e.g., Killingsworth (1983, Ch. 6) and Atkinson and Stiglitz (1980, Lecture 2) for surveys. In order to analyse the effects of such a non-linear constraint, it is often convenient to linearize it around optimal points (see, e.g. Killingsworth, 1983, Ch. 6). The slope of the resulting linearized budget constraint is equal to the real after-tax marginal wage rate and varies with hours supplied. This makes the after-tax marginal wage rate endogenous. However, in the theoretical context of this study it is not necessary to pursue these linearizations.

${ }^{26}$ This negative income tax is a special case of more general transfer schedules the implications of which for labour supply are analysed by, e.g. Hanoch and Honig (1978) and Killingsworth (1983. Sec. 6.3). Hanoch and Honig give also an illustration for a Cobb-Douglas utility function. 
Resulting budget lines are depicted in Figure 2.2. They have a kink at the point $K^{1}, K_{r}$ or $K^{2}$, respectively, where the constraint switches from the transfer regime to the right to the no-transfer regime to the left.

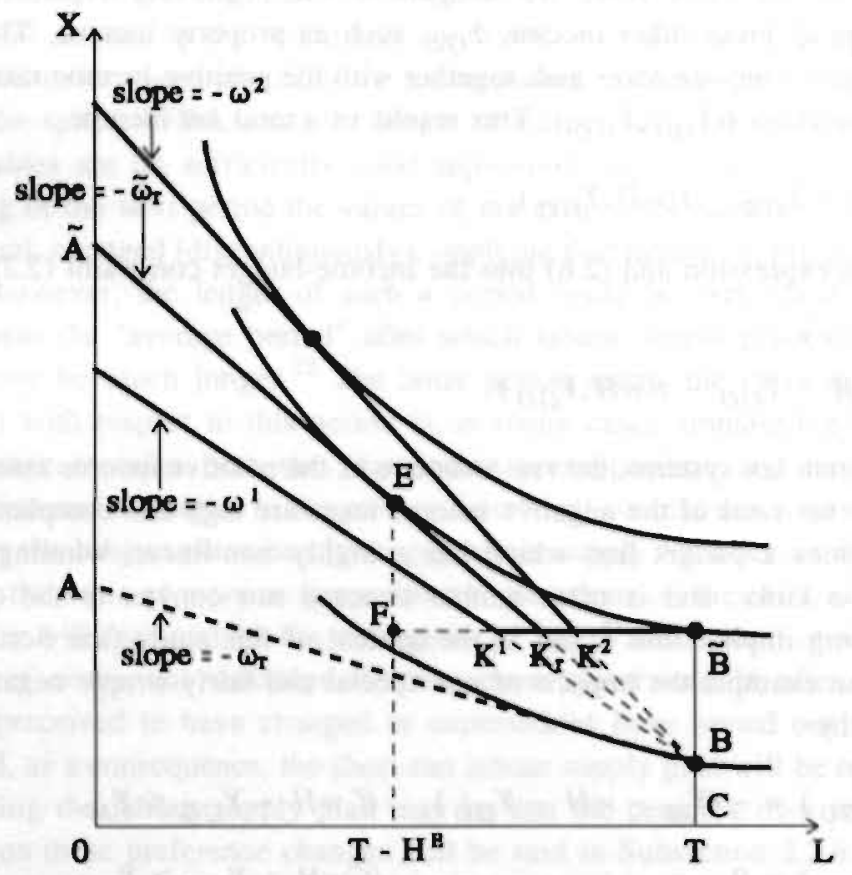

Figure 2.2 Determination of labour supply under a negative income tax of 100 percent. The solid bold lines indicate non-concave budget lines and the dashed bold line is a standard budget line without the negative income tax.

Analogously to the standard model, there is for a sufficiently high real minimum income $Y_{M} / p\left(\bar{B} C\right.$ in Fig. 2.2) and a sufficiently low real wage $w / p \equiv \omega$, e.g. $\omega^{1}$, a corner solution $\bar{B}$ with no participation. However, when, at constant $Y_{G O} / p(B C$ in Fig. 2.2) and constant $Y_{M} / p$, $\omega$ rises, the no-transfer segment of the budget line rotates around the point $B$ and becomes, for $\omega=\widehat{\omega}_{r}$, tangent to the indifference curve through $\vec{B}$ not at $\vec{B}$, but at an interior point $E$. Thus, as a consequence of the budget line being not concave to the ori$\operatorname{gin}^{27}$. we have here a situation with two optimal points. Moreover, the transfer segment of the budget line can never be tangent to an indifference curve, since its slope is always zero, whereas the slopes of the indifference curves, i.e. minus the marginal rates of substitution, are assumed to be negative (see Sec. 2.1 ). ${ }^{28}$ Consequently, there are no interior solutions on the transfer segment of the budget line and there is no comer solution $\tilde{B}$ with tangency

${ }^{27}$ In the literature this is usually referred to as nonconvexity of the budget set. See footnote 9.

28 Note that this impossibility of tangency is typical of an implicit tax rate of 100 percent, since for an implicit tax rate of less than 100 percent the slope of the no-transfer segment of the budget lire is negative for positive real wages. 
of the segment to the indifference curve through $\bar{B}$. For real wage levels above $\omega_{r}$ there is one optimal point on the no-transfer segment of the budget line, implying that $\omega_{r}$ is the real reservation wage $\mathrm{e}^{29}$ and that above this wage the labour supply curve is the same as in the standard model. We may then have a backwand-bending labour supply curve as in Figure 2.3 with a higher reservation wage than in the standard case and a discontinuous jump of hours supplied at that wage.

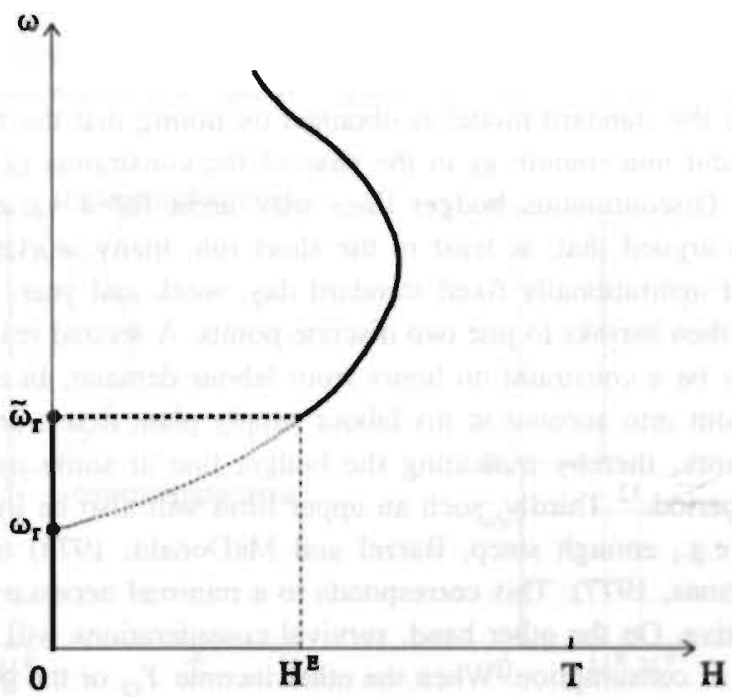

Figure 2.3 Backward-bending labour supply curve under a negative income tax of 100 percent (bold). $\omega_{r}=$ real reservation wage without negative income tax. $\omega_{r}=$ real reservation wage with negative income tax.

Interesting questions are $(i)$ what determines the size of the increase $\omega_{r}-\omega_{r}$ in the reservation wage due to the introduction of the transfer scheme (A $\bar{A} / T$ in Fig. 2.2) and (ii) what determines the size of the positive difference between the total income $\sigma_{r} H^{E}+Y_{G O} / p$ for which people just want to work, and the guaranteed minimum income $Y_{M} / p$ (EF in Fig. 2.2). Regarding question (i) Figure 2.2 suggests that the increase in the reservation wage is larger as the guaranteed minimum income is higher and as the indifference curves are more bowed, or equivalently, as consumption is less substitutable for leisure. ${ }^{30}$ Moreover, Figure 2.2 also suggests that such a relation may hold with respect to question (ii), provided a higher reservation wage implies a lower optimal $L$, i.e., provided the discontinuity in the

${ }^{29}$ Note that this reservation wage is not, as in the standard model, determined as the marginal rate of substitution for $H=0$, but as the wage for which the maximal utility leve! of working without transfer $(E)$ is equal to the utility level of not working with transfer (E). Thus, in this case of a budget line which is not concave to the origin, a local marginal utility analysis around $H=0$ is not sufficient to investigate the labour force participation decision, but a globai utility comparison analysis is also necessary (see Hausman, 1980, Sec. 2).

${ }^{30}$ Cf. Killingsworth (1983, pp. 364-365). 
labour supply curve occurs, as in Figure 2.3, in a forward-sloping part of it. ${ }^{31}$

Of course, a negative income tax is usually financed by a positive income tax. This lowers the no-transfer segment of the budget line, implying an even higher reservation wage. A negative income tax could also be financed by a tax on consumption prices. It can easily be seen from (2.6) and (2.11) that, if this tax is a uniform percentage of all consumption prices, it has the same effects as a corresponding proportional positive income tax.

\subsubsection{Rationings and endogenous wages}

A third extension of the standard model is obtained by noting that the budget line may not only be non-linear and non-smooth as in the case of the constraints (2.11) and (2.13), but also discontinuous. Discontinuous budget lines may arise for a variety of reasons. For example, it is often argued that, at least in the short run, many workers can only choose between working an institutionally fixed standard day, week and year, and not working at all. The budget line then shrinks to just two discrete points. A second reason of a discontinuous budget line may be a constraint on hours from labour demand. In as far as the subject takes such a constraint into account in his labour supply plan, it sets an upper limit on his planned hours of work, thereby truncating the budget line at some maximum number of working hours per period. ${ }^{32}$ Thirdly, such an upper limit will also be imposed by considerations of survival (e.g., enough sleep, Barzel and McDonald, 1973) or the health of the labour supplier (Parsons, 1977). This corresponds to a minimal necessary number of leisure hours which is positive. On the other hand, survival considerations will also dictate a minimal necessary level of consumption. When the other income $Y_{O}$ or the guaranteed minimum income $Y_{M}$ divided by $p$ is smaller than this level, it implies a positive under limit on planned working hours which is inversely proportional to the wage rate $w$.

Killingsworth (1983, Sec. 2.4) distinguishes two kinds of approaches to these discontinuous budget lines, namely disequilibrium and equilibrium approaches. In disequilibrium analyses the discontinuous constraints are treated as exogenously given "rationings" of labour supply. Assuming that such a rationing is taken into account in the labour supply plan of the subject, it will, for example in the case of a two-point budget line, typically lead to either non-participation with a higher reservation wage than without the rationing, or overemployment, or underemployment. ${ }^{33}$ This is illustrated for the case of a backward-bending labour supply curve under a negative income tax of 100 percent (Fig. 2.3) in Figures 2.4a and $2.4 \mathrm{~b}$. In Figure $2.4 \mathrm{a}$ the labour supply ration $H^{k}$ falls outside the discontinuity in the original labour supply curve, whereas in Figure $2.4 \mathrm{~b}$ it is within this discontinuity. This results in different rationed labour supply schedules with no overemployment regime in the

${ }^{31}$ It would be interesting to investigate these questions for the case of a CES utility function, but this leads to complex analytical problems which are beyond the scope of this thesis (cf. Hanoch and Honig. 1978. pp. 11-14, for the case of a Cobb-Douglas utility function).

32 Strictly spoken, the resulting budget line is not discontinuous, but, following Killingsworth (1983, p. 47), we regard it as such.

${ }^{33}$ See, e.g. Deaton and Muellbauer (1980, pp. 283-284) and Killingsworth (1983, pp. 48-52). The resuits are not based on a marginal utility analysis, but on a utility comparison analysis as in the case of non-concave budget lines (see footnote 29). 
case of Figure 2.4b. As can be deduced from Figure 2.2, the reservation wages $\omega_{r}$ are higher than the reservation wage $\omega_{r}$ without the rationing, since the rationed values $H^{R}$ of $H$ are not equal to its optimal value $H^{E}$ at $\omega_{r}$, and hence, at and in some interval above $\omega_{r}$ yield lower utility than the non-participation value 0 of $H$ at $B$. At $\omega_{r}$ the utility of $H^{R}$ has become equal to the utility of non-participation, leading to a discontinuous jump from $H=0$ to $H=H^{R}$ for $\omega>\hat{\omega}_{r}$. In the case of Figure 2.4a the latter rationed labour supply can be argued to be initially larger and for higher $w$ smaller than the optimal $H$ on the standard labour supply curve. This implies successively overemployment and underemployment, whereas in the case of Figure $2.4 \mathrm{~b}$ there is underemployment only. ${ }^{34}$

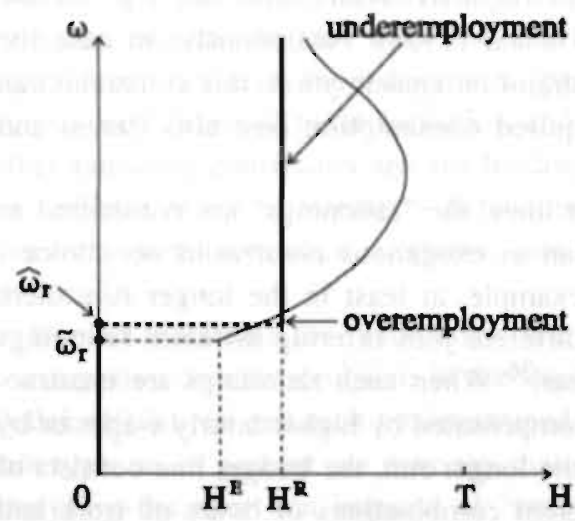

Case

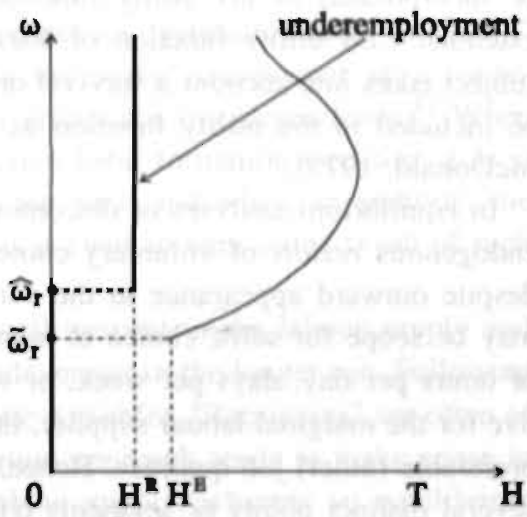

Case b

Figure 2.4 Labour supply schedules under a negative income tax of 100 percent and a two-points labour supply rationing at $H=0$ and $H=H^{R}$ (bold lines). $\omega_{r}=$ real reservation wage without rationing. $\omega_{r}=$ real reservation wage with rationing.

In the case of an upper limit on hours worked from labour demand, usually the disequilibrium theory of, among others. Clower (1965) is applied. ${ }^{35}$ However, in this theory ex ante effective labour supply plans take into account, by definition, consumption demand rationings (spill-overs), but not labour supply rationings, whereas the latter rationings affect ex ante effective consumption demand plans. Only ex post a labour supply rationing affects realized labour supply and makes it equal to the minimum of effective labour supply and demand. When the effective labour supply and demand curves are continuous, this seems a plausible procedure, but when the effective labour supply curve is discontinuous and the effective labour demand curve crosses the discontinuity, it tums out to be incorrect. For example, in the case where the effective labour demand is inelastic and is given by the

${ }^{34}$ See, for an analysis of labour supply decisions under a social security system with a three-point budget line and a CES utility function, Zabalza et al. (1980).

35 See, e.g., Ashenfelter (1977), Deaton and Muellbauer (1980, pp. 290-293) and Killingsworth (1983, pp. 53-56). 
vertical rationing line in Figure 2.4b, realized labour supply can be shown to be given by the rationed labour supply schedule drawn, and, as a consequence of the reservation wage being raised by the rationing, this labour supply schedule clearly does not satisfy the minimum condition. The fundamental reason of this phenomenon is that, at and in some interval above the original reservation wage, the rationed values of $H$ yield lower utility than the nonparticipation value 0 of $H$ (implying that "something is not better than nothing"), whereas the minimum condition implicitly assumes that higher values of the decision variable yield higher utility (implying that "something is better than nothing").

In the case of an upper limit on hours worked from a survival or health constraint the same analysis can be applied. When the subject takes such a constraint into account, it can be incorporated in his utility function as minimal required leisure time as, e.g., in the extended CES utility function of Barzel and McDonald (1973). Analogously, in case the subject takes into account a survival or health constraint on consumption, this constraint can be included in the utility function as minimal required consumption (see also Barzel and McDonald, 1973).

In equilibrium analyses of discontinuous budget lines, the "rationings" are considered as endogenous results of voluntary choices rather than as exogenous constraints on choice despite outward appearance to the contrary. For example, at least in the longer run, there may be scope for some choice of hours between different jobs offering different rationings of hours per day, days per week, or weeks per year. ${ }^{36}$ When such rationings are unattractive for the marginal labour supplier, they will be compensated by higher hourly wages or by preferable (other) job qualities. Hence, at least in the longer run, the budget line consists of several distinct points or segments reflecting different combinations of hours of work and wage rates. Models of optimal employment contracts suggest that also the variability of the wage rate over time and, in relation to that, the probability of unemployment during periods of low product demand may be part of the job package that workers "buy" in exchange for labour time, when they take a particular type of job with a particular type of firm. Furthermore, a full-fledged analysis should include the effects of different job qualities such as working conditions. We will come back to this in the next subsection.

This example of endogenous "rationings" suggests that in a market cross section the hourly wage rates reflect to some extent the other attributes of the job packages of which they are a part. In that case the wage rate is a function of the other job attributes, which forms a second fundamental reason besides income taxation and overtime payment for treating the (after-tax marginal) wage rate as endogenous in cross-section studies of the supply of hours (and which could be considered as a fourth extension of the standard model). ${ }^{37}$ Moreover, at least in the longer run, these job attributes or, equivalently. job types are choice variables. As indicated above, such a choice variable may be the rationing of hours worked such as implied by full-time and part-time work. ${ }^{38}$ Furthermore, (com-

${ }^{36}$ See Deaton and Muellbauer (1980, pp. 286-287) and Killingsworth (1983. pp. 58-56).

${ }^{37}$ See, e.g. Deaton and Muellbauer (1980, pp. 286-287) and Killingsworth (1983, pp. 21-23).

${ }^{38}$ In that case the conventional substitution and income effects do not apply (see Killingsworth, 1983. pp. 65-66). See for recent empirical applications, for instance, Tummers and Woittiez (1988) and Van Soest et al. (1989). 
binations of) rationings and wage rates and, more generally, job packages are not given once and for all for every job, but are, at least in the longer run, subject to contract negotiations by labour suppliers themselves or trade unions (e.g., reduction of working hours and contract wages) or are subject to legislation (e.g., the standard working week and measures on wages). Trade unions as well as the legislator can then be expected to take some account of the preferences of individual labour suppliers (as well as of the employer's labour demands). ${ }^{39}$

Finally, in the case of survival or health constraints on hours worked from minimal required leisure and consumption, the person may choose either to respect such constraints or not to respect them and consequently to get ill or to starve ${ }^{40}$. More importantly, the minimal required leisure and consumption may be larger than those dictated by the survival or health constraints: they may not only contain an exogenous "physiologically necessary" component, but also an endogenous "psychologically necessary" component as a consequence of individual habit formation and behavioural preference interdependence. ${ }^{41}$ When other rationing constraints are not binding, this collective habit formation (see Sec. 1.3) is the indirect result of voluntary behaviour choices in the past, and when, in addition, the individual habit formation is rational (see Sec. 1.1), this is even an anticipated result of such choices. $^{42}$

In such situations the "rationings" are exogenous with respect to the labour supply and consumption demand decisions in the short run, but endogenous in the longer run. Following Pollak (1978), we call such predetermined variables state variables. "Rationings" are often of this type, so in general we may say that a disequilibrium approach tends to make sense in analysing the short-run impacts of "rationings" on labour supply, whereas an equilibrium approach is often more adequate in an analysis of the long-run impacts of "rationings" on labour supply.

\subsubsection{Psychic income of labour time}

In many cases a job is chosen not only because of its combination of rationing of hours worked and wage rate, but also because of other attributes, i.e. job qualities such as working conditions and career perspectives. Preferable job qualities yield by definition higher utility, but this is not accounted for by the standard model, since the utility function in this model depends only on consumption $X$ and leisure $L$. Therefore, in a fifth extension of the standard model, the utility function should depend also on a vector of one or more job quality variables $q_{H}$ and, since more labour time $H$ is likely to increase the effects on utility of many of these quality variables, on $H^{43}$ Thus, we obtain a utility function

${ }^{39}$ See Atkinson and Stiglitz. (1980, p. 44 and Lecture 10).

${ }^{40}$ Compare a hunger strike.

41 See Pollak (1970, p. 749). Phlips (1978), Deaton and Muellbauer (1980, pp. 374-375), Johnson and Pencavel (1984) and Kapteyn and Woittiez (1990).

42 See Subsection 2.2.6, Section 4.6 and Subsection 5.1.3 for more details.

${ }^{43}$ Sec, e.g., Woittiez and Kapteyn (1987, p. 22) for the case of dirty versus clean jobs. 
$\tilde{U}\left(X, L, H, q_{H}\right)$, where the total effect of $H$ and $q_{H}$ on $\tilde{U}$ is usually associated with "psychic income" of labour time. ${ }^{44}$

Such a utility function can be relevant in several kinds of choice situation. First, in job choice models $X, L$ and $H$ and one or more components of $q_{H}$ may all be treated as simultaneous (and interdependent) choice variables. ${ }^{45}$ The budget line could then be taken as continuous with respect to $X, L$ and $H^{46}$, but, as we have seen in the previous subsection, for a given job the labour time $H$ may, at least in the short run, also be rationed at one fixed value $H^{R}$. Job package combinations of the hourly wage rate $w$, rationed labour time $H^{R}$ and job qualities $q_{H}$ can then be considered as discrete choice alternatives. Moreover, the alternative of non-participation with $H^{R}=0$ and no $w$ and $q_{H}$ could be added.

In a second situation the job a labour supplier has or, more generally, the job qualities $q_{H}$ are given in the short run because of their attractivity, job rationing by labour demand or search and moving $\operatorname{costs}^{47}$, whereas there is some scope for varying the labour time, e.g., for working less days per week. In that case $X, L$ and $H$ are the (interdependent) choice variables, whereas $q_{H}$ is exogenous with respect to the short-run choice. On the other hand, $q_{H}$ may be endogenous in the longer run, e.g., as a result of job choice, so in this choice situation $q_{H}$ is a state variable in the sense of Pollak (1978).

In a third, but related choice situation the job qualities $q_{H}$ are not given in the short run, but they vary with the labour time $H$ in a way which is given in the short run. For example, available full-time jobs may have more attractive job qualities (such as better career perspectives) than part-time jobs. This might explain why in the Netherlands many women holding a full-time job would like to work part-time, but nevertheless do not quit their current jobs (Allaart et al, 1989,1991$)$. In such a situation the job qualities $q_{H}$ are functions $q_{H}(H, \alpha)$ of $H$ with parameter(s) $\alpha$ which are exogenously given in the short run. In a longer run, however, these parameters may be endogenous, so they can be regarded as state variables (instead of the job qualities themselves in the second choice situation above).

Substituting the functions $q_{H}(H, \alpha)$ in the above utility function $\hat{U}\left(X, L, H, q_{H}\right)$ yields a utility function $\hat{U}(X, L, H ; \alpha)$, where "; " denotes that $\alpha$ is given in the short run. Thus, in this as well as the second choice situation short-run labour supply is described as the result of maximizing a utility function $\hat{V}(X, L, H)$ for given values of the state variable(s) ( $\alpha$ or $q_{I I}$, respectively). In comparison with a standard utility function $U(X, L)$ such a utility function has interesting implications, which can be derived as follows.

Substituting $H=T-L$ into the utility function $\hat{U}(X, L, H)$, we obtain a reduced utility function $U(X, L)$. This, however, is not a standard utility function. As easily seen, its marginal utility $U_{L}$ is related to the marginal utilities $\hat{V}_{L}$ and $\hat{U}_{H}$ of the original utility function as $U_{L}=U_{L}-U_{H}$. We call $U_{L}$ the net marginal utility or net "psychic income" of $\mathrm{H}$. At

44 See, e.g.. Becker (1965, p. 498), Hamermesh (1974), Lucas (1977), Goedhant and Kapteyn (1978), Van de Stadt (1981) and Siebert (1985). Such utility of labour time was already recogrized in the previous century by the early marginalists Gossen, Jevons and Marshall (see, e.g., Siegers, 1985. Sec. II.3.2).
45 See, e.g.v Woittiez and Kapteyn (1987).
46 Sce, e.g., Woittiez and Kapteyn (1987).
47 See Deaton and Muellbauer (1980, p. 286). 
diminishing marginal utilities ${ }^{48}$ of $L$ and $H, \hat{O}_{L}$ may be smaller than $\hat{O}_{H}$ for sufficiently high $L$ and hence sufficiently low $H$, implying that $U_{L}$ is negative (oversatiation with respect to $L$ ). In the usual case of a positive marginal utility $U_{X}=\hat{U}_{X}$ of consumption, this implies a negative marginal rate of substitution $-d X / d L$ (see (2.9)) for sufficiently high $L$, and hence indifference curves like that in Figure 2.5 (see Ehrenberg and Smith, 1982, pp. 170-171). These indifference curves are assumed to be smooth and strictly convex to the origin as in the standard case and to represent increasing utility levels when moving upwards from the $L$-axis (now not from the origin).

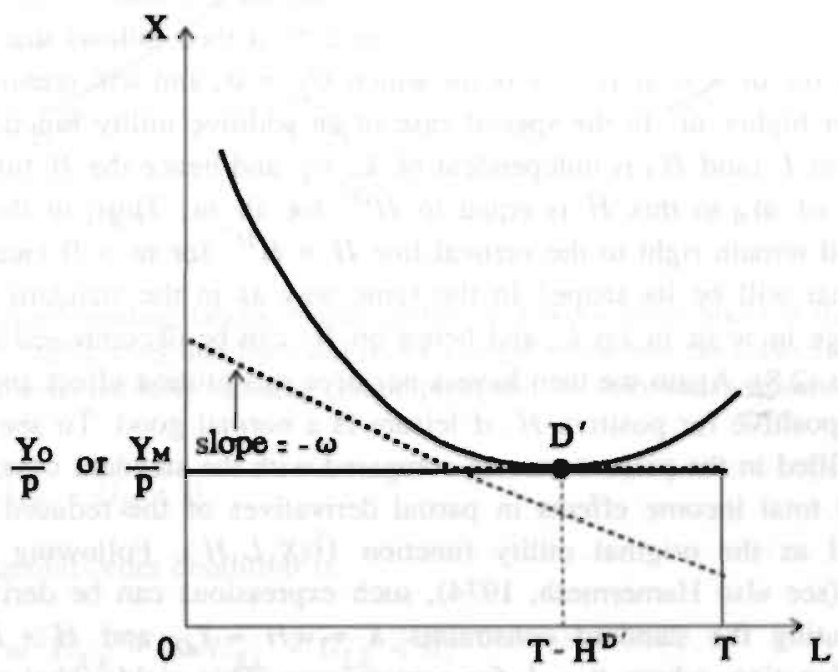

Figure 2.5 Determination of labour supply of a work lover under a standard constraint for $\omega=0$ (solid bold line) and under a negative income tax of 100 percent for low 0 (dashed bold line).

Confronting such indifference curves with standard budget lines, we obtain for zero wage rate $w$ (and positive other income $Y_{O}$ ) with a horizontal budget line an interior optimal point $D^{\prime}$ with $H^{D^{\prime}}>0$. This describes (unpaid) volunteer work. Moreover, the real reservation wage as being equal to the marginal rate of substitution for $L=T$ is negative, i.e., the subject is prepared to give up money income in exchange of the positive net psychic

${ }^{48}$ Strictly spoken, a derivation of labour supply requires only ordinal utility, for which the concept of diminishing marginal utility has no meaning. However, is make interpretations easier we regard the utility function as cardinal, so that we can speak in terms of diminishing marginal utilities. 
income of labour time $H^{49}$ Similar results are obtained for the budget lines in Figure 2.2, describing a 100 percent negative income tax as defined by (2.12). For zero or sufficiently low real wage $\omega$ we have a unique interior optimal point $D^{\prime}$ with $H^{D^{\prime}}>0$ on the transfer segment of the budget line. This describes work while retaining full or partial unemployment benefits. ${ }^{50}$

How will labour supply curves look in these cases? First consider the case without the negative income tax. According to (2.9) the first-order condition for an interior optimum is then given by $U_{L}=\omega U_{X}$. This implies $U_{L}=0$ for $\omega=0$ and, in the usual case where $U_{X}>0, U_{L}>0$ for $\omega>0$. Assuming $U_{L L}<0$ for all $L$ (which is consistent with the diminishing marginal rates of substitution in Figure 2.5), it then follows that a labour supply curve will begin for $\omega=0$ at $H^{D^{\prime}}>0$ for which $U_{L}=0$, and will remain right to the $H$ with $U_{L}=0$ for higher $\omega$. In the special case of an additive utility function, so when the marginal utility of $L$ (and $H$ ) is independent of $X, U_{L}$ and hence the $H$ for which $U_{L}=0$ are independent of $\omega$, so this $H$ is equal to $H^{D^{\prime}}$ for all $\omega$. Thus, in this case a labour supply curve will remain right to the vertical line $H=H^{D^{\prime}}$ for $\omega>0$ (see Fig. 2.6a).

However, what will be its shape? In the same way as in the standard model the total effect of a change in $w$ or $\omega$ on $L$, and hence on $H$, can be decomposed according to the Slutsky equation (2.8). Again we then have a negative substitution effect and a total income effect which is positive for positive $H$, if leisure is a normal good. To see when the latter condition is fulfilled in the present case as compared with the standard case, we express the substitution and total income effects in partial derivatives of the reduced utility function $U(X, L)$ as well as the original utility function $\hat{U}(X, L, H)$. Following Henderson and Quandt (1958) (see also Hamermesh, 1974), such expressions can be derived in a simple way by substituting the standard constraints $X=w H+Y_{O}$ and $H+L=T$ into the reduced utility function, where $p=1$ for convenience. This yields what we call the constrained utility function

$$
U^{c}\left(H ; w, Y_{O}, T\right)=U\left(w H+Y_{O}, T-H\right) .
$$

This utility function is maximized with respect to $H$ for given $w$ and $Y_{O}$. For an interior maximum the first-order condition is

${ }^{49}$ In practice, however, people will at most be prepared to make little costs like travel cosis for volunteering, since they would consider more costs as very unjust. This can be described by a strong suppressing effect of a negative ral wage income $\omega H$ on the "psychic income" of $H$, making the indifference curve in Figun 2.5 almost flat at the right of point $\boldsymbol{B}$ for negative $\omega$. More generally. "psychic income" of $H$ is likely to depend heavily on $\omega H$ and hence on $\omega$. since real wage income is perceived as a measure of the social value and status of a persori's labour (see also Sec. 8.2). The resulting wage dependence of labour supply preferences is similar to price-dependence of consumption preferences (see, e.g., Leibenstein, 1950, and Pollak, 1977) and may lead to "conspicuous eamings" in analogy to conspicuous consumption (Veblen, 1899).

${ }^{50}$ Ehrenberg and Smith (1982) call persons who do so "workaholics", even when they work only a few hours a week. This representation of "workaholics" does not seem very adequate. A more dynamic model of the behaviour of "workaholics" is presented in Chapter 3. 


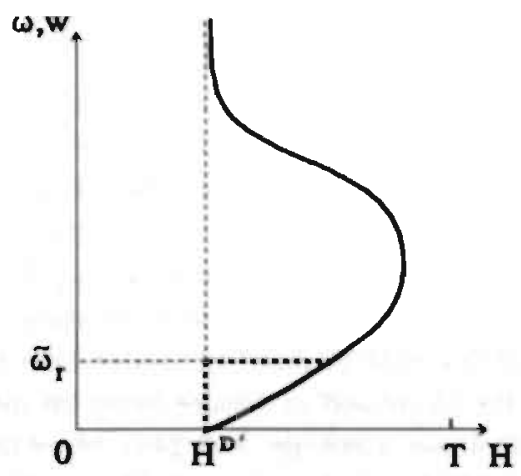

(a)

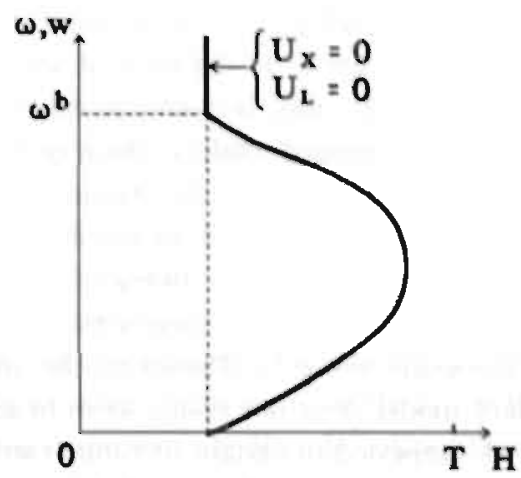

(b)

Figure 2.6 Backward-bending labour supply curves of a work lover under a standard constraint (solid bold curves and line) and under a negative income tax (also dashed bold line in (a)) for (a) not fully satiating consumption and (b) fully satiating consumption.

$$
U_{H}^{c}=w U_{X}-U_{L}=0
$$

(or (2.9)). The second-order condition is

$$
U_{H H}^{c}=w^{2} U_{X X}-2 w U_{X L}+U_{L L}<0 .
$$

Differentiating the first-order condition with respect to $w$ yields $U_{H w}^{c}+U_{H H}^{c} H_{w}=0$ (see Varian, 1984, p. 328) or, equivalently, since $U_{H H}^{c}=-\left|U_{H H}^{c}\right| \neq 0$ by virtue of (2.16),

$$
H_{w}=\frac{U_{H w}^{c}}{\left|U_{H H}^{c}\right|}=\frac{U_{X}+\left(w U_{X X}-U_{L X}\right) H}{\left|w^{2} U_{X X}-2 w U_{X L}+U_{L L}\right|},
$$

where we substituted from (2.16). (Of course, all second-order derivatives have to be evaluated at the optimal point.) Since differentiating the time constraint with respect to $w$ yields $L_{w}=-H_{w}$, (2.17) implies an expression for the Slutsky equation (2.8).

Since, analogously $L_{Y_{F}}=-H_{Y_{F}}$ and

$$
H_{Y_{F}}=H_{Y_{O}}=\frac{U^{c}{ }_{H Y_{O}}}{\left|U_{H H}^{c}\right|}=\frac{w U_{X X}-U_{L X}}{\left|w^{2} U_{X X}-2 w U_{X L}+U_{L L}\right|} \text {, }
$$

the second term in the numerator of (2.17) corresponds to the total income effect in (2.8), and hence the first term in the numerator to the substitution effect. Usually, the substitution effect on $H$ is indeed positive in both the standard and extended model. Assuming $U_{X X}<0$ (which is consistent with the second-order condition and the diminishing marginal rates of 
substitution in Figure 2.5), the total income effect on $H$ is negative, or leisure is a normal good, if $U_{L X}>w U_{X X}$, i.e., if leisure and consumption are not too strong (net) substitutes in the Edgeworth sense. In the case of an additive utility function with $U_{L X}=0$, for which Figure 2.6 holds, this is automatically true, so then there is no difference between the standard and extended models. More generally, the condition $U_{L X}>w U_{X X}$ seems plausible for sufficiently high $w$ in the standard model ${ }^{51}$, but also in the extended model, since the condition is then equivalent to the condition $\hat{U}_{L X}-\hat{U}_{H X}>w \hat{U}_{X X}$ for the original utility function and since $\hat{V}_{H X}$ is likely to be small in absolute value relative to $w \hat{U}_{X X}$ for sufficiently high consumption (corporate time and consumption seem then rather independent in the Edgeworth sense ${ }^{52}$ ). Therefore, the addition of "psychic income" of labour time to the standard model does not really seem to enhance the likelihood of leisure being an inferior good as suggested in certain literature (see, e.g., Wolfson, 1988, pp. 243-244). More importantly, just as the standard model, the extended model can describe a backward-bending labour supply curve as indicated in Figure 2.6a.

In the presence of a negative income tax this curve becomes slightly different (see also Fig. 2.6a). For $\omega$ or $w$ rising from zero $H$ remains equal to $H^{D^{\zeta}}$ until for $\omega=\bar{\omega}_{r}$ the budget line becomes tangent to the indifference curve through $D^{\prime}$ at a second interior point on the no-transfer segment like $E$ in Figure 2.2. For $\omega$ rising beyond $\tilde{\omega}_{r}$ the labour supply jumps discontinuously to the labour supply curve without negative income tax, and follows this eurve for higher $\omega$. Thus, whereas for $\omega<\tilde{\omega}_{r}$ the person prefers to work a limited number of hours while retaining full or partial unemployment benefits, she prefers for (1) $>\omega$, to work a higher number of hours without unemployment benefits. Note, however, that in this model the person does not take into account possible disadvantages of unemployment benefits in the form of stigmatization effects (see Moffitt, 1983, and Sec. 8.2). Such disadvantages would lower $\omega_{r}$.

Another interesting modification results when, just as the marginal utility of leisure, the marginal utility of consumption is allowed to become negative for high levels of consumption, describing oversatiation with respect to $X$ (see also Miller, 1988). Then we may have situations of fully satiating consumption with $U_{X}=0$ at the optimal point where the income constraint is no longer binding. The consumption and labour supply decisions are then unlinked, implying that the first-order condition for an interior optimum for $H$ and $L$ is given by $U_{L}=0$. Thus, an interior optimum with fully satiating consumption also describes fully satiating leisure and labour time. Such an interior "double bliss" optimum is impossible in an extension of the standard model with $U_{X}<0$ for high $X$, but $U_{L}>0$ for all $L$. Then an optimum with $U_{X}=0$ can occur only at a corner point for $H=0$, when the other income $Y_{O}$ is so high as to allow fully satiating consumption without work, so when $U_{X}\left(Y_{O}\right)<0$. In such a case there is no incentive to do (paid) work, no matter how high the wage rate, so the labour supply curve coincides with the vertical $H=0$ axis. This may describe the behaviour of a person living off his interest.

On the other hand, when we also allow $U_{L}<0$ for high $L$ as in the extension of this

${ }^{51} \mathrm{Cf}$. Abbott and Ashenfelter (1976).

52 For low consumption levels this does not seem to hold, since working is more pleasant with a full stomach than with an empty stomach. 
section, there may be an interior "double bliss" optimum for high $\omega$ and hence high $X$. Then the labour supply curve coincides with the $U_{L}=0$ line or curve for $\omega$ above a certain $\omega^{b}$, as in Figure 2.6b (see Sec. 4.5 for an example). This describes a fully satisfied employee who does no longer change her consumption, leisure and labour time at a rise in income, so both consumption and leisure have ceased to be normal goods, but without becoming inferior! (See Scitovsky, 1952, pp. 87-88.)

Also in this case it is possible that the other income $Y_{O}$ is so high as to allow fully satiating consumption without work, so $U_{X}\left(Y_{O}\right)<0$. Now, however, there is still an incentive to do (paid) work which stems from the derived "psychic income". On the other hand, there is no incentive to work more than the number of hours for which $U_{L}=0$, no matter how high the wage rate, so the labour supply curve coincides with the $U_{L}=0$ line or curve. In the case of additive utility functions this is the vertical $H=H^{D^{\prime}}$ line. This may describe the behaviour of a millionaire who works for fun.

However, fully satiating consumption implies that the surplus of income will be saved, and usually such savings will have some utility. This can be taken into account to a certain extent (see below) by including savings as delayed consumption into $X$, but then full satiation with respect to $X$ becomes less plausible. This brings us back from the labour supply curve of Figure 2.6b to that of Figure 2.6a. On the other hand, the former labour supply curve may still serve as an "idealtypical" and interesting approximation of the latter curve. Other implications of savings are that they raise the other income $Y_{O}$ in the next period (e.g., interest income) and, if this is anticipated by the subject, that they act as arguments of an intertemporal utility function with an intertemporal income constraint. However, as indicated in Subsection 2.2.1, an analysis of such intertemporal effects is beyond the scope of this study. Moreover, I do not expect these effects to imply short-run labour supply curves which are qualitatively different from those derived in this book.

\subsubsection{Preference variations and dynamics of state variables}

A sixth extension of the standard model results from the observation that different individuals facing the same constraints may nevertheless exhibit different labotit supply behaviour and, moreover, that the same individual facing the same constraints may exhibit different labour supply behaviour over time. So, labour supply curves could differ among individuals and over time, even if, for each relevant exogenous wage rate, the constraints and, in particular, the values of the other exogenous variables governing them (e.g., gross other income and tax rates) are identical. These phenomena can only be explained by variations of labour supply preferences over individuals and time, respectively. Such preference variations can be expressed by adding a discrete index $i$ for individuals or groups of individuals and a discrete or continuous time index $t$ as exogenous arguments to the utility function. One or more parameters of the utility function may then be considered as deterministic functions of $i$ or $t$ (e.g., a time trend, see Stone, 1964, p. 71). Alternatively, in socalled second-generation studies of static labour supply models ${ }^{53}$, interpersonal preference differences are accounted for by a stochastic and unobservable error term or "taste shifter"

${ }^{53}$ See Killingsworth (1983, Ch. 4). 
$\varepsilon_{i}$. Of course, allowing for preference changes over time, this error term could also be made dependent on $t$. However, such $i$ or $t$-dependent "taste parameters" and "taste shifters" do not explain which kind of factors the preference variations can be attributed to. Accordingly, the ways they vary over $i$ and/or $t$ (including the distribution of $\varepsilon_{i}$ ) are usually treated as exogenous in the short as well as longer run. Therefore, primarily for consumption demand, explanatory theories of preference variation across individuals and/or over time have been developed, which could be interpreted as making the variations of the "taste parameters" and "taste shifters", at least in the longer run, endogenous. Such theories are often referred to as theories of endogenous tastes or preferences ${ }^{54}$ or as theories of preference formation.

Best known of the theories of preference formation are the theories of (individual) habit formation and (behavioural) preference interdependence. ${ }^{55}$ In the habit formation approach an individual's consumption demand and labour supply preferences depend on her own consumption demand and labour supply behaviour in the past. Preference interdependence can be interpreted as meaning that the preferences mentioned depend on the corresponding past behaviour of relevant others. In the present study extensions of these two preference formation theories are applied to labour supply. Both theories imply that the arguments of the utility function should be extended with a vector of state variables, which are exogenous with respect to the labour supply and consumption demand decision in the short run, but which are endogenous in the longer run. In general we denote such a vector of state variables by $s$.

Many other kinds of state variables than those mentioned above can play a role in preference variations. For example, in the second choice situation of Section 2.2 .5 the job quality variables $q_{H}$ are state variables. In this example a preference change as a result of the longer-run choice of another job stems from a change in "psychic income" of labour time. More generally, changes in labour supply preferences are associated with changes in the marginal utilities of consumption, leisure and labour time. In particular, this holds for the preference shifts of "emancipating" married women with respect to their participation in the labour market as mentioned in Chapter 1. Therefore, this study considers utility functions $\hat{D}(X, L, H ; s)$ (see Sec. 2.3 for more details). In terms of the short-run choice variables $X, L$ and $H$ these utility functions can be regarded as conditional on (the values of) the state variables $s .^{56}$

In the longer run the state variables may be endogenous. For example, in the habit formation case mentioned above, one or more state variables representing an individual's behaviour in the past are the result of a choice of the individual at that time. This implies a lagged feedback from her choice behaviour to the state variable(s), which makes the state variable(s) lagged endogenous or endogenous in the longer run with respect to the short-run choice. This results in a (for the case of consumption demand) well-known dynamic analysis in terms of short-run and long-run demand or supply functions (see Chs. 3 and 4). However,

$54 \mathrm{C}$. theories of endogenous technical change as opposed to exogenous technical change.

55 See for surveys of both kind of theories in relation to each other Pollak (1978). Deaton and Muellbauer (1980, pp. 373-377) and Kapteyn (1985, pp. 8-18).

${ }^{56}$ See Pollak (1976a, 1977,1978) for the corresponding distinction between conditional and unconditional preferences. 
an important point to be noted here is that the dynamic character of this analysis is independent of whether the planning horizon of the utility maximizing subject covers one or multiple periods. ${ }^{57}$ On the other hand, our assumption made in Subsection 2.2.1 of a planning horizon of one period implies that the habit formation is supposed to be myopic, i.e.n the subject does not take into account the feedback of her present decision on future levels of the state variable(s), and hence on her future preferences (see Sec. 1.1). Similar kinds of reasonings can be held with regard to other types of preference formation, e.g., preference interdependence. We will come back to these points in the next chapters.

\subsubsection{Family labour supply}

Particular kinds of constraint and preference interdependencies which have been neglected so far, but which can play an important role in the behaviour of non-single labour suppliers, are the constraint and preference interdependencies of family members. Killingsworth (1983, Sec. 2.1) distinguishes three main kinds of models in the literature of the relation between family membership and labour supply. The first and simplest one is what he calls the male chauvinist model. In this model the husband plans his labour supply without reference to his wife's labour supply plans and, therefore, in the same way as in the standard model. On the other hand, the labour supply plans of the wife deviate from the standard model in including the exogenous total income of her husband in her other income $Y_{O}$ and in including the consumption of the other family members in her consumption variable $X$. Thus, $X$ now represents the total family consumption. ${ }^{58}$ However, apart from these reinterpretations of the variables $Y_{O}$ and $X$, the analysis of the labour supply behaviour of the wife is the same as in the standard model.

The second kind of model, which is by far the most popular one, is the family utilityfamily budget constraint model. Here the total utility of the family is maximized as a function $U^{F}\left(X, L_{1}, \ldots, L_{n}\right)$ of total family consumption $X$ and the leisure times of each of the family members $L_{1}, \ldots, L_{n}$. Furthermore, the total incomes of all the family members are pooled into one total family income $\sum_{i} w_{i} H_{i}+Y_{O}$. where $w_{i}$ is the wage rate and $H_{i}$ the labour time of the $i$ th family member and where $Y_{O}$ is the total other than labour income of the family. This results into one family (income-)budget constraint.

This model can be related to the standard model as follows. Suppose that all family members' wage rates change by the same index $w$. Then, analogously to the procedures in Section 2.1, the family members' leisure times $L_{1}, \ldots, L_{n}$ can be aggregated to a Hicks composite leisure time $L \equiv \sum_{i} w_{0 i} L_{i}$, where $w_{0 i}$ is a base period wage rate of the $i$ th family member. This $L$ should be interpreted as the total "expenditure" of full income on (that is, earnings forgone for) leisure times of the family members in constant prices of the base period. Next, $U^{F}\left(X, L_{1}, \ldots, L_{n}\right)$ may be maximized with respect to $L_{1}, \ldots, L_{n}$ under the constraint formed by the above definition of $L$ for each relevant $X$ and $L$. This results in a

${ }_{58}^{57}$ This will clarify footnote 17 .

58 This important necessary modification is not mentioned by Killingsworth. Moreover, it is implicitly assumed that there are a husband and a wife in the family and that they are the only family members who have an income. 
standard utility function $U(X, L)$, where the leisure budget $L$ is supposed to be optimally allocated to leisure times of the family members. In a second stage, $U(X, L)$ can then be maximized under the family incorne-budget constraint $p X+w L \leq w T^{\prime}+Y_{O}$, where $T^{\prime}=\sum_{i} w_{0 i} T_{i}$. This constraint is of the same form as the constraint (2.7) in the standard model, which makes the second stage maximization analogous to that model.

In general, however, the family members' wage rates will not change by the same index. Then, there are not one but two kinds of substitution effect that are relevant to the labour supply of any given family member $i$. First, we have the positive own substitution effect on i's labour supply of an increase in i's own wage. Second, there is also the cross-substitution effect on i's labour supply of a (for the family) income-compensated rise in the wage of some other family member $j$. This effect is positive or negative, depending on whether the leisure times of $i$ and $j$ are complements or substitutes, respectively. If, as is often done, the cross-substitution effects are assumed to be zero, the only effect on $i$ 's labour supply of a rise in $j$ 's wage is a pure income effect. ${ }^{59}$

Regardless of specific assumptions about the cross-substitution effects, the family utilityfamily budget constraint model neglects effects on the total family utility of the distribution of the family's total consumption among its different members. Moreover, it is silent as to the process that actually generates the "household" preference structure determining the labour supplied by its members. Partly as a consequence of these considerations, a third group of models has appeared, which emphasize the individual rather than the family as an important decision maker in a family context. Leuthold (1968) introduced what might be called the individual utility-family budget constraint model. In this model each individual family member maximizes her or his own individual utility as a function of family consumption and the individual's own leisure time subject to a family budget constraint. In doing so, the husband and wife will, analogously to duopolists, react to each other's plans until a consistent and stable (long-run) equilibrium has been reached. A sufficient condition for the existence of such a stable equilibrium is that the composite consumer good is a normal good for both spouses. Outside equilibrium there are no cross-substitution effects, but instead indirect income effects on family member i's labour supply of a rise in family member $j$ 's wage rate via a change in $j$ 's labour supply. ${ }^{60}$ If leisure is a normal good for $i$, these indirect income effects are negative. However, when a stable equilibrium has been reached, the model can be aggregated to a special case of the family utility-family budget constraint model with neither complementarity nor substitutability of the leisure times of the different family members, in which model the indirect income effects have become zero cross-substitution effects.

Ashworth and Ulph (1981) have extended the Leuthold model by allowing the individual

59 The statement of Killingsworth $(1983$, p. 33$)$ that in this case the family utility-family budget constraint model for a given individual $i$ reduces, at least for graphical purposes, to the standard model, is confusing, since another family member $f$ s leisure time $L_{j}$ remains dependent on $i$ 's wage rate $w_{i}$ via the pure income effect. This implies that a change of $w_{i}$ has not only the substitution and income effects on $L_{i}$ of the standard model, but also leads to a change of $j$ 's eamings, and hence of $i$ 's other income including it.

${ }^{60}$ See Killingsworth (1983, p.36) for details. 
utility functions to be conditional on the leisure times of the other family members. ${ }^{61}$ Then there are not only indirect income effects, but aiso so-called preference effects on family member i's labour supply of a rise in family member $j$ 's wage rate via a change in $j$ 's labour supply. These preference effects can be positive, negative or zero. Also in this more general model a consistent and stable equilibritm is possible, but, this equilibrium being reached, the model can only be aggregated to a family utility-family budget constraint model under stringent conditions. ${ }^{62}$ On the other hand, as a consequence of the behavioural preference interdependence, no a priori restrictions are then imposed on the complementarity or substitutability of the leisure times of the family members in the aggregated model.

A major difficulty with the equilibrium mentioned is that it is generally not Pareto optimal. ${ }^{63}$ As has been argued by, among others, Manser and Brown (1980), the family members are then likely to "bargain" with each other in order to reach Pareto optimal solutions for consumption and leisure times of the family members. This can be described by bargaining models on the basis of cooperative game theory. ${ }^{64}$ Again there are indirect income effects rather than cross-substitution effects, but in some bargaining models the total incomes and consumptions of the family members are not pooled. Differences in the distribution by recipient (husband or wife) of a given total amount of property income may then lead to different bargainings strengths of the two partners, and hence to differences in their behaviour. ${ }^{65}$

As all these models suggest, marital status and family situation are major determinants of labour supply. Therefore, these determinants will be taken into acccount in the labour supply models of the following chapters. The next section will formulate the general structure and the comparative statics of the short-run components of these models.

\subsection{The short-run models in this study}

After having given an overview of some important extensions of the standard model of labour supply in the previous section, we are now in the position to formulate the general structure of the short-run components of the labour supply models which will be analysed in the following chapters. We also introduce some modifications in the terminology and derive the implied comparative statics of labour supply with respect to the state variables.

The utility functions are of the form $\hat{U}(X, L, H ; \hat{S})$, where $S$ is a vector of state variables

${ }^{61}$ This is a clear example of behavioural preference interdependence, but is rather a consequence of complementarity or substitutability of the leisure times of the family members than of social con. formity and distinction motives, which are the usual sources of behavioural preference interdepenđence (see Subsec. 5.1.2).

62 A sufficient condition is that the individual utility functions of the different farnily members are identical (see Ashworth and Ulph, 1981, p. 121). See for the case of different individual preferences, e.g., Sarnuelson (1956) and Brown and Chuang (1981).

${ }^{63}$ See, e.g., Manser and Brown (1980) and Kooreman (1986, Ch. 6).

64 See Chiappori (1988), Kooreman (1986, Ch. 6, 1988) and Kooreman and Kapteyn (1989). The last three references also contain empirical applications.

${ }^{65}$ See Killingsworth (1983, p. 37). 
in the context of the preference formation theories which are applied (see Ch. 1). Instead of the usual terms "leisure" and "labour time" for the choice variables $L$ and $H$, this study uses the terms "household time" and "corporate time" for the following reasons. "Leisure" is an inadequate term for the time spent at home by a housewife and "labour time" is an inadequate term for the time spent in a job, since both terms suggest that a housewife does not work. Instead, the terms "unpaid time" and "paid labour time" may be used, but this leads to an intemal contradiction in the modelling of volunteer work as the interior optimal point $D^{\prime}$ in Figure 2.5. According to this model a sufficiently high (net) "psychic income" of paid labour time induces a person to do paid work even when it is not paid, i.e., to do volunteer work. Of course, this is a contradictory description of volunteer work, but it can be remedied by replacing the terms "unpaid time" and "paid labour time" by other terms. An adequate choice seems to be "household time" and "corporate time". Household time is defined as the total time devoted to activities in the private or informal sphere, i.e. to various forms of household production and consumption. Most of these activities are unpaid, but in the case of moonlighting they are paid. On the other hand, corporate time is the total time spent on production and consumption activities in the public or formal sphere (see Atkinson and Stiglitz, 1980, pp. 27 and 47). Mostly the corporate production activities are paid, but not in the case of volunteer work. ${ }^{66}$

In addition to these modifications in terminology, the choice variable $L$ will represent discretionary household time above a minimal necessary level. This exogenous minimal level of household time is due to considerations of physical survival or the health of the labour supplier or her (his) family (see Subsec. 2.2.4) ${ }^{67}$ It should be distinguished sharply from the endogenous minimal required leisure as an increasing function of individual and/or collective habit state variables in the threshold models of Phlips (1978), Johnson and Pencavel (1984), and Kapteyn and Woittiez (1990) (see the end of Subsec. 2.2.4). In the models of the present study the individual and/or collective habit state variables are incorporated in the utility function in a more general and less rigid way.

Given the predetermined state variables $s$, the labour supplier maximizes her utility function $\hat{U}(X, L, H: S)$ with respect to $X, L$ and $H$ under certain constraints. Besides the minimal level constraint we will mostly assume the usual income and time constraints $X \leq w H+Y_{O}$ and $H+L=T$. Here the aggregate consumption price has been equated with $1, w$ is the exogenous (relative) hourly corporate wage rate. $Y_{O}$ is exogenous income other than wage income of the labour supplier ${ }^{68}$, and $T$ is the total available time of 24 hours a day minus the minimal necessary household time. When the labour supplier belongs to a family, $X$ is conceived as the total consumption of the family, whereas $L$ and $H$ remain individual, and $Y_{O}$ contains the assumedly exogenous incomes of the other family members. This amounts to a simplified individual utility-family budget constraint model (see Subsec. 2.2.7) in which the endogeneity of wage incomes of other family members is

66 In the literature also the terms "market time" and "non-market time" are used. but these tems 100 may cause confusion in the cases of volunteer work and moorlighting.

67 We do not similarly assume a minimal necessary level of consumption, since this creates complications which we want to abstract from.

${ }^{68}$ Income from moonlighting is included into this other income. 
neglected. For married women (and assuming no wage income of family members other than the husband and wife), this model coincides with the male chauvinist model (see Subsec. 2.2.7), and especially for them the model may not be a bad approximation of reality. On the other hand, the model allows inconsistencies of the individual labour supply decisions of the family members. Yet, in the present theoretical context and since we treat the individual cases as separate, this does not seem a major problem. Furthermore, both the other income $Y_{O}$ and the total discretionary time $T$ are assumed to be always positive.

Substituting $H=T-L$ into the utility function $\hat{U}(X, L, H ; S)$ reduces it to a utility function $U(X, L ; S)$. However, as shown in Subsection 2.2.5, this is not a standard utility function, but it allows the possibility of negative marginal utilities (oversatiation) of (discretionary) household time $L$ for sufficiently high $L$. This turned out to have interesting implications. Making simplifying assumptions on the state variables corresponding to household time and corporate time, the following chapters make a similar reduction of the utility function with respect to these state variables, yielding a reduced utility function $U(X, L ; s)$.

Maximization of this utility function or the original utility function under the income and time constraints yields the short-run consumption and household time demand functions $X\left(w, Y_{O}, T, s\right)$ and $L\left(w, Y_{O}, T, s\right)$, respectively, and hence the short-run labour supply function $H\left(w, Y_{O}, T, s\right)$. The effects of changes in $w$ and $Y_{O}$ on $L$ or $H$, and hence on $X$, are given by the Slutsky equation (2.8) or (2.17) and the equation (2.18). Similarly to the latter two equations, the effects of changes in the state variables $s$ on the short-run demands and labour supply can be derived in an easy way from the constrained utility function

$$
U^{c}\left(H ; w, Y_{O}, T, s\right)=U\left(w H+Y_{O}, T-H ; s\right) .
$$

This utility function is maximized with respect to $H$ for given $w, Y_{O}, T$ and $s$ in the usual case where consumption is not fully satiating at the optimal point, so where the income constraint holds with equality sign. For an interior maximum the first-order condition is again given by $U_{H}^{C}=w U_{X}-U_{L}=0$. Differentiating this condition with respect to the vector of state variables $s$ yields $U_{H s}^{c}+U_{H H}^{c} H_{s}=0$ (see Varian, 1984, p. 328), or equivalently, since $U_{H H}^{c}=-\left|U_{H H}^{c}\right| \neq 0$ by virtue of the second-order condition (2.16),

$$
H_{s}=\frac{U_{H s}^{c}}{\left|U_{H H}^{c}\right|}=\frac{w U_{X s}-U_{L s}}{\left|w^{2} U_{X X}-2 w U_{X L}+U_{L L}\right|},
$$

where we substituted from (2.16). This equation implies that the effect of a change in a state variable $s_{j}$ on the short-run labour supply $H$ is positive, e.g., if $U_{X s_{j}}$ (as well as $w$ ) is positive and $U_{L s_{j}}$ is non-positive, i.e., if an increase (decrease) in $s_{j}$ induces a rise (fall) in the marginal utility of consumption $U_{X}$ and not a rise (fall) in the marginal utility of household time $U_{L}$ at the optimal point. Since differentiating the time and income constraints to $s$ yields $L_{s}=-H_{s}$ and $X_{s}=w H_{s}$, the expressions for the effecis of changes in the state variables on the household time and consumption demand follow from (2.20). Note that (2.20) and these expressions hold irrespectively of the nature of the state variables in the utility function.

On the other hand, these comparative statics results only hold for the standard time and income consuraints, whereas in many labour supply situations endogenous social security 
benefits (see Subsec. 2.2.3) and/or institutional or demand side rationings of labour supply (see Subsec. 2.2.4) play an important role. For such cases discontinuous short-run labour supply schedules like those in Figures 2.3 and 2.4 hold, implying that the comparative statics becomes essentially different. However, formal and general derivations of these comparative statics and of the ensuing effects of collective habit formation are beyond the scope of this study. Therefore, for these cases the following chapters will only give intuitive and short analyses of simple subcases.

Whereas this section has formulated the general structure and implications of the shortrun labour supply models to be used in the following chapters, those chapters will complement the short-run models with specifications of the endogenous long-run dynamics of state variables in the context of preference formation. The long-run labour supply effects of nonlinear forms of this dynamics will then be analysed. 


\section{CHAPTER 3}

\section{Individual Habit Formation: General Model}

As pointed out by Clark and Summers (1982), persistence of employment status plays an important role in labour supply dynamics. This means that previous (un)employment tends to raise the probability of subsequent (un)employment. One of the mechanisms underlying this phenomenon is individual habit formation (IHF) with respect to consumption and/or household time (or leisure, see Sec. 2.3). Empirical studies by Pllips (1978). Johnson and Pencavel (1984), Hotz et al. (1988), and Kapteyn and Woittiez (1990) suggest that, especially with respect to household time, IHF is rather strong. Still, the IHF is shown to be mostly stable in the sense that the stability conditions for the long-run equilibrium are fulfilled. A simple extension of such a model of stable IHF can explain how after taking a part-time job a person can develop a strong orientation towards corporate work and may even develop into a "workaholic". On the other hand, such a model cannot explain why some long-term unemployed, although having lost their old job involuntarily, become little motivated to get a new job (at the same values of the wage rate and other income). Similarly, such a stable IHF model can give an explanation of the strong orientation of many housewives to household work, but it may not adequately explain how in a process of "emancipation" the same women can develop a strong orientation towards corporate work.

Such profound changes in preferences and behaviour can be modelled as the outcomes of IHF which has an unstable long-run equilibrium between two stable long-run equilibria פs in the addictive consumption model of Becker and Murphy (1988). ${ }^{1}$ This leads to a polarization in behaviour and habit state: on the one hand, when the initial corporate hours are above the unstable equilibrium value, the IHF induces a rise in corporate hours towards a stable equilibrium of relatively high corporate hours and ensuing accustomization. Such an equilibrium may describe the behaviour and habits of an "emancipated" woman with a job or a "workaholic" (when the number of corporate hours is very high), On the other hand, when the initial corporate hours are below the unstable equilibrium value, the IHF causes falls in

${ }^{1}$ Becker and Murphy call IHF addiction, but it seems more appropriate to associate addiction with a habit-dependent threshold for consumption or household time below which a person feels unable to go (see Schokkaert, 1982b, p. 64) and with flip-flops or chaotic reversals of preferences as in the models of Winston (1980) and Day (1986). 
corporate hours towards a stable equilibrium of low or zero corporate hours and similarly ensuing accustomization. The zero corporate hours equilibrium may then correspond to a housewife or a "voluntarily long-term unemployed" person". Since the initial corporate hours depend on the time paths of exogenous variables like the wage rate or rationings in the past, these time paths determine which of the two stable equilibria is actually approached. This 1 call individual hysteresis (see footnote 6 in Sec. 1.1). At certain values of exogenous variables a transition may occur from one equilibrium to another, a point not recognized by Becker and Murphy. Such a transition is referred to as an individual catastrophe in the sense of Thom (1975) (see footnote 7 in Sec. 1.1). Individual catastrophes in behaviour and habits may have occurred (and still occur) in the "emancipation" process of individual married women. In contrast to Becker and Murphy, I assume that the IHF is myopic, which seems plausible (see Sec. 1.1), simplifies the analysis, and facilitates the introduction of more than one habit state variable for consumption, household time and corporate time.

The organization of this chapter is as follows. In Section 3.1 the general model is presented. Section 3.2 contains a graphical analysis of the (in)stability of multiple long-run equilibria and of individual hysteresis dependent on rationings. Section 3.3 presents necessary and sufficient stability conditions in terms of second-order partial derivatives of an arbitrary additive utility function and the wage rate. Next, Section 3.4 gives a general analysis of the differences between short-run and long-run wage (rate) elasticities of labour supply when the stability conditions are met, and Section 3.5 shows how in the case of instability individual catastrophes and hysteresis of the long-run equilibria can occur. Moreover, Section 3.5 compares hysteresis with a phenomenon that I call quasi-hysteresis. Section 3.6 gives an analysis of the effects of IHF in cases of discontinuous short-run labour supply schedules, and Section 3.7 concludes.

\subsection{General model}

Let the one-period preferences of a labour supplier be represented by a utility function $\hat{U}\left(X, L, H: s_{X}, s_{L}, s_{H}\right)$, where $X$ denotes aggregate consumption, $L$ is discretionary household time above an exogenous minimal necessary level (see Sec. 2.3 ), $H$ is corporate time, and $s_{X}, s_{L}$ and $s_{H}$ stand for "psychological stocks" of habits with respect to $X, L$ and $H$ (see Houthakker and Taylor, 1970). These habit stocks are defined as the solutions of the following differential equations which constrain their time paths:

$$
\hat{s}_{L t}=L_{i}{ }^{a}-\delta_{L} s_{L t} \text {, }
$$

where $L_{t}^{a}$ stands for the actual (discretionary) household time at time $\uparrow$, and analogous: equations for $s_{X}$ and $s_{H i}$. Here the positive parameter $\delta_{L}\left(\delta_{X} . \delta_{H}\right)$ is similar to the depreciation rate of a durable good and measures the speed by which habits wear off. These differential equations describe continuous accumulation or reduction of the psychological

${ }^{2}$ Strictly spoken, a long-term unemployed who does no longer want to work and hence does not search for a job, does no longer belong to the labour force, so is not unemployed any more according to the prevailing definition in economics. Still, it is often convenient ro refer to such a person as a "voluntarily long-term. unemployed" person. 
habit stocks. This seems a better approximation of reality than the discrete accumulation or reduction in terms of the usual, rather long, empirical time periods as described by difference equations (see, e.g., Johnson and Pencavel, 1984, and Kapteyn and Woittiez, 1990).

Given her (his) predetermined habit levels $s_{X}, s_{L}$ and $s_{H}$, the labour supplier maximizes her utility function with respect to $X, L$ and $H$ under the usual income and time constraints $X \leq w H+Y_{O}$ and $H+L=T$. Here the aggregate consumption price has been equated with $1, w$ is the exogenous (relative) hourly corporate wage rate, $Y_{O}$ is exogenous income other than wage income of the labour supplier and $T$ is the total available time of 24 hours a day minus the minimal necessary household time. When the labour supplier belongs to a family, $X$ is conceived as the total consumption of the family, whereas $L$ and $H$ remain individual, and $Y_{O}$ contains the assumedly exogenous incomes of the other family members. Both the other income $Y_{O}$ and the total discretionary time $T$ are assumed to be always positive.

A further simplifying assumption is that the decay rates $\delta_{L}$ and $\delta_{H}$ of the household and corporate time habits are equal to each other. As shown by Phlips (1978, eq. (26)), this implies $s_{H}=T / \delta_{L}-s_{L}$. Substituting this expression and $H=T-L$ into the utility function, we obtain a reduced utility function $U\left(X, L ; s_{X}, s_{L}\right)$. As shown in Subsection 2.2.5, this utility function allows the possibility of negative marginal utilities (oversatiation) of household time $L$ for sufficiently high $L$ (see, e.g., Hamermesh, 1974). This may lead to volunteer work and may especially happen in the case of a workaholic who is strongly used and even addicted to a high corporate time $H$. Such a workaholic may even continue to work a high $H$, when his (her) wage rate $w$ falls to zero. ${ }^{3}$

Maximizing the original or reduced utility function under the income and time constraints at time $t$ yields the preferred household time $L_{l}$ and consumption $X_{t}$ as functions $L\left(w_{1}, Y_{O t}, T_{1}, s_{X 1}, s_{L t}\right)$ and $X\left(w_{1}, Y_{O t}, T_{1}, s_{X_{1}}, s_{L t}\right)$. In a labour supply and consumption demand regime these preferred $L_{t}$ and $X_{1}$ are assumed to be immediately realized, so $L_{t}^{a}=L_{t}$ and $X_{t}^{a}=X_{t}{ }^{4}$ Substituting the functions mentioned for $L_{t}^{a}$ and $X_{t}^{a}$ in equation (3.1) and its analogue for $s_{X}$, respectively, leads to

${ }^{3}$ On the other hand, this may only hold in the short run, since in the long run the workaholic may gradually diminish his corporate hours for $w=0$ until a few or zero hours and get used to that (see Secs. 3.4 and 3.5 for such processes). Furthermore. when being used to zero corporate hours, such a person may not be prepared to work as a volunteer. This dynamic description of a workaholic seems more adequate than the static representation by Ehrenberg and Smith (1982, pp. 170-171), which has the implausible implication that every person who is prepared to volunteer for a few hours a week, is a workaholic. However, a more complete modelling of workaholism than done here requires a model of addictive behavior such as that of, e.g.. Winston (1980), but is not needed in the present context.

4 This is a strong assumption, since even in a labour supply regime actual labour supply will only adjust to preferred labour supply in a delayed and stepwise fashion because of labour contracts, commitments, terms of notice and application, search costs and time, and more generally, discontinuous adjustment costs of behaviour (or transaction costs) and uncertainty (Heiner, 1983). These factors form other causes of persistence and hysteresis of labour supply than IHF (cf. Clark and Summers, 1982, Romme, 1990. and Homan, Hagenaars and Van Praag, 1988). However, a brief analysis suggests that these complications do not have an essential effect on the long-run stability properties of our model. 


$$
\begin{aligned}
& s_{L t}=L\left(w_{t}, Y_{O t}, T_{t}, s_{X t}, s_{L t}\right)-\delta_{L} s_{L t}, \\
& s_{X t}=X\left(w_{t}, Y_{O t}, T_{t}, s_{X !}, s_{L t}\right)-\delta_{X} s_{X t} .
\end{aligned}
$$

By virtue of the definition of IHF, $L_{i}$ and $X_{t}$ increase with the habit stocks $s_{L i}$ and $s_{X I}$, respectively, so the partial derivatives $L_{s_{L,}}$ and $X_{s_{X t}}$ are both positive. This implies positive or reinforcing feedbacks of the habit stocks on the behaviour. Moreover, in the usual case where the income constraint is binding, these two equations are linked to each other by the income and time constraints, and hence form a systern of first-order differential equations in $s_{L I}$ and $s_{X I}$.

For constant $w_{t}=w, Y_{O t}=Y_{O}$ and $T_{t}=T$ a long-run (stationary) equilibrium solution $s^{*}=\left(s_{X}^{*}, s_{L}^{*}\right)$ is obtained by putting $s_{L t}=0$ in (3.2a) and $s_{X t}=0$ in (3.2b). The habit stocks will move to such an equilibrium, if it is locally (asymptotically) stable. The next two sections will analyse the conditions under which this happens and when there are multiple long-run equilibria. Then we will also consider the case that labour supply is rationed by labour demand.

\subsection{Multiple equilibria and hysteresis}

For the sake of exposition we first regard IHF with respect to only household time $L_{t}$ (and corporate time $H_{1}$ ), so ignoring the effects of $s_{X}$. Moreover, following most literature, we begin by assuming that $L\left(w, Y_{O}, T, s_{L}\right)$ is linear in $s_{L}$ for $0<L<T$ (omitting the time indices of $L_{1}$ and $s_{L I}$ ).

For constant $w, Y_{O}$ and $T$ the development of a person into a workaholic may then be described by the line of the demand function $L^{A}\left(w, Y_{O}, T, s_{L}\right)$ as a function of $s_{L}$ in Figure 3.1. The intersection $A$ of this line with the equilibrium line $L=\delta_{L} s_{L}$ indicates a unique (long-run) equilibrium. Since the line cuts the equilibrium line at $A$ from above, it is easily seen from (3.2a) that this equilibrium is stable. More formally, an equilibrium point $s_{L}^{*}$ is stable if and only if $L_{s_{L}}^{*}:=L_{s_{L}}\left(w, Y_{O}, T, s_{L}^{*}\right)<\delta_{L}$ (see Appendix $3 A$ for a formal derivation). This condition means that the IHF effect of $s_{L}$ on $L$ at $s_{L}^{*}$ should be sufficiently compensated by the speed $\delta_{L}$ by which the household time habits wear off.

Suppose the person is initially used to unemployment, i.e. $s_{L}=T / \delta_{L}$. Then he (or she) prefers to have household time $L^{0}<T$, so he takes a job of $T-L^{0}$ hours. However, he will start to get used to that household and corporate time, which, by virtue of (3.2a), is described by an (intinitesimally) small horizontal move from the $L^{A}$ line at $L^{0}$ to the left. As a consequence, he will prefer (infinitesimally) less household time and take a job of more hours. This is described by a small vertical move back to the $L^{A}$ line. Again he starts to get used to that and will consequently have less household time and more corporate time, and this process will continue until he spends only $L^{* A}$ hours as household time, so works $T-L^{* A}$ hours of corporate time, and has got used to that (see also footnote 7 in the next section). When the initial household time to which the person is used is even lower than $L^{* A}=\delta_{L} s_{L}^{* A}$, the reverse will happen.

More generally, such a description applies to persons who get used to a certain numbers of corporate hours like "emancipated" married women with a job. However, especially in 


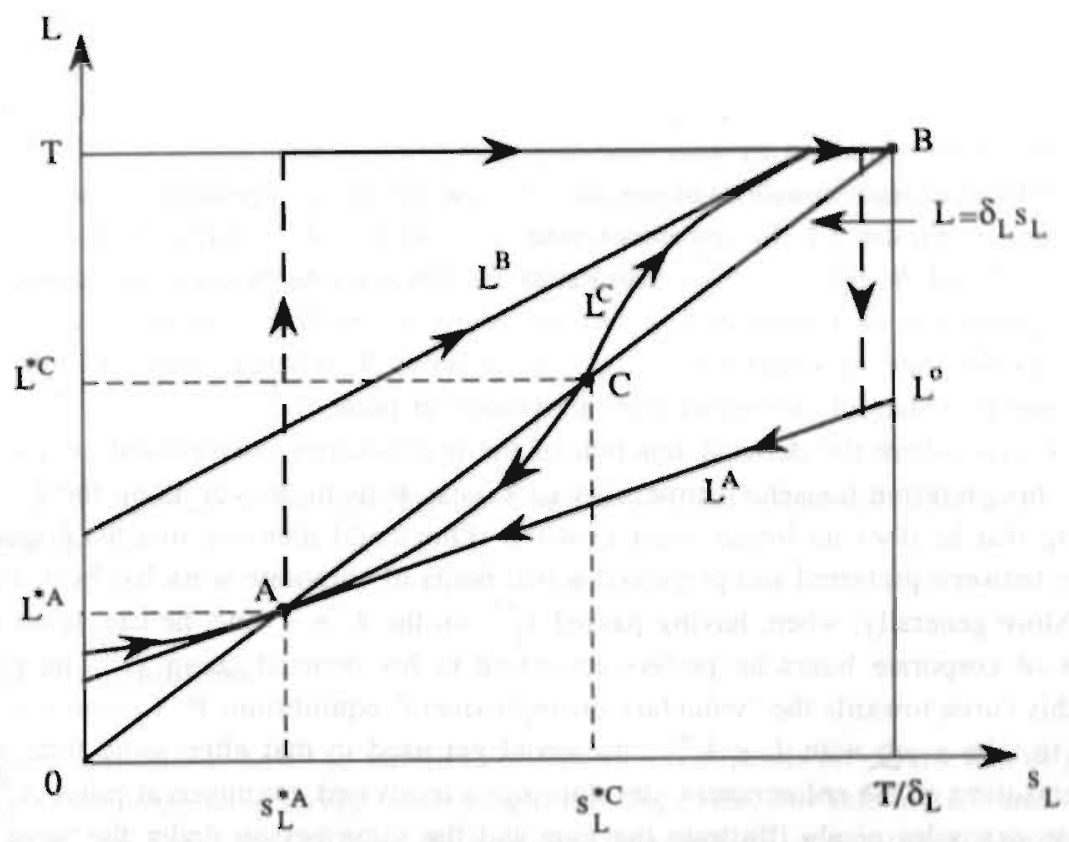

Figure 3.1 Equilibria $A B$ and $C$ as intersections of the equilibrium line $L=\delta_{L} s_{L}$ and household time demand functions $L^{A}, L^{B}$ and $L^{C}$. The dashed lines with arrows indicate possible shifts in $L$ of a temporarily involuntarily unemployed person with demand function $L^{A}$.

that case the household time $L^{* A}$ may be much higher than in the case of a workaholic. In a similar way and assuming the same constant $w, Y_{O}, T$ and $\delta_{L}$, the situation of a person who does not paricipate in the labour market (e.g., a housewife) may be described by the intersection of the line $L^{B}$ and the equilibrium line in Figure 3.1. Now, however, the line $L^{B}$ exhibits a kink at the point where it reaches the boundary $T$ of the feasible domain of the household time $L$, and the stable equilibrium point $B$ is a corner equilibrium for maximal household time or zero corporate time. Thus, when the household time is initially lower than maximal, so when the corporate time is positive, the person will have no rest until she (or he) is no longer employed and can spend all her time as household time.

Consider now the situation of a person at $A$ who involuntarily loses his (or her) job. Then his corporate time $H$ is rationed at zero and he is forced to have actual household time $L_{t}^{a}$ equal to $T$. However, as described by (3.1), his habit state $s_{L t}$ begins to increase from $s_{L}^{* A}$ to $T / \delta_{L}$, i.e., he moves along the $L=T$ line in Figure 3.1 to the right and gets used to his involuntary unemployment. (Note that $L_{t}{ }^{a}$ is then no longer a function of $s_{L t}$ as in (3.2a), so the reinforcing feedback is broken, the differential equation (3.1) is always linear, and the stability condition $\delta_{L}>0$ is always fulfilled.) Nevertheless, when after some time the unemployed person can again work the number of corporate hours he prefers according to his demand line $L^{A}$ (at the same $w, Y_{O}$ and $T$ ), he shifts to this line and subsequently moves back to his original equilibrium $A$. 


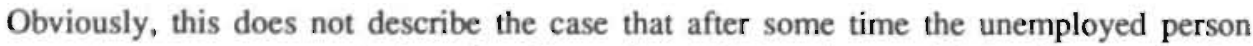
does no longer want to work, i.e., that his preferred $L$ has risen to $T$. In that case he has turned into a "voluntarily unemployed" person with demand function $L^{B}$ and equilibrium $B$. This case can be explained by assuming that both demand functions $L^{A}$ and $L^{B}$ are local (piecewise-)linear approximations of one and the same non-linear sigmoid demand function $L^{C}$ as indicated in Figure 3.1. In agreement with $L^{A}$ and $L^{B}, L^{C}$ implies stable equilibria at the points $A$ and $B$, but now there also exists an unstable intermediate equilibrium at point $C$. This point acts as a watershed or barrier: below $C$ the IHF dynamics leads the labour supplier to the state of a motivated employee at point $A$, whereas above $C$ the dynamics pushes him to a state of "voluntary unemployment" at point $B .^{5}$

In the case where the demand function of the involuntarily unemployed person is given by $L^{C}$, his preferred household time rises to $T$ near $B$ as he moves along the $L=T$ line, implying that he does no longer want to work. (One could then say that his cognitive dissonance between preferred and perceived actual hours of corporate work has been reduced to zero.) More generally, when, having passed $s_{L}^{* C}$ on the $L=T$ line, he can again work the number of corporate hours he prefers according to his demand curve $L^{C}$, he will move along this curve towards the "voluntary unemployment" equilibrium $B$. Conversely, were he forced to take a job with $L<L^{\bullet C}$, he would get used to that after some time and may, after relaxation of the enforcement, develop into a motivated employee at point $A !^{6}$

These examples nicely illustrate that one and the same person under the same external conditions ( $w, Y_{O}$ and $T$ ) may be going to exhibit either the one or the other behaviour, depending on his initial situation (left below or right above $C$ ). In the examples this initial situation is determined by a temporary rationing of his labour supply in the past (involuntary unemployment or labour enforcement), which has a decisive and permanent effect on the behaviour chosen. As a result, the equilibrium which is approached depends on the time path of an exogenous variable (in this case labour demand or forced labour supply) in the past. Our model can also exhibit hysteresis dependent on other exogenous variables. The case of the hourly wage rate $w$ will be dealt with in Section 3.5 .

At first sight the existence of an unstable intermediate equilibrium seems in contradiction with the fact that in the empirical studies mentioned in the introduction of this chapter, the stability conditions are mostly fulfilled. However, these conditions probably apply to averages of stable equilibria like $A$ and $B$ in Figure 3.1 over the persons to which the data employed refer, implying that on average the stability conditions are met without excluding the existence of an unstable intermediate equilibrium.

5 Surprisingly, a similar situation in the context of supply and demand curves for goods has already been observed by Marshall (1920, App. H. Secs. 2 and 3), but only scantily analysed. Such a Keynesian trap situation is much akin to the corridor concept of Leijonhufvod (1973) (see Varian, 1979, p. 2i). Chaos is impossible with differential equations of the form (3.2a) and (3.2b) (or (3.1)), even when $L_{s_{L}}^{*}$ and $X_{s_{X}}^{*}$ were allowed to be negalive for higher $s_{L}$ and $s_{X}$ due to individual long-run satiation as in the difference equation (12) or (14) of Benhabib and Day (1981) (in these equations $s_{L_{t}}=L_{t-1}$ : see also the end of Sec, 3.5).

${ }^{6}$ This suggests a rather right-wing policy recommendation, but other policy recommendations are also possible. However, going into this is beyond the scope of this study. 
When IHF with respect to consumption is added to the analysis given above, nothing essential changes, but the derivation of necessary and sufficient stability conditions becomes more complicated. Household time demand $L$, and hence labour supply $H$, are now also functions of the consumption habit state, and the equilibrium condition $w(T-L)+Y_{O}=X=\delta_{X} s_{X}$ should be added. This is not elaborated here, but in Appendix $3 \mathrm{~A}$ a formal equilibrium and stability analysis is given. This results in a simple necessary and sufficient condition for local stability of an equilibrium point $s^{*}$ which is given by

$$
\frac{X_{s_{X}}^{*}}{\delta_{X}}+\frac{L_{s_{L}}^{*}}{\delta_{L}}<1 .
$$

Note that $X_{s_{X}}^{*}=0$ leads to the stability condition which has been established at the beginning of this section.

\subsection{Stability conditions}

A subsequent question is how the stability condition (3.3) depends on parameters or general properties of the underlying utility function and the wage rate. For interior $L^{*}$ and $X^{*}$ the derivatives $L_{s_{L}}^{*}$ and $X_{s_{X}}^{*}$ in (3.3) can be expressed in terms of second-order derivatives of the utility function and the wage rate as

$$
\begin{aligned}
& L_{s_{L}}^{*}=\frac{U_{L_{s_{L}}}^{*}-w U_{X s_{L}}^{*}}{\left|w^{2} U_{X X}^{*}-2 w U_{X L}^{*}+U_{L L}^{*}\right|}, \\
& X_{s_{X}}^{*}=\frac{w^{2} U_{X s_{X}}^{*}-w U_{L s_{X}}^{*}}{\left|w^{2} U_{X X}^{*}-2 w U_{X L}^{*}+U_{L L}^{*}\right|} .
\end{aligned}
$$

Here the superscript * of the second-order derivatives of $U$ denotes evaluation at $\left(q^{*} ; s^{*}\right)$ with $q^{*}:=\left(X^{*}, L^{*}\right)=\left(\delta_{X} s_{X}^{*}, \delta_{L} s_{L}^{*}\right)$, and the expression between modulus signs in the denominators is negative by virtue of the second-order condition in static theory. (See Sec. 2.3 for a simple derivation of (3.4a) and (3.4b).)

To simplify the analysis I restrict it to utility functions which are additive(ly separable) in the pairs $\left(X, s_{X}\right)$ and $\left(L, s_{L}\right)$, so with $U_{X L}^{*}=U_{X s_{L}}^{*}=U_{L_{s_{X}}}^{*}=0$ (see, e.g., the linear expenditure systems of Phlips, 1978, and Johnson and Pencavel, 1984). Positivity of $L_{s}^{*}$ in (3.4a) and of $X_{s_{X}}^{*}$ in (3.4b) then implies that $U_{L_{s_{L}}}^{*}$ and $U_{X s_{X}}^{*}$ should be positive, i.e., increases in the habit stocks induce rises in the corresponding marginal utilities (at the equilibrium point). 
This is the central mechanism which drives the process of IHF. ${ }^{7}$ Substituting (3.4a) and (3.4b) for additive utility functions into the stability condition (3.3), this condition becomes

$$
\frac{w^{2} \frac{U_{X s_{X}}^{*}}{\delta_{X}}+\frac{U_{L s_{L}}^{*}}{\delta_{L}}}{\left|w^{2} U_{X X}^{*}+U_{L L}^{*}\right|}<1
$$

Assuming $U_{X X}^{*}<0$ and $U_{L L}^{*}<0$ (which are sufficient conditions for the second-order condition with $U_{X L}=0$ at $\left(q^{*}, s^{*}\right)$ ), it follows that the denominator of (3.5) can be written as $w^{2}\left|U_{X X}^{*}\right|+\left|U_{L L}^{*}\right|$, so (3.5) is equivalent to

$$
w^{2} \frac{U_{X s_{X}}^{*}}{\delta_{X}}+\frac{U_{L s_{L}}^{*}}{\delta_{L}}<w^{2}\left|U_{X X}^{*}\right|+\left|U_{L L}^{*}\right| .
$$

This condition holds for all $w$, if $U_{X s_{X}}^{*} / \delta_{X}<\left|U_{X X}^{*}\right|$ and $U_{L s_{L}}^{*} / \delta_{L}<\left|U_{L L}^{*}\right|$, i.e.

$$
\begin{aligned}
& U_{X s_{X}}^{*}<\left|U_{X X}^{*}\right| \delta_{X}, \\
& U_{L s_{L}}^{*}<\left|U_{L L}^{*}\right| \delta_{L} .
\end{aligned}
$$

Thus, in the case of additive utility functions the conditions $U_{X X}^{*}<0, U_{L L}^{*}<0,(3.7 \mathrm{a})$ and (3.7b) are sufficient stability conditions for $s^{*}$. They can be considered as generalizations of the sufficient stability conditions in the additive quadratic model (AQM) of Houthakker and Taylor (1970, Ch. 5, eq. (14)) for the case of two goods. ${ }^{8}$ As formulated by these authors, the conditions (3.7a) and (3.7b) say that "habit formation should not be so strong as to offset the combined effect of diminishing marginal utility and the depreciation rate". I then call the IHF (relatively) weak at $s^{*}$. When (3.7a) or (3.7b) is not fulfilled, I say that the IHF is (relatively) strong at $s * 9$

${ }^{7}$ Assuming that $U_{L s_{L}}$ is also positive at other than equilibrium points $\left(q^{*}, s^{*}\right)$, it follows that when the household time habit stock $s_{L}$ declines, $U_{L}\left(L ; s_{L}\right)$ decreases and hence, for high household time $L$ (so low $U_{L}\left(L ; s_{L}\right)$ ), may become negative for sufficiently low $s_{L}$. This corresponds to the development of a person into a workaholic, in which $s_{L}$ declines as the person gets used to more corporate time and less household time (see Sec. 3.2). Note that indifference curves like those in Figure 2.1 in Section 2.1 then shift to indifference curves like that in Figure 2.5 in Subsection 2.2.5. On the other hand, for $w$ falling to low values, leading to less corporate time and ensuing accustomization, the latter indifference curves may shift back to the former. Then, the person would no longer be prepared to work as a volunteer for zero wage (see footnote 3 ).

${ }^{8}$ In deriving their conditions Houthakker and Taylor depart from the incorrect claim that the $n$ dimensional generalization of the matrix $Q_{s}-D$ (with $D$ being a diagonal matrix with $\delta_{X}$ and $\delta_{L}$ on the diagonal) is symmetric: differentiating the income and time constraints to $s_{L}$ and $s_{X}$, it follows that the off-diagonal elements of this matrix are $X_{s_{L}}=-w L_{s_{L}}$ and $L_{s_{X}}=-X_{s_{X}} / w$. which are in general unequal to each other.

${ }^{9}$ Houthakker and Taylor (p. 196) call this addiction, but it seems more appropriate to use this tem in a different sense (see footnote 1). 
However, as demonstrated below, these sufficient stability conditions are not necessary. Consequently, when one of them is not met (see, e.g., Taylor and Weiserbs, 1972, and Phlips, 1978, for some consumption categories), it is unclear if the equilibrium is unstable (like $C$ in Fig. 3.1). Let us, therefore, take a closer look at the stability condition (3.6), which is both sufficient and necessary, when the sufficient conditions $U_{X X}^{*}<0$ and $U_{L L}^{*}<0$ are fulfilied. Suppose that one of the other two sufficient conditions, say (3.7a), is met. Then (3.6) can be rewritten as a condition for $U_{L s_{L}}^{*}$ as

$$
U_{L s_{L}}^{*}<\left[\left|U_{L L}^{*}\right|+w^{2}\left(\left|U_{X X}^{*}\right|-\frac{U_{X s_{X}}^{*}}{\delta_{X}}\right)\right] \delta_{L} .
$$

In comparison with ( $3.7 \mathrm{~b})$ this condition contains an additional term between the square brackets, which is explicitly proportional to $w^{2}$ and positive. Hence the condition is weaker than (3.7b) and there is an additional stabilizing force the strength of which explicitly depends on $w$. This force can be interpreted as follows. Suppose that, as a consequence of IHF with respect to household time $L, L$ increases by an (infinitesimally) small amount $d L$ sufficiently near the long-run equilibrium point $\left(q^{*}, s^{*}\right)$. This leads not only to a decrease $\left|U_{L L}^{*}\right| d L$ in the marginal utility of household time $U_{L}$, but also, as a consequence of the time and income constraints, to a decrease $w d L$ in the wage income and hence in the levell of consumption $X$. This implies a rise $\left|U_{X X}^{*}\right| w d L$ in the marginal utility of consumption $U_{X}$ : On the other hand, the subject gets used to the lower $X$, which decreases $U_{X}$ by $\left(U_{X s_{X}}^{*} / \delta_{X}\right) w d L$. By virtue of (3.7a) the total effect on $U_{X}^{*}$ is positive and pushes the subject back to a higher $X$ and hence, again as a result of the constraints, to a lower $L$. Translating the force on $X$ into a force on $L$ yields an additional factor $w$. A conclusion from this interpretation is that the time and income constraints are responsible for the force.

Analogously, in the case where the other sufficient condition (3.7b) is fulfilled, assuming positive $w$ and dividing (3.6) by $w^{2}$, it can be reformulated as a condition for $U_{X s_{X}}^{*}$ as

$$
U_{X s_{X}}^{*}<\left[\left|U_{X X}^{*}\right|+\frac{1}{w^{2}}\left(\left|U_{L L}^{*}\right|-\frac{U_{L s_{L}}^{*}}{\delta_{L}}\right)\right] \delta_{X}
$$

Again there is an additional stabilizing force which works through the time and income constraints, but now its strength explicitly depends on $1 / w$, being the relative price of consumption. ${ }^{10}$ When $w=0$ (or $\left.1 / w=\infty\right)$, (3.6) boils down to $(3.7 \mathrm{~b}$ ). In the case where neither of the sufficient conditions (3.7a) and (3.7b) is met, (3.6) does not hold for any value of $w$. The forces of the constraints in (3.8) and (3.9) are then destabilizing.

When the second-order derivatives of $U$ at $\left(q^{*}, s^{*}\right)$ are independent of $w$ as in the AQM, the conditions (3.8) and (3.9) can easily be reformulated as explicit conditions on $w$ or $1 / w$ (see Sec. 4.3), but generally this is not possible. Yet we can formulate:

${ }^{10}$ This stabilizing role of the constraints is overlooked by Houthakker and Taylor (1970), Weiserbs (1974) and Phlips (1974). 
Proposition 3.1 In the case of $I H F$ with respect to both consumption and household time (and corporate time) and of additive utility functions with $U_{X X}^{*}<0$ and $U_{L L}^{*}<0$, an interior equilibrium point $s^{*}$ of $(3.2 a)$ and $(3.2 b)$ is stable if and only if one of the following situations prevails:

(i) weak IHF with respect to both consumption and household time at $s^{*}$,

(ii) weak IHF with respect to consumption and strong IHF with respect to household time at $s^{*}$ which is sufficiently compensated by the stabilizing force of the relative price of household time w as expressed by (3.8),

(iii) weak IHF with respect to household time and strong IHF with respect to consumption at $s^{*}$ which is sufficiently compensated by the stabilizing force of the relative price of consumption $1 / w$ as expressed by (3.9) for $w>0$, or by $w=0$.

Thus, in situations other than these three the interior equilibrium point $s^{*}$ is unstable. This leads to cases as in Figure 3.1 around $C$. However, what happens with such a situation when the hourly wage rate $w$ changes? This is analysed in Section 3.5 .

\subsection{Short and long-run wage elasticities}

As a necessary background for the analysis in Section 3.5, this section discusses the relations and differences between short-run and long-run effects on labour supply of changes in the wage rate $w$ when starting from a stable long-run equilibrium. This means that slopes of short-run labour supply curves $H\left(w ; Y_{O}, T, s^{*}\right)$ for fixed other income $Y_{O}$ and total discretionary time $T$ and fixed equilibrium values $s^{*}$ of the habit states are compared with slopes at the same $w$ of the long-run labour supply curve $H^{*}\left(w ; Y_{O}, T\right):=H\left(w ; Y_{O}, T, s^{*}\left(w ; Y_{O}, T\right)\right)$ for fixed $Y_{O}$ and $T$. The latter curve describes the total effect of a change in $w$ on a person's labour supply, when she (or he) has had the time to fully adjust her habits and behaviour to the new long-run equilibrium.

Suppose that the starting equilibrium describes labour supply $H^{*}$ on a forward-sloping (part of the) short-run labour supply curve. Then not too large a rise in $w$ will induce an increase in $H$, and hence an increase in consumption $X$ and a decrease in household time $L$. The person gets used to all these changes, so $s_{X}$ rises and $s_{L}$ declines, and this leads her to further increase $X$ and decrease $L$, so to increase $H$. Again she gets used to that, implying a further increase in $H$, etc., until a new and higher equilibrium $H^{*}$ is reached. When $w$ declines, the reverse story holds, and in general we can conclude that on a forward-sloping short-run labour supply curve the long-run effects on $H$ of a change in $w$ are larger than the short-run effects. The long-run labour supply curve would then be flatter than the short-run labour supply curves for fixed $s^{*}$.

However, for an $H^{*}$ on a backward-sloping short-run labour supply curve matters are different. Then a rise in $w$ will induce a decrease in $H$, and hence an increase in $L$. On the other hand, $X$ will also increase, provided total consumption $X$ is a nomal good, which is 
very plausible. ${ }^{11}$ The person gets used to both increases, so both $s_{L}$ and $s_{X}$ increase, but the former increase leads to a further decline in $H$, whereas the latter increase induces a rise in $H$. The sign of the total result of this rise and decline is ambiguous and, as a consequence, it is uncertain whether the long-run labour supply curve is flatter or steeper than the short-run curve and even whether its slope is backward or forward (see also Phlips, 1978, pp. 1026-1027, and Johnson and Pencavel, 1984, pp. 367 and 379).

More insight in the relations and differences between these short-run and long-run effects can be obtained by cifferentiating $H^{*}\left(w ; Y_{O}, T\right):=H\left(w ; Y_{O}, T, s^{*}\left(w ; Y_{O}, T\right)\right)$ to $w$ and elaborating it for non-zero $H^{*}$ to the following relation of long-run wage elasticities of labour supply $E_{w}^{H^{*}}$ and short-run wage elasticities $E_{\left.w\right|^{*}}^{H}$ at $s=s^{*}\left(w, Y_{O}, T\right)$ :

$$
E_{w}^{H^{*}}=\frac{E_{\left.w\right|^{*}}^{H}+\frac{X_{s_{X}}^{*}}{\delta_{X}}}{1-\left(\frac{X_{s_{X}}^{*}}{\delta_{X}}+\frac{L_{s_{L}}^{*}}{\delta_{L}}\right)}
$$

(see App. 3B). Here the denominator is positive, since the stability condition (3.3) is assumed to hold. Moreover it is smaller than one, making the multiplier larger than one. Then one easily derives:

Proposition 3.2 In the case of locally stable IHF with respect to consumption and household time

(i) $E_{w}^{H^{*}}$ is positive if and only if $E_{w l^{*}}^{H}$. is larger than $-X_{s_{X}}^{*} / \delta_{X}$,

(ii) $E_{w}^{H^{*}}$ is larger than $E_{w l^{*}}^{H}$ if and only if $E_{\left.w\right|^{*}}^{H}$, is larger than $-\left(X_{s_{X}}^{*} / \delta_{X}\right) /\left(X_{s_{X}}^{*} / \delta_{X}+L_{s_{L}}^{*} / \delta_{L}\right)$.

Surprisingly, the condition under (i) does not depend on the strength $L_{s}^{*} / \delta_{L}$ of the IHF with respect to household time. When it is fulfilled, the stability condition (3.3) implies that the condition under (ii) is also fulfilled. When the condition under (i) is not fulfilled, so when both the short-run and long-run labour supply curves are backward-sloping, it depends on the relative strengths of the IHFs with respect to consumption and household time whether the long-run curve is steeper or flatter than the short-run curve. Moreover, when $X_{s_{X}}^{*}=0$, both conditions are fulfilled only if the short-run labour supply curve is forwardsloping, and the reinforcement by the household time IHF makes the long-run labour supply curve always flatter than the short-run curve. On the other hand, when $L_{s_{L}}^{*}=0$, the lower limit in the condition under (ii) is equal to - 1 , and assuming that consumption is a normal good, it is easily shown that the condition is always fulfilled. This means that the consumption IHF reinforces the short-run effect on $H$ of a change in $w$ at a point where the

11. The derivative $\partial X / \partial w$ can be decomposed into a positive. cross-substitution effect and an income effect which is positive if $\boldsymbol{X}$ is a. nomal good. 
short-run labour supply curve slopes forward and counteracts the short-run effect on $H$ at a point where the short-run labour supply curve slopes backward. If the IHF is sufficiently strong so as to satisfy the condition under $(i)$, the counteraction may even lead to a forwardsloping long-run labour supply curve. ${ }^{12}$

\subsection{Catastrophes, hysteresis and quasi-hysteresis}

A crucial assumption implicit in the analysis of the previous section is that the relevant equilibrium of the state variables and labour supply remains (locally) stable and existing during a change in $w$. In a case of multiple equilibria as in Figure 3.1, however, this does not necessarily hold. Consider, for example, Figure 3.2 and assume that a rise in $w$ can take place on a forward-sloping (part of a) short-run labour supply curve for the whole range of the household time habit $s_{L}$ with household time $L<T$ (so labour supply $H>0$ ). Such a rise leads to an increase in $H$, and hence to a downward movement of the curve of $L\left(w, Y_{O}, T, s_{L}\right)$ as a function of $s_{L}$. Then the unstable equilibrium $C$ moves towards the stable equilibrium $B$. When $w$ keeps rising, $C$ may, at a certain $w^{* 1}$ coincide with $B$ into one unstable equilibrium and subsequently for $w>w^{* 1}$ disappear. Then only the stable equilibrium $A$ at low $L$ is left. Consequently, when the person finds her or himself in or near $B$ in the original situation of Figure 3.2 (so when the person is, for instance, a housewife or a "voluntarily unemployed" person), an infinitesimally small rise in $w$ beyond $w^{* 1}$ will drive the person in stages (see the previous section) to the equilibrium $A$ (so turning her/him into, for instance, a job-oriented woman or a workaholic). This large shift in $s_{L}, L$ and $H$ as a consequence of an infinitesimally small change in $w$ can be called an individual catastrophe in the sense of the catastrophe theory of Thom (1975).

A similar story can be told for declining $w$ on a forward-sloping short-run labour supply curve for the whole range of $s_{L}$ with $L<T$. Then $C$ and $A$ move towards each other, may, at a certain $w^{* 2}$, coincide into one unstable equilibrium, and subsequently for $w<w^{* 2}$ disappear. This leaves only one stable equilibrium $B$ and causes a catastrophic transition from the unstable equilibrium $A=C$ (e.g., job-oriented woman or workaholic) to $B$ (e.g., housewife or "voluntarily unemployed" person). ${ }^{13}$ Both this and the above transition in $s_{L}$ could be regarded as major changes in mentality. Note that by virtue of (3.2a) the vertical distances of the demand curves of $H$ for $w=w^{* 1}$ and $w=w^{* 2}$ to the equilibrium line $L=\delta_{L} s_{L}$ determine the speeds of these mentality shifts. In the present analysis the mentality shifts are triggered by changes in the wage rate, but they can also be induced by

${ }^{12}$ For $H^{*}=0$ the wage elasticities $E_{w}^{H}$. and $E_{w}^{H^{*}}$ are not defined, but then we can consider the short-run and long-run wage derivatives $\partial H /\left.\partial w\right|^{*}$ and $\partial H^{*} / \partial w$, respectively. See App. 3B.

${ }^{13}$ Note that this catastrophe goes together with the curve of $L\left(w, Y_{O}, T, s_{L}\right)$ becoming tangential at $w^{* 2}$ to the long-run equilibrium line $L=\delta_{L} s_{L}$. Then the denominator between brackets in (3.10) for $X_{s_{x}}^{*}=0$ becomes zero, making the long-run wage elasticities of labour supply infinitely large (cf. Becker and Murphy, 1988, Sec. V). However, in the case of the catastrophe at rising w, such a tangency situation does not occur. but $L_{s}^{*}$ at $B$ changes discontinuously from 0 for $w<w^{\bullet 1}$ to a certain positive value larger than $\delta_{L}$ for $w=w^{* 1}$. As a result of this discontinuity the marginal analysis for $X_{\vec{s}_{x}}^{*}=0$ and $H^{*}=0$ breaks down. 


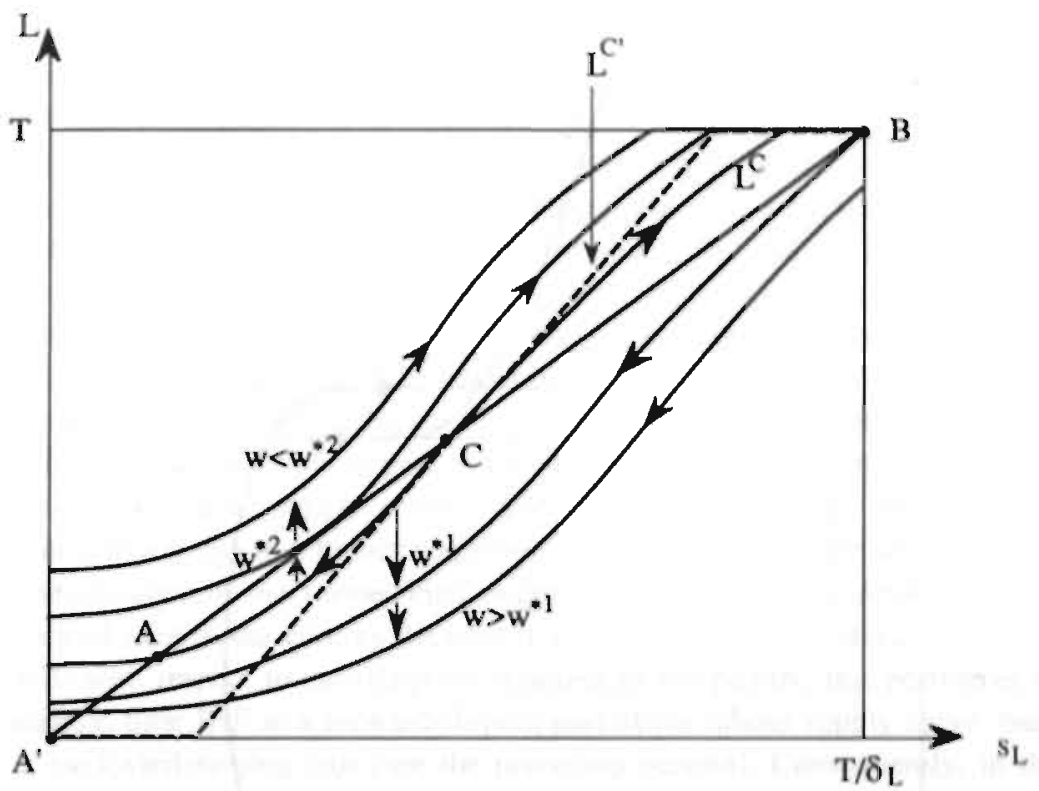

Figure 3.2. Non-linear household time demand functions for various wage rates $w$. The dashed line indicates the piecewise-linear approximation $L^{C^{\prime}}$ of the non-linear demand function $L^{C}$ around $C . A^{\prime}$ is a comer equilibrium for $L^{C^{\prime}}$.

changes in other exogenous variables like other income $Y_{O}$ and the rationings considered in Section 3.2 and even by changes in endogenous variables (see the end of this section). Such individual mentality shifts may have occurred (and still occur) in the "emancipation" process of individual married women from being a housewife towards being a job-oriented woman.

Comparing the two cases for rising and declining $w$, we see that in the latter case the catastrophe occurs at a wage $w^{* 2}$ which is lower than the wage $w^{* 1}$ at which the catastrophe in the former case occurs. This leads to a discontinuous forward-sloping part of the long-run labour supply curve as in Figure 3.3. This figure indicates that a person will enter the labour market at a relatively high reservation wage $w^{* 1}$, since she is used to much discretionary household time (high $s_{L}$ ), whereas she will leave employment at a lower wage $w^{* 2}$, since she is used to no discretionary household time and much corporate time $\left(s_{L}=0\right)$. Thus, Figure 3.3 implies that, in the long run, for the same value of $w$ between $w^{* 2}$ and $w^{* 1}$ a person can either prefer not to participate in the labour market or prefer to be employed (two stable equilibria), and that the alternative which is actually preferred depends on her (or his) history of previous household and corporate time experience. This is an example of individual hysteresis (cf. Sec. 3.2). In particular, it implies that a temporary rise of $w$ from $w^{* 2}<w<w^{* 1}$ to $w>w^{* 1}$ and back drives a non-participating person to 


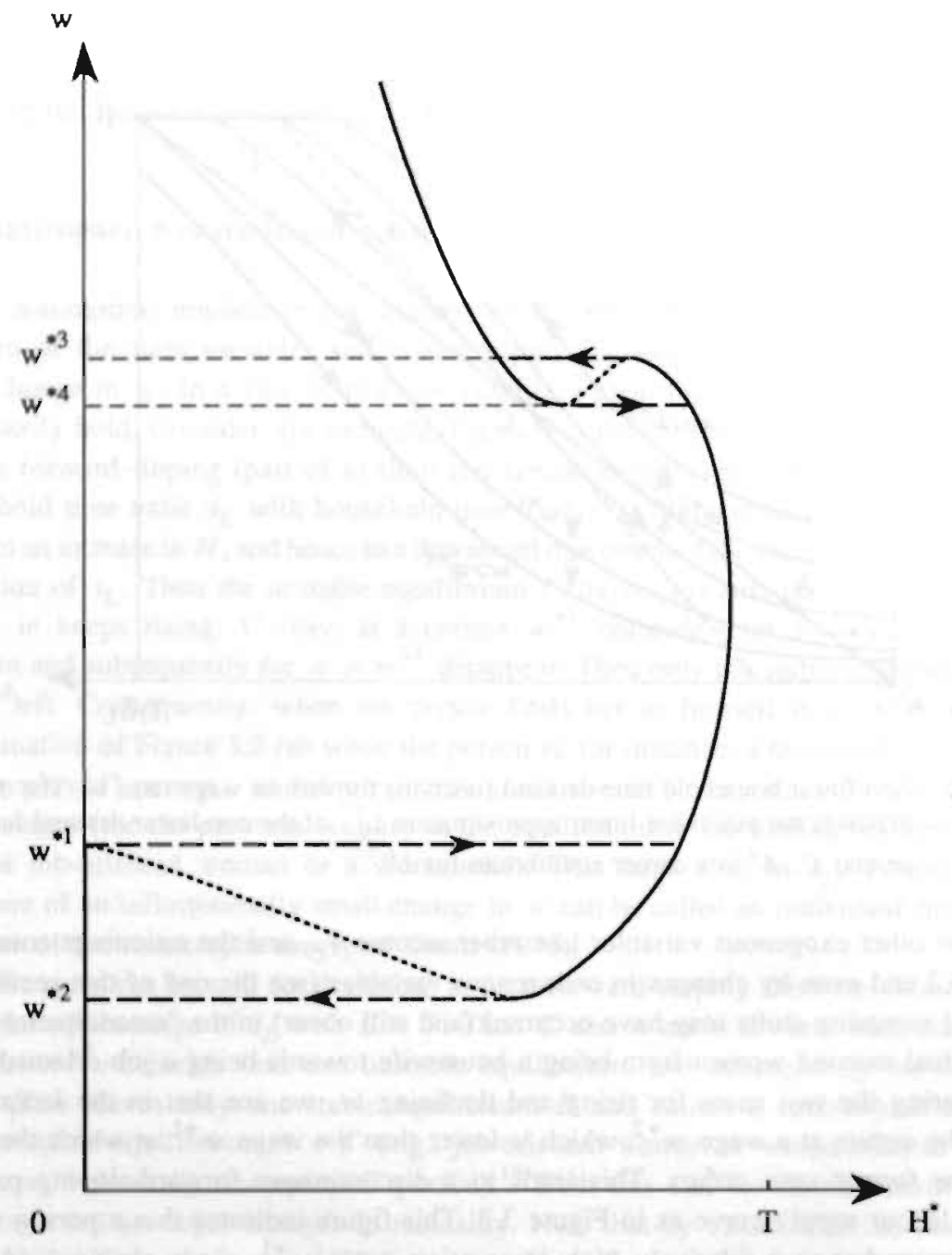

Figure 3.3. Backward-bending long-run labour supply curve with individual catastrophes and hysteresis. The dotted curves indicate the loci of the unstable equilibrium for $H$.

permanent labour market participation. ${ }^{14}$ Moreover, whereas one side of the individual hysteresis coin is persistence of non-participation and employment for $w<w^{* 1}$ and $w>w^{* 2}$, respectively (see Clark and Summers, 1982), the other side is catastrophic transitions between non-participation and employment beyond these ranges. More generally, the latter side does not seem to be recognized by the bulk of the recent (macro-economic) literature on

14 We do not make a distinction between labour market (or labour force) participation and employment because of our assumption of immediate realization of preferred labour supply (see Sec. 3.1). 
hysteresis in unemployment with its linear models (see, e.g., Blanchard and Summers, 1987, and Franz, 1990, Secs. 2 and 4.2). Note that individual hysteresis entails discontinuities in the long-run labour supply curve, even when, as in the present case, the short-run labour supply curves are continuous. ${ }^{15}$

In a similar way as described above for the case of a forward-sloping short-run labour supply curve, hysteresis also seems possible at changes in $w$ in a backward-sloping short-run labour supply curve (see, e.g., the upper half of Fig. 3.3). Then a rising $w$ leads to an upward movement of the curve of $L\left(w, Y_{O}, T, s_{L}\right)$ in Figure 3.2 and may, at a certain wage $w^{* 3}$, induce a catastrophic transition from more to less corporate work. Conversely, at a certain wage $w^{* 4}$ lower than $w^{* 3}$, a catastrophic transition from less to more corporate work may occur. Such individual catastrophes are likely to be smaller than individual catastrophes in the forward-sloping part of the labour supply curve, since in the former case a similar change in labour supply implies a relatively higher change in wage income and hence in consumption due to a higher wage rate. When extended to the social level (see Chs. 6 and 7), catastrophes of the former kind might happen in the now inelastic and persistent labour supply of men in the coming decades (e.g., from five to four days a week).

When IHF with respect to consumption is added to the picture, this reinforces the effect of the household time IHF in a forward-sloping part of the labour supply curve, but counteracts it in a backward-sloping part (see the preceding section). Consequently, in the former case the same individual hysteresis phenomena may occur as in the lower half of Figure 3.3, so being used to more or less consumption is then an additional cause of individual hysteresis in labour supply. On the other hand, in the latter case individual hysteresis seems only possible, when the steepening effect on the labour supply curve of the consumption IHF is weaker than the flattening effect of the household time IHF. If the former effect is stronger, it may even deprive the long-run labour supply curve of its backward-sloping part (see Prop. 3.2 and App. 3B).

In this section it has been assumed implicitly, that when $w$ keeps changing, the person has the time to (almost) fully adjust to the new values of $w$. In that case successive changes in $w$ occur at moments when the person is (in sufficiently good approximation) in a moving stable long-run equilibrium. In reality, however, $w$ may substantially change in nearly every period and the adjustment within one period could be very partial. Then the (ceteris paribus) actual path of labour supply can deviate considerably from the long-run labour supply curve. ${ }^{16}$ For instance, also in the absence of hysteresis in the long-run curve, a cyclical

${ }^{15}$ The discontinuities in the long-run $H^{*}$ and hence in $L^{*}$ and $X^{*}$ as funclions of $w$ should be distinguished sharply from the discontinuity in short-run consumption as a function of the habit state: variable as found by Becker and Murphy (1988, Sec. VII) for very strong IHF. This voluntary "cold turkey" phenomenon does not seem to entail hysteresis and cannot occur in our myopic IHF model, since voluntary cold turkey requires the person to be forward-looking (technically speaking: complex. roots of the differential equation(s) for the state variable(s), which are responsible for the phenomenon. cannot occur in the case of (3.2a) and (3.2b), see App. 3.1). However, enforced cold turkey like: the temporary rationings considered in Section 3.2 is certainly possible in our model. On the other hand, individual catastrophes and hysteresis as found in this section can also appear in the rationall IHF model of Becker and Murphy, but are not recognized by them.

${ }^{16}$ See Vendrik (1985,1986) for an analysis of similar deviations in the context of a dynamic model of adjustment costs and interrelated factor demands. 
variation in $w$ will cause actual labour supply to develop along a loop "around" the long-run curve, which looks like a hysteresis loop (cf. macroeconomic cycles in a Phillips curve as, in Hansen (1970)). This phenomenon is, however, essentially different from hysteresis, since hysteresis is a property of the evolution of multiple long-run equilibria, whereas we have here a disequilibrium phenomenon arising from a combination of short-run adjustment towards one long-run equilibrium and changes in an exogenous variable. Such a phenomenon could be called path-dependence of adjustment or quasi-hysteresis. It has already been observed by Marshall (1920, app. H, Sec. 3) and can, in contrast to hysteresis, be described by linear IHF models. ${ }^{17}$ Since, on a macro level, many models of hysteresis in unemployment describe a similar linear dynamics, they seem capable of explaining quasi-hysteresis, but not genuine hysteresis.

To make matters even more complex, quasi-hysteresis may go together with (individual) hysteresis. This results in a hysteresis figure like that of the ferromagnet in physics (Fig. 1 in Franz, 1990) in which the discontinuities in the long-run labour supply curve of Figure 3.3 have been "smoothed out". ${ }^{18}$ As a consequence, empirical discrimination of hysteresis as path dependence of equilibria from quasi-hysteresis as path dependence of adjustment seems difficult (but nevertheless important).

Finally, I remark that in the very long run catastrophic mentality shifts as found above dependent on the wage rate may be triggered by an endogenous long-run tendency to satiation with respect to household time and corporate time. This long-run satiation could be described by another and more slowly varying state variable than the habit state variables as in the overeating-dieting models of Bordley (1986) and Becker and Murphy (1988, Sec. VII). For a housewife or a "long-term unemployed" person accumulation of satiation with her/his large amount of househoid time may eventually lower her/his entry wage $w^{* 1}$ (see Fig. 3.3) under the prevailing market wage and induce her/him to enter the labour market. On the other hand, for an employee accumulation of satiation with working hard may in the end (of his/her life) raise his (her) exit wage $w^{* 2}$ above his market wage and impel him to retire from the labour market. ${ }^{19}$

\subsection{Discontinuous short-run labour supply schedules and hysteresis}

Although the model developed in the previous sections yields interesting theoretical insights, it can only be considered as a first step towards more realistic models. An important flaw of the model is its neglect of institutional or demand side rationings of labour supply other than

${ }^{17}$ Georgescu-Roegen (1971. Sec. 5.3) discusses hysteresis in general and speaks of the hysteresis effect upon the saving ratio as found by Duesenberry and Modigliani. However, in my view this effect is rather related to quasi-hysteresis.

18 Other. but related kinks of disequilibrium paths of consumption in the presence of multiple equilibria are considered by Becker and Murphy, 1988, Sec. VI.

${ }^{10}$ Connected in series such catastrophic transitions could even leadl to a kind of psychological long cycle of behaviour and mentality shifts. The mechanism would then be similar to that of the Kaldoir business cycle of Varian (1979) and George (1981) with the habit and satiation state variables and the behaviour variables comesponding to national income, the capital stock and net investment, respectively, in the latter model. 
the involuntary unemployment or labour enforcement considered in Section 3.2. Another shortcoming is that endogenous social security benefits are not taken into account. As shown in Subsections 2.2 .3 and 2.2.4, both complications lead to discontinuous short-run labour supply schedules like those in Figures 2.3 and 2.4. In terms of our terminology, these shorirun schedules display individual catastrophes, but not individual hysteresis. This means that in the short run the entry catastrophe occurs at the same value of the wage rate (and of other exogenous variables) as the exit catastrophe. On. the other hand, in the long run this property changes as IHF comes into play. In the following I give an analysis for the case of only household time IHF, an additive utility function and a two-points rationing at $H=0$ and $H=H^{R}$ as in Figure 2.4. Successively we consider the subcases without and with an endogenous social security benefit.

The additivity of the utility function $U\left(X, L ; s_{L}\right)$ implies that it can be written as the sum of a utility of consumption $U^{1}(X)$ and a utility of household time $U^{2}\left(L ; s_{L}\right)$. The reservation wage $\hat{w}_{r}$ is then that value of the wage rate for which the positive difference in consumption utility between having a job and staying at home $U^{1}\left(w H^{R}+Y_{O}\right)-U^{1}\left(Y_{O}\right)$ just compensates for the negative difference in household time utility $U^{2}\left(T-H^{R^{\prime}} ; s_{L}\right)-U^{2}\left(T ; s_{L}\right)$. The latter difference in household time utility is equal to the integral of the marginal household time utility $U_{L}^{2}\left(L ; s_{L}\right)$ over household time $L$ from $T-H^{R}$ to $T$. This implies that, since $U_{L}^{2}\left(L ; s_{L}\right)$ is an increasing function of the household time habit stock $s_{L}$, the difference in household time utility is more negative as $s_{L}$ is larger. In the long run the household time habit stock of a person who stays at home is equal to $T / \delta_{L}$, since she (or he) is then fully used to her maximall household time. On the other hand, when the same person works $H^{\hat{R}}$ corporate hours, her household time habit stock is, in the long run, equal to $s_{L}=\left(T-H^{R}\right) / \delta_{L}$ as she is fully used to her limited household time. Thus, when the person stays at home, the long-run household time habit stock is larger, and hence the long-run difference in household time utility between having a job and staying at home is more negative than when she has a job. Therefore, the compensating difference in consumption utility which determines the long-run reservation wage should be larger in the situation of non-participation, and this implies that the long-run reservation wage is higher in that situation. In other words, the long-run entry wage $\hat{w}^{* 1}$ is higher than the long-run exit wage $\hat{w}^{* 2}$. Thus, we obtain a discontinuous long-run labour supply schedule which resembles the lower half of Figure 3.3 and which describes individual hysteresis and separate individual entry and exit catastrophes.

The same reasoning applies in the subcase of an endogenous social security benefit (see Fig. 2.4), in which the difference in consumption utility between having a job and staying at home is given by $U^{1}\left(w H^{R}+Y_{G O}\right)-U^{1}\left(Y_{M}\right)$. More generally, the same result can be derived, when consumption IHF is added to the analysis and the utility function is non-additive, and in the non-rationing case of an endogenous social security benefit (see Fig. 2.3). However, the derivations are then much more complex and are not given here. In all cases we obtain a discontinuous long-run labour supply schedule like (the lower half of) Figure 3.3, but describing individual hysteresis even if the consumption and household time IHFs are only weak. Then the combination of discontinuous rationing or social security benefit and weak IHF(s) has the same kind of inertial effect on labour supply as strong JHF(s). Moreover, such a combination may be a more plausible explanation of individual hysteresis than strong $\operatorname{IHF}(\mathrm{s})$. 


\subsection{Concluding remarks}

This chapter has shown that, under certain conditions in terms of the strengths of IHFs with respect to consumption and household time (and corporate time), individual hysteresis and catastrophes dependent on rationings and the wage rate may occur. Moreover, it has been established that the consumption and household time IHFs have both reinforcing effects on a forward-sloping part of the labour supply curve, but reinforcing and counteracting effects, respectively, on a backward-sloping part. To obtain these results a general model has been analysed with consumption and household time demand functions time being non-linear in the habit state variables. Finally, a two-points labour supply rationing and an endogenous social security benefit have been shown to reinforce the effects of the IHFs.

In general, individual hysteresis and catastrophes presuppose the existence of an unstable interior long-run equilibrium for some range of the relevant exogenous variable such as the wage rate. In Sections 3.2 and 3.3 we have discussed stability conditions which determine when an interior equilibrium is unstable, but we did not consider conditions in terms of the underlying utility function and constraints under which an interior equilibrium exists. The analysis in Section 3.4 and 3.5 implies that such existence conditions may be fulfilled only for values of the wage rate above a long-run reservation wage or exit wage $\left(w^{* 2}\right.$ in Fig. 3.3). On the other hand, in situation (ii) of Proposition 3.1 in Section 3.3 such values may be so high as to stabilize the interior equilibrium and hence to exclude instability. Therefore, we should determine whether and, if so, when both existence and instability conditions for an interior equilibrium can be fulfilled.

However, even this combination of conditions is not sufficient for individual hysteresis and catastrophes as in Figure 3.3 to occur. In order to derive these phenomena we assumed in Section 3.5 that a rise in $w$ can take place on a forward-sloping (part of the) short-run labour supply curve for the whole range of the household time habit stock for which the household time demand is less than maximal. This implied a downward movement of the curve of the household time demand function in Figure 3.2. More importantly, we implicitly assumed that this continues to hold when $w$ keeps rising, allowing the stable equilibrium $B$ in Figure 3.2 successively to coincide with the unstable equilibrium $C$ for $w=w^{* 1}$ and to disappear for $w>w^{* 1}$. This has been shown to imply an entry catastrophe. However, it is also possible that before this can happen, the short-run labour supply curve for low values of the habit stock becomes backward-sloping. Then, for these habit stock values the household time demand function starts to move upwards at rising $w$, and in that situation the stable equilibrium $A$ in Figure 3.2 may successively coincide with the unstable equilibrium $C$ for $w=w^{* 3}$ and disappear for $w>w^{* 3}$ before the equilibrium $B$ can coincide with $C$ and disappear. In that peculiar case there can occur an exit catastrophe at $w=w^{* 3}$ from positive to zero corporate hours (cf. Fig. 3.3). but the equilibrium $B$ remains stable and an entry catastrophe does not occur (see Sec. 4.4 and App. 4B for details).

Conditions that exclude such cases as well as existence conditions for an interior equilibrium can be derived only for particular specifications of the utility function. An eligible candidate is the additive quadratic utility function of Houthakker and Taylor (1970). Therefore. the implications of this specification will be analysed in the next chapter. Necessary and sufficient conditions for individual hysteresis and catastrophes like those in Figure 3.3 
will be derived, and the additive quadratic specification will also be used to investigate aggregation problems.

Besides incorporating rationings and social security benefits, extensions to make the IHF models of this chapter and the next more realistic are adding intertemporal effects of savings (see, e.g., Becker and Murphy, 1988) and/or behavioural preference interdependence. The latter preference formation will be modelled in Chapter 5 and combined with the IHF models in Chapters 6 and 7.

\section{Appendix 3A Formal equilibrium and stability analysis}

The system of differential equations (3.2a) and (3.2b) can be written in matrix form as

$$
s_{t}=q\left(w_{t}, Y_{O t}, T_{t}, s_{t}\right)-D s_{t},
$$

where $s:=\left(s_{X}, s_{L}\right), q:=(X, L)$, and $D$ is a diagonal matrix with $\delta_{X}$ and $\delta_{L}$ on the diagonal. For constant $w_{t}=w, Y_{O t}=Y_{O}$ and $T_{t}=T$ a long-run stationary solution $s^{*}$ is obtained from the system of algebraic equations $q\left(w, Y_{O}, T, s^{*}\right)=D s^{*}$. When $q\left(w, Y_{O}, T, s^{*}\right)$ is linear in $s$, this system has generically, i.e. in all but accidental cases, at most one solution in the feasible domain, but when $q\left(w, Y_{O}, T, s^{*}\right)$ is piecewise-linear or otherwise nonlinear in $s$, the system may generically have more than one solution.

Linearizing (3A.1) around such a $s^{*}$ in the latter case, a necessary and sufficient condition for local stability of $s^{*}$ is that the eigenvalues of the matrix $Q_{s}\left(w, Y_{O}, T, s^{*}\right)-D$ have negative real parts. Denoting this matrix as $A:=Q_{s}^{*}-D$, its eigenvalues follow from the equation

$$
\operatorname{det}(A-\lambda I)=\lambda^{2}-\left(a_{11}+a_{22}\right) \lambda+a_{11} a_{22}-a_{12} a_{21}=0 .
$$

We can write this as $\lambda^{2}-b \lambda+c=0$ with

$$
\begin{aligned}
& b=a_{11}+a_{22}=X_{s_{X}}^{*}-\delta_{X}+L_{s_{L}}^{*}-\delta_{L}, \\
& c=a_{11} a_{22}-a_{12} a_{21}=X_{s_{X}}^{*} L_{s_{L}}^{*}-\delta_{L} X_{s_{X}}^{*}-\delta_{X} L_{s_{L}}^{*}+\delta_{X} \delta_{L}-X_{s_{L}}^{*} L_{s_{X}}^{*} .
\end{aligned}
$$

In the usual case where the income constraint is binding, differentiating the income and time constraints to $s_{X}$ and $s_{L}$ yields $X_{s_{X}}=-w L_{s_{X}}$ and $X_{s_{L}}=-w L_{s_{L}}$. This implies that the term $-X_{s_{L}}^{*} L_{s_{X}}^{*}$ in (3A.4) is equal to $-X_{s_{X}}^{*} L_{s_{L}}^{*}$, so $c=-\delta_{L} X_{s_{X}}^{*}-\delta_{X} L_{s_{L}}^{*}+\delta_{X} \delta_{L}$. The solutions for the eigenvalues can then be written as $\lambda_{1,2}=\frac{1}{2} b \pm \frac{1}{2} \sqrt{b^{2}-4 c}$. Substituting (3A.3) and (3A.4), it can easily be derived that the discriminant $b^{2}-4 c$ is equal to $\left(a_{11}-a_{22}\right)^{2}+4 X_{s_{L}}^{*} L_{s_{X}}^{*}$. The former term at the right-hand side is non-negative and the latter term is equal to $4 X_{s X}^{*} L_{s_{L}}^{*}$ (see above), and hence positive. Therefore, the discriminant is positive, implying that both the eigenvalues $\lambda_{1}$ and $\lambda_{2}$ are real. Then the stability condition says that $\lambda_{1}$ and $\lambda_{2}$ must be negative. As easily seen, this holds if and only if $b<0$ and $c>0$. 
Substituting (3A.4) into the latter condition, dividing it by the positive $\delta_{X} \delta_{L}$ and rearranging terms, the condition becomes inequality (3.3). This stability condition is not only necessary, but also sufficient, since it implies the condition $b<0$ as follows. First, suppose $\delta_{X} \geq \delta_{L}$. Multiplying (3.3) by $\delta_{X}$ we get $X_{s_{X}}^{*}+\left(\delta_{X} / \delta_{L}\right) L_{s_{L}}^{*}<\delta_{X}$. This implies $X_{s_{X}}^{*}+L_{s_{L}}^{*}<\delta_{X}+\delta_{L}$, and hence $b<0$ (see (3A.3)). When $\delta_{X}<\delta_{L}$, we should multiply (3.3) by $\delta_{L}$ and get the same result. Putting $X_{s_{X}}^{*}=0$ leads to the stability condition $L_{s_{L}}^{*}<\delta_{L}$.

As a generalization of Figure 3.1, stable and/or unstable equilibria can be described as intersections of an equilibrium line in the $\left(s_{L}, s_{X}, L\right)$ space and a two-dimensional surface of the function $L\left(s_{L}, s_{X}\right)$. The equilibrium line is determined by the equilibrium conditions $L=\delta_{L} s_{L}$ and $w(T-L)+Y_{O}=X=\delta_{X} s_{X}$. Along this line we may then have a same kind of situation as in Figure 3.1 with two stable polar equilibria $A$ and $B$ and one unstable intermediate equilibrium $C$.

\section{Appendix 3B Derivation of relations between short and long-run wage effects}

Differentiating $H^{*}\left(w ; Y_{O}, T\right):=H\left(w ; Y_{O}, T, s^{*}\left(w ; Y_{O}, T\right)\right)$ to $w$ yields

$$
\frac{\partial H^{*}}{\partial w}=\left.\frac{\partial H}{\partial w}\right|^{*}+\left.\frac{\partial H}{\partial s_{X}}\right|^{*} \frac{\partial s_{X}^{*}}{\partial w}+\left.\frac{\partial H}{\partial s_{L}}\right|^{*} \frac{\partial s_{L}^{*}}{\partial w},
$$

where "I" means that the "short-run derivatives" of $H\left(w, Y_{O}, T, s\right)$ are evaluated at $s=s^{*}\left(w, Y_{O}, T\right)$. Substituting $\partial s_{X}^{*} / \partial w=\left(\partial X^{*} / \partial w\right) / \delta_{X}=\left(w \partial H^{*} / \partial w+H^{*}\right) / \delta_{X}$, $\partial s_{L}^{*} / \partial w=\left(\partial L^{*} / \partial w\right) / \delta_{L}=-\left(\partial H^{*} / \partial w\right) / \delta_{L}, \quad w \partial H /\left.\partial s_{X}\right|^{*}=\partial X /\left.\partial s_{X}\right|^{*}=: X_{s_{X}}^{*}$, and $-\partial H /\left.\partial s_{L}\right|^{*}=L_{s_{L}}^{*}$ into (3B.1), it follows that

$$
\frac{\partial H^{*}}{\partial w^{*}}=\frac{\left.\frac{\partial H}{\partial w}\right|^{*}+\frac{H^{*} X_{s_{X}}^{*}}{w \delta_{X}}}{1-\left(\frac{X_{s_{X}}^{*}}{\delta_{X}}+\frac{L_{s_{L}}^{*}}{\delta_{L}}\right)} .
$$

For positive $H^{*}$ we can multiply the right and left-hand sides of (3B.2) by $w / H^{*}$ yielding (3.10).

When $H^{*}=0$, the second term of the numerator on the right-hand side of (3B.2) is zero. If $w$ is below the short-run reservation wage $w_{r}$. the short-run wage effect $\partial H /\left.\partial w\right|^{*}$ is also zero, implying that the long-run wage effect $\partial H^{*} / \partial w$ is zero as well. If $w$ is equal to $w_{r}$, the short-run effect is positive. Since the multiplier in (B3.2) is positive and larger than one, it then follows that the long-run effect is also positive (so the long-run reservation wage is equal to the short-run reservation wage) and larger than the short-run effect. 


\section{CHAPTER 4}

\section{Individual Habit Formation: Additive Quadratic Model and Aggregation}

"Everything should be made as simple as possible, but not simpler."

Einstein

In this chapter the general analysis which has been given in the preceding chapter is specialized to the particular case of the additive quadratic model (AQM) of Houthakker and Taylor (1970). This specification serves three purposes. First, it provides an illustration of the general analysis. Second and more importantly, it allows a derivation of necessary and sufficient conditions for individual hysteresis and catastrophes like those in Figure 3.3 to occur. These conditions consist not only of conditions for instability of an interior long-run equilibrium, but also of conditions for existence of such an equilibrium as well as another condition, which excludes certain cases (see Sec. 3.7). Thirdly, the specification is used to analyse aggregation problems. The $\mathrm{AQM}$ is chosen despite its less favourable performance than the linear expenditure system (LES) in the empirical consumption studies of Taylor and Weiserbs (1972) and Weiserbs (1974), since it allows in contrast to the LES a backwardbending labour supply curve and the, in my view, plausible possibilities of negative marginal utilities of large amounts of household time and consumption.

This chapter is organized as follows. First, Sections $4.1-4.4$ analyse the short and longrun effects on individual labour supply of individual habit formation (IHF) with respect to consumption and household time. Section 4.1 derives possible short-run labour supply curves. Section 4.2 explains how, in the long run, individual hysteresis and catastrophes in a forward-sloping part of the labour supply curve like those in Figure 3.3 can arise in the AQM. In preparation to deriving necessary and sufficient conditions for such phenomena, Section 4.3 considers the standard case of an always stable unique long-run equilibrium and derives for that case long-run labour supply curves of the same type as corresponding shortrun curves. However, cases of stable as well as unstable multiple equilibria are also possible and are analysed in Section 4.4, which classifies all possible cases. On the basis of this classification we can then formulate necessary and sufficient conditions for individual

${ }^{1}$ Such an AQM specification is also used by Becker and Murphy (1988) in the context of rational habit formation with respect to consumption. 
hysteresis and catastrophes like those in Figure 3.3. Moreover, as boundary cases of these conditions the existence and properties of so-called bifurcation points are demonstrated. Section 4.5 adds IHF with respect to corporate time to the short and long-run models. It is shown that this IHF can describe a weak and strong variant of "workaholism" as well as related cases. Section 4.6 shows that the general long-run behaviour can be rationalized as the result of the constrained maximization of an additive quadratic "long-run utility function". However, it is demonstrated that in the case of two (or more) stable long-run equilibria this "long-run utility function" is not globally, but locally maximized. This property is crucial for the existence of hysteresis, but is not recognized in the usual long-run utility function approach (see, e.g., Anderson and Blundell, 1982).

In order to trace the macro-economic implications of the short and long-run individual labour supply behaviours thus derived, these behaviours should be aggregated over individuals. This is done in Sections 4.7 and 4.8 under various assumptions regarding the individual variation in utility function parameters, exogenous variables and habit stocks. In the case of long-run labour supply, hysteresis and catastrophes in individual labour supply turn out to imply hysteresis, but no catastrophes in average (i.e. per capita) labour supply. Moreover, the aggregation leads to a continuum of possible long-run equilibria within the hysteresis loop. Again, the equilibrium which is reached depends on the development of exogenous variables like the wage rate in the past. Finally, Section 4.9 concludes.

\subsection{Short-run labour supply}

In terms of aggregate consumption $X$ and discretionary household time $L$ the AQM assumes a quadratic specification of the utility function $U\left(X, L ; s_{X}, s_{L}\right)$ which is additive(ly separable) in $X$ and the consumption habit stock $s_{X}$ on the one hand and $L$ and the household time habit stock $s_{L}$ on the other hand:

$$
\begin{aligned}
U\left(X, L ; s_{X}, s_{L}\right)= & \alpha_{X} X+\beta_{X} s_{X}+\frac{1}{2} \alpha_{X X} X^{2}+\frac{1}{2} \beta_{X X} s_{X}^{2}+\gamma_{X X} X s_{X}+ \\
& \alpha_{L} L+\beta_{L} s_{L}+\frac{1}{2} \alpha_{L L} L^{2}+\frac{1}{2} \beta_{L L} s_{L}^{2}+\gamma_{L L} L s_{L}
\end{aligned}
$$

In the usual case of not fully satiating consumption at the optimal point, in which the income constraint is binding, a specification of the short-run labour supply function $H\left(w, Y_{O}, s_{X}, s_{L}\right)$ can be derived from the first-order condition $w U_{X}-U_{L}=0$ (see (2.15)). Therefore we differentiate (4.1) to $X$ and $L$, yielding the linear expressions

$$
\begin{aligned}
& U_{X}\left(X ; s_{X}\right)=\alpha_{X}+\alpha_{X X} X+\gamma_{X X} s_{X} . \\
& U_{L}\left(L ; \hat{s}_{L}\right)=\alpha_{L}+\alpha_{L L} L+\gamma_{L L} s_{L} .
\end{aligned}
$$

Thus, as a consequence of the additive separability of (4.1), $U_{X}$ does not depend on $L$ and $s_{L}$, and $U_{L}$ does not depend on $X$ and $s_{X}$. Moreover, note that the $\beta$-parameters in (4.1) are absent in (4.2a) and (4.2b). and hence in the first-order condition and the labour supply 
function. Accordingly, for each pair of fixed values of $s_{X}$ and $s_{L}$, the $\beta$-terms in the utility function (4.1) of $X$ and $L$ can be transformed away by an order-preserving translation of this function. ${ }^{2}$ This leaves us with a utility function with six parameters only.

These parameters have the following meanings and signs. According to (4.2a) the parameter $\alpha_{X}$ is equal to $U_{X}(0 ; 0)$, i.e. the marginal consumption utility at zero levels of consumption $X$ and consumption habits $s_{X}$. This marginal utility will be positive, and the parameter $\alpha_{X}$ is called the consumption taste. Analogously, the parameter $\alpha_{L}=U_{L}(0 ; 0)$ in $(4.2 b)$, i.e. the marginal household time utility at zero levels of discretionary household time $L$ and household time habits $s_{L}$, will be positive and is called the household time taste. The parameters $\alpha_{X X}$ and $\alpha_{L L}$ are equal to the second-order derivatives $U_{X X}$ and $U_{L L}$, respectively, and are assumed to be negative (see Sec. 3.3). This implies diminishing marginal utilities of consumption and (discretionary) household time (see footnote 48 in $\mathrm{Ch} .2$ ). Finally, the parameters $\gamma_{X X}=U_{X s_{X}}$ and $\gamma_{L L}=U_{L s_{L}}$ are positive (see Sec. 3.3) and indicate the extents to which the marginal utilities of consumption and household time are raised by increases in the corresponding habit stocks. ${ }^{3}$ They are called the consumption habit sensitivity and household time habit sensitivity.

In order to solve the short-run labour supply $H$ from the first-order condition, we substitute the standard constraints into $U_{X}\left(X ; s_{X}\right)$ and $U_{L}\left(L ; s_{L}\right)$ so that the first-order condition becomes

$$
w U_{X}\left(w H+Y_{O} ; s_{X}\right)-U_{L}\left(T-H ; s_{L}\right)=0 \text {. }
$$

Next, it can easily be derived from (4.2a) and (4l.2b) that

$$
U_{X}\left(w H+Y_{O} ; s_{X}\right)=U_{X}\left(Y_{O} ; s_{X}\right)+\alpha_{X X} w H
$$

and

$$
U_{L}\left(T-H ; s_{L}\right)=U_{L}\left(T ; s_{L}\right)-\alpha_{L L} H .
$$

Substituting (4.4a) and (4.4b) into (4.3), a straightforward derivation yields

$$
H=\frac{a w-b}{c w^{2}+d},
$$

where

${ }^{2}$ See Taylor (1974). However, this equivalence translation does not hold for rational IHF, since then the state variables are also choice variables. In that case the $\boldsymbol{\beta}$-terms determine together with the $\gamma$-terms whether the IHF's are beneficial or harmful (positive or negative $U_{s_{X}}$ and $U_{s_{L}}$, see Becker and Murphy, 1988, p. 684), and affect the behaviour. In connection with this, the equivalence translation can also not be applied in the context of welfare comparisons over time or between persons when the habit stocks at different points in time or of different persons have different values.

${ }^{3}$ When the state variables $s_{X}$ and $s_{L}$ would represent the effects of individual long-run satiation as considered at the end of Section 3.5, the parameters $\gamma_{X X}$ and $\gamma_{L L}$ would be negative. 


$$
\begin{aligned}
& a:=U_{X}\left(Y_{O} ; s_{X}\right)=\alpha_{X}+\alpha_{X X} Y_{O}+\gamma_{X X} s_{X}, \\
& b:=U_{L}\left(T ; s_{L}\right)=\alpha_{L}+\alpha_{L L} T+\gamma_{L L} s_{L}
\end{aligned}
$$

(see (4.2a) and (4.2b)), $c:=-\alpha_{X X}$ and $d:=-\alpha_{L L}$. Thus, the "parameter" $a$ represents the marginal utility of consumption at given habit stock, when one is not employed $\left(X=Y_{O}\right)$. Analogously, the "parameter" $b$ denotes the marginal utility of household time at given habit stock, when one is not employed $(L=T){ }^{4}$ The denominator $c w^{2}+d$ in $(4.5)$ is positive for all $w$, since both diminishing marginal utility parameters $c=-\alpha_{X X}$ and $d=-\alpha_{L L}$ are positive. ${ }^{5}$ Furthermore, (4.5) should, of course, satisfy the restrictions $0 \leq H \leq T$, so holds provided $0 \leq a w-b \leq T\left(c w^{2}+d\right)$.

What can we say on the signs of $a$ and $b$ ? Since the left-hand side of (4.4a) is positive for any optimal $H$ by virtue of the above assumption of not fully satiating consumption at the optimal point, and since the second term on the right-hand side of (4.4a) is non-positive, $a:=U_{X}\left(Y_{O}, s_{X}\right)$ should be positive. This imposes restrictions on (4.6a) in the sense that the positive first and third terms on the right-hand side of (4.6a) should dominate the negative second term (the feasible domain of $s_{X}$ is defined by $s_{X} \geq Y_{O} / \delta_{X}>0$ ). Usually this holds, but the reverse can also be true and then we have a non-positive $a$. To that case we will come back below.

In the present case of a positive $a$ the restriction $a w-b \geq 0$ mentioned above implies $w \geq b / a$. This lower limit of $w$ represents the reservation wage $w_{r}$. Usually the marginal household time utility $b:=U_{L}\left(T ; s_{L}\right)$ is positive, implying a positive reservation wage $w_{r}=b / a$. However, analogously to the case of a positive $a$, this imposes non-trivial restrictions on (4.6b) in the sense that the positive first and third terms should dominate the negative second term. Usually this holds, but the reverse can also be true, implying a nonpositive $b$ and hence a non-positive reservation wage $w_{r}=b / a$. Then the marginal household time utility at a maximal level of household time $b$ is non-positive. This seems rather exceptional, but when "psychic income" of corporate time is added to the AQM as done in Section 4.5 , it becomes more plausible. This case of a non-positive $b$ will be analysed in Section 4.5.

Thus, we are left here with the case that $c, d, a$ and $b$ are all positive. In this case the above-mentioned restriction $H \leq T$ on (4.5) can be shown to be not binding for any (nonnegative) $w$, when $c$ is larger than or equal to a certain positive value $c_{o}$ (which depends on other quantities, but not on $d$ ), so when the marginal utility of consumption diminishes sufficiently fast (relative to other quantities). ${ }^{6}$ This subcase represents an example of the standard model as described in Section 2.1 and leads to a backward-bending labour supply curve: for sufficiently low $w>w^{r}=b / a$ the numerator in (4.5) dominates, whereas for

${ }^{4}$ Note that $a w-b=0$ represents the first-order condition for $H=0$.

5 Positivity of $\mathrm{cw}^{2}+d$ represents the second-order condition $(2.16)$ will $U_{X i}=0$.

${ }^{6}$ Proots of this and following results for short-run labour supply are straightforward and analogous to proofs of similar results for long-run labour supply. which are given in Section 4.3 and Appendix 4.A. The latter results are formulated in Section 4.3 in a more explicit way than the former results in the present section. 
higher $w$ the denominator dominates (and, for $w$ going to infinity, drives $H$ to zero without ever reaching it).

However, when $c$ is smaller than $c_{o}$, so when the person is relatively little satiable in her (or his) consumption, the restriction $H \leq T$ on (4.5) is binding for $w$ between certain positive $w_{-}$and $w_{+}$(cf. Sec. 4.3). For these $w$ we have comer solutions $H=T$ leading to a backward-bending labour supply curve with an inelastic segment for $H=T$.

In contrast, in the case of a non-positive $a(4.4 \mathrm{a})$ implies that consumption is satiating at any optimal $H$. Again $b$ may be either positive or non-positive and the latter case will be considered in Section 4.5. The case of a non-positive $a$ and a positive $b$ is an example of the more general case of fully satiating consumption and not fully satiating household time as analysed in Subsection 2.2.5. There it has been shown that the labour supply curve then coincides with the vertical $H=0$ axis. This is consistent with the fact that in this example the first-order condition $U_{L}=0$ for an interior labour supply optimum leads, by virtue of (4.4b), to $L=b / \alpha_{L L}=-b / d$, which is smaller than 0 .

\subsection{Catastrophes and hysteresis}

In order to analyse the long-run labour supply behaviour which is implied by the AQM we consider the short-run labour supply function (4.5) as a function of the habit stocks $s_{X}$ and $s_{L}$. Since the "parameters" $a$ and $b$ in (4.5), as given by (4.6a) and (4.6b), are linear in $s_{X}$ and $s_{L}$, respectively, labour supply $H$ and hence household time demand $L$ and consumption demand $X$ are also linear in $s_{X}$ and $s_{L}$ for $0<H<T$. ${ }^{7}$ As a consequence, $X$ and $L$ as functions of $s_{X}$ and $s_{L}$ can represent linear or piecewise-linear demand functions like $L^{A}$ and $L^{B}$ in Figure 3.1 in Section 3.2 for the case of individual habit formation (IHF) with respect to household time only. However, they cannot represent non-(piecewise-) linear demand functions like $L^{C}$ in Figure 3.1 and can at best serve as piecewise-linear approximations of such functions. Such an approximation is depicted in Figure 3.2 in Section 3.5 as $L^{C^{\prime}}$ for the case of household time IHF only. This $L^{C^{\prime}}$ is also represented as the middle household time demand function in Figure 4.1.

Just as $L^{C}$, its approximation $L^{C^{\prime}}$ describes an unstable intermediate equilibrium at $C$, since the slope $L^{C^{\prime}}{ }_{s_{L}}$ of the middle linear piece of $L^{C^{\prime}}$ is $\operatorname{larger}$ than $\delta_{L}$. Using $L=T-H$, (4.5) and (4.6b), this sufficient condition for instability can be expressed in terms of the AQM parameters and the wage rate as

$$
\frac{\gamma_{L L}}{\left|\alpha_{X X}\right| w^{2}+\left|\alpha_{L L}\right|}>\delta_{L} .
$$

This condition says that the household time habit sensitivity $\gamma_{L L}$ should be sufficiently strong to offset the stabilizing effects of the diminishing marginal utilities and the wage rate: as well as of the depreciation rate. The condition is equivalent to the negation of the stability

7 This also holds for the specifications used by Phelps (1978), Johnson and Pencavel (1984), and Kapteyn and Woittiez (1990). 


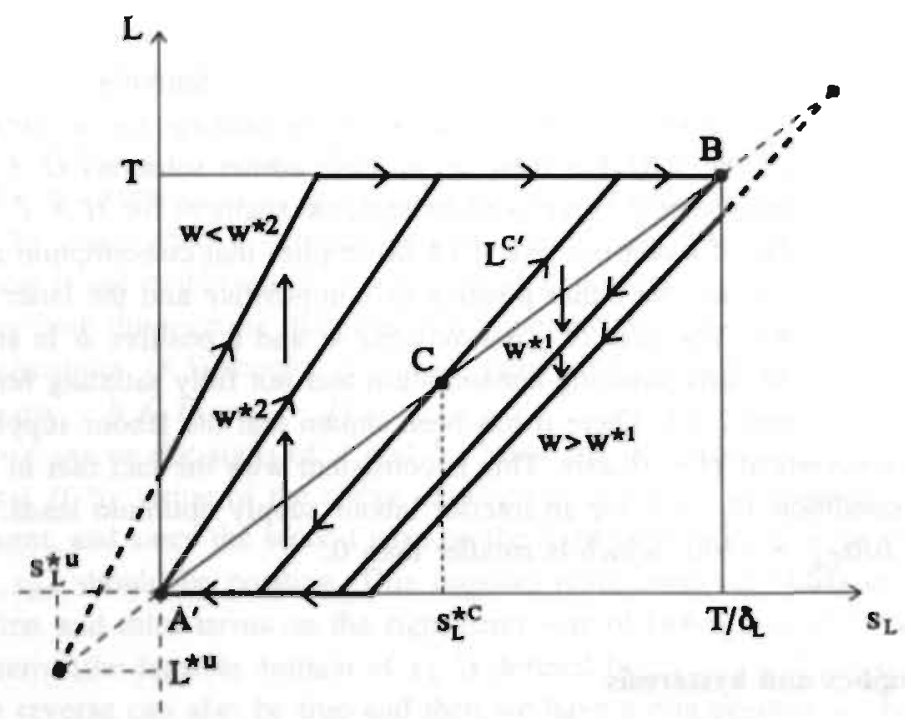

Figure 4.1 Piecewise-linear household time demand functions in the AQM for various wage rates. At $w^{* 1}$ an eniry catastrophe occurs and at $w^{* 2}$ an exit catastrophe.

condition (3.5) in Section 3.3 for the present AQM case with $U_{X_{S_{X}}}^{*}=0$ (except for an equality sign in (4.7)).

Furthermore, just as the non-linear $L^{C}$, the piecewise-linear $L^{C^{\prime}}$ also describes two stable polar equilibria $\left(A^{\prime}\right.$ and $B$ ), but now these equilibria are both comer equilibria. For many corporate workers the corner equilibrium $A^{\prime}$ with $L^{*}=0$ and $H^{*}=T$ can be a good approximation, but for less single-minded job holders like married women with a part-time job this may not hold (see Sec. 3.2), implying that $A^{\prime}$ is not a good approximation in those cases. $^{8}$ Analogously to the analysis in Section 3.2 , the middle situation in Figure 4.1 can be shown to lead to individual hysteresis with $H^{*}=0$ or $H^{*}=T$ as a result of a temporary rationing of labour supply in the past (involuntary unemployment or labour enforcement). Moreover, in a similar way as in the non-linear model of Section 3.5 changes in the wage rate $w$ may lead to individual hysteresis and individual entry and exit catastrophes in a forward-sloping part of the labour supply curve. This can be explained by considering Figure 4.1.

Assume that a rise in $w$ can take place on a forward-sloping (part of a) short-run labour supply curve for the whole range of the household time habit stock $s_{L}$ for which the household time demand $L$ is less than maximal (so for which the labour supply $H$ is positive). Such a rise leads to an increase in $H$, and hence to a downward movement of the middle

${ }^{8}$ Note, however. that the total discretionary fime $T$ does not generally coincide with the standard full-time working week, but may be larger or smaller. In the latter case $H^{\bullet}=T$ comesponds to a part-time job, but such a case in which a married woman spends all her discretionary time in a parttime job, seems in general implausible. 
segment of the household time demand function $L^{C^{\prime}}$ in Figure 4.1. ${ }^{9}$ Then the unstable equilibrium $C$ moves towards the stable comer equilibrium $B$. When $w$ keeps rising, $C$ may, at a certain $w^{* 1}$, coincide with $B$ into one unstable comer equilibrium, and subsequently for $w>w^{* 1}$ disappear outside the feasible domain. Then only the stable corner equilibrium $A^{\prime}$ with $L^{*}=0$ and $H^{*}=T$ is left. Consequently, when the person finds him or herself in or near $B$ in the original situation of Figure 4.1. (so when the person is, for instance, "voluntarily unemployed"), an infinitesimally small rise in $w$ beyond $w^{* 1}$ will drive the person in stages to the long-run equilibrium $A^{\prime}$. This represents an individual entry catastrophe from $L^{*}=T$ and $H^{*}=0$ to $L^{*}=0$ and $H^{*}=T$ at $w=w^{*}$, which describes how an unemployed person takes a job, gets used to that, works more corporate hours, gets used to that, etc., and finally develops into a motivated employee working maximall corporate hours (cf. Secs. 3.4 and 3.5).

An analogous story can be told for declining $w$ on a forward-sloping short-run labour supply curve for the whole range of the household time habit stock $s_{L}$ for which the household time demand $L$ is less than maximal. Then the middle segment of the household time demand function $L^{C^{\prime}}$ in Figure 4.1 moves upwards, so that the unstable equilibrium $C$ moves towards the stable corner equilibrium $A^{\prime}$. When $w$ keeps declining, $C$ may, at a certain $w^{\bullet 2}$, coincide with $A^{\prime}$ into one unstable corner equilibrium, and subsequently for $w<w^{* 2}$ disappear outside the feasible domain. This leaves only one stable comer equilibrium $B$ and induces an individual exit catastrophe from the unstable comer equilibrium $A^{\prime}=C$ with $H^{*}=T$ to $B$ with $H^{*}=0$, describing how an employee working maximal corporate hours starts to work fewer and fewer hours and finally develops into a "voluntarily unemployed" person.

Comparing the two cases we see that the exit catastrophe occurs at an exit wage $w^{* 2}$ which is lower than the entry wage $w^{* 1}$ at which the entry catastrophe occurs. This leads to a discontinuous forward-sloping part of the long-run labour supply curve as in Figure 4.2. In the long run we then have a situation in which for the same value of $w$ between $w^{* 2}$ and $w^{* 1}$ a person can either prefer to be non-participating or prefer to be employed (two stable equilibria) and in which the alternative which is actually preferred depends on his/her history of previous household and corporate time experience. This is an example of individual hysteresis (see Sec. 3.5). In a similar way as described above for the case of a forwardsloping short-run labour supply curve, individual hysteresis and catastrophes also seem possible at changes in $w$ in a backward-sloping short-run labour supply curve. However, Section 4.4 will derive that the AQM excludes this possibility, i.e., a backward-sloping part of a long-run labour supply curve is always continuous as in Figure 4.2.

When consumption IHF is added to the picture, this reinforces the effect of household time IHF in a forward-sloping part of the labour supply curve, but counteracts it in a backward-sloping part (see Sec. 3.4). Consequently, in the former case the same individual hysteresis and entry and exit catastrophes may occur as in Figure 4.2, so being used to more or less consumption then is an additional cause of these phenomena. On the other hand, the exclusion of such phenomena in a backward-sloping part of the labour supply curve by the $\mathrm{AQM}$ is only corroborated by the addition of the consumption IHF. Moreover, as shown in

${ }^{9}$ The slope of the segment as given by the left-hand side of $(4.7)$ then also declines. 


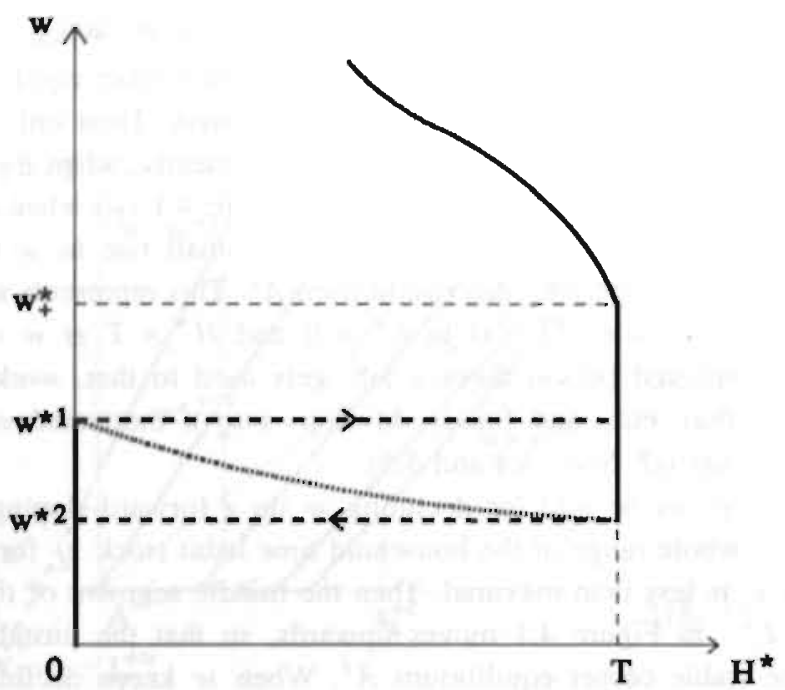

Figure 4.2 Long-run labour supply schedule with individual hysteresis and catastrophes in the AQM. The dotied curve indicates the loci of the unstable equilibrium for $H$.

Section 4.4, a strong consumption IHF (see Sec. 3.3) even deprives the long-run labour supply curve of its backward-sloping part (see Prop. 3.2 in Sec. 3.4).

The rather intuitive analysis of individual hysteresis and individual entry and exit catastrophes in this section leaves us with the question what are necessary and sufficient conditions for such phenomena to occur in the AQM case of IHF with respect to both household time and consumption. This question will be answered in Section 4.4.

\subsection{Always stable long-run case}

As a necessary basis for the analysis in Section 4.4, this section considers the standard case that the unique interior long-run equilibrium for labour supply is stable (like the equilibrium $A$ as described by the linear demand function $L^{A}$ in Fig. 3.1 in Sec. 3.2). When consumption is not (fully) satiating at any optimal point, a specification of the long-run labour supply function $H^{*}\left(w, Y_{O}\right):=H\left(w, Y_{O}, s^{*}\left(w, Y_{O}\right)\right)$ is then obtained by substituting $H^{*}$ for $H, s_{X}^{*}=X^{*} / \delta_{X}=\left(w H^{*}+Y_{O}\right) / \delta_{X}$ for $s_{X}$ and $s_{L}^{*}=L * / \delta_{L}=\left(T-H^{*}\right) / \delta_{L}$ for $s_{L_{*}}$ in the first-order condition (4.3). Next, analogously to (4.4a) and (4.4b), it can easily derived from $(4.2 a)$ and $(4.2 b)$ that 


$$
\begin{aligned}
& U_{X}\left(w H^{*}+Y_{O} ; \frac{w H^{*}+Y_{O}}{\delta_{X}}\right)=U_{X}\left(Y_{O} ; \frac{Y_{O}}{\delta_{X}}\right)+\alpha_{X X} w H^{*}+\gamma_{X X} \frac{w H^{*}}{\delta_{X}}= \\
&=U_{X}\left(Y_{O} ; \frac{Y_{O}}{\delta_{X}}\right)+\left(\alpha_{X X}+\frac{\gamma_{X X}}{\delta_{X}}\right) w H^{*} \\
& \begin{aligned}
U_{L}\left(T-H^{*} ; \frac{T-H^{*}}{\delta_{L}}\right) & =U_{L}\left(T ; \frac{T}{\delta_{L}}\right)-\alpha_{L L} H^{*}-\gamma_{L L} \frac{H^{*}}{\delta_{L}}= \\
& =U_{L}\left(T ; \frac{T}{\delta_{L}}\right)-\left(\alpha_{L L}+\frac{\gamma_{L L}}{\delta_{L}}\right) H^{*} .
\end{aligned}
\end{aligned}
$$

The expressions in (4.8a) and (4.8b) are of the same fom as those in (4.4a) and (4.4b), respectively. Therefore, substituting $(4.8 \mathrm{a})$ and $(4.8 \mathrm{~b})$ into the first-order condition (4.3) yields analogously

$$
H^{*}=\frac{a^{*} w-b *}{c^{*} w^{2}+d^{*}}
$$

where

$$
\begin{aligned}
& a^{*}:=U_{X}\left(Y_{O} ; \frac{Y_{O}}{\delta_{X}}\right)=\alpha_{X}-c^{*} Y_{O}, \\
& b^{*}:=U_{L}\left(T ; \frac{T}{\delta_{L}}\right)=\alpha_{L}-d^{*} T, \\
& c^{*}:=\left|\alpha_{X X}\right|-\frac{\gamma_{X X}}{\delta_{X}} \\
& d^{*}:=\left|\alpha_{L L}\right|-\frac{\gamma_{L L}}{\delta_{L}} .
\end{aligned}
$$

Thus, the "parameter" $a^{*}$ represents the marginal utility of consumption, when one is not employed and has got used to the corresponding consumption level $Y_{D}$. Analogously, the "parameter" $b^{*}$ denotes the marginal utility of household time, when one is not employed and has got used to the corresponding amount of household time $T$.

Positivity of the denominator in (4.9), i.e.

$$
c^{*} w^{2}+d^{*}>0
$$

is equivalent to the stability condition (3.6) for the present case of the AQM. It is also equivalent to the negation of the instability condition (4.7) without equality sign. Condition (4.11) holds for all $w$, if both $c^{*}$ and $d^{*}$ are positive. These parameter restrictions are cquivalent to the sufficient stability conditions (3.7a) and (3.7b), respectively, for the AQM as 
derived by Houthakker and Taylor (1970, Ch. 5, eq. (14)). ${ }^{10}$ Hence, these restrictions define (relatively) weak IHF with respect to consumption and household time, respectively. However, as demonstrated in Section 3.3, these sufficient conditions are not necessary for stability to occur. The various situations in which (4.11) and hence stability are fulfilled are summarized in Table 4.1. When $c^{*}>0$ and $d^{*} \leq 0$, so when the consumption IHF is weak, but the household time IHF is strong, (4.11) is equivalent to $w>\sqrt{-d^{*} / c^{*}}$. This condition is a reformulation of (3.8) for the AQM. It says that $w$ should be so high as to compensate the strong household time IHF (case (ii) of Proposition 3.1). Analogously, when $d^{*}>0$ and $c^{*} \leq 0$, so when the household time and consumption IHF's are weak and strong, respectively, (4.11) is, for $w>0$, equivalent to $1 / w>\sqrt{-c^{*} / d^{*}}$, which is a reformulation of (3.9). For $w=0$ (or $1 / w=\infty)(4.11)$ boils down to $d^{*}>0$. These conditions say that $1 / w$ should be so high as to compensate the strong consumption IHF, including the subcase that $w=0$ or $1 / w=\infty$ (case (iii) of Proposition 3.1). When, in addition, $c^{*}<0$, the conditions can also be written as a condition on $w$, namely $w<\sqrt{-d^{*} / c^{*}}$, whereas for $d^{*}>0$ and $c^{*}=0$ they are fulfilled for any (non-negative) $w$. However, when $c^{*} \leq 0$ and $d^{*} \leq 0$, so when both the consumption and household time IHF's are strong, (4.11) does not hold for any $w$. Below the implications of all these conditions will be clarified.

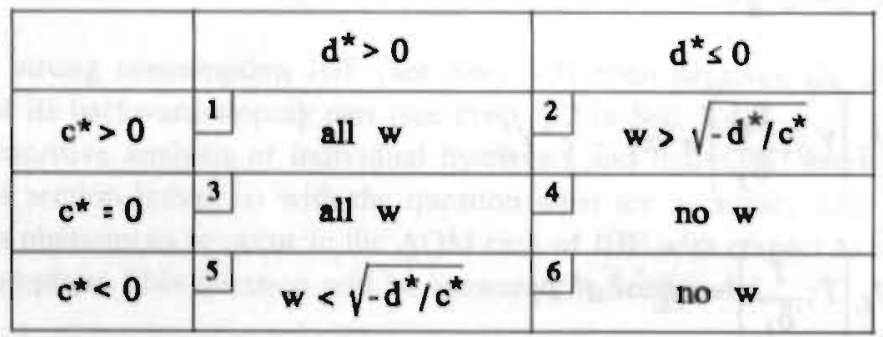

Table 4.1 All situations in which the stability condition $(4,10)$ is fulfilled. $w$ is non-negative.

In addition to these conditions, (4.9) should again satisfy the restrictions $0 \leq H^{*} \leq T$, so holds provided $0 \leq a^{*} w-b^{*} \leq T\left(c^{*} w^{2}+d^{*}\right)$. In order to establish when these restrictions are fulfilled, we first examine the signs of $a^{*}$ and $b^{*}$ as given by (4.10a) and (4.10b). From (4.10a) it follows that the marginal consumption utility $a^{*}$ is positive when $c^{*} \leq 0$ (strong consumption IHF) or, if $c^{*}>0$, when the consumption taste $\alpha_{X}$ is sufficiently high and $Y_{O}$ and $c^{*}$ are not too large. More precisely, $a^{*}$ is positive if and only if $c^{*}<\alpha_{X} / Y_{O}$. Thus, when $c^{*} \geq \alpha_{X} / Y_{O}, a^{*}$ is non-positive. Analogously, (4.10b) implies that the marginal houschold time utility $b^{*}$ is positive when $d^{*} \leq 0$ (strong household time IHF) or, if $d^{*}>0$. when the household time taste $\alpha_{L}$ is sufficiently high and $d^{*}$ is not too large. More precisely, $b^{*}$ is positive if and only if $d^{*}<\alpha_{L} / T$. Thus, when $d^{*} \geq \alpha_{L} / T, b^{*}$ is non-positive. Again, the two cases with $b^{*} \leq 0$ and $a^{*}>0$ and $a^{*} \leq 0$, respectively, are 
relegated to Section 4.5. This leaves us with the two cases with $b^{*}>0$ and $a^{*}>0$ and $a^{*} \leq 0$, respectively.

Moreover, this section restricts to an analysis of the always stable standard Case 1 in Table 4.1 with $c^{*}>0$ and $d^{*}>0$, so with weak (or no) IHF with respect to both consumption and household time. An analysis of the other three stable situations of Table 4.1 is postponed to the next section. Consider first the case with $a^{*}>0$ and $b^{*}>0$, so with $0<c^{*}<\alpha_{X} / Y_{O}$ and $0<d^{*}<\alpha_{L} / T$, i.e., both the corsumption and household time IHFs are weak, but not very weak. Then the restriction $a^{*} w-b^{*} \geq 0$ is fulfilled if and only if $w \geq b^{*} / a^{*}$. Just as in the corresponding short-run case of the previous section, this lower limit of $w$ represents a positive long-run reservation wage $w_{r}^{*}$. The restriction $a^{*} w-b^{*} \leq T\left(c^{*} w^{2}+d^{*}\right)$ is more complicated. Substituting (4.10b) into this restriction, it can be rewritten as

$$
T c * w^{2}-a * w+\alpha_{L} \geq 0 .
$$

Note that this restriction is independent of $d^{*}$, so independent of the household time habit sensitivity $\gamma_{L L} \cdot{ }^{11}$ Moreover, note that the left-hand side of (4.12) is a quadratic form in $w$. Since $c^{*}$ is positive, it describes a "valley parabola".

Hence, when the discriminant $D=a^{* 2}-4 T c * \alpha_{L}$ of the quadratic form is non-positive, implying no or one root of (4.12) with equality sign, the restriction (4.12) is fulfilled for all w. Substituting (4.10a) into the discrimant formula, it is easily derived that $D$ is non-positive if and only if $c^{*}$ is larger than or equal to a certain positive value $c_{o}^{*}$ between 0 and $\alpha_{X} / Y_{O}$, which depends on $\alpha_{X}, Y_{O}, \alpha_{L}$ and $T$, but not on $d^{*}$ (see App. 4A for this and following results). Then the restriction $H^{*} \leq T$ is not binding for any $w$ and the long-run labour supply curve has a usual backward-bending shape with a positive reservation wage $w_{r}^{*}=b^{*} / a^{*}$ (like the corresponding short-run labour supply curve described in the previous section). Moreover, in the subcase with $c^{*}>c_{o}^{*}, H^{*}$ is always smaller than $T$, whereas for $c^{*}=c_{o}^{*}$ and hence $D=0, H^{*}$ attains $T$ for $w$ equal to the unique root $w_{o}^{*}=\sqrt{\alpha_{L} /\left(T c_{o}^{*}\right)}$ of (4.12) with equality sign. This $w_{o}^{*}$ is independent of $d^{*}$, since (4.12) is independent of $d^{*}$. Thus, we have obtained two subcases with moderately weak consumption IHF and not very weak household time IHF, which are represented in Table 4.2 as the Subcases $1^{\mathrm{a}}$ and $1^{\mathrm{b}}$.

In Subcase $1^{c}$ of Table 4.2 with $0<c^{*}<c_{o}^{*}$, so when the consumption IHF is weak, but not too weak, we again have a positive reservation wage $w_{r_{*}^{*}}^{*}=b^{*} / a^{*}$, but now the discriminant mentioned above is positive. This implies two roots $w_{-}^{*}$ and $w_{*}^{*}$ of (4.12) with equality sign, which are given by

11 The reason of this independence is that (4.12) with equality sign is fulfilled for the values of $w$ for which the first-order condition (4.3) with $H=H^{*}, s_{X}=s_{X}^{*}$ and $s_{L}=s_{\dot{L}}^{*}$ yields $H^{*}=T$. Due to the additive separability of the utility function, this condition can depend on $d^{*}$ only via the. marginal household time utility $V_{L}\left(T-H^{*} ; s_{i}^{*}\right)$, and for $H^{*}=T$ this marginal utility is equal to the household time taste $\alpha_{L}=U_{L}(0 ; 0)$, which is independent of $d^{*}$. 


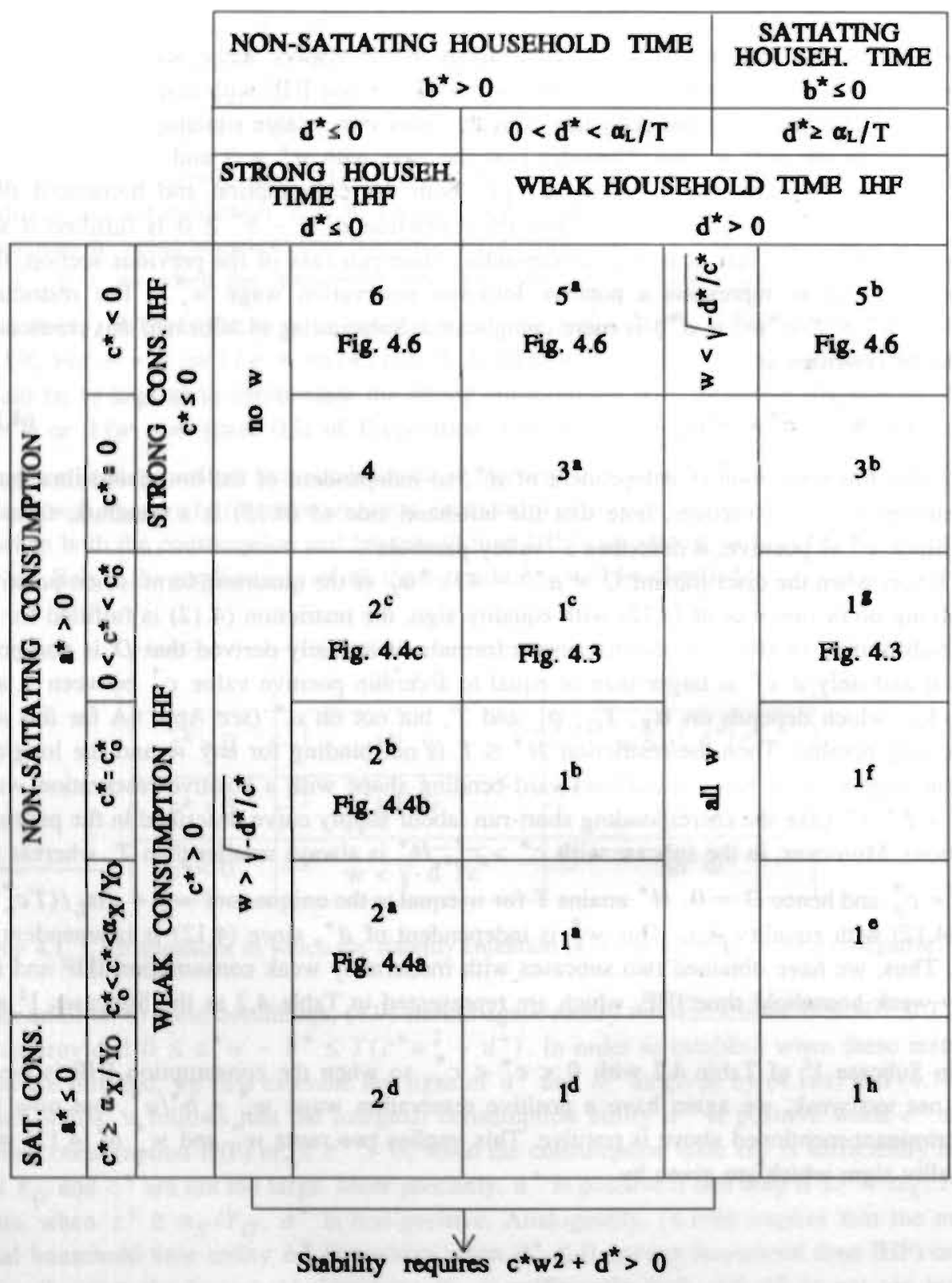

Table 4.2 Subcases of sirong and weak consumption IHF $\left(c^{*}\right)$ and household time IHF ( $\left.d^{*}\right)$. 


$$
w_{ \pm}^{*}=\frac{a^{*} \pm \sqrt{a^{* 2}-4 \alpha_{L} T c^{*}}}{2 T c^{*}} .
$$

Then the restriction (4.12) is not fulfilled for $w$ between $w_{-}^{*}$ and $w_{+}^{*}$, so the restriction $H^{*} \leq T$ is binding in that range. Note that $w_{-}^{*}$ and $w_{*}^{*}$ are independent of $d^{*}$, so independent of the household time habit sensitivity $\gamma_{L L}$, since (4.12) is independent of $d^{*}$. Thus, we obtain a backward-bending long-run labour supply curve with an inelastic segment for $H=T$.

For these three subcases $w_{r}^{*}=b^{*} / a^{*}$ implies that

$$
w_{r}^{*}=\frac{\alpha_{L}-T d^{*}}{\alpha_{X}-Y_{O} c^{*}}=-\frac{T}{\alpha_{X}-Y_{O} c^{*}} d^{*}+\frac{\alpha_{L}}{\alpha_{X}-Y_{O} c^{*}} .
$$

Consequently, when (the positive) $d^{*}$ becomes smaller, so when the (weak) household time IHF becomes stronger, the reservation wage $w_{r}^{*}$ rises. In contrast, when the consumption IHF gets stronger $\left(c^{*}\right.$ smaller), $w_{r}^{*}$ declines. This is in agreement with intuition. Moreover, as shown in (4.14), $w_{r}^{*}$ can be written as a linear function of $d^{*}<\alpha_{L} / T$ for given positive $c^{*}<\alpha_{X} / Y_{O}$. The negative slope of this function becomes more negative when $c^{*}$ falls. For Subcase $1^{\mathrm{c}}$ the function is indicated in Figure 4.3. This figure also displays $w_{-}^{*}$ and $w^{*}$. as constant functions of $d^{*}$ and indicates regimes of interior and corner solutions for $\mathrm{H}^{*}$.

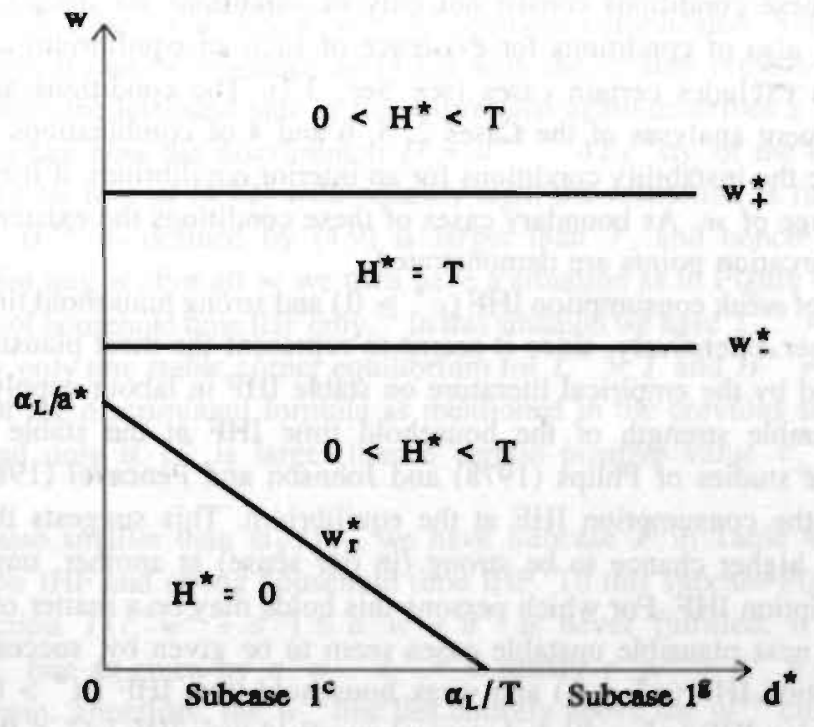

Figure 4.3 Graphs of $w_{r}^{*}, w_{-}^{*}$ and $w_{*}^{*}$ as functions of positive $d^{*}$ for $0<c^{*}<c_{a}^{*}$ (not too weak consumption IHF). Regimes of an interior or comer equilibrium $H^{*}$ are indicated.

In Subcase $1^{d}$ of Table 4.2 with $c^{*} \geq \alpha_{X} / Y_{O}$ and $0<d^{*}<\alpha_{L} / T$ the consumption IHF is very weak and/or the marginal consumption utility diminishes very fast (relative to $\alpha_{X} / Y_{O}$ ), 
and the household time IHF is not very weak. This results in a non-positive $a^{*}$ and a positive $b^{*}$. Since $U_{x}^{*}=a^{*}-c^{*} w H^{*}$ by virtue of $(4.8 \mathrm{a}),(4.10 \mathrm{a})$ and $(4.10 \mathrm{c})$, it then follows that $U_{x}^{*} \leq 0$ for any $H^{*}$. Appendix 4A proves that this long-run case of (fully) satiating consumption and non-satiating household time leads, in analogy to the short-run case, at the end of Section 4.1 , to a long-run labour supply curve which coincides with the vertical $H^{*}=0$ axis.

In all these subcases of Case 1 in Table 4.1, the stability condition (4.11) is fulfilled for all $w$. Hence, in a range of $w$ for which the long-run labour supply function (4.9) satisfies the restrictions $0 \leq H^{*} \leq T$, this function describes a moving equilibrium which is stable. Yet, in the Cases 2, 4, 5 and 6 of combinations of $c^{*}$ and $d^{*}$ in Table 4.1 such an equilibrium may be unstable for at least a range of $w$. The previous section has explained that this may lead to a long-run labour supply schedule with individual hysteresis and individual entry and exit catastrophes. In which situations will this occur? This question will be answered in the next section.

\section{4" Unstable long-run cases}

This section will derive necessary and sufficient conditions for individual hysteresis and individual entry and exit catastrophes to occur in a forward-sloping part of the labour supply curve as in Figure 4.2 in the AQM case of IHF with respect to both household time and consumption. These conditions consist not only of conditions for instability of an interior equilibrium, but also of conditions for existence of such an equilibrium as well as another condition which excludes certain cases (see Sec. 3.7). The conditions are derived as the result of subsequent analyses of the Cases 2, 5,6 and 4 of combinations of $c^{*}$ and $d^{*}$ in Table 4.1, where the instability conditions for an interior equilibrium, if it exists, are fulfilled for at least a range of $w$. As boundary cases of these conditions the existence and properties of so-called bifurcation points are demonstrated.

First, Case 2 of weak consumption IHF $\left(c^{*}>0\right)$ and strong household time $\operatorname{IHF}\left(d^{*} \leq 0\right)$ is discussed rather extensively, since it seems to represent the most plausible unstable case. This is suggested by the empirical literature on stable IHF in labour supply, which typically finds a considerable strength of the household time IHF at the stable equilibrium, that moreover, in the studies of Phlips (1978) and Johnson and Pencavel (1984), is larger than the strength of the consumption IHF at the equilibrium. This suggests that the household time IHF has a higher chance to be strong (in our sense) at another, unstable equilibrium than the consumption IHF. For which persons this holds may be a matter of personal character or age. The next plausible unstable cases seem to be given by, successively, Case 5 of strong consumption IHF $\left(c^{*}<0\right)$ and weak household time IHF $\left(d^{*}>0\right)$, and Case 6 of strong consumption IHF $\left(c^{*}<0\right)$ and strong household time IHF $\left(d^{*} \leq 0\right)$. These cases are discussed together after and more concisely than Case 2. The least plausible unstable case is the accidental transition case 4 of just strong consumption IHF $\left(c^{*}=0\right)$ and strong household time IHF ( $d^{*} \leq 0$ ). This case closely resembles the Cases 5 and 6 . 
Case 2: $c^{*}>0$ and $d^{*} \leq 0$

In this case of weak consumption IHF and strong household time IHF the equilibrium $H^{*}$ as given by (4.9) is stable if and only if $c^{*} w^{2}+d^{*}>0$, or equivalently, $w>\sqrt{-d^{*} / c^{*}}$ (see Table 4.1). Conversely, $H^{*}$ as given by (4.8) forms a unique unstable equilibrium $H^{* \|}$ if and only if $c^{*} w^{2}+d^{*}<0$, or $w<\sqrt{-d^{*} / c^{*}}$. ${ }^{12}$ Accordingly, the latter upperbound for $w$ is denoted as $w^{* t}$. As a function of non-positive $d^{*}$ for given positive $c^{*}$ it is represented in Figures 4.4a-c.

However, in order to define an unstable equilibrium $H^{* u}$ in the feasible domain, (4.9) should also satisfy the restrictions $0 \leq H^{*} \leq T$. Since the denominator $c^{*} w^{2}+d^{*}$ in (4.9) should now be negative, these restrictions are now equivalent to $T\left(c^{*} w^{2}+d^{*}\right) \leq a^{*} w-b \leq 0$. Note that the latter restrictions differ from the corresponding restrictions in the stable case as formulated under Table 4.1 in the previous section. There it has also been established that the present restriction $d^{*} \leq 0$ implies that the marginal household time utility $b^{*}$ is positive, and that the marginal consumption utility $a^{*}$ is also positive if and only if $c^{*}<\alpha_{X} / Y_{O}$.

In the latter case the restriction $a^{*} w-b^{*} \leq 0$ is fulfilled if and only if $w \leq b^{*} / a^{*}$. Now the last positive upperbound is not the long-run reservation wage $w_{r}^{*}$ as in the stable case of the previous section, but will turn out to have another meaning. On the other hand, since the expression is the same, the upperbound can be considered as the continuation of $w_{r}^{*}$ into the unstable domain. As a function of non-positive $d^{*}$ for given positive $c^{*}$ it is represented in Figures $4.4 \mathrm{a}-\mathrm{c}$.

The restriction $T\left(c^{*} w^{2}+d^{*}\right) \leq a^{*} w-b^{*}$ is more complicated. Substituting (4.10b) into this restriction, it can be rewritten as (4.12) with the $\geq$ sign replaced by the $\leq$ sign. Since $c^{*}$ is positive, the left-hand side of this expression again describes a "valley parnbola" in $w$. However, when now the discriminant $D^{\prime}=a^{* 2}-4 T c^{*} \alpha_{L}$ of the quadratic form is negative, implying no root of (4.12) with equality sign, the restriction is fulfilled for no $w$. This means that $H^{* u}$ as defined by (4.9) is larger than $T$, and hence falls outside the feasible domain for any $w$. For all $w$ we then have a situation as in Figure 4.1 for $w<w^{* 2}$ in the special case of household time IHF only. ${ }^{13}$ In this situation we have $L^{* n}=T-H^{* n}<0$, and consequently only one stable corner equilibrium for $L^{*}=T$ and $H^{*}=0$. According to the elaboration of the discriminant formula as mentioned in the previous section, this situation occurs if and only if $c^{*}$ is larger than a certain positive value $c_{\bar{D}}^{*}$ between 0 and $\alpha_{X} / Y_{O}$

When $c^{*}$ is also smaller than $\alpha_{X} / Y_{O}$, we have Subcase $2^{a}$ in Table 4.2 of moderately weak consumption IHF and strong household time IHF. To this subcase Figure 4.4a applies. Since the restriction $T\left(c^{*} w^{2}+d^{*}\right) \leq a^{*} w-b^{*}$ is never fulfilled, it follows that all points on the $w_{r}^{*}$ line as given by $a^{*} w-b^{*}=0$ satisfy $c^{*} w^{2}+d^{*}>0$, so are located in the stable domain. Therefore, the $w_{r}^{*}$ line lies entirely above the $w^{* \|}$ curve and describes: a long-run reservation wage for all $d^{*}$. Hence, above the $w^{* / 1}$ curve we have the same kind

12 When $w=\sqrt{-d^{*} / c^{*}}$, or $c^{*} w^{2}+d^{*}=0,(4.9)$ is not defined and the first-order condition (4.3) tums out to have either no solutions or all feasible $H$ as solutions. Such cases are discussed below.

${ }^{13}$ No consumption IHF can be considered as a special case of weak consumption IHF. 


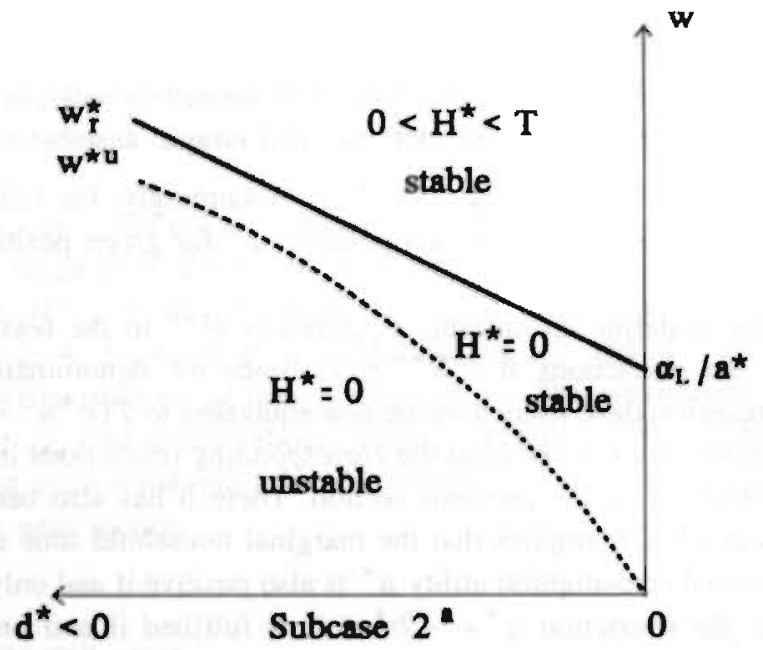

(a) $0<c_{o}^{*}<c^{*}<\boldsymbol{\alpha}_{X} / Y_{O}$ (moderately weak consumption IHF)

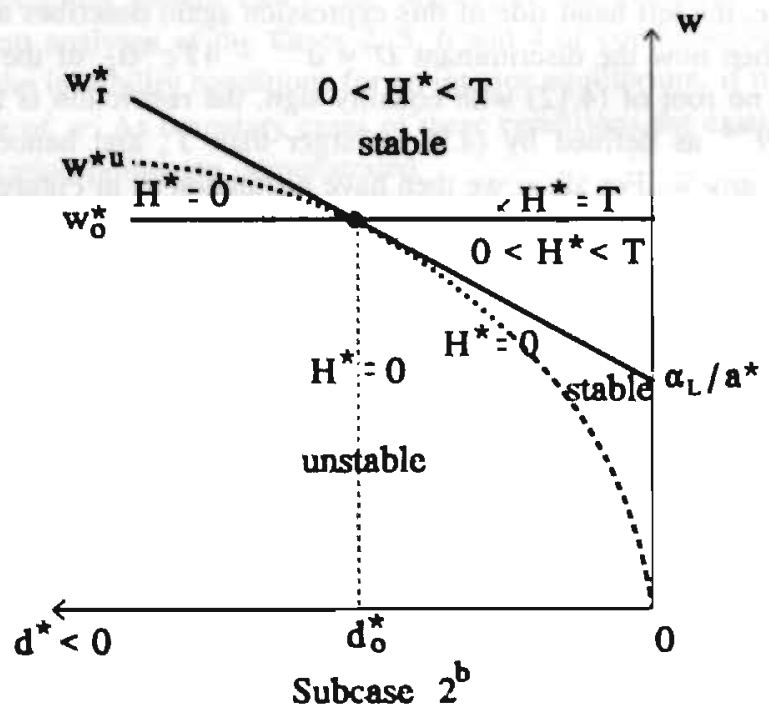

(h) $c^{*}=c^{*}$ 


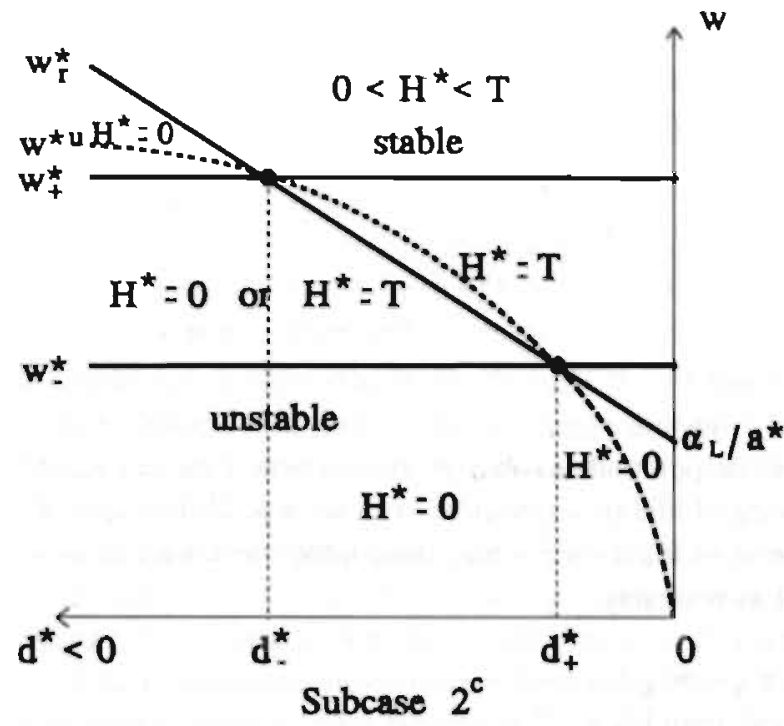

(c) $0<c^{*}<c_{o}^{*}$ (not too weak consumption IHF)

Figure 4.4 Graphs of $w_{r}^{*}, w_{o}^{*}, w_{-}^{*}, w_{+}^{*}$ and $w^{* u}$ as functions of non-positive $d^{*}$. Domains of stable equilibria $H^{*}$ ane indicated.

of situation as in the always stable Subcase $1^{\mathrm{a}}$. Under the $w^{* u}$ curve there is only one stable corner equilibrium for $H^{*}=0$, as established above. Finally, on the $w^{* u}$ curve, so when $c^{*} w^{2}+d^{*}=0,(4.9)$ is not defined and the first-order condition (4.3) has no solutions provided $a^{*} w-b^{*} \neq 0$, i.e., $w$ is not on the $w_{r}^{*}$ line (see App. 4B for this and following results). In the present subcase this holds for all $w$ on the $w^{\text {"h }}$ curve. This corresponds to a situation in which the household time demand line in Figure 4.1 for $w<w^{* 2}$ has become parallel to the equilibrium line while remaining above it. This implies one stable comer solution $H^{*}=0$. As a result of all this, the instability for $w \leq w^{* \|}$ of the equilibrium given by (4.9) does not have any effect on the long-run labour supply curve. This curve has, for all non-positive $d^{*} \leq 0$, a backward-bending shape similar to that in Subcase $1^{\mathrm{a}}$, only having a higher long-run reservation wage and being more bowed due to the stronger household time IHF.

However, for $c^{*}=c_{o}^{*}$ (Subcase $2^{\mathrm{b}}$ in Table 4.2) and $0<c^{*}<c_{a}^{*}$ (Subcase $2^{\mathrm{c}}$ ) matters are different. In the former subcase the discriminant mentioned above is zero, implying one root of (4.12) with equality sign. This root is given by the same $w_{0}^{*}$ as in Subcase $1^{b}$. Only for this $w_{o}^{*}$ the restriction $H^{* u} \leq T$ is just fulfilled with equality sign, whereas for all other $w$ it is not fulfilled. For $w=w_{0}^{*}$ we then have a situation as in Figure 4.1 for $w=w^{* 2}$ with $L^{* u}=T-H^{* u}=0$ and consequently only one stable corner equilibrium for $L^{*}=T$ and $H^{*}=0$. Thus, whereas $H^{*}=T$ for $w=w_{o}^{*}$ in the stable domain, $H^{*}=0$ for $w=w_{o}^{*}$ in the unstable domain. Still, we can consider one horizontal $w_{o}^{*}$ line in the stable as well unstable domain as indicated in Figure $4.4 \mathrm{~b}$. This line goes over from the stable into the 
unstable domain by crossing the $w^{* u}$ curve at a $d_{o}^{*}$, which fulfils $c_{0}^{*} w_{o}^{* 2}+d_{o}^{*}=0$ and is therefore equal to $-c_{o}^{*} w_{o}^{* 2}=-c_{o}^{*} \alpha_{L} /\left(T c_{o}^{*}\right)=-\alpha_{L} / T$. It then follows that for nonpositive $d^{*} \neq d_{o}^{*}$ the long-run labour supply curve has the same backward-bending shape as in Subcase $2^{\text {a }}$, but now it attains $H^{*}=T$ at $w=w_{o}^{*}$ for $d_{o}^{*}<d^{*} \leq 0$ in the same way as for $d^{*}>0$.

On the other hand, for $d^{*}=d_{o}^{*}$ the long-run labour supply curve is qualitatively different. Then we have for $w=w_{o}^{*}$ the accidental situation that both the denominator $c^{*} w^{2}+d^{*}$ and, since the restriction $T\left(c^{*} w^{2}+d^{*}\right) \leq a^{*} w-b^{*}$ is just fulfilled with equality sign, the numerator $a^{*} w-b^{*}$ of (4.9) are zero. This implies that $w_{o}^{*}=w^{* u}=w_{r}^{*}$, so the $w_{o}^{*}$ line, the $w^{* u}$ curve and the $w_{r}^{*}$ line all meet each other at the same $\left(d_{o}^{*}, w_{o}^{*}\right)$. Moreover, this point is the only meeting point, and hence a tangency point of the $w^{* u}$ curve and the $w_{r}^{*}$ line. In that special point all feasible $H$ are unstable long-run equilibria. In the context of Figure 4.1 the demand line then coincides with the equilibrium line. As: a result we obtain a long-run schedule as in Figure 4.5 with catastrophic transitions at $w=w_{o}^{*}$ from $H^{*}=0$ to $H^{*}=T$ as well as reversely.

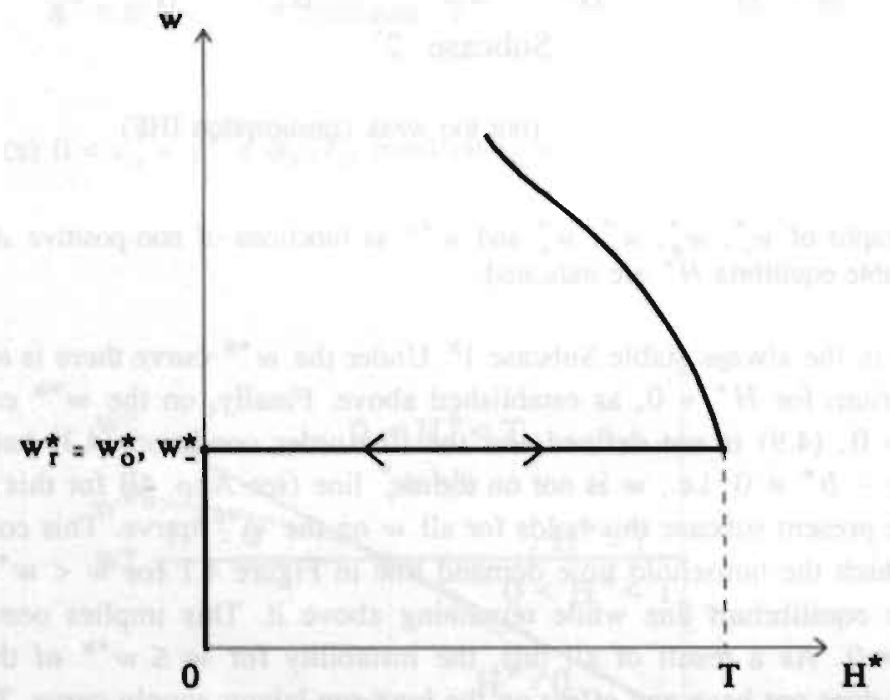

Figure 4.5 Long-run labour supply schedule with entry and exit catastrophe at the same $w_{o}^{*}$ or $w_{-}^{*}$ for $c^{*}=c_{o}^{*}>0$ and $d^{*}=d_{o}^{*}<0$ or for $0<c^{*}<c_{o}^{*}$ and $d^{*}=d_{+}^{*}<0$ (not too weak consumption IHF and moderately strong houselwold time IHF).

Subcase $2^{\mathrm{C}}$ in Table 4.2 of not too weak consumption IHF and strong household time IHF is represented by Figure $4.4 \mathrm{c}$. Then the discriminant mentioned above is positive, implying two roots of (4.12) with equality sign. These roots are given by the same $w_{*}^{*}$ and $w_{*}^{*}$ in (4.13) as in Subcase $1^{\mathrm{c}}$. The restriction $H^{* 14} \leq T$ is then fulfilled for $w_{-}^{*} \leq w \leq w_{+}^{*}$ only. When, moreover, $H^{* u} \geq 0$ or $w \leq w_{r}^{*}$, we then have situations as in Figure 4.1 for $w=w^{* 2}$, $w^{* 2}<w^{* 1}$ and $w^{*}=w^{* 1}$, where $w^{* 2}=w_{-}^{*}$ and $w^{* 1}=w_{r}^{*}$, provided $w_{r}^{*}<w_{+}^{*}$. In the middle situation in Figure 4.1 for $w^{\bullet 2}<w<w^{* 1}$, there is one unstable interior e- 
quilibrium $C$ in addition to two stable corner equilibria $A^{\prime}$ and $B$. From Figure $4.4 \mathrm{c}$ we can see for which range of the household time IHF parameter $d^{*}$ this situation can occur.

In this figure a horizontal $w_{-}^{*}$ line and a horizontal $w_{+}^{*}$ line cross the $w^{*}$ curve at points $\left(d_{+}^{*}, w_{-}^{*}\right)$ and $\left(d_{-}^{*}, w_{+}^{*}\right)$, respectively, where $d_{+}^{*}$ and $d_{-}^{*}$ are equal to $-c^{*} w_{-}^{* 2}$ and $-c^{*} w_{+}^{* 2}$, respectively, and hence depend on $c^{*}, \alpha_{X}, \alpha_{L}, Y_{O}$ and $T$ in a complicated way. Moreover, just as the point $\left(d_{o}^{*}, w_{o}^{*}\right)$ in Figure $4.4 \mathrm{~b}$, these points are also meeting points of the $w^{* u}$ curve and the $w_{r}^{*}$ line, but now these curve and line cross each other. Thus, when coming from Subcase $2^{\mathrm{a}}$ in Figure $4.4 \mathrm{a}, c^{*}$ declines to $c_{o}^{*}$ and beyond it, the $w^{* u}$ curve in Figure 4.4a turns upwards around the origin and the $w_{r}^{*}$ line turns downwards around the point $\left(d^{*}, w\right)=\left(\alpha_{L} / T, 0\right)$ (see (4.14)) until they touch for $c^{*}=c_{o}^{*}$ (Fig. 4.4b) and intersect for $c^{*}<c_{o}^{*}$ (Fig. 4.4c). Since a situation like the middle situation in Figure 4.1 requires $w<w^{* u}$ (unstable domain), w $w w_{r}^{*}\left(H^{* u}>0\right)$ and $w_{-}^{*}<w<w_{+}^{*}\left(H^{* u}<T\right)$, the configuration in Figure $4.4 \mathrm{c}$ implies that such a situation with two stable corner equilibria $H^{\circ}=0$ and $H^{*}=T$ can occur only for $d^{*}<d_{+}^{*}$, so for sufficiently strong household time IHF.

Section 4.2 has shown that, starting from the middle situation in Figure 4.1 , changes in the wage rate $w$ may lead to individual hysteresis and individual entry and exit catastrophes between the corner equilibria when $w$ rises beyond $w^{* 1}$ or declines beyond $w^{* 2}$, Can this occur in the present case and, if so, under which conditions? Therefore, we should first analyse what happens for $w$ for which an unstable interior equilibrium does not exist. For $\left(d^{*}, w\right)$ below the $w_{-}^{*}$ line and above the $w_{*}^{*}$ line we have the same kind of situation as in Figure 4.4a, implying $H^{*}=0$ for $w \leq w_{r}^{*}$ and $0<H^{*}<T$ for $w>w_{r}^{*}$. For $\left(d^{*}, w\right)$ in the band between the $w_{-}^{*}$ and $w_{+}^{*}$ lines which lie above the $w^{*}$ curve, $H^{*}$ as given by (4.9) is stable and larger than $T$. This implies a stable corner solution $H^{*}=T$. On the other hand, for $\left(d^{*}, w\right)$ in the band which lie under the $w^{*}$ curve, $H^{*}$ as given by (4.9) is unstable and smaller than $T$. Moreover, when such a $\left(d^{*}, w\right)$ lies above the $w_{r}^{*}$ line, the unstable $H^{* u}$ is also smaller than 0 . Then we are in a situation as in Figure 4.1 for $w>w^{* 1}$, implying a unique stable corner equilibrium for $L^{*}=0$ and $H^{*}=T$. When a $\left(d^{*}, w\right)$ in the band lies on the $w^{* u}$ curve, so above the $w_{r}^{*}$ line, $H^{*}$ as given by (4.9) does not exist. Then the demand line in Figure 4.1 for $w>w^{* 1}$ is parallel to the $L=\delta_{L} s_{L}$ line while remaining below it, again implying one stable comer equilibrium for $H^{*}=T$.

Now we can see what happens for $d^{*}<d_{*}^{*}$ when $w$ rises from below $w_{-}^{*}$ or declines from above $w_{r}^{*}$ or $w_{+}^{*}$. First, we consider the (sub-)subcase with $d_{-}^{*}<d^{*}<d_{+}^{*}$, i.e. with moderately strong household time IHF. To the unstable domain with $w<w^{* u}$ Figure 4.1 then applies with $w^{* 2}=w_{-}^{*}$ and $w^{* 1}=w_{r}^{*}$. From this figure we see that when $w$ rises from below $w^{* 2}$. a person will stay in the stable corner equilibrium $B$ for $L^{*}=T$ and $H^{*}=0$ until this equilibrium becomes unstable for $w=w^{* 1}$ and disappears outside the feasible domain for $w>w^{* 1}$. This induces an individual entry catastrophe from $L^{*}=T$ and $H^{*}=0$ to $L^{*}=0$ and $H^{*}=T$ at $w=w^{* 1}$, as described in Section 4.2. Analogous ly, when $w$ declines from above $w^{* 1}$ (but below $w_{*}^{*}$ ), a person will stay in the stable corner equilibrium $A^{\prime}$ for $L^{*}=0$ and $H^{*}=T$ until this equilibrium becomes unstable for $w=w^{* 2}$ and disappears for $w<w^{* 2}$. This induces an individual exit catastrophe from $H^{*}=T$ to $H^{*}=0$ at $w=w^{* 2}$, as described in Section 4.2. As a result, we obtain a longrun labour supply schedule as in Figure 4.2 with individual hysteresis and indvidual entry 
and exit catastrophes between $H^{*}=0$ and $H^{*}=T$ in a "forward-sloping" part and a continuous backward-sloping curve for $w$ above $w_{+}^{*}$. In Figure 4.2 the loci of the unstable equilibrium $H^{* \|}$ in the feasible domain are also indicated.

However, for $d^{*}<d_{-}^{*}$, so for very strong household time IHF, matters are different. Then, to the unstable domain with $w<w^{\star u}$ Figure 4.1 only partially applies. For $w$ rising from below $w_{-}^{*}$, a new equilibrium $A^{\prime}$ with $H^{*}=T$ emerges at $w=w^{* 2}=w_{-}^{*}$, but at a certain $w$ between $w^{* 2}$ and $w^{* 1}$ the short-run labour supply curve becomes backwardsloping for the habit stock value $s_{L}{ }^{C}$ (see App. $4 \mathrm{~B}$ for a derivation). This implies that, for $w$ rising beyond this point, a left part of the demand line including $C$ starts to move upwards instead of downwards. For $w$ rising further this leads, at $w=w_{+}^{*}$ and beyond, back to situations like those in Figure 4.1 for $w=w^{* 2}$ and $w<w^{* 2}$, respectively. ${ }^{14}$ As a result, the corner equilibrium $B$ with $H^{*}=0$ remains stable for all $w$ in the unstable domain, so an entry catastrophe does not occur and the person enters the labour market only beyond the high long-run reservation wage $w_{r}^{*}$ in the stable domain (since she is very strongly used to non-participation). In this stable domain the long-run labour supply curve has a continuous and rather pronounced backward-bending shape. On the other hand, for $w$ between $w_{-}^{*}$ and $w_{+}^{*}$ in the unstable domain, $H^{*}=T$ is a second stable equilibrium in addition to $H^{*}=0$, implying individual hysteresis. When the person happens to occupy this equilibrium and when $w$ declines below $w_{-}^{*}$ or rises above $w_{+}^{*}$, the equilibrium becomes unstable and disappears, inducing in both cases an individual exit catastrophe to $H^{*}=0$. Thus, in this peculiar subcase we have no individual entry catastrophe, but two individual exit catastrophes instead. The resulting labour supply schedule is presented in Appendix 4B, which also provides some more details.

As a transition case between the last and preceding subcase, we have, for $d^{*}=d_{-}^{*}$, a long-run labour supply schedule with a coinciding individual entry and exit catastrophe between $H^{*}=0$ and $H^{*}=T$ at $w_{r}^{*}=w_{*}^{*}$ as in Figure 4.5, but also with an individual exit catastrophe at $w_{-}^{*}$ and individual hysteresis as in Figure 4.2 . For $d^{*}=d_{+}^{*}$ we have a schedule as given in Figure 4.5, and for $d^{*}>d_{+}^{*}$ we obtain a same kind of continuous backward-bending labour supply curve as in Subcase $1^{\mathrm{C}}$ in Figure 4.3 with a positive reservation wage and an inelastic segment for $H^{*}=T$.

Consider now in Figure $4.4 \mathrm{c}$ a simultaneous change of $d^{*}$ and $w$ from a point to the right of $d_{*}^{*}$ through the point $\left(d_{*}^{*}, w_{-}^{*}\right)$ towards a point in the domain of multiple equilibria to the left of $d_{*}^{*}$. Such a change may occur as a person gets older and less flexible and hence more susceptible to habit formation, implying a rising household time habit sensitivity $\gamma_{L L}$, so a declining $d^{*}$ (see (4.10d)). In passing $\left(d_{*}^{*}, w_{-}^{*}\right)$, a unique stable equilibrium then bifurcates into two stable comer equilibria. Therefore the point $\left(d_{+}^{*}, w_{-}^{*}\right)$ can be considered as a piecewise-linear model approximation of a so-called bifurcation point, which plays a

${ }^{14}$ For $d^{*} \geq d_{-}^{*}$ too. points of the demand line for low habit stock values will start to move upwants at certain values of $w$ between $w_{-}^{*}$ and $w_{r}^{*}$ as the short-run labour supply curve becomes backward-sloping for these stock values. Then. however, $s_{L}{ }_{L}^{C}$ is not included into these stock values, so that the demand line continues to move downwards at $C$. and hence this equilibrium continues to move upwards towards $B$, at rising $w$. 
key role in the non-linear catastrophe theory. ${ }^{15}$ When only $w$ changes at given $d^{*}=d_{+}^{*}$, there occurs also a catastrophe at this variant of a bifurcation point, as indicated in Figure 4.5. ${ }^{16}$ For $d^{*}$ becoming more negative than $d_{+}^{*}$, this catastrophe splits into a separate entry catastrophe and a separate exit catastrophe as in Figure 4.2. When $\left(d^{*}, w\right)$ changes from the unique interior equilibrium domain for $d^{*}>d_{+}^{*}$ and $w_{r}^{*}<w<w_{-}^{*}$ towards the multiple equilibria domain for $d^{*}<d_{+}^{*}$ through a point slightly below the bifurcation point. the person retires from the labour market (e.g., early retirement). On the other hand, a similar change of $\left(d^{*}, w\right)$ through a point slightly above the bifurcation point, so at a slightly higher $w$, renders the person a motivated employee working maximal corporate hours. Thus, a small difference in the historical time path of $\left(d^{*}, w\right)$ near the bifurcation point can lead to a large difference in the equilibrium which is reached. This hysteresis property of a bifurcation point is called divergence in catastrophe theory. It can describe a polarization within a rather homogeneous group of persons as the result of passing a bifurcation point at different sides. In the present context a special bifurcation point is given by the point $\left(c_{o}^{*}, d_{o}^{*}, w_{o}^{*}\right)$ in Figure $4.4 \mathrm{~b}$. At a suitable simultaneous change in $c^{*}, d^{*}$ and $w$ a unique stable equilibrium for $c^{*}>c_{o}^{*}$ (Fig. 4.4a) bifurcates at this point into two stable corner equilibria for $c^{*}<c_{o}^{*}$ (Fig. 4.4c).

Finally, we have to deal with Subcase $2^{d}$ in Table 4.2. Then the consumption IHF is very weak and/or the marginal consumption utility diminishes very fast (relative to $\alpha_{X} / Y_{O}$ ) and the household time IHF is strong. Just as in Subcase $1^{\mathrm{d}}$ this results in a non-positive $a^{*}$, a positive $b^{*}$, and $U_{X}^{*} \leq 0$ for any $H^{*}$. Appendix 4B proves that also this long-run case of satiating consumption and non-satiating household time leads to a long-run labour supply curve which coincides with the vertical $H^{*}=0$ axis.

\section{Cases 5 and 6: $c^{*}<0$ and $d^{*}>0$ or $d^{*} \leq 0$}

These cases of strong consumption IHF and weak or strong household time IHF are represented by the right and left quadrant of Figure 4.6, respectively. The parameter $a^{*}$ is always positive, whereas $b^{*}$ and hence $w_{r}^{*}=b^{*} / a^{*}$ are positive if and only if $a^{*}<\alpha_{L} / T$ (Subcase $5^{\mathrm{a}}$ and Case 6). Again the subcase with $d^{*} \geq \alpha_{L} / T$ is relegated to the next section. In comparison with (the combination of) Figures 4.3 and $4.4 c$, the $w_{r}^{*}$ and $w_{-}^{*}$ lines in Figure 4.6 are lower and intersect for a positive instead of a negative $d_{*}^{*}$, the $w_{*}^{*}$ line has disappeared into the region of negative $w$, and the $w^{* u}$ curve is now located in the right instead of the left quadrant (see Tab, 4.1 and see App. $4 \mathrm{C}$ for this and following results). Moreover, the equilibrium $H^{*}$ as given by (4.9) is now stable if and only if $\left(d^{*}, w\right)$ lies below instead of above this $w^{* u}=\sqrt{-d^{*} / c^{*}}$ curve. Then we have the sarne kind of situation as in Figure. 4.3 under the $w_{+}^{*}$ line. For $\left(d^{*}, w\right)$ above and left to the $w^{*}$ curve, so when $c^{*} w^{2}+d^{*}<0$, the equilibrium $H^{*}$ as given by (4.9) is an unstable $H^{* \|}$. Then the situation is similar to that in Figure 4.4c for $d^{*}$ between $d_{-}^{*}$ and $d_{*}^{*}$ and for $w$ tinder the $w^{* u}$ curve. Finally, on

${ }^{15}$ See, e.g., Zeeman (1977). Piecewise-linear model approximations of the curves of the so-called cusp in catastrophe theory are given by the $w_{5}^{*}$ and $w_{-}^{*}$ lines for $d_{-}^{*}<d^{*} \leq d_{*}^{*}$. See also Subsection 5.5.3.

${ }^{16}$ This does not hold for the non-linear bifurcation point in catastrophe theory. 
the $w^{* u}$ curve, so when $c^{*} w^{2}+d^{*}=0$, we are in a similar position as on the $w^{* u}$ curve in Figure $4.4 \mathrm{c}$ for $d^{*}>d_{-}^{*}$.

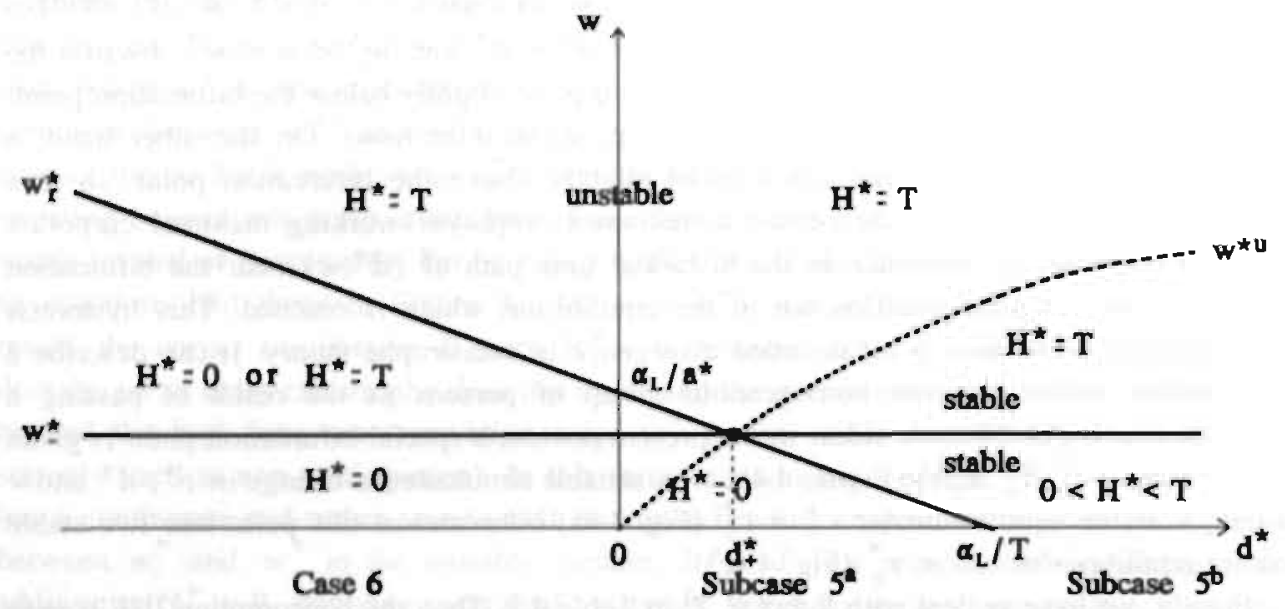

Figure 4.6 Graphs of $w_{r}^{*}, w_{-}^{*}$ and $w^{*}$ as functions of $d^{*}$ for $c^{*}<0$ (strong consumption IHF).

For $d_{+}^{*}<d^{*}<\alpha_{L} / T$ all this results in a continuous long-run labour supply curve with a forward-sloping, but no backward-sloping part, since the strong consumption IHF keeps labour supply on its maximal hours $T$ for $w$ above $w_{-}^{*}$ (cf. Sec. 3.4). For $d^{*}<d_{+}^{*}$ we obtain a similar long-run labour supply schedule as in Figure 4.2 with individual hysteresis and individual entry and exit catastrophes between $H^{*}=0$ and $H^{*}=T$ in a "forwardsloping part", but without a backward-sloping part. Finally, in the transition case $d^{*}=d_{*}^{*}$ we have for $w=w^{* u}=w_{r}^{*}=w_{-}^{*}$ the accidental situation that all feasible $H$ are unstable long-run equilibria. This results, for $d^{*}=d_{+}^{*}$, in a long-run labour supply schedule with coinciding entry and exit catastrophes as in Figure 4.5, but without a backward-sloping part. Again $\left(d_{+}^{*}, w_{-}^{*}\right)$ is a bifurcation point.

Case 3 with $c^{*}=0$ and $d^{*}>0$ and Case 4 with $c^{*}=0$ and $d^{*} \leq 0$ are transition cases describing just strong consumption IHF. The $w^{* / t}$ curve then coincides with the $d^{*}=0$ axis (see Table 4.1) as a transition case between the $w^{* \|}$ curves in Figures $4.4 a-c\left(c^{*}>0\right)$ and the right quadrant of Figure $4.6\left(c^{*}<0\right)$. Right to the $d^{*}=0$ axis we then have the always stable Case 3 of weak household time IHF. and left to and including the $d^{*}=0$ axis, the always unstable Case 4 of strong household time IHF. Apart from this configuration these cases do not yield new situations as compared to the Cases 5 and 6 , and are therefore not further discussed here.

After having considered all the possible cases we can conclude that: 
Proposition 4.1 Individual hysteresis and individual entry and exit catastrophes in a "forward-sloping part" of the long-run labour supply schedule as in Figure 4.2 occur in the following situations:

$$
0<c^{*}<c_{o}^{*}<\alpha_{X} / Y_{O} \text { and } d_{-}^{*}<d^{*}<d_{+}^{*}<0 \text {, where } c_{o}^{*}, d_{-}^{*} \text { and } d_{+}^{*} \text { depend on }
$$
$\alpha_{X}, Y_{O}, \alpha_{L}$ and $T$, and $d_{-}^{*}$ and $d_{+}^{*}$ also on $c^{*}$.

(iii) $c^{*} \leq 0$ and $d^{*} \leq 0$.

Moreover, in situation (i) only the long-run labour supply schedule is also backward-bending (see Fig. 4.2), whereas in the siruations (ii) and (iii) labour supply remains equal to $T$ for higher $w$.

In these situations the consumption and household time IHFs at an interior point are (i) not too weak and moderately strong, respectively (subcase of Subcase 1 ${ }^{\mathrm{C}}$ ), or (ii) strong and not too weak, respectively (subcase of Subcase $5^{\mathrm{a}}$ ), or (iii) both strong (Cases 4 and 6 ). In the last two situations the strong consumption IHF keeps labour supply on its maximal hours $T$ for higher $w$.

This section has considered the effects on long-run labour supply $H^{*}$ of variations in the exogenous variable $w$ and the parameters $c^{*}$ and $d^{*}$. According to $(4.10 \mathrm{c})$ and $(4.10 \mathrm{~d})$ these parameters cover the influence of the diminishing marginal utility parameters $\alpha_{X X}$ and $\alpha_{L L}$ and the habit sensitivities $\gamma_{X X}$ and $\gamma_{L L}$ in the utility function (4.1). This leaves the effects of two relevant parameters, namely the consumption and household time taste $\alpha_{X}$ and $\alpha_{L}$, undiscussed. However, these parameters affect only $a^{*}$ and $b^{*}$, respectively, but not $c^{*}$ and $d^{*}$. As a consequence, a change in $\alpha_{X}$ or $\alpha_{L}$ does not lead to a change in the position of the $w^{* u}$ curve in the pertinent situations, but has only effects on the $w_{r}^{*}$ line and other lines. Therefore, the effect of such a change on $H^{*}$ can easily be analysed. Here we will not elaborate on that, but in Sections 4.7 and 4.8 and in Section 9.11 we will consider situations in which the household time taste $\alpha_{L}$ varies over individuals and over time, respectively. The latter variation will be modelled as the endogenous outcome of satiation with respect to household time in the very long run. By affecting the entry wage $w_{r}^{*}=b^{*} / a^{*}$ or the exit wage $w_{-}^{*}$ as given by (4.13), such an endogenous variation over time can trigger individual catastrophes and could even lead to a kind of psychological long cycle of behaviour and mentality shifts as explained at the end of Section 3.5 .

\section{5* Workaholism}

This section adds individual habit formation (IHF) with respect to corporate time $H$ to the $\mathrm{AQM}$ which has been analysed in the previous sections. This extension has interesting implications. In particular, the dependence of utility on corporate time makes cases of net (fully) satiating household time more plausible (see Subsec. 2.2.5).

Consider a utility function $\hat{U}\left(X, L, H ; s_{X}, s_{L}, s_{H}\right)$ which is additive(ly separable) in $\left\{X, s_{X}\right\},\left\{L, s_{L}\right\}$ and $\left\{H, s_{H}\right\}$. Transforming away terms which are independent of the choice variables $X, L$ and $H$ (see Sec. 4.1), this additive specification can be written as 


$$
\begin{aligned}
\hat{U}\left(X, L, H ; s_{X}, s_{L}, s_{H}\right)= & \hat{\alpha}_{X} X+\frac{1}{2} \hat{\alpha}_{X X} X^{2}+\hat{\gamma}_{X X} s_{X} X+ \\
& \hat{\alpha}_{L} L+\frac{1}{2} \hat{\alpha}_{L L} L^{2}+\hat{\gamma}_{L L} s_{L} L+ \\
& \alpha_{H} H+\frac{1}{2} \hat{\alpha}_{H H} H^{2}+\hat{\gamma}_{H H} s_{H} H
\end{aligned}
$$

with $\alpha_{X}>0, \hat{\alpha}_{X X}<0, \hat{\gamma}_{X X}<0, \alpha_{L}>0, \hat{\alpha}_{L L}<0, \hat{\gamma}_{L L} \geq 0, \alpha_{H}>0, \alpha_{H H}<0$ and $\hat{\gamma}_{H H}=0$. Assuming equal decay rates $\delta_{L}$ and $\delta_{H}$ of the household time and corporate time habits, this utility function can be reduced to a utility function $U\left(X, L ; s_{X}, s_{L}\right)$ by substituting $H=T-L$ and $s_{H}=T / \delta_{L}-s_{L}$ (see Sec. 3.1). This yields a specification of the same form as (4.1) without the $\beta$-terms, where $\alpha_{X}=\alpha_{X}, \alpha_{X X}=\alpha_{X X}, \gamma_{X X}=\hat{\gamma}_{X X}$, and

$$
\begin{aligned}
& \alpha_{L}=\alpha_{L}-\alpha_{H}+\hat{d}_{H}^{*} T \text { with } \hat{d}_{H}^{*}=\left|\alpha_{H H}\right|-\frac{\hat{\gamma}_{H H}}{\delta_{H}}, \\
& \alpha_{L L}=\alpha_{L L}+\alpha_{H H}<0, \\
& \gamma_{L L}=\hat{\gamma}_{L L}+\hat{\gamma}_{H H} \geq 0 .
\end{aligned}
$$

Equation (4.16a) can be interpreted as follows. The "net" household time taste $\alpha_{L}$ is equal to $U_{L}(0 ; 0)=\hat{U}_{L}(0 ; 0)-\hat{U}_{H}\left(T ; T / \delta_{L}\right)$, i.e. the difference between the marginal utilities of the first (discretionary) household time unit and the last corporate time unit when being used to zero household time and maximal corporate time. The former marginal utility represents the "pure" household time taste $\hat{\alpha}_{L}$, and, since $\hat{\theta}_{H}\left(H ; s_{H}\right)=\hat{\alpha}_{H}+\hat{\alpha}_{H H} H+\hat{\gamma}_{H H} s_{H}$, the latter marginal utility can be written as $\alpha_{H}-\hat{d}_{H}^{*} T$. Here $\alpha_{H}=\hat{V}_{H}(0 ; 0)$ is the "pure" corporate time taste, and the parameter $\hat{d}_{H}^{*}$ is analogous to the IHF parameter $d^{*}$ as defined by ( $4.10 \mathrm{~d})$ in Section 4.3 and measures minus the relative strength of the corporate time IHF. When $\alpha_{H}$ is positive and $\hat{d}_{H}^{*}$ is negative, i.e., when the corporate time IHF is (relatively) strong, the "net" household time taste $\alpha_{L}$ is smaller than the "pure "household time taste $\alpha_{L}$ and may even be negative. Especially the latter possibility has interesting implications for the long-run labour supply (see below).

\section{Shart-run labour supply}

In the usual situation of non-satiating consumption at the optimal point, short-run labour supply is again given by (4.5) in Section 4.1 with the same definitions of the "parameters". Now, however, the "parameter" $b:=U_{L}\left(T ; s_{L}\right)$ may more easily be negative, since it is equal to $\hat{U}_{L}\left(T ; s_{L}\right)-\hat{U}_{H}\left(0 ; s_{H}\right)$. i.e. the difference between the marginal utilities of the last possible household time unit and the first corporate time unit at given habit levels, and since the latter marginal utility may be positive. This stronger tendency of $b$ to negativity also follows from (4.2b) for $L=T$, since the "net" household time taste $\alpha_{L}$ may be smaller than the "pure" household time taste $\alpha_{L}$ and even be negative (see above). This implies that 
the net marginal utility of household time as given by (4.2b) may more easily become negative for high $L$ and low $s_{L}$. This corresponds to the development of a person into a "workaholic", in which the household time habit stock $s_{L}$ declines as the person gets used to more corporate time and less household time (see footnote 7 in Sec. 3.3). In particular, $b:=U_{L}\left(T ; s_{L}\right)$ then becomes negative and, by virtue of (4.5), this implies that the corporate time $H$ has the positive value $-b / d$ for zero wage rate $w$. This describes volunteer work.

In the subcase where the parameter $a:=U_{X}\left(Y_{O} ; s_{X}\right)$ is positive "we then get, for $w$ rising from zero, a backward-bending short-run labour supply curve. The restriction $H \leq T$ can be shown to be not binding for any non-negative $w$ if and only if $-b / d$ is smaller than $T$ (or $\alpha_{L}+\gamma_{L L} s_{L}$ is positive) and $c:=\left|U_{X X}\right|$ is larger than or equal to a certain positive value $c_{o}$ (see Sec. 4.1). Then a short-run labour supply curve will look as indicated in Figure 2.6b in Subsection 2.2.5. Since the expression in (4.5) approaches zero for $w$ going to infinity, there is a $w^{b}$ at which the expression becomes equal to $H^{D^{\prime}}=-b / d$ with $U_{L}=0$. At this point $U_{X}$ is also zero by virue of the first-order condition $U_{X}=U_{L} / w$ for an interior optimum, implying a "double bliss" optimum of a fully satisfied corporate worker. For $w$ rising above $w^{b}$ this worker will not reduce her (or his) corporate time as described by (4.5) and as implied by (4.3), but stick to the same corporate time, household time and consumption, since she does not want her marginal utilities of household time and consumption to become negative as implied by (4.3) (which assumes a binding income constraint).

In the subcase where both the parameters $a:=U_{X}\left(\boldsymbol{Y}_{O} ; s_{X}\right)$ and $b$ are negative, we are in the situation of a millionaire who works for fun, as described in Subsection 2.2.5. Then the short-run labour supply curve coincides with the vertical $H=-b / d$ line. The subcases where $a$ or $b$ is zero are straightforward transition cases between positive or negative $a$ or b.

\section{Long-run labour supply}

In the usual situation of non-satiating consumption at the optimal point, long-run labour supply can be derived as follows. First, consider the case where the unique interior equilibrium is stable. Long-run labour supply is then again given by (4.9) in Section 4.3 with the same definitions of the parameters, but now the parameter $b^{*}:=U_{L}\left(T ; T / \delta_{L}\right)=\alpha_{L}-d^{*} T$ may more easily be negative, since it is equal to $\hat{U}_{L}\left(T ; T / \hat{\delta}_{L}\right)-\hat{U}_{H}(0 ; 0)$, i.e. the difference between the marginal utilities of the last household time unit and the first corporate time unit when being used to maximal household time and zero corporate time, and since the latter "pure" corporate time taste may be positive. This stronger tendency of $b^{*}$ to negativity is also implied by the possibilities that the "net" household time taste $\alpha_{L}$ is smaller than the "pure" household time taste $\alpha_{L}$ and that it is even negative (see above).

The IHF parameter $d^{*}:=\left|U_{L L}\right|-U_{L s_{L}} / \delta_{L}$ in the above formula for $b^{*}$ is equal to the sum of the separate IHF parameters $\hat{d}_{L}^{*}:=\left|\hat{v}_{L L}\right|-\hat{\theta}_{L s_{L}} / \hat{\delta}_{L}$ and $\hat{d}_{H}^{*}:=\left|\hat{\theta}_{H H}\right|-\hat{\theta}_{H s_{H}} / \hat{\delta}_{H}$, so measures minus the total (relative) strength of the household and corporate time IHFs. Substituting this relation and (4.16a) into $b^{*}=\alpha_{L}-d^{*} T$, it follows that $b^{*}=\alpha_{L}-a_{H}-\lambda_{L}^{*} T$ This implies that the long-run reservation wage $w_{r}^{*}=b^{*} / a^{*}$ is lowered by a positive 
corporate time taste $\hat{\alpha}_{H}$, but is independent of the strength $-\hat{d}_{H}^{*}$ of the corporate time IHF. Just as the independence of the $w_{ \pm}^{*}$ in (4.13) in Section 4.3 of the strength $-\hat{d}_{L}^{*}$ of the household time IHF, this is implied by the particular feature of the AQM that zero values of a behaviour variable and the corresponding habit stock have no effect on the labour supply. In contrast, the $w_{ \pm}^{*}$ at which the restriction $H^{*} \leq T$ becomes binding, are affected by the corporate time taste $\alpha_{H}$, but also by the strength $-\hat{d}_{H}^{*}$ of the corporate time IHF.

These features have some interesting implications for diagrams like those in Figures 4.3, 4.4a-c and 4.6 in Sections 4.3 and 4.4. Assume a positive $\alpha_{H}$ and first consider only changes in $d^{*}=\hat{d}_{L}^{*}+\hat{d}_{H}^{*}$ at constant $\hat{d}_{H}^{*}$. Then modifications in Figures $4.3,4.4 \mathrm{a}-\mathrm{c}$ and 4.6 are al lower, but parallel position of the $w_{r}^{*}$ line and, for non-negative $\alpha_{L}$, of the $w_{-}^{*}$ line and a higher, but parallel position of the $w_{+}^{*}$ line. As a result, the value $d^{*}=\alpha_{L} / T$ for which the $w_{r}^{*}$ line meets the $w=0$ axis in Figures 4.3 and 4.6, is lower and the width of the band in which there is a long-run equilibrium for $H^{*}=T$ is larger. The former result makes the case of a negative $b^{*}$, describing volunteer work in the long run, more likely. For positive $a^{*}$ in Table 4.2 in Section 4.3, we then obtain a backward-bending long-run labour supply curve as in Figure 2.6a (Subsec. 2.2.5) in Subcases $1^{e}$ and $1^{f}$, and a similar labour supply curve with an inelastic piece for $H^{*}=T$ in Subcase $1^{g}$ (see Fig. 4.3), and a forward-sloping labour supply curve with an inelastic piece for $H^{*}=T$ and higher $w$ in the Subcases $3^{\mathrm{b}}$ and $5^{\mathrm{b}}$ (see Fig. 4.6). When $a^{*}$ is non-positive (Subcase $1^{\mathrm{b}}$ ), we are in the situation of a millionaire who works for fun, as described in Subsection 2.2.5. Then the long-run labour supply curve coincides with the vertical $H=-b^{*} / d^{*}$ line.

For $\alpha_{L}<0$ or $\hat{\alpha}_{L}<\alpha_{H}+\left(-\hat{d}_{H}^{*}\right) T$ (see (4.16a)), i.e., when the "pure" household time taste is lower than the "pure" corporate time taste plus the net marginal utility effect of corporate time IHF and short-run satiation tendency, there are some additional and drastic modifications. Since $\alpha_{L}=U_{L}(0 ; 0)$, the net marginal utility of the first household time unit when being used to zero household time, is then negative, and this implies that, for a person who is used to maximal corporate time and zero household time, there is no incentive to work less than maximal corporate hours, no matter how low or high the wage rate $w$. In all situations this implies the existence of a stable long-run equilibrium $H^{*}=T$ for any $w$. In Figures $4.3,4.4 \mathrm{c}$ and 4.6 the $w_{-}^{*}$ line has then disappeared to negative $w$ and the $w_{+}^{*}$ line has become irrelevant, because it holds only for the case of a binding income constraint for $H^{*}=T$ and hence a positive $\alpha_{L}=U_{L}(0 ; 0)=w_{+}^{*} U_{X}\left(w_{+}^{*} T+Y_{O}:\left(w_{+}^{*} T+Y_{O}\right) / \delta_{X}\right)$ (see (4.3)). Moreover, the value $d^{*}=\alpha_{L} / T$, for which the $w_{r}^{*}$ line meets the $w=0$ axis, has become negative. This eliminates the $w_{r}^{*}$ line in Figure 4.3, leaving only a region with $H^{*}=T$, and it leaves us in Figures 4.4a-c and 4.6 with only a region with $H^{*}=T$ above the $w_{r}^{*}$ line and a region with $H^{*}=0$ or $H^{*}=T$ under this line.

Thus, when the net household time taste $\alpha_{L}$ is negative, all long-run labour supply schedules describe that for $w=0$ the subject prefers to volunteer for the maximal corporate time and that for positive $w$ he (or she) prefers to keep his labour supply on the same maximal level. So, even when the consumption IHF is only weak $\left(c^{*}>0\right)$, the labour supply schedule is not backward-bending. However, for $d^{*}<\alpha_{L} / T$, so when the household time and corporate time IHF is rather strong. the preference for volunteering holds only when the subject has been used to maximal corporate time. Then there is for sufficiently low $w$ also another long-run equilibrium $H^{*}=0$, describing that the person is strongly used to 
zero corporate hours and maximal household time, and hence prefers to remain non-participating (see Figure 4.7). Only beyond a certain positive entry wage $w^{* 1}=w_{r}^{*}$ he will enter the labour market and develop into a person who works maximal corporate time and is strongly used to that. When $w$ then falls below $w^{* 1}$, he will not reduce his corporate time, even when $w$ falls to zero.

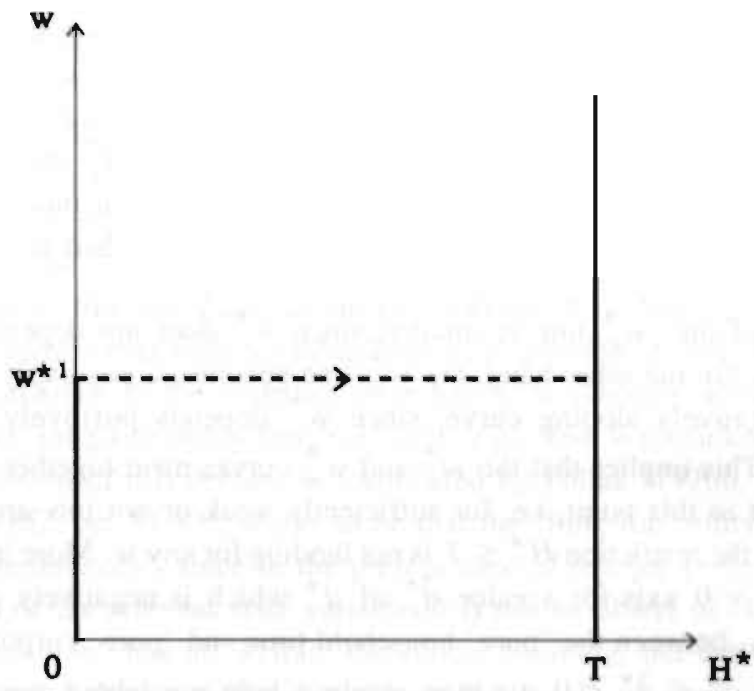

Figure 4.7 Long-run labour supply schedule for negative net household time taste $\alpha_{L}$ and strong household time and corporate time IHF with $d^{*}<\alpha_{L} / T$.

Thus, in this case as well as for $d^{*} \geq \alpha_{L} / T$ there is no positive long-run exit wage. Therefore, both cases seem to model strong forms of "workaholism" in which there is not only no positive short-run exit wage, but also no positive long-run exit wage (see the description of a "workaholic" in Sec. 3.2) and, for $d^{*} \geq \alpha_{L} / T$, no positive long-run entry wage. So, when $w$ is reduced to zero, this workaholic will continue to work the maximal corporate time in the short as well as long run. However, workaholism suggests a not too weak corporate time IHF (so a not too positive $\hat{d}_{H}^{*}$ ), whereas, $\alpha_{L}<0$ being equivalent to $\hat{\alpha}_{L}<\hat{\alpha}_{H}-\hat{d}_{H}^{*} T$ (see above), both cases can also occur for very weak or no corporate time: IHF (a high $\hat{d}_{H}^{*}$ ), provided the "pure" corporate time taste $\alpha_{H}$ is sufficiently larger than the "pure" household time taste $\hat{Q}_{L}$ and, for the case of $d^{*}=\hat{d}_{L}^{*}+\hat{d}_{H}^{*}<\alpha_{L} / T$, provided the household time IHF is sufficiently strong $\left(\hat{d}_{L}^{*}<\alpha_{L} / T-\hat{d}_{H}^{*}\right)$. These subcases of a strong exogenous attachment to corporate work relative to household time ${ }^{17}$ could be seen as, alternative explanations of the described long-run labour supply behaviour besides workahol-

${ }^{17}$ Note that this can even hold for unhappy persons with a negative corporate time taste and a sufficiently more negative (discretionary) household time taste. 
ism involving not too weak endogenous corporate time IHF. ${ }^{18}$ An example of any of these subcases may be given by retired professors, entrepreneurs and the like who continue to work maximal or almost maximal corporate hours after their formal retirement. ${ }^{19}$

In discussing the modifications of Figures 4.3, 4.4a-c and 4.6 only the effects of changes in the total IHF parameter $d^{*}=\hat{d}_{L}^{*}+\hat{d}_{H}^{*}$ at a constant value of the IHF parameter $\hat{d}_{H}^{*}$ for corporate time have been considered. However, it seems more appropriate to analyse the effects of simultaneous changes in $\hat{d}_{L}^{*}$ and $\hat{d}_{H}^{*}$. To obtain again two-dimensional diagrams, we could assume that these IHF parameters vary in a fixed positive proportion, i.e.., as a person is more (less) sensitive to household time IHF, he (or she) is also more (less) sensitive to corporate time IHF. Then the separate IHF parameters $\hat{d}_{L}^{*}$ and $\hat{d}_{H}^{*}$ are fixed positive fractions of the total IHF parameter $d^{*}$ and we get additional modifications in Figures 4.3, 4.4a-c and 4.6. We mention the most important ones only and do not fully elaborate them.

First, the slope of the $w_{r}^{*}$ line is smaller, since $w_{r}^{*}$ does not depend on the corporate time parameter $\hat{d}_{H}^{*}$. On the other hand, the $w_{-}^{*}$ line becomes a positively sloping curve and the $w^{*}$, line a negatively sloping curve, since $w_{-}^{*}$ depends positively and $w_{+}^{*}$ depends negatively on $\hat{d}_{H}^{*}$. This implies that the $w_{-}^{*}$ and $w_{+}^{*}$ curves meet together for a certain value of $d^{*}$, so that right to this point, i.e. for sufficiently weak or not too strong household and corporate time IHF, the restriction $H^{*} \leq T$ is not binding for any $w$. More importantly, the $w_{-}^{*}$ curve meets the $w=0$ axis for a value $\breve{d}^{*}$ of $d^{*}$ which is negatively proportional to the difference $\hat{a}_{L}-\hat{a}_{H}$ between the "pure" household time and "pure" corporate time taste. For $a_{L}-\sigma_{H}>0$ and $d^{*} \leq \check{d}^{*}<0$ we then obtain a long-run labour supply schedule as in Figure 4.7 and for $\alpha_{L}-\alpha_{n}<0$ and $d^{*} \leq \check{d}>0$ we obtain the same schedule for sufficiently negative $d^{*}$ and a schedule with merely $H^{*}=T$ for higher $d^{*}$. Thus, just as found above, a schedule as in Figure 4.7 requires sufficiently strong total IHF with respect to household and corporate time, but because of the assumed fixed positive proportions and hence equal signs of $\vec{d}_{L}^{*}$ and $\vec{d}_{H}^{*}$, this implies now also sufficiently strong corporate time IHF and hence workaholism. Moreover, $\hat{d}_{L}^{*}$ and $\hat{d}_{H}^{*}$ having the same sign is also responsible for the finding that a schedule with merely $H^{*}=T$ can occur only for $\alpha_{L}-\alpha_{H}<0$, i.e., when the "pure" corporate time taste is higher than the "pure" household time taste.

However, although both cases of a negative net household time taste $\alpha_{L}$ may occur in reality, they are rather extreme. For all other cases we can conclude that Proposition 4.1 as formulated in the previous section continues to hold for the combination of household and corporate time IHF instead of pure household time IHF.

18 I do not simply associate workaholism with strong corporate time IHF, since I consider workaholism as a kind of addiction and associate addiction with a habit-dependent threshold rather than with strong IHF as defined here (see foomole $I$ in Ch. 3). In fact, the model of workaholism in Section 3.2 presupposes stable household and corporate time IHF. which can be weak.

${ }^{19}$ Then also the pension should be taken into account in the income constraint, but this does not make a difference for the labour supply behavior. In the case of a professor like the economist Tinbergen I would not speak of workaholism, but rather of a vocation. Still, such a vocation may have strongly been reinforced by one's lifetime experience with corporate work. 


\subsection{Long-run utility functions}

In the always stable long-run case as analysed in Section 4.3 , the fact that the form of the long-run labour supply function (4.9) is similar to the form of the short-run labour supply function (4.5) in Section 4.1, implies that the former function can be rationalized as the result of the constrained maximization of an additive quadratic "long-run utility function". The specification of this utility function is

$$
U^{*}(X, L)=\alpha_{X} X+\alpha_{X X}^{*} X^{2}+\alpha_{L} L+\alpha_{L L}^{*} L^{2},
$$

where $\alpha_{X X}^{*}:=-c^{*}:=\alpha_{X X}+\gamma_{X X} / \delta_{X}$ and $\alpha_{L L}^{*}:=-d^{*}:=\alpha_{L L}+\gamma_{L L} / \delta_{L}$. In Case 1 in Table 4.1 with positive $c^{*}$ and $d^{*}$ this describes "mountain parabolas" in consumption $X$ and household time $L$ like the short-run utility function (4.1). More generally, Schokkaert (1982, pp. 79-80) proves that such a rationalization is possible if and only if the short-run utility function is additive. In the seventies there has been a debate on the (welfare-theoretical) meaning of the "long-run utility function" (see, e.g., Von Weizsïcker, 1971, and Pollak, 1976b). The conclusion of this debate, as formulated by Pollak (1976b, Secs. 5 and 6) (see also Schokkaert, 1982, pp. 81-83), seems to be that the "long-run utility function" does not reflect "long-run preferences", since in the present case of myopic preference formation the long-run evolution of the relevant state variable(s) is not an object of choice. Therefore the "long-run utility function" has no welfare-theoretical meaning, but can merely serve as an indicator of long-run behaviour. To obtain long-run levels of cardinal utility indicating individual welfare for various given $X$ and $L$, we should add the $\beta$-terms in (4.1) with $s_{X}=X / \delta_{X}$ and $s_{L}=L / \delta_{L}$ (describing full habituation) to (4.17) (see footnote 2). The resulting long-run utility function can be written in the same form as (4.17), but the parameters are then generally different.

In the present context the meaning of the "long-run utility function" (4.17) can be clarified as follows. First, we remark that the rationalization of the long-run labour supply function (4.9) by the "Iong-run utility function" (4.17) also holds for the three stable situations other than Case 1 in Table 4.1. Then either $c^{*}$ or $d^{*}$ is non-positive, but, along the standard budget line $X=w(T-L)+Y_{O}$ in the $(X, L)$ plane, (4.17) again describes a mountain parabola. This can be seen by substituting the standard constraints into (4.17) (cf. (4.8a) and (4.8b)), yielding the constrained "long-run utility function"

$$
U^{* c}\left(H ; w, Y_{O}, T\right)=\left(a^{*} w-b^{*}\right) H-\frac{1}{2}\left(c^{*} w^{2}+d^{*}\right) H^{2} .
$$

When $c^{*} w^{2}+d^{*}$ is positive, i.e., when the stability condition (4.11) is fulfilled, this function describes a mountain parabola in corporate time $H$ (which can be regarded as parametrizing the budget line in the $(X, L)$ plane). Applying the first-order condition $U^{* C} H^{=0}$ is then easily seen to yield the long-run labour supply function (4.9).

When $c^{*} w^{2}+d^{*}$ is negative, so when (4.9) stands for an unstable long-run equilibrium $H^{* u}$, the constrained "long-run utility function" (4.18) describes a "valley parabola" in $H$. Then (4.9) minimizes (4.18) and, when it describes an interior $H^{* u}$, so when $w^{* 2}<w<w^{* 1}$ in Figure 4.2, we have for $w$ near $w^{* 2}\left(w \geq w^{* 2}\right)$ a graph of the constrained "long-run 
utility function" (4.18), as indicated by the lowest curve in Figure 4.8. (Recall that $a^{*} w-b^{*}<0$ for $w<w^{* 1}=b^{*} / a^{*}$.) According to this graph the two stable long-run equilibria $H^{*}=0$ and $H^{*}=T$ are local comer maximum points of the "long-run utility function". Whereas only the equilibrium $H^{*}=0$ is also a global maximum point, both equilibria can be realized dependent on the historical time paths of exogenous variables (see Sec. 4.2). Thus, this hysteresis of multiple long-run equilibria is described by local and not global maximization of the "long-run utility function". This local maximization is equivalent to local minimization of a Lyapunov function which is defined as minus the constrained "long-run utility function" and which is similar to the potential functions considered in applications of catastrophe theory (see below). On the other hand, a global maximization or minimization model like the short-run utility maximizing model can describe catastrophes, but no hysteresis (see Sec. 3.6 and see Vendrik, 1979a and $b$, for the corresponding variant of catastrophe theory). In particular, this holds for the usual "long-run utility" maximizing model (see, e.g., Anderson and Blundell, 1982). Moreover, I find this behaviour model implausible, since it cannnot give a proper account of a decrease in long-run utility or welfare of a person who myopically gets used or even addicted to a harmful behaviour like smoking (see Pollak, 1976b, Sec. 5 , and see below).

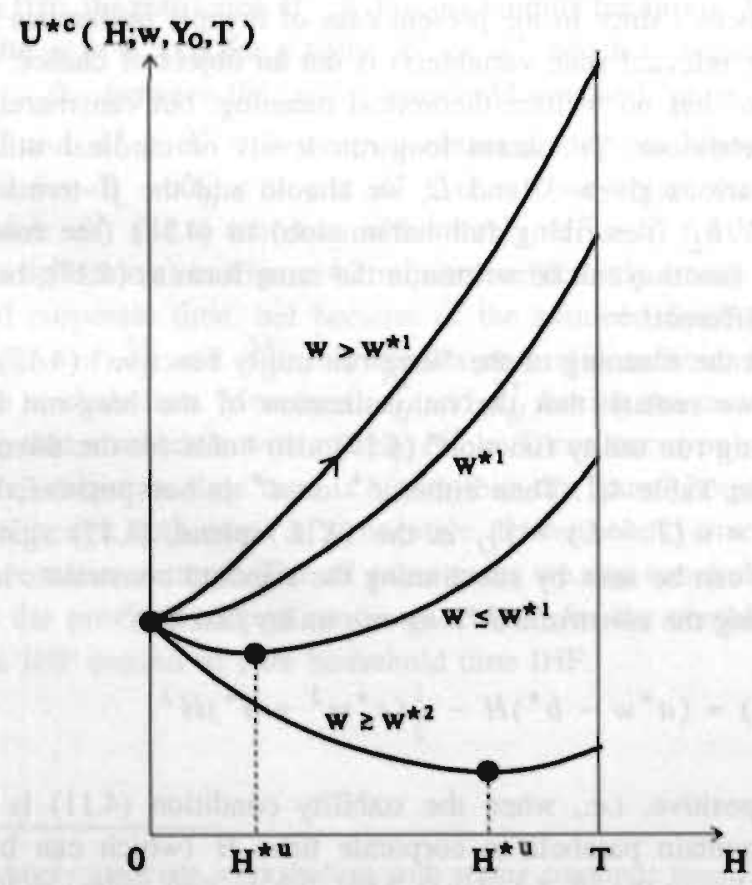

Figure 4.8 Graphs of constrained "long-run utility function" with unstable equilibrium $H^{* u}$ for various values of the wage rate. 
In order to gain more insight into the working and interpretation of the local "long-run utility" maximizing model, we consider the effects of a rising wage rate and suppose that at $w \geq w^{* 2}$ in Figure 4.8 the person is non-participating $\left(H^{*}=0\right)$. As the wage rate rises, the "long-run utility" for positive corporate hours $H$ increases because of the consumption gains, so for $w$ approaching the long-run entry wage $w^{* 1}\left(w \leq w^{* 1}\right)$ the graph of the constrained "long-run utility function" looks as indicated in Figure 4.8. This graph suggests that, in the long run, the utility of high corporate hours is then higher than the utility of nonparticipation. This, however, does not need to hold, since for such a global utility or welfare comparison the $\beta$-terms in (4.1) with $s_{X}=X / \delta_{X}=\left(w H+Y_{O}\right) / \delta_{X}$ and $s_{L}=L / \delta_{L}=(T-H) / \delta_{L}$ should be added to (4.18) (see above). When the consumption IHF and the corporate time IHF are more harmful or less beneficial than the household time IHF (see footnote 2), the long-run utility of high corporate hours may then still be lower than the utility of nonparticipation. On the other hand, the "long-run utility function" (4.18) for $w \leq w^{* 1}$ merely describes that (in the long run) the person prefers to stay at home, since she (or he) is strongly used to that and hence would lose utility in the short run when she would enter the labour market (remember that the person is myopic).

However, the unstable equilibrium $H^{* u}$ approaches the stable non-participation equilibrium and will for $w=w^{* 1}$ coincide with it, making it unstable, and subsequently for $w>w^{* 1}$ disappear (see Sec. 4.2). Accordingly, $H^{*}=0$ becomes a local minimum point of the "long-run utility function" for $w \geq w^{* 1}$ with a positive marginal "long-run utility" for $w>w^{* 1}$ (see Fig. 4.8). This describes that for $w \geq w^{* 1}$ the person prefers to enter the labour market, since she can gain utility in the short run by doing so. Moreover, the positive marginal "long-run utility" of all corporate hours describes that when the person has got used to a part-time job, she wants to work more corporate hours, etc. until she works maximal corporate hours $\left(H^{*}=T\right)$. The person engages into this entry catastrophe irrespectively of whether her genuine long-run utility as given by the welfare extension of (4.18) rises or falls by working an increasingly number of corporate hours. This holds, since the person is: myopic and hence does not take into account the long-run utility costs or benefits of harmful or beneficial IHF in her short-run labour supply decisions.

When the wage rate again declines, the person working maximal corporate hours will engage into an exit catastrophe only beyond a long-run exit wage $w^{* 2}$, which is lower than the long-run entry wage $w^{* 1}$. In Figure 4.8 the local maximum at $H^{*}=T$ has then turned into a local minimum and the result is individual hysteresis. Individual hysteresis also occurs. when the person has become aware of the long-run utility costs or benefits of hamnful or beneficial IHF and takes them more or less into account in her labour supply decisions. A simplified picture of this more or less rational IHF (see Sec. 1.1) is provided by the assumption that the person will then consider her genuine long-run utility as given by the welfare extension of (4.18). The graphs of this constrained long-run utility function of corporate time $H$ can be similar to those in Figure 4.8, but the corresponding values of the wage rate are generally different. Moreover, a non-participating person will already enter in a situation like that for $w \leq w^{* 1}$ in Figure 4.8, when the long-run utility gains of working a high number of corporate hours compensate for the short-run adjustment costs of going through a "valley of habituation". Similarly, a person working maximal corporate hours will already start to work less corporate hours in a situation like that for $w \geq w^{* 2}$ in Figure 4.8 . Nevertheless, again this results in individual entry and exit catastrophes as well as individual 
hysteresis. Furthermore, the long-run entry wage in this case of rational IHF will be lower or higher than the long-run entry wage $w^{* 1}$ in the case of myopic IHF dependent on whether the anticipated formation and/or erosion of habits is more or less beneficial or harmful. For the long-run exit wages in the two cases an analogous relation holds. When the anticipated formation and/or erosion of habits is very harmful, a long-run entry or exit wage may even not exist in the rational IHF case. A severe shortcoming of this rational IHF model is the implicit assumption that the short-run adjustment process towards a new long-run equilibrium is assumed to be "quasi-static", i.e., the adjustment of the corporate hours is assumed to be so slow as to allow approximately full adjustment in the short run of the habits to the changing corporate hours. Thus, the model does not describe the possible advantages of going "cold turkey", i.e., for a non-participant, of starting to work the maximal corporate hours at once. This requires a full-blown intertemporal utility maximizing model like that of Becker and Murphy (1988) (see also Frank, 1989). As already suggested by Pollak (1976b, p. 296), such a model may also provide more appropriate and dynamic indicators of welfare.

Finally, we remark that non-linear demand functions like $L^{C}$ in Figure 3.1 in Section 3.2 are obtained by adding fourth-order terms in the behaviour and habit variables to the longrun utility function (4.17), and hence to (4.18) (cf. Becker and Murphy, 1988, p. 683). One or more of the local maxima describing stable equilibria then become interior. Similar potential functions with fourth or even higher order terms, implying two or more, merely interior, stable equilibria, are considered and classified in catastrophe theory (see, e.g., Thom, 1975, and Zeeman, 1977). In cases of more than two interior stable equilibria, related, but more complicated configurations of catastrophes and hysteresis than found above are derived.

\subsection{Aggregation: short run}

So far we have analysed individual labour supply behaviour. However, in order to trace the macro-economic implications of this behaviour we should aggregate it over individuals. The most simple procedure is to assume that in the social group or population under consideration the individual preferences and constraints are identical. Thus, in a "continuous" labour supply regime everyone faces the same wage rate $w$ and has the same other income $Y_{O}$ and habit stocks $s_{X}$ and $s_{L}$, and the individual short and long-run labour supply functions $H\left(w, Y_{O}, T, s_{X}, s_{L}\right)$ and $H^{*}\left(w, Y_{O}, T\right)$ are identical over individuals. As a consequence, average (or per capita) short and long-run labour supply functions are exact replicas of the corresponding individual functions.

Unfortunately, reality shows that these assumptions are much too simple: individuais face widely diverging $w$ and $Y_{O}$ and have different preferences. As a result, individual labour supply functions are not identical and average short and long-run labour supply in the social group can generally be considered as functions of the distributions over individuals of $w$, $Y_{O}, T$, and state variables and taste parameters in the utility function. Since, at least at the kink of an individual labour supply curve at the reservation wage, an individual labour supply function is non-linear in these variables and parameters, an exact analysis is complicated. Therefore, our discussion of the aggregation issue will focus on a particular specifica- 
tion of the general model, namely the AQM, and next try to generalize its conclusions to other specifications. Aggregation of short-run labour supply curves is discussed in the present section and aggregation of long-run labour supply schedules in the next section.

First, consider individual variation in only the "parameters" $a:=U_{X}\left(Y_{O}^{\prime} ; s_{X}\right)$ and $b:=U_{L}\left(T ; s_{L}\right)$ of formula (4.5) for the short-run labour supply curve for $0<H<T$ in the standard case of $c, d, a$ and $b$ all being positive. The "parameters" $a$ and $b$ are chosen since they appear in (4.5) in a linear way. According to (4.6a) and (4.6b) and assuming no variations in the utility function parameters $\alpha_{X X}=-c, \gamma_{X X}, \alpha_{L L}=-d$ and $\gamma_{L L}$, the variations in $a$ and $b$ may be due to individual differences in $\alpha_{X}, Y_{O}, s_{X}, \alpha_{L}, T$ and $s_{L}$. At first sight average short-run labour supply $\bar{H}$ is given by ${ }^{20}$

$$
\frac{1}{N} \sum_{i=1}^{N} \frac{a_{i} w-b_{i}}{c w^{2}+d}=\frac{\bar{a} w-\bar{b}}{c w^{2}+d} .
$$

However, this neglects the fact that different individuals have different positive reservation wages $w_{r i}=b_{i} / a_{i}$, which are in general unequal to $\bar{b} / \bar{a}$. In the subcase where $c$ is larger than or equal to $c_{o i}$ for all individuals ${ }^{21}$, the correct expression for $\bar{H}$ is

$$
\bar{H}=\frac{1}{N} \sum_{\substack{i \\ w_{r i}<w}} \frac{a_{i} w^{\prime}-b_{i}}{c w^{2}+d} .
$$

(Note that for $w_{r i} \geq w, H_{i}=0$.) For $0 \leq w \leq \min w_{r i}=\min b_{i} / a_{i}, \vec{H}$ is zero, but when $w$ rises beyond this interval, $\vec{H}$ will rise with $w$ as more persons enter the labour market (see Figure 4.9).

Using (4.19) we can write (4.20) as

$$
\bar{H}=\frac{\bar{a} w-\bar{b}}{c w^{2}+d}-\frac{1}{N} \sum_{\substack{i \\ w_{r i}>w}} \frac{a_{i} w-b_{i}}{c w^{2}+d} .
$$

Since the terms under the summation sign are negative, it follows that $\bar{H}$ is larger than the first term on the right-hand side. However, when $w$ rises to high values, the "correction" terms under the summation sign go to zero as less persons stay at home, implying that $\bar{H}$ approaches the first term. When the number of individuals $N$ is large and the simultaneous distribution of $a_{i}$ and $b_{i}$ can be approximated by a continuous and unimodal density func-

${ }^{20}$ Note that we aggregate the individual labour supply functions directly, i.e., we do not firsi aggregate the individual utility functions or the individual household time demand functions of the habit stocks and next apply schemes of consistent aggregation (see, however, the next section ard] Secs. 6.1 and 6.2).

${ }^{21}$ See Section 4.1. When $c$ is smaller than $c_{o i}$ for a number of individuals, (4.19) also neglects the fact that these individuals have different $\boldsymbol{w}_{-i}$ and $\boldsymbol{w}_{, i}$ between which the restriction $H_{i} \leq T_{i}$ is binding. For that case a similar average labor supply curve can be derived as for the case where $c \geq c_{o i}$ for all individuals, but the analysis is more complicated (see also Subsec. 5.5.1). 


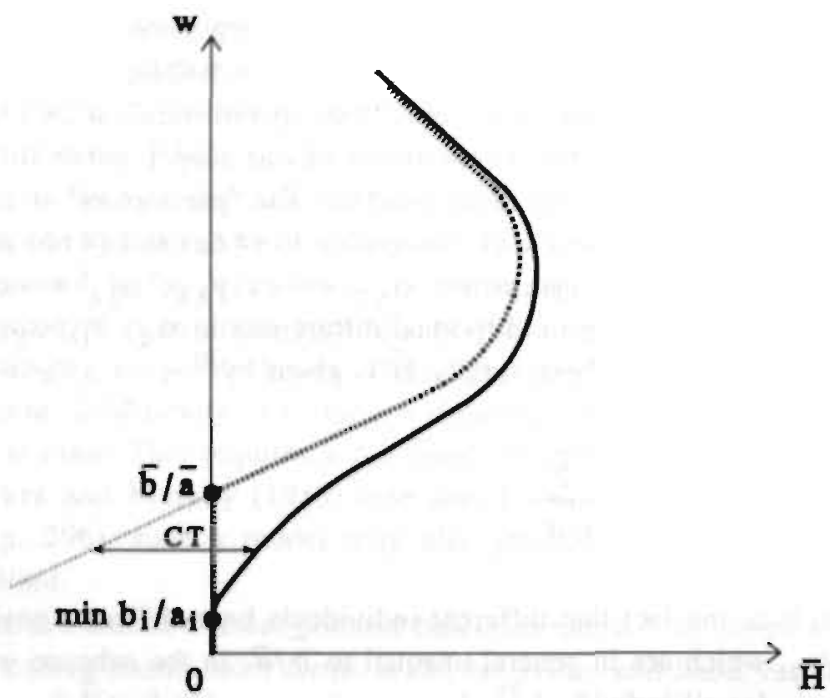

Figure 4.9 Average and representative short-run labour supply curve in the AQM, indicated by solid and dotted bold curve, respectively.

tion, this can be shown usually to result into a smooth average labour supply curve such as in Figure 4.9.22

The first term in (4.21) can be interpreted as describing for $\bar{a} w-\bar{b} \geq 0$, or $w \geq \bar{b} / \bar{a}$, a representative labour supply curve. This curve is indicated as a dotted bold curve in Figure 4.9. The average labour supply curve as indicated by a solid curve in Figure 4.9 deviates from this representative curve, but approaches it for high $w$. For $w \geq \bar{b} / \bar{a}$ the deviation is given by the "correction" terms CT in (4.21), but for $w<\bar{b} / \bar{a}$ the deviation is smaller than $\mathrm{CT}$ by the negative first term in (4.21).

What happens if also the parameters $c$ and $d$ of the short-run labour supply curve (4.5) and the wage rate $w$ vary over individuals? Expression (4.5) is not linear in these parameters and variable, implying that averaging (4.5) over individuals does not result in a simple formula like that in (4.19). Not only the averages of the distributions of $c_{i}, d_{i}$ and $w_{i}$ will play a role, but also (co)variances and higher-order (co)moments of these distributions as well as covariances and higher-order comoments of the distributions of $c_{i}, d_{i}$ and $w_{i}$ with the distributions of $a_{i}$ and $b_{i}$. Nevertheless, when $c_{i}, d_{i}$ and $w_{i}$ do not vary too much, we could, as a first-order approximation, neglect these (co)variances and higher-order (co)moments and replace $c_{i}, d_{i}$ and $w_{i}$ in (4.5) by their averages $\bar{c}, \bar{d}$ and $\bar{w}$. Then we are in the same situation as above with $\bar{c}, \bar{d}$ and $\bar{w}$ instead of $c, d$ and $w$ in (4.19)-(4.21). This

22 "Smooth" means that $\bar{H}(w)$ is twice continuously differentiable. The shape of the average labour supply curve in Figure 4.1 follows from laborious standard derivations of $\partial \bar{H} / \partial w$ and $\partial^{2} H / \partial w^{2}$. implying that both derivatives are positive for sufficiently low $w$ such that the negative income effects of the denominator $c w^{2}+d$ in (4.17) are small relative to the level of the simultaneous density function of $a$ and $b$ for the marginal entrants. 
implies representative and average short-run labour supply curves as functions of $\bar{w}$ which are of the same shape as the curves described by (4.19) for $w \geq \bar{b} / \bar{a}$ and (4.21), respectively, in Figure 4.9. For other than AQM specifications of continuous individual labour supply curves with varying positive reservation wages $w_{r i}$ one can make a similar analysis, implying that the kinks at $w_{r i}$ in the individual curves are "smoothed out" by "correction terms" like those in (4.21) in the average curve. ${ }^{23}$

Finally, as discussed in Section 4.5 , the parameters $b_{i}$ and/or $a_{i}$ may be negative for some individuals. This yields additional complications. For example, when $b_{i}$ is negative and $a_{i}$ is positive, the individual short-run labour supply curves may be given by Figure $2.6 \mathrm{~b}$ in Subsection 2.2 .5 with a non-positive reservation wage and a kink at $w_{i}^{b}$. Aggregating such labour supply curves to an average labour supply curve, we obtain a curve which is similar to that in Figure $2.6 a$, but which has a vertical piece for $w$ above $\max w_{i}^{b}$. Combining this curve with the average labour supply curve of the other "standard" labour suppliers results in an overall average shorl-run labour supply curve according to which $\bar{H}$ is already equal to a positive value $\bar{H}(0)$ for $w=0$, which is less backward-sloping for higher $w$ than the curve in Figure 4.9, and which approaches $\vec{H}(0)$ for $w$ going to infinity.

\subsection{Aggregation: long run}

For individual long-run labour supply curves of the form (4.9) (Sec. 4.3) with $c^{*}, d^{*}, a^{*}$ and $b^{*}$ all being positive, aggregation is analogous to that for the corresponding short-run curves of the form (4.5). However, for individual long-run labour supply schedules with hysteresis and catastrophes as in Figure 4.2, matters become different. We again assume positive $a^{*}$ and $b^{*}$ and first consider individual variation in only the household time taste component $\alpha_{L}$ of the "parameter" $b^{*}$ and strong consumption IHF for all persons $\left(c^{*} \leq 0\right)$. The last assumption implies that the individual long-run labour supplies $H_{i}^{*}$ remain equal to $T$ for higher $w$. Moreover, we assume that all $\alpha_{L i}$ are positive.

What happens with the average long-run labour supply $\bar{H}^{*}$, when the wage rate $w^{\prime}$ is rising from zero? Since $b_{i}^{*}, a_{i}^{*}$ and $\alpha_{L i}$ are positive and $c^{*}$ is negative, it follows that both the long-run entry wages $w_{i}^{* 1}=w_{r i}^{*}:=b_{i}^{*} / a^{*}$ and the long-run exit wages $w_{i}^{* 2}=w_{-i}^{*}$ as given by (4.13) in Section 4.3 are positive. This implies that at $w=0$ all persons in the social group are in the long run always non-participating and used to that. Then $\bar{H}^{*}$ is zero, and, for $w$ rising from zero, this will remain so for $w \leq \min w_{i}^{* 1}=\left(\min b_{i}^{*}\right) / a^{*}$, which is the long-run entry wage $w_{1}^{* 1}$ of the person(s) with the lowest household time taste $\alpha_{L 1}$. For $w$ rising beyond $w_{1}^{* 1}$, first persons with low household time tastes and next persons with higher household time tastes will enter and work in the long run the maximal feasible number of corporate hours $T$. This leads to an average long-run labour supply which is given by

${ }^{23}$ One should then treat and/or approximate the parameters and variables in the individual labour supply functions analogously to those in the AQM specification above. 


$$
\bar{H}^{*}=\frac{1}{N} \sum_{\substack{i \\ w_{i}^{* 1}<w}} r .
$$

For large $N$ we can consider $\alpha_{L i}$ and hence $b_{i}^{*}$ as continuous variables $\alpha_{L}$ and $b^{*}$, and define $\varepsilon:=\alpha_{L}-\bar{\alpha}_{L}=b^{*}-\bar{b}^{*}$ with density function $f(\varepsilon)$. Substituting from this definition into $w^{* 1}=b^{*} / a^{*}<w,(4.22)$ can be approximated as

$$
\bar{H}^{*}=\int_{-\infty}^{a^{*} w-\bar{b}^{*}} T f(e) d \varepsilon=T F\left(a^{*} w-\bar{b}^{*}\right),
$$

where $F$ is the (cumulative) distribution function of $\boldsymbol{e}$. (Note that $f(\varepsilon)=0$ for $b^{*} \leq 0$ or $\varepsilon \leq-\bar{b}^{*}$.) When $f(\varepsilon)$ is continuously differentiable and unimodal, the curve of $F\left(a^{*} w-\bar{b}^{*}\right)$ and hence of $\bar{H}^{*}$ as a function of $w$ can easily be shown to have a smooth sigmoid shape. This results in the upper curve in Figure 4.10. We call such an upper curve the entry curve.

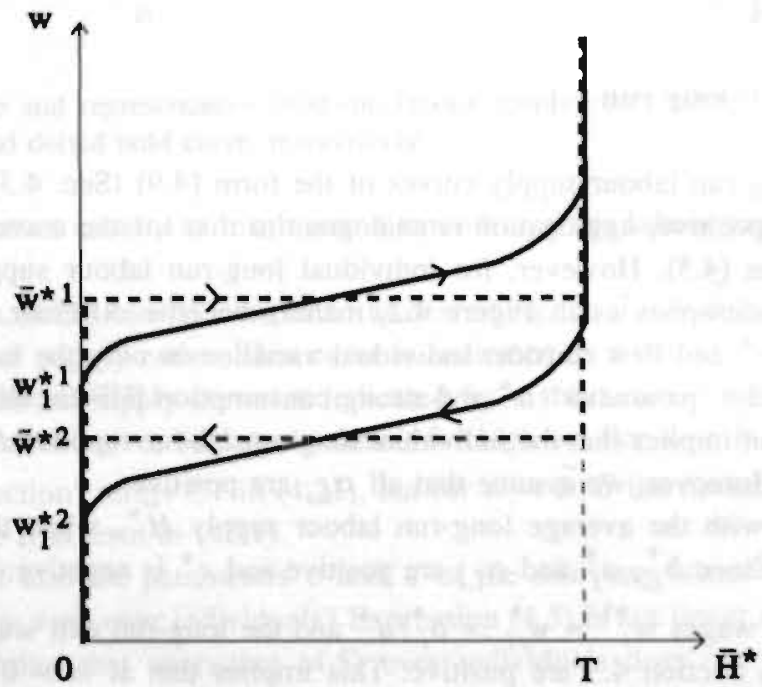

Figure 4.10 Average and representative long-run labour supply schedule with collective hysteresis and catastrophes in the $\mathrm{AQM}$ with strong consumption IHF, indicated by solid curve and dashed bold line, respectively.

For sufficiently high $w$ all persons in the social group will have entered the labour market. ${ }^{24}$ This implies a long-run situation in which all group members are employed at the maximal number of hours $T$ and are used to that. When in such a situation $w$ starts to decline, the group members will leave the labour market in the long run only at an exit wage

${ }^{24}$ Note that the number of persons $N$ being finite implies that the distribution of $\alpha_{L}$, and hence the distribution of the entry wages $w^{* 1}$, are bounded. 
$w_{i}^{* 2}=w_{-i}^{*}$ as given by (4.13) which is lower than the entry wage $w_{i}^{* 1}$. In (4.22) this entry wage should therefore be replaced by the exit wage, which varies with the parameter $\alpha_{L i}$. In the continuous approximation for large $N$ with $\varepsilon:=\alpha_{L}-\bar{\alpha}_{L}$ this can be derived to lead to a formula like (4.23) (see App. 4D), but now the argument of the distribution function $F$ depends on $w$ in a quadratic way. Nevertheless, when $f(\varepsilon)$ is continuously differentiable and unimodal, we usually obtain again a smooth sigmoid average long-run labour supply curve. This is represented by the lower curve in Figure 4.10, and we call such a curve the exit curve. It reaches $\bar{H}^{*}=0$ for $\min w_{i}^{* 2}=w_{1}^{* 2}$ of the person(s) with the lowest household time taste $\alpha_{L_{1}}$. Together with the entry curve the exit curve describes hysteresis, which we refer to as collective hysteresis. On the other hand, the entry and exit curve do not describe catastrophes, since the discontinuities in the individual long-run labour supply curves have been "smoothed out" in the average curve by the individual variation. ${ }^{25}$ This implies a substantial deviation of the average curve from the representative long-run labour supply curve for $b^{*}=\bar{b}^{*}$. The latter curve has the same shape as the individual curves given by Figure 4.2 in Section 4.2 without backward-bending part, and it has discontinuities between 0 and $T$ at the average entry wage $\bar{w}^{* 1}=\bar{b}^{*} / a^{*}$ and the exit wage $\bar{w}^{* 2}=w^{* 2}$ for $\alpha_{L}=\bar{\alpha}_{L}$. This is indicated in Figure 4.10 by dashed bold lines for lower $w$.

When the consumption IHF is weak $\left(c^{*}>0\right)$, but the household time IHF is strong $\left(d^{*} \leq 0\right)$, the individual long-run labour supply schedules may again display individual hysteresis and catastrophes, but are also backward-bending as in Figure 4.2. This makes a derivation of an average long-run labour supply schedule more complicated. If we assume that Figure $\mathbf{4 . 2}$ holds for all persons, an average long-run labour supply schedule as represented in Figure 4.11 can be derived. On the one hand, the lower half of this schedule is analogous to that of Figure 4.10, displaying a collective hysteresis loop, but no catastrophes. On the other hand, the schedule in Figure 4.11 has a backward-sloping part for $w$ above $w_{+N}^{*}$. This $w_{+N}^{*}$ is the $w_{*}^{*}$ in Figure 4.2 of the last entrant $N$ at rising $w$, who has the highest household time taste $\alpha_{L N}$. Since $w_{+i}^{*}$ as given by $(4.13)$ in Section 4.3 declines with increasing $\alpha_{L i}$, this last entrant is the first to reach the backward-sloping part of his (or her) long-run labour supply schedule for $w$ rising towards high levels. 26 Moreover, as more persons reach the backward-sloping part of their long-run labour supply schedule, the average long-run labour supply approaches the backward-sloping part of the representative longrun labour supply schedule for $b^{*}=\bar{b}^{*}$ (dotted curve for $w$ above $\bar{w}_{+}^{*}=w_{*}^{*}$ for $\alpha_{L}=\bar{\alpha}_{L}$ in Fig. 4.11).

${ }^{25}$ The shape of the hysteresis figure is similar to that of the ferromagnet in physics (see Fig. 1 in Franz, 1990). Note that in this case discontinuities in the individual labour supply curves are "smoothed out", whereas in the case of continuous individual labour supply curves kinks are "smoothed out".

${ }^{26}$ For the last entrant the conditions in situation (i) of Proposition 4.I in Section 4.4 for Figure 4.2 to hold are the strongest. This can be seen from the diagram in Figure $4.4 \mathrm{c}$. Because the $w$, line in Figure $4.4 \mathrm{c}$ moves upwards for increasing $\alpha_{L i}$, whereas the $w^{* u}$ curve remains fixed for given $c^{*}$, the under limit $d_{-}^{*}$ and the upper limit $d_{*}^{*}$ in the condition $d_{-}^{*}<d^{*}<d_{*}^{*}$, as determined by the intersections of this line and curve, move towards each other for increasing $\alpha_{L i}$. This implies that the conditions are the strongest for the last entrant at rising $w$, who has the highest household time taste $\alpha_{L N}$. 


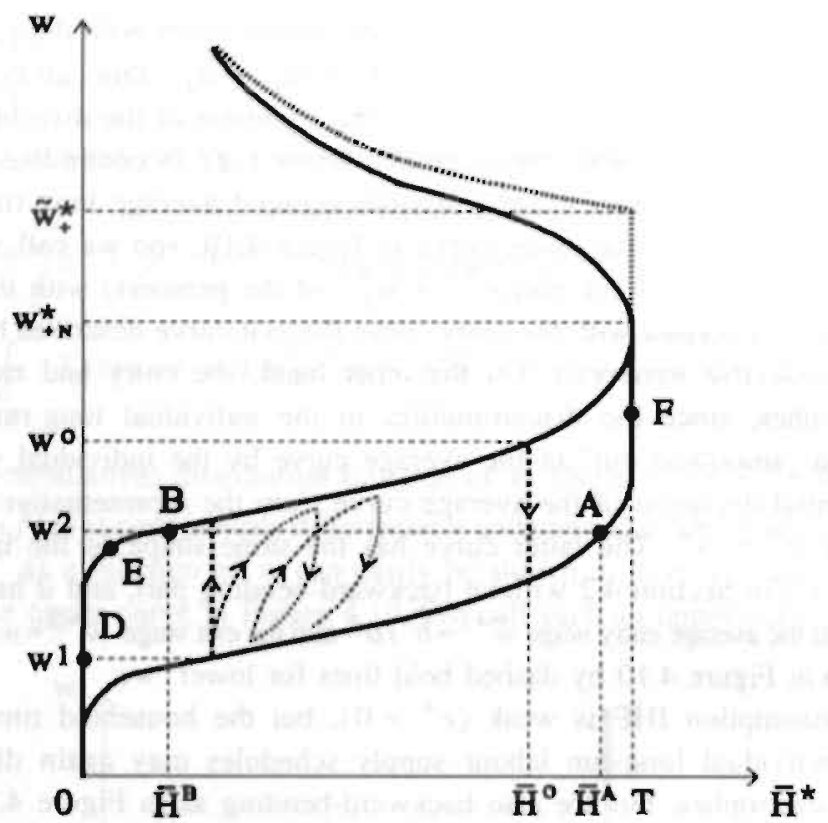

Figure 4.11 Average long-run labour supply schedule in the AQM with strong household time IHF and weak consumption IHF. The entry and exit curve are indicated by solid lines and curves, the trajectories within the collective hysteresis loop by dashed bold lines and dotted curves, and the backward-sloping part of the representative long-run labour supply schedule by a dotted bold curve.

In deriving the entry curve in Figure 4.10 or 4.11 it has been assumed that $w$ rises from zero to a sufficiently high level so as to make all group members employed before it will start to decline. In reality. however, this will often not be the case. Consider, for instance, in Figure 4.11 a rise in $w$ from zero till $w^{0}$ and, after the long-run equilibrium $\bar{H}^{0}$ being established, adecline in $w$. What will happen then? At $\bar{H}^{0}$ a majority of the population has switched from zero to the maximal number of corporate hours, but the remaining minority is still not participating. The employed have got accustomed to their employment, so when $w$ declines, they will switch back to non-participation only at their relatively low exit wages $w_{i}^{* 2}$. The first to switch back is the last person who switched to employment when $w$ reached $w^{0}$, since she (or he) has not only the highest entry wage $w_{i}^{* 1}$ and hence the highest $\alpha_{L i}$ of the employed, but also the highest exit wage $w_{i}^{* 2}$. This exit wage lies on the lower curve at the same participation rate or average labour supply $\bar{H}^{0}$. since, in the abovementioned descent of the lower curve for declining $w$, it is then the turn of the person to leave the labour market. For $w$ declining from $w^{0}$, this implies that the average long-run labour supply will be (perfeetly) inelastic (as indicated by a vertical dashed line) before the lower curve is reached, and that it will follow this curve afterwards.

However, in reality $w$ will not decline to zero before it will again start to rise. Suppose $w$ starts to rise from a long-run equilibrium at, say, $w^{1}$ on the lower curve. Then the long- 
run average labour supply will again follow a vertical inelastic line below the upper curve. In general, average long-run labour supply is inelastic within the collective hysteresis loop. This implies, that when $w$ changes within the hysteresis loop between time $t-d t$ and time $t, \vec{H}_{t}^{*}=\bar{H}_{t-d t}^{*}$ for any initial $\bar{H}_{t-d t}^{*}$. Adding a stochastic disturbance term $\varepsilon_{t} d t$ with $E \varepsilon_{t}=0$, we then have the continuous version of a random walk process ${ }^{27}$, so in the presence of such stochastic disturbances the average long-run labour supply will follow a random walk within the hysteresis loop. On the other hand, when the wage rate reaches one of the borders of the loop, the average long-run labour supply suddenly becomes highly elastic. More generally, by missing the latter very important feature the existing (macroeconomic) random walk models of hysteresis in unemployment seem at most adequate as local approximations within hysteresis loops (see also the end of Sec. 10.3).

The inelastic long-run labour supply within the hysteresis loop implies, for every given $w$ in a wide range of wage rates (e.g., $w^{2}$ in Figure 4.11), a continum of possible long-run equilibria between the entry and exit curve (e.g., between $B$ and $A$ for $w=w^{2}$ ). Moreover, the equilibrium which is reached depends on the development of the wage rate in the past. It is interesting to compare these features of the average long-run labour supply schedule with those of the individual long-run labour supply schedule in Figure 4.2 in Section 4.2, of which the average labour supply schedule in Figure 4.11 is an aggregate. For every given $w$ between $w^{* 2}$ and $w^{* 1}$, the individual schedule describes two stable long-run equilibria at $H^{*}=0$ and $H^{*}=T$ and one unstable long-run equilibrium at an interior $H^{*}$. Moreover, the stable equilibrium which is reached, again depends on the development of the wage rate in the past.

In the case of household time IHF only, the three equilibria correspond to the equilibria $B, A^{\prime}$ and $C$, respectively, in Figure 4.1 in Section 4.2 , which are implied by the piecewiselinear individual short-run demand function $L^{C^{\prime}}$ of the individual habit stock $s_{L}$. Then the question arises whether in a similar way the continuum of equilibria between $B$ and $A$ in the average labour supply schedule of Figure 4.11 can be described by a graph of the average short-run household time demand $L$ as a function of the average habit stock $\bar{s}_{L}$ for given $w, Y_{O}$ and $T$. In order to answer this question we aggregate the differential equations (3.2a) for $s_{L i}$ without $s_{X i}$ (omitting the time index $t$ ), so in particular the individual household time demand functions. From (4.21), (4.6b), and $\bar{b}=\bar{\alpha}_{L}+\alpha_{L L} T+\gamma_{L L} \bar{s}_{L}$ it follows that the average short-run household time demand $\bar{L}$ is a function not only of $w, Y_{O}, T$ and the average habit stock $\bar{s}_{L}$ (mainly the first term of the right-hand side of (4.21)), but also of the degree of variation of the individual habit stocks $s_{L i}$ around $\bar{s}_{L}$ (the "correction terms" in (4.21)). Assuming no individual variation in the depreciation rate $\delta_{L}$, the differential equations ( $3.2 \mathrm{a})$ can then exactly be aggregated to

27 Writing the random walk equation as $\bar{H}_{t}^{*}-\bar{H}_{t-d t}^{*}=\varepsilon_{t} d t$, dividing both sides by $d t$ and taking the limit for $d t \rightarrow 0$, we obxain the stochastic differential equation $\bar{H}_{i},=\varepsilon$, However, when the stochastic disturbance in the average long-run labour supply is derived from a stochastic disturbance with zero expectation in the individual short-run labour supply, the former disturbance can be shown to be strongly positively biased for $\left(\bar{H}_{t-d t}^{*}, w\right)$ near the entry curve and strongly negatively biased for $\left(\bar{H}_{t-d t}^{*}, w\right)$ near the exit curve. In that case a random walk specification is not a good approximation near the entry and exit curve. 


$$
\dot{\bar{s}}_{L}=\bar{L}\left(w, Y_{O}, T, \bar{s}_{L}, \underline{s}_{L}^{\prime}\right)-\delta_{L} \bar{s}_{L},
$$

where $\underline{s}_{L}^{\prime}=\left(s_{L 1}, \ldots, s_{L N-1}\right)$. The $N$ th habit stock is omitted, since it is determined by the $N-1$ other habit stocks and the average habit stock. The differential equation (4.24) for the average habit stock is linked to the differential equations (3.2a) for the individual habit stocks. Thus, in general aggregation yields a system of $N$ differential equations which cannot be reduced to one equation and which describes a complex dynamics of the average habit stock.

However, for $w$ and $\bar{H}$ within the hysteresis loop of Figure 4.11 matters become much simpler. Consider, for example, the decline in $w$ from a long-run equilibrium on the entry curve at $w^{0}$. In the initial situation all individual habit stocks are in a long-run equilibrium with $L_{i}=\delta_{L} s_{L i}^{0}$. For the majority that works maximal corporate hours, we have $s_{L i}^{0}=0$ and for the remaining non-participating minority $s_{L i}^{0}=T / \delta_{L}$. When $w$ remains inside the hysteresis loop, so when $w$ remains above the exit wages of all employed persons, these individual equilibria continue to exist and to be stable. As a result, the $L_{i}$ do not change. For $w=w^{2}$ we then have $\bar{L}=\delta_{L} \bar{s}_{L}^{0}$. More generally, this holds for all $\bar{L}$ between $B$ and $A$ in Figure 4.11 , so in this range the average short-run household time $\bar{L}$ is a simple linear function of the initial average habit stock $\bar{s}_{\bar{L}}^{0}$ which coincides with the long-run equilibrium line (see (4.24) for $\dot{\bar{s}}_{L}=0$ ). This is indicated in Figure 4.12. We then have a continuum of long-run equilibria between $A$ and $B$.

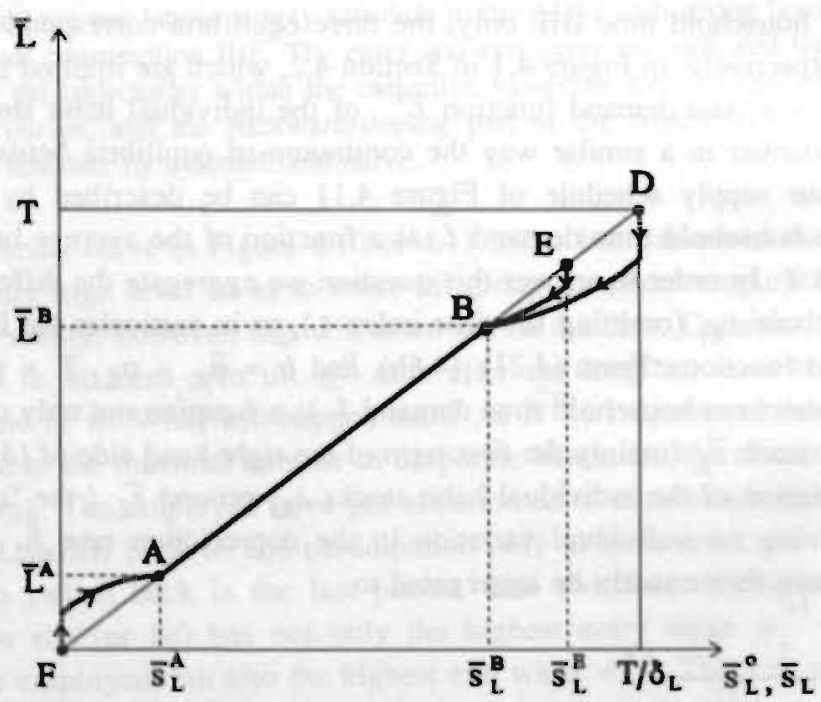

Figure 4.12 Short-run average household time demand $\bar{L}$ as a function of the average habil stocks $\bar{s}_{L}^{o}$ and $\bar{s}_{L}$ for given $w=w^{2}$ and $Y_{O}$ and conditional on initial long-run equilibrium distributions on the entry and exit curve in Fig. 4.11 , indicated by solid line and curves. 
In contrast, for $w$ and $\bar{H}$ outside the hysteresis loop of Figure 4.11 matters are different. Suppose, for instance, that the initial situation is given by a long-run equilibrium point $D$ on the entry curve for $w=w^{1}$ and $\vec{H}=0$. Then everybody is non-participating and used to that, so $\bar{s}_{L}^{D}=T / \delta_{L}>\bar{s}_{L}^{B}$. When $w$ rises to $w^{2}$, some individual non-participation equilibria for $L_{i}=T$ become unstable and disappear (see Fig. 4.1 in Sec. 4.2 for $w=w^{* 1}$ and $\left.w>w^{* 1}\right)$. As a result, these individuals will reduce their household time and enter the labour market. This leads to an adjustment of the habit stocks of these persons from $T / \delta_{L}$ to 0 , and hence to an adjustment of the average habit stock from $T / \delta_{L}$ to $\bar{s}_{L}^{B}$. The resulting adjustment path is indicated in Figure 4.12 (see App. 4E).

Suppose now that the initial situation is given by a long-run equilibrium point $E$ on the entry curve for $w<w^{2}$ and $\bar{H}>0$. Then the initial $\bar{s}_{L}^{E}$ lies between $\bar{s}_{L}^{B}$ and $T / \delta_{L}$, and for $w$ rising to $w^{2}$ we obtain a similar adjustment path of $\bar{s}_{L}$ from its initial value to $\bar{s}_{L}^{B}$ as above. However, as indicated in Figure 4.12, this adjustment path does not form a part of the adjustment path from $\bar{s}_{L}^{D}=T / \delta_{L}$, but lies slightly higher (as a result of different distributions of the individual habit stocks in the adjustment process, see App. 4E). Thus, in general the position of an adjustment path of $\bar{s}_{L}$ to $\bar{s}_{L}^{B}$ depends on the initial value of $\bar{s}_{L}$. When the initial situation is given by a long-run equilibrium point $F$ on the exit curve above $A$ with $\bar{s}_{L}^{F}=0<\bar{s}_{L}^{A}$ and when $w$ declines to $w^{2}$, we have a similar dynamics. Some individual employment equilibria become unstable and disappear, implying increases in household time and moves towards non-participation (equilibrium $B$ in Fig. 4.1). This leads to an adjustment path of the average habit stock from 0 to $\bar{s}_{L}^{A}$ as indicated in Figure 4.12. The position of this path again depends on the initial $\bar{s}_{L} F=0.28$

Comparing the aggregated Figure 4.12 with the individual piecewise-linear demand function $L^{C^{\prime}}$ in Figure 4.1, we see that the three equilibria $A^{\prime}, B$ and $C$ in the latter figure have been replaced by a continuum of equilibria between $A$ and $B$ in the former figure. We may then say that the barrier between $A^{\prime}$ and $B$ in the individual case is completely "smoothed out" by individual variation in the aggregated case. Moreover, in contrast with $A$ 'in Figure 4.1, $A$ in Figure 4.12 is an interior equilibrium and we do not have single adjustment paths to the left of $A$ and to the right of $B$. These differences are typical consequences of aggregation of individual catastrophes and hysteresis in the presence of individual variation.

However, when, in long-run catastrophic labour supply, not only individual variation in the household time taste $\alpha_{L}$, but also variations in $T, \alpha_{X}, Y_{O}, c^{*}, d^{*}$ are allowed, the story becomes more complex. If only $w$ varies, the same kind of results as above can be derived with $\bar{w}$ instead of $w$, but if one or more of the other variables and parameters vary, the order in which the members of the social group leave the labour market at declining $\bar{w}$, may not be the reverse of the order in which they enter at rising $\bar{w}$. This may especially

${ }^{28}$ Note that Figure 4.12 is conditional on the initial distributions of the individual habit stocks being Iong-run equilibrium distributions on the forward-sloping part of the entry or exit curve. These distributions are of the form $\underline{s}_{i}^{*}=\left(0, \ldots, 0, T / \delta_{i}, \ldots, T / \delta_{L}\right)$ in order of entry. Under such a condition the initial average habit stock uniquely determines the distribution of the individual habit stocks (so is a sufficient statistic). This implies unique adjustment paths of the average habit stock from given initial values $\bar{s}_{L}^{0}$. 
happen when, in addition to $\alpha_{L}$, the (relative) strength $-d^{*}$ of the household time IHF varies. A larger $-d_{i}^{*}$ increases the entry wage $w_{i}^{* 1}$, but does not affect the exit wage $\boldsymbol{w}_{i}^{* 2}$. Therefore, when the variation in $-d_{i}^{*}$ is so strong as to overrule the variation in $\alpha_{L i}$ in determining the order of the entry wages, the order of the exit wages as determined by the variation in $\alpha_{L i}$ may not be its reverse. Then it can be shown that, when $w$ rises from $w^{1}$ after a decline as in Figure 4.11, persons who are less subject to habit formation than on average and who therefore have a lower entry wage, may enter the labour market before the higher curve is reached. This implies a deviation from the straigth vertical line and may, at subsequent rises and declines in $w$, lead to a complex trajectory of circling small collective hysteresis loops within the large collective hysteresis loop, as indicated in Figure 4.11. However, a precise analysis of this interesting complication is beyond the scope of this study. 29

Further complications arise when the parameters $b_{i}^{*}, a_{i}^{*}$ and/or $\alpha_{L i}$ are negative for some individuals (see Sec. 4.5). For example, when $\alpha_{L i}$ is negative, $b_{i}^{*}$ and $a_{i}^{*}$ are positive and $d_{i}^{*}$ is negative, the individual long-run labour supply schedules are given by Figure 4.7 with no positive exit wage and no backward-sloping part. Aggregating such labour supply curves together with those according to Figure 4.2 , we obtain an average long-run labour supply schedule which is similar to that in Figure 4.12 , but in which the exit curve reaches the $w=0$ axis at a positive $\bar{H}^{*}$ and which has a less backward-sloping part for higher $w$. An additional complication results when, together with the net household time taste $\alpha_{L_{i}}$ and the strength $-\hat{d}_{L}^{*}$ of the household time IHF, the strength $-\hat{d}_{H}^{*}$ of the corporate time IHF varies over the individuals in a fixed positive proportion to $-\hat{d}_{L}^{*}$ (cf. the end of Sec. 4.5). As a counterpart of the effects of a larger $-\hat{d}_{L}^{*}$ (see above), a larger $-\hat{d}_{H}^{*}$ decreases the exit wage $w_{i}^{* 2}$, but does not affect the entry wage $w_{i}^{* 1}$. As a consequence, the combination of a larger $-\hat{d}_{L}^{*}$ and a larger $-\hat{d}_{\mu}^{*}$ tends to lead to a later entry at rising $w$, but also to a later exit at declining $w$. This reinforces the complex hysteresis pattern as described in the preceding paragraph.

For other than AQM specifications of individual labour supply curves (including cases of discontinuous short-run labour supply schedules), similar stories hold, except that then also hysteretic patterns in the backward-sloping part of the average long-run labour supply schedule seem possible. In general, catastrophes and barriers in individual short-run and long-run labour supply schedules are "smoothed out" in the aggregated schedule by individual variation, whereas the hysteresis property carries over to more complex hysteretic patterns in the aggregated schedule.

${ }^{20}$ See for an analysis of a similar case in travel mode choice Goodwin (1976), especially Figure 5. It is intriguing that a pattem of circling loops is also found in the empirical developments of the Phillips curve over the last three decades in several OECD countries (see, e.g., McCracken et al., 1978. Charts 15 and 16, and Hall and Taylor, 1991. Ch. 17). 


\subsection{Concluding remarks}

This chapter has derived for the $\mathrm{AQM}$ of Houthakker and Taylor necessary and sufficient conditions for individual hysteresis and catastrophes as in Figure 4.2 to occur. First only household time and consumption IHF were considered, but later corporate time IHF was added to the model. This IHF was shown to describe a weak or strong variant of workaholism, if it is sufficiently strong. Moreover, it was demonstrated that individual hysteresis and catastrophes can be rationalized as the result of local and not global maximization of an additive quadratic "long-run utility function". Next, short and long-run individual labour supply schedules were aggregated to average schedules under various assumptions regarding the individual variation in utility function parameters, exogenous variables and habit stocks. Aggregation of hysteresis and catastrophes in individual long-run labour supply schedules turned out to imply hysteresis and complex trajectories within the hysteresis loop, but no catastrophes in average long-run schedules. Whereas the former individual schedules describe two stable equilibria and one intermediate unstable equilibrium, the latter average schedules were established to imply a continuum of "stable" equilibria within the collective hysteresis loop.

However, as already noted at the end of the previous chapter, both our general and our specific IHF model have a number of shortcomings. An important one is the implicit assumption that individual labour suppliers do not influence each other's preferences. Yet, in reality individual labour supply preferences are affected not only by one's own past behaviour leading to habits, but also by the behaviours of relevant others. Therefore, the resulting behavioural preference interdependence in labour supply is modelled in the next chapter and combined with IHF into integrated models in the subsequent two chapters.

\section{Appendix 4A Derivation of results for Case 1 in Section 4.3}

Substituting (4.9a) into the discriminant $D=a^{* 2}-4 T c^{*} \alpha_{L}$ of the quadratic form (4.12), we obtain

$$
D=Y_{O}^{2} c^{* 2}-\left(2 \alpha_{X} Y_{O}+4 \alpha_{L} T\right) c^{*}+\alpha_{X}^{2} .
$$

This quadratic form in $c^{*}$ describes a "valley parabola". Consequently, $D$ is negative if and only if $c^{*}$ lies between the two roots of the equation $D=0$, which are given by

$$
c_{ \pm}^{*}=\frac{\alpha_{X} Y_{O}+2 \alpha_{L} T \pm \sqrt{\left(\alpha_{X} Y_{O}+2 \alpha_{L} T\right)^{2}-\alpha_{X}{ }^{2} Y_{O}{ }^{2}}}{Y_{O}{ }^{2}} .
$$

Since all parameters and variables in this expression are assumed to be positive, it easily follows that both roots are positive. Moreover, the higher root $c_{*}^{*}$ is larger than $\alpha_{X} / Y_{0}$, so lies outside the considered domain for $c^{*}$ for which $a^{*}$ is positive. Furthermore, denoting the lower root as $c_{G}^{*}$ and elaborating it as 


$$
c_{o}^{\prime \prime}=\frac{\alpha_{X} Y_{O}+2 \alpha_{L} T-2 \sqrt{\alpha_{L}{ }^{2} T^{2}+\alpha_{X} Y_{O} \alpha_{L} T}}{Y_{O}{ }^{2}} .
$$

it is easily seen that $c_{o}^{*}$ is smaller than $\alpha_{X} / Y_{O}$. Thus, Subcase $1^{\mathrm{a}}$ in Table 4.2 is obtained. In Subcase $1^{\mathrm{b}}$ with $c^{*}=c_{o}^{*}$ and hence $D=0,(4.12)$ with equality sign has a unique root

$$
w_{o}^{*}=a^{*} /\left(2 T c_{o}^{*}\right)=2 \sqrt{T c_{o}^{*} \alpha_{L}} /\left(2 T c_{o}^{*}\right)=\sqrt{\alpha_{L} /\left(T c_{o}^{*}\right)} .
$$

In Subcase $1^{d}$ the (full) satiation with respect to consumption at the long-run equilibrium points implies that the labour supply decisions at these points are unlinked from the consumption decisions (see Subsection 2.2.5). As a consequence, the former decisions are affected by the household time IHF only, and then it is easily seen that a necessary and sufficient stability condition is given by $d^{*}>0$. In the present case this condition is fulfilled, and in analogy to the short-run case, the stable long-run solution of the first-order condition $U_{L}^{*}=b^{*}+d^{*} H^{*}=0$ (see $\left.(4.8 \mathrm{~b})\right)$ is given by $-b^{*} / d^{*}<0$. This implies for all $w$ a unique stable corner solution $H^{*}=0$, so the long-run labour supply curve coincides with the vertical $H^{*}=0$ axis.

\section{Appendix 4B Derivation of results for Case 2 in Section 4.4}

Substituting (4.8a) and (4.8b) into the first-order condition (4.3), this condition can be written as $\left(c^{*} w^{2}+d^{*}\right) H^{*}=a^{*} w-b^{*}$. When $c^{*} w^{2}+d^{*}=0$, this equation has no solutions for $H^{*}$ if $a^{*} w-b^{*} \neq 0$, and it has all feasible $H$ as solutions if $a^{*} w-b^{*}=0$ In the latter case all feasible $H$ are long-run equilibria which are unstable in the sense that when a small shock pushes the person away from a long-run equilibrium, she is not pulled back to this equilibrium (as in a random walk).

For $d^{*}<d_{-}^{*}$ in Figure 4.4c we have $w_{r}^{*}>w^{* u}$ and $w_{+}^{*}<w^{* u}$. The former inequality implies that in the unstable domain below the $w^{* u}$ curve the restriction $H^{* u} \geq 0$, or $L^{* u} \leq T$, is always fulfilled, so we can never have situations as in Figure 4.1 for $w=w^{* 1}$ and $w>w^{* 1}$. On the other hand, the inequality $w_{*}^{*}<w^{* u}$ implies that the restriction $H^{* u} \leq T$, or $L^{*} \geq 0$, is fulfilled for $w_{-}^{*} \leq w \leq w_{+}^{*}$, so for both $w^{*}=w_{-}^{*}$ and $w=w_{+}^{*}$ the restriction is just fulfilled, as in Figure 4.1 for $w=w^{* 2}$. Consequently, the unstable $H^{* \prime}$ for $w_{-}^{*} \leq w \leq w_{*}^{*}$ are located as in Figure 4B, and the household time demand functions like those in Figure 4.1 move, for $w$ rising from below $w_{-}^{*}$, such as described in Section 4.4. The resulting labour supply schedule in Figure 4B is also described in Section 4.4. In particular, when for $w_{-}^{*}<w<w_{+}^{*}$ the labour supply of the person happens to be larger than the unstable "watershed" $H^{* \|}$, for instance by coercion or as a consequence of a different preceding regime, and when he (or she) has got sufficiently used to that, the person will adjust his labour supply to the stable maximal hours $T$. In that case he will be very strongly used (negative $d^{*}$ ) to a minimum level of household time, while being weakly used (positive, but not too large $c^{*}$ ) to a relatively high level of consumption. However, when $w$ declines below $w_{-}^{*}$ or rises above $w_{+}^{*}, H^{*}=T$ becomes unstable and disappears, inducing in both cases an individual exit catastrophe to $H^{*}=0$. On the other hand, and this is very remark- 
able, when the person does not participate and has got sufficiently used to that, he (or she) will not engage into an individual entry catastrophe for any $w$ as a result of the very strong household time IHF.

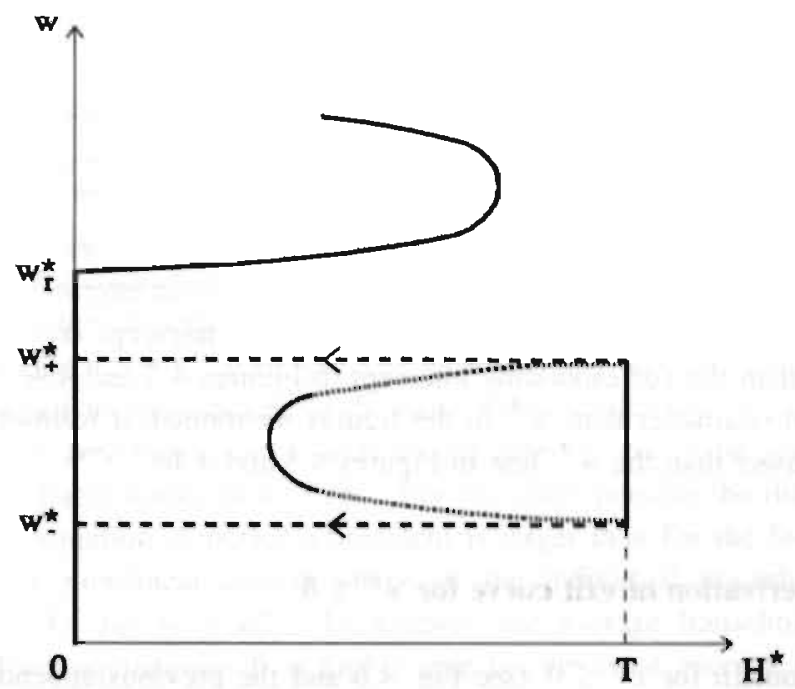

Figure 4B Long-run labour supply schedule with exit catastrophes at $w_{-}^{*}$ and $w^{*}$, and no entry catastrophe for $0<c^{*}<c_{o}^{*}$ and $d^{*}<d_{-}^{*}<0$ (not too weak consumption IHF and very strong household time IHF). The dotted curve indicates the loci of the unstable equilibrium $H^{*}$.

In Subcase $2^{\mathrm{d}}$ in Table 4.2 , the necessary and sufficient stability condition $d^{*}>0$ (see the end of the previous appendix) is not satisfied, and for $d^{*}<0$, the unstable long-run solution $H^{*}=-b^{*} / d^{*}$ of the first-order condition $U_{L}^{*}=b^{*}+d^{*} H^{*}=0$ is positive. From $b=\alpha_{L}+d^{*} T>d^{*} T$ it follows that $H^{* \|}$ is also larger than $T$. Then we are in a situation with $L^{* u}<0$ as in Figure 4.1 for $w<w^{* 2}$, implying one stable corner equilibrium $L^{*}=T$, or $H^{*}=0$, for all $w$. When $d^{*}=0$, the first-order condition has no solutions, since $b^{*} \neq 0$. Then the demand line in Figure 4.1 for $w<w^{* 2}$ is parallel to the $L=\delta_{L} s_{L}$ line while remaining above it. Again this implies one stable corner equilibrium $H^{*}=0$ for all $w$. As a result, the instability of the long-run solution of the first-order condition does not affect the long-run labour supply curve: just as for $0<d^{*}<\alpha_{X} / Y_{D}$, this curve coincides with the $w$-axis for all $d^{*} \leq 0$. Analogously to the situation in Figure $4,4 \mathrm{a}$, this insensitivity to instability is a consequence of the fact that the unstable domain in the $\left(w, d^{*}\right)$ space for $d^{*} \leq 0$ falls outside the region for $d^{*}>\alpha_{L} / T>0$, where $H^{*}>0$.

\section{Appendix 4C Derivation of results for Cases 5 and 6 in Section 4.4}

In the stable domain of Figure 4.6 the restriction $H^{*} \geq 0$ on (4.9) is again fulfilled for $w \geq w_{r}^{*}=b^{*} / a^{*}$. The restriction $H^{*} \leq T$ on $(4.9)$ is then again equivalent to (4.12), but 
due to the negativity of $c^{*}$, the quadratic form in $w$ in (4.12) now describes a "mountain parabola" and its discriminant $D=a^{* 2}-4 T c^{*} \alpha_{\mathcal{L}}$ is now always positive. This implies that (4.12) is not fulfilled for $w$ higher than the higher root or lower than the lower root. These roots are again given by (4.13), but now $w_{+}^{*}$ is negative and hence lower than the positive $w_{-}^{*}$. Thus, (4.12) is not fulfilled for non-negative $w>w_{-}^{*}$ only (see Fig. 4.6).

In the unstable domain of Figure 4.6 the restriction $H^{* u} \geq 0$ is again fulfilled for $w \leq b^{*} / a^{*}=: w_{r}^{*}$. The restriction $H^{* \|} \leq T$ is then again equivalent to (4.12) with the $\geq$ sign replaced by the $\leq$ sign. This restriction is now fulfilled for non-negative $w \geq w_{-}^{*}$, so for $w_{-}^{*}<w<w_{r}^{*}$ there is one unstable interior equilibrium $H^{*}$ alongside two stable corner equilibria $H^{*}=0$ and $H^{*}=T$ (see Fig. 4.6). The $w_{-}^{*}$ line and $w^{* u}$ curve now intersect for positive $d_{+}^{*}=c^{*} w_{-}^{* 2}$, and again $\left(d_{+}^{*}, w_{-}^{*}\right)$ is also an intersection point for the $w_{r}^{*}$ line. According to Figure $4.6 w_{-}^{*}$ is then smaller than the intercept $\alpha_{L} / a^{*}$ of the $w_{r}^{*}$ line, which is smaller than the corresponding intercept in Figures 4.3 and 4.4c. Since, in its turn, the latter intercept is smaller than $w_{-}^{*}$ in the figures mentioned, it follows that the $w_{-}^{*}$ line in Figure 4.6 is lower than the $w_{-}^{*}$ line in Figures 4.3 and 4.4c.

\section{Appendix 4D Derivation of exit curve for $c^{*} \leq 0$}

In the unstable domain for $c^{*} \leq 0$ (see Fig. 4.6 and the previous appendix), the condition for the exit wage $w_{i}^{* 2}=w_{-i}^{*}<w$ in the analogue of (4.22) holds if and only if the value of the "mountain parabola" $T c * w^{2}-a^{*} w+\alpha_{L i}$ is negative. In the continuous approximation for large $N$ with $\varepsilon:=\alpha_{L}-\bar{\alpha}_{L}$ this is equivalent to $\varepsilon<T\left|c^{*}\right| w^{2}+a^{*} w-\bar{\alpha}_{L}$. Analogously to (4.23), it then follows that

$$
\bar{H}^{*}=T F\left(T\left|c^{*}\right| w^{2}+a^{*} w-\bar{\alpha}_{L}\right) \text {. }
$$

where $F$ is the (cumulative) distribution function of $\varepsilon$. (Note that $f(\varepsilon)=0$ for $\alpha_{L} \leq 0$, or e $\leq-\bar{\alpha}_{L}$.) In comparison with (4.23) the argument of $F$ has now an additional quadratic term in $w$. In order to deduce the shape of the exit curve, when $f(\varepsilon)$ is continuously differentiable and unimodal (so quasi-concave), we derive

$$
\begin{aligned}
\frac{\partial \bar{H}}{\partial w}= & T f\left(T\left|c^{*}\right| w^{2}+a^{*} w-\bar{\alpha}_{L}\right)\left(2 T\left|c^{*}\right| w+a^{*}\right), \\
\frac{\partial^{2} \bar{H}}{\partial w^{2}}= & T\left[f^{\prime}\left(T\left|c^{*}\right| w^{2}+a^{*} w-\bar{\alpha}_{L}\right)\left(2 T\left|c^{*}\right| w+a^{*}\right)^{2}+\right. \\
& \left.+f\left(T\left|c^{*}\right| w^{2}+a^{*} w-\bar{\alpha}_{L}\right) 2 T\left|c^{*}\right|\right] .
\end{aligned}
$$

For $w$ between the lowest exit wage $w_{1}^{* 2}$ and the highest exit wage $w_{N}^{* 2}$, the first-order derivative is positive. The second-order derivative is then non-negative for $w$ to the left of the unique maximum of the density function $f$, and for $w$ rising beyond this maximum, the first term between brackets tends to become increasingly negative, whereas the second term becomes less positive for $c^{*}<0$ and is zero for $c^{*}=0$. As a result, the second-order derivative will usually be non-negative for $w$ lower than a value to the right of the maxi- 
mum (for $c^{*}<0$ ) and non-positive for $w$ higher than this value. This implies a smooth sigmoid shape of the exit curve. Note that for $c^{*}=0$ we have the same kind of linear argument of $F$ in (4D.1) as in (4.23) and hence a shape of the exit curve which is identical to the shape of the entry curve.

\section{Appendix 4E Derivation of adjustment paths of the average habit stock in Figure 4.12}

The adjustment paths in Figure 4.12 result from aggregation of the individual household time demand functions in Figure 4.1 for $w>w^{* 1}$ and $w<w^{* 2}$, respectively. The kinks in these functions are then "smoothed out". Along the adjustment path from equilibrium $D$ to equilibrium $B$, a number of persons adjust their household time habit stocks from $T / \delta_{L}$ to 0 . At the intermediate $\bar{s}_{L}=\bar{s}_{L}$ these persons have partially adjusted their habit stocks. On the other hand, when the adjustment to $B$ starts from equilibrium $E$, some of the same persons have already zero habit stocks, whereas the others of the same persons just start the adjustment of their habit stocks from $T / \delta_{L}$. For the latter persons the difference in household time with the situation of partial adjustment is larger than for the former persons as a consequence of the non-linear convex shape of the individual household time demand function in Figure 4.1 for $w>w^{* 1}$. As a result, the average household time along the adjustment path from equilibrium $E$ is higher than for the same average habit stocks along the adjustment path from equilibrium $D$. 


\section{CHAPTER 5}

\section{Behavioural Preference Interdependence}

Whereas the previous two chapters have analysed the short-run and long-run effects on labour supply preferences of one's own behaviour in the past, this chapter will investigate the short and long-run effects on labour supply preferences of the behaviour of relevant others. Provided the latter behaviour is at least partly determined by preferences of the relevant others, we then have a particular kind of interdependence of preferences, which we call behavioural preference interdependence $(B P I)$ to distinguish it from other kinds of preference interdependence (see below). Underlying BPI in labour supply are: various processes of mutual social influencing. These social influence processes can be interpreted as different kinds of social interaction, provided the term "interaction" is conceived in a broader sense than usual in sociology. Here the term "interaction" refers not only to direct influencing among agents, but also to indirect influencing via other agents or institutions. ${ }^{1}$

Interactions among agents in this general sense have been a major concern of economics from its very beginning. Since Adam Smith the focus has been on exchange processes and the struggle for market opportunities, but until the beginning of the twentieth century a good deal of attention was also paid to interactions of a more social nature. Examples in case are the benevolence concept of Smith (1759), the recognition of social wants by Bentham (1789), the class struggle according to Marx (1867), and the strong emphasis on the behaviour motive of distinction by Marshall $(1920)^{2}{ }^{2}$ However, in connection with the completion of the separation of economics and sociology at the beginning of our century and the econometric revolution in the thirties with its need for formalization and measurement, social interactions, i.e. interactions of a more social nature, got out of sight in mainstream economics.

Economists like Duesenberry (1949), Leibenstein (1950), Becker (1974), Pollak (1976a), Hirsch (1976), Kapteyn (1977), Sen (1983) and Frank (1985) have tried to reverse this trend, but nowadays most economists still seem to feel that social interactions belong to the realm of sociology and social psychology and not to that of economics. Nevertheless, in contrast to the division of labour in social science, social reality is not separated in disjunctive fragments. Social interactions play an important role in economic life and should therefore

I Also the term "indirect" here has another meaning than usual in sociology: when agent A influences agent $\mathbf{B}$ and agent $\mathbf{B}$ influences agent $\mathbf{C}$. we say that agent $\mathrm{A}$ indirectly influences agent $\mathrm{C}$.

2 See also Becker (1974). 
be included in its study. An important starting point of such an undertaking, which tends to be neglected by economists working in the field, should be a simple classification of different kinds of social interaction in economic life. In the context of consumer (and labour supply) behaviour attempts to make such a classification have been made by Leibenstein (1950), Becker (1974), Schokkaert (1982) and Baxter (1988). Partly based on these attempts, but adapted to the labour supply context of our study, a possible classification is proposed in Section 5.1.

This classification tries to distinguish social interactions from "economic" or market interactions and primarily focuses on the various kinds of social interaction underlying BPI in labour supply. However, it considers also other important forms of preference interdependence in labour supply as well as social interactions affecting constraints. In Section 5.2 the social interactions underlying BPI in labour supply are summarized in a general model of BPI with respect to both the average (i.e. per capita) consumption and average (discretionary) household time in a social group (as formulated by lannacone (1989) for consumption only). This model is derived from the most general model of BPI with respect to both individual consumption and household time by ten successive simplifying assumptions. Moreover, it is extended so as to include BPI with respect to average corporate time. An attractive feature of the resulting model is that it is analogous in structure to the general individual habit formation (IHF) model which has been developed in Chapter 3 (see also Deaton and Muellbauer, 1980, pp. 374-375). As a consequence, the model has analogous properties like multiple equilibria exhibiting collective hysteresis and catastrophes dependent on rationings and the wage rate. Similarly to the unstable IHF model, such a model of unstable BPI can explain why members of certain subcultures of long-term unemployed, although having lost their old job involuntarily, become little motivated to get a new job. Furthermore, collective catastrophes within social groups may have played (and still play) a role in the strong rise of the labour force participation of married women in many OECD countries since World War II. All this is discussed in the Sections 5.3 and 5.4.

Notwithstanding the analogy in structure of the IHF and BPI models, it should be noted that, whereas the IHF model holds for individual behaviour, the BPI model is formulated on the level of average behaviour in a social group. This implies an additional complication in the BPI model as compared to the IHF model, namely that of individual variation within the social group. The stabilizing effects of this individual variation are illustrated for an additive quadratic specification of the utility function in Section 5.5. For this specification necessary and sufficient conditions for collective catastrophes and hysteresis can then be derived. Finally, Section 5.6 makes a comparison between the different effects on average labour supply of unstable BPI and unstable IHF.

\subsection{Classification of social interactions}

\section{I.I General notions}

This section presents a simple classification of social interactions in the context of labour supply and consumption demand behaviour along the lines of Figure 5.1. These social interactions are distinguished from (usual) market interactions on a labour or consumption 


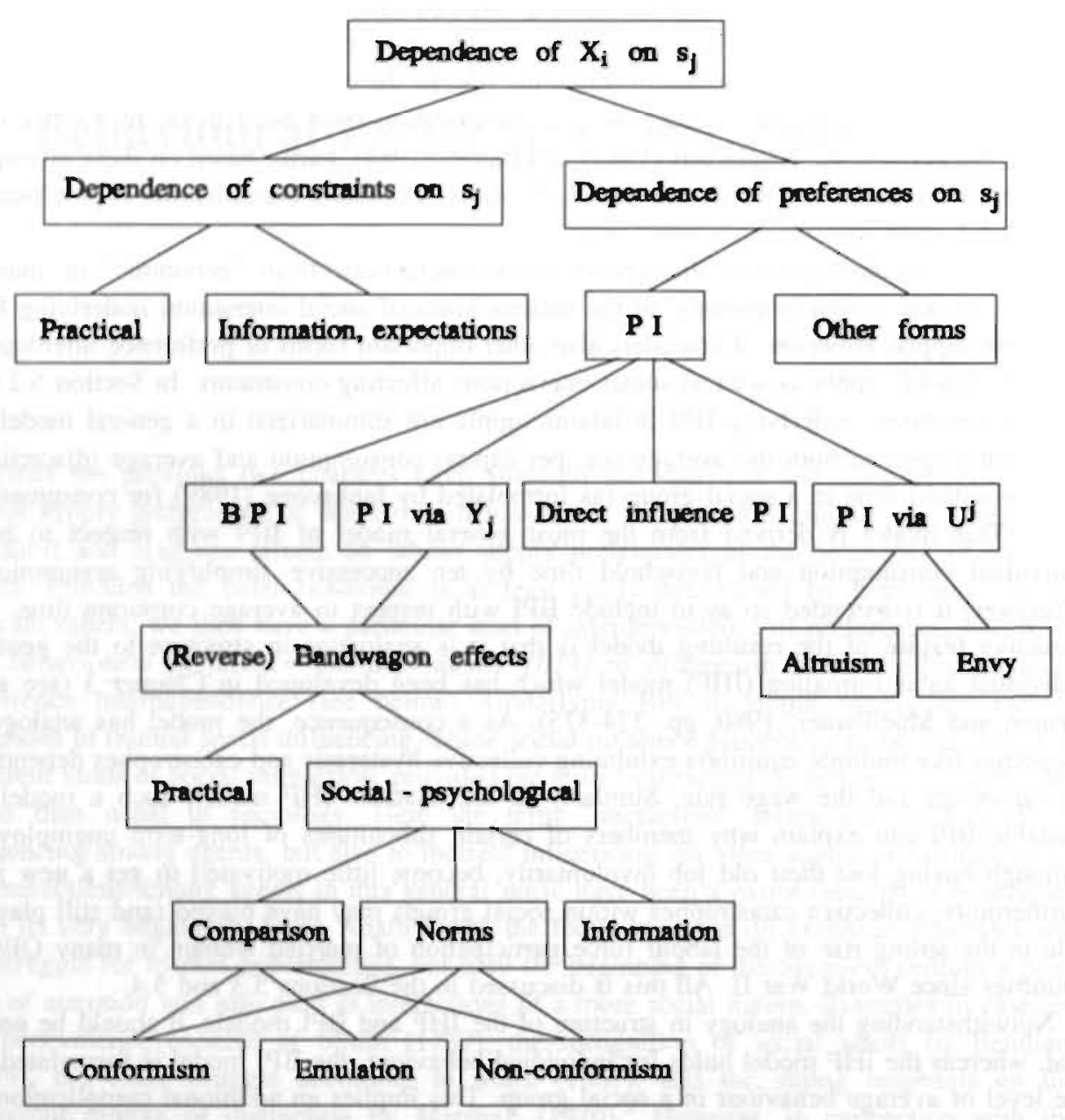

Figure 5.1. Classification of social interactions in context of labour supply and consumption demand behaviour.

good market in order to be able to associate social interactions with extemal effects in welfare theory. ${ }^{3}$ Quite generally, we define a social interaction between labour suppliers or consumers as a process, in which a certain aspect of a person $i$ at time $t$ depends on one or more perceived or expected aspects of one or more other persons $j$ at the same, past or future points in time, and which is not a (usual) market interaction on a labour or

${ }^{3}$ See, e.g. Iannacone (1989. Sec. 4), and see Frank (1989. Sec. IV) for proposats to intemalize such external effects in income taxes. Social interactions are considered as social exchange processes by Becker (1974. 1976) and, more generally. the Chicago School. This seems the main reason why Becker (1974) does not distinguish social interactions from market interactions. However, because of the association of social interactions with external effects, I make a distinction. 
consumption good market (cf. Becker, 1974). Examples of aspects are behaviour, income, preferences, well-being, perceptions, expectations and beliefs.

Our classification starts from social dependencies of a preferred behaviour $X_{i}$ of person $i$ on aspects $s_{j}$ of other persons $j$ and follows Figure 5.1. In the present context $X_{i}$ stands for consumption demand, household time demand or labour supply. Such a preferred behaviour is supposed to be the result of person $i$ maximizing a utility function under certain constraints. This implies two kinds of sources of a dependence of $X_{i}$ on aspects $s_{j}$ of others $j:$ : (i) dependence of the constraints of $i$ on $s_{j}$ and (ii) dependence of the utility function or preferences of $i$ on $s_{j}$. Examples of $(i)$ are the Leuthold model of family labour supply with $s_{j}$ being the labour supply of another family member (see Sec. 2.2.7) and exchange of information and social influences on expectations with respect to state variables in constraints (e.g., labour demand). ${ }^{4} \mathrm{~A}$ well-known example of (ii) is the dependence of the preferences of $i$ on the actual behaviour $X_{j}^{a}$ of others $j$ as perceived or expected by $i$. Usually this is called preference interdependence, since the preferences of $i$ are assumed to depend on the preferences of $j$ via $j$ 's actual behaviour $X_{j}^{a}$. This holds, provided this actual behaviour is at least partly determined by $j$ 's preferences with respect to the behaviour, so provided $j$ 's actual behaviour is not fully determined by rationings. (See for the case of consumption, e.g., Gaertner, 1974, Pollak, 1976a, Kapteyn, 1977, 1985, and Kapteyn and Wansbeek, 1985, and see for the case of household time or labour supply Kapteyn and Woittiez, $1990{ }^{5}$ ).

However, preferences of different persons can also depend on each other in another way than via their actual behaviour. For instance, beliefs in norms and values may be transmitted as a result of direct interpersonal communication without reference to one's own actual behaviour (see Schokkaert, 1982b, Sec. V.B.III, and see Subsec. 5.1.4). Therefore, it seems appropriate to define preference interdependence more generally as a phenomenon in which a person's preferences or an inner determinant of them like a belief or expectation depend on another person's preferences or an inner determinant of them via any kind of social interaction. We note, however, that the distinction between constraints and preferences, and hence that between the above-mentioned categories (i) and (ii) of dependence of constraints and dependence of preferences, is not absolute, but depends on the particular model formulation or interpretation (see Stigler and Becker, 1977, Schokkaert, 1982a, Linđenberg, 1990, and Vendrik, 1988).

\subsubsection{Sources of $B P I$}

Several kinds of preference interdependence can be distinguished. The best-known, already referred to in the previous subsection, is the dependence of a person i's preferences at time $t$ on expected or perceived actual behaviour $X_{j}^{a}$ of other persons $j$ at the same or past points in time. To distinguish it from other types of preference interdependence (to be discussed in Subsec. 5.1.4) we call it behavioural preference interdependence (BPI). In a

\footnotetext{
${ }^{4}$ Note that competitive market interactions on consumption good markets make the prices in these. constraints - at least in the long run - dependent on the behaviours or average costs of others $j$. We distinguish such competitive market interactions from social interactions, and hence exclude them from our classification.

${ }^{5}$ Remarkably, this is the only reference on PI in labour supply that I know.
} 
general linear case (see the next section), the influence of all relevant $X_{j}^{a}$ 's on $i$ 's preferences at time $t$ can be summarized by the influence of a weighted average $s_{\bar{X} i t}$ of the $X_{j}^{a}$ 's over persons $j$ and over the same and past points of time. This can lead to one of two kinds of effect of $s_{\bar{X} i}$ (omitting the time index) on preferred $X_{i}$, namely a "bandwagon effect" with a positive correlation between $X_{i}$ and $s_{\bar{X} i}$ and a "reverse bandwagon effect" (or "snob effect") with a negative correlation (see Leibenstein, 1950, for the case of consumption). Note that similar effects could result from the influence of a weighted average $s_{\bar{X}}$ it of relevant $X_{j}^{a}$ 's in constraints, but the terms of bandwagon and reverse bandwagon effect are usually reserved for influences of an $s_{\bar{X} i t}$ in (usual) preferences.

Both kinds of effect can have practical as well as social-psychological causes (see, e.g., Hirsch, 1976, Sen, 1983, 1987, Granovetter and Soong, 1986, and Frank, 1989, for the consumption case). In the context of labour supply an example of a practical cause is the complementarity or substitutability of the household times of family members, as modelled by Ashworth and Ulph (1981) (see Subsec. 2.2.7). Complementarity implies a bandwagon effect, whereas substitutability causes a reverse bandwagon effect. Another example of a practical bandwagon effect is that married women might be induced to look for a job when her friends have one, since she cannot drink coffee or sherry with them during the daytime.

In the case of social-psychological causes of (reverse) bandwagon effects the preferences of a person are influenced by the behaviour of persons in her/his (social) reference group (Hyman, 1942). Such a group can be defined as a group "to which the individual relates himself as a part or to which he aspires to relate himself psychologically" (Sherif, 1953). This definition implies that the reference group of person $i$ may consist of persons of a membership group of $i$, i.e. a group to which $i$ feels she (or he) belongs, but that it may also consist of persons of other social groups to which $i$ aspires to relate herself in some manner. In the literature usually three kinds of reference group are distinguished, namely the (a) comparative, (b) normative and (c) informational reference group (see, e.g., Park and Lessig. 1977, and, Baxter, 1988, Sec. 2.6.1).

\section{(a) Social comparison}

Social comparison with persons in the comparative reference group enables someone to evaluate her status in society and to enhance or support her self-concept (Hyman, 1942). The comparative reference group may consist of persons of a membership group of similar status as well as of persons of groups of higher or lower status. On the one hand, the person wants to conform her behaviour to the behaviour of persons in her membership group or in a higher status group (imitation), but on the other hand, she wants to distinguish her behaviour in a status-enhancing way from that of persons in her membership group or in a lower status group (differentiation). ${ }^{6}$ Thus, with respect to the membership group both a conformity and

${ }^{6} \mathrm{Cf}$. the unitomity and discrimination tendency in the case of capacities according to the social comparison theory of Festinger (1954). See also Thierry and Koopman-Iwerna (1984. Sec. 2.3.2). Baxter (1988, Sec. 2.6.3) and Schokkaen (1982b, Ch. 5). My interpretation of the concepts of comparative reference group and membership group is broader than the interpretation by, for example. Runciman (1966), since this is more appropriate to the general use I want to make of the concept of reference group in the next sections and chapters. Moreover, my interpretation seems to fit in well with the interpretations by economists like Kapteyn. Van Praag and Van Herwaarden (1978). 
distinction tendency may be active, whereas with respect to the higher status group a conformity tendency and with respect to the lower status group a distinction tendency may be at work.

The distinction tendency can express itself either as "more of the same behaviour" (e.g., more consumption of the same good) or as "less of the same behaviour and more of a different behaviour" (e.g., less consumption of the same good and more of another good). ${ }^{7}$ We call the former distinction tendency emulation and the latter non-conformism. Emulation can be considered as a transition case between conformism and non-conformism: it is conformistic with regard to the kind of behaviour, but non-conformistic with regard to the level of behaviour (e.g., consumption). Both conformism and emulation cause a bandwagon effect, whereas non-conformism implies a reverse bandwagon effect. Within a membership group a combination of emulation and conformism may lead to the phenomenon that people want to surpass others just a little (cf. Festinger, 1954). This implies a satiation point for emulation, which is usually neglected in economic models of social interaction with their one-sided emphasis on emulation (see, e.g., Becker, 1974, Frank, 1984, 1989, and Kapteyn and Wansbeek, 1985). ${ }^{8}$ In the context of labour supply and consumption demand behaviour this point should be taken into account in the choice of a specification of the utility function, but it can be shown not to affect the bandwagon effect. Another point is that conformism and/or emulation may produce bandwagon effects for relatively low levels of the average behaviour $s_{\bar{\gamma} i}$ in the comparative reference group, but turn into non-conformism and hence a reverse bandwagon effect for high $s_{\bar{X}_{i}}$. It is also possible that, analogously to the time hierarchy of individual habit formation and long-run satiation postulated in Section 3.5, a bandwagon effect works in a shorter run, whereas a reverse bandwagon effect becomes manifest in a longer run (see Sec. 9.11 for an example).

\section{(b) Social norms}

A second social-psychological cause of a (reverse) bandwagon effect is the adoption of (beliefs in) social norms from a normative reference group (Newcomb, 1947), in as far as the process of this adoption or the social norm beliefs which are transmitted are determined (in the long run) by the behaviour of others. In the context of labour supply an example is the adoption of work ethics norms from other labour suppliers. These labour suppliers may belong to the same membership group (e.g., the same family, age or sex), but they may also belong to another social group (e.g., other families, ages or sex; cf. Kapteyn and Woittiez, 1990). The actual behaviour of such relevant others can induce the adoption of social norms as follows. When a social norm is actually obeyed by a majority of the relevant others, this may act as a signal that this majority also believes in the social norm, and hence make adoption of the social norm, i.e. the development of one's own belief in the norm, attractive. Alternatively, the social norm may be adopted as a resuit of direct interpersonal com. munication on the social nom without reference to actual behaviour (see Schokkaert, 1982b,

\footnotetext{
${ }^{7}$ This important distinction may not be recognized in the literature on social comparison.

${ }^{8}$ A related remark on the neglect of the effect of conformity in the economic tradition is made: by Schokkaert (1982b, p. 166) in connection with the formation of iife style groups. A striking example. of the neglect is the implicit assumption of Frank (1989, p. 84) that living as a rich person in a poor neighborhood has no social price of a lack of belonging.
} 
Sec. V.B.III), but in the long run the social norm beliefs of the relevant others may be determined by the extent to which the norm is actually obeyed by the relevant others themselves, by the generation of their parents, or by members of the membership group to which the norm applies (e.g., the wives, when the relevant others are husbands). Such a long-run determination of social norm beliefs by the actual norm behaviour is elaborated in Section 8.5. Another variant of the resulting normative BPI is obtained, when adoption of a social norm as a result of direct interpersonal communication is affected by the actual norm behaviour of trendsetters (see Sec. 10.3).

Another kind of normative preference interdependence is a dependence of person i's preferences on the opinion of $i$ held by person $j$ (Becker, 1974) or $i$ 's reputation with person $j$ (Akerlof, 1980). This opinion or reputation $R_{i}^{j}$ depends on $i$ 's behaviour as well as on norm beliefs $n_{j}$ of $j$, which are outside $i$ 's control (in the short run). As elaborated in Section 8.1, this provides a motive for $i$ to conform her behaviour to the norm beliefs of $j$ (see the conformity concept of "it-is dangerous-not-to-conform" of Asch, 1952, the compliance process of Kelman, 1961, and Jahoda, 1972). The persons $j$ whose norm beliefs are relevant for $i$ can be said to form a normative reference group of $i$, but this reference group does not necessarily coincide with the normative reference group from which $i$ adopts norms as considered above (see Sec. 8.5 for the distinction). Again the reference group may consist of members of the same membership group to which the norn(s) applies (apply), but also of persons of other social groups (e.g., the husband when the membership group consists only of women). Although the social norm beliefs of these relevant others are exogenous state variables in the short run, in the long run they are likely to be determined or affected by the actual norm behaviour of themselves or others as a result of the mechanisms described above. This makes the normative preference interdependence behavioural in the long run.

Usually the normative BPI's as described above lead to a conformity tendency, but when the social norms prescribe emulous behaviour (e.g., scoring high in a publication competition of research economists) or non-conformist behaviour (e.g., in protest movements), it may induce also such behaviour. On the other hand, norms of non-conformism may mainly apply with respect to people outside the membership group, and hence induce a reverse bandwagon effect only with respect to these "outsiders" (e.g., revolutionaries vis-a-vis the bourgeois). Within the membership group normative interactions can then generally be assumed to lead to conformism and/or emulation and hence to bandwagon effects.

\section{(c) Exchange of information}

Bandwagon effects may also occur because the behaviour of other persons is used as an "informational reference source" (Ofshe, 1972) on the quality of the behaviour (e.g., consumption good). One then relies on others' experiences as a screening device (see Nelson's (1970) distinction between "search" and "experience" goods). A labour supply example is the exchange of information on experiences with jobs by married women. When these experiences are favourable, a bandwagon effect will result, but when the experiences are less favourable, a reverse bandwagon effect may occur.

A kind of preference interdependence (PI) which is very similar to BPI, is a dependence of the preferences of person $i$ on perceived or expected incomes $Y_{j}$ of others $j$. In the case 
of not fully satiating consumption and zero savings, in which $Y_{j}$ coincides with the total consumption of $j$, this PI is even identical to a BPI. More generally, this. PI leads to a dependence of the preferred behaviour $X_{i}$ on relative income and has the same kind of bandwagon and reverse bandwagon effects as BPI (see, e.g., Duesenberry, 1949, Deaton and Muellbauer, 1980, Sec. 12.2, Baxter, 1988, Sec. 3.1, and Frank, 1989, Sec. II).

\subsubsection{Endogenous dynamics of $B P I$}

In the previous subsections we have discussed only the comparative statics of (reverse) bandwagon effects of the actual behaviours $X_{j}^{a}$ of persons $j$ in reference groups of person $i$ on $i$ 's preferred behaviour $X_{i} \cdot{ }^{9}$ However, each actual $x_{j}^{a}$ is assumed to be at least partly determined by $j$ 's preferences with respect to that behaviour. In their turn, when $i$ belongs to a reference group of $j, j$ 's preferences are influenced by $i$ 's actual behaviour $X_{i}^{a}$ and hence, assumedly, by $i$ 's preferences with respect to that behaviour. Thus we have a positive feedback of $i$ 's preferences on $X_{j}^{a}$, making the dynamics of this aspect of $j$ endogenous. Usually it is assumed that this feedback dynamics is lagged (see, e.g., Pollak, 1976a, and the next section). This implies that $j$ 's actual behaviour $X_{j}^{a}$ is predetermined for $i$ 's preferences, and hence is a state variable. More generally, we can assume that the average behaviour $s_{\bar{X}} i$ in the reference group(s) of $i$ is a state variable which is exogenous with respect to $i$ 's preferences in the short run, but endogenous with respect to $i$ 's preferences in the longer run. In the next and subsequent sections of this chapter the endogenous long-run dynamics of the state variable $s_{\bar{X} i}$ will be analysed.

However, it is also possible that the person $i$ anticipates in her choice of behaviour the feedback of that behaviour on her social environment and takes this feedback into account in her decision. Analogously to rational habit formation, this involves endogenous (rational) expectations with respect to the future behaviours of others and could be called rational behavioural preference interdependence. However, since the behaviours of others may be the result of similar rational choices, rational BPI will in general become very complicated and may not have a unique solution, as suggested by game theory. More importantly, the feedback of $i$ 's behaviour on the average behaviour of a reference group as a whole may be negligible (see Deaton and Muellbauer, 1980, p. 375, and the next section), and/or the high degree of $i$ 's awareness of that feedback which is required for rational BPI seems in general implausible. An exception may the BPI of family members. Then cooperative rational decisions taking into account the BPI can lead to a Pareto-optimal solution (see, e.g., Chiappori, 1988, Kooreman and Kapteyn, 1989, and Subsec. 2.2.7). Nevertheless, this study abstracts from such effects. Another factor which makes the relevant behaviour of others endogenous and which is neglected here, is the selection of reference groups by the person herself (see, e.g., Baxter, 1988, pp. 104-106, and Frank, 1989, p. 84). In line with practically all economic research on BPI, reference groups are supposed to be exogenously given.

\footnotetext{
${ }^{9}$ For convenience we also speak of reference groups to denote groups of persons $j$ whose actua! behaviours affect the preferred behaviour of person $i$ by virtue of practical causes of (reverse) bandwagon effects.
} 


\subsubsection{Other forms of preference (inter)dependence}

In Subsection 5.1.2 we have considered the adoption of a social norm as a result of direct interpersonal communication on the social norm without reference to one's own actual behaviour. Such direct interpersonal communication can also play an important role in social comparison and in exchange of information and expectations (see Schokkaert, 1982a, Sec. V.B.III). In all cases we may then speak of a direct influence preference interdependence (PI), in which the preferences of person $i$ or an inner determinant of them like a belief or expectation depend directly on the preferences or an inner determinant of them of (an)other person(s) $j$ at past points in time (influencing takes time). In the normative case of Subsection 5.1.2 this direct influence PI has been argued to be a part of broader BPI processes. This holds even for a case like that of highschool girls influencing each other's opinions on future labour force participation when they will be married. This direct influence PI cannot be based on own experiences with participation, but it is likely to be strongly affected by participation experiences of mothers, older sisters, etc.. Thus, the direct influence PI is embedded in a broader BPI. More generally, this embeddedness seems to be pervasive in normative as well as other cases of direct influence PI as mentioned above (see Sec. 10.3 for a normative case). ${ }^{10}$

A kind of PI which seems more separate from BPI is a dependence of $i$ 's preferences on the well-being or preferences $U^{j}$ of another person $j$ at the same point in time as perceived or expected by $i$. This describes altruism or envy (see the extensive altruism literature and, in particular, Tomes, 1983). In order to imply PI the perceived or expected well-being of person $j$ should at least partially be determined by (revealed) preferences of $j$ or an inner determinant of them like one or more needs. Mostly this will hold. Analogously to the reputation case in Subsection 5.1.2, the perceived or expected well-being or preferences $U^{j}$ may depend on i's behaviour as well as on a degree of dependence $d_{i}^{j}$ of $U^{j}$ on $i$, which is outside $i$ 's control in the short run (Becker, 1974). The latter degree of dependence can then be considered as a state variable.

Besides all these kinds of PI there are dependencies of preferences on aspects $s_{j}$ of other persons $j$ which are not forms of PI. An example is given by a dependence of the labour supply preferences of a person $i$ on the zero actual labour supply of an involuntarily unemployed person $j$. In the latter case the zero actual labour supply of $j$ is fully determined by a rationing by labour demand and does not reflect $j$ 's labour supply preferences. However, as argued in Section 3.2, $j$ may turn into a "voluntarily unemployed" person in the long run, i.e., his (or her) zero labour supply gets supponed by his labour supply preferences. In that case the influence of $j$ 's zero labour supply on $i$ s labour supply preferences may become stronger than before and we can again speak of a PI between the persons $i$ and $j$.

10 This makes the sharp distinction between BPI and direct PI which is made by Schokkaent (1982b, Sec. V.B.II) somewhat questionable. 


\subsection{General model}

In this section and subsequent ones a general model of behavioural preference interdependence (BPI) in labour supply is constructed and analysed. This model summarizes the various BPI processes which have been discussed in the previous section. Its structure is analogous to the structure of the general individual habit formation model developed in Chapter 3. It can be considered as a kind of canonical model in the same spirit as the market model of perfect competition: the basic assumptions are simple and perhaps too unrealistic for certain purposes, but it describes important mechanisms in a transparant way and can serve as a basis for more realistic, but less transparant extensions. For the case of BPI with respect to one consumption good the model is formulated by Iannacone (1989). Here we assume BPI with respect to aggregate consumption $X_{i}$ as well as discretionary household time $L_{i}$ (see Sec. 2.3 for definitions of these variables). At the end of this section we shall extend the model so as to include BPI with respect to corporate time. ${ }^{11}$

This section demonstrates how the canonical model can be obtained from the most general model of BPI with respect to $X_{i}$ and $L_{i}$ by successive simplifying assumptions. In the latter model the one-period preferences of a labour supplier $i$ are represented by a utility function

$$
\tilde{U}^{i}\left[X_{i i}, L_{i t},\left\{X_{j t-\tau}^{a}, L_{j t-\tau}^{a} / j \varepsilon R G_{i t-\tau}, \tau z 0\right\}, t\right],
$$

where $X_{j t-\tau}^{a}$ and $L_{j t-\tau}^{a}$ are the actual consumption and the actual household time, respectively, of the members $j$ of $i$ 's total reference group $R G_{i t-\tau}$ at time $i-\tau$ (as perceived or expected by $i$ ). The total reference group $R G_{i t-\tau}$ is defined as the union of all reference groups for consumption as well as household time with respect to the relevant BPI processes, as discussed in the previous section. ${ }^{12}$ The time delays in the BPI can have various causes such as ( $i$ ) delays in diffusion and recognition of information on $X_{j t}^{a}$ and $L_{j i}^{a}$, while expected values of these variables are based on extrapolations of this information (see, e.g., Pollak, 1976a, Deaton and Muellbauer, 1980, p. 330), (ii) dependence of $\bar{U}^{i}$ on permanent rather than transitory changes in $X_{j t}^{a}$ and $L_{j t}^{a}$ (see, e.g., Iannacone, 1989, Sec. 3), (iii) delays in adjustment of $\bar{U}^{i}$ to perceived or expected $X_{j t}^{a}$ and $L_{j t}^{a}$ because of cognitive-affective reasons (see, e.g., Kapteyn and Wansbeek, 1985, Sec. 5), (iv) delays in adjustment of norm

11 A related model of both BPI and individual habit formation (IHF) with respect to only household time or corporate time of husband and wife is elaborated and estimated by Kapteyn and Woittiez (1990). This model is more detailed and more directly aimed at empirical realism than our combined BPI/IHF model (see Chs, 6 and 7), but it neglects BPI and IHF with respect to consumption and it is less transparant. Moreover, it is less general in the sense that it employs a particular specification of the labour supply equations, it does not analyse the effects of multiple equilibria, and it does not consider aggregation problems.

12 The union of two sets is the set which consists of all elements of the two sets. In general i's total reference group for consumption does not necessarily coincide with i's total reference group for houschold time. The effects of some reference variables in (5.i) are then zero. 
beliefs to $X_{j t}^{a}$ and $L_{j t}^{a}$ (see Sec. 8.5). These delays imply that the $X_{j t-\tau}^{a}$ and $L_{13 t-\tau}^{a}$ are predetermined for the choice variables $X_{i t}$ and $L_{i t}$, and hence are state variables. ${ }^{13}$

As simplifying Assumption $I$, we assume that $\bar{U}^{i}$ does not explicitly depend on time $t$ and that the reference group $R G_{i t-\tau}$ does not change with $t-\tau$. This implies that the consumption and household time preferences of person $i$ change with time only via the actual consumptions $X_{j t-\tau}^{a}$ and household times $L_{j t-\tau}^{a}$ in $i$ 's total reference group. When one of the (implicit) parameters of (5.1) is a linear function of the $X_{j t-\tau}^{a}$ and one other parameter is a linear function of the $L_{j t-\tau}^{a}$ (e.g., committed consumption and household time, see Pollak, 1976a), the influence of these state variables on $\tilde{U}^{i}$ can be summarized by the influence of two weighted averages $s_{\bar{X}}{ }_{i l}$ and $s_{\bar{L} i t}$ of $X_{j t-\tau}^{a}$ and $L_{j t-\tau}^{a}$, respectively, over $j$ and $\tau$. This also holds when more than one parameter of (5.1) is a linear function of the $X_{j t-\tau}^{a}$ or the $L_{j \ell-\tau}^{a}$ in a proportional way and in the case of an additive quadratic specification of (5.1) (see Sec. 5.5). The state variables $s_{\bar{X}_{i i}}$ and $s_{\bar{L}}$, represent the relevant social environments of person $i$ and are referred to as social state variables.

Usually averages like $s_{\bar{X}}{ }_{i t}$ and $s_{\bar{L}}{ }_{i t}$ are expressed in terms of discrete time periods, but, analogously to the case of individual habit formation (see Sec. 3.1), social influence processes like those mentioned above look more like continuous processes than like discrete processes in terms of the usual empirical time periods. Therefore, we consider $\tau$ as a continuous time variable and express $s_{\bar{L} \bar{i}}$ as

$$
s_{\bar{L} i t}=\int_{0}^{\infty} \sum_{j \in R G_{i}} \lambda_{L \tau}^{i j} L_{j t-\tau}^{a} d \tau
$$

with

$$
\int_{0}^{\infty} \sum_{j \ell R G_{i}} \lambda_{L \tau}^{i j} d \tau=1
$$

for all $i=1, \ldots, N$, where $N$ is the number of individuals in the population. An analogous formula holds for $s_{\bar{X} i t}$. This leaves us as simplifying Assumption $I I$ with a utility function $U^{i}\left(X_{i r}, L_{i t}, s_{\bar{X}_{i t}}, s_{L_{i t}}\right)$ and means that the distributions of the $X_{j t-\tau}^{a}$ and the $L_{j t-\tau}^{a}$ over the reference group and current and past points in time only affect $\tilde{U}^{i}$ via weighted averages of these distributions. ${ }^{14}$

Assumption III brings a separability structure into the weights $\lambda_{L \tau}^{i j}$ and $\lambda_{X \tau}^{i j}$ by assuming that they can be written as $\pi_{L \tau}^{i} \rho_{L}^{i j}$ and $\pi_{X \tau}^{i} \rho_{X}^{i j}$. respectively, where $\rho_{L}^{i j}{ }_{i}$ and $\rho_{X}^{i j}$ are $\tau$ independent reference weights assigned by person $i$ to person $j$, and where $\pi_{L \tau}^{i}$ and $\pi_{X \tau}^{i}$ are $j$-independent memory weights. These weights satisfy

13 This can also be assumed for $\tau=0$ as representing delays within a discrete time period or an infinitesimally small delay in the case of continuous time.

14 When a parameter of $(5.1)$ is a non-linear function of $X_{j t-\tau}^{a}$ and/or $L_{j t-\tau}^{a}$, also weighted (co)variances and other higher-order moments of the distributions will play a role fef. Kapteyn. 1977. and Kapteyn and Wansbeek. 1985, eq. (11)). 


$$
\begin{aligned}
& \sum_{j \varepsilon R G_{i}} \rho_{L}^{i j}=1, \\
& \int_{0}^{\infty} \pi_{L \tau}^{i} d \tau=1,
\end{aligned}
$$

and analogous adding-up conditions for $\rho_{X}^{i j}$ and $\pi_{X \tau}^{i}$ for all $i$. This separation of the weights implies that (5.2) can be formulated as

$$
s_{\bar{L} i t}=\int_{0}^{\infty} \pi_{L \tau}^{i} \sum_{j \varepsilon R G_{i}} \rho_{L}^{i j} L_{j t-\tau}^{a} d \tau=: \int_{0}^{\infty} \pi_{L \tau}^{i} \bar{L}_{t-\tau}^{a i} d \tau,
$$

where $\bar{L}_{t-\tau}^{a i}$ is the weighted average of $L_{j t-\tau}^{a}$ over $i$ 's reference group at time $t-\tau$. Again an analogous formula holds for $s_{\bar{X}}{ }_{i t}{ }^{15}$

Assumption $I V$ equates the memory weights $\pi_{L \tau}^{i}$ and $\pi_{X \tau}^{i}$ with exponentially declining weights $\delta_{\overline{L i}} e^{-\delta_{\bar{L} i} \tau}$ and $\delta_{\bar{X} i} e^{-\delta_{\bar{X} i} \tau}$, respectively. ${ }^{16}$ The positive parameters $\delta_{\bar{L} i}$ and $\delta_{\bar{X} i}$ measure the influence of recent average reference group behaviours relative to the more distant past. Substituting $t-\tau=s$ and $\tau=t-s$ into (5.6) and differentiating to $t$, this assumption turns out to be equivalent to the following first-order differential equations for $s_{\bar{L} i t}$ and $s_{\bar{X}}{ }_{i t}$ :

$$
\begin{aligned}
& \dot{s}_{\bar{L} i t}=\delta_{\bar{L} i}\left(\bar{L}_{t}^{a i}-s_{\bar{L} i t}\right), \\
& \dot{s}_{\bar{X} i t}=\delta_{\bar{X} i}\left(\bar{X}_{t}^{a i}-s_{\bar{X} i t}\right) .
\end{aligned}
$$

These equations describe how at each time $t$ the social state variables are adapted to new realizations of average reference group behaviours (cf. the adaptive expectations specification (6) of Iannacone (1989)). In a labour supply regime the actual household time and consumption demands $L_{j i}^{a}$ and $X_{j t}^{a}$ of persons $j$ in $i$ 's reference group, of which $\bar{L}_{i}^{a i}$ and $\bar{X}_{i}^{a i}$ are weighted averages, are assumed to be equal to $j$ 's preferred household time and consumption $L_{j 1}$ and $X_{j l}$, respectively, at the same time $t$ (Assumption $V$, see Sec. 3.1). In their turn (see Subsec. 5.1.3), these $L_{j t}$ and $X_{j t}$ may be influenced by the preferred $L_{i t}$ and $X_{i t}$ of person $i$, and hence by $i$ 's social state variables $s_{\bar{L} i t}$ and $s_{\bar{X} i t}$. Besides that, the $L_{j i}$ and $X_{j t}$ will be influenced by the $L_{j^{\prime}}$ and $X_{j^{\prime} t}$ of other persons $j^{\prime} \neq i$, and hence by their social state variables $s_{\bar{L}_{j} t}$ and $s_{\bar{X}_{j} j_{t}}$. Since these state variables do not generally coincide with $s_{\bar{L} i t}$ and $s_{\bar{X} i t}$, we obtain in general a system of $2 N$ linked differential equations (5.7a) and (5.7b) for $s_{\bar{L} i t}$ and $s_{\bar{X} i t}, i=1, \ldots, N$.

Without further simplifications such a huge dynamic system of $2 \mathrm{~N}$ interacting social environments is very difficult to be analyzed. A first simplification is to assume that the

${ }^{15}$ See Kapteyn (1977) and Kapteyn and Wansbeek (1985) for foundations of this structure by a theory of preference formation.

${ }^{16} \mathrm{Cf}$. Kapteyn and Wansbeek (1985, Sec. 7.3) and Iannacone (1989, eq. (6)). 
population can be partitioned into a limited number $K$ of social groups (e.g., status groups or age cohorts) such that $s_{\bar{L}}{ }_{i t}$ and $s_{\bar{X}_{i t}}$ are independent of $i$ within each social group, i.e., are the same for each member of such a group. (Such a group may be interpreted as a membership group.) This reduces the differential system to a system of $2 K$ equations. In the realistic case that $L_{j t-\tau}$ and $X_{j t-\tau}$ vary with $j$ and $t-\tau$ within each social group, it then follows from (5.6), its analogue for $s_{\bar{X}}{ }_{i t}$, and Assumptions IV and V that the adjustment speeds $\delta_{\bar{L} i}$ and $\delta_{\bar{X}_{i}}$, the reference group $R G_{i}$ and the reference weights $\rho_{L}^{i j}$ and $\rho_{X}^{i j}$ should all be independent of $i$ within each social group.

A further reduction of the differential system can be achieved by neglecting the social interactions between the social groups (Assumption VI). This leaves us with only two interlinked differential equations (5.7a) and (5.7b) for each sociai group, thereby allowing a separate analysis of the BPI in each group. Consider social group $S G_{1}$ with individuals $i=1, \ldots, N_{1}$. Assumption $V I I$ then states that $\delta_{\bar{L} i}=\delta_{\bar{L}}$ and $\delta_{\bar{X} i}=\delta_{\bar{X}}$ for all $i$ (see above). Moreover, Assumption VIII equates the reference group $R G_{i}$ of person $i$ with the social group $S G_{1}$ minus the person $i$ herself and assumes equivalent neighbor interactions. This means that every person $i$ assigns the same reference weights $\rho_{L}^{i j}$ and $\rho_{X}^{i j}$ to every other person $j$ in the social group. ${ }^{17}$ In combination with the adding-up condition (5.4), it implies that $\rho_{L}^{i j}=\rho_{X}^{i j}=1 /\left(N_{1}-1\right)$ for all $i$ and $j$ in $S G_{1}$. The reference weights are then independent of $i$ as required above, but the reference group $R G_{i}=S G_{1}-\{i\rangle$ still depends on $i$. Therefore, Assumption $I X$ says that $N_{1}$ is so large as to make the contribution of $i$ 's actions to the average social group demands $\bar{L}_{t-\tau}$ and $\bar{X}_{t-\tau}$ negligible. This implies that in (5.6) (omitting the superscript $a$ )

$$
\bar{L}_{t-\tau}^{i}=\frac{1}{N_{1}-\mathbf{i}} \sum_{\substack{j=1 \\ j \neq i}}^{N_{1}} L_{j t-\tau}=\frac{1}{N_{1}} \sum_{j=1}^{N_{1}} L_{j t-\tau}=: \bar{L}_{t-\tau},
$$

which is indeed independent of $i$. Analogously, we obtain $\bar{X}_{t-\tau}^{i}=\bar{X}_{i-\tau}$.

Given all the assumptions made so far, the canonical BPI model describes individuals $i$ each maximizing a utility function $U^{i}\left(X_{i t}, L_{i t} ; s_{\bar{X}}, s_{\bar{L} t}\right)$ with respect to $X_{i t}$ and $L_{i t}$ at predetermined values of $s_{X_{i}}$ and $s_{L_{i}}$ under the usual budget constraint $X_{i t} \leq w_{i t}\left(T_{i t}-L_{i t}\right)+Y_{\text {Oit }}$. Here $w_{i t}$ is the exogenous (relative) hourly corporate wage rate prevailing for individual $i$ at time $t, T_{i t}$ is her total discretionary time, and $Y_{O i t}$ is exogenous income other than her own wage income (see Sec. 2.3). This implies household time and consumption demand functions. $X_{i}\left(w_{i t}, Y_{O i t}, T_{i t}, s_{\bar{L} t}, s_{\bar{X}}\right)$ and $L_{i}\left(w_{i t}, Y_{O i t}, T_{i t}, s_{\bar{L}}, s_{\bar{X} t}\right)$, respectively. Note that these functions vary in general with $i$ due to individual variation in parameters of the utility function $U^{i}$ and in the exogenous constraint variables $w_{i t}, T_{i t}$ and $Y_{O i t}$, whereas $s_{L_{t}}$ and $s_{\bar{X}}$, are assumed to be the same for all $i$. Thus the canonical model describes individual variation in

17 I adopt the term of equivalent neighbor interactions (ENVI) from statistical physics, in which it is used to denote uniform interactions between atoms or molecules in a solid or liquid. These interactions can serve as a foundation of the so-called mean-field approximation (see, e.g., Vendrik, 1979a). The present canonical model is similar to that approximation. In line with this approximation the ENI can also be postulated with respect to the whole population, but I prefer to restrict the ENI to a social. group within this population, since this offers a more adequate basis for adding interactions between social groups to the model (see Sec. 9.5). 
behaviour, while assuming no individual variation in the social environments of the individuals. In particular, the same social environment may have different impacts on different individuals (see Sec. 5.5 for details).

According to (5.6), its analogue for $s_{\bar{X}}$, and subsequent assumptions, these social environments are determined by the average social group demands $\bar{L}_{t-\tau}$ and $\bar{X}_{t-\tau}$. However, since at least at the kinks of the individual labour supply curves at reservation wages, the individual demand functions are non-linear in the exogenous constraint variables, these individual functions cannot be exactly aggregated to average demand functions $\bar{L}\left(\bar{w}_{t}, \bar{Y}_{O t}, \bar{T}_{t}, s_{L_{l}}, s_{\bar{X} t}\right)$ and $\bar{X}\left(\bar{w}_{t}, \bar{Y}_{O t}, \bar{T}_{l}, s_{\bar{L}_{t}}, s_{\bar{X}}\right)$, when $w_{i t}, Y_{O i t}$ and/or $T_{i t}$ vary with $i$ (see Sec. 5.5 for the case of an addlitive quadratic specification of the utility function and cf. Sec. 4.7). Then the average demands depend on the entire vectors of the $i$-dependent exogenous variables like $\left(w_{1}, \ldots, w_{N}\right){ }^{18}$ These vectors can be completely characterized by the averages of their elements and by vectors of absolute deviations of the elements from these averages like $\left(w_{1},-\bar{w}_{1}, \ldots, w_{N-1},-\bar{w}_{1}\right)$ or vectors of relative deviations like $\left(\left(w_{1 t}-\bar{w}_{t}\right) / \bar{w}_{t}, \ldots,\left(w_{N-1 t}-\bar{w}_{t}\right) / \bar{w}_{t}\right)$. Assumption $X$ assumes that the latter absolute or relative deviation vectors are constants. Changes in individual values of the exogenous variables are then completely determined by changes in their averages, implying average demand functions as mentioned. ${ }^{19}$

As a result of this and preceding assumptions, we can rewrite the differential equations (5.7a) and (5.7b) for social group $S G_{1}$ as

$$
\begin{aligned}
& s_{\bar{L}_{t}}=\delta_{\bar{L}}\left[\bar{L}\left(\bar{w}_{t}, \bar{Y}_{O t}, \bar{T}_{t}, s_{\bar{L}_{t}}, s_{\bar{X} t}\right)-s_{\bar{L}_{t}}\right], \\
& \left.s_{\bar{X}_{t}}=\delta_{\bar{X}}\left[\bar{X}_{\left(\bar{w}_{t}\right.}, \bar{Y}_{O t}, \bar{T}_{t}, s_{\bar{L} t}, s_{\bar{X}_{t}}\right)-s_{\bar{X} t}\right] .
\end{aligned}
$$

When the partial derivative $\bar{L}_{s_{\bar{L}}}$ or $\bar{X}_{s_{\bar{X}}}$ is positive, we have a (net) bandwagon effect, and when it is negative, a (net) reverse bandwagon effect is active. An interesting feature of this differential system is that, up to scaling factors $\delta_{\bar{L}}$ and $\delta_{\bar{X}}$ of $s_{\bar{L}_{1}}$ and $s_{\bar{X}_{1}}{ }^{20}$, it has the same structure as the differential system (3.2a) and (3.2b) for individual habit formation. In the next section we will see that this implies that social bandwagon effects in the canonical BPI model display analogous kinds of dynamics as individual habit formation in the model of Chapter $3 .^{21}$ Note, however, the important difference that the present BPI model is formulated in terms of average demands, whereas the model of Chapter 3 describes in-

${ }_{19}^{18}$ Cf. Iannacone (1989, p. 441 ).

${ }^{19}$ Note that Assumption X implies that the (co)variances and/or higher-order (co)moments of the distributions of the $i$-dependent exogenous variables and the covariances and/or higher-order comoments of these distributions with the distributions of the $i$-dependent utility function parameters, are constants, which are in general not zero (in contrast to the approximation for an AQM specification at the end of Sec. 4.7).

${ }^{20}$ Choosing units for $s_{\bar{L},}$ and $s_{\bar{X}}$, which are $1 / \delta_{\bar{L}}$ and $1 / \delta_{\bar{X}}$, respectively, as high as the original units, $s_{\bar{L}_{t}}$ and $s_{\bar{X}}$ in $(5.9 \mathrm{a})$ and $(5.9 \mathrm{~b})$ should be multiplied by $\delta_{\bar{L}}$ and $\delta_{\bar{X}}$, respectively. Then dividing both sides of $(5.9 \mathrm{a})$ and $(5.9 \mathrm{~b})$ by $\delta_{\bar{L}}$ and $\delta_{\bar{X}}$. respectively, yields equations of the same form as (3.2a) and (3.2b).

${ }^{21}$ See also Deaton and Muellbauer (1980, pp. 374.375). 
dividual demands. Another interesting point is that, just like the prices in the perfect competition model, the social environments as represented by the social state variables $s_{\bar{L}}$ and $s_{\bar{X} t}$ are exogenous with respect to the individual behaviours in the short run, but endogenous with respect to the aggregates of the individual behaviours in the long run. ${ }^{22}$ Furthermore, like the prices in the perfect competition model, the social environments are assumed to be the same for all individuals within a social group. In the above derivation this assumption has been made to keep the model manageable as well as transparant. Moreover, it may not be a bad approximation of reality.

Analogously to the procedure in Section 3.2, the canonical BPI model as derived in this section can be extended so as to include BPI with respect to corporate time. A generalization of the derivation in this section which includes this BPI, leads to individual utility functions $\hat{U}^{i}\left(X_{i t}, L_{i t}, H_{i t} ; s_{\bar{X} t}, s_{\bar{L}}, s_{\bar{H} t}\right)$, where $H_{i t}$ denotes the corporate time of individual $i$ at time $t$ and where $s_{\bar{H}} r$, analogously to $s_{\bar{L}}$, and $s_{\bar{X} t}$, represents the social corporate time environment at time $t$. Making the simplifying assumption that the adjustment rate $\delta_{\bar{H}}$ of this social environment (see (5.9)) is (approximately) equal to the adjustment rate $\delta_{\bar{L}}$ of the social household time environment $s_{\bar{L} t}$, it easily follows that $s_{\bar{H} t}=T-s_{\bar{L} t}$. Substituting this expression and $H_{i t}=T_{i t}-L_{i t}$ into the utility functions, we obtain reduced utility functions $U^{i}\left(X_{i t}, L_{i t} ; s_{\bar{X}}, s_{L_{t}}\right)$. Their marginal utilities $U_{L_{i}}^{i}$ are again related to the marginal utilities $\hat{V}_{L_{i}}^{i}$ and $\hat{U}_{H_{i}}^{i}$ of the original utility functions as $U_{L_{i}}^{i}=\hat{O}_{L_{i}}^{i}-\hat{O}_{H_{i}}^{i}$ and may therefore be negative for sufficiently high $L_{i l}$ and hence sufficiently low $H_{i r}$. This may lead to volunteer work and may especially happen in the case of a social group of "collective workaholics" who are addicted to a high $H$ as a consequence of social control by the group. Such "collective workaholics" may even continue to work a high $H$, when their wage rate $w$ falls to zero. $^{23}$

Maximizing the original or reduced utility functions under the usual budget constraints again yields the individual and average demand functions given above and leads to the differential equations (5.9a) and (5.9b). In the usual case where the budget constraint is binding, these equations are linked to each other, and hence form a system of two first-order differential equations in $s_{L_{t}}$ and $s_{\bar{X}}$. For constant $\bar{w}_{t}=\bar{w}_{,} \bar{Y}_{O t}=\bar{Y}_{O}$ and $\bar{T}_{t}=\bar{T}$ a longrun stationary equilibrium solution $s^{*}:=\left(s_{\bar{L}}^{*}, s_{\bar{X}}^{*}\right)$ is obtained by putting $s_{\bar{L} t}=0$ in (5.9a) and $s_{x_{1}}=0$ in $(5.9 b)$. The next two sections will analyse the conditions under which such an equilibrium is locally (asymptotically) stable and when there are multiple equilibria.

${ }^{22}$ Note, however, that in the canonical BPI model the average relative wage rate $\bar{w}$ is exogenous in both the short and long run. This implies that the effects of competitive market interactions on prices in labour and consumption good markets are neglected. See Granovelter and Soong (1986) for the addition of market effects on consumption good prices to a BPI model. In a similar way market effects on wage rates could be incorporated. See also Section 10.3.

${ }^{23}$ On the other hand, this may only hold in the short run, since in the long run the "collective workaholics" may collectively and gradually diminish their corporate hours for $w=0$ until a few or zero hours and adapt their expectation of the average corporate time in their social group to that (see Sec. 5.4 for such processes). Thus, such persons whose long-run corporate hours for $w=0$ are zero. would, in the long run. no longer be prepared to work as volunteers. Again, a more complete modelling of "collective workaholism" requires a model of addictive behaviour such as that of, e.g., Winston (1980), but is not needed in the present context. 


\subsection{Multiple equilibria, hysteresis and stability conditions}

Analogously to Section 3.2, we first regard behavioural preference interdependence (BPI) with respect to only average household time $\bar{L}_{t}$ (and average corporate time $\bar{H}_{t}$ ), so ignoring the effects of $s_{\bar{X}}$. Moreover, we begin by assuming that the individual household time demand functions $L_{i}\left(w_{i}, Y_{O}, T_{i}, s_{L}\right)$ are linear in the social household time environment $s_{L}$ for $L_{i}$ in the interior of its feasible domain, i.e. for $0<L_{i}<T_{i}$ (omitting the time indices of $L_{i l}$ and $\left.s_{L_{l}}\right)^{24}$

For a social group of persons with sufficiently low (net) tastes for household time, the last inequality may hold at the prevailing $w_{i}, Y_{O i}$ and $T_{i}$ for all values of $s_{L}^{-}$and all group members $i$. This leads to average household time demand functions $\bar{L}\left(\bar{w}, \bar{Y}_{O}, \bar{T}, s_{L}\right)$ with $0<\bar{L}<\bar{T}$ which are also linear in $s_{\bar{L}}$. An example for the case of a (net) bandwagon effect $\left(\bar{L}_{s_{\bar{L}}}>0\right)$ is given by the average demand $\bar{L}^{-A}$ as a function of $s_{L}$ in Figure 5.2. The intersection $A$ of the line of this function with the equilibrium line $\bar{L}=s_{\bar{L}}^{-}$(see (5.9a) for $s_{\bar{L}}=0$ ) indicates a unique long-run (stationary) equilibrium. Analogously to Section 3.2, this equilibrium is stable, since the necessary and sufficient stability condition $\bar{L}_{s_{\bar{L}}}^{*}:=\bar{L}_{s_{\bar{L}}}\left(\bar{w}_{,} \bar{Y}_{O}, \bar{T}_{,} s_{\bar{L}}^{*}\right)<1$ is fulfilled. This condition means that the preference interdependence effect of the expected or perceived social environment $s_{\bar{L}}$ on the average household time demand $\bar{L}$ at the long-run equilibrium is smaller than one. Note that in this moving average case (see (5.6)) as opposed to the psychological stock case of Section 3.2, the stability condition is independent of the speed of adjustment of the social environment to the average household time demand $\delta_{\bar{L}}$ (which corresponds to the depreciation rate in the psychological stock case). In the case of a bandwagon effect the stability condition may not always be fulfilled, but in the case of a reverse bandwagon effect, which is described by a negative slope of the demand line, it always holds. ${ }^{25}$

Consider again the example of the average demand function $\bar{L}^{A}$ in Figure 5.2 of the social group of persons with sufficiently low household time tastes. These persons can develop into motivated corporate workers or even "collective workaholics" in the following way. Suppose that all the group members are initially unemployed and the expectations (or perceptions) of the average group behaviour have been fully adapted to that, i.e. $s_{\bar{L}}^{-}=\bar{T}$. Then the group members prefer on average a household time of $\bar{L}^{0}<\bar{T}$ hours, so they take jobs of, on average, $\bar{T}-\bar{L}^{0}$ hours. However, they will start to adapt their expectations $s_{\bar{L}}$

${ }^{24}$ Examples of such linear household time demand functions follow from the specification of the labour supply equations of Kapteyn and Woittiez (1990) and from the additive quadratic model as formulated in Section 5.5 .

${ }^{25}$ This excludes the possibility of chaos in contrast with the case where the dynamics of $s_{i}$ is described by a difference equation instead of the differential equation (5.9a). For the case of consumption Granovelter and Soong (1986) show that a combination of a bandwagon effect for relatively low levels of the average consumption $s_{\bar{X} t}=\bar{X}_{t-1}$ of others and a reverse bandwagon efifects for high $s_{\bar{X}}$ may, under certain conditions, lead to a chaotic behaviour pattem. On the other hand, Iannacone (1989) argues that, in the presence of an unstable behaviour pattem, an adaptive expectations specification of $s_{\bar{X}}$ is more plausible than the myopic expectations specification of Granovelter and Soong and demonstrates for the former specification that a chaotic consumption pattem becomes less likely as the weights of recent points of time in $s_{\bar{X}}$, decline. 


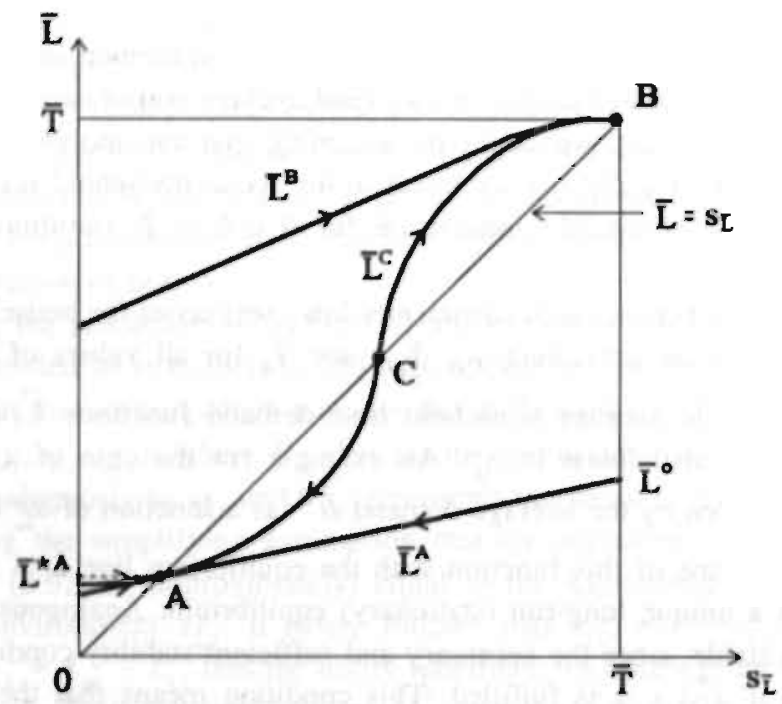

Figure 5.2 Equilibria $A B$ and $C$ as intersections of the equilibrium line $\bar{L}=s_{\bar{L}}$ and average household time demand functions $\bar{L}^{A}, \bar{L}^{B}$ and $\bar{L}^{C}$.

of the average household time to that, which, by virtue of $(5.9 \mathrm{a})$, is described by an (infinitesimally) small horizontal move from the $\bar{L}^{A}$ line at $\bar{L}^{0}$ to the left. As a consequence, the group members will on average prefer (infinitesimally) less household time and take jobs of more hours. This is described by a small vertical move back to the $\bar{L}^{A}$ line. Again the group starts to adapt its expectation of the average household time to that and will consequently have less average household time and more average corporate time, and this process will continue until the group spends (on average) only $\bar{L}^{* A}$ hours as household time, so works $\bar{T}-\bar{L}^{* A}$ hours of corporate time, and has adapted its expectation of the average household time to that. If $\bar{T}-\bar{L}^{* A}$ is high, so $\bar{L}^{* A}$ small, the group may have developed into a group of "collective workaholics". When the initial expectation of the average household time is even lower than $\bar{L}^{* A}$, a reverse process towards $\bar{L}^{* A}$ hours will take place. ${ }^{26}$

In a similar way and assuming the same constant $\bar{w}, \bar{Y}_{O}$ and $\bar{T}$, the situation of a social group of persons who do not participate to the labour market (e.g.. housewives) may be described by the long-run comer equilibrium $B$ in Figure 5.2. Now, however, the individual household time demand functions display a kink at the point where they reach the boundary $T_{i}$ of the feasible domain of $L_{i}$. Since this point varies in general with $i$, we have a same kind of situation as in the case of an $i$-dependent reservation wage of individual labour supply curves. Analogously to the average labour supply curve in Figure 4.9, this can be

${ }^{26}$ Note that, for the sake of brevity, the reasoning given switched from speaking in terms of the group members to speaking in terms of the group. We can even go beyond that and consider the average behaviour of the group members as if it is the behaviour of one average labour supplier who is subject to individual habit formation. 
shown to lead to a non-linear average household time demand function $\bar{L}^{B}$ of $s_{L}^{-}$in which the individual kinks have been "smoothed out". 27 The intersection of the curve of $\bar{L}^{B}$ and the equilibrium line then forms the stable equilibrium $B$ for $\bar{L}=\bar{T}$. This implies that when $\bar{L}$ is initially lower than $\bar{T}$, so when at least some group members are employed, the social influence of the group will lead to a situation in which all members are non-participating and spend all their time as household time.

Consider now the situation of a social group of corporate workers at $A$ who involuntarily lose their jobs. Then their average corporate time $\bar{H}$ is rationed at zero and they are forced to have average actual household time $\bar{L}_{i}^{a}$ equal to $\bar{T}$. This is described by a vertical switch from $A$ to the $\bar{L}=\bar{T}$ line. Analogously to Section 3.2 , they will adapt their expectation $s_{\bar{L}}$ of the average household time to that, so move to the right along the $\bar{L}=\bar{T}$ line. However, when after some time they can again work the average number of corporate hours they prefer according to their average household time demand line $\bar{L}^{A}$ (at the same $\bar{w}, \bar{Y}_{O}$ and $\bar{T}$ ), they shift to this line and subsequently move back to their original long-run equilibrium A. However, it is also possible that after some time unemployed persons do not want to do corporate work any more, i.e., their average preferred household time $\bar{L}$ has risen to $\bar{T}$. ${ }^{28}$ This is described by a non-linear sigmoid demand function $\bar{L}^{-C}$ as indicated in Figure 5.2. Again this demand function implies stable equilibria at the points $A$ and $B$, but also an unstable intermediate equilibrium at $C$, acting as a watershed or barrier.

Analogously to Section 3.2, the average demand function $\bar{L}^{-}$can also explain how labour supply enforcement of a social group of non-participants at equilibrium $B$ may turn them into a group of motivated employees at equilibrium $A$. More in general, the average demand function $\bar{L}^{C}$ explains how a temporary rationing of the average labour supply of a social group in the past (involuntary unemployment or labour enforcement) can have a permanent effect on the average labour supply of the group in the future. This holds, since the long-run equilibrium which is approached depends on the time path of the rationing in the past, i.e., we have hysteresis of average labour supply dependent on rationings. We call this collective hysteresis.

Adding behavioural preference interdependence (BPI) with respect to consumption to the analysis does not essentially change it and results in a simple necessary and sufficient stability condition which is given by

$$
\bar{L}_{s_{\bar{L}}^{*}}^{*}+\bar{X}_{s_{\bar{X}}^{*}}^{*}<1
$$

${ }^{27}$ One should then treat and/or approximate the parameters and variables in specifications of the individual household time demand functions of $s_{L}$ analogously to those in the $A Q M$ specification of the individual labour supply functions of $w$ in Section 4.7. See Subsection 5.5 .2 for the case of an AQM specification of the individual household time demand functions of $s_{\bar{L}}$.

28 One could then say that the cognitive dissonance of the group members between preferred and perceived actual hours of corporate work has been reduced to zero by the mutual influencing among the group members. This mechanism should be distinguished from the reduction of cognitive dissonance by individual habit formation as modelled in Section 3.2. More generally, the interpersonal mechanism of BPI is essentially different from the intrapersonal mechanism of individual habit formation. 
In the usual case where the budget constraint is binding, this, stability condition can be expressed in terms of second-order derivatives of the individual utility functions and the wage rate(s) as follows. First,

$$
\bar{L}_{s_{\bar{L}}}^{*}+\bar{X}_{s_{\bar{X}}}^{*}=\frac{1}{N} \sum_{i=1}^{N}\left(L_{i_{s_{\bar{L}}}^{*}}^{*}+X_{i_{s_{\bar{X}}}^{*}}^{*}\right) .
$$

Next, analogously to the comparative statics formulas (3.4a) and (3.4b) in Section 3.3, for $L_{i}^{*}$ and $X_{i}^{*}$ for which the restrictions $H_{i} \sqsubset 0$ and $H_{i} \leq T_{i}$ are not binding, the summands in (5.11) are equal to the corresponding $i$-specific right-hand sides of (3.4a) and (3.4b) with $s_{L}$ and $s_{X}$ replaced by $s_{\bar{L}}$ and $s_{\bar{X}}$, respectively. For $L_{i}^{*}$ and $X_{i}^{*}$ for which one of the restrictions $H_{i} \succeq 0$ and $H_{i} \leq T_{i}$ is binding and which are therefore comer points in flat pieces of the individual demand functions of the social environments (e.g., the non-participants mentioned above), the summands in (5.11) are zero. Thus we have a similar aggregation problem as in Section 4.7. For individual utility functions which are additive in the pairs $\left(L_{i}, s_{\bar{L}}^{-}\right)$and $\left(X_{i}, s \bar{\chi}\right)$, we therefore first allow only individual variations in parameters and exogenous variables which do not affect (5.11), and in the second-order derivatives $U_{L_{i} s_{i}^{i}}^{i s}$ and $U_{X_{i}}^{i *} s_{X^{\prime}}{ }^{\prime}$ which appear in the analogues of (3.4a) and (3.4b) for the present case in a linear way.

For additive utility functions the stability condition then becomes

$$
\frac{N^{u *}}{N} \frac{\overline{U_{L_{s_{L}}^{*}}^{*}}+w^{2} \overline{U_{X}^{*}} \| *}{\left|U_{L_{\bar{X}}}^{*}+w^{2} U_{X X}^{*}\right|}<1,
$$

where $N^{u *}$ denotes the long-run equilibrium number of unrestricted group members, i.e. the group members for which the restrictions $0 \leq H_{i} \leq T_{i}$ are not binding. Indices $i$ have been omitted in (5.12) to indicate averages or independence of $i$ and the superscripts $u *$ of the averages indicate averaging over the long-run unrestricted group members. Furthermore, we note that for additive utility functions positivity of $\overline{U_{L_{s_{\bar{L}}}^{*}}^{*}}$ or $\overline{U_{X_{s_{\bar{X}}}^{*}}^{*}}$ implies a net bandwagon effect, whereas negativity of $\overline{U_{L_{s_{\bar{L}}}^{*}}^{*}}$ or $\overline{U_{X_{s_{\bar{X}}}^{*}}^{*}}$ leads to a net reverse bandwagon effect.

Up to the factor $N^{u *} / N$, the stability condition (5.12) has the same form as the condition (3.5) in Section 3.3 for $\delta_{L}=\delta_{X}=1$. Therefore, when the factor $N^{11 *} / N$ equals one, i.e., when in long-run equilibrium all group members are unrestricted such as the motivated corporate workers at equilibrium $A$ in Figure 5.2, an ensuing analysis can be made which is fully analogous to that in Section 3.3. In particular, we can distinguish (relatively) weak and strong BPI with respect to household (and corporate) time and consumption at the long-run equilibrium, and we can formulate a proposition analogous to Proposition 3.1.

However, due to individual variation, often some group members are restricted in equilibrium (e.g., non-participants), whereas others are not. Then the factor $N^{u *} / N$ is smaller than one (but positive), implying that the stability condition (5.12) is more easily fulfilled. This stabilizing effect is a consequence of the impossibility for the non-participants to work less than zero corporate hours and/or the impossility for "collective workaholics" to work 
more than maximal corporate hours. When binding, these restrictions make the labour supply behaviours of non-participants and "collective workaholics" insensitive to small changes in the average labour supply in the social group to which they belong. This suppresses the average sensitivity to such changes in the social group, and hence makes the average labour supply dynamics more easily stable.

In this case we say that the BPI with respect to household (and corporate) time or consumption is (relatively) weak at a long-run equilibrium, when

$$
\frac{N^{u *}}{N} \overline{U_{L s}^{*} u^{*}}<\left|U_{L L}^{*}\right|
$$

or an analogous inequality for consunption, respectively. When (5.13) or its analogue for consumption is not fulfilled, we call the BPI (relatively) sirong at the long-run equilibrium. Thus, the strength of the destabilizing force of BPI is compared not only to the strength of the stabilizing force of diminishing marginal utility, but also to that of individual variation. Apart from this modification the analysis is then again analogous to that in Section 3.3. In Subsection 5.5 .2 we give an elaboration for an additive quadratic specification of the utility function.

When there are also individual variations in the second-order derivatives $U_{L_{i} L_{i}}^{i *}$ and $U_{X_{i} X_{i}}^{i *}$ and in the wage rate $w_{i}$, an exact analysis becomes much more complicated, but, as a first approximation, we could replace $U_{L_{i} L_{i}}^{i \bullet}, U_{X_{i} X_{i}}^{i *}$ and $w_{i}$ in the stability condition by their averages. In such an approximation the analysis is then also analogous to that in Section 3.3. Thus, the present section has shown many analogies between the analysis of BPI and the analysis of individual habit formation (IHF) in Chapter 3. These analogies, however, do not alter the fact that the interpersonal mechanism of BPI and the intrapersonat mechanism of IHF are essentially different and have different effects in a non-linear model (as opposed to a linear model like that of Pollak (1976a)). Whereas the differential system (3.2a) and (3.2b) for IHF models the dynamics of individual labour supply, the analogous differential system (5.9a) and (5.9b) for BPI models the dynamics of the average labour supply in a social group. Consequently. when we want to compare the effects of IHF and BPI, the effects of IHF on individual labour supply should first be aggregated to effects on the average labour supply in a social group. This has been done in the Sections 4.7 and 4.8 and it has been demonstrated there that individual variation in parameters and variables makes the average labour supply essentially different from a representative labour supply. As will be shown at the end of this chapter this leads to essentially different effects of locally unstable IHF and locally unstable BPI on average labour supply. Moreover, in contrast with the case of IHF, the individual variation forms an additional stabilizing force in the case of BPI (see (5.12)).

\subsection{Wage elasticities, catastrophes and hysteresis}

First we consider the relations and differences between short-run and long-run effects on average labour supply of changes in the average wage rate when starting from a long-nin equilibrium. We then compare slopes of short-run average labour supply curves $\bar{H}\left(\bar{w} ; \bar{Y}_{O}, s^{*}\right)$ 
with slopes of the long-run average labour supply curve $\bar{H}^{*}\left(\bar{w} ; \bar{Y}_{O}\right):=\bar{H}\left(\bar{w} ; \bar{Y}_{O}, s^{*}\left(\bar{w}^{*} \bar{Y}_{O}\right)\right)$ at the same $\bar{w}$, where $s^{*}$ now denotes the vector of long-run equilibrium values $s_{\bar{L}}^{*}$ and $s_{\bar{X}}^{*}$ of the social environments. When the members of the social group face diverging wage rates, the income constraint is, in line with Assumption $\mathrm{X}$ in Section 5.2, approximately aggregated to $\bar{X}=\bar{w} \bar{H}+\bar{Y}_{O}$. Then the reasoning is fully analogous to that in Section 3.4. In particular, we can derive for non-zero $\bar{H}^{\bullet}$ a relation between long-run and short-run wage elasticities of average labour supply which is of the same form as (3.10) with $\delta_{L}=\delta_{X}=1$. This implies a proposition which is analogous to Proposition 3.2. A difference with Section 3.4 is that here $\bar{H}^{*}$ is already non-zero when only one member of the group has entered the labour market. In the case where the relevant long-run equilibrium of the social environments and labour supply remains (locally) stable and existing during a change in $w$, this leads to a long-run average labour supply curve which may have the same shape as the short-run curve in Figure 4.9 in Section 4.7, but which is flatter in its forward-sloping part and flatter or steeper in its backward-sloping part. If the BPI with respect to consumption is sufficiently strong, it may even deprive the long-run curve of its backward-sloping part.

However, in a similar way as in Section 3.5, a stable long-run equilibrium like $A$ or $B$ in Figure 5.2 (for the case of BPI with respect to only household time) may become unstable and subsequently disappear, when $\bar{w}$ rises beyond a certain $\bar{w}^{* 1}$ or falls below a certain $\tilde{w}^{* 2}$. This causes catastrophic transitions in the average labour supply of the social group between an equilibrium $B$ in which all group members are non-participants and an equilibrium $A$ in which they are motivated corporate workers. These collective catastrophes form discontinuities in the forward-sloping part of the long-run average labour curve which are similar to the discontinuities in the forward-sloping part of the individual curve in Figure 3.3. A slight modification may be that in equilibrium $B$ most, but not all group members are non-participating (see the end of the previous section). Then $B$ is an interior equilibrium with a positive average labour supply, and the long-run average labour supply curve has a forward-sloping part as indicated in Figure 5.3. According to this figure most group members will collectively enter the labour market at a relatively high entry wage $\bar{w}^{* 1}$, since they are discouraged from doing so at lower wages by the high average household time in the group (high $s_{L}$ ). Such collective entries within social groups may have played (and still play) a role in the strong rise of the labour force participation of married women in many OECD countries since World War II. On the other hand, most group members will collectively leave employment at an exit wage $\bar{w}^{* 2}$ which is lower than $\bar{w}^{* 1}$, since they are kept from doing so at higher wages by the low average household time in the group (low $s_{\bar{L}}$ ). This is an example of collective hysteresis. Further note that Figure 5.3 assumes that after the collective exit still some group members are employed. At $w$ declining further, these group members leave the labour market at long-run reservation wages at which they also enter at rising $w{ }^{29}$

${ }^{29}$ Figure 5.3 also assumes that the lowest individual reservation wage $w_{r l}^{*}$, and hence the colleclive exit wage $\bar{w}^{* 2}$ and the collective entry wage $\bar{w}^{\bullet 1}$, are all positive. However, $w_{r i}^{*}$ can also be non-positive when one or mone group members have such strong corporate time tastes that they would volunteer for $w=0$ despite the social influence of a majority who would stay at home. Moreover, in a similar way as in the two cases at the end of Section 4.5, it can be shown that $\bar{w}^{\bullet 2}$ and even $w^{\cdot 1}$ can be non-positive, when a majority of the social group has strong corporate time 


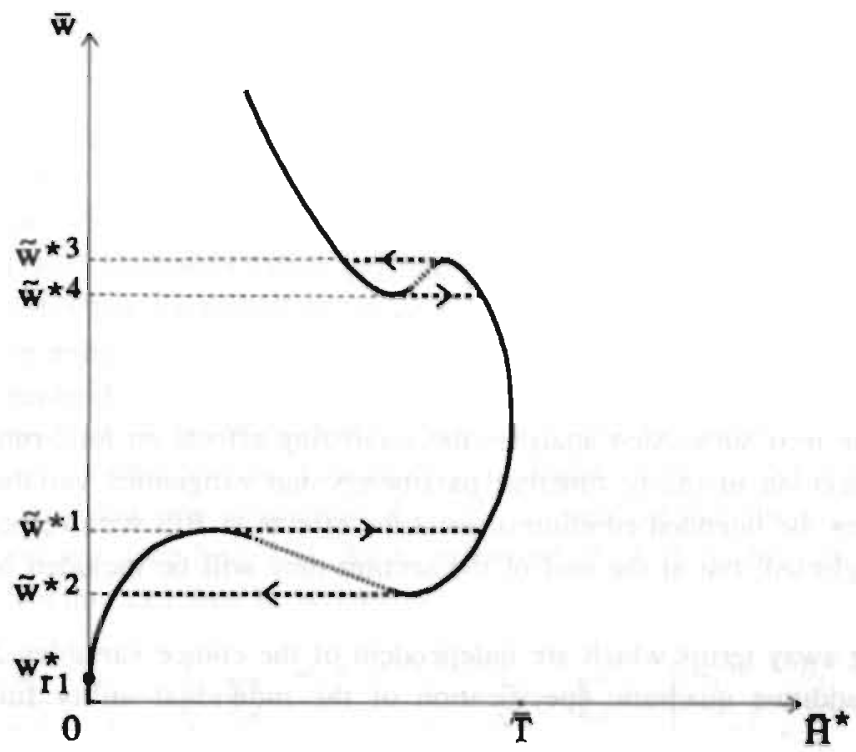

Figure 5.3 Backward-bending long-run average labour supply schedule with collective catastrophes and hysteresis. The dotted curves indicate the loci of the unstable equilibrium for $H$.

In a similar way collective hysteresis and catastrophes seem also possible in a backwardsloping part of the average labour supply curve. This is also indicated in Figure 5.3. When BPI with respect to consumption is added to the picture, this is easily seen to create an additional cause of collective hysteresis in a forward-sloping part of the average labour supply curve. On the other hand, it diminishes the probability of collective hysteresis in a backward-sloping part (which part may even not exist, see above).

In general, necessary and sufficient conditions for collective hysteresis and catastrophes as in Figure 5.3 to occur consist not only of conditions for instability of an interior long-run equilibrium, but also of other conditions. The latter conditions can be investigated only for particular specifications of the utility function. Just as in the case of individual habit formation (see Ch. 4), an eligible candidate is an additive quadratic specification of the utility function. Therefore and for the purpose of illustration of the general analysis given above, the implications of this specification will be analysed in the next two sections.

tastes and/or displays a strong form of "collective workaholism" (see also the end of the next section). 


\subsection{Additive quadratic model}

\subsection{Short-run labour supply}

Analogously to the additive quadratic model ( $\mathrm{AQM}$ ) of Chapter 4 , this section and the next consider a quadratic specification of the individual utility functions which is additive(ly separable) in individual consumption $X_{i}$ and the social consumption environment $s_{\bar{X}}$ on the one hand and individual household time $L_{i}$ and the social household time environment $s_{\bar{L}}$ on the other hand. Again the main purpose is to derive necessary and sufficient conditions for hysteresis and catastrophes as in Figure 5.3 to occur. As a preparation to that as well as for its own sake, this subsection derives and aggregates individual short-run labour supply functions and the next subsection analyses the stabilizing effects on long-run labour supply of individual variation in utility function parameters and exogenous variables. Subsection 5.5.3 then derives the intended conditions. First the effects of BPI with respect to corporate time will be neglected, but at the end of the section they will be included into the present AQM.

Transforming away terms which are independent of the choice variables $X_{i}$ and $L_{i}$ (see Sec. 4.1), the additive quadratic specification of the individual utility functions can be written as

$$
\begin{aligned}
U^{i}\left(X_{i}, L_{i} ; s_{\bar{X}}, s_{\bar{L}}\right)= & \alpha_{X i} X_{i}+\frac{1}{2} \alpha_{X X i} X_{i}^{2}+\gamma_{X \bar{X} i} s_{\bar{X}} X_{i}+ \\
& \alpha_{L i} L_{i}+\frac{1}{2} \alpha_{L L i} L_{i}{ }^{2}+\gamma_{L \bar{L} i} s_{\bar{L}} L_{i}
\end{aligned}
$$

with $\alpha_{X i}>0, \alpha_{X X i}<0, \gamma_{X \bar{X} i} \geq 0, \alpha_{L i}>0, \alpha_{L L i}<0$ and $\gamma_{L \overline{L i}}<0$. Analogously to the derivation in Section 4.1, maximization of these individual utility functions under the budget constraints leads to individual short-run labour supply functions

$$
H_{i}=\frac{a_{i} w_{i}-b_{i}}{c_{i} w_{i}^{2}+d_{i}} .
$$

where

$$
\begin{aligned}
& a_{i}:=U_{X_{i}}^{i}\left(Y_{O i}: s_{\bar{X}}\right)=\alpha_{X i}+\alpha_{X X i} Y_{O i}+\gamma_{X \bar{X} i} s_{\bar{X}} . \\
& b_{i}:=U_{L_{i}}^{i}\left(T_{i}: s_{L}\right)=\alpha_{L i}+\alpha_{L L i} T_{i}+\gamma_{L \overline{L i}} s_{\bar{L}} . \\
& c_{i}:=-U_{X_{i} X_{i}}^{i}=-\alpha_{X X i}>0 . \\
& d_{i}:=-U_{L_{i} L_{i}}^{i}=-\alpha_{L L i}>0 .
\end{aligned}
$$

Of course. (5.15) should satisfy the restrictions $0 \leq H_{i} \leq T_{i}$, so holds provided 
$0 \checkmark a_{i} w_{i}-b_{i} \checkmark T_{i}\left(c_{i} w_{i}{ }^{2}+d_{i}\right)$. The parameters $\gamma_{X \bar{X} i}$ and $\gamma_{L \bar{L} i}$ in (5.16a) and (5.16b) are equal to the second-order derivatives $U_{L_{i} s_{\bar{L}}}^{i *}$ and $U_{X_{i} s_{\bar{X}}}^{i *}$, respectively, so describe the extents to which the marginal utilities of consumption and household time are affected by changes in the corresponding social environments. We call the parameters $\gamma_{X \bar{X} i}$ and $\gamma_{L \bar{L} i}$ the susceptibilities to social influence of the preferences of person $i$ with respect to consumption and household time, respectively. Note that individual variations in these susceptibilities imply different impacts of the same social environments $s_{\bar{X}}$ and $s_{\bar{L}}$ on the labour supplies $H_{i}$ of different individuals.

To be able to aggregate the interior labour supply functions (5.15) exactly in terms of averages of utility function parameters and exogenous variables, we now assume only individual variation in the parameters $\alpha_{X i}, \gamma_{X \bar{X} i}, \alpha_{L i}$ and $\gamma_{L \bar{L} i}$ and the variables $Y_{O i}$ and $T_{i}$. Moreover, we assume that the parameter $c$ is smaller than $c_{o i}$ for a number of group members, implying that the restriction $H_{i} \checkmark T_{i}$ is binding for these individuals for $w$ between $w_{-i}$ and $w_{+i}$ (see Sec. 4.1). As an extension of (4.21) in Section 4.7, the average labour supply function can then be written as

$$
\bar{H}=\frac{\bar{a} w-\bar{b}}{c w^{2}+d}-\frac{1}{N} \sum_{\substack{i \\ w_{r i}>w}} \frac{a_{i} w-b_{i}}{c w^{2}+d}-\frac{1}{N} \sum_{\substack{i \\ w_{-i}<w<w_{+i}}}\left(\frac{a_{i} w-b_{i}}{c w^{2}+d}-T_{i}\right) .
$$

The terms under the summation signs are negative and positive "correction terms" $C T$ that are due to the $i$-dependent restrictions $H_{i} \geq 0$ and $H_{i} \leq T_{i}$, respectively. When $c$ is larger than or equal to $c_{o i}$ for all group members, the second kind of $C T$ in (5.17) are absent as in (4.21). Moreover, the average "parameters" $\bar{a}$ and $\bar{b}$ are given by

$$
\begin{aligned}
& \bar{a}=\bar{\alpha}_{X}+\alpha_{X X} \bar{Y}_{O}+\bar{\gamma}_{X \bar{X}} \bar{X}_{\bar{X}}, \\
& \bar{b}=\bar{\alpha}_{L}+\alpha_{L L} \bar{T}+\bar{\gamma}_{L \bar{L}} \bar{s}_{\bar{L}} .
\end{aligned}
$$

The first term at the right-hand side of (5.17) again describes a representative short-run labour supply curve and, in a similar way as in Figure 4.9 , the $C T$ lead to a "smoothing out" of kinks in this curve at $w=\bar{b} / \bar{a}(\bar{H}=0)$ and at $w=w_{-}$and $w=w$, for $a=\bar{a}$ and $\alpha_{L}=\bar{\alpha}_{L}(\bar{H}=\bar{T})$.

\subsubsection{Long-run labour supply}

In order to analyse the long-run labour supply behaviour which is implied by our AQM, we can consider the average labour supply function in (5.17) as a function of the social environments $s_{\bar{X}}$ and $s_{\bar{L}}$. Since the average "parameters" $\bar{a}$ and $\bar{b}$ in (5.17) as given by (5.18a) and (5.18b) are linear in $s_{\bar{X}}$ and $s_{\bar{L}}$. the first term at the right-hand side of (5.17), and hence the corresponding representative consumption demand and household time dernand, are also linear in $s_{\bar{X}}$ and $s_{\bar{L}}^{-}$for $0<\bar{H}<T$. For the case of BPI with respect to average household time only a graph of the latter representative household time demand function for given $w, \bar{Y}_{O}$ 
and $\bar{T}=T^{30}$ may look like the piecewise-linear graph of $\bar{L}^{\prime}$ in Figure 5.4 (dashed bold line). Note that $\bar{L}^{C^{\prime}}$ is equal to $T-(\bar{a} w-\bar{b}) /\left(c w^{2}+d\right)$ for interior $\bar{L}$. This representative $\bar{L}^{C^{\prime}}$ describes an unstable long-run equilibrium at $C^{\prime}$, since the slope of $\bar{L}^{C^{\prime}}$ at $C^{\prime}$ is larger than one (see the stability condition (5.10) with $\bar{X}_{s_{\bar{X}}}^{*}=0$ ). Moreover, $\bar{L}^{C^{\prime}}$ describes two stable comer equilibria $A^{\prime}$ and $B$ for $\bar{L}=0$ and $\bar{L}=T$, respectively.

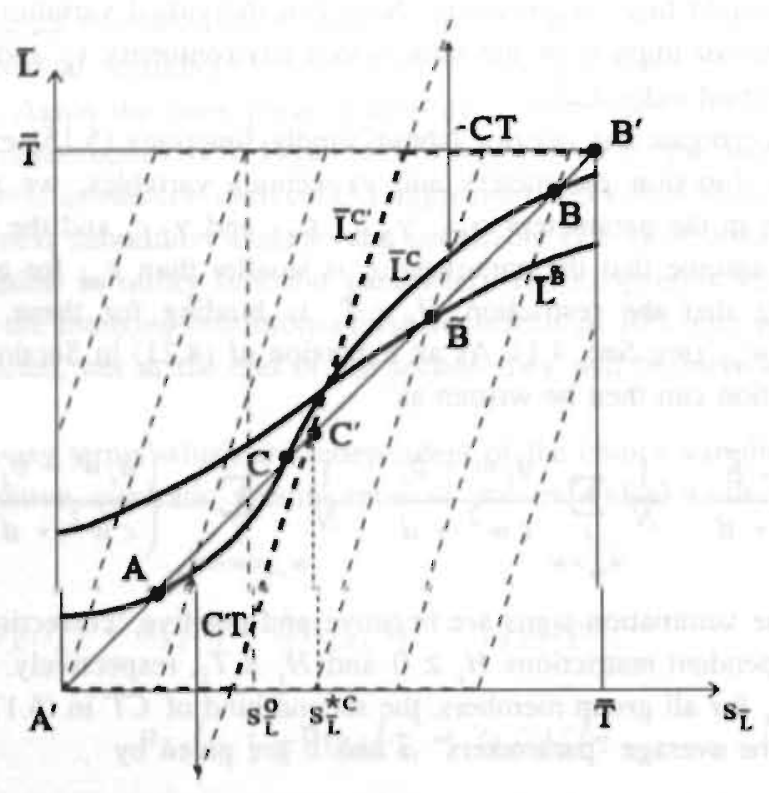

Figure 5.4 Average houschold time demand functions $\bar{L}^{C}$ and $\bar{L}^{-\bar{B}}$ as aggregates of clistributions of individual demand functions around the representative demand function $\bar{L}^{C^{\prime}}$.

However, this representative household time demand function $\bar{L}^{C^{\prime}}$ deviates from the average household time demand function $\bar{L}^{C}$ by virtue of the "correction terms" $C T$ in (5.17), since $\bar{L}^{C}$ is given by $T-(\bar{a} w-\bar{b}) /\left(c w^{2}+d\right)+C T$ for interior $\bar{L}$. What are the effects of the $C T$ on this average household time demand function and on the positions and (in)stabilities of the long-run equilibria in Figure 5.4? To answer these questions we consider for the sake of simplicity the special case where only the household time taste parameter $\alpha_{L i}$, and hence only the "parameter" $b_{i}$ in (5.15), vary over the group members. The individual household time demand functions of the social household time environment have then all the same slope, but have different levels for group members with different household time tastes. As a result, these individual demand functions as indicated by dashed lines in Figure 5.4 are "distributed" around the representative demand function and display different values of the social environment (or perceived average household time) $s_{L}-$ for which the restrictions $H_{i} \geq 0$, or $L_{i} \leq T$, and $H_{i} \leq T$, or $L_{i} \geq 0$, become binding. For a given $s_{\bar{L}}$ (e.g. $s_{L}^{o}$ ), there may then be some group members for which the restriction $L_{i} \leq T$ is

${ }^{30}$ For the sake of simplicity $T$ is assumed to be the same for all group members. 
binding, whereas the restriction $L_{i} \geq 0$ may be binding for other group members. The former binding restriction gives rise to the first kind of $C T$ in (5.17), whereas the latter binding restriction accounts for the second kind of $C T$ in (5.17). As $s_{\bar{L}}$, and hence $L_{i}$, are higher, the restriction $L_{i} \leq T$ is binding for more group members and the restriction $L_{i} \geq 0$ is binding for fewer group members. Therefore, for higher $s_{\bar{L}}^{-}$the first kind of $C T$ dominate the second kind of $C T$, so the total $C T$ is negative. Moreover, this $C T$ becomes more negative as $s_{L}^{-}$rises (see Fig. 5.4). On the other hand, for lower $s_{L}^{-}$the reverse holds, so the total $C T$ is positive and becomes more positive as $s_{L}^{-}$declines.

When the number of group members $N$ is large, we can consider the household time taste $\alpha_{L i}$ and hence the "parameter" $b_{i}$ as continuous variables $\alpha_{L}$ and $b$, and define $\varepsilon:=\alpha_{L}-\bar{\alpha}_{L}=b-\bar{b}$ with density function $f(\varepsilon)$ and zero mean. Assuming that this density function is continuous and unimodal, the average household time demand function $\bar{L}^{-C}$ can be derived to have a smooth sigmoid shape like, for instance, that in Figure 5.4. ${ }^{31}$. Thus, the kinks in the graph of the representative household time demand function have been "smoothed out" by the $C T$ in the graph of the average demand function (cf. Fig. 4.9).

In Figure 5.4 it has been assumed that the individual variation in the household time taste is sufficiently weak, and hence the $C T$ are sufficiently small, so as to preserve the existence of the three long-run equilibria (see below). However, the positions of the equilibria for the average demand function are generally different from the positions of their representative counterparts. The intermediate equilibrium $C$ may deviate only slightly from its representative counterpart $C^{\prime}$ because of relatively small $C T$, but the other two equilibria $A$ and $B$ may differ substantialiy from the corner equilibria $A^{\prime}$ and $B^{\prime}$. Both equilibria can be interior (as in Fig. 5.3 for $w$ between $\bar{w}^{* 2}$ and $\bar{w}^{* 1}$ ), since the average household time demand can be positive for zero social environment and less than maximal for maximal social environment. As indicated in Figure 5.4, this is due to group members who prefer to have more than zero (discretionary) household hours even when the perceived average household hours in the group are zero, or who prefer to have less than maximal household hours even when the perceived average household hours are maximal.

Just as the equilibrium $C^{\prime}$, the equilibrium $C$ is unstable in Figure 5.4 , since the slope of the average household time demand function $\bar{L}^{-C}$ at $C$ is assumed to be larger than one. However, it is smaller than the slope of the representative demand function $\bar{L}^{\prime}$ at $C^{\prime}$, since at $s_{\bar{L}}^{-}=s_{\bar{L}}^{\star C}$ some group members are bound by the restriction $L_{i} \leq T$ or the restriction $L_{i} \geq 0$, and hence do not contribute to the slope of the average demand function. More

${ }^{31}$ The shape of $\bar{L}^{C}$ follows from the signs of the first and second-order derivatives $\partial L / \partial s_{\bar{H}}$ and $\partial^{2} L / \partial s_{\bar{H}}$. The former slope of $\bar{L}^{-}{ }^{2}$ is given by the positive left-hand side of $(5.20)$ with $\gamma_{X} \bar{X}=0$ (see below), where the slope-depressing factor $N^{*} / N$ is given by an expression analogous to $(5.19)$. Since only this factor depends on $s_{\bar{L}}$ and since $\partial \bar{b} / \partial s_{\bar{L}}=\gamma_{L \bar{L}}$ according to (5.18b). the secondorder derivative is positively proportional to the difference in density function value

$f\left(a w-\bar{b}-T\left(c w^{2}+d\right)\right)-f(a w-\bar{b})$. For sufficiently low values of $s_{\bar{L}}$ and hence of $\bar{b}$, the argument $a w-\bar{b}$ will be so much higher than the maximum point of the unimodal density function as to make the corresponding density function value lower than the other one and hence the secondorder derivative positive. On the other hand, for sufficiently high values of $s_{\bar{L}}$ and hence of $\bar{b}$, the reverse holds. Together, this implies a sigmoid shape of $\bar{L}^{C}$. This shape is also smooth because of the continuity of the density function and hence of the second-order derivative. 
specifically, the slope of $\bar{L}^{C}$ at $C$ is $N^{\mu * C} / N$ times the $i$-independent slope of the individual demand functions, or $N^{u * C} / N$ times the slope of $\bar{L}^{C^{\prime}}$ at $C^{\prime}$, where $N^{u * C} / N$ denotes the long-run equilibrium proportion of "unbounded" group members at $C$ (see (5.12)). This slope-depressing factor $N^{u * C} / N$ is equal to the proportion of group members for which $e=b-\bar{b}$ (see above) is such that the labour supply function (5.15) with $\gamma_{X \bar{X} i}=0$ in (5.16a) and $s_{\bar{L}}=s_{\bar{L}}^{*} C^{C}$ in (5.16b) satisfies the restrictions $0 \leq H_{i} \leq T$, or $0 \leq a w-\bar{b}-\varepsilon \leq T\left(c w^{2}+d\right)$, i.e.

$$
\begin{aligned}
\frac{N^{u * c}}{N} & =P\left[a w-\bar{b}^{*} C-T\left(c w^{2}+d\right) \leq e \leq a w-\bar{b} \cdot c\right]= \\
& =F\left(a w-\bar{b}^{*} \cdot C\right)-F\left(a w-\bar{b} \cdot c-T\left(c w^{2}+d\right)\right),
\end{aligned}
$$

where $\bar{b}^{* C}$ is the long-run equilibrium value of $\bar{b}$ at $C$ and $F$ denotes the (cumulative) distribution function of $\varepsilon$. In the situation of Figure 5.4, in which the equilibrium $C$ lies vertically above the middle positively-sloping part of the representative demand line, the lower and upper limit of $\varepsilon$ in the proportion can easily be shown to be negative and positive, respectively. Then, as the individual variation in the household time taste $\alpha_{L}$, and hence the variance of $\varepsilon$ is larger (smaller), the range $T\left(c w^{2}+d\right)$ of $\varepsilon$ in the proportion being fixed, the proportion can be expected to be smaller (larger) due to thicker (thinner) tails of the density function $f(\varepsilon)$. This is in agreement with intuition on the basis of Figure 5.4.

However, when the individual variation in $\alpha_{L}$ is sufficiently strong. the slope-depressing effect may be so strong as to make the equilibria $C$ and $A$ or $C$ and $B$ disappear. Then we are left with an average demand function like $\vec{L}^{\vec{B}}$ in Figure 5.4 or $\bar{L}^{A}$ in Figure 5.2 describing one stable equilibrium $\bar{B}$ or $A$, respectively. At this equilibrium the stability condition (5.12) is fulfilled, which in the present case of variation in $\alpha_{L}$ only can be expressed in terms of the utility function parameters and exogenous variables as

$$
\frac{N^{u *}}{N} \frac{\gamma_{X \bar{X}} w^{2}+\gamma_{L \bar{L}}}{\left|\alpha_{X X}\right| w^{2}+\left|\alpha_{L L}\right|}<1 .
$$

Hereby $N^{1 *} / N$ is given by an expression analogous to that for $N^{u * \mathcal{C}} / N$ in (5.19) and $\gamma_{X \bar{X}}$ is zero in the present case of BPI with respect to household time only. A sufficient condition for $(5.20)$ to be fulfilled is given by

$$
\frac{N^{u *}}{N} \gamma_{L L}<\left|\alpha_{L L L}\right|
$$

i.e. (5.13) for the present case of variation in $\alpha_{L}$ only. This defines (relatively) weak household time BPI at the equilibrium. Conversely, in the absence of consumption BPI, the negation of (5.21). or strong household time BPI, is necessary, but not sufficient for making the equilibrium $C$ in Figure 5.4 unstable. A necessary as well as sufficient condition for instability is given by the negation of (5.20) with $\gamma_{X \bar{X}}=0$. This instability condition implies that the destabilizing force of the susceptibility to social household time influence $\gamma_{L L}$ should 
vercome not only the stabilizing forces of diminishing marginal utilities and the wage rate, ut also that of individual variation.

Fulfilment of such an instability condition for a range of the wage rate $w$ is a necessary condition for collective catastrophes and hysteresis as in Figure 5.3 to occur. To be able to establish for which values of utility function parameters (and exogenous variables) this condition can be met, we should derive parameter conditions under which the negation of (5.20) has solutions for $w$ (cf. Table 4.1). However, even in the simplest case of only individual variation in the household time taste and only household time BPI, the expression of the factor $N^{u *} / N$ in terms of $w$ as given by (5.19) depends on the specific distribution of the household time taste and tends to be complex for the usual distribution functions $F$. Therefore, a derivation of precise parameter conditions like those in Table 4.1 is bound to be restricted to very particular cases as well as laborious. In addition, instability of an interior equilibrium for a range of the wage rate $w$ is not a sufficient condition for collective catastrophes and hysteresis as in Figure 5.3, so other conditions should also be derived.

All this makes a derivation of precise parameter conditions rather unattractive. Fortunately, however, more qualitative, but also more general parameter conditions can be obtained by following an alternative and less cumbersome approach. This approach is explained in the next section.

\section{$5.5 .3^{*}$ Conditions for catastrophes and hysteresis}

This subsection will derive necessary and sufficient conditions for collective catastrophes and hysteresis as in Figure 5.3 to occur. These conditions are rather qualitative, but also rather general in the sense of allowing individual variation in the parameters $\alpha_{X i}, \gamma_{X \overline{\delta_{i}}}, \alpha_{L i}$ and $\gamma_{L \bar{L} i}$, and in the variables $Y_{O i}$ and $T_{i}$.

Following a so-called perturbation method as often used in physics, we consider the case of individual variation in which the slope-depressing factor $N^{1 *} / N$ in $(5.20)$ is smaller than one (IV case), as a perturbation of or deviation from the case of no individual variation with $N^{u *} / N=1$ (NIV case). In the NIV case all group members behave identically in the short and long run, so average short and long-run labour supply functions are exact replicas of the corresponding individual functions (the "correction terms" in (5.17) are zero). Substituting (5.16a) and (5.16b) into the individual short-run labour supply functions (5.15), the social environments $s_{\bar{x}}$ and $s_{\bar{L}}$ appear in the same way as the individual habit stocks $s_{X}$ and $s_{L}$, respectively, in (4.5) in Section 4.1, when substituting (4.6a) and (4.6b) into it. Moreover, the social environments are adjusted to the same average or individual demands according to (5.9a) and (5.9b) in Section 5.2 as the individual habit stocks according to (3.2a) and (3.2b) in Section 3.1, and up to scaling factors $\delta_{\bar{L}}$ and $\delta_{\bar{X}}$ of $s_{\bar{L}}$ and $s_{\bar{X}}{ }^{32}$, the former equations describe the same kind of adjustment paths as the latter equations. Therefore, in spite of the essentially different mechanisms of individual habit formation (IHF) and BPI, their effects on individual and average labour supply in the NIV case are the same for identical values of $\gamma_{L L}$ and $\gamma_{L \bar{L}}$, of $\delta_{L}$ and $\delta_{\bar{L}}$, and of the corresponding parameters for consumption.

${ }^{32}$ See footnote 20. 
The short and long-run labour supply schedules for the additive quadratic model (AQM) with BPI have the same shape as corresponding labour supply schedules for the AQM with IHF as derived in Sections 4.1 - 4.4. In particular, we may have a long-run labour supply schedule as in Figure 4.2 with collective and individual catastrophes between zero and maximal corporate hours in which all group members are involved. Necessary and sufficient conditions for collective and individual catastrophes and hysteresis to occur are fully analogous to those for individual catastrophes and hysteresis in the case of IHF as formulated in Proposition 4.1 at the end of Section 4.4. We should only replace the IHF parameters $\gamma_{X X}$ and $\gamma_{L L}$ by the BPI parameters $\gamma_{X \bar{X}}$ and $\gamma_{L \bar{L}}$, respectively, so $c^{*}$ and $d^{*}$ by $\bar{c}^{*}:=\left|\alpha_{X X}\right|-\gamma_{X \bar{X}}$ and $\bar{d}^{*}:=\left|\alpha_{L L}\right|-\gamma_{L}$, respectively. ${ }^{33}$ Accordingly, the parameters $\bar{d}_{-}^{*}$ and $\bar{d}_{*}^{*}$, which depend on $\bar{c}^{*}$, take the place of the parameters $d_{-}^{*}$ and $d_{+}^{*}{ }^{*}$

For the sake of exposition we consider BPI with respect to average household time only, to which Figure 5.4 applies (so $\gamma_{X \bar{X}}=0$ ). In particular, the representative agent graph of $\bar{L}^{C^{\prime}}$ hold's for the analogue of situation (i) in Proposition 4.1 for the NIV case, so for $0<\bar{c}^{*}<c_{o}^{*}<\alpha_{X} / Y_{O}$ and $\bar{d}_{-}^{*}<\bar{d}^{*}<\bar{d}_{+}^{*}$, and for $\bar{w}^{* 2}<w<\bar{w}^{* 1}$, where $\bar{c}^{*}=\left|\boldsymbol{\alpha}_{X X}\right|$ and where $\bar{w}^{* 1}$ and $\bar{w}^{* 2}$ indicate the entry and exit wage in the NIV case, respectively. ${ }^{35}$ The former conditions mean that the short-run satiation tendency of consumption is not too strong and that the household time IHF is moderately strong. At the entry and exit wages we have constellations such as in Figure 4.1 for $w=w^{* 1}$ and $w=w^{* 2}$, respectively, implying collective catastrophes beyond the entry and exit wages such as in Figure 4.1 for $w>w^{* 1}$ and $w<w^{* 2}$, respectively.

What happens with these constellations when individual variations come into play and increase from zero? The representative agent graph of $\bar{L}^{\prime}$ is then transformed into a graph like that of $\bar{L}^{-}$in Figure 5.4, and for such a graph and for sufficiently strong individual variation, collective catastrophes will occur beyond an entry wage $\bar{w}^{* 1}$ and an exit wage $\vec{w}^{* 2}$ (see Fig. 5.3) at which the curve of $\bar{L}^{c}$ is tangent to the equilibrium line (like the curve of $L^{C}$ in Figure 3.2 for $w=w^{* 2}$ ). The left-hand side of the stability condition (5.20) is then equal to one. As indicated at the end of the previous section, it is generally difficult to solve this equation for $w$, but from the position of the curve of $\bar{L}^{C}$ vis-a-vis the line of $\bar{L}^{-} C^{\prime}$ it is easily seen that, at rising $w$ and declining $L^{C}$ and $L^{C}$. a tangency situation for $L^{C}$ is reached sooner than a situation as in Figure 4.1 for $\bar{L}^{-}$at $w=w^{* 1}$. Thus, the entry wage $\bar{w}^{* !}$ in the IV case is lower than the entry wage $\bar{w}^{* 1}$ in the NIV case. Analogously, the exit wage $\bar{w}^{* 2}$ in the IV case is higher than the exit wage $\vec{w}^{* 2}$ in the NIV case.

As functions of $\bar{d}^{*}:=\left|\alpha_{L L}\right|-\bar{\gamma}_{L L}$ for given values of $\alpha_{L L}$ and the other parameters and variables, these entry and exit wages are represented in Figure 5.5. The graphs for the

${ }^{33}$ Note that the adjustment parameters $\delta_{\bar{x}}$ and $\delta_{\bar{L}}$ do not appear in the definitions of $\bar{c}^{\bullet}$ and $\bar{d}^{*}$ because of the moving average instead of psychological stock specifications of the social environments in (5.6) in Section 5.2.

${ }^{34}$ Note that the bars in $\bar{c}^{*}, \bar{d}^{*}, \bar{d}_{-}^{*}$ and $\bar{d}_{+}^{*}$ do not indicate averages of the corresponding IHF parameters. The parameter $c_{o}^{*}$ in Proposition 4.1 does not have to be replaced by a parameter $\vec{c}_{o}^{*}$. since $c_{o}^{*}$ depends only on $\alpha_{X}, \alpha_{L}, Y_{O}$ and $T$.

35 Again these entry and exit wages are here not averages of the entry and exit wages in the IHF case as in Section 4.8 . 
NIV case are analogous to those for the IHIF Subcase $2^{\mathrm{c}}$ in Figure $4.4 \mathrm{c}$. The entry wages $\bar{w}^{* 1}$ are given by the part of the $\bar{w}_{r}^{*}$ line between its intersections with the $\bar{w}_{+}^{*}$ line and the $\bar{w}_{-}^{*}$ line (and below the $\bar{w}^{* u}$ curve), so for $\bar{d}^{*}$ between $\bar{d}_{-}^{*}$ and $\bar{d}_{+}^{*}$ (solid in Fig. 5.5). The corresponding exit wages $\bar{w}^{\cdot 2}$ are described by the part of the $\bar{w}_{-}^{*}$ line in the same interval for $\bar{d}^{*}$. Thus, only for $\bar{d}_{-}^{*}<\bar{d}^{*}<\bar{d}_{+}^{*}$, i.e. for a sufficiently, butt not too strong (average) susceptibility to social influence $\bar{\gamma}_{L \bar{L}}$, the NIV case allows collective catastrophes and hysteresis in a forward-sloping part of the labour supply curve such as in Figure 4.2.

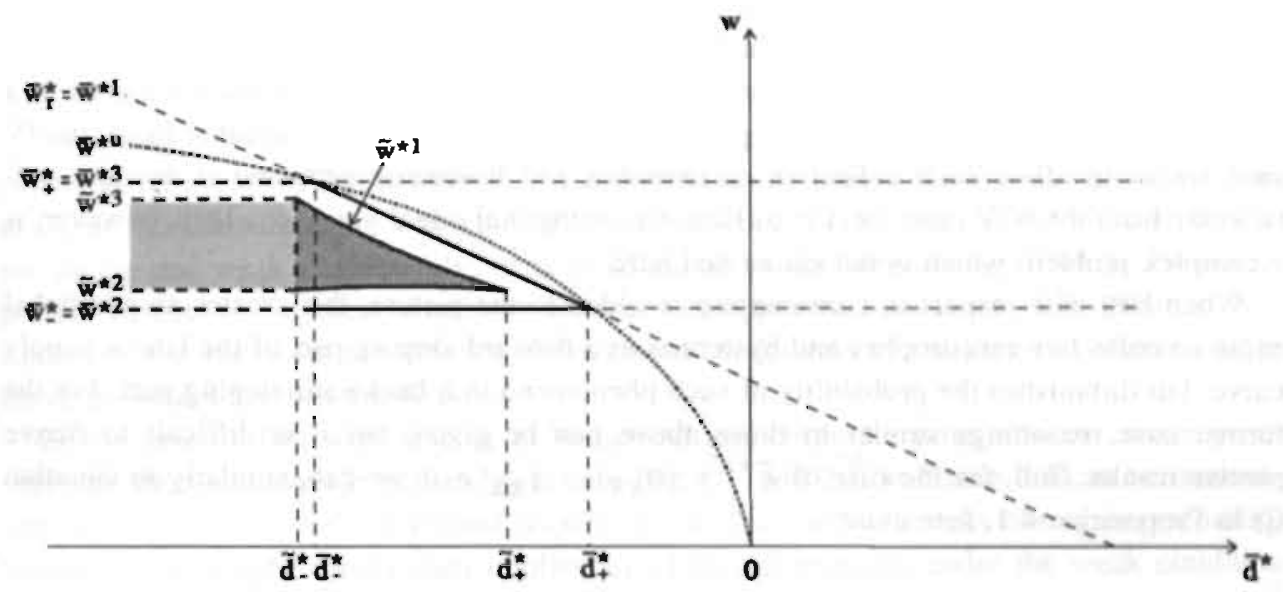

Figure 5.5 Graphs of entry and exit wages as functions of $\bar{d}^{*}:=\left|\alpha_{L L}\right|-\bar{\gamma}_{L \bar{L}}$ for given $\left|\alpha_{L L}\right|$ and $0<\bar{c}^{*}:=\left|\alpha_{X X}\right|-\bar{\gamma}_{X \bar{X}}<\bar{c}_{o}^{*}<c_{o}^{*}$ in the IV as compared to the NIV case. The hatched surface indicates the instability region in the IV case and the cross-hatched part of it corresponds to Figure 5.3.

Similarly, in the IV case the entry wage $\bar{w}^{* 1}$ and the corresponding exit wage $\tilde{w}^{* 2}$ as functions of $\bar{d}^{*}$ are described by a $\bar{w}^{* 1}$ curve and the part of a $\bar{w}^{* 2}$ curve, respectively, for $\bar{d}^{*}$ between $\bar{d}_{-}^{*}$ and $\bar{d}_{+}^{*}$. The $\bar{w}^{* 2}$ curve also holds for $\bar{d}^{*} \leq \bar{d}_{*}^{*}$ for which we have two exit wages $\bar{w}^{* 2}$ and $\bar{w}^{* 3}$ like $w_{-}^{*}$ and $w_{*}^{*}$ in Figure 4B in Appendix 4B. In contrast to the NIV case, the $\bar{w}^{* 1}$ curve is not a part of a $\bar{w}_{r}^{*}$ curve, the $\bar{w}^{* 2}$ curve is not a part of a $\bar{w}_{-}^{*}$ curve and the $\tilde{w}^{* 3}$ curve is not a part of a $\bar{w}_{*}^{*}$ curve, but together the three former curves constitute the frontier of "threshold wages" $\bar{w}^{* u}$ for the existence of an unstable intermediate equilibrium within this frontier (cf. Case 2 in Sec. 4.4) ${ }^{36}$ The instability region within the frontier is indicated by the hatched surface in Figure 5.5. The part of that region which yields a labour supply schedule like that in Figure 5.3 is cross-hatched.

${ }^{36}$ The $\bar{w}^{* 2}$ branch of this frontier corresponds to the branch of unfeasible negative threshold wages $w^{* u}=-\sqrt{-\bar{d}^{*} / \bar{c}^{*}}$ in the NIV case. Note that in the present IV case the entry wage $\tilde{w}^{* 1}$, at which most group members collectively enter, is higher than the "aggregate reservation wage" or lowest individual reservation wage $w_{r 1}$ beyond which the average long-run labour supply $\ddot{H}^{*}$ becomes positive at rising $w$ (see Fig. 5.3). 
As a consequence of the relative positions of the $\bar{w}^{* 1}$ curve and the $\bar{w}^{* 2}$ curve vis-à-vis. the corresponding lines in the NIV case, $\bar{d}_{+}^{*}$ is more negative than $\bar{d}_{+}^{*}$. Thus, with individual variation the average susceptibility to social influence $\bar{\gamma}_{L \bar{L}}$ should be stronger than without individual variation in order to allow collective catastrophes and hysteresis in a forwardsloping part of the labour supply curve as in Figure 5.3. On the other hand, since in a similar way as above the $\bar{w}^{* 3}$ curve can be shown to be located below the $\bar{w}^{* 3}$ line in the NIV case, it is ambiguous whether $\bar{d}_{-}^{*}$ at the meeting point of the $\bar{w}^{* 1}$ curve and the $\bar{w}^{* 3}$ curve is more or less negative than $\vec{d}_{-}^{*}$.

An important difference between the NIV and IV case is the following. In analogy to the IHF case of Section 4.4, the NIV case excludes collective catastrophes and hysteresis in a backward-sloping part of the labour supply curve as in Figure 5.3. On the other hand, the IV case seems to allow such collective catastrophes and hysteresis provided it deviates sufficiently from the NIV case, i.e. for sufficiently strong individual variation. This, however, is a complex problem which is not elaborated here.

When BPI with respect to consumption is added to the picture, this creates an additional cause of collective catastrophes and hysteresis in a forward-sloping par of the labour supply curve, but diminishes the probability of such phenomena in a backward-sloping part. For the former case reasonings similar to those above can be given, but it is difficult to derive precise results. Still, for the case of $\bar{c} \bullet:=\left|\alpha_{X X}\right|-\bar{\gamma}_{X \bar{X}}>0$ we can, similarly to situation (i) in Proposition 4.1, formulate

Proposition 5.1 In a situation of not too strong individual variation collective catastrophes and hysteresis in a forward-sloping part of the labour supply curve such as in Figure 5.3 occur for $0<\bar{c}^{*}<\bar{c}_{o}^{*}$ and $\bar{d}_{-}^{*}<\bar{d}^{*}<\bar{d}_{+}^{*}<0$, where $\bar{c}_{o}^{*}, \bar{d}_{-}^{*}$ and $\bar{d}_{+}^{*}$ are complex and unknown functions of the distributions of $\alpha_{X i}, \alpha_{L i}, \gamma_{X \bar{X} i}, \gamma_{L \bar{L} i}, Y_{O i}$ and $T_{i}$ and of $\alpha_{X X}$ and $\alpha_{L L}$, except that $\bar{c}_{o}^{*}$ does not depend on $\bar{\gamma}_{X \bar{X}}$ and $\bar{\gamma}_{L \bar{L}}$, and $\bar{d}_{-}^{*}$ and $\bar{d}_{+}^{*}$ not on $\bar{\gamma}_{L \bar{L}}$. Moreover, $\bar{c}_{o}^{*}$ is smaller than $c_{o}^{*}$ in the NIV case and $\bar{d}_{+}^{*}$ is more negative than $\bar{d}_{+}^{*}$ in the NIV case.

The restriction $\bar{c}^{*}=\left|\alpha_{X X}\right|-\bar{\gamma}_{X \bar{X}}>0$ is equivalent to the average susceptibility to social influence $\bar{\gamma}_{L \bar{L}}$ being smaller than the short-run tendency to satiation $\left|\alpha_{X X}\right|$. This implies $\left(N^{\prime \prime *} / N\right) \bar{\gamma}_{X \bar{X}} \| *<\left|\alpha_{X X}\right|$, since $N^{\| *} / N$ times the average susceptibility to social influence of the unrestricted group members ${\overline{\gamma_{X X}}}^{1 *}$ can easily be shown to be smaller than the average susceptibility of all group members $\bar{\gamma}_{X \bar{X}}$. According to the analogue of (5.13) in Section 5.3 for consumption, this defines (relatively) weak BPI with respect to consumption at the intermediate equilibrium. Together with the stability condition (5.12) (cf. (5.20)), this implies that in order to allow collective catastrophes and hysteresis, the BPI with respect to household time should then be strong at the intermediate equilibrium, i.e. 
$\left(N^{u *} / N\right){\overline{\gamma_{L L}}}^{u *} \geq\left|\alpha_{L L}\right|^{37}$ According to Proposition 5.1 this holds in an interval of $w$ for $\bar{\gamma}_{L \bar{L}}>\left|\alpha_{L L}\right|+\left|\bar{d}_{*}^{*}\right|$, and in addition we should have $\bar{\gamma}_{L \bar{L}}<\left|\alpha_{L L}\right|+\left|\bar{d}_{-}^{*}\right|$. On the other hand, as $\bar{c}^{*}$ increases, so as $\bar{\gamma}_{X \bar{X}}$ declines or $\left|\alpha_{X X}\right|$ rises, the interval for $\bar{d}^{*}$ narrows and the entry and exit wage curves in Figure 5.5 approach each other until the interval vanishes and the two curves coincide into one point for the value $\tilde{c}_{o}^{*}$ of $\bar{c}^{*}$. The crosshatched surface in Figure 5.5 has then shrunk to zero. However, when the individual variation is too strong, we have for all positive $\bar{c}$ a situation in which a graph like that of $\bar{L}^{\prime}$ in Figure 5.4 is transformed into a graph like that of $\bar{L}$, describing a stable equilibrium. In that case the surface in Figure 5.5 is already zero for a non-positive $\bar{c}_{o}^{*}$. Therefore, Proposition 5.1 holds only for not too strong individual variation.

Analogously to the situations (ii) and (iii) in Proposition 4.1 at the end of Section 4.4, collective catastrophes and hysteresis in a forward-sloping part of the labour supply curve as in Figure 5.3 can also occur when the BPI's with respect to consumption and household time are strong and weak, respectively, or both strong at an intermediate equilibrium. However, precise conditions for these situations are more complicated than those in Proposition 5.1. Likewise, statements on the absence of backward-bendedness of the long-run labour supply curve in these situations are difficult.

In the case of BPI with respect to household and corporate time only or consumption only, the short-run average demand functions like $\bar{L}^{C}$ and $\bar{L}^{\vec{B}}$ in Figure 5.4 can be rationalized as the result of the constrained maximization of an additive short-run aggregate utility function. Catastrophe theory then implies an additional property under the weak condition that an average household time or consumption demand function of the corresponding social environment like $\bar{L}^{C}$ in Figure 5.4 is three times continuously differentiable $\left(C^{3}\right)$. This property is formulated in the following proposition for the case of household time BPI, but an analogous proposition holds for consumption BPI.

Proposition 5.2 Consider the class of $C^{3}$ average household time demand functions of the social environment $s_{\bar{L}}^{-}$which have a sigmoid shape similar to that of $\bar{L}^{C}$ in Figure 5.4 , and for which the collective entry and exit catastrophes involve interior tangency of the curves of these demand funcions to the equilibrium line. For all demand functions in this class it holds that, in first approximation for $\bar{d}^{*}$ near and left to $\bar{d}_{*}^{*}$, the difference between the long-run entry and exit wages is proportional to $\left|\bar{d}^{*}-\bar{d}_{*}^{*}\right|^{3 / 2}=\left(\bar{\gamma}_{L} \bar{L}^{-}-\bar{\gamma}_{L} \bar{L}_{*}\right)^{3 j 2}$ (at constant $\left|\alpha_{L L}\right|$ ). Here $\bar{\gamma}_{L, L_{*}}$ is the value of the average social susceptibility to social influence that corresponds to $\vec{d}_{+}^{*}$.

${ }^{37}$ This can be seen by rewriting the stability condition (5.12) for the present AQM case, analogcusly to (4.11) in Section 4.3, as $\bar{c}^{*} w^{2}+\bar{d}^{*}>0$. where $\bar{c}^{*}:=\left|\alpha_{X X}\right|-\left(N^{* *} / N\right) \overline{\gamma_{X} \bar{X}}{ }^{* *}$ and $\bar{d}^{*}:=\left|\alpha_{L L}\right|-\left(N^{\mu *} / N\right){\overline{\gamma_{L L}}}^{u *}$. Instability of the intermediate equilibrium then requires that $\bar{c}^{*} w^{2}+\bar{d}^{*} \leq 0$, or $\bar{d}^{*} \leq-\bar{c}^{*} w^{2}$, so when the consumption BPI is weak at the intermediate equilibrium, or $\bar{c}^{*}>0$, we should have $\bar{d}^{*} \leq 0$, or strong household time BPI at the intermediate: equilibrium. 
The power $3 / 2$ is a so-called invariant, i.e., it holds for all demand functions in the class mentioned (see Vendrik, 1979b, p. 520). Note that the corresponding power in the linear NIV case is 1. For BPI with respect to both household time and consumption an analogous proposition can be expected to hold, but this is not implied by catastrophe theory, since the short-run average demand functions can then not be rationalized by an additive short-run aggregate utility function as a consequence of the factor $N^{u} / N$ depending on both social environments (cf. Sec. 4.6). Therefore, an investigation of this case is beyond the scope of this study.

When BPI with respect to corporate time $H$ is added to the analysis in this section, not much changes. Analogously to Section 4.5 , we consider a quadratic specification of the individual utility functions $\hat{U}^{i}\left(X_{i}, L_{i}, H_{i} ; s_{\bar{X}}, s_{\bar{L}}, s_{\bar{H}}\right)$ which is additive (ly separable) in $\left\{X_{i}, s_{\bar{X}}\right\},\left\{L_{i}, s_{\bar{L}}^{-}\right\}$and $\left\{H_{i}, s_{\bar{H}}\right\}$. Assuming equal adjustment rates $\delta_{\bar{L}}$ and $\delta_{\bar{H}}$ of the sociall household and corporate time environments, this specification can be reduced to a specification of the individual utility functions $U^{i}\left(X_{i}, L_{i} ; s_{\bar{X}}, s_{\bar{L}}\right)$ by substituting $H_{i}=T_{i}-L_{i}$ and $s_{\bar{H}}=\bar{T}-s_{\bar{L}}$ (see Sec. 5.2). Assuming $T_{i}=T$, this yields a specification of the same form as (5.14), where $\alpha_{X i}=\alpha_{X i}, \alpha_{X X i}=\alpha_{X X i}, \gamma_{X \bar{X} i}=\hat{\gamma}_{X \bar{X} i}$ and

$$
\begin{aligned}
& \alpha_{L i}=\alpha_{L i}-\alpha_{H i}+\hat{d}_{\bar{H} i}^{*} T \text { with } \hat{d}_{\bar{H} i}^{*}=\left|\alpha_{H H i}\right|-\hat{\gamma}_{H \bar{H} i}, \\
& \alpha_{L L i}=\alpha_{L L i}+\alpha_{H H i}<0, \\
& \gamma_{L \overline{L i}}=\hat{\gamma}_{L \overline{L i}}+\hat{\gamma}_{H \overline{H i}} \geq 0 .
\end{aligned}
$$

The parameter $\hat{d}_{\vec{\mu}}^{*}$ in (5.22a) measures minus the (relative) strength of the corporate time BPI for individual $i$. When the "pure" corporate time taste $\hat{\alpha}_{H i}$ is positive and $\hat{d}_{\bar{H} i}^{*}$ is negative, i.e., when the corporate time BPI is (relatively) strong for $i$, the "net" household time taste $\alpha_{L i}$ is smaller than the "pure" household time taste $\hat{\alpha}_{L i}$ and may even be negative. These possibilities imply interesting modifications of individual and average short and long-run labour supply schedules and of diagrams like Figure 5.5. These modifications are similar to those discussed in Sections 4.5, 4.7 and 4.8 and are not elaborated here. I mention only one of them.

When $\alpha_{L i}<0$, or $\alpha_{L i}<\alpha_{H i}+\left(-\hat{d}_{\vec{H}}^{*}\right) T$ (see (5.22a)), for all individuals, i.e., when the "pure" household time taste of each individual is lower than his (or her) "pure" corporate time taste plus the net marginal utility effect of his corporate time BPI and satiation tendency, maximal average employment $(\underline{H}=T)$ is a stable long-run equilibrium for any $w$ (cf. Fig. 4.7). In such a situation of a strong exogenous attachment to corporate work relative to household time and/or strong endogenous "collective workaholism", there is not only no positive short-run exit wage, but also no positive individual or collective long-run exit wage (see the description of "collective workaholics" at the end of Sec. 5.2), and, when $\bar{d}_{i}^{*}:=\left|\alpha_{L L L i}\right|-\gamma_{L \overline{L i}}>\alpha_{L i} / T$ for all individuals, no positive individual or collective longrun entry wage. However, apart from such rather extreme cases, Propositions 5.1 and 5.2. continue to hold for the combination of household and corporate time BPI instead of pure household time BPI. 


\subsection{Concluding remarks}

This chapter has derived and analysed a general model of BPI with respect to both consumption and household and corporate time in labour supply. This model turned out to be, in part, analogous to the general individual habit formation model which was developed in Chapter 3. As a consequence, the model has analogous properties like multiple equilibria exhibiting collective hysteresis and catastrophes dependent on rationings and the wage rate. An additional complication, however, is formed by the stabilizing force of individual variation in parameters and exogenous variables. As elaborated in the previous section for an additive quadratic (AQM) specification of the utility function, this makes parameter conditions for collective catastrophes and hysteresis stronger. Moreover, it implies relations between collective entry and exit wages on the one hand and BPI parameters on the other hand which differ from those without individual variation.

A more important implication of individual variation in parameters and exogenous variables is that it makes the effects on average labour supply of locally unstable individual habit formation (IHF) essentially different from those of locally unstable BPI (see the end of Sec. 5.3). In order to see this, we should compare the average labour supply graphs for BPI as derived in this chapter for an AQM to corresponding aggregated labour supply graphs for IHF in Section 4.8. Comparing Figure 4.11 and Figure 5.3, we see that collective hysteresis can occur in both the IHF and BPI cases, but, whereas in the former case catastrophes in the individual long-run labour supply curve are "smoothed out" in the aggregated long-run curve by individual variation, we have in the latter case collective catastrophes in the aggregated long-run curve. This means that when members of a social group strongly influence each other's preferences, the labour market entries of most members are "synchronized" and the individual variation in the reservation wages of these members in the absence of this social influence is reduced to zero. Another important difference between the IHF and BPI cases is that whereas locally unstable IHF leads to a continuum of possible equilibria (Fig. 4.12), and hence to movements within the collective hysteresis loop (Fig. 4.11), locally unstable BPI polarizes the long-run expectations and behaviours into two distinct stable equilibria (Fig. 5.4) and consequently precludes a continuum of equilibria, and hence long-run trajectories within the collective hysteresis loop (Fig. 5.3). We can then summarize the differences between the effects of locally unstable IHF and those of locally unstable BPI in Table 5.1. On the one hand, locally unstable BPI leads to collective hysteresis and catastrophes and, as a consequence, to individual hysteresis and catastrophes. On the other hand, locally unstable IHF implies individual catastrophes, but, as a result of individual variation, never collective catastrophes.

However, the last difference holds only for the long-run curve, so when the group members are in a state of long-run equilibrium. As argued in Section 3.5, in a world of ever changing wage rates labour suppliers will be outside long-run equilibrium most of the time. In particular, a cyclical variation in $\bar{w}$ will lead to a quasi-hysteresis phenomenon and collective catastrophes in the long-run average labour supply curve are "smoothed out" in an "actual" average labour supply curve. Then the last mentioned difference between the effects of locally unstable BPI and those of locally unstable IHF is not so clear any more, but still we can expect a more pronounced flattening of the average labour supply curve by BPI than by IHF. 


\begin{tabular}{|l|c|c|}
\hline & $\begin{array}{c}\text { locally unstable } \\
\text { IHF }\end{array}$ & $\begin{array}{c}\text { locally unstable } \\
\text { BPI }\end{array}$ \\
\hline individual hysteresis & yes & yes \\
\hline individual catastrophes & yes & yes \\
\hline collective hysteresis & yes & yes \\
\hline collective catastrophes & no & yes \\
\hline $\begin{array}{l}\text { trajectories within collective } \\
\text { hysteresis loop }\end{array}$ & yes & no \\
\hline
\end{tabular}

Table 5.1 Hysteresis and catastrophe phenomena at locally unstable IHF as compared to locally unstable BPI.

A subsequent question is what a combination of the effects of (locally unstable) BPI and those of (locally unstable) IHF may look like. This will be dealt with in the next two chapters. 


\section{CHAPTER 6}

\section{Collective Habit Formation: BPI and Stable IHF}

This chapter and the next analyse labour supply models which combine the effects of (bandwagon) behavioural preference interdependence (BPI) with those of individual habit formation (IHF). For consumption demand, integrations of a BPI model and a IHF model have been made by Pollak (1976a) and Alessie and Kapteyn (1991). For labour supply, such an integration has only been carried out by Kapteyn and Woittiez (1990) (see footnote 11 in Sec. 5.2). For the sake of brevity, we refer to these integrated models as models of collective habit formation $(\mathrm{CHF}) .^{1}$ All the CHF models mentioned imply or are estimated to have a unique stable long-run equilibrium for the combined IHF/BPI process. However, just as the separate stable IHF or stable BPI models, such a stable CHF model cannot explain why members of certain subcultures of long-term unemployed, although having lost their old job involuntarily, become little motivated to get a new job. Similarly, such a stable CHF model may not adequately explain how, in a collective process of "emancipation", social groups of former housewives can develop a strong orientation towards paid work. To model such profound changes in labour supply preferences in a more complete way than the IHF models of Chapters 3 and 4 and the BPI models of Chapter 5, we integrate these two kinds of models into (unstable) CHF models.

First, Section 6.1 gives a general stability analysis for the case of BPI and stable IHF with respect to household time only. For two moderately general (sub)cases which can occur when the individual household time demand functions are linear or piecewise-linear in the individual habit states, simple necessary and sufficient (in)stability conditions are derived. However, these conditions turn out not to be the relevant (in)stability conditions in the case of BPI and separately unstable IHF. For that case a modified relevant (in)stability condition is derived in Chapter 7. Accordingly, Section 6.2 and subsequent sections of the present chapter are restricted to the case of BPI and separately stable IHF, whereas Chapter 7 analyses the case of BPI and separately unstable IHF.

In order to investigate the labour supply dynamics implied by the stability conditions in Section 6.1. Section 6.2 considers an additive quadratic specification of the CHF model

${ }^{1}$ This term has been inspired by the connection between IHF and BPI as shown by Deaton and Muellbauer (1980, pp. 374-375). 
(AQM). It is shown that, in the presence of exogenous individual variation, the IHF not only reinforces the destabilizing effect of BPI, but also counteracts it by amplifying the stabilizing individual variation (a point not recognized by Kapteyn and Woittiez). Moreover, it is demonstrated that, as a generalization of the case of unstable BPI and no IHF as treated in the previous chapter, collective catastrophes and hysteresis may occur for changing values of the wage rate. Necessary and sufficient conditions for the occurrence of such phenomena are formulated. Sections 6.3 and 6.4 add BPI and stable IHF with respect to consumption and corporate time to the models, and Section 6.5 concludes.

\subsection{Stability conditions}

\section{I.I General analysis}

Consider individuals $i=1, \ldots, N$ maximizing a utility function $U^{i}\left(X_{i}, L_{i} ; s_{L i}, s_{\bar{L}}\right)$ with respect to consumption $X_{i}$ and household time $L_{i}$ at predetermined levels of their individual habit state $s_{L i}$ and social environment $s_{L}$ under the usual budget constraint $X_{i} \leq w_{i}\left(T_{i}-L_{i}\right)+Y_{O i}$. This implies individual consumption demand functions $X_{i}\left(w_{i}, Y_{O i}, T_{i}, s_{L i}, s_{L}\right)$ and individual household time demand functions $L_{i}\left(w_{i}, Y_{O i}, T_{i}, s_{L i}, s_{L}\right)$. In a labour supply regime the individual labour supplies, and hence the individual household time demands, are assumed to be immediately realized. Next, thus realized, the individual household time demands are assumed to affect, in the long run, the individual habit states according to the differential equations

$$
s_{L i}=\delta_{L i}\left[L_{i}\left(w_{i}, Y_{O i}, T_{i}, s_{L i}, s_{\bar{L}}\right)-s_{L i}\right] . \quad i=1, \ldots, N .
$$

Since these equations are to be combined with a differential equation for the social environment like (5.9a) in Section 5.2, they are specified in an analogous way and model the individual habit states as moving averages of current and past individual behaviour. These specifications differ from the psychological stock specifications (3.2a) in Section 3.1 by scaling factors $\delta_{L i}$ of the state variables (see footnote 20 in Sec. 5.2) and by the demand functions having the social environment $s_{L}^{-}$as additional arguments.

To be able to specify the differential equation for the social environment, we should aggregate the individual household time demand functions to an average demand function. Since, at least at kinks at the reservation wage, these individual demand functions are nonlinear, they can, in general, be exactly aggregated only to an average demand function $\bar{L}\left(\underline{w}, \underline{Y}_{O}, \underline{T}, \underline{s}_{L}, s_{L}\right)$ which depends on the entire vectors $\underline{w}, \underline{Y}_{O}, \underline{T}$ and $\underline{s}_{L}$ of the individual variables for $i=1, \ldots, N$. In a labour supply regime this average dernand affects the longrun evolution of the (expected or perceived) social environment $s_{\bar{L}}$ as motelled by the differential equation

$$
s_{\bar{L}}=\delta_{\bar{L}}\left[\bar{L}\left(\underline{w}, \underline{Y}_{O}, \underline{T}, \underline{s}_{L}, s_{\bar{L}}\right)-s_{\underline{L}}\right] .
$$

This equation is linked to the $N$ individual habit equations (6.1), and the latter equations are linked to each other via the former equation. Thus, we have a huge system of $N+1$ differen- 
tial equations in the individual habit states $s_{L i}, i=1, \ldots, N$, and the social environment $s_{\bar{L}}$. For constant $\underline{w}, \underline{Y}_{O}$ and $\underline{T}$ a long-run stationary equilibrium $\left(\underline{s}_{L}^{*}, s_{\bar{L}}^{*}\right)$ of this system is given by the equations $L_{i}\left(w_{i}, Y_{O i}, T_{i}, s_{L i}^{*}, s_{\bar{L}}^{*}\right)=s_{L i}^{*}, i=1, \ldots, N$, and $\bar{L}\left(\underline{w}, \underline{Y}_{O}, \underline{T}, \underline{s}_{L}^{*}, s_{\bar{L}}^{*}\right)=s_{\bar{L}}^{*}$. However, in general it is very difficult to derive necessary and sufficient conditions for stability of such an equilibrium like the stability conditions (3.3) and (3.5) in the case of IHF and (5.10) and (5.12) in the case of BPI (see App. 6A). For that purpose the system has to be simplified.

First, we can make the same assumption as Assumption $X$ in Section 5.2, namely that the vectors of absolute or relative deviations of the exogenous variables $w_{i}, Y_{O i}$ and $T_{i}$ from their averages $\bar{w}, \bar{Y}_{O}$ and $\bar{T}$ are constant. Then the vectors $\underline{w}, \underline{Y}_{O}$ and $\underline{T}$ in the average demand function in (6.2) can be replaced by $\bar{w}_{,} \bar{Y}_{O}$ and $\bar{T}$. However, an analogous assumption for the endogenous habit state vector $\underline{s}_{L}$ is dubious, since the individual habit state equations (6.1) generally imply not only an endogenous dynamics of the average habit state $\bar{s}_{L}$, but also an endogenous dynamics of the absolute or relative deviations of the individual habit states from this average habit state. As a result, these deviations do not generally remain constant at changes in the average exogenous variables. Therefore, the individual househoid time demand functions can not generally be aggregated to an average demand function $\bar{L}\left(\bar{w}, \bar{Y}_{O}, \bar{T}, \bar{s}_{L}, s_{\bar{L}}\right)$, so the differential system can not generally be simplified in this way.

Nevertheless, there are at least two moderately general cases in which a derivation of a simple necessary and sufficient stability condition is still possible. These cases are dealt with in the next two subsections.

\subsubsection{Case of one uniform habit slope}

Consider the case where the slopes ${L_{i}{ }_{s_{L i}}^{*}}$ of the individual demands as functions of the individual habit states at a long-run equilibrium point are the same for all members of the social group. At varying values of the exogenous variables this can hold for linear or piecewise-linear individual demand functions like $L_{i}^{A}, L_{i}^{B}$ and $L_{i}^{C^{\prime}}$ in Figure 6.1, provided either all individual equilibria $L_{i}^{*}=s_{L i}^{*}$ remain points in positively sloping segments of the demand functions, like the interior equilibria $A_{i}$ and $C_{i}$, or all individual equilibria remain corner points in flat segments of the demand functions, like $B$ and $A^{\prime}$. (These demand functions and equilibria are also indicated in Figs, 3.1 and 3.2.) In the former subcase all individual equilibria describe situations in which the restrictions $0 \leq H_{i} \leq T_{i}$ are not binding and the slopes of the individual demand functions have the same positive value. ${ }^{2}$ On the other hand, in the latter subcase all individual equilibria are restricted to zero or maximal corporate hours and the slopes of the individual demand functions are zero (so we

${ }^{2}$ Note that, in a limiting situation of this subcase, some individual equilibria may be comer points of positively sloping segments of the individual demand functions (see Fig. 4.1 in Sec. 4.2 for $w=w^{\bullet 1}$ and $w=w^{\cdot 2}$ ). The restrictions $0 \leq H_{i} \leq T_{i}$ are then just not binding. 
may have a combination of individual equilibria at zero corporate hours and individual equilibria at maximal corporate hours). ${ }^{3}$

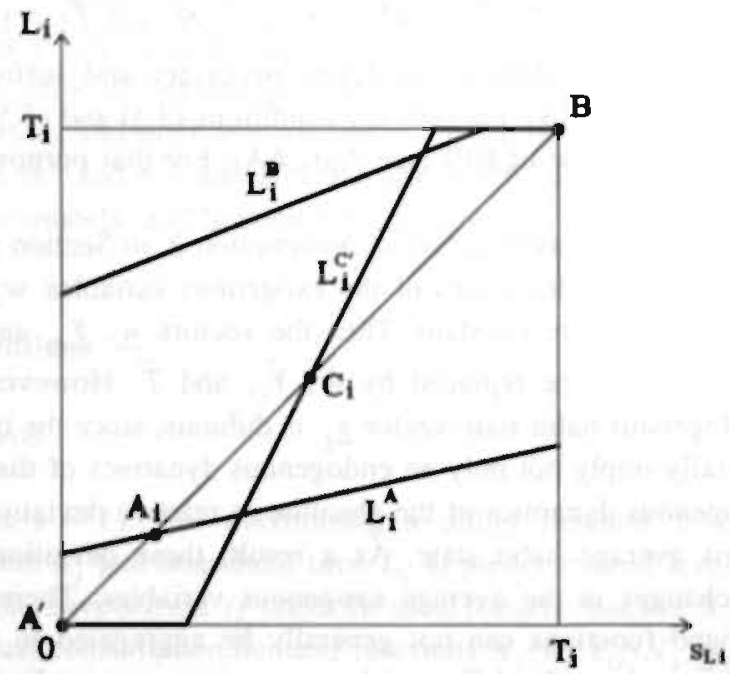

Figure 6.1 Linear and piecewise-linear individual household time demand functions of the individual habit state $L_{i}^{A}, L_{i}^{B}$ and $L_{i}^{C^{\prime}}$, implying individual long-run equilibria $A_{i}, B, C_{i}$ and $A^{\prime}$ (at given long-run equilibrium values of the social environmeni).

Examples of these subcases follow from the specification of the labour supply equations of Kapteyn and Woittiez (1990) and from the additive quadratic model (AQM) as analysed in the next section. For the case of disaggregated consumption demands, examples of the former subcase with only interior solutions are implied by the models of Gaerner (1974) and Pollak (1976a). Such subcases hold only for ranges of the exogenous variables $\bar{w}, \bar{Y}_{O}$ and $\bar{T}$ for which the individual long-run equilibria under consideration are either all points in positively sloping segments of the individual demand functions or all corner points in flat segments. For values of the vector $\left(\underline{s}_{L}, s_{\bar{L}}\right)$ in a sufficiently small neighborhood of the overall long-run equilibrium $\left(\underline{s}_{L}^{*}, s_{\vec{L}}^{*}\right)$, all group members are then in the same linear segment of their (piecewise-)linear demand functions with either all the same positive slope or all the same zero slope. ${ }^{4}$ Consequently, in these ranges of the exogenous variables and in this neighborhood of endogenous variables, the individual demand functions can be exactly aggregated to an average demand function $\bar{L}\left(\bar{w}, \bar{Y}_{O}, \bar{T}, \bar{s}_{L}, s_{L}\right)$ which is linear in the average

${ }^{3}$ Note that the restrictions do not prevent the individuals from working positive or less than maximal corporate hours, but exclude negative or higher than maximal corporate hours.

${ }^{a} \mathrm{~A}$ deviation of an individual habit state $s_{L i}$ from its long-run equilibrium value $s_{L i}$ implies a shift along the individual demand line of person $i$ (see Fig. 6.1). whereas a deviation of the social environment $s_{\bar{L}}^{-}$from its long-run equilibrium value $s_{\bar{L}}^{*}$ causes a shift of $i$ 's individual demand line. When both deviations are sufficiently small, the individual habit state $s_{L i}$ can be seen to remain in the same linear segment of $i$ 's individual demand line as its long-run equilibrium $s_{i}{ }_{i}$ at the long.nun equilibrium value of the social environment $s_{\dot{L}}^{*}$. 
habit state $\bar{s}_{L}$ with the same slope as the individual demand functions of $s_{L i}$. The differential equation (6.2) for the social environment $s_{L}^{-}$can then locally be simplified to

$$
s_{\bar{L}}=\delta_{\bar{L}}\left[\bar{L}\left(\bar{w}, \bar{Y}_{O}, \bar{T}, \bar{s}_{L}, s_{\bar{L}}\right)-s_{\bar{L}}\right]
$$

This equation has the same form as $(5.9 \mathrm{a})$ in Section 5.2 with the average habit state $\bar{s}_{L}$ instead of the social environment $s_{\bar{X}}$ as an argument of the average demand function.

The local evolution of the average habit state $\bar{s}_{L}$ follows from local aggregation of equation (6.1). Neglecting the effects of (co)variation in the individual adjustment parameter $\delta_{L i}$, we obtain

$$
\dot{s_{L}}=\bar{\delta}_{L}\left[\bar{L}\left(\bar{w}, \bar{Y}_{O}, \bar{T}, \bar{s}_{L}, s_{L}\right)-\bar{s}_{L}\right]
$$

This equation is similar in form to $(4.24)$ in Section 4.8 with the social environment $s_{L}^{-}$ instead of the individual habit vector $\underline{s}_{L}^{\prime}$ (and apart from a moving average instead of a psychological stock specification). ${ }^{5}$ According to $(6.3)$ and (6.4) both the social environment $s_{L}^{-}$and the average habit state $\bar{s}_{L}$ are adapted to the same average demand $\bar{L}$ with adjustment speeds $\delta_{L}$ and $\bar{\delta}_{L}$, respectively, which will generally differ. Thus, the system of $N+1$ differential equations has locally been reduced to a system of two differential equations for one social environment and one average habit state with respect to household time.

Chapter 8 will analyse a piecewise-linear model in which the concepts of social environment and average habit state coincide. Then the equations (6.3) and (6.4) are automatically identical with identical adjustment speeds $\delta_{\bar{L}}$ and $\bar{\delta}_{L}{ }^{6}$ This reduces the system of two differential equations to only one differential equation, which is similar in form to equation (5.9a) in Section 5.2 without $s_{\bar{X}}$ for pure BPI as well as to equation (4.24) in Section 4.8 without $s_{L}^{\prime}$ for pure IHF. This suggests that, under certain conditions, the dynamics of $s_{L} \equiv \bar{s}_{L}$ may be described by average household time demand functions of $s_{\bar{L}} \equiv \bar{s}_{L}$ which resemble those in Figure 5.2 or those in Figure 4.12 or combinations of those functions. In general, however, social environments and average habit states are different entities with different adjustment speeds. Figure 5.2 should then be extended to a three-dimensional picture in the $\left(s_{\bar{L}}, \bar{s}_{L}, L\right)$ space in which the stable and unstable equilibria are described as intersections of an equilibrium line $\bar{L}=s_{L}=\bar{s}_{L}$ and a two-dimensional surface of the average demand function $\bar{L}\left(s_{\bar{L}}, \bar{s}_{L}\right)$. Along the equilibrium line we may then have the same kind of situations as in (combinations of) Figures 5.2 and 4.12.

Similarly to the derivation of a necessary and sufficient stability condition for IHF with respect to both household time and consumption in Appendix $3 \mathrm{~A}$, it is easily shown that, for

5 The individual habit vector does not show up in (6.4), since (6.4) holds only for ranges of exogenous and endogenous variables in which all group members are in the same segment of their (piecewise-)linear demand functions. This implies that kinks, and hence correction terms as in (4.21) in Section 4.7, do not play a role in the aggregation.

${ }^{6}$ Identity of the social environment and average habit state also follows from the disaggregated linear difference equation models of Pollak (1976a) for consumption goods and of Kapteyn and Woittiez (1990) for labour supply. In particular, (approximately) aggregating the latter model implies $s_{L_{t}} \equiv \bar{s}_{L_{t}} \equiv \bar{L}_{t-1}$ with $\delta_{\bar{L}}=\bar{\delta}_{L}=1$ for both males and females. 
the present special case of IHF and BPI with respect to household time only, a necessary and sufficient stability condition is given by

$$
\bar{L} \dot{s_{L}}+\bar{L}_{s \bar{L}}<1
$$

(see also App. 6A). The "average slope" $\bar{L}_{\overline{s_{L}}}$ is equal to the uniform individual slope $L_{i_{s_{L i}}}$. In the subcase where all individual long-run equilibria are not bound by the restrictions $0 \leq H_{i} \leq T_{i}$, both this slope and the slope $L_{s_{L}}$ with respect to the social environment are supposed to be positive. Then the IHF and the BPI reinforce each other's effect. Accordingly, fulfilment of the stability condition (6.5) implies fulfilment of the stability condition $L_{i_{s_{L i}}}=\bar{L}_{\bar{s}_{L}}^{*}<1$ for IHF at given long-run equilibrium value $s_{\bar{L}}^{*}$ of the social environment as well as fulfilment of the stability condition $\bar{L}_{s_{L}}^{*}<1$ for BPI at given longrun equilibrium value $\bar{s}_{L}^{*}$ of the average habit state. ${ }^{7}$ Consequently, in the present case separate (conditional local) stability of IHF and BPI are necessary, but not sufficient conditions for stability of their combination. For the sub-subcase where the last stability holds, the mutual reinforcement of the effects of IHF and BPI is also found in the linear models of Pollak (1976a) and Kapteyn and Woittiez (1990).

When the combined effect of IHF and BPI is sufficiently strong to make the left-hand side of (6.5) larger than one and hence to violate (6.5), the equilibrium is unstable (like $C$ in Fig. 5.2). This can happen for IHF and BPI which may be separately unstable as weil as separately stable. Whatever be the case, the social environment and average habit state may then move to another equilibrium like $A$ and $B$ in Figure 5.2. However, when the social environment and average habit state move to a comer equilibrium like $B$ in Figure 5.2, the individual habit states all move to an individual corner equilibrium $\left(\bar{L}^{*}=\bar{T}\right.$ implies $L_{i}=T_{i}$ for all $i$. In all but accidental situations this can happen only when the individual demand functions of the individual habit states move towards a piecewise-linear function like $L_{i}^{B}$ or $L_{i}^{C^{\prime}}$ in Figure 6.1 due to the changing social environment. Thus, we end up in the subcase where all individual equilibria describe non-participation or employment at maximal hours in flat segments of the individual demand functions. All individual slopes $L_{i^{S} i}{ }^{*}$, and hence the "average slope" $\bar{L}_{\bar{s}_{L}}^{*}$ as well as the other slope in (6.5), are then zero, implying that (6.5) is always fulfilled. Thus, in this rather trivial subcase the long-run equilibrium of individual habit states and social environment is always stable.

On the other hand, when the average habit state and social environment nove towards an interior equilibrium like $A$ in Figure 5.2 , some individual habit states may move towards an interior individual equilibrium, but others may develop towards a corner individual equilibrium at maximal corporate hours in a flat segment of the individual demand function. Similarly, we may have an interior equilibrium $B$ at which most, but not all group members are non-participating in a flat segment of the individual demand function (see Fig. 5.4 in Subsec. 5.5.2). When the individual variation is not very weak or the BPI is not very strong, such a "mixed" equilibrium in which a part of the social group is restricted to zero corporate

${ }^{7}$ Since the given long-nun equilibrium values of the social environment and the average habit state can be considered as exogenous, they do not affect the forms of the stability conditions for separate IHF and separate BPI, respectively. 
hours and another part is not bound by the restrictions $0 \checkmark H_{i} \checkmark T_{i}$, is likely to exist for a broad range of the exogenous variables (e.g., for $w_{r l}^{*} \leq \bar{w}<\bar{w}^{* 1}$ in Fig. 5.3 in Sec. 5.4). In this case the zero slopes of the individual demand functions of the former subgroup are unequal to the positive slopes of the demand functions of the latter subgroup, so the above derivation of the stability condition (6.6) does not hold for this case. Nevertheless, if we make some simplifying assumptions, still a stability condition similar to (6.5) can be derived. This is shown in the next subsection.

\section{1 .3 Case of two uniform habit slopes}

Consider a mixed equilibrium in which a part of the social group is restricted to zero corporate hours and another part is not bound by the restrictions $0 \leq H_{i} \leq T_{i}$. Assume for the unrestricted subgroup a uniform habit slope of the individual demand functions and a uniform habit adjustment parameter $\delta_{L}$. Appendix $6 \mathrm{~A}$ proves that in this case a necessary and sufficient stability condition is given by

$$
L_{s_{L}}^{* u}+\bar{L}_{s_{\bar{L}}}^{*}<1 \text {. }
$$

where the first term denotes the uniform individual habit slope $L_{i_{s_{L i}}}{ }^{*}$ in the unrestricted subgroup. The second term, indicating the overall average social environment slope, is equal to $\left(N^{u *} / N\right) \overline{L_{s_{L}}^{*}}$, where $N^{u *}$ denotes the long-run equilibrium number of unrestricted group members and $\overline{L_{s}^{*}}$ * stands for the average positive social environment slope in the unrestricted subgroup in long-run equilibrium (see Sec. 5.3). Thus, in contrast with the habit slope, the social environment slope in (6.6) is suppressed by the factor $N^{u *} / N$. This is due to the stabilizing effect on the dynamics of the social environment of the impossibility for the non-participants to work less than zero hours, whereas the stable adjustment of the individual habit states of the restricted non-participants to their fixed long-run equilibrium household times $T_{i}$ does not affect the stability of the overall system. In the limiting case that the factor $N^{u *} / N$ is equal to one, i.e., all group members are unrestricted with the same positive habit slope, we have one of the two subcases which have been analysed above, and the stability condition (6.6) reduces to $(6.5) .^{8}$ More generally, we may have a mixed equilibrium in which one part of the social group is restricted to zero corporate hours, another part is not bound by the restrictions $0 \leq H_{i} \leq T_{i}$, and the remaining part is restricted to maximal corporate hours (see, e.g., Sec. 5.5). The necessary and sufficient stability condition (6.6) as derived in Appendix 6.2 applies to this case too.

In both cases the IHF and BPI reinforce each other's effect, but in Section 6.2 we will see that the IHF also counteracts the effect of the BPI by making the factor $N^{\| *} / N$ smaller as the IHF becomes stronger. Analogously to the case of (6.5), separate (conditional local) stability of the IHF of the unrestricted group members and separate stability of the BPI are

${ }^{8}$ In the stability analysis in Appendix 10E of Kapteyn and Woittiez (1990) it is implicitly assumed that all individuals are unrestricted. This is in contradiction with the data employed as described in Table 10F.2, according to which more than half of the females is non-employed. On the other hand, Kapteyn and Woittiez need only a sufficient stability condition and the condition (6.6) with $N^{u *} / N=1$ is sufficient for (6.6) with $N^{\mu *} / N<1$. 
necessary, but not sufficient conditions for stability of their combination. ${ }^{9}$ Again the combined effect of IHF and BPI may be so strong as to make the left-hand side of (6.6) larger than one, and hence to violate (6.6), implying that the equilibrium is unstable. This can happen for the following combinations of separately unstable or separately stable IHF and BPI.

First, the unrestricted habit slope $L_{s_{L}}^{* u}$ may be smaller than one, implying separately stable IHF. The social environment slope $\bar{L}_{s_{L}}^{*}$ should then be larger than $1-L_{s_{L}}^{* u}$, which is smaller than one. Hence, it is sufficient that the social environment slope is larger than one, implying separately unstable BPI. This, however, is not necessary: the social environment slope may also be smaller than one, so the BPI may be separately stable, provided it is sufficiently strong. As a generalization of the subcase of no IHF and unstable BPI, it can be argued that in both subcases of separately stable IHF and separately unstable or sufficiently strong BPI, the social environment and average habit state may move to one of two other equilibria like $A$ and $B$ in Figure 5.2 in Section 5.3. Section 6.2 gives an argumentation for an additive quadratic specification of the combined IHF/BPI model. Section 6.2 also demonstrates that for changing values of the exogenous variables collective catastrophes and hysteresis may occur as in Figure 5.3 in Section 5.4 and that collective catastrophes go together with the left-hand side of (6.6) becoming equal to one in the case of an internal unstable equilibrium as in Figure 5.3 and larger than (or equal to) one in the case of a corner unstable equilibrium. Moreover, the collective catastrophes and hysteresis imply individual catastrophes and hysteresis.

The left-hand side of the stability condition (6.6) is also larger than one, when the unrestricted habit slope $L_{s_{L}}^{* n}$ is larger than one, implying separately unstable IHF. This holds for separately stable as well as unstable BPI. As a generalization of the subcase of unstable IHF and no BPI, it can be shown that in the subcase of separately unstable IHF and separately stable BPI examples are given by equilibria like $A$ and $B$ in Figure 4.12 in Section 4.8. Then all individual habit states are polarized into one of two long-run comer equilibria 0 and $T_{i}$, but one or more of these individual equilibria are unstable as in Figure 4.1 in Section 4.2 for $w=w^{* 1}$ and $w=w^{* 2}$. The latter equilibria are not bound by the restrictions $0 \leq H_{i} \leq T_{i}$ and the corresponding unrestricted habit slope $L_{s_{L}}^{* \|}$ is larger than one. At a slight change in an exogenous variable the unstable individual equilibria may disappear as in Figure 4.1 for $w>w^{* 1}$ and $w<w^{* 2}$. implying individual catastrophes from maximal to zero household hours or the reverse. This happens for $w$ rising along the entry curve or declining along the exit curve in Figure 4.11 in Section 4.8 for the subcase of no BPI. Then the equilibrium on the entry or exit curve like $A$ or $B$ in Figures 4.11 and 4.12 moves inwards. More generally, this holds in the presence of BPI. In fact, for large $N$ the factor $N^{u *} / N$ is very small, so the social environment slope $\bar{L}_{s \bar{L}^{*}}^{*}$ which is equal to $\left(N^{u * / N}\right) \bar{L}_{s_{\bar{L}}^{*}}^{u *}$. is negligible. This implies that the BPI is separately "very stable", and it turns out to be a

${ }^{9}$ Note that the IHF's of the persons who ane restricted to zero or maximal corponate hours are always separately stable, since these persons are in flat pieces of their individual demand functions (see, e.g. $L_{i}^{C^{\prime}}$ in Fig. 6.1). 
consequence of the counteracting effect of the strong IHF in combination with individual variation.

In Section 5.6 we concluded from Figure 4.11 that in the AQM without BPI unstable IHF implies individual catastrophes, but, as a consequence of individual variation, never collective catastrophes, no matter how strong the IHF. Combined with the implication of separately "very stable" BPI, this suggests that collective catastrophes are unlikely in the presence of separately unstable IHF. However, Chapter 7 will demonstrate that under sufficiently strong social influencing the individual catastrophes can trigger subsequent individual catastrophes to such an extent that collective catastrophes arise. This is not reflected in the social environment slope in (6.6), since this slope holds for given long-run equilibrium values of the individual habit states, and hence does not take into account the discontinuous shifts in these values as a result of the individual catastrophes. The social environment slope can be adjusted for this effect and then a collective catastrophe turns out to go together with the adjusted slope becoming equal to one. ${ }^{10}$

Finally, analogously to the cases of IHF only and BPI only, the stability conditions (6.5) and (6.6) can be expressed in terms of second-order derivatives of the individual utility functions, the wage rate and other parameters and exogenous variables (cf. (3.5) in Sec. 3.3 and (5.12) in Sec. 5.3). Moreover, propositions which are analogous to Proposition 3.1 in Section 3.3 can be derived. This, however, is not elaborated here, but only some elements of it are used in the additive quadratic model of the next section.

\subsection{Additive quadratic model}

\subsection{Short-run labour supply}

This section analyses the combined effects of BPI and (separately) stable IHF with respect to household time for an additive quadratic specification of the individual utility functions. Thus, we assume that these utility functions are additive(ly separable) in individual consumption $X_{i}$ on the one hand and individual household time $L_{i}$, individual (household time) habits $s_{L i}$ and social (household time) environment $s_{L}^{-}$on the other hand. This subsection derives and aggregates individual short-run labour supply functions. The next two subsections analyse the two uniform habit slope cases which have been considered in the previous section. In the second case an interesting interaction between the exogenous individual variation and the IHF is investigated. Finally. Subsection 6.2.4 derives necessary and sufficient conditions for collective catastrophes and hysteresis to occur.

Transforming away terms which are independent of the choice variables $X_{i}$ and $L_{i}$ (see Sec. 4.1), the additive quadratic utility functions can be specified as

${ }^{10}$ However, this tangency condition should not be confused with the "degenerated" tangency situation between $A$ and $B$ in Figure 4.12. 


$$
\begin{aligned}
U^{i}\left(X_{i}, L_{i} ; s_{L i}, s_{L}\right)= & \alpha_{X i} X_{i}+\frac{1}{2} \alpha_{X X i} X_{i}{ }^{2}+\alpha_{L i} L_{i}+\frac{1}{2} \alpha_{L L i} L_{i}{ }^{2}+ \\
& +\gamma_{L L i} s_{L i} L_{i}+\gamma_{L \bar{L} i} s_{L} L_{i}
\end{aligned}
$$

with $\alpha_{X i}>0, \alpha_{X X i}<0, \alpha_{L i}>0, \alpha_{L L i}<0, \gamma_{L L i} \succeq 0$ and $\gamma_{L \overline{L i}} \succeq 0$. Analogously to the derivation in Section 4.1, maximization of these utility functions under the budget constraints leads to individual short-run labour supply functions

$$
H_{i}=\frac{a_{i} w_{i}-b_{i}}{c_{i} w_{i}^{2}+d_{i}},
$$

where

$$
\begin{aligned}
& a_{i}:=U_{X_{i}}^{i}\left(Y_{O i}\right)=\alpha_{X i}+\alpha_{X X i} Y_{O i}, \\
& b_{i}:=U_{L_{i}}^{i}\left(T_{i} ; s_{L i}, s_{L}^{-}\right)=\alpha_{L i}+\alpha_{L L i} T_{i}+\gamma_{L L i} s_{L i}+\gamma_{L \overline{L i}} s_{L}, \\
& c_{i}:=-U_{X_{i} X_{i}}^{i}=-\alpha_{X X i}>0, \\
& d_{i}:=-U_{L_{i} L_{i}}^{i}=-\alpha_{L L i}>0 .
\end{aligned}
$$

Of course, (6.8) should satisfy the constraints $0 \leq H_{i} \checkmark T_{i}$, so holds provided $0 \leq a_{i} w_{i}-b_{i} \checkmark$ $T_{i}\left(c_{i} w_{i}^{2}+d_{i}\right)$.

For the purpose of exact aggregation of the interior labour supply functions (6.8), we assume, just like in Subsection 5.5.1, individual variation in only the parameters $\alpha_{X i}, \alpha_{L i}$ and $\gamma_{L} \bar{L}_{i}$ and the variables $Y_{O i}$ and $T_{i}$. Analogously to (4.19) in Section 4.7 and (5.17), the average labour supply function can then again be written as

$$
\bar{H}=\frac{\bar{a} w-\bar{b}}{c w^{2}+d}+C T,
$$

where $C T$ denotes a sum of correction terms due to the $i$-dependent restrictions $0 \leq H_{i} \checkmark T_{i}$. Moreover, the average parameters $\bar{a}$ and $\bar{b}$ are given by

$$
\begin{aligned}
& \vec{a}=\bar{\alpha}_{X}+\alpha_{X X} \bar{Y}_{O}, \\
& \bar{b}=\bar{\alpha}_{L}+\alpha_{L L} \bar{T}+\gamma_{L L} \bar{s}_{L}+\bar{\gamma}_{L L} s_{\bar{L}} .
\end{aligned}
$$

Since the individual parameters $b_{i}$, and hence the individual household time demands $L_{i}=T_{i}-H_{i}$ are linear in $s_{L i}$ according to (6.9b) and (6.8), and since, moreover, the slopes $\gamma_{L L} /\left(c w^{2}+d\right)$ are the same for all group members, this model can describe the two cases of linear or piecewise-linear individual household time demand functions of the individual habit states as considered in the previous section. The next subsection will analyse the first case and the subsequent subsection the second case. 


\subsubsection{Case of one uniform habit slope}

In this case either all individual long-run equilibria $L_{i}^{*}=s_{L i}^{*}$ describe situations in which the restrictions $0 \leq H_{i} \checkmark T_{i}$ are not binding and the individual demand functions have the same positive slope, or all individual long-run equilibria are restricted to zero or maximal corporate hours and the individual demand functions have a zero slope. In the former subcase the sum of correction terms $C T$ in $(6.10)$ is zero. Therefore, since the parameter $\bar{b}$ is linear in the average habit state $\bar{s}_{L}$ and the social environment $s_{\vec{L}}$, the average short-run labour supply and hence the average short-run household time demand $\bar{L}=\bar{T}-\bar{H}$ are linear functions of $\vec{s}_{L}$ and $s_{L}$, which functions do not depend on the absolute or relative deviations of the individual habit states from $\bar{s}_{L}$. For the special case in which $\bar{s}_{L}$ and $s_{L}$ coincide, examples of such linear average household time demand functions of $\bar{s}_{L} \equiv s_{L}$ are given by $\bar{L}^{A}$ with slope smaller than one and the interior segment of $\bar{L}^{C^{\prime}}$ with slope larger than one in Figure 5.2 in Section 5.3. The slope being smaller or larger than one determines whether the interior equilibrium is stable or unstable, respectively, and is equal to the sum of the two slopes in the stability condition (6.5).

More generally, this stability condition holds for the case in which the average habit state and the social environment are different entities. In the present subcase, substituting (6.11a), (6.11b), (6.9c) and (6.9d) into the right-hand side of $(6.10)$ with $C T=0$ and differentiating this to $\bar{s}_{L}$ and $s_{\bar{L}}$, the stability condition for the interior equilibrium becomes

$$
\frac{\gamma_{L L}+\bar{\gamma}_{L \bar{L}}}{\left|\alpha_{X X}\right| w^{2}+\left|\alpha_{L L}\right|}<1 .
$$

This condition implies a perfect mutual reinforcement of the effects of IHF and BPI. In fact, despite the essentially different mechanisms of IHF and BPI, their aggregate effects are completely interchangeable in the present linear model in the sense that, for given values of $\gamma_{L L}$ or $\bar{\gamma}_{L L}$ in (6.11b) and of the adjustment parameter $\bar{\delta}_{L}$ or $\delta_{L}$ in (6.4) or (6.3), it does not make any difference for the short-run and long-run average labour supplies whether these supplies are determined by the dynamics of an average habit state $\bar{s}_{L}$ or by that of a social environment $s_{\bar{L}}$. Thus, the reinforcing effect on average labour supply of the household time habit formation of individual labour suppliers is qualitatively the same as the reinforcing effect on average labour supply of the mutual influencing of each other's household time preferences. $^{11}$

However, this conclusion holds only for the two subcases of either all individual long-run labour supplies being unrestricted or all individual long-nun labour supplies being restricted to zero or maximal corporate hours, and these subcases apply only if there is no individual variation (NIV) in parameters and exogenous variables or, in the case of individual variation (IV), for certain intervals of the wage rate (and the other exogenous variables). In the NIV (sub)case all individual labour suppliers behave identically in the short and long run, so average short and long-run labour supply functions are exact replicas of the corresponding

${ }^{11}$ Such a result is also found in the linear consumption model of Pollak (1976a). However, it does not hold for the individual supplies or demands, since the individual habit states generally vary over individuals, whereas the social environment does not. 
individual functions. Now not only the aggregate effects of IHF and BPI on average labour supply, but also their effects on the individual labour supplies as being identical to the average labour supply are completely interchangeable. As a consequence, the short and longrun labour supply schedules have the same shape as corresponding labour supply schedules for the AQM with IHF only as derived in Sections 4.1-4.4. In particular, we may have a long-run labour supply schedule as in Figure 4.2 with individual and collective catastrophes between zero and maximal corporate hours in which all group members are involved. Just as in the NIV case in Subsection 5.5.3, necessary and sufficient conditions for individual and collective catastrophes and hysteresis to occur are fully analogous to those for individual catastrophes and hysteresis in the case of IHF with respect to household time only as formulated under situation (i) in Proposition 4.1 in Section 4.4. We should only put $\gamma_{X X}=0$ and replace the IHF parameter $\gamma_{L L}$ by the sum of IHF and BPI parameters $\gamma_{L L}+\gamma_{L \bar{L}}$, so replace $c^{*}$ and $d^{*}$ by $\bar{c}^{*}:=\left|\alpha_{X X}\right|$ and $\bar{d}^{*}:=\left|\alpha_{L L}\right|-\gamma_{L L}-\gamma_{L \bar{L}}$, respectively. ${ }^{12}$ Accordingly, the parameters $\bar{d}_{-}^{*}$ and $\bar{d}_{*}^{*}$, which depend on $\bar{c}^{*}$, take the place of the parameters $d_{-}^{*}$ and $d_{+}^{*} .^{13}$

On the other hand, in the IV case matters are much more complicated. Then different individuals will generally have different long-run reservation wages or different entry and exit wages. This implies that the subcase in which all individual long-run labour supplies are uniestricted, can exist only for levels of the wage rate which are higher than or equal to the highest long-run reservation, entry and/or exit wage of all individuals. Similarly, the subcase in which all individual long-run labour supplies are restricted to zero or maximal corporate hours may hold only for wage rates which are lower than the lowest individual long-run reservation wage, entry and/or exit wage (when the restriction $H_{i} \leq T_{i}$ is not binding for any group member and any $w$, as in Figure 4.9 in Section 4.7 and, possibly, in Figure 5.3 in Section 5.4). Only for such ranges of the wage rate average labour supply schedules coincide with representative ones, and the Figures 4.9 and 4.11 suggest that these ranges may be very limited. More importantly, we are interested in what happens around reservation, entry and exit wages such as individual and collective catastrophes, and this brings us to the second case of mixed equilibria which has been analysed in the previous section.

\section{$6.2 .3^{*}$ Case of wo uniform habit slopes}

In this case a part of the social group is unrestricted with a uniform positive habit slope and another part is restricted to zero or maximal corporate hours with a uniform zero habit slope. Then the sum of correction terms $C T$ in (6.10) as describing the effects of the individual variation in the kinks of the individual demand functions, becomes non-zero and should be taken into account. As a consequence, the average short-run labour supply as expressed by (6.10), and hence the average short-run household time demand. is a function not only of the average variables $\bar{Y}_{O}, \bar{T}$ and $\bar{s}_{L}$, but also, via the non-zero $C T$, of the vectors of absolute or relative deviations of the individual variables $Y_{O i}, T_{i}$ and $s_{i i}$ from their averages. As

12 Note that the adjustment parameters $\delta_{L}$ and $\delta_{L}$ do not appear in the definition of $\bar{d}^{*}$ because of the moving average instead of psychological stock specifications of the habit states and social environment according to (6.1) and (6.2).

${ }^{13}$ Note that we use the same symbols as in the case of BPI only in Subsection 5.5.3. 
argued in the previous section, the deviation vectors of the exogenous variables $Y_{O i}$ and $T_{i}$ can be assumed to be constant, but an analogous assumption for the deviation vector of the endogenous habit states $s_{L i}$ is dubious. Therefore, we are in a more complex situation than above to which stability condition (6.6) applies. In the present case this condition becomes

$$
\frac{\gamma_{L L}+\frac{N^{u *}}{N} \overline{\gamma_{L \bar{L}}} u *}{\left|\alpha_{X X}\right| w^{2}+\left|\alpha_{L L}\right|}<1 .
$$

This condition differs from the simpler condition (6.12) by the replacement of the overall average susceptibility to social influence $\bar{\gamma}_{L L}$ by the factor $N^{u *} / N$ times the average susceptibility in the unrestricted subgroup $\overline{\gamma_{L L}} \bar{L}^{*}$. Note that the habit slope term does not have changed. The condition (6.14) can be rewritten as

$$
\gamma_{L L}+\frac{N^{u *}}{N}{\overline{\gamma_{L L}}}^{u *}<\left|\alpha_{L L}\right|+w^{2}\left|\alpha_{X X}\right|
$$

This condition says that the combined destabilizing effect of IHF and BPI as expressed by the habit rigidity $\gamma_{L L}$ and the average susceptibility to social influence $\overline{\gamma_{L} \vec{L}} \| *$, should be not so strong as to offset the stabilizing effects of diminishing marginal utilities and the wage rate as well as of the individual variation as expressed by the depressing factor $N^{\text {"**}} / N$ (cf. the condition (3.6) or (3.8) in Sec. 3.3 with $U_{X_{s_{X}}}=0$ and $\delta_{L}=1$ ). A sufficient condition for (6.14) to be fulfilled is given by

$$
\gamma_{L L}+\frac{N^{\mu *}}{N} \overline{\gamma_{L i}} u *<\left|\alpha_{L L}\right|
$$

As a generalization of weak IHF and weak BPI with respect to household time (see Secs. 3.3 and 5.3), this defines a (relatively) weak combination of IHF and BPI with respect to household time, taking into account the stabilizing effects of individual variation. Conversely, when the condition (6.15) does not hold, we have a (relatively) strong combination of IHF and BPI. Yet, from (6.14) it will be clear that this strong combination of IHF and BPI is not generally sufficient to make the equilibrium unstable, since then also the stabilizing effect $w^{2}\left|\alpha_{X X}\right|$ of the diminishing marginal utility of consumption should be compensated.

To obtain more insight in the stabilizing effects of individual variation, we should, just as in Subsection 5.5.2, express the factor $N^{\| *} / N$ in the stability condition (6.14) in terms of the density function(s) of the $i$-dependent parameters and exogenous variables. For the sake of simplicity we again consider the special case where only the household time taste parameter $\alpha_{L i}$ varies over the group members. Now, however, this exogenous individual variation induces also an endogenous individual variation in the individual habit states $s_{L, i}$, since the variation in $\alpha_{L i}$ leads to a variation in the individual household time demands, and hence to a variation in the individual habit states as they are adapted to these demands. Again only the parameter $b_{i}$ varies in (6.8). Considering $\alpha_{L i}, s_{L i}$, and hence $b_{i}$, for a large $N$ as continuous variables $\alpha_{L}, s_{L}$ and $b$, and defining $e:=b-\bar{b}$ with density function $f(\varepsilon)$ and zero mean, it follows from (6.9b) and (6.11b) that $\varepsilon=\left(\alpha_{L}-\bar{\alpha}_{L}\right)+\gamma_{L L}\left(s_{L}-\bar{s}_{L}\right)$. The factor $N^{u *} / N$ is then given by (5.19) in Subsection 5.5.2 except that the definitions of $a$ 
and $\bar{b} \cdot C$ are now different and $\varepsilon$ should be replaced by its long-run equilibrium value $\varepsilon^{* C}$, since $\boldsymbol{e}$ is now endogenous via $s_{L}$. Moreover, the distribution function of $\boldsymbol{e}^{* C}$ is then also endogenous and is denoted as $F^{\circ}$. Furthermore, (5.19) has been formulated for the intermediate long-run equilibrium $C$ in Figure 5.4, but, mutatis mutandis, the same expression holds for any long-run equilibrium. For the case of an intermediate equilibrium the present expression has the same kind of properties as those explained under (5.19).

On the other hand, now the variation in the long-run equilibrium values of the individual habit states $s_{L}$ amplifies the variance of $\varepsilon$ which is due to merely the variation in the household time taste $\alpha_{L}$, since the former variation is positively correlated to the latter variation. This can be seen as follows. A higher household time taste $\alpha_{L}$ implies, according to (6.9b), a higher $b$, and hence, by virtue of (6.8), a higher short-run reservation wage $b / a$ and above this reservation wage a lower labour supply $H$, so a higher household time demand $L$. In the longer run a higher $L$ leads to a higher value of the habit state $s_{L}$ as this is adapted to the higher $L$, and the higher habit state feeds, according to (6.9b) and (6.8), back on a lower $H$, so a higher $L$, etc.. Thus, we can conclude that in a long equilibrium persons with a higher household time taste will have a higher value of their habit state (i.e., be used to more household hours) above or at their long-run reservation wage, or at least have an equal value $(T)$ of their habit state below their long-run reservation wage. This implies a positive correlation of the household time taste $\alpha_{L}$ and the long-run equilibrium habit state $s_{L}^{* C}$, and hence a variance of $\varepsilon^{* C}=\left(\alpha_{L}-\bar{\alpha}_{L}\right)+\gamma_{L L}\left(s_{L}^{* C}-\bar{s}_{L}\right)$ which is larger than the variance of $\alpha_{L}$ by a long-run multiplier effect.

If the intermediate equilibrium $C$ does not deviate too much from the intermediate representative agent equilibrium (like $C^{\prime}$ in Fig. 5.4), it then follows that the IHF will further depress the slope of the average shor-run household time demand function. Moreover, the variance of $e^{* C}$ is larger, and hence the slope-depressing effect is stronger, as the habit sensitivity $\gamma_{L L}$ is higher. This has two reasons. First, in the short run a rise in $\gamma_{L L}$ implies an enlargement of the variance of $\varepsilon=\left(\alpha_{L}-\bar{\alpha}_{L}\right)+\gamma_{L L}\left(s_{L}-\bar{s}_{L}\right)$, since the second term in the last expression is directly proportional to $\gamma_{L L L}$. Secondly, this enlargement of the variance of $\varepsilon$ leads to a larger variance of the individual household time demands, and hence in the longer run to an enlargement of the variance of the individual habit states $s_{L}$, as they are adapted to these demands. In its turn, the last enlargement feeds back on the variance of $\varepsilon$, implying a long-run multiplier effect on the variance of $\varepsilon^{-C}$.

Thus, both a rise in the variance of the household time taste $\alpha_{L}$ and a rise in the level of the habit sensitivity $\gamma_{L L}$ have a positive long-run multiplier effect on the variance of $e^{* C}=\left(\alpha_{L}-\bar{\alpha}_{L}\right)+\gamma_{L L}\left(s_{L}^{* C}-\bar{s}_{L}\right)$, and hence on the variance of the long-run individual household time demand, via the variance of the long-run individual habit state $s_{L}^{* C}$. Moreover, either rise reinforces the multiplier effect of the other. In this way the IHF weakens the effect of BPI by amplifying the counteracting effect of individual variation. The intuition of this amplification is that, when persons start to behave differently from each other due to differences in personal characteristics, they tend to behave more "extremely", and hence more differently, as they get used to their behaviour.

On the other hand, the habit sensitivity $\gamma_{L L}$ directly raises the slope of the average household time demand function via the first term of the numerator on the left-hand side of (6.13). This implies a reinforcenent of the effect of the BPI. Thus, in the presence of individual variation, IHF has both a reinforcing and a weakening effect on the working of 
BPI. In general, the net result of these two effects is ambiguous. However, this ambiguity disappears, when the individual variation in household time taste and/or the IHF are sufficiently weak to make the variance of the long-run individual household time demands, and hence the variance of the long-run individual habit states (which are equal to the demands), small. Then the individual variation enlarging effect of $\mathrm{IHF}$ will be small and be dominated by the reinforcing effect of $\mathrm{IHF}$.

Whatever be the case, in order to imply an unstable overall equilibrium the combined effect of the BPI and stable IHF should be so strong as to make the left-hand side of (6.13) larger than or equal to one. This necessary and sufficient condition for instability is equivalent to the negation of (6.14). Fulfilment of this instability condition for an interior long-run equilibrium for a range of the wage rate $w$ is again a necessary condition for collective hysteresis and catastrophes like those in Figure 5.3 in Section 5.4 to occur. To be able to establish for which values of utility function parameters (and exogenous variables) this condition can be met, we should derive parameter conditions under which the negation of (6.14) has solutions for $w$ (cf. Table 4.1 in Sec. 4.3). However, as a consequence of the individual variation and in contrast to the conditions (3.7a) and (3.7b) defining weak IHF, (6.14) depends, in general, not only on $w$ via the factor $w^{2}$ on the right-hand side of (6.14), but also via the factor $N^{u *} / N$ and the averaging of $\gamma_{L \bar{L}}$ over the unrestricted subgroup on the left-hand side of (6.14). Therefore, to determine when precisely the negation of this condition has solutions for $w$, we should express the factor $N^{u *} / N$ and the average $\overline{\gamma_{L} \vec{L}} u *$ in $w$. However, even in the simplest case of exogenous individual variation in the household time taste only, the expression of the factor $N^{u *} / N$ in terms of $w$ as given by the modified (5.19), depends on the specific exogenous distribution of the household time taste and tends to be complex for usual specifications of the endogenous distribution function $F^{\bullet} C$. Consequently, just as in Subsection 5.5.2, a derivation of precise parameter conditions like those in Table 4.1 is bound to be restricted to very particular cases as well as laborious. In addition, instability of an interior equilibrium for a range of the wage rate $w$ is not a sufficient condition for collective hysteresis and catastrophes like those in Figure 5.3, so other conditions should also be derived.

All this makes a derivation of precise parameter conditions rather unaturactive. Fortunately, however, more qualitative, but also more general parameter conditions can again be obtained by following an alternative and less cumbersome approach. This approach is explained in the next subsection.

\section{$6.2 .4^{*}$ Conditions for collective catastrophes and hysteresis}

This subsection will derive necessary and sufficient conditions for collective catastrophes and hysteresis like those in Figure 5.3 in Section 5.4 to occur. These conditions are rather qualitative, but also rather generai in the sense of allowing individual variation in the parameters $\alpha_{X i}, \alpha_{L i}$ and $\gamma_{L L_{i}}$ and in the exogenous variables $Y_{O i}$ and $T_{i}$.

Just as in Subsection 5.5.3, we can consider the IV case of individual variation in which the slope-depressing factor $N^{\mu *} / N$ in (6.13) is smaller than one, as a perturbation of or deviation from the NIV case of no individual variation with $N^{\| *} / N=1$, which case has been discussed in the previous subsection. In the NIV case we have a simultaneous dynamics of only the average habit state $\bar{s}_{L}$ and the social environment $s_{\bar{L}}$, and for the sake of exposition we consider the special case in which $\bar{s}_{L}$ and $s_{\bar{L}}$ coincide. The dynamics, of 
$\bar{s}_{L} \equiv s_{L}$ is then described by $(6.3)=(6.4)$ with average household time demand functions. of $\bar{s}_{L} \equiv s_{\bar{L}}$ like the linear $\bar{L}^{A}$ in Figure 5.2 in Section 5.3 or the piecewise-linear $\bar{L}^{\prime}$ in Figure 5.4 .

On the other hand, the IV case implies a simultaneous dynamics of $\bar{s}_{L} \equiv s_{\bar{L}}^{-}$and a vector $\underline{d}_{s_{L}}:=\left(s_{L 1}-\bar{s}_{L}, \ldots, s_{L N-1}-\bar{s}_{L}\right)$ of absolute deviations of the individual habit states from their average (cf. eq. (4.24) in Sec. 4.8). Such a dynamics can be described by the following $N$ differential equations as straightforwardly derived from the system of first-order differential equations (6.1) and (6.2):

$$
\begin{aligned}
& \left.\dot{s}_{L}=\delta_{L}\left[\bar{L}_{(w,} \bar{Y}_{O}, \bar{T}_{,} \bar{s}_{L}, \underline{d}_{s_{L}}\right)-\bar{s}_{L}\right], \\
& \dot{d}_{s_{L}}=\delta_{L}\left[\underline{d}_{L}\left(w, \bar{Y}_{O}, \bar{T}, \bar{s}_{L}, \underline{d}_{s_{L}}\right)-\underline{d}_{s_{L}}\right] .
\end{aligned}
$$

Here $\underline{d}_{L}$ denotes a vector $\left(L_{1}-\bar{L}, \ldots, L_{N-1}-\bar{L}\right)$ of absolute deviations of the individual household time demands from their average. Thus, the dynamics of $\bar{s}_{L} \equiv s_{\bar{L}}$ and $\underline{d}_{s_{L}}$ is determined by the combination of an average household time demand function and $N-1$ individual household lime deviation functions of both $\bar{s}_{L}$ and $\underline{d}_{s_{L}}$. This implies a $2 N$. dimensional configuration, which is hard to visualize.

However, there is a way out. Given $w, \bar{Y}_{O}$ and $\bar{T}$, the first-order differential equations (6.16a) and (6.16b) imply, for each given vector of initial values $\left(\bar{s}_{L}^{0}, \underline{d}_{s_{L}}^{0}\right)$, a unique adjustment path in the $\left(\bar{s}_{L}, \underline{d}_{s_{L}}, \bar{L}_{,} \underline{d}_{L}\right)$ space towards a long-run equilibrium. As the given vector of initial values we could consider an infinitesimally small deviation from an unstable long-run equilibrium. The system will then follow a unique adjustment path to another equilibrium. ${ }^{14}$ We now consider the projection of such an adjustment path from the $2 \mathrm{~N}$ dimensional $\left(\bar{s}_{L}, \underline{d}_{s_{L}}, \bar{L}_{,} \underline{d}_{L}\right)$ space onto the two-dimensional $(\bar{s}, \bar{L})$ space. For sufficiently weak exogenous individual variation (EIV) in parameters and variables and/or for sufficiently weak IHF ${ }^{15}$. examples of such a projection could be given by the two segments of the curve of $\bar{L}^{C}$ in Figure 5.4 between $C$ and $B$ and between $C$ and $A$, as being perturbations of the corresponding segments of $\bar{L}^{C^{\prime}}$ in the NIV case. We can then say that the average habit state or social environment moves from its unstable equilibrium $C$ towards one of two other equilibria $\boldsymbol{A}$ and $\boldsymbol{B}$. More generally, such a situation can hold when the average habit state and social environment do not coincide.

${ }^{14}$ Note that if the vector of initial values would exactly coincide with the unstable equilibrium, the system would stay in that equilibrium. The unique adjustment path would then be a point.

is Following Assumption $X$ in Section 5.2, the vectors of absolute or relative deviations of the exogenous variables $Y_{O}$ and $T_{i} a^{*}$ from their averages have been assumed to be constant. These deviations are therefore implicitly incorporated in the average household time demand function and the individual household time deviation functions. In genera!, the individual variation enlarging effect of IHF, which has been demonstrated in the previous subsection for the case of EIV in household time taste only, holds also for EIV in other parameters and variables. 
Nevertheless, there are two important differences with the IV case of BPI only and no IHF as analysed in Chapter 5. First, analogously to the adjustment paths from $D$ and $E$ to $B$ in Figure 4.12 in Section 4.8 for the IV case of IHF only and no BPI, projected adjustment paths from initial points between $C$ and $B$ to $B$ and from initial points between $C$ and $A$ to $A$ in Figure 5.4 do not generally form a part of the corresponding projected adjustment paths from (infinitesimally small deviations of) $C$ on the curve of $\vec{L}^{-} \dot{C}$, but lie higher or lower. The positions of the former adjustment paths depend on the initial values $\underline{d}_{s_{L}}^{0}$ of the individual habit deviations.

Secondly, the projection of adjustment paths from the $\left(\bar{s}_{L}, \underline{d}_{s_{L}}, \bar{L}_{,} \underline{d}_{L}\right)$ space onto $\bar{L}^{C}$ implies that the deviation vector $\underline{d}_{s_{L}}$ in the original average household time demand function in (6.16a) becomes an implicit function of $\bar{s}_{L} \equiv s_{\bar{L}}$. Thus, $\bar{L}^{C}$ is a function of $\bar{s}_{\bar{L}} \equiv s_{\bar{L}}$ not only directly, but also indirectly via $\underline{d}_{s_{L}}$. This implies that the slope of $\bar{L}^{C}$ at $C$ is, in general, not simply obtained by multiplying the NIV slope in (6.12) by the depressing factor $N^{u} \% / N$ as in $(5.20)$. Moreover, due to the projection, the slope is generally neither equal to the value of the left-hand side of the IV stability condition (6.13) at $C$. On the other hand, what is preserved by the projection, is instability with slope larger than one, i.e., the slope of $\bar{L}^{C}$ at $C$ is larger than one if and only if the left-hand side of (6.13) at $C$ is larger than one.

This equivalence is especially useful when we consider what happens with the curve of $\bar{L}^{C}$ in Figure 5.4 when the wage rate changes. Analogously to the IV case of BPI only, the curve may then rise or fall until it becomes tangent to the equilibrium line at a certain $\tilde{w}^{* 1}$ or $\bar{w}^{* 2}$ and next loses contact with this line for $w$ beyond $\bar{w}^{* 1}$ or $\bar{w}^{* 2}$ (as in Fig. 3.2 in Sec. 3.5 for $w=w^{* 2}$ ). This induces collective catastrophes from $B=C$ to $A$ or from $A=C$ to $B$, and hence collective hysteresis as in Figure 5.3. In the tangency situation the slope of $\bar{L}^{C}$ at $C=B$ or $C=A$ is equal to one and, as a consequence of the equivalence mentioned above and a continuity argument, this will go together with the left-hand side of (6.13) being equal to one. In the case of weak EIV and/or weak IHF, and hence a small deviation of $\bar{L}^{C}$ from $\bar{L}^{C^{\prime}}$, it is also possible that the collective catastrophe from $B=C$ to $A$ occurs when $B$ is still a comer equilibrium (as in Fig. 3.2 for $w=w^{* 1}$ ). Then a same kind of reasoning as above implies that this will go together with the left-hand side of (6.13) being larger than one.

Analogously to Subsection 5.5.3, we can now construct a figure similar to Figure 5.5 and formulate a proposition like Proposition 5.1 on necessary and sufficient conditions for collective catastrophes and hysteresis to occur. Define $\bar{c}^{*}:=\left|\alpha_{X X}\right|$ and $\bar{d}^{*}:=\left|\alpha_{L L}\right|-\gamma_{L L}-\bar{\gamma}_{L \bar{L}}$. Then

Proposition 6.1 In a situation of sufficiently weak EIV andior sufficiently weak IHF, collective catastrophes and hysteresis in a forward-sloping part of the labour supply curve such as in Figure 5.3 occur for $0<\bar{c}^{*}<\bar{c}_{o}^{*}$ and $\bar{d}_{-}^{*}<\bar{d}^{*}<\bar{d}_{+}^{*}<0$, where $\bar{c}_{a}^{*}, \bar{d}_{-}^{*}$ and $\bar{d}_{+}^{*}$ are complex and unknown functions of the distributions of $\alpha_{X i}, \alpha_{L i}, \gamma_{L \overline{L i}}, Y_{O i}$ and $T_{i}$ and of $\alpha_{X X}, \alpha_{L L}$ and $\gamma_{L L}$, except that $\tilde{c}_{o}^{*}$ does not depend on $\alpha_{X X}$ and $\bar{\gamma}_{L \bar{L}}$, and $\bar{d}_{-}^{*}$ and $\ddot{d}_{+}^{*}$ not on $\bar{\gamma}_{L \bar{L}}$. Moreover, $\bar{c}_{o}^{*}$ is smaller than $c_{o}^{*}$ in the NIV case and $\bar{d}_{+}^{*}$ is more negative than 
$\bar{d}_{+}^{*}$ in the NIV case.

In this case of no IHF and BPI with respect to consumption the stability condition (6.14) implies that in order to allow collective catastrophes and hysteresis, the combination of IHF and BPI with respect to household time should be strong at the intermediate equilibrium, i.e. $\gamma_{L L}+\left(N^{u *} / N\right) \overline{\gamma_{L L}}{ }^{u *} \geq\left|\alpha_{L L}\right|$. According to Proposition 6.1 this holds in an interval of $w$ for $\bar{\gamma}_{L \bar{L}^{-}}>\left|\alpha_{L L}\right|-\gamma_{L L^{+}}\left|\bar{d}_{+}^{*}\right|$, and in addition we should have $\bar{\gamma}_{L \bar{L}}<\left|\alpha_{L L}\right|-\gamma_{L L^{+}}\left|\bar{d}_{-}^{*}\right|$. On the other hand, as the short-run tendency to consumption satiation $\bar{c}^{*}:=\left|\alpha_{X X}\right|$ increases, the interval for $\bar{d}^{*}$ narrows and the entry and exit wage line in Figure 5.5 approach each other until the interval vanishes and the two lines coincide into a point for the value $\bar{c}_{a}^{*}$ of $\bar{c}^{*}$. The cross-hatched surface in Figure 5.5 has then shrunk to zero. However, when at given sufficiently weak IHF, the EIV is not sufficiently weak, we have for all positive $\bar{c}^{*}$ a situation in which a graph like that of $\bar{L}^{\prime}$ in Figure 5.4 is transformed into a graph like that of $\bar{L}^{B}$, describing a stable equilibrium. In that case the surface in Figure 5.5 is already zero for a non-positive $c_{o}^{*}$.

On the other hand, when at given sufficiently weak EIV, the IHF is not sufficiently weak, the individual variation enlarging effect of IHF becomes important alongside the reinforcing effect of IHF. In particular, when the IHF is separately unstable at an intermediate equilibrium, all individual habit states can be shown to be polarized into one of two long-run corner equilibria 0 and $T_{i}$ (see the next chapter). At most some of these individual equilibria are not bound by the restrictions $0 \leq H_{i} \leq T_{i}$ as in Figure 4.1 in Section 4.2 for $w=w^{* 1}$ and $w=w^{* 2}$. For large $N$ this makes the slope-depressing factor $N^{k *} / N$, and hence the second term in the stability condition (6.14), negligibly small. As a consequence, this case deviates too much from the NIV case with $N^{\prime *} / N=1$ for Proposition 6.1 to apply to it, so the IHF in Proposition 6.1 should at least be separately stable. According to Section 3.3 a $w$-independent sufficient condition for stability demands that the separate IHF is (relatively) weak, i.e. $\gamma_{L L}-\left|\alpha_{L L}\right|<0^{16}$, but, dependent on the EIV, a sufficient condition on the IHF for Proposition 6.1 to apply may still be stronger. The resulting sufficient conditions for collective catastrophes and hysteresis to occur are given under Case II in Table 6.1 , where $\dot{d}_{i}^{*}$ depends on the EIV in an unknown way, but is expected to decline with increasing EIV and usually to be negative. Table 6.1 displays four relevant eases of combinations of, on the one hand, weak or strong (separate) IHF and, on the other hand, weaker or stronger BPI relative to EIV. Case I will be discussed below and Cases III and IV will be considered in Chapter 7.

${ }^{16}$ See foomote 7 and Section 4.3. The relative strength $\gamma_{L Z}-\left|\alpha_{L L}\right|$ of the IHF increases by a rise in the habit sensitivity $\gamma_{L L}$ as well as by a decline in the short-run rendency to household time satiafion $\left|\alpha_{L L}\right|$. In the tatter case the range $T\left(\kappa w^{2}+d\right) \equiv T\left(\left|\alpha_{X X}\right| w^{2}+\left|\alpha_{L L}\right|\right)$ of $\mathbf{e}^{\circ C}$ in the proportion of the analogue of $(5.19)$ for the present case decreases. while the variance of $\varepsilon$ remains the same. Again this is likely to imply a decrease in the proportion, i.e. in the slope-depressing factor $N^{u * C} / N$. 


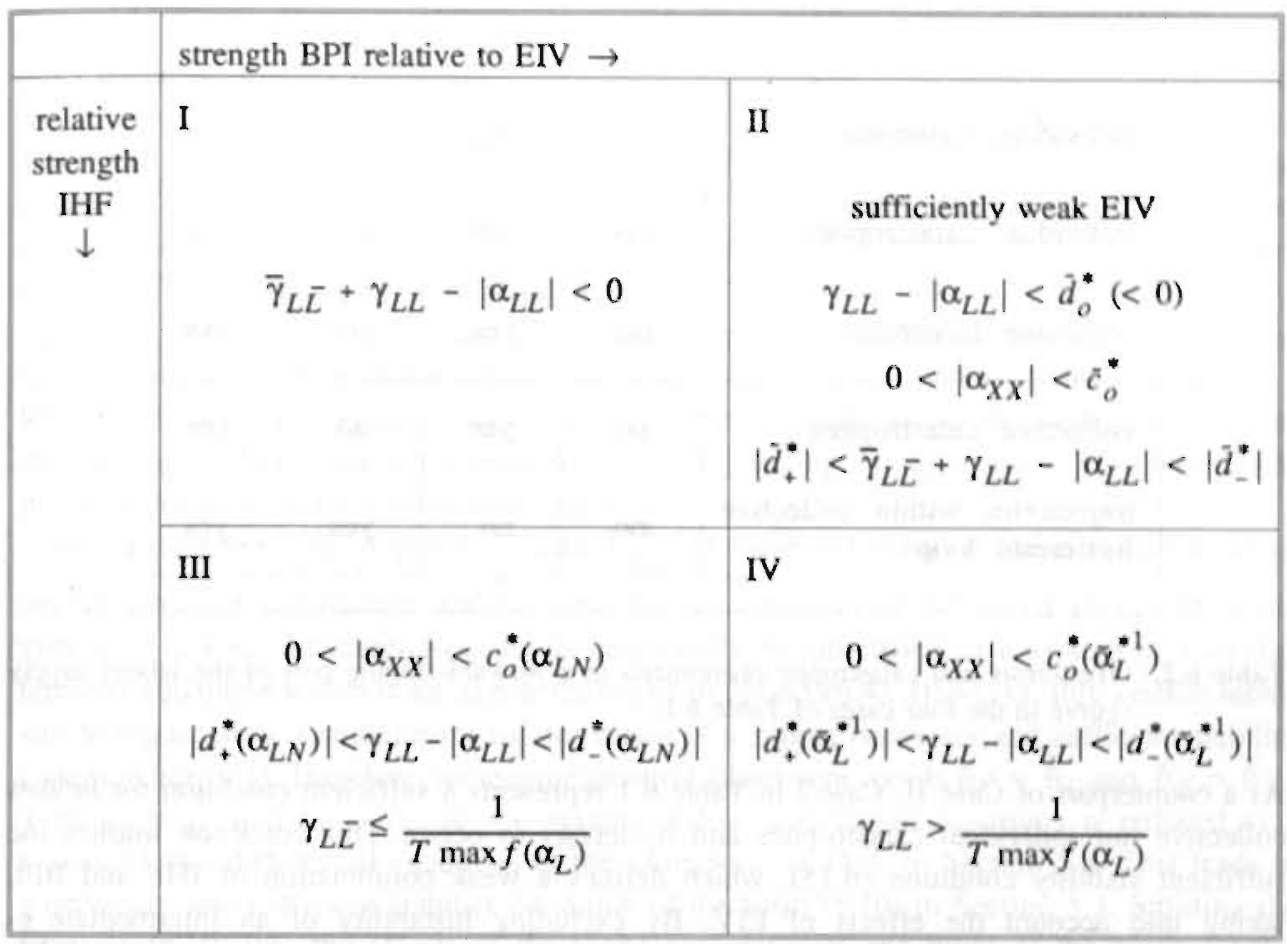

Table 6.1 Four cases of combinations of, on the one hand, weak or strong IHF and, on the other hand. weakei or stronger BPI relative to exogenous individual variation (EIV). In the Cases III and IV there is EIV in the household time taste $\alpha_{L}$ only.

In the present Case II a collective catastrophe again induces individual catastrophes and hysteresis, but in this case of separately stable IHF, a number of individual catastrophes will occur not between individual corner equilibria for zero and maximal corporate hours, but between zero hours and an interior equibrium for less than maximal corporate hours, or between two interior equilibria. Moreover, in contrast to the case of unstable IHF, there are no long-run trajectories within the collective hysteresis loop. The hysteresis and catastrophe phenomena in this Case II are summarized in Table 6.2, which summarizes also the hysteresis and catastrophe phenomena in the other three cases of Table 6.1 (see below). Analogously to Proposition 5.2, an additional property is suggested by catastrophe theory, but, just as in the case of BPI with respect to both household time and consumption, it is not implied by this theory, since the short-run average household time demand functions of the individual habit states and the social environment cannot be rationalized by an additive shortrun aggregate utility function as a consequence of the factor $N^{i} / N$ depending on both the individual habit states and the social environment (cf. Sec. 4.6). Therefore, an investigation of this case is again beyond the scope of this study. 


\begin{tabular}{|l|c|c|c|c|}
\hline & I & II & III & IV \\
\hline individual hysteresis & no & yes & yes & yes \\
\hline individual catastrophes & no & yes & yes & yes \\
\hline collective hysteresis & no & yes & yes & yes \\
\hline collective catastrophes & no & yes & no & yes \\
\hline $\begin{array}{l}\text { trajectories within collective } \\
\text { hysteresis loop }\end{array}$ & no & no & yes & yes \\
\hline
\end{tabular}

Table 6.2. Hysteresis and catastrophe phenomena in a forward-sloping part of the labour supply curve in the four cases of Table 6.1.

As a counterpart of Case II, Case I in Table 6.1 represents a sufficient condition for neither collective nor individual catastrophes and hysteresis to occur. This condition implies the sufficient stability condition (6.15), which defines a weak combination of IHF and BPI, taking into account the effects of EIV. By excluding instability of an intermediate equilibrium the latter condition also excludes collective catastrophes. Moreover, the condition in Table 6.1 implies $\gamma_{L L}-\left|\alpha_{L L}\right|<0$, so weak separate IHF, and this excludes instability of intermediate individual equilibria, and hence individual catastrophes. As a result, we then have neither individual nor collective hysteresis, and hence no long-run trajectories within a collective hysteresis loop. All this is summarized in Table 6.2. This Case I corresponds to the labour supply model of Kapteyn and Woittiez (1990) and the consumption demand models of Gaertner (1974) and Pollak (1976a) (who, however, use linear specifications other than the $A Q M$ ).

Both Case I and Case II hold for separately stable IHF. What happens for separately unstable IHF? Above we have seen that then, for large $N$, the second term in the stability condition (6.14) is negligibly small. However, the remaining inequality represents a necessary and sufficient stability condition for the separate IHF at unrestricted individual equilibria and is therefore violated in the case of separately unstable IHF. In particular, the few unrestricted individual comer equilibria as in Figure 4.1 in Section 4.2 for $w=w^{* 1}$ and $w=w^{* 2}$ are unstable, since the (uniform) unrestricted slope of the individual demand functions of the individual habit states is larger than one. At a slight change in an exogenous variable these unstable individual corner equilibria may disappear, as in Figure 4.1 for $w>w^{* 1}$ and $w<w^{* 2}$, implying individual catastrophes from maximal to zero household hours or the reverse. This implies individual and collective hysteresis, but generally no collective catastrophe. However, the next chapter demonstrates that, for sufficiently high values of the susceptibilities to social influence $\gamma_{L \bar{L}}$, the individual catastrophes can trigger subsequent individual catastrophes to such an extent that collective catastrophes arise. 


\subsection{BPI and stable IHF in consumption}

\subsubsection{General stability conditions}

When the general model of BPI and stable IHF with respect to household time as analysed in Section 6.1 is extended with BPI and stable IHF with respect to consumption, the system of $N+1$ differential equations (6.1) and (6.2) is augmented to a system of $2 N+2$ differential equations in the individual habit states $s_{H_{i}}, s_{X i}, i=1, \ldots, N$, and the social environments $s_{\bar{H}}$ and $s_{\bar{X}}$ for household time and consumption, respectively. Again, there are two moderately general cases in which a simple necessary and sufficient condition for stability of a long-run equilibrium of this system seems to exist. These cases can occur when the individual demand functions for household time and consumption are linear or piecewise-linear in the corresponding individual habit states.

In the first case the slopes $L_{i_{s_{L i}}}{ }^{*}$ and $X_{i_{s_{X i}}}{ }^{*}$ of these individual demand functions at an overall long-run equilibrium are the same for all members of the social group. Then the system of $2 N+2$ differential equations can locally be simplified to a system of four differential equations which is an augmentation of (6.3) and (6.4). However, this system seems still to big to allow a straightforward derivation of a simple necessary and sufficient stability condition like (6.5). Therefore, we assume identical adjustment speeds $\delta_{\bar{L}}=\bar{\delta}_{L}$ and $\delta_{\bar{X}}=\bar{\delta}_{X}$. Then $s_{\bar{L}} \equiv \bar{s}_{L}$ and $s_{\bar{X}} \equiv \bar{s}_{X}$, so the system of four differential equations is reduced to a system of two differential equations of the same form as (5.9) in Section 5.2. This leads to a necessary and sufficient stability condition of the form (5.10) in Section 5.3. Splitting the terms in (5.10) into the effects of the social environments and those of the average habit states, the stability condition becomes

$$
\bar{L}_{\overline{s_{L}}}^{*}+\bar{L}_{s_{\bar{L}}}^{*}+\bar{X}_{\overline{s_{X}}}^{*}+\bar{X}_{s_{\bar{X}}}^{*}<1 .
$$

This condition can be considered as an extension of stability condition (6.5), which has been derived for the general case that the social environment and average habit state with respect to household time are different entities with different adjustment speeds. A plausible conjecture then seems to be that $(6.17)$ is a necessary and sufficient stability condition also in the general case that the social environments and average habit states with respect to household time as well as consumption are different entities with different adjustment speeds. This, however, seems hard to be proven. Anyhow, an ensuing analysis which is analogous to that following (6.5) can be given.

This analysis leads to the second case in which a simple necessary and sufficient stability condition seems to exist. In this case we have an unrestricted subgroup with uniform positive habit slopes $L_{i_{s_{L i}}}^{*}$ and $X_{i_{s_{X i}}}^{*}$ alongside a restricted subgroup with zero habit slopes. Then, even when we assume identical adjustment speeds of the social environments and the corresponding average habit states, a derivation of a necessary and sufficient stability condition seems very difficult. Again, however, we can make a plausible conjecture, namely that the stability condition is a straightforward extension of (6.6) analogous to the extension (6.17) of (6.5), which is given by 


$$
L_{s_{L}}^{* u}+\bar{L}_{s_{\bar{L}}^{*}}^{*}+X_{s_{X}}^{* u}+\bar{X}_{s_{\bar{X}}}^{*}<1 .
$$

Here the first and third terms denote the uniform individual habit slopes in the unrestricted subgroup. Moreover, analogously to the second term, the fourth term, indicating the overall average social environment slope with respect to consumption, is equal to $\left(N^{u *} / N\right) \overline{X_{s_{\bar{X}}}^{*}} u *$, where $N^{u *}$ denotes the long-run equilibrium number of unrestricted group members and $\overline{X_{s}^{5}} u^{*}$ stands for the average positive social consumption environment slope in the unrestricted subgroup in long-run equilibrium. Again, taking for granted that (6.18) is indeed correct, an ensuing analysis which is analogous to that following (6.6) can be given.

\subsubsection{Additive quadratic model}

In order to obtain more insight in the implied dynamics of the social environments and average habit states, we can, just as in Section 6.2, resort to an additive quadratic specification of the individual utiity functions. Thus, we assume that the utility functions $U^{i}\left(X_{i}, L_{i} ; s_{X i}, s_{L i}, s_{\bar{X}}, s_{\bar{L}}\right)$ are quadratic as weli as additive(ly separabile) in $\left\{X_{i}, s_{X i}, s_{\bar{X}}\right\}$ and $\left\langle L_{i}, s_{L i}, s_{\bar{L}}\right\}$. In comparison with the specification (6.7) in Section 6.2 this implies two additional terms, namely $\gamma_{X X i} s_{X i} X_{i}+\gamma_{X} \bar{X} i s \bar{X} X_{i}$. Again, maximization of these utility functions under the budget constraints leads to individual utility functions of the form (6.8), but now $a_{i}$ is analogous to $b_{i}$ in $(6.9 \mathrm{~b})$. Next an analysis can be given which is analogous to that following (6.9d).

In the case of separately stable IHF with respect to both household time and consumption and individual variation in the parameters $\alpha_{X i}, \alpha_{L i}, \gamma_{X \bar{X} i}$ and $\gamma_{L \bar{L} i}$ and the variables $Y_{O i}$ and $T_{i}$, this analysis leads to the necessary and sufficient stability condition

$$
\frac{\left(\gamma_{X X}+\left(N^{u *} / N\right) \overline{\gamma_{X \bar{X}}}{ }^{* *}\right) w^{2}+\gamma_{L L}+\left(N^{u *} / N\right) \overline{\gamma_{L L}} u *}{\left|\alpha_{X X}\right| w^{2}+\left|\alpha_{L L}\right|}<1 .
$$

This condition ean be considered as an extension of the stability condition (5.20) in Subsection 5.5.2 as well as of the stability condition (6.13) in Subsection 6.2.3. Sufficient conditions for (6.19) to be fulfilled are given by (relatively) weak combinations of IHF and BPI with respect to household time and consumption, where the latter combination is defined analogously to the former according to (6.15) in Subsection 6.2.3.

On the one hand, by adding terms in (6.19) as compared with (6.13), the addition of collective habit formation with respect to consumption makes the system more easily unstable. On the other hand, this extension creates an additional suppressing effect on the factor $N^{u *} / N$ by endogenous individual variation in the individual consumption habit states alongside that in the individual household time habit states. The implications of these additional destabilizing and stabilizing effects are not elaborated here, but we can expect similar resuits as in the case of collective habit formation with respect to household time only. In particular, we can construct a similar figure as Figure 5.5 in Subsection 5.5 .3 and formulate a proposition like Proposition 5.1 and Proposition 6.1 on necessary and sufficient conditions for collective catastrophes and hysteresis to occur. Differences with Proposition 6.1 are that 
now $\bar{c}^{*}:=\left|\alpha_{X X}\right|-\gamma_{X X}-\bar{\gamma}_{X \bar{X}}$ and that the parameters $\bar{c}_{o}^{*}, \bar{d}_{-}^{*}$ and $\bar{d}_{+}^{*}$ also depend on $\gamma_{X X}$ and the distribution of $\gamma_{X \bar{X}}$. This leads to a case like Case II in Table 6.1 with probably weak separate IHF with respect to household time as well as consumption, displaying the same hysteresis and catastrophe phenomena in a forward-sloping part of the labour supply curve as summarized in Table 6.2. As a counterpart we then have Case I with the additional condition $\bar{\gamma}_{X \bar{X}}+\gamma_{X X}-\left|\alpha_{X X}\right|<0$. This condition implies a sufficient stability condition like (6.15), defining a weak combination of IHF and BPI with respect to consumption, taking into account the effects of individual variation. As a result, we then have neither individual nor collective hysteresis and catastrophe phenomena in a forward-sloping part of the labour supply curve, as summarized in Table 6.2.

Thus, adding BPI and stable IHF with respect to consumption leads to similar results as without this addition. On the other hand, analogously to the situations (ii) and (iii) in Proposition 4.1 in Section 4.4, collective catastrophes and hysteresis in a forward-sloping part of the labour supply curve as in Figure 5.3 in Section 5.4 seem also possible when the combinations of IHF and BPI with respect to consumption and household time are strong and weak, respectively, or both strong at an intermediate equilibrium. Moreover, the long-run labour supply curve is likely to be not backward-bending in these situations. However, a derivation of precise conditions for these situations is beyond the scope of this study.

\subsection{BPI and stable IHF in corporate time}

BPI and stable IHF with respect to corporate time $H$ can be added to the analysis by combining the procedures in Section 3.2 and at the end of Section 5.2. Consider individual utility functions $\hat{U}^{i}\left(X_{i}, L_{i}, H_{i} ; s_{X i}, s_{L i}, s_{H i}, s_{\bar{X}}, s_{\bar{L}}, s_{\bar{H}}\right)$, where $s_{H i}$ is the corporate time habit state of individual $i$ and $s_{\vec{H}}$ represents the social corporate time environment. Again making the simplifying assumptions that the adjustment rates $\delta_{H i}$ and $\delta_{\bar{H}}$ of this habit state and this social environment are (approximately) equal to the adjustment rates $\delta_{L i}$ and $\delta_{\bar{L}}$ of the household time habit state $s_{L i}$ and the social household time environment $s_{\bar{L}}^{-}$, respectively, it easily follows that $s_{H i}=T_{i}-s_{L i}$ and $s_{H}=\bar{T}-s_{L}$. Substituting these expressions and $H_{i}=T_{i}-L_{i}$ into the utility functions, we obtain reduced utility functions

$U^{i}\left(X_{i}, L_{i} ; s_{X i}, s_{L i}, s_{\bar{X}}, s_{L}\right)$. Their marginal utilities $U_{L_{i}}^{i}$ are again related to the marginal utilities $\hat{U}_{L_{i}}^{i}$ and $\hat{U}_{H_{i}}^{i}$ of the original utility functions as $U_{L_{i}}^{i}=\hat{U}_{L_{i}}^{i}-\hat{U}_{H_{i}}^{i}$, and may therefore be negative for sufficiently high $L_{i}$ and hence sufficiently low $H_{i}$. This may lead to volunteer work and may especially happen in the case of a social group of "collective workaholics" who are addicted to a high $H$ as a consequence of corporate time IHF as well as social control by the group. Such "collective workaholics" may even continue to work a high $H$, when their wage rate $w$ falls to zero (see footnotes 3 in Sec. 3.1 and 23 in Sec. 5.2).

A more detailed view of this extension can be obtained by considering a quadratic specification of the original utility functions $\hat{U}^{i}\left(X_{i}, L_{i}, H_{i} ; s_{X i}, s_{L i}, s_{H i}, s_{\bar{X}}, s_{\bar{L}}, s_{\bar{H}}\right)$ which is additive(ly separable) in $\left\{X_{i}, s_{X_{i}}, s_{\bar{X}}\right\},\left\{L_{i}, s_{L_{i}}, s_{\bar{L}}\right\}$ and $\left\{H_{i}, s_{H_{i}}, s_{H}-\right.$. Along the above lines and assuming $T_{i}=T$, this specification can be reduced to a specification of the same form as the extension of (6.7) with the two consumption terms (see the previous section), where 


$$
\begin{aligned}
\alpha_{X i}= & \hat{\alpha}_{X i}, \alpha_{X X i}=\alpha_{X X i}, \gamma_{X X i}=\hat{\gamma}_{X X i}, \gamma_{X \bar{X} i}=\hat{\gamma}_{X \bar{X} i} \text { and } \\
& \alpha_{L i}=\alpha_{L i}-\alpha_{H i}+\hat{d}_{\overline{H i}}^{*} T \quad \text { with } \quad \hat{d}_{\bar{H}}^{*}=\left|\alpha_{H H i}\right|-\hat{\gamma}_{H H i}-\hat{\gamma}_{H \bar{H} i} \\
& \alpha_{L L i}=\alpha_{L L i}+\alpha_{H H i}<0 \\
& \gamma_{L L i}=\hat{\gamma}_{L L i}+\gamma_{H H i} \geq 0 \\
& \gamma_{L \overline{L i}}=\gamma_{L \overline{L i}}+\hat{\gamma}_{H \bar{H} i} \geq 0 .
\end{aligned}
$$

The parameter $\hat{d}_{\bar{H}_{i}}^{*}$ in (6.20a) measures minus the (reiative) strength of the combination of corporate time IHF and BPI for individual $i$. When the "pure" corporate time taste $\hat{\alpha}_{H i}$ is positive and $\hat{d}_{\bar{H}}^{*}$ is negative, i.e., when the combined corporate time IHF and BPI are (relatively) strong for $i$, the "net" household time taste $\alpha_{L i}$ is smaller than the "pure" household time taste $\boldsymbol{Q}_{L i}$ and may even be negative. These possibilities imply interesting modifications of individual and average shor and long-run labour supply schedules and of diagrams like Figure 5.5 in Subsection 5.5.3. These modifications are similar to those discussed in Sections 4.5, 4.7 and 4.8 and at the end of Section 5.5, and are not elaborated here.

However, apart from rather extreme cases like those mentioned at the end of Section 5.5, Proposition 6.1 and Cases $I$ and II in Tables 6.1 and 6.2 as well as their extensions for consumption IHF and BPI continue to hold for the combinations of household and corporate time IHF and BPI instead of pure household time IHF and BPI.

\subsection{Concluding remarks}

This chapter has analysed labour supply models which combine the effects of BPI with those of separately stable IHF. As a preparation to that, a general stability analysis for the case of BPI and IHF with respect to household time only was given. For two moderately general subcases of this case simple necessary and sufficient (in)stability conditions were derived. It was argued that these conditions are the relevant ones only for the case of BPI and separately stable IHF. For that case, the implications of these stability conditions for the labour supply dynamics were investigated for an additive quadratic specification of the combined IHF/BPI (or CHF) model. It was shown that, in the presence of exogenous individual variation (EIV), the IHF not only reinforces the destabilizing effect of BPI, but also counteracts it by amplifying the stabilizing individual variation. Moreover, it was demonstrated that. when the net result of this complicated interaction between BPI. IHF and EIV implies instability of an interior long-run equilibrium, collective catastrophes and hysteresis may occur for changing values of the wage rate (as a generalization of the case of unstable BPI and no IHF as treated in the previous chapter). Necessary and sufficient conditions for the existence of such phenomena were formulated. Finally, the results were extended so as to account for the additional effects of BPI and separately stable IHF with respect to consumption as well as corporate time.

However, the interesting cases of BPI and separately anstable IHF are not covered by the analysis in this chapter. The reason is that, although these cases can also display collective 
catastrophes and hysteresis, the mechanism underlying these phenomena is different. This implies a modification of the (in)stability conditions and another kind of analysis than in this chapter. This analysis is presented in the next chapter.

\section{Appendix 6A Derivation of stability condition (6.6)}

Consider the system of $N+1$ differential equations (6.1) and (6.2) in the individual habit states $s_{L, i}, i=1, \ldots, N$, and the social environment $s_{\bar{L}}$. Linearizing this system around a long-run equilibrium $\left(s_{L 1}^{*}, \ldots, s_{L N}^{*}, s_{\bar{L}}^{*}\right)$, we obtain the system

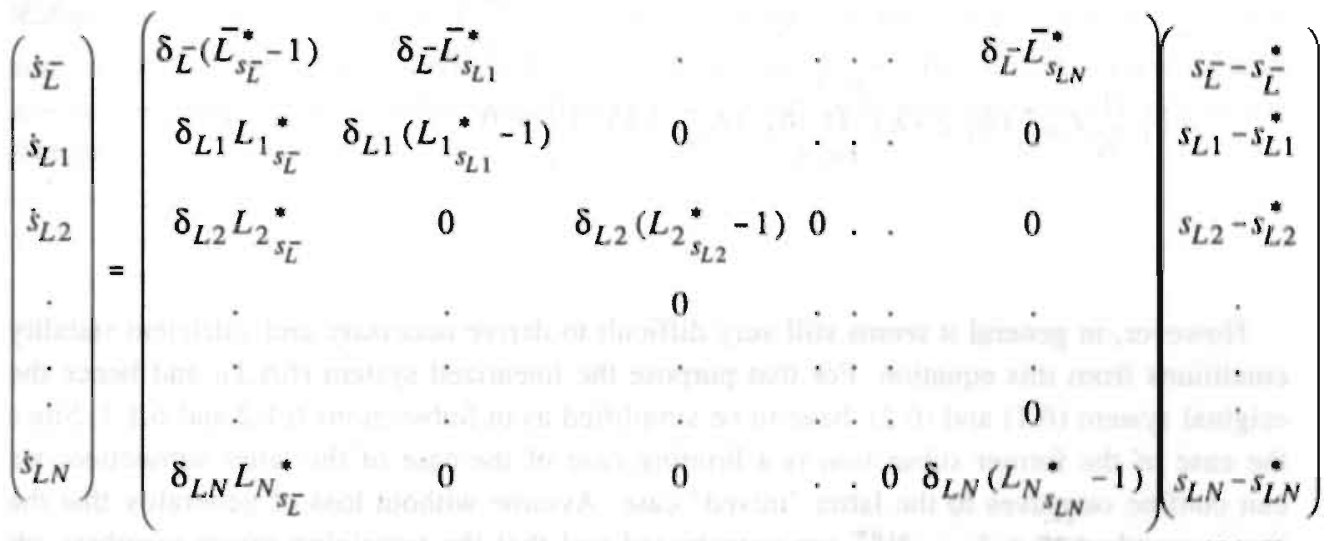

where we have reversed the order of (6.1) and (6.2) for convenience's sake and where we have omitted a constant vector term. A necessary and sufficient condition for local stability of the long-run equilibrium $\left(s_{L 1}^{*}, \ldots, s_{L N}^{*}, s_{\bar{L}}^{*}\right)$ is that the eigenvalues of the matrix in (6A.1) have negative real parts. Denoting this matrix as $A$, its eigenvalues follow from the equation

$$
\begin{aligned}
& \operatorname{det}(A-\lambda I)=\left[\delta_{L_{L}}-\left(\bar{L}_{s_{L}}^{*}-1\right)-\lambda\right] \prod_{i=1}^{N}\left[\delta_{L i}\left(L_{i_{s_{L i}}^{*}}-1\right)-\lambda\right]- \\
& -\delta_{L 1} L_{1_{s_{L}}}^{*} \delta_{\bar{L}} \frac{1}{N} L_{1_{s_{L 1}}}^{*} \prod_{i=2}^{N}\left[\delta_{L i}\left(L_{i_{s_{L i}}^{*}}^{*}-1\right)-\lambda\right]- \\
& -\delta_{L 2} L_{2}^{*} \delta_{s_{L}}^{*} \delta_{L} \frac{1}{N} L_{2}{ }_{s_{L 2}}^{*} \prod_{i \neq 2}^{N}\left[\delta_{L i}\left(L_{i_{s_{L i}}^{*}}^{*}-1\right)-\lambda\right]- \\
& -\delta_{L N} L_{N_{s_{L}}^{*}}^{*} \delta_{\bar{L}} \frac{1}{N} L_{N_{s_{L N}}^{*}} \prod_{i=1}^{N-1}\left[\delta_{L i}\left(L_{i_{s_{L i}}^{*}}^{*}-1\right)-\lambda\right]=0 .
\end{aligned}
$$

where we have substituted $\bar{L}_{s_{L i}}^{*}=(1 / N) L_{i_{s i}}^{*}, i=1, \ldots, N$. Rewriting the factors $\delta_{L i} L_{i_{s}{ }^{*}}^{*}$ 
in front of the last $N$ multiple products as $\delta_{L i}\left(L_{i_{s_{L i}}{ }^{*}}-1\right)-\lambda+\delta_{L i}+\lambda$ and rearranging terms, (6A.2) can be simplified to

$$
\begin{aligned}
& \left(\delta_{\bar{L}}+\lambda\right) \prod_{i=1}^{N}\left[\delta_{L i}\left(L_{i_{s_{L i}}^{*}}^{*}-1\right)-\lambda\right]+ \\
& +\delta_{\bar{L}} \frac{1}{N} L_{1_{s_{L}}}^{*}\left(\delta_{L 1}+\lambda\right) \prod_{i=2}^{N}\left[\delta_{L i}\left(L_{i_{s_{L i}}^{*}}^{*}-1\right)-\lambda\right]+ \\
& +\delta_{\bar{L}} \frac{1}{N} L_{2}^{*}\left(\delta_{s_{L}}^{*}+\lambda\right) \prod_{i \neq 2}^{N}\left[\delta_{L i}\left(L_{i_{s_{L i}}^{*}}^{*}-1\right)-\lambda\right]+ \\
& +\ldots . . . . . \\
& +\delta_{\bar{L}} \frac{1}{N} L_{N_{s_{L}}^{*}}^{*}\left(\delta_{L N}+\lambda\right) \prod_{i=1}^{N-1}\left[\delta_{L i}\left(L_{i_{s_{L i}}}^{*}-1\right)-\lambda\right]=0 .
\end{aligned}
$$

However, in general it seems still very difficult to derive necessary and sufficient stability conditions from this equation. For that purpose the linearized system (6A.1), and hence the original system (6.1) and (6.2), have to be simplified as in Subsections 6.1.2 and 6.1.3. Since the case of the former subsection is a limiting case of the case of the latter subsection, we can confine ourselves to the latter "mixed" case. Assume without loss of generality that the group members $i=1, \ldots, N^{u *}$ are unrestricted and that the remaining group members are bound by the restrictions $0<H_{i}<T_{i}$. Under the assumptions of Subsection 6.1.3 we then have $L_{i_{s_{L i}}^{*}}^{*}=L_{s_{L}}^{* u}>0$ and $L_{i_{s_{L}}}^{*}>0$ for $i=1, \ldots, N^{u *}, L_{i_{s_{L i}}^{*}}^{*}=0$ and $L_{i_{s_{L}}{ }^{*}}^{*}=0$ for $i=N^{u *}+1, \ldots, N$ and $\delta_{L i}=\delta_{L}$ for all $i$. Substituting these identities into (6A.3), we obtain the equation

$$
\left\{\left(\delta_{\bar{L}}+\lambda\right)\left[\delta_{L}\left(L_{s_{L}}^{* \prime \prime}-1\right)-\lambda\right]+\left(\delta_{L}+\lambda\right) \delta_{\bar{L}} \bar{L}_{s_{L}}^{\cdot}\right\}\left[\delta_{L}\left(L_{s_{L}}^{* \prime \prime}-1\right)-\lambda\right]^{N^{\mu *}-1}\left(-\delta_{L}-\lambda\right)=0 .
$$

The left-hand side of this equation consists of three factors, so the equation holds if and only if one of these three factors is zero.

The first factor being zero is equivalent to the equation $\lambda^{2}-b \lambda+c=0$ with

$$
\begin{aligned}
& b=\delta_{L}\left(\bar{L}_{s_{L}}^{*}-1\right), \\
& c=\delta_{\bar{L}} \delta_{L}\left(1-L_{s_{L}}^{* \prime \prime}-\bar{L}_{s_{\bar{L}}^{*}}^{*}\right) .
\end{aligned}
$$

The solutions for the eigenvalues can then be written as $\lambda_{1,2}=\frac{1}{2} b \pm \frac{1}{2} \sqrt{b^{2}-4 c}$. The discriminant $b^{2}-4 c$ can be positive, but can be also non-positive for positive $c$. In the former case the eigenvalues $\lambda_{1}$ and $\lambda_{2}$ are both real and must be negative in order to fulfil the stability condition. It is easily seen that this holds if and only if $b<0$ and $c>0$. In the case of a non-positive discriminant for $c>0$, the eigenvalues are both complex (imply- 
ing oscillations!) with the same real part $\frac{1}{2} b$. The stability condition says that this real part must be negative, so we end up with the same conditions $b<0$ and $c>0$ as in the case of a positive discriminant.

Since the adjustment parameters $\delta_{L}$ and $\delta_{L}$ are positive, these conditions are equivalent to $L_{s_{L}^{*}}^{-*}<1$ and $L_{s_{L}}^{* u}+\bar{L}_{s_{\bar{L}}}^{*}<1$, respectively. The former condition is implied by the latter, and the latter condition is the condition (6.6). In order to establish that this stability condition is not only necessary, but also sufficient, we should consider also the other eigenvalues of the matrix $A$ in (6A.1) as given by the second and third factor in (6A.4). The second factor being zero defines the real eigenvalue $\lambda_{3}=\delta_{L}\left(L_{s_{L}}^{* u}-1\right)$, and this eigenvalue is negative if and only if $L_{s_{L}}^{* u}<1$. This condition is again implied by (6.6). The thind term in (6A.4) being zero defines the real eigenvalue $\lambda_{4}=-\delta_{L}$ and this eigenvalue is always negative. Thus, stability condition (6.6) is indeed not only necessary, but also sufficient. 


\section{CHAPTER 7}

\section{Collective Habit Formation: BPI and Unstable IHF}

This chapter investigates what happens in the additive quadratic model (AQM) of the previous chapter in the case of BPI and (separately) unstable IHF. This case is considered as an extension of the AQM case of unstable IHF only and no BPI as analysed in Chapter 4. In the former just as in the latter case, the unstable IHF polarizes the individual habits into two long-run corner equilibria at zero and maximal corporate hours or into one of these equilibria (see Fig. 4.1 and footnote 8 in Sec. 4.2). This makes the situation resemblant to, but still essentially different from that in cases of BPI and dichotomous short-run decisions (see, e.g., Iannacone, 1989. App., for the case of BPI in dichotomous consumption demand). For our more complex model, this chapter will demonstrate that, at changing values of exogenous variables, collective catastrophes and hysteresis can arise as results of a sort of "two-stage rocket". In the first stage the unstable IHF induces individual catastrophes of some group members from zero to maximal corporate hours or the reverse. The second stage fires when, due to a sufficiently strong BPI, these individual catastrophes trigger subsequent individual catastrophes of other group members to such an extent that collective catastrophes arise. Then, for instance, a rocketing number of group members start to work a rocketing number of corporate hours.

Such collective catastrophes, however, are not described by the (in)stability conditions derived in Section 6.1, but by a modified (in)stability condition, which is derived in Section 7.1. The implied labour supply dynamics is analysed in the next sections. First considering unstuble IHF and BPI with respect to household time only. two subcases are distinguished, namely Case $A$ of individual variation only in household time taste and IHF strength and Case B of individual variation only in susceptibility to social influence. For Case A Section 7.2 derives a necessary and sufficient condition for the existence of a collective catastrophe and for Case B Section 7.3 demonstrates that a collective catasirophe already occurs under weak regularity conditions. For both subeases this leads to an interesting long-run average labour supply schedule with two domains of catastrophic long-run behaviour alongside a domain of persistent and hysteretic long-run behaviour. Section 7.4 shows how the necessary and sufficient conditions for (separately) unstable IHF in Case A depend on the collective catastrophe condition in that case. Seccions 7.5 and 7.6 add BPI and unstable IHF with respect to consumption and corporate time to the models. Finally. Section 7.7 concludes. 


\subsection{General analysis}

As a generalization of the AQM case of unstable IHF and no BPI as analysed in Chapter 4, separately unstable IHF at given long-run equilibrium value $s_{\bar{L}}^{*}=\vec{L}^{*}$ of the social household time environment (see under (6.5)) polarizes the individual habit states into two longrun corner equilibria at zero and maximal $\left(T_{i}\right)$ corporate hours or into one of these equilibria (see Fig. 4.1 in $\mathrm{Sec}_{.} 4.2$ ). Accordingly, at changing values of exogenous variables and at the given predetermined social environment $s_{\bar{L}}^{*}$, some group members may then engage into individual catastrophes from zero to maximal corporate hours or the reverse. As a result, the social household time environment will start to change somewhat, but since the implied BPI feedback on the supply of corporate hours is reinforcing (see Sec. 5.3), this will only reinforce the individual catastrophes. In addition, this reinforcing feedback will trigger subsequent individual catastrophes of other group members. Moreover, as will be shown in this chapter, when the social influencing is sufficiently strong, the reinforcing feedback can even lead to a rocketing number of group members engaging into individual catastrophes, i.e. to a collective catastrophe. We then have a "two-stage rocket" as described in the introduction of this chapter. Whereas the individual catastrophes of the first stage are connected with the habit slope term in the stability condition (6.6) in Subsection 6.1.3, the collective catastrophes of the second stage are not connected with the negligible social environment slope term in (6.6), since this slope holds for given long-run equilibrium values of the individual habit states, and hence does not take into account the discontinuous shifts in these values as a result of the individual catastrophes.

In order to adjust the social environment slope for this effect, we should make a clear distinction between stable individual long-run equilibria of the habit states ( 0 or $T_{i}$ corporate hours) at a given value of the social environment on the one hand and a long-run overall equilibrium of both the individual habit states and the social environment on the other hand. We define $N^{m}$ as the number of group members in stable individual equilibrium who prefer a maximal number of household hours and hence do not participate to the labour market at a given value of the social environment $s_{\bar{L}}$. When $s_{\bar{L}}$ changes by $d s_{\bar{L}}$ from a long-run equilibrium value $s_{\bar{L}}^{*}$, individual catastrophes from zero to maximal corporate hours and the reverse may arise (see Subsec. 6.1.3). As a result, the number of non-participating persons $N^{m}$ will, in the continuous approximation for large $N$, change by $N_{s}^{m *} d s_{\bar{L}}$. Hence, assuming a uniform maximal number of corporate hours $T$, the average household time $\bar{L}$ in long-run individual equilibrium will change by $\frac{1}{N} T N^{m *} s_{L} d s_{\bar{L}}$. This change should be added to the change according to the social environment slope in (6.6), but, for large $N$ and assuming that no employed person is on the backward-sloping part of her (or his) long-run labour supply curve and hence is unrestricted, the latter change is negligible (see Subsec. 6.1.3). Dividing by $d s_{\bar{L}}$, the adjusted social environment slope at $s_{\bar{L}}^{*}$ then becomes

$$
\bar{L}_{s_{\bar{L}}}^{*}=T \frac{N_{s_{\bar{L}}}^{m^{*}}}{N} \text {. }
$$

In this slope the individual habit states as having stable long-run equilibrium values 0 or $T$ have become implicit functions of the social environment. Thus, the stability problem has 
been reduced to a problem in terms of one state variable only, namely the social environment with respect to household time. Accordingly, the superscripts * in (7.1) denote evaluation of the derivatives at a long-run equilibrium value of this social environment, and a necessary and sufficient condition for stability of such an equilibrium is given by the slope in (7.1) being smaller than one. Similar stability conditions are found in the literature on BPI in dichotomous consumption demand (see, e.g., Granovetter and Soong, 1986, and lannacone, 1989).

Moreover, analogously to the case of BPI only in Sections 5.4 and 5.5, it can be shown (for particular cases) that the collective catastrophes of the second stage of the two-stage rocket go together with the slope in (7.1) becoming equal to one. However, before this can be shown, we should first demonstrate how collective catastrophes can arise in the present case of separately unstable IHF in combination with BPI. Therefore, we consider this case as an extension of the aggregate case of unstable IHF only and no BPI, which has been analysed in Section 4.8. There we directly aggregated the individual long-run labour supply schedules to an average long-run labour supply schedule with entry and exit curves as displayed in Figure 4.11.

What happens with such an aggregate schedule when BPI comes into play? In answering this question we first regard a general situation in which all parameters and variables except the wage rate $w$ and the total discretionary time $T$ (and, of course, the social household time environment $s_{\bar{L}}$ ) vary over individuals. In the case of a considerable BPI the term $\gamma_{L \bar{L} i} s_{\bar{L}}$ in $(6.9 \mathrm{~b})$ in Section 6.2 becomes important. Assuming that both $a_{i}$ and $b_{i}$ are positive, this affects the individual short-run reservation wages $w_{r i}=b_{i} / a_{i}$. On the other hand, this social influence is exogenous to an individual group member in the short as well as long run (see Assumption IX in Sec. 5.2). Therefore, in order to analyse the long-run effects of BPI in the case of separately unstable IHF, we can start from the individual longrun entry and exit wages in that case at given long-run equilibrium values $s_{\bar{L}}^{*}=\bar{L}^{*}$ of the (expected) social environment. In this way the first stage of the two-stage rocket is incorporated into the second stage.

The individual long-run entry wage $w_{i}^{* 1}$ coincides with the short-run reservation wage $w_{r i}=b_{i} / a_{i}$ of person $i$ when he has fully got used to non-participation or maximal household time $\left(s_{L i}=T\right)$. Therefore, an expression for the entry wage $w_{i}^{* 1}$ at given long-run equilibrium value of the social environment is obtained by substituting $s_{L i}=T$ and $s_{\bar{L}}=\bar{L}^{*}$ into (6.9b) and substituting (6.9a) and (6.9b) into $w_{r i}=b_{i} / a_{i}$. This results in

$$
w_{i}^{* 1}=\frac{\alpha_{L i}+\left(\gamma_{L L i}-\left|\alpha_{L L i}\right|\right) T+\gamma_{L \overline{L i}} \bar{L}^{*}}{\alpha_{X i}-\left|\alpha_{X X i}\right| Y_{O i}} .
$$

Now the crucial point is that each person's entry wage depends positively on the long-run average household time $\bar{L}^{*}$ in her group and that $\bar{L}^{*}$ is equal to $T$ times the proportion $N^{m *} / N$ of group members who have not yet entered the labour market (assuming that no employed person is on the backward-sloping pan of her long-run labour supply curve, the long-run household time of all the employed persons is zero). When most group members are still at home, the average household time $\bar{L}^{*}$ is large, so the entry wages of persons who would enter the labour market in the absence of BPI is substantially increased. In other 
words, these persons are deterred from entering the labour market by most members of their social group staying at home. On the other hand, as the wage rate rises and more people enter, the long-run average household time in the group decreases, and hence the entry wages decline. Thus, whereas forerunners enter the labour market at much higher entry wages than without BPI due to the social influence of the stay-behinds, the latter enter at lower entry wages than without predecessors due to the social influence of these predecessors. As a consequence, the variance of the entry wages over the group members decreases.

A similar story can be told on the long-run exit wages $w_{i}^{* 2}$. These are given by (4.13) for $w_{-}^{*}$ in Section 4.3 with the household time taste parameter $\alpha_{L}$ being replaced by the social(ly influenced) household time taste $\alpha_{L i}^{*}:=\alpha_{L i}+\gamma_{L \bar{L}} \bar{L}^{*}$ and with $c^{*}=\left|\alpha_{X X i}\right|$ and $a^{*}=\alpha_{X i}-\left|\alpha_{X X i}\right| Y_{O i}$. For the sake of exposition we consider a first-order approximation of this formula for $4 \alpha_{L i}^{*} T\left|\alpha_{X X i}\right| \ll a_{i}^{* 2}$ which is given by

$$
w_{i}^{* 2} \approx \frac{\alpha_{L i}+\gamma_{L \overline{L i}} \bar{L}^{*}}{\alpha_{X i}-\left|\alpha_{X X i}\right| Y_{O i}}
$$

(see Appendix 7A). Note that this expression differs only from the formula for the long-run entry wages in (7.2) by the positive habit formation minus satiation tendency term $\left(\gamma_{L L i}-\left|\alpha_{L L i}\right|\right) T$. In fact, the expression represents the short-run reservation wage $w_{r i}=b_{i} / a_{i}$ of person $i$ when he has fully got used to maximal employment or zero household time $\left(s_{L i}=0\right)$. This short-run reservation wage is lower than the long-run exit wage. ${ }^{1}$ When $w$ falls below it, the person will leave the labour market directly (i.e. not in stages as in the case of a decline of $w$ below the long-run exit wage, but above the short-run reservation wage). In an analogous way as the distribution of the long-run entry wages, the distribution of these reservation wages, or, approximately, long-run exit wages, will be compressed by the BPI.

When the social influence as geared by the susceptibility to social influence $\gamma_{L \bar{L}}$ is sufficiently strong, it may even decrease the variance in the entry or exit wages of a number of group members to zero. This results in a collective catastrophe at a certain entry or exit wage. Then the individual variation is, at least for a part of the social group, compensated by the social influence process. This phenomenon is derived below for the particular case of exogenous individual variation only in the household time taste $\alpha_{L i}$ and the (relative) IHF strength $\gamma_{L L i}-\left|\alpha_{L L i}\right|$ (Case A) and for the case of exogenous individual variation only in the susceptibility to social influence $\gamma_{L \bar{L}}$ (Case B). The phenomenon has implications for the long-run average labour supply schedules in these two cases which are also derived. We assume that the IHF is such that each group member goes through individual entry and exit catastrophes as described by Figure 4.2 in Section 4.2 . In Section 4.4 we will come back to this assumption.

${ }^{1}$ This reservation wage corresponds to the intercept $\alpha_{L} / a^{*}$ of the long-run reservation wage $w_{r}^{*}$ as a function of $d^{*}$ in Figure $4.4 \mathrm{c}$ in Section 4.4. This intercept is smaller than the long-run exit wage $w^{* 2}=w_{-}^{*}$ for $d^{*}<d_{*}^{*}$. 


\subsection{Case A: variation in household time taste and IHF strength}

First, we consider exogenous individual variation in the positive household time taste $\alpha_{L i}$ only. We regard $\alpha_{L i}$ as a continuous variable $\alpha_{L}$ with density function $f\left(\alpha_{L}\right)$. What happens with long-run average labour supply when the wage rate is rising from zero? Since the susceptibility to social influence $\gamma_{L L}$ is never negative, the long-run exit wages $w_{i}^{* 2}$ according to (7.3), and hence the long-run entry wages $w_{i}^{* 1}$ according to (7.2), are positive, implying that at $w=0$ all group members are, in the long run, always non-participating and (fully) collectively used to that. ${ }^{2}$ Then the long-run average household time $\bar{L}^{*}$ is equal to $T$, so the long-run entry wage $w_{1}^{* 1}$ of the first entrant at $w$ rising from zero has the positive numerator min $\alpha_{L i}+\left(\gamma_{L L}-\left|\alpha_{L L}\right|\right) T+\gamma_{L L} T$. Thus, at rising $w$, nobody will enter the labour market for $w$ below $w_{1}^{* 1}$ due to positive minimal household time tastes (the first term of the numerator), habit sensitivity (the second term) and social influence by the other group members (the third term). This is indicated by the long-run average labour supply schedule in Figure 7.1.

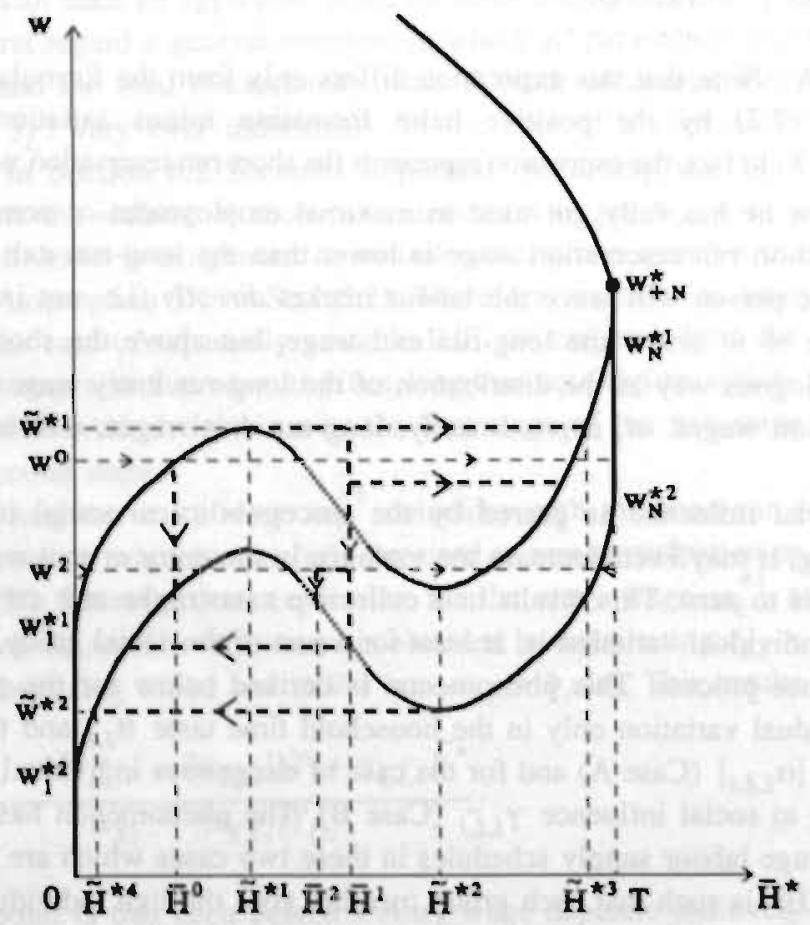

Figure 7.1 Long-run average labour supply schedule for AQM in Case A of exogenous individual variation in household time taste only.

${ }^{2}$ By "(fully) collectively used" we mean that both the individual habit states and the expected or perceived social environment have been fully adapted to their long-run equilibrium values. 
However, as $w$ rises beyond $w_{1}{ }^{* 1}$, forerunners will enter and work, in the long run, the maximal number of corporate hours. First persons with low household time tastes will enter and next persons with higher household time tastes. This leads to increases in the long-run average labour supply, and hence to decreases in the long-run average household time $\bar{L}^{*}$. and this lowers the entry wages of the stay-behinds. The long-run average household time, or $T$ times the proportion $N^{m *} / N$ of stay-behinds, is equal to $T$ times the proportion of the social group for which the household time taste $\alpha_{L}$ is higher than the household time taste $\alpha_{L i}$ of the last or marginal entrant(s). This proportion can be written as $1-F\left(\alpha_{L i}\right)$, where $F\left(\alpha_{L}\right)$ is the distribution function of $\alpha_{L}$. Then, as $\alpha_{L i}$ rises by an infinitesimally small amount $d \alpha_{L i}$, the long-run average household time $\bar{L}^{*}$ declines by $T F_{\alpha_{L}}\left(\alpha_{L i}\right) d \alpha_{L i}=T f\left(\alpha_{L i}\right) d \alpha_{L i}$, so the total change in the long-run entry wage $w_{i}^{* 1}$ as given by (7.2) is positively proportional to $\left[1-\gamma_{L L}-T f\left(\alpha_{L i}\right)\right] d \alpha_{L i}$.

This change becomes negative when $\gamma_{L L} T f\left(\alpha_{L i}\right)$ becomes larger than one, so when the product of the susceptibility to social influence $\gamma_{L \bar{L}}$ and the proportion of marginal entrants $f\left(\alpha_{L i}\right)$ is sufficiently large. This implies that the long-run entry wage of the next entrants is lower than that of their predecessors as a consequence of the social influence of these predecessors. In Figure 7.1 this happens at $w=\bar{w}^{* 1}$ and $\bar{H}^{*}=\bar{H}^{* 1}$. For the case where the density function $f\left(\alpha_{L}\right)$ is continuously differentiable and unimodal, the long-run entry wage of the marginal entrants as a function of the average long-run labour supply $\bar{H}^{*}$ just before their entrance is given by the upper sharply sigmoid curve in Figure $7.1 .^{3}$ This function behaves qualitatively in the same way as the long-run entry wage $w_{i}^{* 1}$ of the marginal entrant(s) as a function of their household time taste $\alpha_{L i}$ as considered above, since $\bar{H}^{*}=T-\bar{L}^{*}$ is a monotonously increasing function of $\alpha_{L i}$. The condition under which these two functions start to decline at $w=\bar{w}^{* 1}$, can be reformulated as saying that the value $f\left(\alpha_{L i}\right)$ of the density function at the household time taste of the marginal entrant(s) becomes larger than $1 /\left(\gamma_{L L}-T\right)$. According to Figure 7.2 this happens at the "marginal household time taste" $\alpha_{L}^{1}$.

From this figure it also follows that the "entry wage function" in Figure 7.1 will again start to rise at a marginal household time taste $\alpha_{L}^{2}$, or an average labour supply $\hat{H}^{* 2}$, for which $f\left(\alpha_{L i}\right)$ becomes again smaller than $1 /\left(\gamma_{L L}-T\right)$. The entry wage function then rises until at $\tilde{H}^{* 3}$ the same level is reached as at $\tilde{H}^{* 1}$. Appendix $7 \mathrm{~B}$ shows that this corresponds to a marginal household time taste $\alpha_{L}^{3}$ in Figure 7.2 for which the surface area under the $1 /\left(\gamma_{L L}-T\right)$ line between $\alpha_{L}^{1}$ and $\alpha_{L}^{3}$ is equal to the surface area under the curve of the density function between $\alpha_{L}^{1}$ and $\alpha_{L}^{3}$. (The surface areas $S_{1}$ and $S_{2}$ are then equal to each other.) This surface area indicates the proportion of group members whose long-run entry wages are lower than the entry wage $\bar{w}^{* 1}$ of predecessors and who are therefore involved in a collective catastrophe from $\tilde{H}^{* 1}$ to $\tilde{H}^{\star 3}$ at that wage. We call these group members conformists and all their predecessors trendsetters. Following physics, the proportion of

${ }^{3}$ Thus, in contrast to the entry curve in Figure 4.11 in Section 4.8, this curve describes not only (stable and unstable) $\bar{H}^{*}$ as a function of the wage rate, but also, because of the feedback of $\bar{H}^{*}$ on the entry wages, the entry wage of marginal entrants as a function of $\bar{H}^{*}$. 


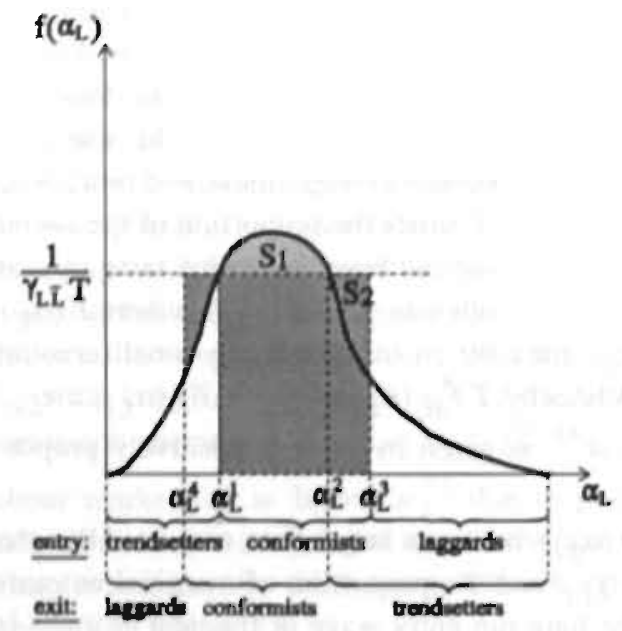

(a)

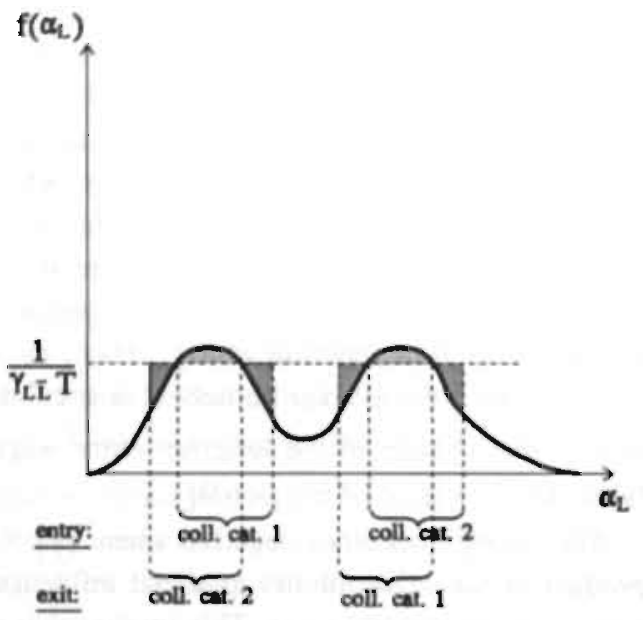

(b)

Figure 7.2 Household time tastes of marginal entrants at which collective entry and exil catastrophes start and end for an $(a)$ unimodal and $(b)$ bimodal continuously differentiable density function.

trendsetters $\ddot{H}^{* 1} / T$ which is required for a collective catastrophe to occur can be referred to as a critical mass. It is indicated by the surface area under the curve of the density function left to $\alpha_{L}^{1}$. After the collective catastrophe has occurred, there are still some group members. left who stay at home. For $w$ rising at levels above $\bar{w}^{* 1}$, these stay-behinds will enter. We call them laggards. Their proportion is given by the surface area under the curve of the density function right to $\alpha_{L}^{3}$.

It is important to note that the entry wages of the conformists in Figure: 7.1. being lower than the entry wage $\bar{w}^{* 1}$ of predecessors are conditional on the presence of these predecessors entering at $w^{* 1}$. Accordingly, at rising $w$ the conformists will only enter after these predecessors have entered at $\bar{w}^{* 1}$. As a result, the conformists enter at the same effective long-run entry wage $\bar{w}^{* 1}$. This implies that the distribution of the (effective) long-run entry wages over the individuals collapses to a distribution with an infinitely high and infinitely narrow peak at $\bar{w}^{* 1}$ as displayed in Figure $7.3 \mathrm{a}^{4}$ This peak represents the conformists, whereas the left tail of the distribution refers to the trendsetters with low household time tastes and the right tail to the laggards with high household time tastes. The surface areas of the left and right tail indicate the proportions of trendsetters and laggards, respectively. ${ }^{5}$

\section{Technically, such a peak is called a $\delta$-function or Dirac function.}

${ }^{5}$ When the density function $f\left(\alpha_{L}\right)$ is symmetric, it follows from Figure 7.2a that the proportion of trendsetters, and hence the surface area of the left tail of the density function $h\left(w^{\circ 1}\right)$, is larger than the proportion of laggards, and hence the surface area of the right tail of $h\left(w^{* 1}\right)$. Moreover, it can be proven that $h\left(w^{* 1}\right)$ goes to infinity for $w^{* 1}$ approaching $\tilde{w}^{* 1}$ from below, but goes to a finite value for $w^{* 1}$ approaching $\hat{w}^{* 1}$ from above. 


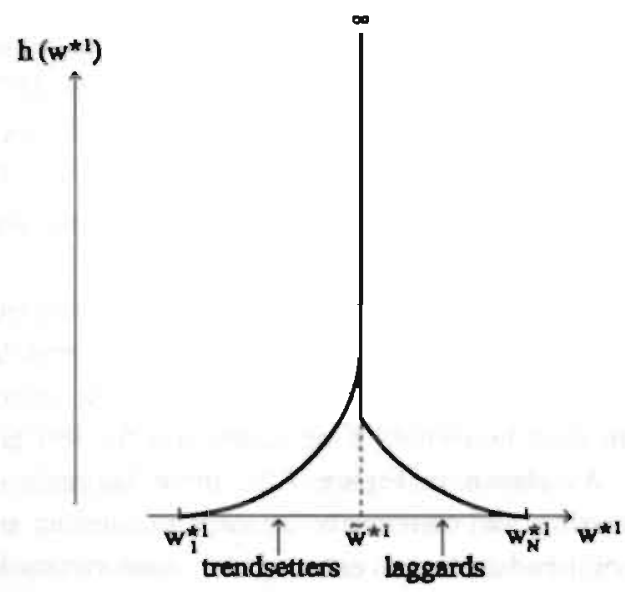

(a)

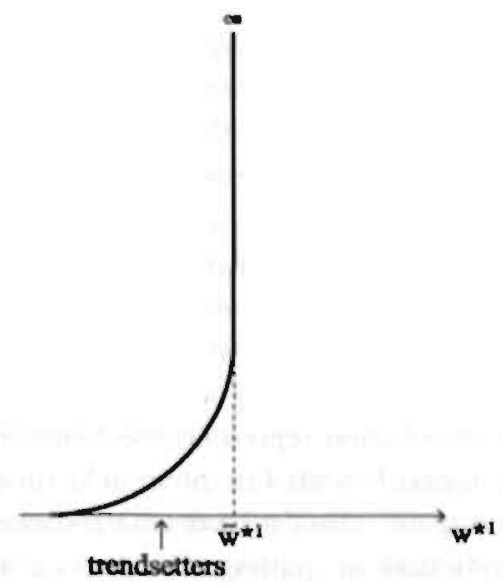

(b)

Figure 7.3 Distributions of effective long-run entry wages in (a) Case A and (b) Case $\mathbf{B}$.

For $w$ rising above $\bar{w}^{* 1}$, the entrance of laggards leads to a further increase in the longrun average labour supply till its maximal value $T$ for $w$ above $w_{N}^{* 1}$, at which all laggards have entered (see Fig. 7.1). When $w$ rises further, the first to reach the backward-sloping part of her individual long-run labour supply schedule is the group member with the lowest $w_{+i}^{*}$ in Figure 4.2 as given by (4.13), where the household time taste $\alpha_{L}$ does not need to be replaced by the social household time taste $\alpha_{L i}^{*}:=\alpha_{L i}+\gamma_{L} \bar{L}^{*}$, since the average household time $\bar{L}^{*}$ is now equal to zero. This group member has the highest household time taste, and hence is the last entrant $N$. As a consequence, the average long-run labour supply schedule becomes backward-sloping for $w=w_{+N}^{*}$.

For $w$ above $w_{+N}^{*}$ all group members have a job and are collectively used to that. When $w$ then starts to decline, the average long-run labour supply $\bar{H}^{*}$ will first increase, next remain equal to its maximal value $T$, and start to decrease only at the exit wage $w_{N}^{* 2}$ of the first leaver or last entrant, which is lower than her entry wage $w_{N}^{* 1}$ as implied by (7.2) and (7.3). First persons with high household time tastes will quit and next persons with lower household time tastes. However, analogously to the case of the entry wages, the resulting increases in the average long-run household time $\bar{L}^{*}$ raise the long-run exit wages $w_{i}^{* 2}$ as given by (7.3). For the case where the density function $f\left(\alpha_{L}\right)$ is continuously differentiable and unimodal, the long-run exit wage of the marginal entrants as a function of the average longrun labour supply $\bar{H}^{*}$ just before their entrance is given by the lower sharply sigmoid curve in Figure 7.1. The condition under which this function starts to rise at $w=\tilde{w}^{* 2}$ and $\vec{H}^{*}=\hat{H}^{* 2}$. again states that the value $f\left(\alpha_{L i}\right)$ of the density function at the household time taste of the marginal entrants becomes larger than $1 /\left(\gamma_{L L}-T\right)$. Now, however, this does not hold in Figure 7.2 for rising marginal household time taste at $\alpha_{L}^{1}$, but for deciining marginai household time taste at $\alpha_{L}^{2}$. At decreasing $\bar{H}^{*}$ the "exit wage function" in Figure 7.1 starts again to decline at $\mathscr{H}^{* 1}$ corresponding to $\alpha_{L}^{1}$ in Figure 7.2. At $\tilde{H}^{* 4}$ in Figure 7.1, or $\alpha_{L}^{4}$ in Figure 7.2 , the same exit wage level is reached as at $\hat{H}^{* 2}$, with an analogous equality of 
surface areas in Figure 7.2 as in the entry case. In a similar way these surface areas indicate the proportion of group members whose long-run exit wages are higher than the exit wage $\tilde{w}^{* 2}$ of predecessors and who are therefore involved in a collective catastrophe from $\bar{H}^{* 2}$ to $\bar{H}^{* 4}$ at that wage. Again, there may be laggards who quit only at lower exit wages. The exit wage $w_{1}{ }^{2}$ of the last laggard, or first entrant, has the positive numerator min $\alpha_{L i}+\gamma_{L L}-T$, implying that below $w_{1}^{* 2}$ all group members are, in the long run, non-participating and collectively used to that.

As a consequence of the collective catastrophe, the distribution of the effective long-run exit wages collapses to a distribution with an infinitely high and infinitely narrow peak at $\bar{w}^{\bullet 2}$, which is analogous to the distribution in Figure $7.3 \mathrm{a}$. However, now the right tail of the distribution represents the trendsetters with high household time tastes and the left tail the laggards with low household time tastes. As shown in Figure 7.2a, these laggards in leaving the labour market are trendsetters in entering, and conversely laggards in entering are trendsetters in quitting. However, a number of trendsetters in entering are conformists. in. quitting and some trendsetters in quitting are conformists in entering. Thus, some but not all conformists in entering are also conformists in quitting, and the reverse. From the analysis given above it follows that such collective catastrophes in entering and leaving the labour market will occur if and only if

$$
\gamma_{L \bar{L}}>\frac{1}{T \max f\left(\alpha_{L}\right)} .
$$

Here $\max f\left(\alpha_{L}\right)$ denotes the maximal value of the density function.

However, just as in Section 4.8, we may wonder what happens when $w$ rises from zero till a level $w^{0}$ at which only some trendsetters have entered the labour market (see Fig. 7.1) and, after the long-run equilibrium $\bar{H}^{\circ}$ being established, again declines to zero. Then, analogously to Section 4.8 , the trendsetters will switch back to non-participation only at their relatively low exit wages $w_{i}^{* 2}$ as given by the exit curve in Figure 7.1. Therefore, the longrun average labour supply of the social group will be inelastic for $w$ declining from $w^{o}$ (ass indicated by a vertical dashed line) until the exit curve is reached, and follow this highly elastic curve afterwards. In general, long-run average labour supply is inelastic between the solid "stable" parts of the entry and exit curve in Figure 7.1, whereas it becomes suddenly highly elastic, when the wage rate reaches one of these parts. ${ }^{6}$

However, what about the region around the dashed parts of the entry and exit curve? Can. this region be reached by sticcessive rises and declines in $w$ ? Starting from a long-run equilibrium point, it cannot because of the collective catastrophes in the entry and exit curve. On the other hand, these collective catastrophes take time to work out themselves. Consider. for example, a situation in which conformists are involved in a collective catastrophe on the entry curve, so are collectively increasing the average labour supply in their social group from $h^{* 1}$ to $h^{* 3}$. Suppose that at a certain level $\bar{H}^{1}$ of that average labour supply the wage rate declines. In the short run, the average labour supply then also declines, but in the long run, several things happen. On the one hand, the decline of the average labour supply is

6 The "stable" parts of the entry and exit curves consist of long-run equilibria like $A$ and $B$ in Figure 4.12 for given $w$. 
reinforced by the BPI. On the other hand, the individual household time demands as functions of the individual habit states rise, so that a number of the demands of the conformists obtain an interior unstable equilibrium like $C_{i}$ in Figure 6.1. When an individual habit state is to the left side of such a watershed, it will continue its individual catastrophe towards zero household hours, but when it is to the right side of the watershed, it will return to maximal household hours. The net result of these short- and long-run effects for the average labour supply is ambiguous. If $w$ remains sufficiently high above the dotted part of the entry curve, most individual catastrophes, and hence the collective catastrophe, will continue, but a "stable" long-run equilibrium of average labour supply is then reached at a lower level on the solid part of the entry curve. If $w$ falls near or below the dotted part of the entry curve, but still sufficiently far above the dotted part of the exit curve (e.g., to $w^{2}$ ), the average labour supply adjusts to a long-run value $\bar{H}^{2}$, which tends to be lower than $\bar{H}^{\mathrm{i}}$, but which, at further changes of $w$ between the entry and exit curve, remains inelastic. Finally, if $w$ declines near or below the dotted part of the exit curve, a collective catastrophe back to nonparticipation will set in.

Thus, generally we can distinguish a band of persistent and hysteretic long-run behaviour between the entry and exit curve and two zones of catastrophic long-run behaviour outside this band. Note that this configuration forms a combination of features of the long-run average labour supply schedule in the case of only unstable IHF with respect to household time in Figure 4.11 in Section 4.8 and features of the labour supply schedule in the case of only unstable BPI with respect to household time in Figure 5.3 in Section 5.4. Accordingly, the band of persistent and hysteretic long-run behaviour is not found in models of only unstable BPI with respect to dichotomous short-run behaviour (see, e.g., Iannacone, 1989. App., and Granovetter and Soong, 1986).

Such a configuration is also obtained when we use the exact expression for the long-run exit wage according to the pertinent variant of (4.13) instead of its first-order approximation (7.3). The only difference is that the band width between the entry and exit curve in Figure 7.1 is then no longer a constant with as numerator the habit formation minus satiation tendency tem $\left(\gamma_{L L}-\left|\alpha_{L L}\right|\right) T$, but varies with $\bar{H}^{*} \cdot 7$ A second modification results when the susceptibility to social influence is so strong and/or the maximum of the density function $f\left(\alpha_{L}\right)$ in Figure 7.2a is so high as to make the surface area $S_{1}$ large. Then we may have situations in which there are no laggards in entry or exit, implying that $w_{N}^{* 1}$ in Figure 7.1 becomes. smaller than $\tilde{w}^{=1}$ and Figure 7.3a tums into Figure 7.3b.

A third modification is given by the case where the density function $f\left(\alpha_{L}\right)$ is continuously differentiable and bimodal. Then we may have a situation as in Figure $7.2 \mathrm{~b}$, implying two collective catastrophes in both the entry curve and the exit curve of the long-run average labour supply schedule in which different minorities of the social group are involved. This leads to entry and exit wage distributions which have two infinitely high and narrow peaks. Moreover, the laggards of the first collective catastrophe go smoothly over into the trendsetters of the second one. However, similarly to the second modification mentioned above, we

${ }^{7}$ Since the approximation (7.3) underestimates the exit wage (see footnote 1), it overestimates the band width between the entry and exit curves. This is due to the underestimation of the stabilizing effect of the diminishing marginal utility of consumption as expressed by the parameter $c^{*}=\left|\alpha_{X X}\right|$ (see the approximation condition for (7.3) to hold). 
may also have situations in which laggards in entry or exit are absent and in which the two collective catastrophes even melt together into one. From Figures $7.2 \mathrm{a}$ and $7.2 \mathrm{~b}$ it can be deduced that a collective catastrophe in which a majority of the social group is involved will occur more easily as the susceptibility to social influence is stronger or as the variance of the household time taste is smaller.

So far we have considered exogenous individual variation in the household time taste $\alpha_{L i}$ only. What happens if we allow individual variation in the IHF strength $\gamma_{L L i}-\left|\alpha_{L L i}\right|$ too? This affects the long-run entry wages according to (7.2), but not the long-run exit wages according to (7.3) or the pertinent variant of (4.13). In (7.2) the first two terms of the numerator then vary and we can formulate

Proposition 7.1 Assume that all group members display unstable IHF and individual catastrophes as in Figure 4.2. Further assume exogenous individual variation only in the household time taste $\alpha_{L}$ and the IHF strength $\gamma_{L L}-\left|\alpha_{L L}\right|$ and continuously differentiable and unimodal or bimodal density functions $f\left(b_{o}^{*}\right)$, where $b_{o}^{*}=\alpha_{L}+\left(\gamma_{L L}-\left|\alpha_{L L}\right|\right) T$ for entries and $b_{o}^{\prime}=\alpha_{L}$ for exits. Then a collective catastrophe in entry or exit can occur if and only if

$$
\gamma_{L \bar{L}}>\frac{1}{T \max f\left(b_{o}^{*}\right)}
$$

The marginal $b_{o i}^{*}=b_{o}^{* 1}$ or $b_{o}^{* 2}$ at which the collective catastrophe sets in, is given by the condition $\gamma_{L L} T f\left(b_{o i}^{*}\right)=1$, and the marginal $b_{o i}^{*}=b_{o}^{* 3}$ or $b_{o}^{* 4}$ at which the collective catastrophe ends, is either determined in the same way as $\alpha_{L}^{3}$ or $\alpha_{L}^{4}$, respectively, in Figure 7.2 or is equal to the highest $b_{o i}^{*}=b_{o N}^{*}$ or lowest $b_{o i}^{*}=b_{o l}^{*}$, respectively.

As a result, we obtain entry and exit curves and distributions of effective long-run entry and exit wages similar to those in Figure 7.1 and Figure $7.3 \mathrm{a}$ or $7.3 \mathrm{~b}$, respectively. A difference for the entries, but not for the exits is that now the trendsetters have low household time tastes and/or low habit formation strengths and laggards high household time tastes and/or high IHF strengths. As a consequence, the long-run average labour supply schedule is similar to the schedule in Figure 7.1 , but the values of average labour supply at which the exit curve starts to rise or decline, do not generally coincide with the corresponding values for the entry curve. More importantly, analogously to Section 4.8, long-run trajectories within the band between the entry and exit curve may not be straight vertical lines, but may consist of circling small hysteresis loops like those in Figure 4.11.

\subsection{Case B: variation in susceptibility to social infuence}

In the case where only the non-negative susceptibility to social influence $\gamma_{L_{L}} \bar{L}_{i}$ varies over individuals, matters are partially different from those in the case of the previous section. We regard $\gamma_{L} \bar{L}_{i}$ as a continuous variable $\gamma_{L \bar{L}}$ with density function $g\left(\gamma_{L} \bar{L}\right)$. Since the household time taste $\alpha_{L}$ is positive, the long-run exit wages $w_{i}{ }^{22}$ according to (7.3), and hence 
the long-run entry wages according to (7.2), are positive, implying that at $w=0$ all group members are, in the long run, always non-participating and collectively used to that. The long-run average household time demand $\bar{L}^{*}=T$ is then equal to $T$, so the long-run entry wage $w_{1}^{* 1}$ of the first entrant at $w$ rising from zero has the positive numerator $\alpha_{L}+\left(\gamma_{L L}-\left|\alpha_{L L}\right|\right) T+\min \gamma_{L L i} T$. Thus, at $w$ rising from zero, nobody will enter the labour market for $w$ below $w_{1}^{* 1}$. This is indicated by the long-run average labour supply schedule in Figure 7.4.

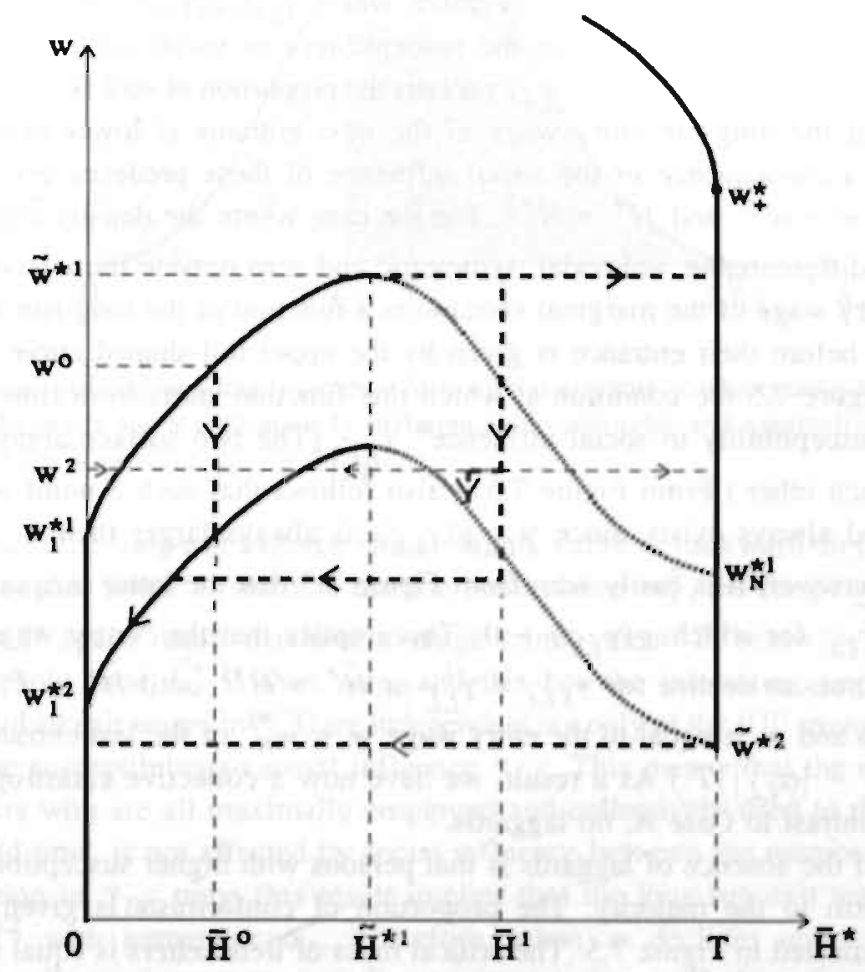

Figure 7.4 Long-run average labour supply schedule for AQM in Case B of exogenous individual variation in susceptibility to social influence only.

However, as $w$ rises beyond $w_{1}{ }^{-1}$, forerunners will enter and work, in the long run, the maximal number of corporate hours. First persons with low susceptibilities to social infiuence will enter and next persons with higher susceptibilities. This leads to increases in the long-run average labour supply, and hence to decreases in the long-run average household time $\bar{L}^{*}$. lowering the entry wages of the stay-behinds. The long-run average household time, or $T$ times the proportion of stay-behinds, is equal to $T$ times the proportion of the social group for which the susceptibility to social influence $\gamma_{L L}$ is higher than the susceptibility $\gamma_{L \bar{L} i}$ of the last or marginal entrant(s). This proportion can be written as $1-G\left(\gamma_{L \bar{L} i}\right)$, where $G\left(\gamma_{L L}\right)$ is the distribution function of $\gamma_{L \bar{L}}$. Then, as $\gamma_{L \bar{L} i}$ rises by an infinitesimally 
small amount $d \gamma_{L \bar{L}}$, this leads directly to a rise in the long-run entry wage $w_{i}^{* 1}$ as, given by (7.2), which rise is positively proportional to $d \gamma_{L \overline{L i}} T\left[1-G\left(\gamma_{L \overline{L i}}\right)\right]$. On the other hand, it leads also indirectly via $\bar{L}^{*}$ to a decline in $w_{i}^{* 1}$, which is positively proportional to $\gamma_{L \bar{L} i} T g\left(\gamma_{L \bar{L} i}\right) d \gamma_{L \bar{L} i}$. Thus, the total change in $w_{i}^{* 1}$ is given by

$$
d w_{i}^{* 1} \propto\left\{T\left[1-G\left(\gamma_{L \bar{L} i}\right)\right]-\gamma_{L \overline{L i}} T g\left(\gamma_{L \overline{L i}}\right)\right\} d \gamma_{L \overline{L i}},
$$

where $\propto$ denotes positive proportionality.

This total change in $w_{i}^{\cdot 1}$ becomes negative when $\gamma_{L \overline{L i}} g\left(\gamma_{L \bar{L} i}\right)$ becomes larger than $1-G\left(\gamma_{L \overline{L i}}\right)$, so when the product of the susceptibility to social influence $\gamma_{L \bar{L} i}$ and the proportion of the marginal entrants $g\left(\gamma_{L \bar{L}}\right)$ exceeds the proportion of stay-behinds $1-G\left(\gamma_{L \bar{L}}\right)$. This implies that the long-run entry wage of the next entrants is lower than that of their predecessors as a consequence of the social influence of these predecessors. In Figure 7.4 this happens at $w=\bar{w}^{* 1}$ and $\bar{H}^{*}=\bar{H}^{* 1}$. For the case where the density function $g\left(\gamma_{L L}\right)$ is continuously differentiable, unimodal, symmetric and zero outside the interval $\left[0,2 \bar{\gamma}_{L L}\right]$, the long-run entry wage of the marginal entrants as a function of the long-run average labour supply $\bar{H}^{\bullet}$ just before their entrance is given by the upper hill-shaped curve in Figure 7.4. According to Figure 7.5 the condition at which this function starts to decline is fulfilled at the "marginal susceptibility to social influence" $\gamma_{L \bar{L}}^{1}$. (The two surface areas indicated are then equal to each other.) From Figure 7.5 it also follows that such a point where the condition is fulfilled always exists, since $\gamma_{L \bar{L}} g\left(\gamma_{L \bar{L}}\right)$ is always larger than $1-G\left(\gamma_{L \bar{L}}\right)$ for $\gamma_{L \bar{L}}=\bar{\gamma}_{L \bar{L}}$. Moreover, it is easily seen from Figure 7.5 that the latter inequality holds also for all $\gamma_{L \bar{L}}>\bar{\gamma}_{L \bar{L}}$ for which $g\left(\gamma_{L L}\right)>0$. This implies that the "entry wage function" in Figure 7.4 continues to decline for $\gamma_{L \bar{L} i}>\gamma_{L \bar{L}}^{1}$ or $\bar{H}^{*}>\hat{H}^{* 1}$ until $\bar{H}^{*}=T$. (At this point the slope is zero and $w$ is equal to the entry wage $w=w_{N}^{* 1}$ of the last entrant with numerator $\alpha_{L}+\left(\gamma_{L L}-\left|\alpha_{L L}\right|\right) T$.) As a result, we have now a collective catastrophe from $\bar{H}^{* 1}$ to $T$ with, in contrast to Case A, no laggards.

The reason of the absence of laggards is that persons with higher susceptibilities to social influence conform to the majority. The proportion of conformists is given by the equal surface areas indicated in Figure 7.5. The critical mass of trendsetters is equal to the complement of these surface areas $G\left(\gamma_{L L}^{1}\right)$. It is smaller, and hence the proportion of conformists is larger, as the variance of the susceptibilities to social influence is lower. (The surface area $G\left(\gamma_{L L^{-}}^{1}\right)$ is then smaller relative to the square surface area $\gamma_{L L^{\prime}}^{1} g\left(\gamma_{L L^{-}}^{1}\right)$.) Moreover, for the specific kind of density function in Figure 7.5, this critical mass is always smaller than a half.

The conformists enter at the same effective long-run entry wage $\bar{w}_{1}^{* 1}$. This implies that the distribution of the (effective) long-run entry wages over the individuals looks as shown in Figure 7.3b. The peak represents the conformists, whereas the left and only tail of the distribution refers to the trendsetters with low susceptibilities to social influence. For higher $w$ all employees reach the backward-sloping part of their long-run labour supply curve at the same $w_{+}^{*}$ (see Fig. 4.2 in Sec. 4.2), since the long-run average household time $\bar{L}^{*}$, and hence (the individual variation in) the social influence term $\gamma_{L L^{-}} \bar{L}^{*}$ in $(6.9 \mathrm{~b})$ has become 


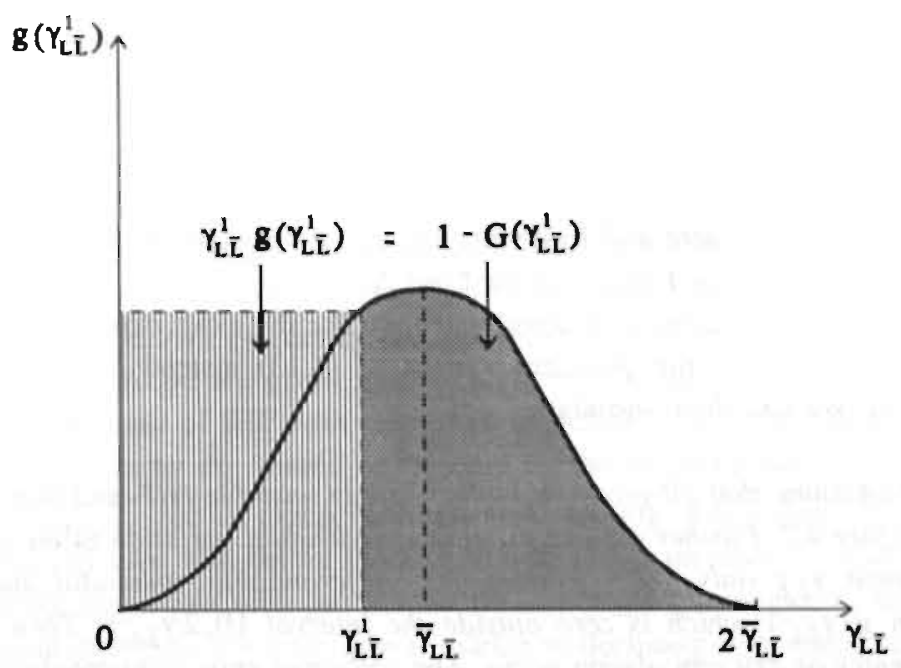

Figure 7.5 Susceptibility to social influence of marginal entrants at which collective entry catastrophe starts for a continuously differentiable, unimodal and symmetric density function which is zero for $\gamma_{L \bar{L}}>2 \bar{\gamma}_{L \bar{L}}$.

zero. As a result, the long-run average labour supply curve is backward-sloping beyond $w_{+}^{*}$.

This implies that when $w$ starts to decline from above $w_{*}^{*}$, the long-run average labour supply $\bar{H}^{*}$ will again reach maximal average employment $T$ below $w_{+}^{*}$. The long-run average household time $\bar{L}^{-}$is then zero, and this has the interesting implication that the long-run individual exit wages in (7.3) are independent not only of the IHF strength $\gamma_{L L}-\left|\alpha_{L L}\right|$, but also of the susceptibility to social influence $\gamma_{L \bar{L}}$. This means that the exit behaviour of group members who are all maximally employed and collectively used to the corresponding zero household time, is not affected by social influence between the members. In the present case of variation in $\gamma_{L}-$ only, this result implies that the long-run exit wages are equal to the same $\vec{w}^{* 2}$ with numerator $\alpha_{L}$. Therefore, when $w$ declines under $\dot{w}^{* 2}$, all group members will collectively start to work fewer corporate hours. Their motivation to do so will be reinforced by the other group members doing the same, implying a rise in $\bar{L}^{*}$, and hence a rise in the exit wage $w^{* 2}$. As a result, the group members will not stop reducing their corporate hours until they are all non-participating, so $\bar{H}^{*}=0$. Thus, the exit behaviour displays a collective catastrophe at $\ddot{w}^{* 2}$ which does not require a critical mass and in which all group members are involved.

Just as in Case A we may wonder what happens when $w$ rises from zero till a level $w^{0}$ at which only some trendsetters have entered the labour market and, after the long-run equilibrium $\bar{H}^{\circ}$ being established, again declines to zero. The long-run average labour supply of the social group will then be inelastic until an exit curve is reached, and follow this highly elastic curve afterwards. Analogously to Case A, the exit curve represents the long-run exit wage of marginal entrant(s) as a function of the average long-run labour supply $\bar{H}^{*}$ just before their entrance, and is parallel to the entry curve. This exit curve also indicates 
what happens when, in the midst of a collective entry catastrophe at $\bar{H}^{1}$, the wage rate declines under the entry curve. For $w$ sufficiently high above the exit curve (e.g. $w=w^{2}$ ), the average labour supply adjusts to a long-run inelastic value $\bar{H}^{2}$, which tends to be lower than $\bar{H}^{1}$. On the other hand, when $w$ declines near or below the exit curve, a collective catastrophe back to non-participation sets in, which tends to end at a "stable" equilibrium on the solid part of the exit curve at which still a number of exit laggards are fully employed. Note that the band of persistent and hysteretic behaviour between the entry and exit curve has now another shape than in Figure 7.1 for Case $\mathbf{A}$.

Again, a similar configuration is obtained when we use the exact expression for the longrun exit wage according to the pertinent variant of (4.13) instead of its first-order approximation (7.3). We can then formulate

Proposition 7.2 Assume that all group members display unstable IHF and individual catastrophes as in Figure 4.2. Further assume exogenous individual variation in the susceptibility to social influence $\gamma_{L}$ only and a continuously differentiable, unimodal and symmetric density function $g\left(\gamma_{L} \bar{L}\right)$ which is zero outside the interval $\left[0,2 \bar{\gamma}_{L L}\right]$. Then a collective catastrophe in entry or exit can always occur. The collective entry catastrophe sets in at the marginal $\gamma_{L \bar{L} i}=\gamma_{L \bar{L}}^{1}$ for which $\gamma_{L \bar{L}} g\left(\gamma_{L \overline{L i}}\right)=1-G\left(\gamma_{L \overline{L i}}\right)$, and ends at the highest $\gamma_{L \bar{L}}=\gamma_{L \overline{L N}}$ of the last entrant. The collective exit catastrophe sets in at all group members simultaneously and ends with the first entrant with the lowest $\gamma_{L L_{i}}=\gamma_{L \bar{L}_{1}}{ }^{8}$.

More generally, such a proposition and a long-run average labour supply schedule similar to that in Figure 7.4 presumably hold for a large class of density functions $g\left(\gamma_{L} \bar{L}\right)$, no matter how small the average $\bar{\gamma}_{L \bar{L}}$ of the susceptibilities to social influence or how large their variance.

\subsection{Conditions and configurations of equilibria}

When we have simultaneous variation in both the household time taste $\alpha_{L i}$ (or $b_{o i}^{*}$ ) and $\gamma_{L \bar{L} i}$, matters become much more complex. This holds even in the special case in which the distributions of these parameters are independent of each other. Therefore, this case is not elaborated here and we only mention some global results. Under certain collective catastrophe conditions, the long-run average labour supply schedule and the distribution of the entry wages are similar to the schedule in Figure 7.1 and the distribution in Figure $7.3 \mathrm{a}$ or $7.3 \mathrm{~b}$. On the one hand, there are a number of trendsetters in entry with low household time tastes, low habit sensitivities and/or low susceptibilities to social influence. On the other hand, there may be laggards in entry with high household time tastes, high habit sensitivities and/or high susceptibilities to social influence. However, many laggards may have low susceptibilities to social influence, since laggards are facing a low average household time $\bar{L}^{*}$ and hence a

${ }^{8}$ This holds, since the speed of an individual exit catastrophe is lower as the susceptibility to social influence is weaker. 
low social influence $\gamma_{L \bar{L} i} \bar{L}^{*}$ on their behaviour. This makes the labour supply of laggards rather insensitive to variation in $\gamma_{L \bar{L}_{i}}$.

As a result of the analysis of Case $\mathbf{A}$, this case can be decomposed in two subcases. Assuming individual variation in household time taste only, these two subcases are indicated as Case III and IV in Table 6.1 in Subsection 6.2.4. In both (sub)cases all individuals are assumed to display unstable IHF and individual hysteresis and catastrophes between nonparticipation and maximal employment as in Figure 4.2. Moreover, in both cases we then have a collective hysteresis loop and long-run equilibrium trajectories within this loop (see Table 6.2 in Subsec. 6.2.4). Necessary and sufficient conditions under which these phenomena show up in the AQM are given by the conditions in situation (i) of Proposition 4.1 in Section 4.4 for the case of IHF with respect to household time only except that the household time taste parameter $\alpha_{L}$ should be replaced by the social household time taste

$\alpha_{L i}^{*}:=\alpha_{L i}+\gamma_{L L^{2}} \bar{L}_{i}^{*}$ for all group members (see also Fig. 4.4c). Here $\bar{L}_{i}^{*}$ is the long-run average household time just before the entrance of group member $i$. This is equal to the long-run average household time just before the exit of group member $i$, since the order in which the group members leave the labour market at declining $w$ is the reverse of the order in which they enter at rising $w$.

Because the $w_{r}^{*}$ line in Figure $4.4 c$ moves upwards with increasing $\alpha_{L}$, whereas the $w^{*}$ curve remains fixed for given $c^{*}$, the under limit $d_{-}^{*}$ and the upper limit $d_{*}^{*}$ in the condition $d_{-}^{*}<d^{*}<d_{+}^{*}$, as determined by the intersections of this line and curve, move towards each other with increasing $\alpha_{L}$. This implies that the conditions are the strongest for a group member with the highest, or maximal, social household time taste $\alpha_{L}^{* m}$. Therefore, the conditions for all group members are equivalent to the conditions for $\alpha_{L}^{* m}$. Since the entry wage $w_{i}^{* 1}$ of the marginal entrant is increasing with $\alpha_{L i}^{*}, \alpha_{L}^{* m}$ corresponds to a maximal $w_{i}^{* 1}$. When collective catastrophes do not occur as in Figure 4.11 , the maximal entry wage is given by $w_{N}^{* 1}$, so $\alpha_{L}^{* m}=\alpha_{L N}^{*}=\alpha_{L N}=\alpha_{L}^{m !}$, since $\bar{L}_{N}^{*}=0$. The resulting conditions are indicated under Case III in Table 6.1. The collective catastrophe condition (7.4) for the susceptibility to social influence $\gamma_{L \bar{L}}$ and the density function $f\left(\alpha_{L}\right)$ of the individual household time tastes is then not fulfilled.

On the other hand, when collective catastrophes do occur, there are two possibilities. If there are entry laggards as in Figure 7.1 , the maximal entry wage is again given by $w_{N}^{* 1}$, so $\alpha_{L}^{* m}=\alpha_{L}^{m}$. If there are no entry laggards, the maximal entry wage is given by $\bar{w}^{* 1}$, at which the collective catastrophe sets in, so $\alpha_{L}^{* m}$ is equal to the corresponding social household time $\tilde{\alpha}_{L}^{* 1}$. The existence of $\bar{\alpha}_{L}^{* 1}$ depends on the fulfilment of the collective catastrophe condition (7.4), and its value is determined by $\gamma_{L \bar{L}}$ and $f\left(\alpha_{L}\right)$. When, for example, $\gamma_{L \bar{L}}$ rises, $\tilde{\alpha}_{L}^{* 1}$ can be shown also to rise. This makes the conditions for $\left|\alpha_{X X X}\right|$ and $\gamma_{L L}-\left|\alpha_{L L}\right|$ more stringent. However, from a theoretical point of view, the resulting mutual interdependence of conditions for collective entry and exit catastrophes to occur is not a problem, since $0<c_{o}^{*}\left(\tilde{\alpha}_{L}^{* 1}\right)$ and $\left|d_{*}^{*}\left(\bar{\alpha}_{L}^{* 1}\right)\right|<\left|d_{-}^{*}\left(\bar{\alpha}_{L}^{* 1}\right)\right|$ hold for any value of $\bar{\alpha}_{L}^{* 1}$. In the subcase of entry laggards we can even make the conditions somewhat weaker by replacing $\alpha_{L}^{m}$ by $\bar{\alpha}_{L}^{* 1}$. A number of laggards with the highest household time tastes may then behave as indicated by Figure $4 \mathrm{~B}$ in Appendix $4 \mathrm{~B}$ and we end up with the same conditions as in the subcase of no entry laggards. These conditions are indicated under Case IV in Table 6.1. 
If we allow individual variation in the IHF strength too, we can derive similar conditions for Case III, but for Case IV the conditions become somewhat more complicated. As argued in Section 4.8, the order in which the group members leave the labour market at declining $w$, may then not be the reverse of the order in which they enter at rising $w$. This makes the long-run average household times just before the entrance of group member $i$ and just before the exit of group member $i$, and hence the corresponding social household time tastes, unequal to each other. This case is not elaborated here. In Case B of individual variation in the susceptibility to social influence $\gamma_{L \bar{L}}$, the conditions for the IHF strength are identical to those under Case IV in Table 6.1 with $\bar{\alpha}_{L}^{* 1}$ now being equal to $\alpha_{L}+\bar{\gamma}_{L \bar{L}}^{* 1} \bar{L}^{* 1}$. On the other hand, in order to allow a collective catastrophe, the density function $g\left(\gamma_{L L}\right)$ has at most to satisfy some regularity conditions (see Proposition 7.2), so in this case we can distinguish only a Case IV.

In Section 7.1 we mentioned that collective catastrophes can be shown (in particular cases) to go together with the slope in (7.1) becoming equal to one. In particular, this should hold for the Cases A and B as analysed in the two previous sections. Therefore, we should consider the short-run average household time demand $\bar{L}$ as a function of the social environment $s_{L}$ (recall from Section 7.1 that the individual habit states have become implicit functions of the social environment). Just as Figure 4.12 in Section 4.8 can be derived from Figure 4.ii, graphs of this household time demand function for various values of the wage rate can be read off Figures 7.1 and 7.4. For Case A such graphs for $w=w^{2}, w=w^{o}$ and $w=\bar{w}^{* 1}$ are represented by Figure 7.6. The graph for $w=w^{2}$ displays the interesting phenomenon that the continuum of long-run equilibria of Figure 4.12 for the case of only unstable IHF has been split by the unstable BPI into three disjunct segments which are interconnected by an entry catastrophe for lower $s_{\bar{L}}$ and $\bar{L}$ (so higher $\bar{H}$ ) and by an exit catastrophe for higher $s_{\bar{L}}$ and $\bar{L}$. Comparing this graph with the graph of $\bar{L}^{C}$ in Figure 5.4 in Subsection 5.5.2 for the case of unstable BPI only, we can also say that the three discrete long-run equilibria $A, B$ and $C$ have been stretched out by the separately unstable IHF into three continua of long-run equilibria. Note that, in contrast to equilibrium $C$, the interior equilibria of the middle continuum are not unstable in the same way as $C$. For Case B of Figure 7.4 a similar phenomenon can be derived for $w=w^{2}$ except that then the continuum for lower $s_{\bar{L}}$ is replaced by a discrete equilibrium for $\bar{L}^{*}=0$. This more restricted variant of the phenomenon has also been found by Naylor (1989) in an application of the theory of social custom of Akerlof (1980) and is referred to as a "trimodal outcome" by him (see Section 8.7).

However, when $w$ rises from $w^{2}$, the exit catastrophe becomes smaller and the higher two continua approach each other until the exit catastrophe disappears and the two continua become connected at $\hat{H}^{* 1}$ for a certain $w$. For $w$ rising further to $w^{o}$, this configuration remains qualitatively the same, but the interconnected continuum shrinks, the entry catastrophe grows and the other continuum reaches a coner equilibrium $\bar{L}^{*}=0$ for $w=w_{N}^{* 2}$. The resulting graph for $w=w^{0}$ is displayed in Figure 7.6b. When $w$ rises above $w^{0}$, the higher continuum further shrinks until it turns into a point at $\bar{L}=T-\bar{H}^{* 1}$ for $w=\bar{w}^{* 1}$ as shown in Figure 7.6c. For $w$ rising beyond $\tilde{w}^{* 1}$ this discrete equilibrium disappears, inducing a collective entry catastrophe towards the lower continuum. Since the household time 


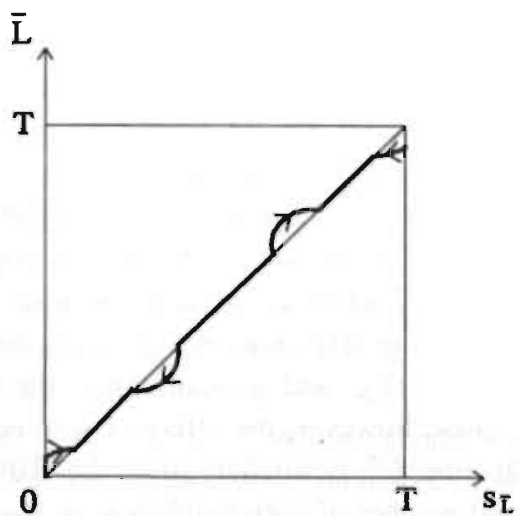

(a) $w=w^{2}$

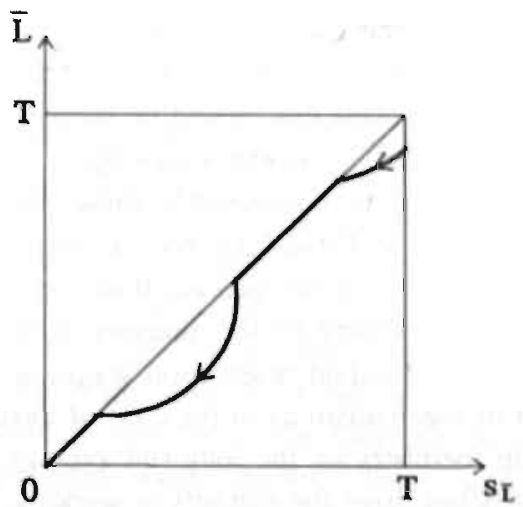

(b) $\mathbf{w}=\mathbf{w}^{0}$

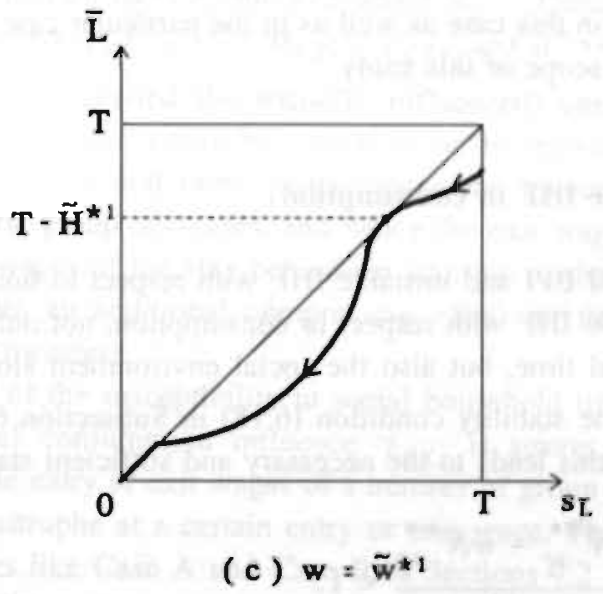

Figure 7.6. Short-run average household time demand as a function of the social environment for various values of the wage rate, indicated by bold lines and curves.

demand function is smooth for $w>\bar{w}^{* 1}$, it is, for continuity reasons, smooth for $w=\bar{w}^{* 1}$ too, and this implies a tangency condition for the collective entry catastrophe with the slope in (7.1) being equal to one.

For $w$ declining from $w^{2}$, an analogous story can be told with an identical tangency condition for a collective exit catastrophe from a discrete equilibrium at $\bar{L}=T-\bar{H}^{* 2}$ for $w=\tilde{w}^{* 2}$. For collective entry and exit catastrophes in Case B of Figure 7.4, a similar reasoning holds except that both catastrophes go on till a corner equilibrium and except that also the exit catastrophe starts from a comer equilibrium. In the latter case too a tangency condition can be shown to apply, implying that the slope in (7.1) is equal to one. This, however, seems exceptional for a collective catastrophe from a corner equilibrium. The case 
of BPI and stable IHF of Section 6.2. suggests that more generally such a catastrophe would be expected to go together with the slope in (7.1) being larger than one. Furthermore, we note that in the Cases A and B the condition that the slope in (7.1) is equal to one, should coincide with the corresponding condition as formulated in the Propositions 7.1 and 7.2 , respectively. This can indeed be shown to hold true, but proofs are not given here.

Finally, the collective catastrophe conditions which have been derived in the previous sections, may be compared to those derived in Subsection 6.2.4 for the case of stable IHF (see Case II in Table 6.1). We can then conclude that the former conditions are less general, but also much more precise than the latter conditions. Therefore, we may wonder what happens according to the analysis in this section when the IHF is stable. In that case the long-run individual reservation wages are again given by (7.2) and we can expect the same kind of mechanism as in the case of unstable IHF. Now, however, the effect of entrance by group members on the long-run average household time $\bar{L}^{*}$ is smaller, since the IHF will usually not drive the entrants to working the maximal number of corporate hours in the long run. Therefore, in the particular case of individual variation in the household time taste and the IHF strength, the susceptibility to social influence $\gamma_{L L}$ should be larger than according to condition (7.5) in order to induce a collective catastrophe. Unfortunately, an exact analysis looks very complicated in this case as well as in the particular case of variation in $\gamma_{L \bar{L}}$, and is therefore beyond the scope of this study.

\subsection{BPI and unstable IHF in consumption}

So far we have analysed BPI and unstable IHF with respect to household time only. When we add BPI and unstable IHF with respect to consumption, not only the social environment slope $\bar{L}_{s_{i}}^{*}$ for household time, but also the social environment slope $\bar{X}_{s_{\bar{X}}}^{*}$ for consumption should be adjusted in the stability condition (6.18) in Subsection 6.3.1. As an extension of the derivation of (7.1), this leads to the necessary and sufficient stability condition

$$
\bar{L}_{\bar{s}_{\bar{L}}}^{*}+\bar{X}_{s_{\bar{X}}}^{*}=T \frac{N^{m *} s_{\bar{L}}^{m}-w N^{m *} s_{\bar{X}}^{m}}{N}<1 .
$$

Here $N^{m}$ is the number of group members in stable individual equilibrium who spend a maximal number of household hours, so are non-participating, at given values of the social environments $s_{\bar{L}}^{-}$and $s_{\bar{X}}$. Again, it can be shown (for particular cases) that collective catastrophes go together with the slope in (7.7) becoming equal to one, but before that, it should be demonstrated how these collective catastrophes can arise.

As an extension of the analysis in the previous sections, this can be done by considering the individual long-run entry and exit wages at given long-run equilibrium values $s_{\bar{L}}^{*}=\bar{L}^{*}$ and $s_{\bar{X}}^{*}=\bar{X}^{*}$ of the (expected) social environments. The long-run entry wages $w_{i}^{* 1}$ are obtained by substituting $s_{L i}=T, s_{\bar{L}}=\bar{L}^{*}, s_{X i}=Y_{O i}$ and $s_{\bar{X}}=\bar{X}^{*}$ into the formulas for $b_{i}$ and $a_{i}$, respectively (see $\left.(6.9 \mathrm{~b})\right)$, and by substituting the resulting expressions into $w_{r i}=b_{i} / a_{i}$, yielding 


$$
w_{i}^{* 1}=\frac{\alpha_{L i}+\left(\gamma_{L L i}-\left|\alpha_{L L i}\right|\right) T+\gamma_{L \overline{L i}} \bar{L}^{*}}{\alpha_{X i}+\left(\gamma_{X X i}-\left|\alpha_{X X i}\right|\right) Y_{O i}+\gamma_{X \bar{X} i} \bar{X}^{*}}
$$

(cf. (7.2)). Thus, each person's entry wage depends positively on the long-run average household time $\bar{L}^{*}$ in her social group, but negatively on the long-run average consumption $\bar{X}^{*}$. This average consumption is equal to $w T\left(N-N^{m *}\right) / N+\bar{Y}_{O}$, where $\left(N-N^{m *}\right) / N$ denotes the proportion of group members who have already entered the labour market (assuming that none of these group members is on the backward-sloping part of her long-run labour supply curve). When most people are still at home, $\bar{X}^{\bullet}$ is relatively small, so the entry wages of forerunners is only slightly lower than in the absence of consumption BPI. On the other hand, as the wage rate rises and more people enter, the long-run average consumption in the group increases, and hence the entry wages decline. This implies a suppressing effect on the entry wages of the stay-behinds in addition to that due to the household time BPI, and hence an additional compressing effect on the variance of the entry wages between the group members.

A similar story can be told on the long-nun exit wages $w_{i}^{* 2}$. These are given by (4.13) in Section 4.3 with $\alpha_{L}=\alpha_{L i}+\gamma_{L, \bar{L} i} \bar{L}^{*}, c^{*}=\left|\alpha_{X X i}\right|=\gamma_{X X i}$ and $a^{*}=\alpha_{X i}+\gamma_{X X \bar{X} i} \bar{X}^{*}-c^{*} Y_{O i}$, where $\alpha_{X i}+\gamma_{X \bar{X} i} \bar{X}^{*}$ can be called the social(ly influenced) consumption taste. These expressions for the exit wages can easily be shown to be decreasing in $a^{*}$, and hence in $\bar{X}^{*}$. As the wage rate declines and more people leave the labour market, the long-run average consumption in the group decreases, and hence the exit wages rise. This implies a raising effect on the exit wages of the stay-behinds in addition to that due to the household time BPI, and hence again an additional compressing effect on the variance of the exit wages between the group members.

When the combination of the susceptibility to social household time influence $\gamma_{L \bar{L} i}$ and the susceptibility to social consumption influence $\gamma_{X} \bar{X}_{i}$ is strong enough, it may even decrease the variance in the entry or exit wages of a number of group members to zero. This results in a collective catastrophe at a certain entry or exit wage. This phenomenon can be derived for particular cases like Case A and Case B in Sections 7.2 and 7.3. The resulting collective catastrophe conditions for $\gamma_{L \bar{L}(i)}$ and $\gamma_{X} \bar{X}_{(i)}$ are weaker, but much more complex than those in Propositions 7.1 and 7.2. This, however, is not elaborated here, and we confine ourselves to the following remarks.

Cases like Case A can be decomposed in subcases like Case III and IV in Table 6.1 in Subsection 6.2.4. In cases like Case B collective catastrophes in entry or exit can practically always occur, so we can then distinguish only a Case IV. The long-run average labour supply schedules seem similar to those in Figures 7.1 and 7.4 for Case $A$ and B, respectively, except for two differences. First, there will be no backward-sloping part for a sufficiently strong combination of IHF and BPI with respect to consumption.

Second, in the case of variation in the susceptibility to social consumption influence $\gamma_{X \bar{X}}$ only, the forward-sloping part is likely to look like that in Figure 7.1 except that there are no laggards in exit. Thus, in contrast to Figure 7.4 for Case B, there are then laggards in entry and trendsetters in exit. The latter trendsetters have the lowest susceptibilities to social consumption influence, and hence are most inclined to reduce their corporate time, and 
hence their consumption, at a declining wage rate. At a certain $\bar{w}^{* 2}$ a critical mass of exit trendsetters is reached and a collective catastrophe towards non-participation sets in. This collective transition will not leave behind laggards, since persons with higher susceptibilities to social consumption influence are likely to conform to the majority. On the other hand, such persons are trendsetters in entering the labour market, because for them a higher consumption level, and hence corporate work, are more important than for persons with lower susceptibilities. At a certain $\tilde{w}^{* 1}$ a critical mass of entry trendsetters is reached and a collective catastrophe to maximal employment occurs. Now, however, this collective transition may leave behind laggards with lower susceptibilities to social consumption influence.

In this and all other cases we may again have, for a certain interval of the wage rate, a "trimodal outcome" of either three continua of long-run equilibria or two continua and a discrete equilibrium. In addition, collective entry and exit catastrophes are again characterized by tangency conditions with the left-hand side of (7.7) being equal to one as in Figure $7.6 \mathrm{c}$.

\subsection{BPI and unstable IHF in corporate time}

When we add BPI and unstable IHF with respect to corporate time to the BPI and unstable IHF with respect to household time, we can again follow the reduction procedure explained in Section 6.4. This yields a reduced utility function with the same arguments as in the case of BPI and unstable IHF with respect to household time only. As a consequence, we have a "two-stage rocket" with the same stability condition (7.1) and the same formulas (7.2) and (7.3) for the long-run entry wages $w_{i}^{* 1}$ and the long-run exit wages $w_{i}^{* 2}$.

Therefore, in Case A of individual variation in the household time taste $\alpha_{L i}$ and the household time IHF strength $-d_{L i}^{*}:=\gamma_{L L i}-\left|\alpha_{L L i}\right|$, Proposition 7.1 continues to hold, implying a long-run average labour supply schedule like that in Figure 7.1. Now, however, $\alpha_{L i}$ is a "net" household time taste which, by virtue of $(6.20 \mathrm{a})$, can be written as

$$
\alpha_{L i}-\alpha_{L i}-\alpha_{H i}-\left(-\hat{a}_{H i}^{*}\right) T-\hat{\gamma}_{H \overline{H i}} T \text {. }
$$

Here $\hat{\alpha}_{L i}$ and $\hat{\alpha}_{H i}$ are the "pure" household and corporate time tastes, $-\hat{d}_{H i}^{*}:=\hat{\gamma}_{H H i}-\left|\alpha_{H H i}\right|$ is the "pure" corporate time IHF strength, and $\hat{\gamma}_{H \bar{m}}$ is the susceptibility to "pure" social corporate time influence. Moreover, $-d_{L i}^{*}$ now is a total household time IHF strength which. by virtue of $(6.20 \mathrm{c})$ and $(6.20 \mathrm{~b})$ in Section 6.4 , is equal to the sum $\left(-\hat{d}_{L i}^{*}\right)+\left(-\hat{d}_{H i}^{*}\right)$ of the "pure" household time and corporate time IHF strengths.

These equations imply that when in Proposition 7.1 not only $\hat{\alpha}_{L i}$ and $-\hat{d}_{L i}^{*}$, but also $\alpha_{H i}$ and $-\hat{d}_{H i}^{*}$ are allowed to vary among the group members, $b_{o}^{*}$ as representing the $i$ dependent terms in the numerator of (7.2) or (7.3) is given by the first three terms of (7.9) for exits. whereas $b_{o}^{*}$ for entries is equal to $\alpha_{L i}-\alpha_{h i}+\left(-\hat{d}_{L i}^{*}\right) T$. Thus, the exit wages as well as the entry wages vary with an IHF strength. Whereas trendsetters in entry have low "pure" household time tastes, high "pure" corporate time tastes and/or low "pure" household time IHF strengths, trendsetters in exit have high "pure" household time tastes, low "pure" corporate time tastes and/or low "pure" corporate time IHF strengths. Furthermore, laggards 
in entry and exit have characteristics which are opposite to those of trendsetters. In addition, it seems plausible to assume that group members with a low (high) "pure" household time IHF strength $-\hat{d}_{L i}^{*}$ also have a low (high) "pure" corporate time IHF strength $-\hat{d}_{H i}^{*}$ (see the ends of Secs. 4.5 and 4.8 ). Then both trendsetters in entry and trendsetters in exit have low $-\hat{d}_{L i}^{*}$ as well as low $-\hat{d}_{H i}^{*}$, whereas both laggards in entry and exit have high $-\hat{d}_{L i}^{*}$ as well as high $-\hat{d}_{H i}^{*}$. The collective catastrophe condition is again given by (7.5), where according to (6.20d) in Section 6.4, the susceptibility to social household time influence $\gamma_{L \overline{L i}}$ is now equal to the sum $\hat{\gamma}_{L \bar{L} i}+\hat{\gamma}_{H \bar{H} i}$ of the susceptibilities to "pure" social household time and corporate time influence.

In Case B of individual variation in $\gamma_{L \bar{L} i}$, matters become more complicated when not only $\hat{\gamma}_{L \overline{L i}}$, but also $\hat{\gamma}_{H \bar{H}}$ is allowed to vary among the group members. Substituting (6.20d) in Section 6.4 into (7.2) and (7.3) and combining it with the expression (7.9) for $\alpha_{L i}$, we obtain two $i$-dependent terms $\hat{\gamma}_{L \bar{L} i} \bar{L}^{*}-\hat{\gamma}_{H \bar{H}_{i}} \bar{H}^{*}$ in the numerator of (7.2) as well as (7.3). However, forerunners in entry face a low average employment $\bar{H}^{*}$, so their entry wages are almost independent of the susceptibility to "pure" social corporate time influence $\hat{\gamma}_{H \bar{H}}$. Therefore, the entry situation of forerunners is similar to that in Proposition 7.2, and since the endogenous increase in $\bar{H}^{*}$ reinforces the positive feedback of the endogenous decrease in $\bar{L}^{*}$, a collective entry catastrophe can practically always occur. The trendsetters will then have (relatively) low susceptibilities to "pure" social household time influence $\hat{\gamma}_{L \overline{L i}}$, but may, in principle, have high as well as low $\hat{\gamma}_{H \bar{H}_{i}}$. However, analogously to the above assumption for IHF strengths, it seems plausible to assume that group members with a low (high) $\hat{\gamma}_{L \bar{L} i}$ have also a low (high) $\hat{\gamma}_{H \bar{H}}$. Then trendsetters in entry will have low $\hat{\gamma}_{L \bar{L} i}$ as well as low $\hat{\gamma}_{H \bar{H}}$.

On the other hand, when, as in Figure 7.4 , potential quitters face a maximal average employment $\bar{H}^{*}$, and hence a zero average household time $\bar{L}^{*}$, their exit wages are independent of $\hat{\gamma}_{L \bar{L} i}$. Nevertheless, their exit wages still depend on the variation in $\hat{\gamma}_{H \bar{H}}$, so they will not all quit at the same exit wage $\tilde{w}^{* 2}$ as in Figure 7.4. Yet, analogously to the entry case, a collective exit catastrophe can practically always occur as a result of a positive feedback of an endogenous decrease in $\bar{H}^{*}$ reinforced by an endogenous increase in $\bar{L}^{*}$. The trendsetters will then have (relatively) low $\hat{\gamma}_{H \bar{H}}$ as well as low $\hat{\gamma}_{L \bar{L}}$, so have the same characteristics as the trendsetters in entry. Therefore, we can draw the interesting conclusion that, in this case of variation in susceptibilities to social household and corporate time influence only, the trendsetters in exit more or less coincide with the trendsetters in entry.

A combination of this entry and exit behavior seems to lead to a long-run labour supply schedule like that in Figure 7.1. However, in contrast to this figure, there will be no laggards in entry and exit, when, as assumed above, group members with a low (high) $\hat{\gamma}_{L \bar{L}} \overline{\text { have a }}$ low (high) $\hat{\gamma}_{H \bar{H}}$ too. The reason is that for laggards in entry the average corporate time $\bar{H}^{*}$ would be high, and hence the average household time $\bar{L}^{*}$ low, implying that the variation in $\hat{\gamma}_{H \bar{H}}$ would be more important than the variation in $\hat{\gamma}_{L \bar{L}}$. Therefore, laggards in entry should be persons with a low $\hat{\gamma}_{H \bar{H}}$, but because of the assumed positive correlation with a low $\hat{\gamma}_{L \bar{L}}$, such persons would already have entered as trendsetters or early conformists. Analogously, laggards in exit should be persons with a low $\hat{q}_{L \overline{L i}}$, but because of the assumed positive correlation with a low $\hat{\gamma}_{H \bar{H}}$, such persons would already have quit as trendsetters or early conformists. 
This leaves us with one substantial difference of the long-run labour supply schedule with that in Figure 7.4, namely the presence of trendsetters in exit as a consequence of the individual variation in the susceptibility to "pure" social corporate time influence. In addition, the conditions for the marginal $\hat{\gamma}_{L \bar{L} i}$ and $\hat{\gamma}_{H \bar{H}}$ at which collective entry and exit. catastrophes set in, are much more complex than the pertinent conditions in Proposition 7.2, but are not elaborated here.

Finally, so far it has been assumed implicitly that the long-run exit wages $w_{i}^{* 2}$ as given by (7.3) (or (4.13) with $\alpha_{L i}+\gamma_{L \bar{L} i} \bar{L}^{*}$ instead of $\alpha_{L}$ ) are all positive. This implies that the exit wage $\tilde{w}^{* 2}$ at which the coilective exit catastrophe sets in, is positive. However, in the present case this exit wage may be negative, which can be seen as foliows. The numerator of the expression in (7.3) is equal to the "net" social(ly influenced) household time taste $\alpha_{L i}^{*}:=\alpha_{L i}+\gamma_{L \overline{L i}} \bar{L}^{*}$. Using (7.9) and (6.20d) in Section 6.4, this household time taste can be expressed as

$$
\alpha_{L i}^{*}=\alpha_{L i}-\alpha_{H i}-\left(-\hat{d}_{H i}^{*}\right) T+\hat{\gamma}_{L \overline{L i}} \vec{L}^{*}-\hat{\gamma}_{H \overline{H i}} \bar{H}^{*} .
$$

Figure 7.1 suggests that at $w=\bar{w}^{* 2}$ and $\bar{H}^{*}=\bar{H}^{* 2}$, at which the collective exit catastrophe sets in, $\bar{H}^{*}$ is larger than $\bar{L}^{*}$. Then it follows that, when at this point the "pure" corporate time taste $\alpha_{H i}$ is sufficiently high relative to the "pure" household time taste $\hat{\alpha}_{L i}$, the "pure" corporate time IHF strength $-\hat{d}_{H i}^{*}$ is sufficiently large, and $\hat{\gamma}_{L \overline{L i}}$ is sufficiently large relative to $\hat{\gamma}_{H \bar{H}}$ (recall that $\hat{\gamma}_{L \bar{L} i}$ and $\hat{\gamma}_{H \bar{H}}$ have been assumed to be positively correlated), the expression in (7.10) at $w=\bar{w}^{* 2}$ and $\bar{H}^{*}=\bar{H}^{* 2}$, and hence $\bar{w}^{* 2}$, are negative. In that case no collective exit catastrophe occurs for positive $w$, so the majority will, even in the long run, remain maximally employed when $w$ falls to zero. This can describe a strong form of "collective workaholism" (see the description of "collective workaholics" at the end of Sec. 5.2 and ef. the end of Subsec. 5.5.3).

\subsection{Concluding remarks}

This chapter has analysed an AQM for labour supply which combines the effects of behavioural preference interdependence (BPI) with those of unstable individual habit formation (IHF). The first four sections considered BPI and unstable IHF with respect to household time only. For two cases of individual variation in household time taste. IHF strength and susceptibility to social influence, (necessary and) sufficient conditions for the existence of collective catastrophes and hysteresis as well as long-run average labour supply schedules were derived. Such labour supply schedules were found to display two domains of catastrophic long-run behaviour alongside a band of persistent long-run behavior.

The last two sections added BPI and unstable IHF with respect to consumption and corporate time, respectively, to the BPI and unstable IHF with respect to household time. Generally speaking, this did not lead to essentially different results. but there were some interesting enrichments. Especially the addition of BPI and unstable IHF with respect to corporate time implied more symmetric entry-exit pictures. However, what has not been 
considered so far, is the combined effects of adding BPI and unstable IHF with respect to both consumption and corporate time. We can then expect a combination of the effects of the separate additions. Taking all individual variation cases together, a long-run labour supply schedule will then resemble the schedule in Figure 7.1, with collective entry and exit catastrophes and with trendsetters and laggards in entry as well as exit.

The expected tendencies in characteristics of these trendsetters and laggards in terms of the varying "pure" taste parameters, IHF strengths and susceptibilities to social influence are summarized in Table 7.1. From this table we see that the characteristics of laggards always tend to be opposite to those of the corresponding trendsetters. At first sight, one would expect that trendsetters in exit and entry tend to have the same characteristics as laggands in entry and exit, respectively, but the table shows that this holds only for the taste parameters, the consumption IHF strength and the susceptibility to social consumption influence. Whereas laggards in entry tend to exhibit high household time and corporate time IHF strengths and high susceptibilities to social household time and corporate time influences, trendsetters in exit score oppositely on these characteristics, and hence equally to trendsetters in entry. Analogously, the latter trendsetters score oppositely on these characteristics to laggards in exit. In particular, the opposition in IHF strengths is responsible for complex trajectories

\begin{tabular}{|c|c|c|c|c|}
\hline & \multicolumn{2}{|c|}{ Entry } & \multicolumn{2}{|c|}{ Exit } \\
\hline & Trendsetters & Laggards & Trendsetters & Laggards \\
\hline$\hat{Q}_{\mathrm{u}}$ & low & high & high & low \\
\hline $\boldsymbol{Q}_{\mathbf{H}}$ & bigh & low & low & high \\
\hline $\mathbb{Q}_{x}$ & high & low & low & high \\
\hline$P_{w}-\left|2_{L u}\right|$ & low & high & low & high \\
\hline$\hat{P}_{\mathrm{HHi}}-\left|\hat{\mathbf{Q}}_{\mathrm{HHI}}\right|$ & low & high & low & high \\
\hline$\hat{\gamma}_{x x a}-\left|\hat{a}_{x x a}\right|$ & high & low & low & high \\
\hline$\hat{\gamma}_{L \bar{u}}$ & low & high & low & high \\
\hline$P_{\text {Hat }}$ & low & high & low & high \\
\hline$P_{x R}$ & high & low & low & high \\
\hline
\end{tabular}

Table 7.1 Characteristics of trendsetters and laggards in entry and exit in terms of "pure" taste parameters, IHF strengths and susceptibilities to social influence for household time, corporate time and consumption. 
within the band of persistent long-run behavior in a long-run average labour supply schedule like that in Figure 7.1 (see Sec, 4.8). ${ }^{9}$

When other parameters or exogenous variables than those in Table 7.1 vary among the group members such as, for instance, the (discretionary) income of the husband (component of $Y_{O}$ ) for married women, this will have the same kind of effects as variation in taste parameters. In particular, the expected tendencies in characteristics of trendsetters and laggards in terms of the varying parameter(s) or variable(s) are similar.

Furthermore, we remark that the labour supplies, and hence the incomes of the husbands of married women, may be high and persistent as a result of strong collective habit formation by these husbands with respect to (family) consumption, corporate time and household time (cf. Sec. 3.5). This has a suppressing effect on the long-run labour supply by married women. Conversely, low levels of the latter labour supply will, in the long run, reinforee high levels of the male labour supply (which is then a state variable for female labour supply). On the other hand, collective catastrophes in either labour supply will, in the long run, affect the other one and may even trigger collective catastrophes in the other one. This implies a fascinating dynamics of interacting collective habit formations in male and female labour supply. From the discussion in Subsection 2.2 .7 it follows that there are more sources of such a dynamics than the interaction via family income. However, an elaboration of the resulting dynamics is beyond the scope of this study.

Finally, in Section 3.6 it has been argued that the effects of institutional or demand side rationings of labour supply and/or the effects of endogenous social security benefits should be added to our IHF models of labour supply. This holds also with respect to our BPI and combined IHF/BPI models. As shown in Subsections 2.2.3 and 2.2.4, both complications lead to discontinuous short-run individual labour supply schedules like those in Figures 2.3 and 2.4. This means that discontinuous rationings and/or social security benefits polarize individual labour supply in a way which resembles the long-run polarization by unstable IHF (see also other models of dichotomous short-run behaviour like the participation model in Ch. 9). A difference is that unstable IHF, in contrast to the former phenomena, implies also individual hysteresis. However, adding only weak IHF with respect to household time, consumption or corporate time to the discontinous short-run individual labour supply schedules, we obtain discontinuous long-run individual labour supply schedules like (the lower half of) Figure 3.3 in Section 3.5 or Figure 4.2 in Section 4.2, which describe individual hysteresis in addition to individual catastrophes. Combining these models with BPI, we are in a same kind of situation as in the models of unstable IHF and BPI analysed in this chapter. More specifically, we can distinguish two cases like Cases III and IV in Table 6.1 in Subsection 6.2.4 without the upper pairs of conditions for unstable IHF. Necessary and sufficient conditions for a collective catastrophe to occur are then likely to resemble the lower condition (7.4) in Case IV, but are not derived here. On the other hand, Section 9.10 will derive such a condition for a dichotomous participation model.

${ }^{9}$ Note that the equal scores in Table 7.1 on the household and corporate time IHF strengths as well as the equal scores on the susceptibilities to social household and corporate time influences are implied by the assumed positive correlations of these characteristics for household time and corporate time. 


\section{Appendix 7A Derivation of (7.3)}

The expression for $w_{i}^{* 2}=w_{-i}^{*}$ according to the pertinent variant of (4.13) can be rewritten as

$$
w_{i}^{* 2}=\frac{a_{i}^{*}}{2 T\left|\alpha_{X X i}\right|}\left[1-\sqrt{1-\frac{4 \alpha_{L i}^{*}\left|\alpha_{X X i}\right|}{a_{i}^{* 2}}}\right] \text {. }
$$

For $4 \alpha_{L i}^{*} T\left|\alpha_{X X i}\right| \ll a_{i}^{* 2}$ this formula can be approximated as

$$
w_{i}^{* 2}=\frac{a_{i}^{*}}{2 T\left|\alpha_{X X i}\right|}\left[1-\left(1-\frac{2 \alpha_{L i}^{*} T\left|\alpha_{X X i}\right|}{a_{i}^{* 2}}\right)\right]=\frac{\alpha_{L i}^{*}}{a_{i}^{*}},
$$

which is identical to (7.3).

\section{Appendix 7B Derivation of equality of surface areas $S_{1}$ and $S_{2}$ in Figure 7.2}

The average labour supply $\tilde{H}^{* 3}$ in Figure 7.1 and the corresponding marginal household time taste $\alpha_{L}^{3}$ in Figure 7.2 are determined by the condition that the long-run entry wage $w_{i}^{* 1}$ of the corresponding marginal entrant(s) as given by (7.2) is equal to the "collective entry wage" $\bar{w}^{* 1}$. In the present case of exogenous individual variation in the household time taste $\alpha_{L}$ only, this holds if and only if

$$
\alpha_{L}^{3}+\gamma_{L L} L^{* 3}=\alpha_{L}^{1}+\gamma_{L L} L^{* 1} \text {, }
$$

where $\breve{L}^{* i}$ is the average household time corresponding to $\mathscr{H}^{* 3}$ for $i=1,3$. Since $L^{* i}$ is equal to $T\left[1-F\left(\alpha_{L}^{i}\right)\right]$ for $i=1,3$ (see Sec. 7.2), (7B.1) is equivalent to

$$
\alpha_{L}^{3}-\alpha_{L}^{1}=\gamma_{L L} T\left[F\left(\alpha_{L}^{3}\right)-F\left(\alpha_{L}^{1}\right)\right] \text {. }
$$

or

$$
\left(\alpha_{L}^{3}-\alpha_{L}^{1}\right) \frac{1}{\gamma_{L L} T^{T}}=F\left(\alpha_{L}^{3}\right)-F\left(\alpha_{L}^{1}\right)
$$

In Figure 7.2 the left-hand side of this equation represents the surface area under the $1 /\left(\gamma_{L L} T\right)$ line between $\alpha_{L}^{1}$ and $\alpha_{L}^{3}$ and the right-hand side is equal to the surface area under the curve of the density function between $\alpha_{L}^{1}$ and $\alpha_{L}^{3}$. It then follows easily that the surface areas $S_{1}$ and $S_{2}$ are equal to each other. 


\section{CHAPTER 8}

\section{Dynamics of Social Norms}

"... I took pleasure in the same vices not only for the enjoyment of what I did", but also for the applause I won."

The Confessions of Augustine, Book II, Sec. IIII

A theory of preference formation which is related to the theories of individual habit formation and behavioural preference interdependence considered in the previous chapters, is the theory of social custom (TSC) of Akerlof (1980). This theory modeis the impacts of beliefs in social norms on economic behaviour as well as a long-run feedback of that behaviour on the social norm beliefs. The former impacts represent the effects of internalized social norms and social control as well-known in sociology. The latter feedback describes processes of internalization and "externalization" of a social norm, by which beliefs in that social norm change.

In this chapter a variant of the TSC along the lines of Romer (1984) is applied to labour supply. In preparation to this application, Section 8.1 reviews the basic assumptions of the TSC and Sections 8.2 and 8.3 give an overview of social norms which seem to play a role in male and female labour supply. Only the implications of social norms in female labour supply are elaborated, but the implications of social norms in male labour supply are similar. Sections 8.4 and 8.5 show that the application of the TSC to a so-called household norm is analogous in structure to a limiting case of Case B of the AQM of collective habit formation in Section 7.3. This is with the proviso that the intergenerational process by which social norm beliefs change in the conception of Akerlof is replaced by an intrapersonal belief changing process. On a conceptual level, this relativizes the distinction between social norms and habits as made by Elster $(1989, p$. 100). If we envision the concept of habit in a broader sense as general attitude or habitus (cf. Bourdieu and Passeron, 1971) and with the qualification just mentioned, the effects and dynamics of social norms in the TSC can then be interpreted as one of the possible "channels" through which unstable IHF and BPI may proceed. Moreover, it is argued that the parameter restrictions on the AQM which correspond to the labour supply application of the TSC, are implausible. This is important, since, as analysed in Section 8.6, the insights and conditions which have been obtained from the AQM, turn out to be sensitive to these parameter restrictions on a number of essential points. One of these points is that the restrictions automatically imply anstable IHF, and hence individual catastrophes and individual and collective hysteresis as well as very weak 
conditions for collective catastrophes, whereas in the AQM stable IHF and absence of catastrophes and hysteresis are certainly possible. ${ }^{\text {I }}$

In Section 8.7 a comparison with other applications of the TSC by Akerlof (1980), Romer (1984), Booth (1985) and Naylor (1989) makes clear that the present application adds two pairs of essential elements to the overall picture, namely the pair of individual and collective catastrophes and the pair of individual and collective hysteresis. However, the extended model has still a number of drawbacks and should therefore be further extended. This is done in the Sections $8.8-8.10$ by introducing the effects of a social nom of financial independence in the female labour supply model. Finally, Section 8.11 concludes.

\subsection{Theory of social custom}

In an article under the title "A theory of social custom, of which unemployment may be one consequence" (1980), George Akerlof presents a theory which explains why social norms are obeyed even when that is disadvantageous from the usual economic point of view. ${ }^{2}$ Akerlof uses this theory to explain the persistence of a fair rather than a market-clearing wage, implying an explanation of involuntary unemployment. Here we apply Akerlof's theory to labour supply.

Akerlof starts from the following assumptions. First, he assumes the existence of a social norm in a community, with a fraction of that community (denoted $m$ ) subscribing to this norm. Second, it is assumed that persons $i$ care about their reputation $R_{i}$ in their community in addition to caring about their consumption $X_{i}$ of goods and services and, for believers in the social norm, in addition to caring about the agreement of their actions with that norm. This leads to individual utility functions

$$
\tilde{U}\left(X_{i}, R_{i}, A_{i} ; n_{i}, \varepsilon_{i}\right)
$$

where $A_{i}$ is a dummy variable equal to one if individual $i$ obeys the social norm and equal to zero if $i$ disobeys the social norm, $n_{i}$ is a dummy variable equal to one if $i$ believes in the norm and equal to zero if $i$ does not believe in the norm, and $\varepsilon_{i}$ represents $i$ 's personal tastes. The variables $X_{i}, R_{i}$ and $A_{i}$ are choice variables, whereas $n_{i}$ is a state variable (see below) and $\varepsilon_{i}$ is an exogenous parameter.

However, the reputation $R_{i}$ and the social norm behaviour $A_{i}$ are not independent choice variables. According to Akerlof's third assumption the reputation of an individual depends on her obedience of the social norm and also on the fraction $m$ of community members who believe in that norm. The larger the number of believers, the more reputation is lost by disobeying the norm. The reputation of person $i$ can therefore be written as a function

${ }^{1}$ See also the labour supply model of Kapteyn and Woittiez (1990) and the consumption demand model of, e.g., Pollak (1976a) (who, however, use linear specifications other than the AQM).

${ }^{2}$ Akerlof speaks of social customs and codes of honour or codes of behaviour, but following De Neubourg (1988), I prefer to use the term of "social norm", since the latter term has the advantage of denoting a concept which is commonly used in sociology. Its meaning covers that of code of behaviour as meant by Akerlof, whereas the behaviour which is prescribed by a social norm, can be considered as a social custom. 
$R\left(A_{i} ; m\right)$ which depends positively on $A_{i}$ and negatively on $m$ (and the parameters of which are assumed independent of $i$ ). This describes the effect of social control as wellknown in sociology.

Substituting the reputation functions in the utility functions (8.1), we see that the individual utilities depend on the social norm behaviour $A_{i}$ in two ways. First, indirectly via the sanction of loss of reputation in case of disobedience of the norm, and second, for believers in the norm, directly since believers care about the agreement of their action with the norm. ${ }^{3}$ If believers do not follow the norm, they suffer from a utility loss due to a discrepancy between their behaviour and their own norm belief. This utility loss can be associated with the concept of cognitive dissonance of Festinger (1957) ${ }^{4}$ and with feelings of guilt (cf. Earl, 1983). It may be conceived as consisting of losses of, among other things, self-esteem (or "reputation with oneself"!), self-confidence and personal identity and meaning (cf. Lutz and Lux, 1988). These inner sanctions of not obeying the norm are the result of internalization of the norm, which is another key concept of sociology.

In order to analyse the implications of such a model, Akerlof considers a particular specification of the individual utility functions ( 8.1 ) which is given by

$$
\hat{U}\left(X_{i}, R_{i}, A_{i} ; n_{i}, \varepsilon_{i}\right)=\alpha X_{i}+\beta \varepsilon_{i} R_{i}-\gamma n_{i}\left(1-A_{i}\right)
$$

(omitting an irrelevant constant). Here $\alpha, \beta, \varepsilon_{i}$ and $\gamma$ are all positive parameters. The taste parameter $\varepsilon_{i}$ indicates one's sensitivity to reputation and $\gamma$ represents the loss of utility from (fully) disobeying the social norm $\left(A_{i}=0\right)$ by a (full) believer in the norm $\left(n_{i}=1\right)$. The reputation function is specified as

$$
R\left(A_{i}, m\right)=-\rho m\left(1-A_{i}\right)
$$

Here $\rho$ is a positive parameter denoting the loss of reputation from (fully) disobeying the social norm $\left(A_{i}=0\right)$ when everybody else (fully) believes in the norm $(m=1)$. Substituting (8.3) into (8.2) yields

$$
U_{i}=\alpha X_{i}-\beta \varepsilon_{i} \rho m\left(1-A_{i}\right)-\gamma n_{i}\left(1-A_{i}\right) .
$$

The second and third term represent the sanctions of loss of reputation and cognitive dissonance, respectively, and model the effects of social control and internalization of the social norm, respectively. The sanctions introduce elements of, respectively, social-psychological and individual-psychological inertia into the behaviour of individuals.

Specification (8.4) can be simplified by "transforning away" terms which are independent of the short-run choice variables $X_{i}$ and $A_{i}$ (see Sec. 4.1). This leads to

$$
U_{i}=\alpha X_{i}+\beta \varepsilon_{i} \rho m A_{i}+\gamma n_{i} A_{i}
$$

Now the second and third term do not represent sanctions of utility losses, but can be interpreted as rewards of utility gains in terns of reputation and cognitive consonance for

\footnotetext{
${ }^{3}$ The implied dualistic motivation structure of the nom behaviour resembles that according to the attitude/subjective nom theory of Ajzen and Fishbein (1977).

${ }^{4}$ See also Akerlof and Dickens (1982). Tazelaar and Wippler (1981) and De Neubourg (1985).
} 
obeying the social norm. This implies the interesting conclusion that the social norm behaviour of the individuals is independent of whether it is stimulated by sanctions of utility losses or by rewards of utility gains! The reason of this equivalence relation is that we have here a model of myopic behaviour, in which individuals do not take account of future utility gains or losses in their decision (cf. Sec. 4.6).

In the short run, the community fraction of believers $m$ and the individual norm beliefs $n_{i}$ are exogenous with respect to the choice variables. Given these beliefs, the threats of loss of reputation and cognitive dissonance motivate the members of the community to conform their behaviour to the social norm. However, since the community members also care about their consumption, it may be advantageous for some members not to conform to the norm. Akerlof assumes that, in the long run, this undermines the belief in the social norm in the community. If there are fewer persons obeying the norm in the current generation than there are believers, the number of believers in the next generation will fall to the number of obedients. Conversely, community members who do not believe in the social norm, but are induced to conform to it by fear of loss of reputation, are assumed to reinforce the belief in the social norm in the community. If there are more persons obeying the norm in the current generation than there are believers, the number of believers in the next generation will rise to the number of obedients. This assumption of Akerlof can be interpreted as describing that the childrens' generation internalizes social norms according to the social norm behaviour of the parents' generation. Thus, the internalization of norms by children is not determined by what educators tell them, but by what these educators actually do, i.e. by the examples they make. This seems quite plausible and implies a normative kind of BPI where the parents' generation forms normative reference groups of the children (see Subsec. 5.1.2). As a particular specification of this preference formation process Akerlof assumes the differential equation

$$
\dot{m}=\delta(F-m) \text {, }
$$

where $F$ is the fraction of the community which obeys the norm and $\delta$ is a positive adjustment parameter. When the individuals are not rationed in their choice behaviour, $F$ is a function of the fraction of believers $m$. In general this function is non-linear, implying that the differential equation (8.6) is non-linear.

In order to apply this model to labour supply we should specify the content of the social norm in relation to labour supply. There are then several possibilities for male as well as female labour supply. These possibilities are discussed in the next two sections.

\subsection{Social norms in male labour supply}

In Western societies a strongly dominant social norm in relation to labour supply is given by the work ethic norm that a person should work for a living. This norm goes back as far as biblical wisdom telling Adam "In the sweat of thy face shalt thou eat bread". 5 Notwithstan-

5 This norm should, however, not be identified with the related, but stronger work ethic norm which is implied by the Protestant Work Ethic as conceived by Max Weber. See Fumham (1990) for 
ding the existence of elaborated systems of social security providing transfer incomes of different types, this work ethic norm is still adhered to by vast majorities of the populations. It is sustained and reinforced by the fact that not only money income, but also status, prestige and power are distributed among the population according to whether or not a persons works and the type of work he/she does.

It is, however, clear that the work ethic norm does not apply to the entire population equally: the norm recognizes legitimate reasons for non-application. The most obvious groups within the population for which the work ethic norm is believed to be irrelevant or less relevant, are the very young members of the population, the older members, those who are unable to work, those who follow full-time education, and those who have income from property ${ }^{6}$. Since the nom is said not (or less) to apply to the members of these groups, they are not expected to obey the norm and their legitimized "disobedience" must not be interpreted as potentially undermining the work ethic norm. Therefore, these groups are left out from the analysis. Crudely put, the remaining part of the population can be described as the individuals aged between 16 and 64 , except those who are unable to work, follow full time education or have income from property. However, even this group cannot be regarded as homogeneous in relation to the work ethic norm. At least two major groups have to be distinguished: men and women. The first group will be considered in this section and the second group in the next section.

The norm that an individual should work for a living applies in full force to the male members of the restricted population. In our societies this means that male individuals are supposed to have a job or at least to actively look for a job. Thus, obedience or disobedience to this norm is a positive function of the individual labour supply in terms of paid corporate hours $H_{i}$. Then, however, the question arises how many paid corporate hours should be worked in order to obey the norm, or how large (small) $H_{i}$ should be to make $A_{i}=1$ $\left(A_{i}=0\right)$. A simple assumption is to define $A_{i}=0$ for $H_{i}=0$ and $A_{i}=1$ for $H_{i}>0$, but this does not seem very adequate, since it is implausible that already a very small number of paid corporate hours is sufficient to (fully) obey the norm.

A more plausible alternative is to suppose that the work ethic norm for men states not only that a man should have a job, but also that he should eam enough money in this job (or jobs) to make a (decent) living for himself and/or his family without needing a supplementary social security benefit. We call this the breadwinner norm. This norm can be assumed to be (fully) obeyed $\left(A_{B_{i}}=1\right)$ if and only if the total non-benefit income is at least equal to the social minimum $Y_{S M i}$ (which depends on the size of $i$ 's family). Supposing that the non-labour income of the man as well as the incomes of the other family members are zero $\left(Y_{O i}=0\right)$, the total non-benefit income is equal to the man's labour income $w_{i} H_{i}$. Then the breadwinner nom variable $A_{B i}$ as a function of the man's paid corporate hours $H_{i}$ can be specified as

a recent survey of psychological research on the Protestant Work Ethic; see also Scitovsky (1977) and see below.

"It is interesting to note that in some cases and during specific periods in history (e.g., among the propertied classes in 18 th and 19 th century England) stigma attached to working (at least in "trade") rather than to idleness. Although the mechanisms that are responsible for this important exception. are fascinating, we disregard such cases in order to simplify the present analysis. 


$$
\begin{aligned}
& A_{B i}\left(H_{i}\right)=1, \quad \text { if } w_{i} H_{i} \geq Y_{S M i}, \text { or } H_{i} \geq \frac{Y_{S M i}}{w_{i}}, \\
& A_{B i}\left(H_{i}\right)=0, \quad \text { if } w_{i} H_{i}<Y_{S M i}, \text { or } H_{i}<\frac{Y_{S M i}}{w_{i}} .
\end{aligned}
$$

This sharp dichotomy between fully obeying and not obeying the breadwinner norm can be shown to be consistent with empirical results of Moffiti (1983) on the stigmatization effects of participation in a welfare program in the U.S.A.. In Moffitt's model the disutility of participation has a flat stigma component arising from participation per se as well as a variable stigma component which varies with the size of the benefit. An empirical test of this model suggests a strong flat stigma, but no variable stigma. However, Moffitt has some data problems and his results seem less plausible for social security systems which are less stigmatizing than the welfare program considered in his paper. ${ }^{7}$ Then it seems to matter for the breadwinner norm whether a man who draws a supplementary benefit, earns a larger or smaller part of his income in a job. In such a case it seems more realistic to conceive the breadwinner norm variable $A_{B i}$ as a continuous variable with values in the range $[0,1]$, indicating to what extent a person is obeying the breadwinner norm (see also Romer, 1984). The discrete specification (8.7) may then be replaced by the continuous specification

$$
\begin{array}{ll}
A_{B i}\left(H_{i}\right)=\left(\frac{w_{i} H_{i}}{Y_{S M i}}\right)^{\eta_{B}}, & \text { if } H_{i} \leq \frac{Y_{S M i}}{w_{i}}, \\
A_{B i}\left(H_{i}\right)=1, & \text { if } H_{i} \geq \frac{Y_{S M i}}{w_{i}} .
\end{array}
$$

Here $\eta_{B}$ is a positive elasticity parameter which depends on how the norm obedience is perceived. When it is judged as important whether a man needs a (supplementary) social security benefit per se (cf. the flat stigma of Moffitt), the norm obecience for $H_{i}$ in the transfer regime will be relatively low, which is described by a value of $\eta_{\nexists l}$ larger than one (see Fig. 8.1). In particular, when $\eta_{B}$ goes to infinity, the continuous specification (8.8) approaches the discrete specification (8.7). On the other hand, when the extent of nom obedience is judged only according to the size of the benefit needed (cf. the variable stigma

${ }^{7}$ For that welfare program the participation rate within the eligible population was estimated by Moffitt to be only about $\mathbf{4 5}$ percent. This is cenainly much lower than the corresponding participation rate for most European social security systems and seems to be related to stronger stigmatization effects of the U.S.A. welfare program. In fact, our treatment of the breadwinner norm implicitly assumes that when a person is eligible for a (supplementary) social security benefit, he will take it. 
of Moffitt), the norm obedience for low levels of the benefit will be relatively high, which is described by a value of $\eta_{B}$ equal to one or even smaller than one. ${ }^{8}$

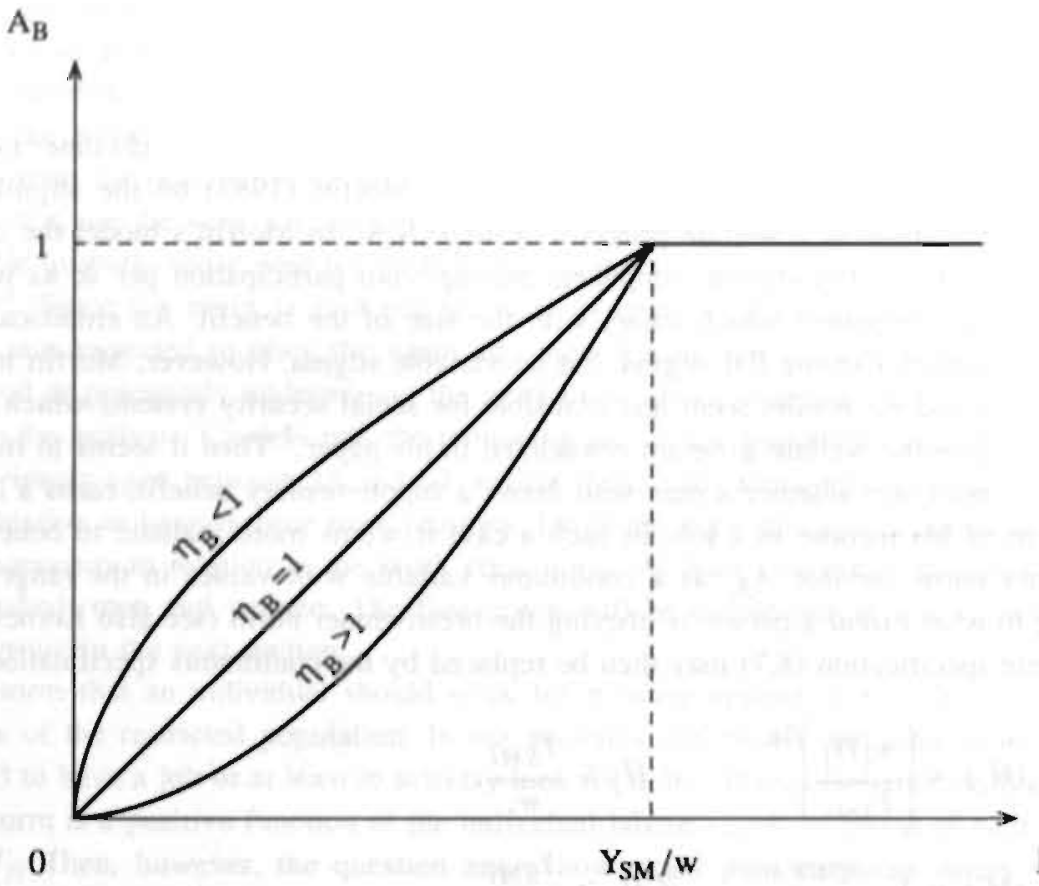

Figure 8.1 Extent of obedience to the breadwinner norm as a function of corporate time.

When a man needs a (supplementary) benefit for his family, and hence does not fully obey the breadwinner norm $\left(A_{B i}<1\right)$, he will suffer from a loss of reputation as a breadwinner (negative $R_{B i}$ as implied by (8.3)) and, if he believes himself in the breadwinner norm (positive $n_{B i}$ ), from cognitive dissonance (negative third term on the right-hand side of (8.2)). It is also possible that a man is supposed to obey an even stronger work ethic norm, namely that he should earn a labour income which is as high as possible given the normal full-time working week and given his earning capacity. There is at least one underlying reason for such a norm. A "good family man" (in a traditional sense) may be expected to provide his family with the highest possible wealth given the normal full-time working week. (There may be a second underlying reason: a "good citizen" might be supposed to make the highest possible contribution to the total production in his society as measured by his labour income.) Whatever be the underlying reason, this work ethic norm implies that a

s It is also possible that in the own perception of a person who does not fully obey the nom, the extent of nom obedience for $H_{i}$ in the transfer regime is higher, and hence the elasticity parameter $\eta_{B}$ lower, than in the perception of relevant others who do fully obey the norm. This may be due to reduction of cognitive dissonance and implies a difference in specification of the norm variable as function of $H_{i}$ in the second and third terms of the utility function (8.5). 
man should work full-time in a job (or jobs). We call it the (good) family man norm. Its implications do not differ very much from those of the breadwinner norm. In terms of the specification (8.8), $Y_{S M i} / w_{i}$ has to be replaced by the number of hours in a normal full-time working week with the side condition that the hourly wage rate $w_{i}$ should be as high as possible and at least positive. In this case a man already suffers from a reputation loss and, if he believes himself in the norm, from cognitive dissonance, when he works less than fulltime.

One further step may be to assume that the contents of the work ethic norms are related not only to simple subsistence or to the standard full-time working week, but also to the implicit norms governing the career process (or the implicit norms defining whether a man can be considered as belonging to a certain group such as, e.g., "the dedicated workers"). In order to make a career (or to be accepted as a member of a certain group), it may be necessary to do more than just work the standard number of corporate hours. It may be required that one spends all or practically all his discretionary time on his corporate work. This social norm may be called the "macho norm". The social values underlying it may be related to the Protestant Work Ethic as conceived by Max Weber (see Maccoby and Terzi, 1979, and Fumham, 1990). Again, the analysis is not fundamentally different from the previous cases. The macho norm may be fully obeyed only above, say, 60 weekly hours of corporate work. ${ }^{9}$ Consequently, unless a man works more than 60 corporate hours per week, he will lose reputation as a macho and, if he believes himself in the macho norm, suffer from cognitive dissonance.

Although the hypothetical work ethic norms which have been considered in this section, are rather traditional, they seem still to play a dominant role in modern societies. This holds especially for the breadwinner norm and the farnily man norm, whereas the macho norm seems important only in (certain) social groups. On the other hand, there are indications that these norms have been eroding in subcultures of long-term unemployed and of certain other social groups. In the latter groups the macho norm is absent and the family man and breadwinner norms have been relativized in conjunction with changing role definitions of the male and female partners in the family. As a result of these role changes, a new norm for men may be emerging, which will be explained after an analysis of social norms in female labour supply in the next section.

\subsection{Social norms in female labour supply}

In the case of social norms in female labour supply a sharp distinction should be made between single and non-single women. Just as for men, for single women (between 16 and 64 years old) the breadwinner norm holds, i.e., a single woman is expected to make a (decent) living for herself and/or her child(ren) by a job without needing a supplementary social security benefit, unless a legitimate reason makes the norm inapplicable. The family man norm, prescribing full-time corporate work, may apply to a lesser degree than for men

${ }^{9}$ Formally, one is then often paid for a standard workweek only, but the overtime can be considered as being implicitly paid for by a higher hourly wage rate. 
and the macho norm does not generally seem to apply (when it does apply, it could be called the virago norm).

Non-single women are mostly married. Therefore and since we want to start the analysis with traditional norms, we consider first the traditional work ethic norm for married women. The general work ethic norm that a person should work for a living (see the beginning of the previous section) can be said to apply to married women too. However, traditionally this general norm has been interpreted for married women in a quite different sense than for men. Whereas in a traditional role definition the husband is expected to earn the family income in a job, the wife is supposed to take care of the household and the children. In fact, the wife is even supposed to devote ali her discretionary time to the household and the children and not to be involved in paid work elsewhere. This norm, which we refer to as the household norm, played a dominant role in many OECD countries during the fifties and sixties.

Obviously, obedience or disobedience of the household norm $A_{L i}$ is a positive function of the number of discretionary hours which is spent in the household, so of the household time $L_{i}$. Moreover, the household nom is fully obeyed $\left(A_{L i}=1\right)$ only when all the wife's discretionary time $T_{i}$ is spent in the household. This amount of discretionary time may be conceived as depending negatively on the number of children of woman $i$ and positively on the available home help and day care (see also Sec. 2.3 ; thus, $T_{i}$ may be larger or smaller than a full-time working week). We could then assume $A_{L i}=0$ for $L_{j}<T_{i}$ and $A_{L i}=1$ for $L_{i}=T_{i}$, but, just as in the case of work ethic norms for men, such a sharp dichotomy between disobeying and obeying the norm does not look very realistic. Analogously to (8.8) in the previous section, a more realistic specification of $A_{L i}\left(L_{i}\right)$ would then be

$$
A_{L i}\left(L_{i}\right)=\left(\frac{L_{i}}{T_{i}}\right)^{\eta_{L}}
$$

where $\eta_{L}$ is a positive elasticity parameter which depends on how the norm obedience is perceived (cf. Fig. 8.1). This specification implies that the nom obedience $A_{L i}$ increases continuously from 0 for $L_{i}=0$ to 1 for $L_{i}=T_{i}$.

More specific variants of the household norm may also play a role. the obedience of which is a positive function of the amount of time devoted to household production or of the results of household production such as clean windows, ironed shirts and/or a hot meal each day. When, however, the straightforward household norm is the dominant and society-wide accepted norm for married women, married women do not lose utility due to a loss of reputation as a housewife and mother and due to cognitive dissonance if and only if they spend all their time in the household, so when they do not have a job.

Confronting this theoretical conclusion with the empirical fact of a strong increase in the labour force participation of married women in many OECD countries since World War II (see Sec. 9.1 for details), we may explain the latter decrease in obedience of the household norm by strong decreases in the average belief in the household norm in that period. In fact. empirical evidence for the Netherlands supports such an explanation (see Sec. 9.2). At the same time new work ethic norms as advocated by women's liberation movements may have been emerging. The most important one is a financial independence norm, stating that a nonsingle woman should have a job in which she earns so much as to be financially independent 
of her (male) partner. Analogously to the continuous specification (8.8) of the extent of obedience to the breadwinner norm (and neglecting effects of the tax system), the extent of obedience to the financial independence norm $A_{F i}$ as a continuous function of the woman's paid corporate hours $H_{i}$ could be specified as

$$
\begin{array}{ll}
A_{F i}\left(H_{i}\right)=\left(\frac{w_{i} H_{i}}{Y_{I M i}}\right)^{\eta_{F}}, & \text { if } H_{i} \leq \frac{Y_{I M i}}{w_{i}}, \\
A_{F i}\left(H_{i}\right)=1, & \text { if } H_{i} \geq \frac{Y_{I M i}}{w_{i}} .
\end{array}
$$

Here $Y_{I M i}$ is now the individual minimum necessary for woman $i$ to be financially independent (which may depend on the social class of woman $i$ ). Graphically, this specification can be represented by a graph similar to Figure 8.1 with $Y_{I M i} / w_{i}$ instead of $Y_{S M i} / w_{i}$.

Alongside or instead of financial independence, self-fulfilment in and by means of a job may have become a new work ethic norm for non-single women. The extent of obedience to this self-fulfilment norm may be specified analogously to the continuous specification (8.10) of the extent of obedience to the financial independence norm with $Y_{I M i} / w_{i}$ replaced by the minimal number of corporate hours which are judged to be necessary for self-fulfilment, $H_{M i}$ (which may depend on the kind of job of woman $i$ ). Graphically, this can again be represented by a graph similar to Figure 8.1 with $H_{M i}$ instead of $Y_{S M i} / w_{i}$.

In a liberal social group in which a belief in an "emancipation" norm of financial independence and/or self-fulfilment bas developed, a woman will suffer from a loss of reputation and cognitive dissonance as an "emancipated" woman, when she does not have a job of sufficient hours to be financially independent and/or to fulfil herself. At the same time, however, such a woman may belong also to another social group (e.g., her next of kin) several members of which still believe in the household norm. Moreover, the belief in the household may not completely have disappeared in the liberal group and/or in the woman herself. When in such a situation the woman has a job in order to avoid a loss of reputation and cognitive dissonance as an "emancipated" woman, she may still suffer from a loss of reputation and/or cognitive dissonance as a housewife and mother. Thus, whatever she does, she will never be without reputation loss and cognitive dissonance. This describes (external) role conflicts as well-known in sociology.

The changes in role definitions of the male and female partners in the family, which induce such role conflicts, may also lead to an extension of the breadwinner norm to nonsingle women in the sense that both partners are supposed to contribute to making a (decent) living for themselves and/or their children. As a counterpart of this norm there can then emerge an extension of (a weak variant of) the household norm to men in the sense that both partners are expected to contribute to the care of the household and/or the children.

\subsection{Household norm and collective habit}

Having specified the possible contents of social noms in relation to male and female labour supply in the previous two sections, we can elaborate the implications of Akerlof's theory of 
social custom, as described in Section 8.1, for male and female labour supply. In this study, however, only the effects and dynamics of social norms in female labour supply are elaborated. This and the subsequent two sections derive the implications of the household norm, whereas Sections 8.8-8.10 add the effects of the financial independence norm.

In order to derive first some general implications of the household norm, we substitute its specification (8.9) into the general individual utility functions (8.1) and into the general reputation function (as specified by, e.g., (8.3)). Substituting the reputation function into (8.1), we then obtain individual utility functions $U\left(X_{i}, L_{i} ; n_{L i}, m_{L}, \varepsilon_{L i}\right)$. Maximization of these utility functions with respect to $X_{i}$ and $L_{i}$ under the usual income constraints $X_{i} \checkmark w_{i}\left(T_{i}-L_{i}\right)+Y_{O i}$ yields individual consumption and household time demand functions $X\left(w_{i}, Y_{O i}, T_{i}, n_{L i}, m_{L}, \varepsilon_{L i}\right)$ and $L\left(w_{i}, Y_{O i}, T_{i}, n_{L i}, m_{L}, \varepsilon_{L i}\right)$, respectively. When these demands are not rationed, they determine the short-run behaviour of married women for given values of the individual and overall household norm beliefs $n_{L i}$ and $m_{L}$, respectively.

In the long run, these norm beliefs are assumed to change endogenously according to the differential equation (8.6). However, in order to be able to express the overall nom obedience $F$ in this equation in terms of the individual household times, we should first reinterpret it in line with the reinterpretation of the individual household norm obedience $A_{\mathrm{Li}}$ as at continuous variable in the previous section. This reinterpretation is that $F_{L}$ represents the average extent of obedience to the household norm $\bar{A}_{L}$ in a community or social group of married women. ${ }^{10}$ When the elasticity parameter $\eta_{L}$ in (8.9) happens to equal one, implying a linear specification, $\bar{A}_{L}$, and hence $F_{L}$, is approximately equal to the average household time $\bar{L}$ in the social group divided by the average discretionary time $\bar{T}$. Substituting this into the equation (8.6), multiplying the left and right-hand sides of (8.6) by $\bar{T}$, and aggregating the individual to average household time demand functions as in equations (4.24) and (5.9), we obtain for the non-rationing case

$$
\bar{T} \dot{m}_{L}=\delta_{L}\left[\bar{L}\left(\bar{w}, \bar{Y}_{O}, \bar{T}_{,} \underline{n}_{L}, m_{L}\right)-\bar{T} m_{L}\right] .
$$

Here the absolute or relative deviations of the $i$-dependent exogenous variables $w_{i}, Y_{O i}$ and $T_{i}$ from their averages (see Assumption $X$ in Sec. 5.2) and the distribution of the personal tastes $\varepsilon_{i}$ are assumed to be constant, and hence omitted from the arguments of the average household time demand function. Furthermore, $\underline{n}_{L}$ is the vector $\left(n_{L 1}, \ldots, n_{L N-1}\right)$ of individual norm beliefs. ${ }^{11}$

These individual norm beliefs as well as the overall norm belief $m_{L}$ should also be reinterpreted. In Section 8.1 equation (8.6) has been interpreted as describing that the childrens' generation internalizes social norms according to the social norm behaviour of the parents' generation. Since this social norm behaviour has now become a continuous variable. it seems adequate to conceive the individual norm belief $n_{L i}$ too as a continuous variable with values in the range $[0,1]$, indicating to what extent the married woman $i$ believes in the household norm (see also Romer, 1984). Then the overall norm belief $m_{L}$ can be inter-

${ }^{10}$ Note that $F$ was already equal to $\bar{A}$ in the discrete case.

11 The Nih individual nom belief $n_{L N}$ is omitted, since it is determined by the $N-1$ other nom beliefs and the average nom belief $\bar{n}_{L}=m_{L}$ (see below). See also Section 6.1. 
preted as the average extent of belief in the household norm in a social group of married women $\bar{n}_{L} \cdot{ }^{12}$

Now it is interesting to compare the general model derived so far with our general model of IHF and BPI with respect to household time only as analysed in Section 6.1. The latter model assumes utility functions $U^{i}\left(X_{i}, L_{i} ; s_{L i}, s_{L}^{-}\right)$and the differential equation (6.2), where the vectors $\underline{w}, \underline{Y}_{O}$ and $\underline{T}$ in the average demand function can be replaced by their averages $\bar{w}, \bar{Y}_{O}$ and $\bar{T}$. These functions and equation happen to be analogous to the utility functions $U\left(X_{i}, L_{i} ; n_{L i}, m_{L}, \varepsilon_{L i}\right)$ and the differential equation (8.11) obtained above, for constant $\bar{T}$, and hence constant $T_{i}, i=1, \ldots, N$ (see above). The individual household time habit $s_{L i}$ then corresponds to $T_{i}$ times the individual household norm belief $n_{L i}$, the social household time environment $s_{L}$ corresponds to $\bar{T}$ times the average household norm belief $m_{L}$, and the adjustment speed $\delta_{\bar{L}}$ corresponds to the adjustment parameter $\delta_{L}$. That this analogy is not sheer coincidence, can be clarified by considering the particular specification (8.5) of Akerlof's utility functions.

Applying specification (8.5) to our labour supply application of the household norm for married women and substituting (8.9) with $\eta_{L}=1$ into (8.5) yields

$$
U\left(X_{i}, L_{i} ; n_{L i}, m_{L}, \varepsilon_{L i}\right)=\alpha X_{i}+\frac{\gamma_{L}}{T_{i}^{2}}\left(T_{i} n_{L i}\right) L_{i}+\frac{\beta_{L} \varepsilon_{L i} \rho_{L}}{\bar{T} T_{i}}\left(\bar{T} m_{L}\right) L_{i}
$$

This specification has been written in a form comparable with the AQM specification (6.7) in Subsection 6.2.1. Putting $s_{L i}=T_{i} n_{L i}$ and $s_{\bar{L}}=\bar{T} m_{L}$, we see that (8.12) corresponds to a limiting case of (6.7) with $\alpha_{X X i}=\alpha_{L i}=\alpha_{L L i}=0$. The parameters $\alpha_{X i}, \gamma_{L L i}$ and $\gamma_{L \bar{L} i}$ should then be equated with $\alpha, \gamma_{L} / T_{i}^{2}$ and $\left(\beta_{L} \varepsilon_{L i} \rho_{L}\right) /\left(\bar{T} T_{i}\right)$, respectively.

The parameter restriction $\alpha_{L i}=0$ is due to the assumption implicit in (8.2), (8.3) and (8.9) that a married woman loses or gains utility from household time only via obeying more or less the household norm. This assumption is very implausible, since a married woman will derive more or less pleasure (or nuisance) other than reputation and cognitive consonance from staying at home. A simple way to take such norm-independent "psychic income" from household time into account is adding the term $\alpha_{L i} L_{i}$ to the utility function (8.12). Moreover, individual differences in household time taste can then be described by the parameter $\alpha_{L i}$.

The other parameter restrictions $\alpha_{X X i}=0$ and $\alpha_{L L i}=0$ exclude diminishing marginal utilities of consumption and household time ${ }^{13}$ and are consequences of the assumed linearity of the utility function (8.2) and the reputation function (8.3) in the choice variables $X_{i}$, $R_{i}$ and $A_{i}$. The linearity of the utility function implies perfect perceived substitutability of consumption, reputation and, among other things, self-esteem. On the one hand, this property may be a good approximation of the observations that a lot of people seem to pursue an increase in reputation as a substitute for a lack of self-esteem, that others may regard self-

12 Note that $m$ was already equal to $\bar{n}$ in the discrete case. See Section 8.7 for a different interpretation of $m$ by Romer (1984, p. 722).

${ }^{13}$ See footnote 48 in Subsec. 2.2.5. 
esteem as a rather good substitute for reputation, and some persons may (consciously or unconsciously) perceive eating more food as a compensation for a lack of reputation or selfesteem. On the other hand, most people will view reputation and self-esteem as insufficient substitutes of consumption and, conversely, eating more food as a poor compensation for a lack of reputation or self-esteem. Therefore, the perceived substitutability of consumption, reputation and self-esteem seems, in general, rather imperfect. Moreover, the implied linearity of the utility function (8.12) in $X_{i}$ and $L_{i}$ has the implausible implications of constant marginal utilities and perfect substitutability of consumption and (discretionary) household time, and of indifference curves being straight lines and hence excluding, for almost all values of the exogenous variables, interior solutions for the individual short-run labour supplies. Rather it seems realistic to assume diminishing marginal utilities of consumption and household time as prescribed by the First Law of Gossen, implying imperfect substitutability of consumption and household time. This can be implemented by relaxing the parameter restrictions mentioned (see Section 8.11 for further extensions).

We can then conclude that the AQM specification (6.7) in Section 6.2 is a more adequate representation of the labour supply preferences of married women than the more restricted Akerlof specification (8.12). ${ }^{14}$ Therefore, the insights and conditions for collective catastrophes and hysteresis to occur which have been obtained from the AQM, seem more reliable than corresponding insights and conditions for the case of the Akerlof specification. Nevertheless, in order to assess the robustness of the insights and conditions according to the AQM to the restrictions in the Akerlof specification, we could compare these insights and conditions with those for the Akerlof specification. This is done in Section 8.6. However, before that we should take into account that there is a difference between the intergenerational process by which social norm beliefs change in the conception of Akerlof and the combination of individual habit formation and adaptation of the social environment as described by our "canonical" model. This is analysed in the next section.

\subsection{Dynamics of social norm beliefs}

In the conception of Akerlof the social nom beliefs change by a specific kind of social influence between generations, whereas in our "canonical" model the individual habits and social environment change by the influence of individuals on themselves and by the social influence within a generation, respectively. Thus, the intergenerational process of Akerlof is not covered by our model. On the other hand, the social norms beliefs may change not only in a very long run by the intergenerational process, but also in a much less long run by intrapersonal and intragenerational processes.

The intrapersonal process may work as follows. Believers in the norm who, nevertheless, prefer to disobey it to a cernain extent, suffer from cognitive dissonance (see (8.4)). In such a situation, in which the members do not want to change their norm-violating behaviour,

${ }^{14}$ Recall, however, from Sections 4.2 and 7.1 that also the AQM does not seem very adequate, since in the case of unstable IHF, it has the implausible implication that married women with a parttime job will, in the long run, either spend all their discretionary time in a (full-time) job or have no job at all. See Sections 8.8-8.11 for possible extensions of the AQM. 
they could reduce their cognitive dissonance in the long run by conforming their norm belief $n_{i}$ to their norm behaviour $\hat{\boldsymbol{A}}_{i}$, so by letting erode their norm belief. Conversely, disbelievers in the norm who, nevertheless, are induced to conform to it to a certain extent, may internalize the norm to the same extent. In the Akerlof model, however, this cannot be the result of reduction of cognitive dissonance, since disbelievers do not suffer from that It should rather be interpreted as the result of an enlargement of cognitive consonance (see (8.5)) as motivated by a need to justify or legitimize one's own behaviour by a social norm in which the person believes herself. As the mirror image of reduction of cognitive dissonance, this can be assumed to lead to a utility gain in terms of increases in self-esteem, self-confidence, personal identity and meaning, etc..

A particular specification of this intrapersonal belief changing process which implies the average belief equation (8.6) with $m=\bar{n}$ and $F=\bar{A}$ (see the previous section) by a simple aggregation, is given by the differential equations

$$
\dot{n}_{i}=\delta\left(A_{i}-n_{i}\right), \quad i=1, \ldots, N .
$$

In the non-rationing case the individual norm behaviour $A_{i}$ is a function of the individual norm belief $n_{i}$ and the average nom belief $m$. This implies that the individual belief equations (8.13) are then linked to each other via the average belief $m$. For constant values of the exogenous variables, a long-run (stationary) equilibrium $\underline{n}^{*}$ of this system of $N$ differential equations is given by the system of $N$ algebraic equations $A_{i}\left(n_{i}^{*}, m^{*}\right)=n_{i}^{*}$, $i=1, \ldots, N$. Thus, in long-run equilibrium all believers in the social norm obey it and all disbelievers disobey it. This is also found by Akerlof and Romer (1984). ${ }^{15}$ Moreover, substituting (8.9) with $\eta_{L}=1$ into (8.13) and multiplying the left and right-hand sides of (8.13) by $T_{i}$, we obtain differential equations which, for constant $T_{i}, i=1, \ldots, N$, are of the same form as the individual habit equations (6.1). Thus, this intrapersonal process by which social norm beliefs change implies a dynamics similar to that of our "canonical" model.

On a conceptual level, this relativizes the distinction between social norms and habits as made by Elster (1989, p. 100). If we envision the concept of habit in a broader sense as general attitude or habitus (cf. Bourdieu and Passeron, 1971), we could interpret individual and average household norm beliefs as specific aspects of individual household time habit(us) states and the social household time environment, respectively. Moreover, the effects of cognitive dissonance and reputation on individual behaviour may be regarded as specific mechanisms through which the influences of individual habits and the social environment are working. Finally, the intrapersonal process by which social norms change could be considered as a specific "channel" through which individual habit(us) formation and adaptation

${ }^{15}$ Akerlof (1980) and Romer (1984) do not consider the theoretical possibility in their models that. in a long-run equilibrium with the number of believers being equal to the number of obedients, some believers with a low sensitivity to reputation disobey the nom., whereas an equal number of disobedients with a high sensitivity to reputation obey the norm. On the other hand, when starting from a situation where all community members are believers as well as obedients, the values of exogenous variables change. the first to break the norm, and hence to lose their belief in it, are the members. with the lowest sensitivity to reputation. This suggests a positive correlation between belief and sensitivity to reputation, which excludes the theoretical possibility mentioned and which is explicitly. assumed by Naylor (1989) (see his Assumption 6). 
of the social environment are proceeding. In other words, we may interpret the former process as a specific mode of collective habit(us) formation as described by our model.

In the case of an intragenerational, but interpersonal belief changing process, matters are different. Then the individual norm beliefs are directly influenced by either the norm beliefs or the norm behaviours of other individuals of the same generation. Such individuals may form a normative reference group of the person from which she adopts social norms (see Subsec. 5.1.2). There may be several motives for this adoption of social norms like " for instance, needs for belongingness and social approval. ${ }^{16}$ Such social benefits can depend positively on the degree of conformity of one's own norm belief to the norm beliefs or norm behaviours of others, but should be distinguished clearly from reputation in the short-run model as depending positively on the degree of conformity of one's own norm behaviour to the norm beliefs of others.

Furthermore, only adoption of social norms from others in as far as this is induced by their norm behaviours, can serve as an micro-underpinning of the long-run macro-equation (8.6), since this requires a feedback of norm behaviours on norm beliefs. Adoption of social norms from others on the basis of merely their norm beliefs can explain how individual extents of norm beliefs $n_{i}$ may converge ${ }^{17}$, but it does not explain how in a community of only full believers $\left(n_{i}=m=1\right)$ the norm belief may erode or how in a community of only disbelievers $\left(n_{i}=m=0\right)$ a norm belief may emerge. ${ }^{18}$ On the contrary, the last two phenomena can be explained by a feedback of norm behaviours on norm beliefs, and a particular specification of an interpersonal variant of such a feedback, which implies the average belief equation ( 8.6 ) by a simple aggregation, is given by the differential equations

$$
\dot{n}_{i}=\delta\left(F-n_{i}\right) \quad i=1, \ldots, N .
$$

In the non-rationing case the average norm behaviour $F$ is, in general, a function of all individual norm beliefs $n_{i}$ and the average norm belief $m$. Hence, the individual belief equations (8.14) are then linked to each other. For constant values of the exogenous variables, a long-run (stationary) equilibrium $\underline{n}^{*}$ of this system of $N$ differential equations is given by $n_{i}^{*}=F\left(\underline{n}^{*}, m^{*}\right), i=1, \ldots, N$. This implies that all individual norm beliefs converge to the same average norm belief $m^{*}$.

However, since the individual norm behaviours may remain different from each other due to different sensitivities to reputation, these behaviours may deviate from the corresponding individual beliefs in long-run equilibrium. This is inconsistent with results found by Akerlof (1980) and Romer (1984) as well as with our individual habit equations (6.1). Therefore, this interpersonal process by which social norm beliefs change, generally implies another kind of

16 See, for example. Newcomb (1947).

${ }^{17} \mathrm{Cf}$. Krelle (1968) and the models of fomation of life style groups of Schokkaer (1982b, Sec. V.B.III).

18 This important limitation also applies to the epidemiological model of the diffusion of social nom beliefs and labour supply behaviours of De Neubourg (1985. 1988). In this model the infected persons seem to be those who neither believe nor obey the social nom. and the non-infected persons seem to be those who both believe and obey the social nom. Thus, all believers obey the nom and vice versa, so $m=F$, and hence $m=0$ in (8.6). This implies that, at least in its present form. the model of De Neubourg describes a long-nun dynamics which is different from that of Akerlofs model. 
dynamics than Akerlof's as well as our model. On a conceptual level it could be considered as a specific mode of behavioural preference interdependence as analysed in Chapter 5 according to which individual norm beliefs as aspects of individual habits are not formed under the direct influence of one's own behaviour, but under the direct influence of the behaviour of others. In the next section, however, we assume only an intrapersonal belief changing process as described above and investigate the implications of the parameter restrictions in the Akerlof specification (8.12) for the occurrence of collective catastrophes and hysteresis.

\subsection{Collective catastrophes and hysteresis}

At first sight, the conditions for collective catastrophes and hysteresis to occur in the case of the restricted Akerlof specification (8.12) seem to be obtained by simply imposing the parameter restrictions on the AQM conditions. However, this is not automatically correct, since, as already referred to in Section 8.4, linearity of the utility function excludes unique interior solutions of the first-order condition as given by the individual short-run labour supply functions (6.8) in Subsection 6.2.1. ${ }^{19}$ On the other hand, this linear case can be shown to form a limiting case of the AQM. Substituting the parameter restrictions into (6.9a) and $(6.9 \mathrm{~b})$, it then follows that there are non-negative individual short-run reservation wages $w_{r i}=b_{i} / a$ which are given by

$$
w_{r i}=\frac{\gamma_{L L i} s_{L i}+\gamma_{L \overline{L i}} s_{\bar{L}}}{\alpha_{X}}=\frac{\gamma n_{L i}+\beta_{L} \varepsilon_{L i} \rho_{L} m_{L}}{T_{i} \alpha} .
$$

For $w$ above $w_{r i}$ there is a unique comer solution $H_{i}=T_{i}$ for the individual short-run labour supply. For $w=w_{r i}$ an indifference line coincides with the budget line, implying that all values of $H_{i}$ are solutions. When the denominator of $(8.15)$ is positive, the reservation wage is positive, and for $w$ below $w_{r i}$ there is a unique corner solution for $H_{i}=0$. This implies a discontinuous short-run individual labour supply curve with a catastrophe between $H_{i}=0$ and $H_{i}=T_{i}$ at $w_{r i}{ }^{20}$

In the long run we then have, for any positive habit sensitivities $\gamma_{L L i}$, a situation similar to that in the AQM case of BPI and unstable IHF with its polarization of individual labour supply to zero and maximal corporate hours (see Sec. 7.1). This corresponds to the Cases III and IV in Table 6.1 in Subsection 6.2.4. (Note that the parameter restriction $\alpha_{L L i}=0$ excludes the Cases I and II, since the parameters $\bar{\gamma}_{L \bar{L}}$ and $\gamma_{L L}$ both are, by definition, nonnegative for all persons.) However, in the present case of the Akerlof specification and assuming. just as in Section 7.1, a uniform $T$, there is exogenous individual variation only in the sensitivity to reputation $\varepsilon_{L i}$, and hence in the susceptibility to social influence

${ }^{19}$ Note that the parameter restrictions imply $c_{i}=d_{i}=0$ and hence a zero denominator in (6.8).

${ }^{20}$ The shape of this short-run labour supply curve is similar to that in the case of an institutional rationing of labour supply at zero and full-time employment (see Subsec. 2.2.4). In the present case, however. the total discretionary time $T_{i}$ of woman $i$ may be larger of smaller than a full-time working week. 
$\gamma_{L \overline{L i}}=\left(\beta_{L}{ }^{e} L i \rho_{L}\right) / T^{2}$, but not in the household time taste $\alpha_{L}=0$ and the habit sensitivity $\gamma_{L L}=\gamma_{L} / T^{2}$. Analogously to Proposition 7.2 in Section 7.3, it then follows that, for a presumably large class of density functions of $\gamma_{L \bar{L}}$ or $\varepsilon_{L}$, there can always occur a collective catastrophe in entering the labour market. This leaves us with a case like Case IV in Table 6.1 in Subsection 6.2.4. Besides to the absence of variation in household time taste and habit sensitivity, this result can be attributed to the parameter restrictions $\alpha_{X X i}=\alpha_{L L i}=0$, which exclude short-run satiation effects of diminishing marginal utilities of consumption and household time (note that satiation counteracts IHF and BPI).

In order to derive long-run average labour supply schedules for a social group of married women (see Fig. 8.2), we first consider the case of $w$ rising from zero and suppose that at $w=0$ all group members do not participate in the labour market and are collectively used to that, c.q. fully believe in the household norm, i.e. $s_{L i}=s_{L \bar{L}}=T$, c.q. $n_{L i}=m_{L}=1$ for all $i$ (cf. Sec. 4.8). ${ }^{21}$ For $w$ below $w_{1}^{* 1}=\left(\gamma_{L}+\beta_{L} \min \varepsilon_{L i} \rho_{L}\right) /(T \alpha)$, nobody will enter the labour market because of sanctions of cognitive dissonance and reputation loss, but for higher $w$ trendsetters will take a job with maximal hours. First women will enter who are the least sensitive to their reputation among their housewive-friends (lowest $\boldsymbol{e}_{L i}$ ) and next women who are more sensitive to their reputation. At a certain $\bar{w}^{* 1}$ a critical mass $\tilde{H}^{* 1} / T$ of trendsetters is reached, and beyond $\bar{w}^{* 1}$ the remaining conformist housewives collectively also take a job with maximal hours. This immediately leads to maximal employment of all women, and in the long run all women will have collectively got used to that, c.q. will have completely lost their belief in the household norm, so $s_{L i}=s_{L \bar{L}}=0$, c.q. $n_{L i}=m_{L}=0$ for all $i$. For higher $w$ the long-run average labour supply remains maximal, since there is no incentive to reduce the individual labour supplies. This is due to the restriction $\alpha_{L}=0$, which implies that household time has no utility other than possible utilities from reputation or cognitive consonance, the latter utilities being zero in the present situation.

For the same reason, in such a maximal employment situation the individual exit wages as given by (8.15) with $n_{L i}=m_{L}=0$ are equal to zero. This implies that, at declining $w$, all group members will remain maximally employed (even for $w=0$ ), i.e., that maximal employment of all group members and the corresponding absence of belief in the household norm $\left(m_{L}=0\right)$ form a (stable) long-run equilibrium for all $w$. Together with the behaviour of entrants to the labour market for $w$ rising from 0 , this leads to a long-run average labour supply schedule as represented in Figure 8.2. This figure displays also a long-run labour supply schedule in the special case that the susceptibility to social influence $\gamma_{L . \bar{L} i}$ or $\beta_{L} \varepsilon_{L i} \rho_{L}$ is zero for all group members. The entry wages are then the same for all group members, and in the present case they are equal to $w^{* 1}=\gamma_{L} /(T \alpha)$. Thus, there are no trendsetters and all women are involved in a collective catastrophe at the uniform entry wage.

However, returning to the more plausible case that at least some married women are susceptible to social influence from their group, suppose that $w$ first rises from zero till a level $w^{\circ}$ at which only some trendsetters have entered the labour market and, after the longrun equilibrium being established, again declines to zero. In the long-run equilibrium at $w^{0}$, the trendsetters who have entered, have collectively got used to that, c.q. have completely lost

${ }^{21}$ By "collectively used" we mean that both the individual habit states and the expected or perceived social environment have been fully adapted to their long-run equilibrium values. 


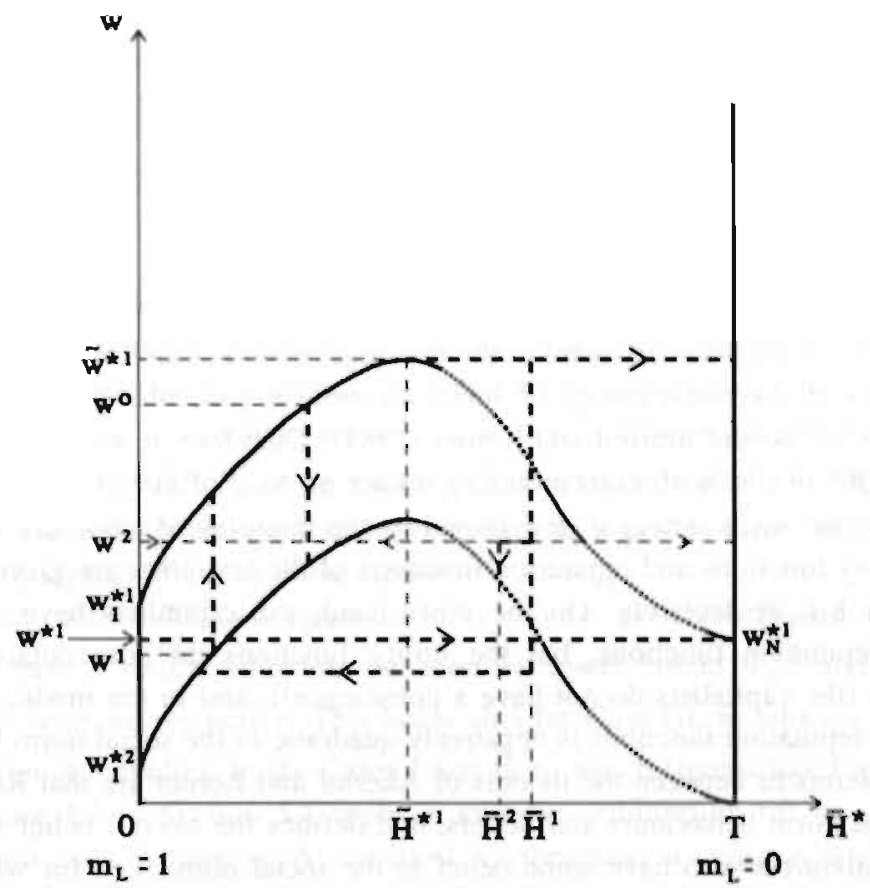

Figure 8.2 Long-run average labour supply schedule for Akerlof specification with household norm, zero household time taste and exogenous individual variation only in sensitivity to reputation (or no sensitivity to reputation).

their belief in the household norm $\left(n_{L, i}=0\right)$. This implies that they do no longer suffer from cognitive dissonance, and hence will leave the labour market only at an exit wage, given by (8.15) with $n_{L i}=0$, which is substantially lower than their entry wage. Therefore, the long-run average labour supply of the married women will be inelastic for $w$ declining from $w^{\circ}$ until the exit wage of those working women is reached who are most sensitive to their bad reputation among their housewife friends (highest $\varepsilon_{L i}$ ). Those women will then quit, and at lower $w$ the other working women will quit in order of decreasing sensitivity to reputation, which is the reverse of their order of entrance. However, if $w$ again starts to rise from a long-run equilibrium at $w^{1}$ before all trendsetters have returned to their households, the trendsetters who have returned, have again be converted to the household norm and will enter only at a higher entry wage because of cognitive dissonance sanctions. The long-run average labour supply of the married women is then again inelastic until at rising $w$ the entry curve is reached. For $w$ rising beyond that point, the elastic entry curve is followed.

It is also possible that in the midst of a collective entry catastrophe at $\bar{H}^{1}$, the wage rate declines under the entry curve. For $w$ above the exit curve, the average labour supply will adjust to a long-run inelastic value $\bar{H}^{2}$ which tends to be lower than $\bar{H}^{1}$, but higher than $\hat{H}^{* 1}$, i.e., a number of entry conformists is maximally employed, whereas other entry conformists are not employed. On the other hand, when $w$ declines below the exit curve, a collective catastrophe back to non-participation sets in, which tends to end at a "stable" 
equilibrium on the solid part of the exit curve at which still a number of exit laggards are maximally employed. Thus, we have again a band of persistent and hysteretic long-run behaviour between the entry and exit curves and two domains of catastrophic long-run behaviour outside this band.

\subsection{Comparison with social custom literature}

More generally, similar results as found in the previous section could be expected to hold for other applications of Akerlof's theory of social custom, in particular for the application to fair wage setting by Akeriof himself and Romer (1984). Therefore, it seems to make sense to compare our AQM results with corresponcing results of Akerlof (1980) and Romer (1984). In their approach the wage setting is determined by the interplay of labourers and owners of capital. The utility functions and reputation functions of the labourers are given by (8.2) and (8.3) in Section 8.1, respectively. On the other hand, the capitalists have similar utility functions and reputation functions, but the utility functions do not contain a cognitive dissonance term (the capitalists do not have a conscience!), and in the model of Romer the utility loss from reputation sanctions is negatively quadratic in the social nom behaviour (cf. (8.4)). Other differences between the models of Akerlof and Romer are that Romer assumes continuous social norm behaviours and beliefs, and defines the overall belief variable $m$ as the fraction of labourers who have some belief in the social norm (i.e., for whom $n_{i}>0$ ). In both models the long-run evolution of $m$ is given by (8.6) and three propositions on the existence and nature of long-run equilibria of $m$ are derived. The propositions of Romer are analogous to those of Akerlof, but slightly stronger. Therefore and since the assumptions of Romer are more close to our assumptions than those of Akerlof, we consider the propositions of Romer. ${ }^{22}$ Omitting details which are less relevant for the comparison with our AQM results, these propositions amount to:

I. A zero fraction $m$ of believing labourers always represents a long-run equilibrium.

II. Assume that the distributions of reputation parameters like $\beta \varepsilon_{j} p$ in (8.4) are bounded. There are then two cases. If the cognitive dissonance sanctions $\gamma n_{i}$ on disobedience to the social norm $\left(A_{i}=0\right)$ by a believer in the norm $\left(n_{i}>0\right.$, see (8.4)) are sufficiently strong relative to the utility gains from a higher consumption by disobedience, there is a positive $m^{\circ}$ such that any $m<m_{o}$ yields a long-run equilibrium. If the cognitive dissonance sanctions are not sufficiently large, $m=0$ is a stable longrun equilibrium.

III. If the parameters of the linear term in the individual utility losses from reputation sanctions of the capitalists are sufficiently large relative to the utility gains from a higher consumption by disobedience to the norm, $m=1$ is a stable long-run equilibrium.

22 In Romer's model the extent of violation of the social norm is described by the absolute difference $|w-\bar{w}|$ between the wage $w$ and the fair wage $\bar{w}$. This comesponds to $1-A_{L i}=1-L_{i} / T=H_{i} / T$ in our female labour supply model. 
Comparing these propositions with our AQM results, we find the following points of agreement and disagreement. First, in the $A Q M$ a long-run equilibrium for $m_{L}=0$, so $\bar{H}^{*}=T$, exists for all values of the exogenous variables only in the limiting case that the household time taste parameter $\alpha_{L i}=0$ for all group members $i$ (see Fig. 8.2), since only then there is no incentive for obeying more or less the household norm when $n_{L i}=m_{L}=0$. In the general situation that $\alpha_{L i}>0$ for all $i$, a distinction should be made between cases like those in Table 6.1 in Subsection 6.2.4. Above we have seen that only a case like Case IV with individual variation only in the susceptibility to social influence $\gamma_{L i}=\left(\beta_{L} \varepsilon_{L i} \rho_{L}\right) / T^{2}$ corresponds to the Akerlof specification. This holds also with respect to the model of Romer, so Figure 7.4 in Section 7.3 applies. According to this figure a long-run equilibrium for $m_{L}=0$, so $\bar{H}^{*}=T$, exists only for $\bar{w}^{* 2} \leq w \leq w_{+}^{*}$. Here $\tilde{w}^{* 2}=\alpha_{L} / a$ with $a=\alpha_{X}-\left|\alpha_{X X}\right| Y_{O}$ and $w_{*}^{*}$ is given by (4.13) in Section 4.3. The latter expression for $w_{+}^{*}$ implies that this upperbound is due not only to a positive $\alpha_{L}$, but also to a positive diminishing marginal utility of consumption $c^{*}=\left|\alpha_{X X}\right|$.

In Case IV with individual variation in $\alpha_{L i}$ only, to which Figure 7.1 in Section 7.2 applies, we have a similar situation, but then the lower bound $w_{N}^{* 2}$ and the higher bound $w_{+N}^{*}$ tend to be more restrictive. This holds also for Case III, to which a figure like Figure 4.11 in Section 4.8 applies. In the Cases I and II, to which figures like Figure 4.9 in Section 4.7 and Figure 5.3 in Section 5.4 apply, a long-run equilibrium for $m_{L}=0$, so $\bar{H}^{*}=T$, does often not exist for any $w$. At the most we then have a long-run equilibrium like $A$ in Figure 5.2 in Section 5.3 at which a majority of the social group does not believe in the social norm, but at which there are still some persons who believe in it to some extent. Thus, in many cases our AQM excludes a pure "neoclassical" equilibrium in which nobody more or less believes in a social norm and in which such a social norm has therefore no effect on the individual behaviour. ${ }^{23}$ We may even have a situation as described by $\bar{L}^{-B}$ in Figure 5.2 in which there is only one equilibrium $B$ at which everybody fully believes in the social norm.

In the first case of Romer's Proposition II, the cognitive dissonance sanctions $\gamma n_{i}$ for $n_{i}>0$ are so strong as to guarantee that in the short run all persons who have some belief in the social norm also fully obey it, independently of any influence from a reputation sanction. Conversely, if the utility losses from reputation sanctions like $\beta_{\varepsilon_{j}} \rho m$ in $(8.4)$ are sufficiently weak, i.e. for $m<m^{\circ}$, in the short run all persons who disbelieve the social norm also disobey it because of consumption gains. Such short-run equilibria in which the persons who obey the norm exactly, coincide with the persons who have some belief in it, are automatically also long-run equilibria. Thus, any initial distribution of individual norm beliefs with $m<m^{\circ}$ forms a long-run equilibrium.

In the labour supply counterpart of Romer's model as represented by Figure 8.2 , this situation occurs for $w \leq w_{N}^{* 1}$, where $w_{N}^{* 1}$ is the entry wage of the last entrant and is given by (8.15) for $n_{L i}=1$ and $m_{L}=0$, so is equal to $\gamma_{L} /(T \alpha)$. This inequality is equivalent to $\gamma_{L} \geq \alpha w T$, expressing that the cognitive dissonance sanction $\gamma_{L}$, for a full believer in the household norm ( $n_{L i}=1$ ) should be at least as strong as the utility gain from the additional 
consumption $w T$ when working maximal corporate hours. ${ }^{24}$ For the specific configuration of Figure 8.2, any point to the right of the dotted part of the exit curve is then located in the band of persistent long-run behaviour, so represents a long-run equilibrium with $m_{L}<m_{L}^{o}$, where $m_{L}^{o}$ is given by the dotted curve. ${ }^{25}$ On the other hand, for $w>w_{N}^{* 1}$ points with sufficiently low positive $m_{L}$ lie in the domain of catastrophic entry behaviour. Then $m_{L}=0$ is a stable long-run equilibrium. In the more general Cases IV of Figure 7.4 and Figure 7.1 in Sections 7.3 and 7.2, a property as in Proposition II holds only for $w \geq \bar{w}^{* 2}=\alpha_{L} / a$ or $w \geq w_{N}^{* 2}=\max \alpha_{L i} / a$, respectively, so for sufficiently high $w$ or $a$ or sufficiently low $\alpha_{L}$ or $\max \alpha_{L i}$. A similar qualification applies to Case III as represented by Figure 4.11 in Section 4.8, and in the Cases I and II as represented by Figure 4.9 in Section 4.7 and Figure 5.3 in Section 5.4 the property disappears.

A stable long-run equilibrium for $m_{L}=1$ as in Proposition III occurs in Figure 8.2 for $w \leq w_{1}^{* 1}$, where $w_{1}^{* 1}$ is the entry wage of the first entrant and is given by (8.15) for $n_{L i}=1, \varepsilon_{L i}=\min \varepsilon_{L i}$ and $m_{L}=1$, so is equal to $\left(\gamma_{L}+\beta_{L} \min \varepsilon_{L i} \rho_{L}\right) /(T \alpha)$. A sufficient condition for this inequality to hold independently of the value of the cognitive dissonance parameter $\gamma_{L}$, is $\beta_{L} \min \varepsilon_{L i} \rho_{L} \geq \alpha w T$ or, equivalently,

$\rho_{L} \geq(\alpha w T) /\left(\beta_{L} \min \varepsilon_{L i}\right){ }^{26}$ This condition is also sufficient for a stable long-run equilibrium for $m_{L}=1$ in the more general Cases IV of Figure 7.4 and Figure 7.1, but then it is moreover sufficient if $w \leq(\min ) \alpha_{L(i)} / a$, or equivalently, (min) $\alpha_{L(i)} \geq a w$, so if the (minimal) household time taste is sufficiently high to induce all women in the social group to conform to the household norm. Both conditions apply also to the other Cases II, I and III.

Results which are similar to those of Akerlof (1980) and Romer (1984) are obtained by Booth (1985) and Naylor (1989) in applications of Akerlof's theory to trade union membership and collective strike action. The analysis of Naylor is the most advanced: as already mentioned in the previous section, he finds a "trimodal outcome" with two continua of longrun equilibria and a discrete equilibrium for $m=0$, and he analyses the effects of changing values of several parameters on this outcome. Our Figures 8.2 and 7.4 display such a trimodal outcome for $w=w^{2}$, and Figure 7.1 exhibits for $w=w^{2}$ a less restricted variant of a trimodal outcome with three continua of long-run equilibria. The effects of a changing wage rate have been analysed in the previous section, showing that a trimodal outcome is at most obtained for a restricted range of the wage rate.

However, in comparison to the analyses of Naylor and the other authors our model adds two major insights. First, it demonstrates how the historical time paths of the exogenous variables determine which individual and overall long-run equilibria out of the broad ranges of possible equilibria are actually approached. This finding is referred to as individual and collective hysteresis. Second, our model shows how at certain values of the exogenous

\footnotetext{
${ }^{24} \mathrm{Cr}$. Proposition II of Akerlof (1980), that considers only cases in which the reputation parameter $\rho$ approaches 0.

${ }^{25}$ For sufficiently small $\beta_{L} \rho_{L}$ the entry wage $w_{N}^{\cdot 1}$ may also be higher than the highest exit wage. In that case we have $m_{L}^{0}=1$ for $w$ above this exit wage. On the other hand, in the model of Romer $m^{\circ}$ is not specified.

${ }^{26}$ Cf. Proposition III of Akerlof (1980).
} 
variables a transition may occur from one individual or overall equilibrium to another. Such transitions are called individual and collective catastrophes, respectively.

Moreover, in order to derive plausible conditions for such phenomena to occur, Akerlof's particular specification of his model has been extended to a continuous additive quadratic model. This has the advantage of making visible that the phenomena which are implied by Akerlof's specification occur only when certain parameter conditions are met. However, the extended model has still a number of drawbacks and should therefore be further extended. Such extensions will be analysed in the next sections.

\subsection{Financial independence norm: Akerlof specification}

Notwithstanding their advantages, the extended TSC as well as the AQM derived in the previous chapter still suffer from the drawback that the implied piecewise-linearity of the individual demand functions in the individual norm beliefs or habit states precludes stable individual equilibria from being interior in the case of separately unstable individual (dis)belief formation or IHF. This seems implausible especially for married women with a part-time job, since it implies that these married women would, in the long run either spend all their discretionary time in one or more jobs or have no job at all (see footnote 8 in Sec. 4.2). One way to remedy this drawback is the following.

As counterparts of the IHF and BPI with respect to consumption and corporate time, the TSC can be extended so as to allow for the effects and dynamics of social "emancipation" norms of financial independence and self-fulfilment, respectively, alongside the household norm in female labour supply. In Section 8.3 it has been noted that, whereas in the fifties and sixties the household norm played a dominant role in many OECD countries, since the end of the sixties the "emancipation" norms came up. An important "emancipation" norm is the financial independence norm, which is specified by (8.10). The coming three sections will analyse the implications of this norm for short and long-run female labour supply according to extensions of the TSC. This section and the next will consider the case where only the financial independence norm plays a role, and Section 8.10 will add the household norm to this case.

Specification (8.10) can be rewritten in terms of the total consumption $X_{i}$ of the family of the female labour supplier by using the usual binding income constraint $X_{i}=w_{i} H_{i}+Y_{O i}$, where $Y_{O i}$ is assumed to be the income of the husband. This implies $w_{i} H_{i}=X_{i}-Y_{O i}$. and substituting this into $(8.10)$ yields

$$
\begin{array}{ll}
A_{F i}\left(X_{i}\right)=\left(\frac{X_{i}-Y_{O i}}{Y_{I M i}}\right)^{\eta_{F}}, & \text { if } X_{i} \leq Y_{O i}+Y_{I M i} \\
A_{F i}\left(X_{i}\right)=1, & \text { if } X_{i} \geq Y_{O i}+Y_{I M i} .
\end{array}
$$

Thus, now the social norm variable $A_{F i}$ is a positive function of consumption $X_{i}$, so consumption cannot play the role of the incentive to breaking the norm as in the TSC. However, this role can be taken over by household time $L_{i}$, which, following specification (8.5), leads to individual utility functions 


$$
\hat{D}\left(L_{i}, A_{F i} ; n_{F i}, \varepsilon_{F i}\right)=\alpha_{L} L_{i}+\beta_{F} \varepsilon_{F i} \rho_{F} m_{F} A_{F i}+\gamma_{F} n_{F i} A_{F i},
$$

where $\alpha_{L}, \beta_{F}, \varepsilon_{F i}, \beta_{F}$ and $\gamma_{F}$ are again positive parameters.

Analogously to the procedure in Section 8.4, we substitute (8.16) with $\eta_{F}=1$ into (8.17). Moreover, since $Y_{O i}$ and $Y_{I M i}$ are assumed to be exogenous, terms which depend on these variables, but not on the behaviour variables, can be transformed away. Assuming that $\boldsymbol{Y}_{I M i}$ is the same for each group member, we then obtain

$$
U\left(L_{i}, X_{i} ; n_{F i}, m_{F}, \varepsilon_{F i}\right)=\alpha_{L} L_{i}+\frac{\gamma_{F}}{Y_{I M}{ }^{2}}\left(Y_{I M} n_{F i}\right) X_{i}+\frac{\beta_{F} \varepsilon_{F i} \rho_{F}}{Y_{I M}{ }^{2}}\left(Y_{I M} m_{F}\right) X_{i}
$$

for $X_{i} \leq Y_{O i}+Y_{I M}$ and

$$
\begin{aligned}
U\left(L_{i}, X_{i} ; n_{F i}, m_{F}, \varepsilon_{F i}\right)= & \alpha_{L} L_{i}+\frac{\gamma_{F}}{Y_{I M}^{2}}\left(Y_{I M} n_{F i}\right)\left(Y_{O i}+Y_{I M}\right)+ \\
& +\frac{\beta_{F} \varepsilon_{F i} \rho_{F}}{Y_{I M}{ }^{2}}\left(Y_{I M} m_{F}\right)\left(Y_{O i}+Y_{I M}\right)
\end{aligned}
$$

for $X_{i} \geq Y_{O i}+Y_{I M}$. Now the second and third term in (8.18a) seem to correspond to the two terms $\gamma_{X X i} s_{X i} X_{i}+\gamma_{X \bar{X} i} s_{\bar{X}} X_{i}$ which are added to the AQM specification (6.7) in Subsection 6.2.1 in order to account for IHF and BPI with respect to consumption (see Sec. 6.3). In the extended (6.7) we should then put $s_{X i}=Y_{I M} n_{F i}, s_{\bar{X}}=Y_{I M} m_{F}, \alpha_{L i}=\alpha_{L}$, $\gamma_{X X i}=\gamma_{F} / Y_{I M}{ }^{2}, \gamma_{X \bar{X} i}=\beta_{F} \varepsilon_{F i} \rho_{F} / Y_{I M}{ }^{2}$ and $\alpha_{X i}=\alpha_{X X i}=\alpha_{L L i}=\gamma_{L L i}=\gamma_{L \overline{L i}}=0$. However, this is not entirely correct, since, in the long run, the state variables $Y_{I M} n_{F i}$ and $Y_{I M} m_{F}$ are not adjusted to the family consumption levels $X_{i}$ and $\bar{X}$ as $s_{X i}$ and $s_{\bar{X}}$, respectively, but to the wife's own income levels $w H_{i}=X_{i}-Y_{O i}$ and $w \bar{H}=\bar{X}-\bar{Y}_{O}$, respectively. Therefore, the formation of (dis)beliefs in the financial independence norm can be interpreted as a specific mode of collective habit formation only with respect to the wife's own income. On the other hand, because of the assumed exogeneity of the income of the husband $Y_{O}$, the dynamics of this collective habit formation is analogous to that of collective habit formation with respect to consumption. This analogy will be used in the following. However, we will then take into account that the analogy holds only for $X_{i} \leq Y_{O i}+Y_{I M}$, or $H_{i} \leq Y_{I M} / w$ (see (8.10) in Sec. 8.3). As shown below, this leads to an interesting modification of the results of the $\mathrm{AQM}$ for consumption IHF and BPI.

For the same kind of reasons as the parameter restrictions in (8.12), the parameter restrictions $\alpha_{X i}=\alpha_{X X i}=\alpha_{L L i}=0$ in (8.18) are implausible and should therefore be relaxed. However, for the sake of comparison, we first analyse this restricted case. Since the utility functions as given by (8.18) are then linear in $L_{i}$ and $X_{i}$ in both regimes, the indifference curves generally consist of two straigh' segments (see Fig. 8.3), with slopes given by the marginal rates of substitution

$$
-\frac{d X_{i}}{d L_{i}}=\frac{U_{L_{i}}}{U_{X_{i}}}=\frac{Y_{I M} \alpha_{L}}{\gamma_{F} n_{F i}+\beta_{F} \varepsilon_{F i} \rho_{F} m_{F}}
$$


for $X_{i} \leq Y_{O i}+Y_{I M}$ and $-d X_{i} / d L_{i}=U_{L_{i}} / U_{X_{i}}=\infty$ for $X_{i} \geq Y_{O i}+Y_{I M}$. Just as in the standard model, the short-run reservation wage $w_{r i}$ is equal to the marginal rate of substitution for $L=T$, and this is given by the marginal rate of substitution in (8.19) (cf. (8.15)). For $w$ below $w_{r i}$, there is a corner solution for $L_{i}=T$ or $H_{i}=0$. For $w$ equal to $w_{r i}$, the right segment of an indifference curve coincides with the opportunity frontier, implying that all values of $H_{i}$ between and including 0 and $Y_{I M} / w_{r i}$ are solutions. Herewith it has been assumed that $Y_{I M} / w_{r i}$ is smaller than the maximum $T$ of $H_{i}$, and this holds for all values of $n_{F i}, m_{F}$ and $\varepsilon_{F i}$ if and only if $\gamma_{F}+\beta_{F} \max \varepsilon_{F i} \rho_{F}<\alpha_{L} T$. For $w$ above $w_{r i}$, there is a unique interior solution for $H_{i}=Y_{I M} / w$. This implies a discontinuous short-run individual labour supply curve with a catastrophe between $H_{i}=0$ and $H_{i}=Y_{I M} / w_{r i}$ at $w_{r i}$ and a backward-sloping part above $w_{r i}$.

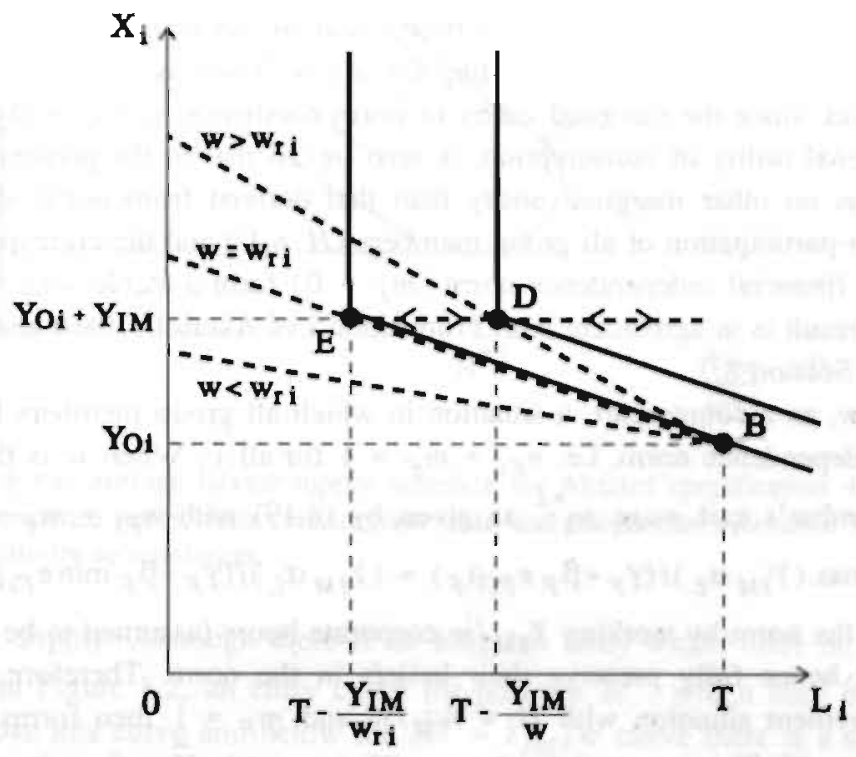

Figure 8.3 Detemination of short-run labour supply in the presence of a financial independence norm and a constant marginal utility of household time under a standard constraint for $w<w_{r i}, w=w_{r i}$ and $w>w_{r i}$ (dashed bold lines). Indifference lines are solid bold. The dashed bold line with arrows indicates the optimal points for $w>w_{r i}$.

However, this story holds only when $n_{F i}$ and/or $m_{F}$ is positive, i.e., when the person herself believes in the financial independence norm to some extent and/or when some other persons in her social (reference) group believe in it. There is then at least one incentive to obey this norm, and this incentive becomes stronger relative to the marginal utility of household time $\alpha_{L}$ as $w$ rises, since one can then (fully) obey the norm for a lower $H_{i}$, so with a smaller loss of household time. Beyond $w=w_{r i}$ the incentive becomes stronger than $\alpha_{L}$, inducing the person to enter the labour market. Moreover, since the marginal utilities of norm obedience and household time are independent of $H_{i}$, it is then advantageous to obey 
the norm fully. On the other hand, it is not advantageous to work more corporate hours than minimally necessary to fully obey the norm, since this would imply a larger loss of household time, whereas consumption does not have a marginal utility above that derived from norm obedience. All this results in a discontinuous jump from $H_{i}=0$ to $H_{i}=Y_{I M} / w_{r i}$. Finally, when $w$ rises further, the number of corporate hours $H_{i}=Y_{I M} / w$ which is needed to fully obey the norm, decreases. This feature is similar to that in the case where a minimal necessary consumption level (see Subsec. 2.2.4) or a breadwinner norm (see Sec. 8.2) in combination with an insufficient other income dictates an positive under limit on $\mathrm{H}_{i}$ which is inversely proportional to $w$.

In contrast to the reservation wage $(8.15)$ in the case of the household norm, the reservation wage given by $(8.19)$ in the present case of the financial independence norm decreases with increasing $n_{F i}$ and $m_{F}$. Conversely, when both $n_{F i}$ and $m_{F}$ are zero, i.e., when nobody in the social group of person $i$ believes in the financial independence norm, the reservation wage in (8.19) is infinite. This means that she as well as the other members of her social group will remain non-participating for any $w$. There is then no incentive to enter the labour market, since the marginal utility of norm obedience $\gamma_{F} n_{F i}+\beta_{F} \varepsilon_{F i} \rho_{F} m$, and hence the marginal utility of consumption, is zero (recall that in the present restricted case consumption has no other marginal utility than that derived from norm obedience). This implies that non-participation of all group members $(\bar{H}=0)$ and the corresponding absence of belief in the financial independence norm $\left(m_{F}=0\right)$ form a stable long-run equilibrium for all $w$. This result is in agreement with Proposition I of Akerlof (1980) and Romer (1984) as discussed in Section 8.7.

Consider now, as a counterpart, a situation in which all group members fully believe in the financial independence norm, i.e. $n_{F i}=m_{F}=1$ for all $i$. When $w$ is then higher than each group member's exit wage $w_{i}^{* 2}$ as given by (8.19) with $n_{F i}=m_{F}=1$, i.e. for $w$ above $w_{1}^{* 2}=\max \left(Y_{I M} \alpha_{L}\right) /\left(\gamma_{F}+\beta_{F} \varepsilon_{F i} \rho_{F}\right)=\left(Y_{I M} \alpha_{L}\right) /\left(\gamma_{F}+\beta_{F} \min \varepsilon_{F i} \rho_{F}\right)$, all persons will fully obey the norm by working $Y_{I M} / w$ corporate hours (assumed to be smaller than $T$ as above), and hence fully preserve their beliefs in the norm. Therefore, this less than maximal employment situation with $\bar{H}=Y_{I M} / w$ and $m_{F}=1$ then forms another stable equilibrium alongside the non-participation equilibrium with $\bar{H}=0$ and $m_{F}=0$. This is indicated in Figure 8.4. When $w$ falls below $w_{1}^{* 2}$, the women who are the less sensitive to their reputation among their working friends (lowest $\varepsilon_{F i}$ ) will leave the labour market. As $w$ further declines, also women who are more sensitive to their reputation quit, and at a certain $\bar{w}^{* 2}$ a critical mass $\left(Y_{I M} / \bar{w}^{* 2}-H^{* 2}\right) /\left(Y_{I M} / \bar{w}^{* 2}\right)$ of trendsetters will be reached such that beyond $\bar{w}^{* 2}$ the remaining conformist jobholders collectively also leave their job. This immediately leads to non-participation of all women, and in the long run, all women will have completely lost their belief in the financial independence norm, so $n_{F i}=m_{F}=0$ for all $w$. There is then no longer any incentive to enter the labour market, so the long.run average labour supply remains zero for any $w$.

The exit curve, describing the long-run exit wage of the marginal quitters as a function of the average long-run labour supply $\bar{H}^{*}$ just before their exit, is analogous to the entry curve in Figure 8.2 in Section 8.6 and may run as indicated in Figure 8.4. Below this curve we have a domain of exit catastrophes and above the curve a band of persistent and hysteretic 


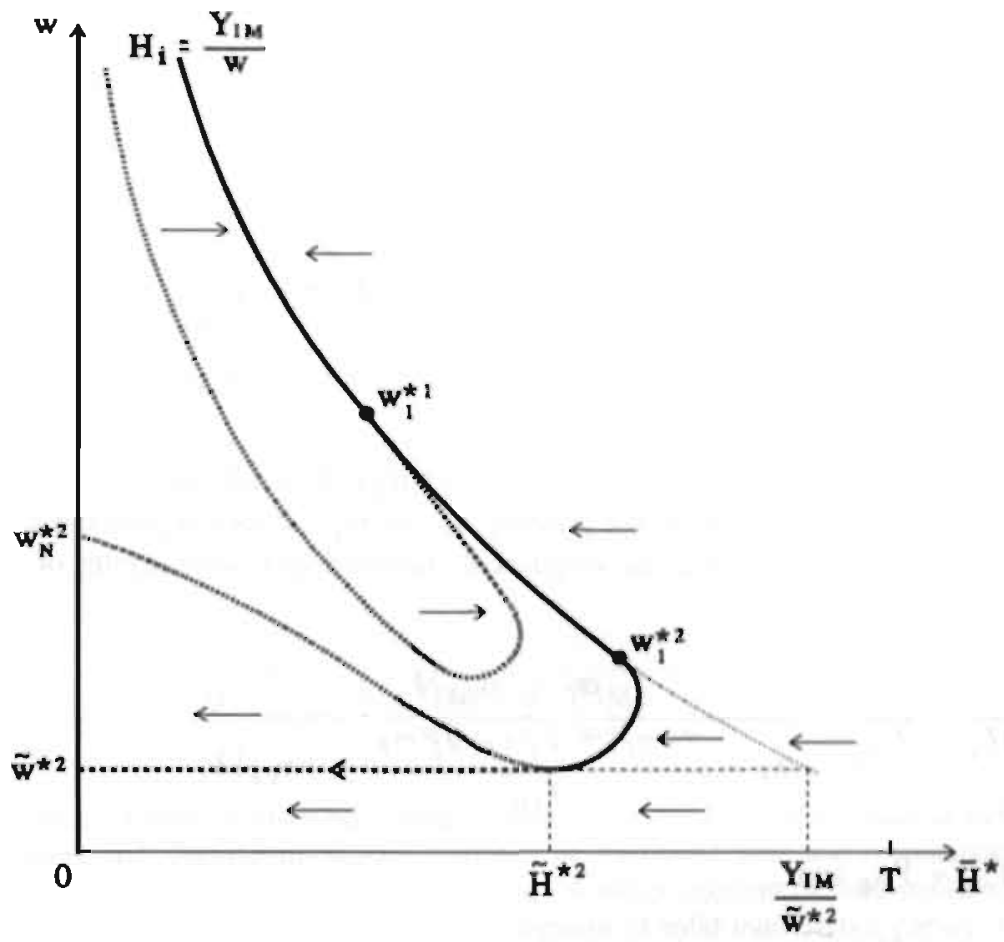

Figure 8.4 Long-run average labour supply schedule for Akerlof specification with financial independence norm, zero consumption taste and exogenous individual variation only in sensitivity to reputation.

long-run labour supply. Although there is no long-run entry wage, there is, analogously to the exit curve in Figure 8.2, an entry curve for positive $\bar{H}^{*}$, which may be located as in Figure 8.4. Above this curve and below the $\bar{H}^{*}=Y_{I M} / w$ curve there is a domain of entry catastrophes, and between the entry and exit curves and below the $\bar{H}^{*}=Y_{I M} / w$ curve we have the band of persistent long-run labour supply. When you turn Figure 8.4 upside-down, you can see its similarity to Figure 8.2.

\subsection{Financial independence norm: AQM}

From the derivation of short and long-run labour supply schedules in the previous section it can be deduced that these schedules are independent of the income of the husband $Y_{O i}$. This holds since obedience to the financial independence norm is, by definition, independent of $Y_{O i}$, while being the only source of the marginal utility of (family) consumption. However, the latter restrictive assumption as well as the assumption of constant marginal utilities of consumption and household time are very implausible. Therefore, these restrictions should be relaxed, which can be done by extending the specification (8.18) to the additive quadratic 
specification

$U\left(L_{i}, X_{i} ; n_{F i}, m_{F}, \varepsilon_{F i}\right)=\alpha_{L} L_{i}+\alpha_{L L} L_{i}^{2}+\left(\alpha_{X}+\frac{\gamma_{F} n_{F i}+\beta_{F} \varepsilon_{F i} \rho_{F} m_{F}}{Y_{I M}}\right) X_{i}+\alpha_{X X} X_{i}{ }^{2}$,

for $X_{i} \leq Y_{O i}+Y_{I M}$ and

$$
\begin{aligned}
U\left(L_{i}, X_{i} ; n_{F i}, m_{F}, \varepsilon_{F i}\right)= & \alpha_{L} L_{i}+\alpha_{L L} L_{i}^{2}+\alpha_{X} X_{i}+\alpha_{X X} X_{i}^{2}+ \\
& \frac{\gamma_{F} n_{F i}+\beta_{F} \varepsilon_{F i} \rho_{F} m_{F}}{Y_{I M}}\left(Y_{O i}+Y_{I M}\right)
\end{aligned}
$$

for $X_{i} \geq Y_{O i}+Y_{I M}$, where $\alpha_{L L}<0, \alpha_{X}>0$ and $\alpha_{X X}<0$. Just as in the case of Figure 8.3, the indifference curves consist, for positive $n_{F i}$ or $m_{F}$, of two segments (see Fig. 8.5), but now they are strictly convex to the origin with diminishing marginal rates of substitution which are given by

$$
-\frac{d X_{i}}{d L_{i}}=\frac{U_{L_{i}}}{U_{X_{i}}}=\frac{\alpha_{L}-\left|\alpha_{L L}\right| L_{i}}{\alpha_{X}+\frac{\gamma_{F} n_{F i}+\beta_{F} \varepsilon_{F i} \rho_{F} m_{F}}{Y_{I M}}-\left|\alpha_{X X}\right| X_{i}}
$$

for $X_{i} \leq Y_{O i}+Y_{I M}$ and

$$
-\frac{d X_{i}}{d L_{i}}=\frac{U_{L_{i}}}{U_{X_{i}}}=\frac{\alpha_{L}-\left|\alpha_{L L}\right| L_{i}}{\alpha_{X}-\left|\alpha_{X X}\right| X_{i}}
$$

for $X_{i} \geq Y_{O i}+Y_{I M}$. Therefore, in contrast with Figure 8.3, there is a unique solution for any $w$, and hence no discontinuity in the short-run individual labour supply curve. In the interior of both regimes this labour supply curve will usually be a part of a standard backward-bending curve (see Figure 8.6), but at the kink in the indifference curves for $X_{i}=Y_{O i}+Y_{I M}$. there is a regime switch from the supply curve for $n_{F i}>0$ or $m_{F}>0$ to the supply curve for $n_{F i}=m_{F}=0 .{ }^{27}$ As implied by Figure 8.5, there is then a range of $w$ between $w_{i}^{1}$ and $w_{i}^{2}$ in which the opportunity frontier is neither tangent to the lower segment of an indifference curve nor to the higher segment. The optimal point then remains on the $X_{i}=Y_{O i}+Y_{I M}$ line in Figure 8.5, implying that short-run labour supply then follows the $H_{i}=Y_{I M} / w$ curve in Figure 8.6.

However, in contrast to the short-run labour supply curve implied by Figure 8.3, short-run labour supply is larger than $Y_{1 M} / w$ for $w>w_{i}^{2}$. The reason is as follows. On the one hand. the marginal urility $\gamma_{F} n_{F i}+\beta_{F} \varepsilon_{F i} \rho_{F} m_{F}$ of obedience to the financial independence norm falls discontinuously to zero at $H_{i}=Y_{I M} / w$, implying a discontinuous drop in the marginal utility of consumption, and hence a discontinuous increase in the marginal rate of substitut-

${ }^{27}$ This does not hold because the positive $n_{F i}$ or $m_{F}$ drop(s) to zero at the regime switch, but because its (their) effect does so. 


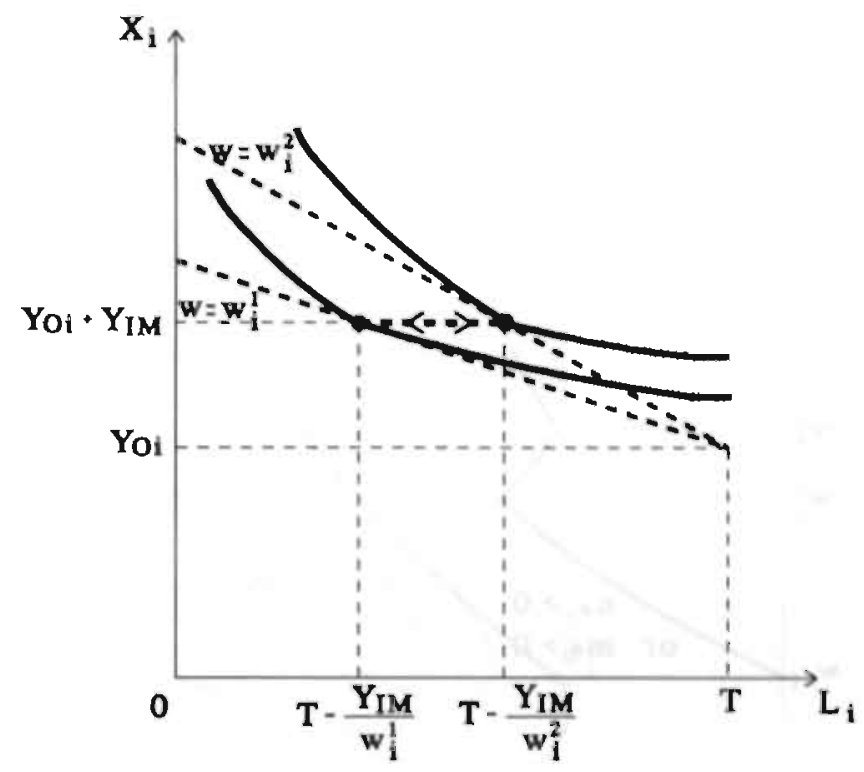

Figure 8.5 Determination of short-run labour supply in the presence of a financial independence. norm and diminishing marginal utilities of houschold time and consumption under a standard constraint for $w=w_{i}^{1}$ (tangency to lower segment of solid indifference curve) and for $w=w_{i}^{2}$ (tangency to higher segment of solid indifference curve). The dashed line with arrows indicates the optimal points for $w_{i}^{1}<w<w_{i}^{2}$.

ion. On the other hand, now the marginal utility of consumption does not fall to zero, and hence the marginal rate of substitution does not go to infinity, since there is now another source of (family) consumption utility than norm obedience which is represented by the consumption taste parameter $\alpha_{X}$ (and the diminishing marginal utility term $-\left|\alpha_{X X}\right| Y_{O i}$ ). Therefore, a sufficiently high rise in $w$ can compensate for the discontinuous increase in the marginal rate of substitution, and hence induce a married woman to work more corporate hours than is necessary for fully obeying the financial independence norm. In addition, in Figure 8.6 it has been assumed that the consumption taste is sufficiently high relative to the satiation tendency $\left|\alpha_{X X}\right| Y_{O i}$ and the individual minimum $Y_{I M}$ so as to imply a forwardsloping labour supply curve just above $w=w_{i}^{2}$.

The reservation wage $w_{r i}$ is given by the marginal rate of substitution in (8.21a) for $L_{i}=T$ and $X_{i}=Y_{O i}$. This implies that even when both $n_{F i}$ and $m_{F}$ are zero, i.e., when nobody in the social group of person $i$ believes in the financial independence norm, there is a finite reservation wage. Assuming that the income of the husband $Y_{O i}$ is (approximately) the same for every group member, it then follows that all group members will collectively enter the labour market for $w$ rising beyond the entry wage 


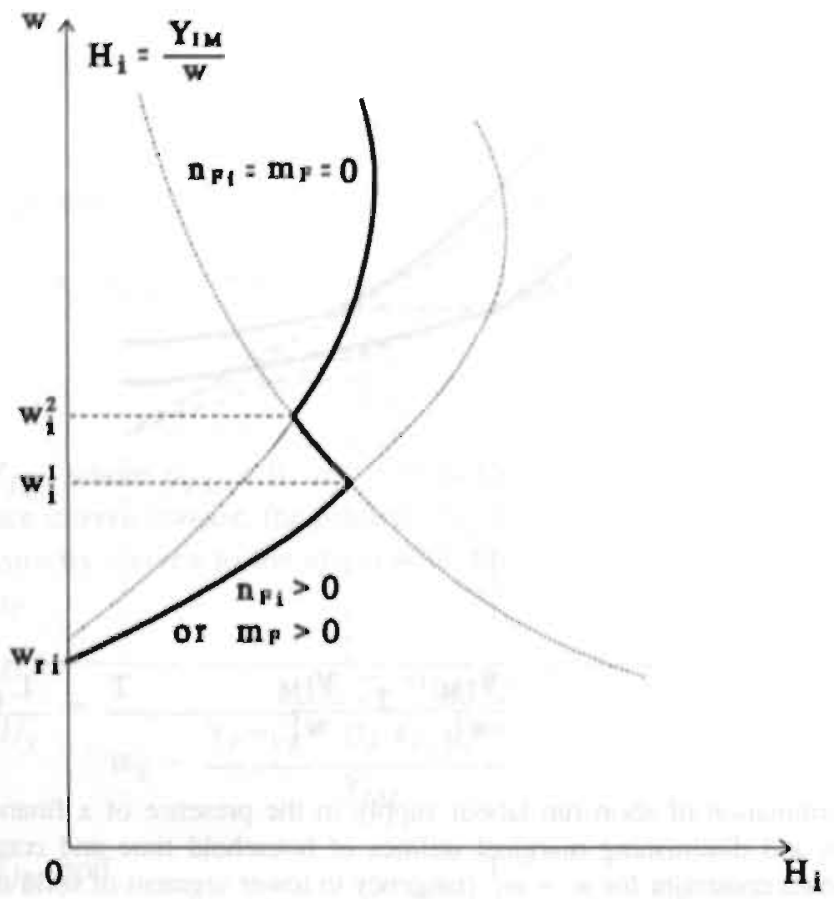

Figure 8.6 Short-run individual labour supply schedule for AQM specification with financial independence norm and positive consumption taste.

$$
\tilde{w}^{* 1}=\frac{\alpha_{L}-\left|\alpha_{L L}\right| T}{\alpha_{X}-\left|\alpha_{X X}\right| Y_{O}}
$$

The denominator of this expression for the entry wage represents the incentive to the entry. Consequently, in contrast to Figure 8.4, non-participation of all group members $(\bar{H}=0)$ and the corresponding absence of belief in the financial independence norm $\left(m_{F}=0\right.$ ) now form a stable long-run equilibrium only for $w$ below $w^{* 1}$. This is indicated in Figure 8.7.

If the norm beliefs would remain zero after entry, the average labour supply would follow a representative standard backward-bending labour supply for $n_{F i}=m_{F}=0$ (see Fig. 8.7). This, however, does not hold, since according to the differential equations (8.13) and (8.6) the group members are assumed to develop a (partial) belief in the financial independence norm as they start to work a number of corporate hours. This development of norm beliefs by the group members themselves as well as by the other group members reinforces their motivation for corporate work and prompts them to work more corporate hours. Assume now that the cognitive dissonance parameter $\gamma_{f}$ is 30 high as to make the process of reinforcement by the group members themselves separately (locally) unstable in analogy to unstable consumption IHF. According to the analogue of the stability condition (3.9) in Section 3.3 with $U_{L_{s_{L}}}^{*}=0$ and $\delta_{X}=1$ for the present (moving-average) case, this holds when $\gamma_{F} / Y_{I M}{ }^{2} \geq\left|\alpha_{X X}\right|+\left|\alpha_{L L}\right| / \bar{w}^{* 12}$, or 


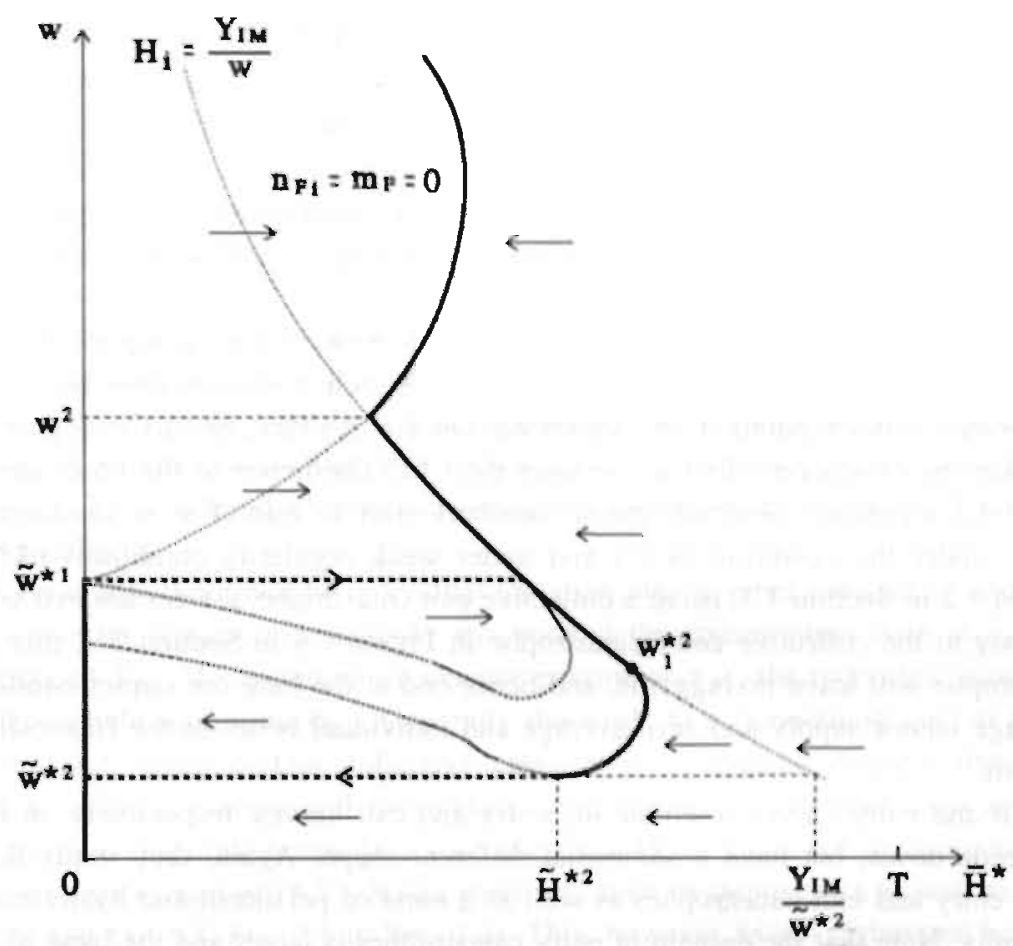

Figure 8.7 Long-run average labour supply schedule for AQM specification with financial independence nom, positive consumption taste and exogenous individual variation only in sensitivity 10 reputation.

$$
\gamma_{F} \geq Y_{I M}^{2}\left(\left|\alpha_{X X}\right|+\frac{\left|\alpha_{L L}\right|}{\bar{w}^{* 12}}\right)
$$

where the collective entry wage $\bar{w}^{* 1}$ is given by (8.22). Since this expression for $w^{* 1}$ is independent of $\gamma_{F},(8.23)$ is already an explicit condition for $\gamma_{F}$. If it is fulfilled, the process of reinforcement by the group members themselves separately induces individual entry catastrophes to full obedience to the norm as well as full belief in it. This holds for all group members simultaneously, since they enter collectively, so the individual entry catastrophes imply a collective entry catastrophe with neither trendsetters nor laggards. This collective catastrophe occurs independently of the strength of the reputation mechanism and is analogous to the collective exit catastrophe in Figure 7.4 in Section 7.3. Just like the latter catastrophe, the former catastrophe with its absence of trendsetters and laggards is characteristic for the present simplified case of individual variation in the sensitivity to reputation $\varepsilon_{F i}$ only.

However, in contrast to the collective exit catastrophe in Figure 7.4 , the collective entry catastrophe in Figure 8.7 ends at an assumedly interior stable equilibrium. Consider $w$ with 
$\bar{w}^{* 1} \leq w \leq w^{2}$, where $w^{2}$ is determined in the short-run schedule in Figure 8.6 for the present case of a uniform $Y_{O}$, and hence a uniform $w^{2}$. Then the interior long-run equilibrium is given by $\bar{H}=Y_{I M} / w$ for the same reasons for which the short-run individual labour supply in Figure 8.6 has this value for $w_{i}^{1} \leq w \leq w_{i}^{2}$. Also for the same reasons the longrun average labour supply above $w^{2}$ coincides with the short-run average or $i$-independent individual labour supply for $n_{F i}=m_{F}=0$. Thus, in comparison with Figure 8.4 for the Akerlof specification in the previous section, the long-run labour supply schedule has an additional backward-bending part.

When in such a situation of full norm belief and obedience by all group members, $w$ falls below $\tilde{w}^{* 1}$, the group members will first not reduce their corporate time because of cognitive dissonance and reputation loss sanctions. On the contrary, just as in Figure 8.4, they will increase their corporate time to continue their full obedience to the norm until beyond $w_{1}^{* 2}$ the least reputation-sensitive group members start to quit. For $w$ declining further, there will, under the condition (8.23) and under weak regularity conditions like those in Proposition 7.2 in Section 7.3, occur a collective exit catastrophe at a certain exit wage $\tilde{w}^{\bullet 2}$. Analogously to the collective entry catastrophe in Figure 7.4 in Section 7.3, this collective exit catastrophe will leave no laggards, and hence end at the long-run corner equilibrium for zero average labour supply and zero average and individual belief in the financial independence norm.

The exit and entry curves resemble the entry and exit curves, respectively, in Figure 7.4 turned upside down, but have a somewhat different shape. Again, they imply domains of collective entry and exit catastrophes as well as a band of persistent and hysteretic long-run labour supply. Note that the domain of entry catastrophes is larger and the band of persistent labour supply smaller than the corresponding domain and band in Figure 8.4 in the previous section. The implied higher entry mobility is due to the positive consumption taste.

Figure 8.7 has been derived under the assumption that the cognitive dissonance parameter $\gamma_{F}$ is so high as to make the process of reinforcement by the group members themselves separately unstable. However, this assumption is stronger than necessary and perhaps plausible. Collective entry and exit catastrophes can also arise in a case like Case II in Table 6.1 in Subsection 6.2.4 in which only the sum of $\gamma_{F}$ and the average product $\beta_{F} \bar{\varepsilon}_{F} \rho_{F}$ of the reputation parameters is so high as to make the combined process of reinforcement by the group members themselves as well as by other group members unstable (in analogy to an unstable combination of consumption IHF and BPI, see Sec. 6.3). In the NIV case of no individual variation in the reputation sensitivity $\varepsilon_{F i}$ this holds, analogously to the above case of high separate $\gamma_{F}$ (cf. Subsec. 6.2.2), when

$$
\begin{gathered}
\gamma_{F} / Y_{I M}{ }^{2}+\left(\beta_{F} \varepsilon_{F} \rho_{F}\right) / Y_{I M}{ }^{2} \geq\left|\alpha_{X X}\right|+\left|\alpha_{L L}\right| / \tilde{w}^{* 12} \text { or } \\
\gamma_{F}+\beta_{F} \varepsilon_{F} \rho_{F} \geq Y_{I M}{ }^{2}\left(\left|\alpha_{X X}\right|+\frac{\left|\alpha_{L L}\right|}{\bar{w}^{* 12}}\right)
\end{gathered}
$$

where the collective entry wage $\bar{w}^{* 1}$ is again given by (8.22). Thus, again (8.24) is already an explicit condition for $\gamma_{F}+\beta_{F} \varepsilon_{F} P_{F}$. If it is fulfilled, the combined reinforcement process induces in entry as well as exit collective and hence individual catastrophes between zero norm obedience and belief and full norm obedience and belief by all group members. 
In the IV case of individual variation in $\boldsymbol{\varepsilon}_{F i}$, collective and hence individual catastrophes in entry and exit occur under a condition for $\gamma_{F}+\beta_{F} \bar{\varepsilon}_{F} \rho_{F}$ in the spirit of Proposition 6.1 in Subsection 6.2.4, which condition is more complicated and stronger than (8.24). Under the present condition there are trendsetters in exit with lower sensitivities to their reputations as financially independent women, as in Figure 8.7, but moreover, the individual entry catastrophes of some of such women may end at individual equilibria for less than $Y_{I M} / w$ corporate hours, so for less than full norm obedience and belief (cf. the end of Subsec. 6.2.4). The collective entry catastrophe will then also end at less than $Y_{I M} / w$ corporate hours. Only when $\gamma_{F}+\beta_{F} \bar{\varepsilon}_{F} \rho_{F}$ fulfils an even stronger condition than the above condition, the collective entry catastrophe will end at an equilibrium with full norm obedience and full norm belief by all group members. In that situation we obtain a similar long-run average labour supply schedule as in Figure 8.7 except that in a case like Case II in Table 6.1 in Subsection 6.2.4, the band of persistent long-run labour supply is reduced to a curve of unstable equilibria.

More generally, there will be individual variation also in other parameters and exogenous variables than the reputation sensitivity $\varepsilon_{F i}$ such as the consumption taste $\alpha_{X i}$, the income of the husband $Y_{O i}$, the cognitive dissonance parameter $\gamma_{F i}$, the individual minimum $Y_{I M i}$ and the household time taste $\alpha_{L i}$. Generally this leads to a "smoothing out" of the kinks in Figure 8.7 and, when certain collective catastrophe conditions stronger than (8.24) are fulfilled, to collective entry and exit catastrophes between more than zero and less than $\bar{Y}_{I M} / w$ average corporate hours. In a case like Case IV in Table 6.1 in Subsection 6.2.4, in which a stronger variant of (8.23) holds, there will then be trendsetters as well as laggards in both entry and exit (cf. Fig. 7.1 in Sec. 7.2). This, however, is not elaborated here.

Another social norm alongside the financial independence norm which may play an important role in the labour supply of "emancipated" women, is the self-fulfilment norm as specified in Section 8.3 below (8.10). This can easily be shown to lead to similar short and long-run labour supply schedules as in the case of the financial independence norm, with the minimal self-fulfilment hours $H_{M}$ instead of $Y_{I M} / w$. A simplification then is that $H_{M}$, unlike $Y_{I M} / w$, is independent of $w$, implying that the $\vec{H}=Y_{I M} / w$ curve is replaced by a vertical $\bar{H}=H_{M}$ line in the average labour supply schedules. Moreover, unlike a very strong working of the financial independence nom, a very strong working of the self-fulfilment norm may imply a non-positive individual or collective long-run exit wage. In such cases women who lose their job and cannot find another one, will individually or collectively volunteer in the long run. Apart from the fact that $H_{M}$ does not need to be high, these cases correspond to the strong forms of individual or collective "workaholism" which have been considered in the Sections 4.5, 5.2, 6.4 and 7.6. However, proofs of these statements on the working of the self-fulfilment norm are beyond the scope of this study.

\subsection{Household norm and financial independence norm}

This section combines our model of the effects on female labour supply of the financial independence norm with that of the effects of the household norm into a model in which both norms can exist. Such a model can be used to describe the erosion of the household 
norm and the (possible) emergence of the financial independence norm in several OECD countries in the last three decades. As an extension of the specifications (8.2) and (8.5) in Section 8.1, the individual utility functions are then specified as

$$
\begin{aligned}
\hat{U}\left(X_{i}, R_{L i}, A_{L i}, L_{i}, R_{F i}, A_{F i}, n_{L i}, \varepsilon_{L i}, n_{F i}, \varepsilon_{F i}\right)= & \alpha_{X} X_{i}+\beta_{L} \varepsilon_{L i} R_{L i}+\gamma_{L} n_{L i} A_{L i}+ \\
& \alpha_{L} L_{i}+\beta_{F} \varepsilon_{F i} R_{F i}+\gamma_{F} n_{F i} A_{F i},
\end{aligned}
$$

where the subscripts " $L$ " and " $F$ " refer to the household and financial independence norm, respectively, and where all parameters are positive. The reputation $R_{L i}$ denotes the reputation of woman $i$ as housewife and mother, whereas $R_{F i}$ stands for her reputation as financially independent woman. These reputations are functions of the corresponding norm obediences $A_{L i}$ and $A_{F i}$ and the corresponding average norm beliefs $m_{L}$ and $m_{F}$, which functions are specified as $R_{L}\left(A_{L i}, m_{L}\right)=\rho_{L} m_{L} A_{L i}$ and $R_{F}\left(A_{F i}, m_{F}\right)=\rho_{F} m_{F} A_{F i}$.

Substituting these reputation functions and the norm obedience functions (8.9) with $\eta_{L}=1$ and (8.16) with $\eta_{F}=1$ into (8.25), transforming away irrelevant terms, and adding two negative quadratic terms in consumption and household time, we obtain the additive quadratic specification

$$
\begin{aligned}
U\left(L_{i}, X_{i} ; n_{L i}, m_{L}, \varepsilon_{L i}, n_{F i}, m_{F}, \varepsilon_{F i}\right)= & \left(\alpha_{L}+\frac{\gamma_{L} n_{L i}+\beta_{L} \varepsilon_{L i} \rho_{L} m_{L}}{T}\right) L_{i}+\alpha_{L L} L_{i}^{2}+ \\
& \left(\alpha_{X}+\frac{\gamma_{F} n_{F i}+\beta_{F} \varepsilon_{F i} \rho_{F} m_{F}}{Y_{I M}}\right) X_{i}+\alpha_{X X} X_{i}^{2},
\end{aligned}
$$

for $X_{i} \leq Y_{O i}+Y_{I M}$ and

$$
\begin{aligned}
U\left(L_{i}, X_{i} ; n_{L i}, m_{L}, \varepsilon_{L i}, n_{F i}, m_{F i}, \varepsilon_{F i}\right)= & \left(\alpha_{L}+\frac{\gamma_{L} n_{L i}+\beta_{L} \varepsilon_{L i} \rho_{L} m_{L}}{T}\right) L_{i}+\alpha_{L L} L_{i}^{2}+ \\
& \alpha_{X} X_{i}+\alpha_{X X} X_{i}^{2}+ \\
& \frac{\gamma_{F} n_{F i}+\beta_{F} \varepsilon_{F i} \rho_{F} m_{F}}{Y_{I M}}\left(Y_{O i}+Y_{I M}\right)
\end{aligned}
$$

for $X_{i} \geq Y_{O i}+Y_{I M}$, where $\alpha_{L L}<0$ and $\alpha_{X X}<0$. Just as in the case of Figure 8.5 in the previous section, the indifference curves consist, for positive $n_{F i}$ or $m_{F}$, of two segments and are strictly convex to the origin with diminishing marginal rates of substitution given by

$$
-\frac{d X_{i}}{d L_{i}}=\frac{U_{L_{i}}}{U_{X_{i}}}=\frac{\alpha_{L}+\frac{\gamma_{L} n_{L i}+\beta_{L} \varepsilon_{L i} \rho_{L} m_{L}}{T}-\left|\alpha_{L L}\right| L_{i}}{\alpha_{X}+\frac{\gamma_{F} n_{F i}+\beta_{F} \varepsilon_{F i} \rho_{F} m_{F}}{Y_{I M}}-\left|\alpha_{X X}\right| X_{i}}
$$

for $X_{i} \leq Y_{O i}+Y_{I M}$ and 


$$
-\frac{d X_{i}}{d L_{i}}=\frac{U_{L_{i}}}{U_{X_{i}}}=\frac{\alpha_{L}+\frac{\gamma_{L} n_{L i}+\beta_{L} \varepsilon_{L i} \rho_{L} m_{L}}{T}-\left|\alpha_{L L}\right| L_{i}}{\alpha_{X}-\left|\alpha_{X X}\right| X_{i}}
$$

for $X_{i} \geq Y_{O i}+Y_{I M}$. Analogously to (8.21), these marginal rates of substitution imply shortrun individual labour supply schedules like those in Figure 8.6 in the previous section. A difference is that for $n_{L i}>0$ and/or $m_{L}>0$, i.e. for positive beliefs in the household norm, the marginal rates of substitution in (8.27) are higher than those in (8.21), since the beliefs enhance the marginal utility of household time. In particular, the reservation wage $w_{r i}$ as given by the marginal rate of substitution in (8.27a) for $L_{i}=T$ and $X_{i}=Y_{O i}$ is then higher (but still finite), and for any given $w$ above the reservation wage, short-run labour supply is lower.

Suppose now that at $w=0$ all group members do not participate and have fully adapted their norm beliefs to that, i.e., they fully believe in the household norm $\left(n_{L i}=m_{L}=1\right.$ for all $i)$ and they do not believe at all in the financial independence norm $\left(n_{F i}=m_{F}=0\right.$ for all $i$ ). The individual long-run entry wages $w_{i}^{* 1}$ are then given by

$$
w_{i}^{* 1}=\frac{\alpha_{L}+\frac{\gamma_{L}+\beta_{L} \varepsilon_{L i} \rho_{L}}{T}-\left|\alpha_{L L}\right| T}{\alpha_{X}-\left|\alpha_{X X}\right| Y_{O i}} .
$$

These entry wages are all positive, so for $w$ rising from zero, the initial situation will remain a long-run equilibrium until $w$ reaches the lowest entry wage $w_{1}^{* 1}$. Assuming that the income of the husband $Y_{O i}$ is (approximately) the same for every group member, this entry wage is higher than the collective entry wage $\bar{w}^{* 1}=\left(\alpha_{L}-\left|\alpha_{L L}\right| T\right) /\left(\alpha_{X}-\left|\alpha_{X X}\right| Y_{O}\right)$ in Figure 8.7 in the previous section because of the cognitive dissonance and reputation loss sanctions with respect to the household norm.

Consider now first the NIV case of no individual variation in the sensitivities $\varepsilon_{L}$ and $\varepsilon_{F}$ to the reputations as housewife and mother and as financially independent woman. The group members then have the same individual entry wage as given by $(8.28)$, implying that they will collectively enter for $w$ rising beyond this entry wage $\bar{w}^{* 1}$. They will then develop a (partial) belief in the financial independence norm, while letting erode their belief in the household norm. This happening in the group members themselves as well as in other group members will reinforce their motivation for corporate work and induce them to work more corporate hours. In its turn, this will again strengthen their belief in the financial independence norm and weaken their belief in the household norm, etc..

Assume now that combined the sums $\gamma_{F}+\beta_{F} \varepsilon_{F} \rho_{F}$ and $\gamma_{L}+\beta_{L} \varepsilon_{L} \rho_{L}$ of cognitive dissonance and reputation parameters for the financial independence norm and the household norm are so high as to induce collective and hence individual entry catastrophes towards full obedience to the financial independence norm as well as full belief in it. This can happen in two situations which correspond to the analogues of Figure $4.4 \mathrm{c}$ and Figure 4.6 in Section 4.4 for the present model, with a financial independence norm instead of collective habit 
formation with respect to consumption. ${ }^{28}$ For example, in the former situation of a strong working of the household norm, an extension of the reasoning in Subsection 6.2.2 for the NIV case leads to the collective catastrophe conditions

$$
\begin{aligned}
& 0<\bar{c}^{*}:=\left|\alpha_{X X}\right|-\gamma_{X X}-\gamma_{X \bar{X}}=\left|\alpha_{X X}\right|-\frac{\gamma_{F}}{Y_{I M}{ }^{2}}-\frac{\beta_{F} \varepsilon_{F} \rho_{F}}{Y_{I M}{ }^{2}}<c_{o}^{*}, \\
& \bar{d}_{-}^{*} \leq \bar{d}^{*}:=\left|\alpha_{L L}\right|-\gamma_{L L}-\gamma_{L \bar{L}}=\left|\alpha_{L L}\right|-\frac{\gamma_{L}}{T^{2}}-\frac{\beta_{L} \varepsilon_{L} \rho_{L}}{T^{2}}<\bar{d}_{*}^{*}<0 .
\end{aligned}
$$

Here $\bar{d}_{-}^{*}$ and $\bar{d}_{+}^{*}$ are increasing and decreasing functions of $\bar{c}^{*}$, respectively, and for convenience we use the same symbols $\gamma_{X X}$ and $\gamma_{X \bar{X}}$ for our collective habit formation parameters with respect to the wife's income (see Sec. 8.8) as in the case of collective habit formation with respect to consumption. The conditions (8.29) can be rewritten as

$$
\begin{aligned}
& \gamma_{F}+\beta_{F} \varepsilon_{F} \rho_{F}>Y_{I M}{ }^{2}\left(\left|\alpha_{X X}\right|-c_{o}^{*}\right), \\
& T^{2}\left(\left|\alpha_{L L}\right|+\left|\bar{d}_{*}^{*}\right|\right)<\gamma_{L}+\beta_{L} \varepsilon_{L} \rho_{L} \leq T^{2}\left(\left|\alpha_{L L}\right|+\left|\bar{d}_{-}^{*}\right|\right) .
\end{aligned}
$$

Moreover, assume that separately the sum $\gamma_{L}+\beta_{L} \varepsilon_{L} \rho_{L}$ of the cognitive dissonance and reputation parameters for the household norm is not sufficiently high to induce collective and hence individual entry catastrophes to complete disobedience to the household norm as well as complete disbelief in it $\left(H_{i}^{*}=T\right)$. In the above situation example this happens when one of the conditions (8.30a) and (8.30b) is not fulfilled for $\gamma_{F}=\beta_{F} \varepsilon_{F} \rho_{F}=0$ and hence $\bar{c}^{*}=\left|\alpha_{X X}\right|$, so when, for instance, for this relatively large value of $\bar{c}^{*}$, the upperbound $\bar{d}_{+}^{*}$ becomes more negative than $\bar{d}^{*}$ or when $\bar{c}^{*}$ itself becomes larger than $c_{o}^{*}$ (this case corresponds to Figure 4.4a). In such cases there will, just as in Figure 8.7, occur individual entry catastrophes and hence a collective entry catastrophe from zero to $Y_{I M} / \bar{w}^{* 1}$ corporate hours. Note that in this equilibrium the group members, while fully believing in the financial independence norm, still belief to some extent in the household norn.

For $w$ rising further, the long-run labour supply will again follow the $Y_{l M} / w$ line until it meets a labour supply curve for $n_{F i}=m_{F}=0$. This curve represents the long-run average labour supply as a result of the working of the household norm, but not of the financial independence norm (this norm does not have an effect in the regime of $(8.30 \mathrm{~b})$ ). Because of the separately stable working of the household norm as assumed above, this curve will be continuous, albeit flatter than corresponding short-run curves (see Sec. 3.5), and it may therefore have a standard, though more pronounced backward-bending shape. For $w$ above a certain $w^{2}$, long-run labour supply is described by such a curve. Note that this $w^{2}$ will be

28 The maximal corporate time $T$ should then be replaced by $Y_{I M} / w$. the long-run reservation wage $w_{r}^{*}$ does no longer depend on the habit sensitivity $\gamma_{X X}$ and the susceptibility to social influence $\gamma_{X \bar{X}}$, and $w_{-}^{*}$ depends on the habit sensitivity $\gamma_{L L}$ and the susceptibility to social influence $\gamma_{L} \bar{L}$. Neither of these modifications leads to qualitatively different effects of changes in $c^{*}$ and $d^{*}$. but they imply other formulas for $w_{r}^{*}, w_{-}^{*}, w_{+}^{*}, d_{-}^{*}$ and $d_{*}^{*}$. 
more near to $\bar{w}^{* 1}$ as the working of the household norm is stronger and hence the corresponding long-run labour supply curve is flatter.

When $w$ again falls below $\tilde{w}^{* 1}$, the group members will first not reduce their corporate time because of cognitive dissonance and reputation loss sanctions in relation to the financial independence norm. On the contrary, they will, just as in Figure 8.7, increase their corporate time to continue their full obedience to the norm until, for $w$ declining beyond a certain $\dot{w}^{* 2}$, all group members collectively start to quit. Under the conditions (8.29) or $(8.30)$ this will lead to a collective exit catastrophe. Analogously to the collective entry catastrophe in Figure 8.7, this collective exit catastrophe will end at the long-run corner equilibrium for zero average labour supply and zero average and individual belief in the financial independence norm, but full average and full belief in the household norm. However, apari from the absence of trendsetters in the present NIV case, a difference with Figure 8.7 is that in the present case the exit wage $\tilde{w}^{* 2}$ tends to be higher as a result of the working of the household norm (see (8.30a) with positive $n_{L i}$ and $m_{L}$ ).

In a special case like Case IV in Table 6.1 in Subsection 6.2.4 with high $\gamma_{L}$ and/or $\gamma_{F}$, the exit and entry curves. look like the left parts of the entry and exit curves in Figure 8.7, again implying domains of collective entry and exit catastrophes and a band of persistent and hysteretic long-run labour supply. However, as a consequence of the additional cognitive dissonance effect of the household norm, the latter band will be broader than in Figure 8.7. On the other hand, in a special case like Case II in Table 6.1 with lower $\gamma_{L}$ and $\gamma_{F}$, the band of persistent long-run labour supply is reduced to a curve of unstable equilibria.

More generally, the household norm counteracts the positive consumption taste in forming a resistance against entry, but when the entry eventually takes place, the erosion of the household norm in combination with the emergence of the financial independence norm may reinforce the entry into a collective catastrophe. On the other hand, the financial independence norm counteracts the positive household time taste in forming a resistance against exit, but when the exit eventually takes place, the erosion of the financial independence norm in combination with the rise of the household norm may reinforce the exit into a collective catastrophe. Contrary to the models for one norm, this extended model of two opposite norms has the interesting implication that a pure "neoclassical" equilibrium in which nobody more or less believes in a social norm $\left(n_{i}=m=0\right.$ for all $i$ ), is always excluded (cf. Sec. 8.7). Every group member always believes to some extent in either the household norm or the financial independence norm or both.

Similar, but more complex results are obtained when we allow individual variation in the reputation sensitivities $\varepsilon_{L i}$ and $\varepsilon_{F i}$ and in other parameters and exogenous variables. In general, individual variation in more parameters and exogenous variables leads to stronger collective catastrophe conditions like those in Table 6.1, which, if fulfilled, imply collective entry and exit catastrophes between more than zero and fewer than $\bar{Y}_{I M} / w$ average corporate hours. The kinks in Figure 8.7 are then "smoothed out". In particular, in a case like Case II in Table 6.1 , the collective catastrophe conditions are obtained by replacing the bounds $c_{o}^{*}, \bar{d}_{-}^{*}$ and $\bar{d}_{+}^{*}$ in $(8.29)$ and $(8.30)$ by more stringent bounds $\vec{c}_{o}^{*}, \bar{d}_{-}^{*}$ and $\bar{d}_{+}^{*}$ as functions of the degrees of individual variation. Variation in the sensitivity to the reputation as housewife and mother $\varepsilon_{L i}$ leads to trendsetters with low $\varepsilon_{L i}$ preceding a collective entry catastrophe as in Figure 7.4 in Section 7.3. As a counterpart, variation in the sensitivity to the reputation as financially independent woman $\varepsilon_{F i}$ leads to trendsetters with low $\varepsilon_{F i}$ 
preceding a collective exit catastrophe as in Figure 8.7. Variation in other parameters and exogenous variables will, in a case like Case IV in Table 6.1, often imply trendsetters as well as laggards in both entry and exit, as in Figure 7.1 in Section 7.2.

Another extension is adding the self-fulfilment norm to the analysis. This may lead to somewhat more complicated results, but these are not essentially different from those found above (see, however, the end of the previous section). This holds also when we add other forms of collective habit formation with respect to household time, consumption, one's own income and corporate time. When the sum total of all these forms of collective habit formation, including the formations of (dis)beliefs in the household and self-fulfilment norm, is only destabilizing in combination with the formation of (dis)beliefs in the financial independence norm, we can again obtain a long-run average labour supply schedule like that in Figure 8.7. As referred to at the beginning of Section 8.8, in the case of married women the individual long-run labour supply schedules underiying such an average schedule are more plausible than schedules like that in Figure 4.2 in Section 4.2, describing individual hysteresis and catastrophes between zero and maximal corporate hours.

\subsection{Concluding remarks}

In this chapter variants of the theory of social custom (TSC) of Akerlof (1980) have been applied to female labour supply, and it has been demonstrated that these applications are largely analogous in structure to limiting cases of the AQM for BPI and unstable IHF with respect to household time and/or consumption. This turned out to have several implications and it has been argued that unrestricted AQM specifications give more adequate representations of the labour supply behaviour of married women than the more restricted Akerlof specifications. Moreover, a comparison with the literature on applications of the TSC has made clear that our applications add two essential pairs of elements to the overall picture, namely the pair of individual and collective catastrophes and the pair of individual and collective hysteresis. Finally, an important extension of the TSC consisted in combining the models for two opposite social norms, namely the household norm and the financial independence norm, into a model in which both norms can exist. Contrary to the TSC, this extension turned out to have the interesting implication of always excluding "pure" neoclassical equilibria in which nobody more or less believes in a social norm.

Although the TSC implies a less adequate representation of the labour supply behaviour of married women than the AQM, it is superior to the general AQM and most other specifications of IHF and BPI in the literature in giving an explanation of a specific mode of BPI by a reputation motive working via a reputation function and the utility function. In the present chapter this explanation has been supplemented by the explanation of a specific mode of IHF by the working of a cognitive consonance/dissonance motive in the utility function. On the other hand, in the general AQM and most other specifications of IHF and BPI in the literature such explanations are absent: the utility function is usually specified as a black box (in 
a rather ad-hoc way) and does not contain explicit motives of IHF and BPI. ${ }^{29}$ Then the question arises whether other or more general modes of collective habit formation than the mode modelled by the TSC can be explained in a similar way. This question is tackled by a general approach to explaining preference formation along the lines of Stigler and Becker (1976) in Vendrik (1988). Unfortunately, a discussion of this approach is beyond the scope of this study.

Anyway, in the present chapter the merits of both the TSC and the general AQM of collective habit formation have been combined by extending the Akerlof specification of the utility function in the TSC to an AQM specification of this utility function. Moreover, in order to remedy the, for married women, implausible exclusion by the $A Q M$ of interior stable individual equilibria in the case of separately (locally) unstable individual (dis)belief formation or IHF, the effects of a financial independence norm have been added to the AQM specification of the TSC for the household norm. Another extension of the AQM which remedies the implausible implication mentioned, is adding higher than second order terms to the utility function in the spirit of catastrophe theory (see Sec. 4.6 and Becker and Murphy, 1988 , p. 683). Such an addition can be rationalized by making in the TSC the very plausible assumption of diminishing marginal utilities of not only consumption, but also of reputation and, among other things, self-esteem. In a utility function like (8.5) this assumption leads to additional mixed fourth-order terms in social norm behaviours and beliefs, and intuitively the assumption can be argued to imply the possibility of one or two interior stable individual equilibria in the case of separately (locally) unstable individual (dis)belief formation (as in Fig. 3.1 in Sec. 3.2). However, this extension of the TSC should be further elaborated.

Another subject which has not been elaborated in the present chapter is the effects and dynamics of social norms in male labour supply. The results of such an elaboration would be partly similar to corresponding results for female labour supply. For example, neglecting the effects of social security benefits on the income constraint, results for the breadwinner norm and the family man or macho norm would be largely analogous to those for the financial independence norm and the self-fulfilment norm, respectively. ${ }^{30}$ On the other hand, for the labour supply of men as opposed to that of married women the availability of social security benefits is important, so its effects on the income constraint should be taken into account. For the case of a minimum social security benefit as in Subsection 2.2.3, the implications of the family man norm for short-run male labour supply are derived by De Neubourg and Vendrik (1993) (see also Sec. 10.3). These implications are similar to those of various levels of "psychic income" of corporate time for the same social security case (see, e.g., Subsection 2.2.5).

A related point which has been neglected in the present chapter, is the likely interaction

${ }^{29}$ A notable intermediate case is given by the lognomal utility function of Van Praag (1971). Contrary to the usual black-box approach, this specification is derived in a rigorous way from assumptions about motives formed by characteristics of goods (sec Van Praag. 1968, and Goedhart and Kapteyn, 1978). On the other hand, the IHF and BPI are specified in a rather ad-hoc way without explicit motives.

${ }^{30}$ Moreover, results for a weak household norm for men as mentioned at the end of Section 8.3 would be related to some extent to the results for the houschold norm for married women. Fortunately, the former norm is not 100 strong, since otherwise this book might not have been written. 
of the formations of (dis)beliefs in social norms by husband and wife. This interaction may proceed via the family income in the family budget constraint (see Sec. 7.7), but it may also be of a more direct type. For example, the husband may belong to a normative reference group of the wife, and the reverse. However, an elaboration of the dynamics of such indirect and direct norm interactions between husband and wife is beyond the scope of this study.

Although the extensions of the TSC and AQM as considered in the present chapter are substantial improvements, they may not be sufficient to meet two other drawbacks of the $\mathrm{AQM}$, namely $(i)$ within the class of additive utility functions the AQM of IHF with respect to consumption categories performs less favorably in empirical consumption studies than a linear expenditure threshold model (Taylor and Weiserbs, 1972, and Weiserbs, 1974), (ii) additivity of the utility function is rejected in many empirical studies (see, e.g., Deaton and Muellbauer, 1980, p. 140). These drawbacks suggest a need for a non-additive specification of the utility function. However, allowing higher than second order terms, such a specification will have too many parameters to be manageable. In order to restrict the number of parameters in a plausible and not-ad-hoc way we need another separability structure of the utility functions than additivity. Such a separability structure is derived in Vendrik (1988) by employing the same general approach which is used to explain preference formation, but should be further elaborated.

Finally, the theoretical results which have been derived in this and previous chapters, should be empirically tested. Although full-blown empirical tests are far beyond the scope of this study, the next chapter will discuss the empirical relevance of some theoretical results of this study. In particular, we will then make preliminary attempts to assess the role of social norms and collective habit formation in the developments of the female labour force participation in the Netherlands and in the U.S.A.. 


\section{CHAPTER 9}

\section{Dynamics of Female Participation}

This chapter investigates the empirical relevance of the theoretical models which have been set forth in the previous chapters, by applying them to the developments of the labour force participation of Dutch and American married women since World War II. Whereas the participation rate of Dutch married women was remarkably low and stable till 1960, it grew strongly after 1960. Econometric time-series analyses of this development have been given by Mol et al. (1988) for the period 1889-1985 and by Romme $(1987,1990)$ for the shorter period 1971-1985. Although the empirical results of the former study seem plausible, the study suffers from the drawback of not explicitly taking into account the probably important impacts of social norms and collective habit formation (CHF). Romme, on the other hand, includes variables for the impacts of social norms and behavioural preference interdependence (BPI) in linear and loglinear logit participation equations for female (birth) cohorts, and finds consistently significant, stable and important coefficients of these variables. For CHF this result is confirmed by cross-section estimates for 1985 of the detailed microeconomic individual labour supply model of Kapteyn and Woittiez (1990). However, Romme's specifications of his participation equation are not systematically derived from an underlying micro-economic labour supply model. Moreover, he does not distinguish the female cohorts according to civil status (married or not) and number and age of children.

As an improvement on that, this chapter derives general and logit participation equations for a social group, cohort and subpopulation of married women with school-going (or small) children on the basis of the theoretical models of the previous chapters. The logit participation equation resembles the loglinear logit participation equation of Romme, so Romme's empirical results can be considered as a support of the empirical relevance of our theoretical model of the impacts of social norm change and CHF on female labour supply. On the other hand, the latter model suggests that the loglinear as well as the linear participation equation of Romme contain severe specification errors. Moreover, our theoretical model implies different specifications of the participation equation for entry and exit behaviour as a result of hysteretic persistence effects of individual habit formation (IHF). The modelling of such effects by Clark and Summers (1982) is shown to be incorrect due to a severe aggregation error.

The endogenous dynamics of CHF and social norm beliefs according to our theoretical model turn out to imply long-run elasticities of the participation rate with respect to the wage rate and other exogenous variables which essentially differ from the corresponding 
short-run elasticities. More specifically, the long-run elasticities vary with the level of the participation rate according to an essentially different hump-shaped profile. This is shown to imply serious doubts on the empirical simulation finding of Hartog and Theeuwes (1985) of a strong and dominant impact of the own wage rate on the development of the participation rate of Dutch married women since World War II. The implications of the hump-shaped participation rate profile of the long-run elasticities for the long-run dynamics of the participation rate of married women are elaborated. If we make a simplifying assumption, the profile is demonstrated to lead, in a regime of constant relative change rates of the exogenous variables encouraging participation, to a sigmoid shape of the time profile of the participation rate which is considerably "sharper" than the sigmoid shape of a logistic curve implied by static labour supply models like that of Hartog and Theeuwes. We call the former curve super-exponential-logistic. More adequately than the logistic curve, such a curve can account for the empirical pattern of a remarkably persistent low participation rate of Dutch married women before 1960 and a strongly growing participation rate of these women after 1960. The persistence of the low participation rate before 1960 is then partially explained by low values of long-run elasticities of exogenous variables, whereas the strong rise of the participation rate after 1960 is partially explained by higher values of such elasticities. In close connection, the persistence of the participation rate of American married women just after World War II (Clark and Summers, 1982) is explained as the result of hysteresis effects of IHF. Such hysteresis effects are also shown to explain differences in as well as convergence of participation rates among countries.

The strong rises in the participation rate of Dutch married women after 1960 and of American married women after 1950 may have been caused by collective catastrophes within social groups, i.e. by large shifts in the participation rates of social groups as a consequence of (infinitesimally) small changes in the exogenous variables. Then the long-run elasticities of the participation rate of the social group become infinite as a result of unstable behavioural preference interdependence (BPI). A necessary and sufficient condition for such a collective catastrophe in the participation rate is derived. Just as in Section 7.2, we obtain a labour supply schedule with a persistent domain, where the long-run participation rate follows (small) hysteresis loops, and two catastrophic domains. Aggregating such schedules to the level of cohorts or (sub)populations is shown to preserve the hysteresis properties, but to "smooth out" the collective catastrophes. Because of the scarcity of disaggregated data on the level of social groups the latter property severely hinders the identification of collective catastrophes on that level. The hysteresis properties imply that the persistence of the participation rate of American married women just after World War II can be explained not only by IHF, but also by unstable BPI within social groups. Finally, it is argued that the development of the participation rate of Dutch married women after World War II may be conceived in a stylized way as the result of a cultural cycle superimposed on an upward labour demand trend. The cultural cycle is caused by a combination of collective habit (and norm belief) formation in a medium long run and collective satiation in a very long run. Since the two mechanisms can be associated with conceptions of Marx and Maslow (1943, 1970), respectively, the cycle is referred to as the Marx-Maslow cycle.

The organization of this chapter is as follows. Section 9.1 reviews the main results of the econometric time-series analysis of the long-run development of the labour force participation of Dutch married women by Mol et al.. Section 9.2 analyses the impacts of social 
norms and collective habits on this development, referring to empirical results of Romme and of Kapteyn and Woittiez. Sections 9.3 and 9.4 derive participation equations for one social group of married women with school-going (or small) children on the basis of the theoretical models of the previous chapters. Section 9.5 aggregates these participation equations to the level of a (birth) cohort or subpopulation of married women with schoolgoing children and adds the effects of BPI between social groups. Section 9.6 discusses Dutch survey proxies for the norm belief variables in these aggregated participation equations and analyses the econometric evidence of Romme on the impacts of such proxies. Moreover, the modelling of the persistence effects of IHF on labour force participation by Clark and Summers is criticized.

Then, Section 9.7 gives a short description of historical evidence on the endogenous dynamics of social norms and collective habits in the participation of Dutch married women. Section 9.8 derives the implied hump-shape of the participation rate profile of the long-run elasticities of the participation rate with respect to the wage rate and other exogenous variables. The ensuing serious doubts on the empirical simulation finding of Hartog and Theeuwes are expressed. Section 9.9 elaborates the implications for the long-run dynamics of the participation rate of married women. In particular, the super-exponential-logistic curve is derived and the hysteresis effects of IHF are analysed. Section 9.10 analyses the possibility of collective entry and exit catastrophes and derives a condition for such catastrophes as well as implied long-run participation rate schedules. Section 9.11 introduces the Marx-Maslow cycle and Section 9.12 concludes.

\subsection{Female labour force participation: the Dutch case}

Since World War II there has been a substantial increase in the labour force participation of women in many OECD countries. This is shown in Table 9.1. Obviously, until recent years

\begin{tabular}{lrrrrrrr}
\hline & 1950 & 1960 & 1970 & 1975 & 1980 & 1985 & 1991 \\
\hline Canada & 26.2 & 32.0 & 41.1 & 50.5 & 57.9 & 63.5 & 69.9 \\
France & 49.5 & 46.4 & 49.8 & 52.9 & 55.6 & 56.4 & 58.1 \\
Germany & 44.3 & 49.2 & 48.1 & 50.8 & 52.8 & 52.9 & 55.9 \\
Japan & 57.6 & 60.0 & 55.4 & 51.7 & 54.9 & 57.2 & 61.5 \\
& & & & & & & \\
Spain & 17.6 & 23.6 & 28.9 & 33.8 & 33.8 & 34.8 & 42.6 \\
Sweden & 35.1 & 55.0 & 60.6 & 68.9 & 75.8 & 79.7 & 82.6 \\
United Kingdom & 40.7 & 46.1 & 53.5 & 58.6 & 61.7 & 63.3 & 68.2 \\
United States & 37.2 & 42.6 & 50.4 & 54.9 & 61.3 & 65.5 & 69.6 \\
& & & & & & & \\
Netherlands & 28.5 & 26.3 & 30.3 & 32.0 & 36.3 & 41.0 & 54.5 \\
\hline
\end{tabular}

Table 9.1 Female labour force participation rates in some OECD countries: 1950-1991 (in \%). Sources: OECD (1979), Demographic Trends 1950-1990. Paris; OECD (1990), Labor Force Statistics 1968-1988, Paris: OECD (1992), Labor Force Statistics 1970-1990. Paris. 
the Dutch female labour force participation rate was far below that in other OECD countries. Only Spain, which was in a less advanced state of economic development, had a similar participation rate.

Moreover, these data indicate that the increase in the Dutch female labour force participation rate started later than in most other OECD countries, namely after 1960 . This is shown in Figure 9.1, which displays the Dutch developments of the participation rate of all. women as well as those of married and unmarried women between 1889 and 1985 . Thus, we see that the time pattern of the overall participation rate is mainly determined by the time pattern of the participation rate of married women. Whereas this participation rate was remarkably stable between 1889 and 1960 , it was growing strongly after that from $7 \%$ in 1960 to $36 \%$ in 1985 . This meant a multiplication by five in 25 years.

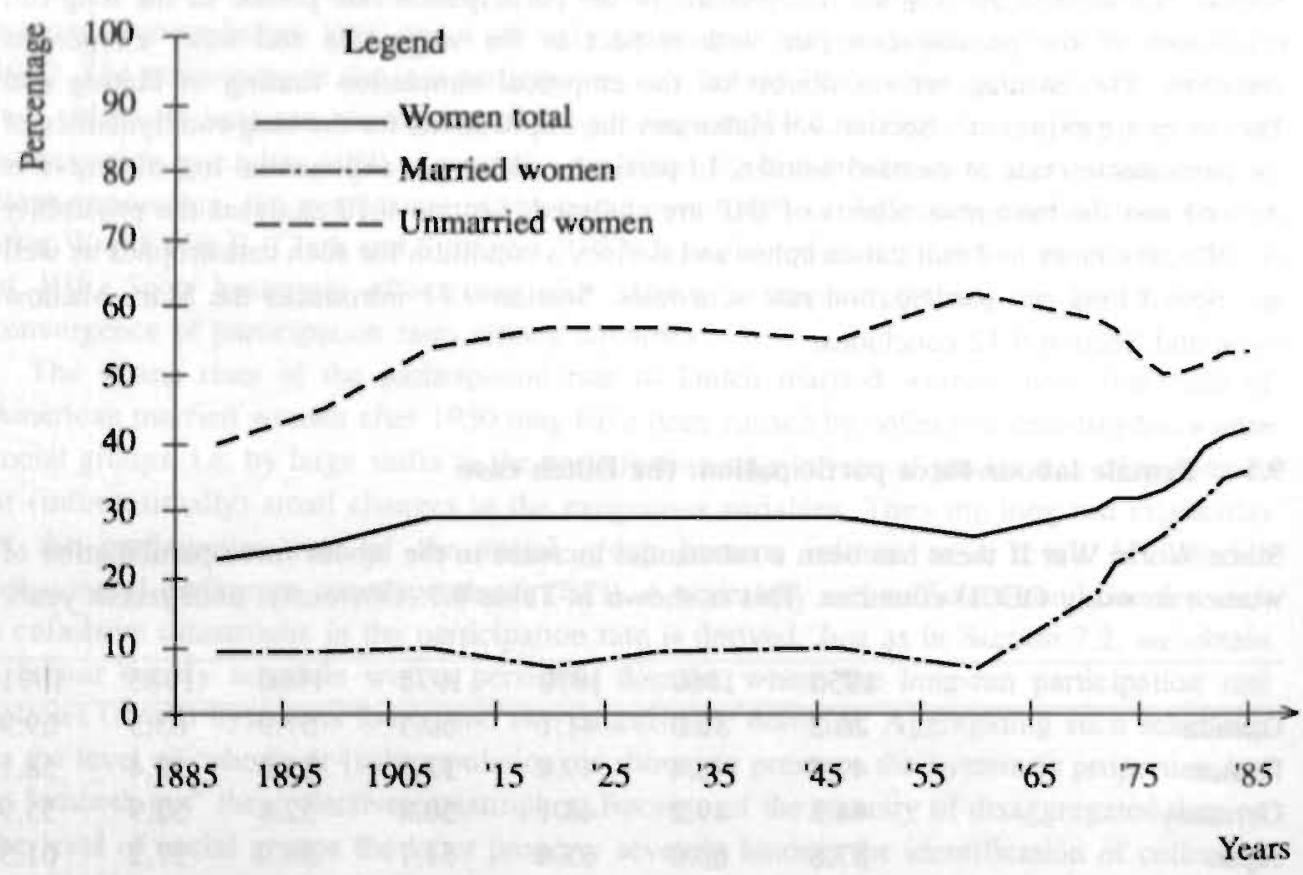

Figure 9.1 Labour force participation rates of married and unmarried Dutch women: 1889-1985 (in \%). Source: data of Mol et al. (1988, Fig. 1.1).

This phenomenon has induced a lot of research on the labour force participation of married women. However, a survey of the most important econometric studies since the midseventies by Mol et al. (1988) reveals that most of these studies are confined to cross-section analyses and that only some recent studies combine such an analysis with a shor time-series analysis. As far as I know, econometric time-series analyses of long-run developments of the labour force participation of Dutch married women have only been carried out by Mol et al. (1988) for the period 1889-1985 and by Romme (1987, 1990) for the much shorter period 1971-1985. Non-econometric analyses of historical trends are given by, among others, 
Hartog and Theeuwes (1985, Sec. II), Kok and De Neubourg (1986, Ch. III) and De Neubourg (1988, Ch. IV). The main results of Mol et al. are dealt with in this section, whereas: the main results of Romme, Kok and De Neubourg, and De Neubourg will be discussed in subsequent sections.

On the basis of their survey and the availability of long-term data, Mol et al. choose a number of explanatory variables on the supply and demand. sides of the labour market. The empirical analysis leads to a reduction of this number of exogenous variables to five. Three of these five variables belong to the supply side, namely age of the women, number of children, and education of the women. Two variables relate to the demand side, namely the share of the service sector in total employment and the share of the number of persons with a part-time job in the total labour force (of men as well as women). Here we discuss only those results with respect to these variables which are of interest in the present context.

First we consider the age effects. Separating the development of the participation rate of married women into the developments of the participation rates in different age groups, we obtain Figure 9.2. In this figure the unimportant age group of 15-19 years has been omitted. For all remaining age groups except that of 60-64 years, the participation rate was again rather stable between 1889 and 1960. After 1960 the participation rate was rocketing in the

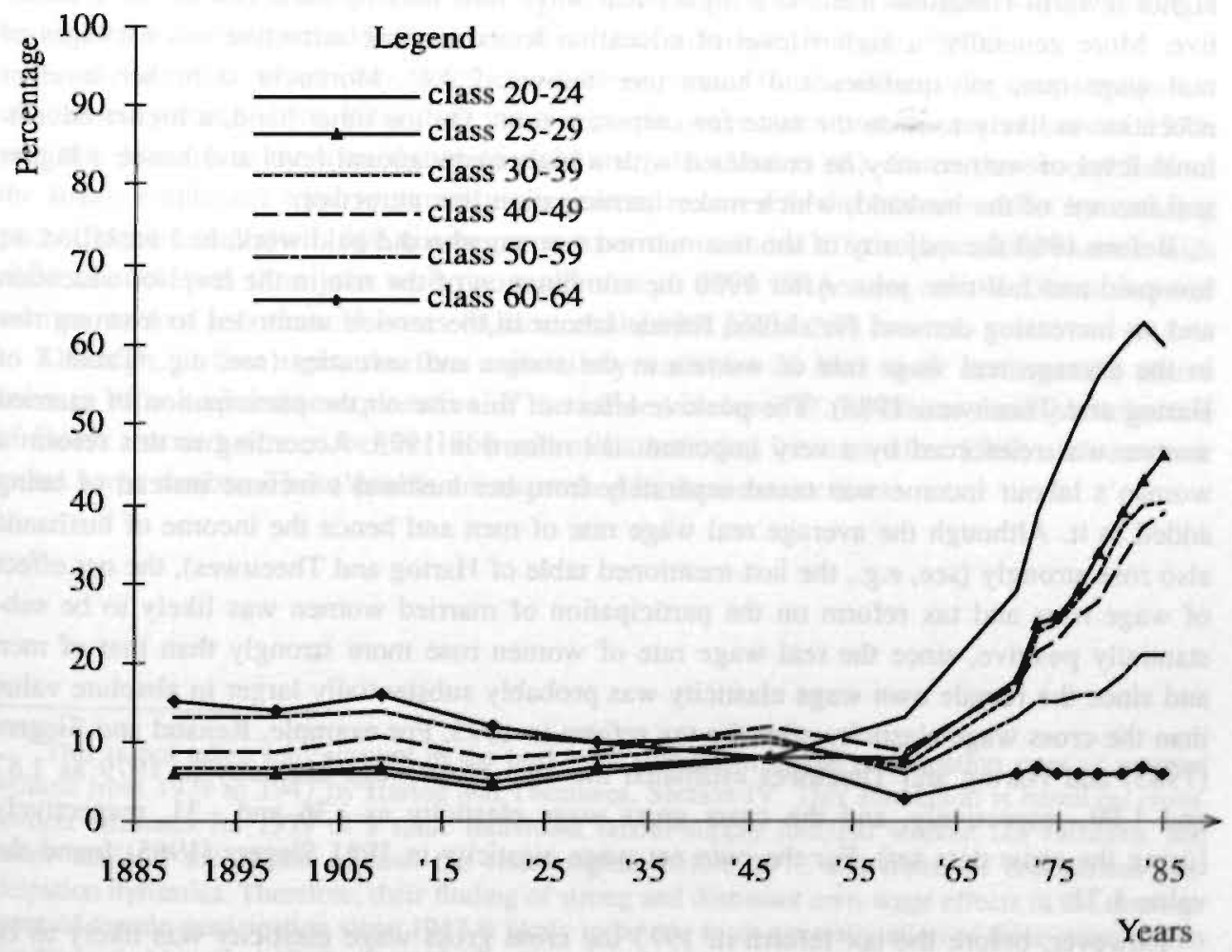

Figure 9.2 Labour force participation rates of Dutch married women, dislinguished into age classes: 1889-1985 (in \%). Source: data of $\mathrm{Mol}$ et al. (1988, Fig. 2.3). 
age group of $20-24$ years from $13 \%$ in 1960 to even $60 \%$ in 1985 and was growing at a somewhat slower pace in the age groups of $25-29,30-39$ and 40-49 years. Remarkably, the last three age groups display a similar time pattern. On the other hand, the participation growth was much lower in the age group of 50-59 years and very low in the age group of 60-64 years.

An important cause of the participation booms in the age group of 20-25 and, to a less extent, in that of 25-29 years may be that after 1960 a growing number of women kept their jobs when marrying and delayed the birth of the first child. More generally, a drop in the birth rate was an important cause of the participation growth in all age groups till 60 years and, in particular, favoured re-entry at older ages. First more women with grown-up children re-entered, next more women with school-going children, and finally, as implied by Figure 2.6 in Mol et al., a growing number of women with children under school age re-entered or kept their jobs after 1977.

Another important cause of the participation growth in all age groups till 60 years was the rising level of education. Especially the number of married women with a higher education increased strongly, and especially the participation of this group of women rose dramatically. In general, the positive effect of the educational level on the participation of women results from the impacts of a number of factors (see, e.g., Siegers, 1983, pp. 417-418). First, a higher level of education leads to a higher real wage rate, making participation more attractive. More generally, a higher level of education leads to more attractive job packages of real wage rate, job qualities and hours (see Subsec. 2.2.4). Moreover, a higher level of education is likely to raise the taste for corporate time. On the other hand, a higher educational level of women may be correlated with a higher educational level and hence a higher real income of the husband, which makes participation less attractive.

Before 1960 the majority of the few married women who did paid work, had unskilled, so low-paid and full-time jobs. After 1960 the combination of the rise in the level of education and an increasing demand for skilled female labour in the service sector led to a strong rise in the average real wage rate of women in the sixties and seventies (see, e.g., Table 7 of Hartog and Theeuwes, 1985). The positive effect of this rise on the participation of married women was reinforced by a very important tax reform in 1973. According to this reform a woman's labour income was taxed separately from her husband's income instead of being added to it. Although the average real wage rate of men and hence the income of husbands also rose strongly (see, e.g., the last-mentioned table of Hartog and Theeuwes), the net effect of wage rises and tax reform on the participation of married women was likely to be substantially positive, since the real wage rate of women rose more strongly than that of men and since the female own wage elasticity was probably substantially larger in absolute value than the cross wage elasticity after the tax reform in 1973. For example, Renaud and Siegers (1983) and Hartog and Theeuwes estimated the own gross wage elasticity in 1979 as 1.87 and 1.99. respectively, and the cross gross wage elasticity as -.36 and -.31 , respectively (using the same data set). For the own net wage elasticity in 1981 Siegers (1985) found the value 1.71 .

However, before the tax reform in 1973 the cross gross wage elasticity was likely to be substantially more negative, since the increase in the income of the husband then led to a higher marginal tax rate for the additional family income of the wife (see Mol et al., p. 29). This implies a smaller or even negative difference between the absolute values of the own 
wage and cross wage elasticities before 1973. Moreover, an endogenous participation dynamics like that explained in the Sections 9.8-9.10 implies smaller absolute values of both wage elasticities by common factors, and hence a smaller difference between these absolute values in the sixties and a much smaller difference in the fifties. Therefore, due to the combination of the fiscal regime before 1973 and forms of endogenous participation dynamics, the wage effect on the participation of married women may have been smaller in the sixties and much smaller in the fifties. ${ }^{1}$

On the other hand, the above-mentioned high estimate 1.71 of the own net wage elasticity in 1981 suggests that the tax reform in 1973 had a strong direct impact on the participation of married women by implying a slump in the marginal tax rate, and hence a jump in the net real wage rate of married women. However, this does not show up as a structural break in the participation developments around 1973 (see Figs. 9.1 and 9.2). This may be related to the first oil crisis. On the other hand, when in 1984 and 1985 the tax reform of 1973 was partially reversed by so-called earning couple measures, about 10.000 women resigned from their job (see Social and Cultural Planning Bureau, 1986, p. 69). This may partially explain the decline in participation of the age group of 20-24 years and the slow-down of the rise in participation of the age groups of 24-59 years between 1983 and 1985 according to Figure 9.2 (see also Fig. 9.1). ${ }^{2}$ Another explanatory factor may be discouragement by the high unemployment, but according to $\mathrm{Mol}$ et al. such a negative discouraged worker effect is dominated by a positive added worker effect (see, however, Romme, 1990).

A crucial factor which may have been more important for the increase in the participation of married women since 1960 than the rise in the wage rates and the tax reform of 1973 . was the increase in the availability of part-time jobs (Mol et al.). Since the second half of the fifties employers tried to attract more married women to the labour market by creating part-time jobs, and after 1960 this began to have the intended effect. Especially women with children took part-time jobs with the result that in 199078 percent of the Dutch married women worked less than 35 hours (Allaart et al., 1991, Tab. 2.7).

Although, at first sight, the five explanatory variables of $\mathrm{Mol}$ et al. as discussed above seem to offer a plausible explanation of the stable low level of the labour force participation of Dutch married women before 1960 and of its booming increase after 1960, a closer look raises some doubts. These doubts are expressed in the next sections.

I This is not taken into account in the backward simulation of the participation rates of married women from 1979 to 1947 by Hartog and Theeuwes, Section IV. This simulation is based on crosssection estimates for 1979 of a static individual labour supply function without tax variables, and hence neglects the effects of both the fiscal regime before 1973 and forms of endogenous participation dynamics. Therefore. their finding of strong and dominant own-wage effects in the development of female participation since 1947 is likely to be due to an overestimation of these wage effects for the period between 1947 and 1973. See Secs. 9.8-9.10 for details on an endogenous participation dynamics.

${ }^{2}$ See Bruyn-Hundt and Van der Linden (1989) for a recent inventarization of government regulations discouraging the labour supply of Dutch women. 


\subsection{Impacts of social norms and collective habits}

The explanation of the long-term development of the participation of Dutch married women by Mol et al. gives rise to a number of questions. First, changes in social norms, collective habits and social attitudes, which seem very important in the Dutch participation history according to historical descriptions (see, e.g., Sec. $2.4 \mathrm{of} \mathrm{Mol} \mathrm{et} \mathrm{al.),} \mathrm{are} \mathrm{not} \mathrm{explicitly} \mathrm{taken}$ into account in the model of Mol et al.. As proxies for the changes in social norms with respect to the role of the married woman Mol et al. introduce the upward trends in the degrees of secularization and urbanization. However, although the degree of secularization is significant in (first-difference) logit participation equations for the whole period 1889-1985 as well as for the period 1960-1985, both proxies do not survive selection exercises.

On the other hand, for the much shorter time period 1971-1985 Romme $(1987,1990)$ introduces a more reliable proxy of the general support of social norms regarding the participation of married women. This proxy is obtained from a questionaire item in the reports of the Dutch Social and Cultural Planning Bureau (1986). In an, after 1978 almost yearly, questionnaire among a representative sample of the Dutch population the following question is asked: "What do you think of a married woman with school-going children who has a job?". The following answers could be given: (1) recommended, (2) not objectionable, or (3) objectionable. As shown in Figure 9.3, the percentages of these answers have changed a lot between 1965 and 1985 (and 1991). Whereas in $196517 \%$ of the Dutch population thought that a married woman with school-going children having a job was recommended or not objectionable, this percentage had risen to $70 \%$ in 1985 . Romme assumes that this combined percentage of "recommended" and "not objectionable" is a good proxy of the general support for a norm in favour of female participation (see Fig. 1 of Romme for the development of this proxy over time and see Sec. $\mathbf{9 . 6}$ for a critical remark on this proxy). Next, regressions of logit participation equations including this proxy yield consistently significant and stable coefficients of this social nom variable. However, if we compare this important result to the corresponding results of $\mathrm{Mol}$ et al., it should be noted that most explanatory variables of Romme are different from those of Mol et al. (see Sec. 9.6 for details).

Some of the explanatory variables of Romme represent the participations of the same and earlier (birth-)cohorts in previous periods and can be associated with the impacts of behavioural preference interdependence. ${ }^{3}$ Also these impacts are significant and very large in the case of a loghinear logit specification of the participation equation (see Secs. 9.4 and 9.6 for details). For micro-economic cross-section data for 1985 these significant impacts of collective habits are confirmed by the detailed micro-economic individual labour supply model of Kapteyn and Woittiez (1990) (see also Kapteyn, Woittiez and Ten Hacken, 1989). They find significant effects of individual habit formation and behavioural preference interdependence on individual labour supply.

A second question concerning the results of Mol et al. is related to the strong impact of the age variable (which is represented by dummies for the age groups). Figure 9.2 shows

${ }^{3}$ Romme introduces these lagged participation variables in his participation equations in order to describe the influence of social noms via social reference groups. However, this interpretation of the lagged participation variables seems wrong, since the influence of social noms is already captured by Romme's social norm variable. 


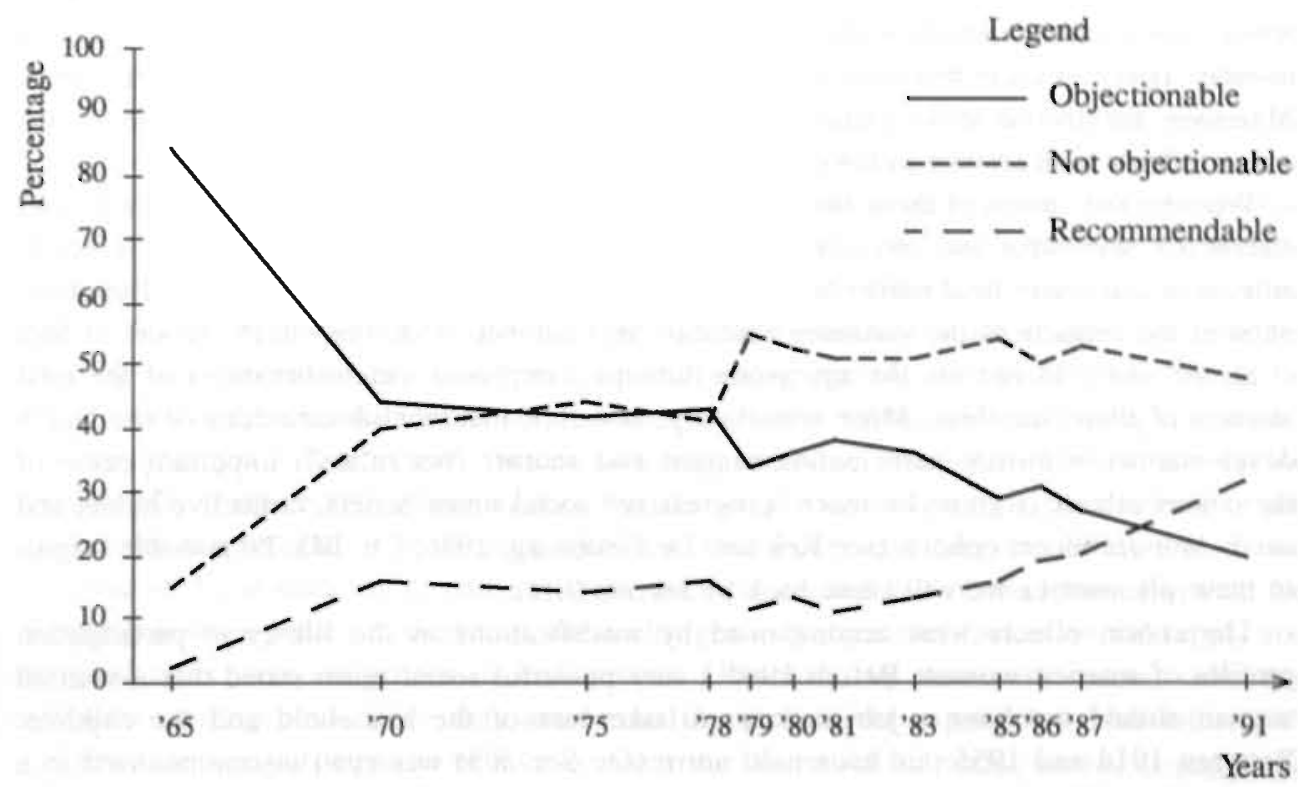

Figure 9.3 Public opinion on married women with school-going children having a job: 1965-1991 (in \%). Source: Social and Cultural Planning Bureau (1986, 1992).

that in the period 1960-1985 the participation in younger age groups was mostly higher than the participation in older age groups. Exceptions are given by the participation in the age group of 30-39 years being somewhat lower than the participation in the age group of 40-49 in 1960 and 1971 (see also Fig. 2.5 of Mol et al.). These empirical facts are the net or total results of two different kinds of effect, namely of so-called (birth-)cohort effects and of lifecycle effects (cf., e.g., Joshi and Overton, 1984). According to the former effects the participation in more recent cohorts was practically always higher than and sometimes equal to the participation in earlier cohorts in all age groups between 20 and 64 in the period 19601985 (see Fig. 2.5 of Mol et al.). Similar results for all women have been obtained for the U.S.A. and Great Britain for much longer time spans (see, e.g., Killingsworth and Heckman, 1986, Sec. 2) and for Sweden. ${ }^{4}$ Thus, in these countries as well as in the Netherlands between 1960 and 1985, more recent female cohorts were generally more oriented towards corporate work than were earlier cohorts. For the Netherlands this explains the above-mentioned finding of higher participation in younger age groups.

On the other hand, life-cycle effects have quite different implications. These effects result from the developments of the participations of individual women over their lifetimes. For cohorts of women who marry and get children, these developments typically imply a bimodal (or multimodal) life-cycle participation profile with a decline at childbearing and childrearing ages and a rise due to re-entries at older ages (see, e.g., Killingsworth and Heckman, 1986, Figs. 2.1 and 2.2). For the Netherlands the latter rise accounts for the above-mentioned

${ }^{4}$ Kok and De Neubourg (1986, Sec. III.3) give some evidence for the period 1963-1983. 
exceptional finding of the participation in the age group of 30-39 years being somewhat lower than the participation in the age group of $40^{\circ}-49$ in $1960^{\circ}$ and 1971 . However, for other re-entry ages and years this life-cycle effect is more than compensated by the cohort effects. Moreover, the decline of the participation at childbearing and childrearing ages reinforces the cohort effects with respect to the differences in participation between age groups.

Whereas the causes of these life-cycle effects are rather obvious, the causes of the cohort effects are somewhat less obvious. Important factors seem to be given by higher levels of education and lower total numbers of children of women in more recent cohorts. Therefore, parts of the impacts of the variables education and number of children in the model of $\mathrm{Mol}$ et al. are likely to run via the age group dummies, implying underestimations of the total impacts of these variables. More importanily, however, historical descriptions of the Dutch developments in female participation suggest that another (but related) important cause of the cohort effects is given by more "progressive" social norm beliefs, collective habits and needs in more recent cohorts (see Kok and De Neubourg, 1986, Ch. III). To possible origins of these phenomena we will come back in Section 9.11.

The cohon effects were accompanied by modifications in the life-cycle participation profiles of married women. Before 1960 a very powerful social norm stated that a married woman should not have a job in order to take care of the household and the children. Between 1914 and 1955 this household norm (see Sec. 8.3) was even institutionalized in a law requiring dismissal of female civil servants on marriage. Only in the fifties the household norm began to lose grounds and, combined with an increasing delay of the birth of the first child and other factors, the further erosion of the norm in the sixties led to a booming practice of women keeping their job when marrying. Moreover, the erosion of the household norm contributed to a strong increase in re-entries by married women with grown-up children.

On the other hand, a weaker variant of the household norm stating that women with school-going children should not have a job, at first remained strong and began to erode only in the mid sixties (see Fig. 9.3). Then, however, it eroded extremely fast between 1965 and 1970 as expressed by the sharp fall in Figure 9.3 of the percentage of the answer "objectionable" from $84 \%$ in 1965 to $44 \%$ in 1970 . As a result, more women with schoolgoing children re-entered. Finally, in the late seventies and eighties the weakest variant of the household nom stating that women with children under school age should stay at home, began to lose its influence. Whereas in $198257 \%$ of the women of 21-37 years were of the opinion that a job and having children under school age cannot be combined, in 1988 a minority of $38 \%$ took that view (De Graaf, 1989). In combination with better day care facilities this contributed to the development after 1977 of a growing number of married women with small children re-entering or keeping their jobs. As a result, among the most recent cohorts there will have been a dampening of the dip in the life-cycle participation profile at childbearing and childrearing ages that was characteristic of earlier cohorts. ${ }^{5}$

A third question regarding the study of $\mathrm{Mol}$ et al. is related to the assumed exogeneity of the variables number of children and education. In fact, in our modern time of broad access

5 This result has also been found for the U.S.A. for earlier cohorts. (see Killingsworth and Heckman, 1986, Sec. 2). 
to birth control methods and education for women, both variables are subject to choice by women and may even be choice variables in simultaneous long-run choices of education, participation and number of children (see for simultaneity of the last two choice variables, e.g., Siegers, 1985, Sec. III.6). In that case education and number of children should be treated as endogenous choice variables in more or less simultaneous long-run preferences of married women with respect to education, participation and number of children. After 1960 these preferences have apparently changed, but then the question arises why? Probably important "objective" opportunity factors are birth control methods, education opportunities, reasonably paid part-time jobs and day care facilities becoming more broadly available for married women. However, a probably equally important role was played by changes in "subjective" preference factors as social norms, collective habits, social attitudes and needs.

Such changes in "subjective" factors may have played an important role not only on the supply side of the female labour market, but also on the demand side in the decrease of job and wage discrimination of women (see, e.g., Schippers and Siegers, 1991) and in the increase of the availability of part-time jobs since the second half of the fifties. But then the question arises whether, in their turn, these changes in "subjective" factors on the supply and demand sides were exogenous or endogenous. Obviously, by their very nature, collective habits are endogenous, since they are formed by (collective) experiences with either participation or non-participation behaviour. Does this also hold for social norms, as suggested by the long-run models in Sections 8.1 and 8.5 , and for social attitudes and needs? These questions are addressed in Section 9.7 for social norms and attitudes and in Section 9.11 for needs. Before that, however, the next three sections derive participation equations for subpopulations of married women which include variables for collective habits and social norms.

\subsection{General participation equation for one social group}

This section and the next two derive equations for the labour force participation rates of specific subpopulations of married women, including variables for collective habits and social norms. First, this section derives a general participation equation for one social group in these subpopulations. Then, the next section analyses a logit specification of the general participation equation. Finally, Section 9.5 aggregates this logit participation equation to the level of (birth) cohorts or subpopulations and adds behavioural preference interdependence between social groups to the equations.

Since observed paths of participation rates will generally deviate from their long-run equilibrium paths (see the end of Sec. 3.5), they can be described only by equations for short-run participation rates, taking into account the actual disequilibrium values of the state variables. In general, the short-run participation rate in a social group $P$ is equal to the fraction of group members $i$ whose short-run reservation wage $w_{r i}$ is lower than the wage rate $w_{i}$ prevailing for them. Combining the $\mathrm{AQM}$ for collective habit formation of Subsection 6.3.2 (and Sec. 6.4) with the AQM for social norms of Section 8.10, the short-run reservation wage $w_{r i}$ can be expressed in the parameters of the thus augmented AQM as 


$$
w_{r i}=\frac{b_{i}}{a_{i}}=\frac{\alpha_{L i}-\left|\alpha_{L L i}\right| T_{i}+\gamma_{L L i} s_{L i}+\gamma_{L \bar{L}} s_{L}+\frac{\gamma_{L i}}{T_{i}} n_{L i}+\frac{\beta_{L i} \varepsilon_{L i} \rho_{L i}}{T_{i}} m_{L}}{\alpha_{X i}-\left|\alpha_{X X i}\right| Y_{O i}+\gamma_{X X i} s_{X i}+\gamma_{X \bar{X} i} s_{\bar{X}}+\frac{\gamma_{F i}}{Y_{I M i}} n_{F i}+\frac{\beta_{F i} \varepsilon_{F i} \rho_{F i}}{Y_{I M i}} m_{F}} .
$$

Here the parameters $\alpha_{L i}$ and $\alpha_{X i}$ represent the household time and family consumption tastes of married woman $i, T_{i}$ is her total discretionary time, $Y_{O i}$ is the income of her husband, $\alpha_{L L i}$ and $\alpha_{X X i}$ describe diminishing marginal utilities of household time and (family) consumption, $\gamma_{L L i}$ and $\gamma_{X X i}$ are the household time and consumption habit sensitivities, $s_{L i}$ and $s_{X i}$ are the corresponding habit states, $\gamma_{L \bar{L} i}$ and $\gamma_{X \bar{X} i}$ are the susceptibilities to the social household time and consumption environments $s_{\bar{L}}$ and $s_{\bar{X}}, \gamma_{L i}$ and $\gamma_{F i}$ are cognitive dissonance parameters with respect to the individual beliefs in the household norm and financial independence norm $n_{L i}$ and $n_{F i}, Y_{I M i}$ is the individual minimum income for being financially independent, and $\gamma_{L i} \varepsilon_{L i} \rho_{L i}$ and $\gamma_{F i} \varepsilon_{F i} \rho_{F i}$ are reputation parameters with respect to the average beliefs in the household norm and financial independence norm $m_{L}$ and $m_{F}$.

The values of the state variables, i.e. the values of the habit states, the social environments and the individual and average norm beliefs, and hence the level of the reservation wage of woman $i$, depend on the kind of situation in which the decision to participate or not is made. In one situation, woman $i$ holds a job and may decide to keep her job when marrying or when getting her first or second or such-and-such child. As mentioned in the previous section, decisions to keep one's job when marrying boomed in the sixties, whereas decisions to keep one's job when getting one's first child have increased since the eighties. In another situation, woman $i$ is a housewife with children, and may decide to (re-)enter the labour market, when the children have grown up or have become school-going. Such (re-)entries have grown considerably in number since the sixties.

First, we consider the latter situation. The short-run reservation wage of woman $i$ is then a short-run entry wage, and the woman is more or less used to maximal household time, no corporate time and a family consumption level equal to the income of her husband. Assuming, for the sake of simplicity, that habits are formed in one year, we can approximate woman $i$ 's habit states averaged over year $t$ as $s_{L i t}=L_{i t-1}^{a}=T_{i t-1}$ and $s_{X i t} \approx X_{i t-1}^{a}=Y_{\text {Oit-1 }}$, where the superscript $a$ stands for "actual value". Similarly, woman $i$ 's social household time and family consumption environments $s_{\bar{L} t}$ and $s_{\bar{X}}$ in year $t$ can be approximated as the average actual household time $\bar{L}_{t-1}^{a}=\bar{T}_{t-1}-\bar{H}_{t-1}^{a}$ and the average family consumption $\bar{Y}_{O t-1}+\bar{w}_{t-1} \bar{H}_{t-1}^{a}$ in year $t-1$ in $i$ 's social reference group. This social reference group can be expected to consist of married women who have grown-up or school-going children and who also share a number of other characteristics with woman $i$ like level of education, age, number of children, region of residence, status, etc. (cf. Kapteyn and Wansbeek, 1985, Sec. 7.2). In this section and the next we assume that the social reference group of woman $i$ coincides with her membership group or social group as defined by characteristics as mentioned, and that every other member of this group has the same social group as social reference group (see Subsec. 5.1.2). 
According to the models for the long-run dynamics of sociall norm beliefs in Sections 8.5 and 8.8 , the individual and average beliefs in the household nom and financial independence norm are also determined by the behaviour in the past. On the other hand, direct information on these beliefs can be obtained from the SCP surveys mentioned in the previous section. Therefore, we do not here express the social norm beliefs in the past behaviours. Again, in this section and the next we assume that any woman $i$ 's normative reference group(s) for the household norm and financial independence norm coincides with her membership group or social group, as being the same for every group member.

In the case of a decision to keep one's job when marrying or when getting a child, the short-run reservation wage of woman $i$ is a short-run exit wage. Then woman $i$ is more or less used to the amounts of household time, corporate time and consumption resulting from her last job. Similarly to above, we can then approximate woman $i$ 's habit states in year $t$ as $s_{L i t}=L_{i t-1}^{a}=T_{i t-1}-H_{i t-1}^{a}$ and $s_{X i t}=X_{i t-1}^{a}=Y_{O i t-1}+w_{i t-1} H_{i t-1}^{a}$, where $X_{i t-1}^{a}$ and $Y_{\text {Oit-1 }}$ may now stand for woman $i$ 's own actual consumption and own other income before her marriage. Especially the corporate work experience will lower the exit wage, whereas a low consumption experience before marriage as compared to the high consumption level resulting from marrying a man with a high income leads to a relatively high exit wage. Again, woman $i$ 's social household time and family consumption environments $s_{\bar{L} t}$ and $s_{\bar{X} t}$ in year $t$ can be approximated as the average actual household time $\bar{L}_{t-1}^{a}=\bar{T}_{t-1}-\bar{H}_{t-1}^{a}$ and the average actual family consumption $\bar{Y}_{O t-1}+\bar{w}_{t-1} \bar{H}_{t-1}^{a}$ in year $t-1$ in $i$ 's social (reference) group. Now this social (reference) group will consist of married women with small children or married women in general. To the individual and average beliefs in the household norm and financial independence norm the same statements as above apply.

Adding a stochastic disturbance term somewhere in our micro-economic labour supply model, we could express the probability of participation of woman $i$ in terms of the distribution function of this stochastic disturbance as well as the expression (9.1) for the reservation wage, and the wage rate. In principle, we could then estimate the resulting expression for the participation probability for micro-economic cross-section data, following the usual probit method (see, e.g., Killingsworth, 1983, Ch. 4, and Hartog and Theeuwes, 1985, p. S245). Here, however, we are interested in long-run developments of participation rates, requiring a time-series analysis. ${ }^{6}$ Since for such a time-series analysis only macro-economic (or meso-economic) data are available and since we want to obtain more insight in the macroeconomic implications of the microeconomic dynamics, the expression for the participation rate should be expressed in terms of macroeconomic averages of the wage rate and of the variables and parameters in the expression (9.1) for the reservation wage. However, this aggregation (cf. Secs. 4.7 and 4.8) should take into account that the theoretical models for collective habit formation and social norms in this study have been aggregated only to the level of social groups. Therefore, we should make a clear distinction between the mesoeconomic level of social groups and the macro-economic level of (birth) cohorts or (sub)populations.

${ }^{6}$ Simulations over time on the basis of cross-section estimates for a particular point in time as carried out by Hartog and Theeuwes (1985) are dubious. See footnote 3. 
First, we consider the aggregation from individual participation decisions in the social group of woman $i$ to the participation rate of that social group. This can be done in several ways, but in a time series context the most appropriate and simple way is expressing the participation rate in terms of the distribution of one continuous deviation variable $\tilde{\boldsymbol{\varepsilon}}$ with mean zero, which distribution can be assumed to be approximately constant over time (cf. Assumption $\mathrm{X}$ at the end of Sec. 5.3). To find such an $\tilde{\boldsymbol{\varepsilon}}$, we first note that the participation rate of a social group can be expressed as the fraction of group members for whom the quotient $w_{r i} / w_{i}$ is smaller than one. The wage rate $w_{i}$ in the denominator of this quotient may be assumed to change over time by roughly the same percentage for each group member (as in Assumption X in Sec. 5.3). Similarly, the reservation wage $w_{r i}$ in the numerator of the quotient may, as a first approximation, be assumed to change over time by roughly the same percentage for each group member. ${ }^{7}$ Then the same statement holds with respect to the quotient itself, and we define $\varepsilon_{i}$ as the relative deviation $\left(q_{i}-\bar{q}\right) / \bar{q}$ of the quotient $q_{i}$ from its group average $\bar{q}=\bar{w}_{r} / \bar{w}$. The participation rate of the social group can then be expressed as the fraction of group members $i$ for whom $\left(\bar{w}_{r} / \bar{w}\right)\left(1+\varepsilon_{i}\right)$ is smaller than one, which is equivalent to $\ln \left(1+\varepsilon_{i}\right)<\ln \bar{w}-\ln \bar{w}_{r}$. If we consider $\ln \left(1+\varepsilon_{i}\right)$, for a large number of group members $N$, as a continuous variable $\tilde{\varepsilon}$ and approximate $\bar{w}_{r}$ as $\bar{b} / \bar{a}$, it then follows that

$$
P=F\left(\ln \bar{w}-\ln \bar{w}_{r}\right)=F(\ln \bar{w}+\ln \bar{a}-\ln \bar{b}),
$$

where $F$ is the (cumulative) distribution function of $\bar{\varepsilon}$. Note that the mean of the distribution of $\tilde{\varepsilon}$ is zero and that its variance and higher-order moments are constant over time as a consequence of the assumptions made above.

\subsection{Logit participation equation for one social group}

Regarding the specification of the distribution function $F$ in (9.2), the most plausible assumption seems to be that the distribution of $\tilde{\varepsilon}$ is normal, but for reasons of simplicity and comparability with the usual logit specifications, it may also be approximated by a logistic distribution. In the latter case the distribution function $F$ is specified as $F(\tilde{\varepsilon})=\left(1+e^{-K \tilde{e}}\right)^{-1}$. where $K$ is a constant that is inversely proportional to the standard deviation of the distribution by a factor $\pi / \sqrt{3} .^{8}$ Applying the usual logit transfonmation $\operatorname{logit} P:=\ln [P /(1-P)]$ to the participation equation $(9.2)$, this equation becomes

${ }^{7}$ On the one hand, this percentage may have the same sign as the percentage of change of the wage rates as a result of a same direction of change of the incones of the husband $\boldsymbol{Y}_{O i}$. On the other hand, the percentage may have the opposite sign, when a rise (fall) of the wage rates leads to a higher (lower) average corporate time in the social group, and hence via the mutual social influence to lower (higher) reservation wages (see Sec. 7.1).

8 This can be read from the expression (11) for the logistic density function in terms of its mean and variance in Kotz and Johnson (1985, p. 124). 


$$
\operatorname{logit} P_{t}=\kappa\left(\ln \bar{w}_{t}-\ln \bar{w}_{r t}\right)=\kappa\left(\ln \bar{w}_{t}+\ln \bar{a}_{t}-\ln \bar{b}_{t}\right),
$$

where the time dependence has been indicated. Now we can substitute the (approximate) averages of the expressions for $a_{i}$ and $b_{i}$ in (9.1) into (9.3). Next, for the two participation situations considered above, the habit states and social environments can be approximated by the individual and average actual household time and family consumption in the previous year, and these behaviours can be expressed in the individual and average actual corporate hours in the previous year.

However, the average actual corporate hours $\bar{H}_{t-1}^{a}$ depend on the endogenous labour force participation rate in the previous year $P_{t-1}$. In order to make the resulting endogenous dynamics visible and to facilitate a comparison with participation equations in the literature (see below), we express $\bar{H}_{t-1}^{a}$ in $P_{t-1}$ as follows. The average actual corponate hours $\bar{H}_{t-1}^{a}$ are equal to the employment ratio $E_{t-1}$ (proportion of group members who are employed) times the average actual corporate hours of the employed group members $\bar{H}_{t-1}^{a e}$. The employment ratio $E_{t-1}$ is, in general, lower than the participation rate $P_{t-1}$, since the latter rate also includes the unemployed group members who search for a job (see Clark and Summers, 1982, p. 833). The number of these unemployed group members as a fraction of all participating group members defines the unemployment rate $u_{t-1}$, and it then easily follows that the employment ratio $E_{t-1}$ is equal to $1-u_{t-1}$ times the participation rate $P_{t-1}$.

A positive unemployment rate $u_{t-1}$ implies that the labour supply of the group members is rationed with respect to the number of jobs. In addition, recent Dutch data for the female labour supply of hours (Allaar et al., 1989, 1991) also indicate overemployment in the sense that many women who hold a job would like to work fewer hours. This implies a rationing of the average preferred corporate hours of the employed group members. Both kind of rationings have not been taken into account in the theoretical models underlying the participation equations (9.2) and (9.3), so we should make ad hoc assumptions on nature and effects of the rationings. Regarding the rationing of the average preferred corporate hours of the employed group members, we assume for the sake of simplicity that the number of job hours are exogenously fixed by the employers. This implies that the average actual corporate hours of the employed group members $\bar{H}_{i-1}^{a e}$ are exogenously given (see, however, below). The average actual corporate hours of all group members $\bar{H}_{t-1}^{a}$ are then equated to $\bar{H}_{t-1}^{a c}\left(1-u_{t-1}\right) P_{t-1}$.

In addition, the rationing of the average corporate hours of the employed group members makes entrance to the labour market less attractive, implying that the individual reservation wages will be higher than $b_{i} / a_{i}$ (see Subsection 2.2.4). Since the size of the increment has not been derived for our $A Q M$ models, we assume ad hoc that in an overemployment situation as mentioned above the average household time taste $\bar{\alpha}_{L,}$ is raised by an increment that is proportional to the positive gap $\bar{H}_{t-1}^{a c}-\bar{H}_{t-1}^{e}$ between the averages of the actual and the preferred corporate hours of the employed group members. For the sake of simplicity we again assume that this gap is exogenously given. Furthermore, as a result of a discouraged worker effect unemployment is assumed to raise the average household time taste, and hence the average reservation wage, by an increment that is proportional to the unemployment rate $u_{t}$. Finally, we conceive the average wage rate $\bar{w}_{t}$ as a real net wage (so we abstract from progressive taxation effects). 
Having made all these assumptions and additions, we can write out the logit participation equation (9.3) for the case of a social group of re-entering married women as

$$
\begin{aligned}
& \operatorname{logit} P_{t}=\mathbf{x}\left\{\ln \bar{w}_{t}+\ln \left[\bar{\alpha}_{X t}-\left|\bar{\alpha}_{X X}\right| \bar{Y}_{O t}+\left(\bar{\gamma}_{X X}+\bar{\gamma}_{X X}\right) \bar{Y}_{O t-1}+\bar{\gamma}_{X \bar{X}} \bar{w}_{t-1} \bar{H}_{t-1}^{a e}\left(1-u_{t-1}\right) P_{t-1}+\right.\right. \\
& \left.+\frac{\bar{\gamma}_{F}}{\bar{Y}_{I M t}} \bar{n}_{F t}^{h}+\frac{\bar{\beta}_{F} \bar{\varepsilon}_{F} \bar{\rho}_{F}}{\bar{Y}_{I M t}} m_{F t}\right]-\ln \left[\bar{\alpha}_{L t}-\left|\bar{\alpha}_{L L}\right| \bar{T}_{t}+\left(\bar{\gamma}_{L L}+\bar{\gamma}_{L L}\right) \bar{T}_{t-1}-\bar{\gamma}_{L L}-\bar{H}_{t-1}^{a e}\left(1-u_{t-1}\right) P_{t-1}+\right. \\
& +\frac{\left.\left.\bar{\gamma}_{L} \bar{n}_{L t}^{h}+\frac{\bar{\beta}_{L} \bar{\varepsilon}_{L} \bar{\rho}_{L}}{\bar{T}_{t}} m_{L t}+\pi_{1}\left(\bar{H}_{t-1}^{a e}-\bar{H}_{t-1}^{e}\right)+\pi_{2} u_{t-1}\right]\right\},}{}
\end{aligned}
$$

Here averages of products of parameters and variables have been approximated by the products of their averages, and all average parameters except the taste parameters $\overline{\boldsymbol{\alpha}}_{X t}$ and $\bar{\alpha}_{L t}$ have been assumed independent of time. The taste parameters can be considered as implicit functions of exogenous variables which are not made explicit in the participation equation. For example, the average consumption taste $\bar{\alpha}_{X}$ is likely to depend positively on the average number and age of children and the qualities of consumption goods, and the average (net) household time taste $\bar{\alpha}_{L,}$ can be expected to depend positively on the average number of children and negatively on the average age of the children, the average level of education of the group members, the average efficiency of household production, and the average working conditions (see also Vendrik, 1988, p. 17).

The wage rate term describes the always positive substitution effects of changes in the average wage rate on the logit of the participation rate, and hence on the participation rate itself. Note that there is no income effect of changes in the wage rate on the participation, since at zero labour supply changes in the wage rate do not affect one's own income (see also Pencavel, 1986, pp. 32-33). The term $-\left|\bar{\alpha}_{X X}\right| \bar{Y}_{O,}$ represents the negative participation effect of the average income of the husband. In particular. when this income declines as a consequence of husbands becoming unemployed, the term describes the added-worker effect. The term $\left(\bar{\gamma}_{X X}+\bar{\gamma}_{X} \bar{X}\right) \bar{Y}_{O t-1}$ models the positive participation effect of collective habit formation with respect to family consumption as far as determined by the incomes of one's own and other women's husbands in the previous year. The next term, which is proportional to the lagged participation rate $P_{t-1}$, describes the positive participation effect of behavioural preference interdependence (BPI) with respect to family consumption as far as determined by the labour incomes of other employed group members. It implies a positive feedback of changes in the participation rate in the previous year on a determinant of the participation rate in the present year, and hence a long-run multiplier effect on the participation rate (see Sec. 9.7). This feedback is stronger as the wage rate $w_{t-1}$ and the average actual corporate hours of the employed group members $\vec{H}_{t-1}^{a e}$ are higher and as the unemployment rate $u_{t-1}$ is lower. Note that there is no participation effect of individual habit formation (IHF) with respect to family consumption as far as determined by the labour income of the woman herself, since this labour income is zero before entry and only reinforces the entry decision 
after entry, so does not lead to an increase in the participation rate. On the other hand, such IHF has a negative effect on exits from the labour market, when after a period of (uniformly) declining $q_{i}=w_{r i} / w_{i}$ and hence entries, the $q_{i}$ again rise. Here, however, we assume that the $q_{i}$ only decline, and we come back to this complication at the end of the section.

The next two terms model the positive participation effects of one's own belief and the average group belief in the financial independence norm as a result of cognitive dissonance and reputation sanctions. The average own belief variable $\bar{n}_{F t}^{h}$ has a superscript $h$ to indicate that for women who have already re-entered the labour market, their belief in the financial independence norm if they would not yet have re-entered, so were still housewives, should be considered. Analogously to the IHF term for zero labour income considered above, the reason of this procedure is that a possible increase in one's own belief in the financial independence norm after and as induced by entry only reinforces the entry decision, so does not raise the participation rate. The remaining positive participation effects of one's own belief and the average group belief in the financial independence norm are larger as the average individual minimum income $\bar{Y}_{I M \mid}$ is lower, since the extent of obedience to the financial independence nom is then more sensitive to variations in the labour income. Recall from the previous section that although the norm belief variables are endogenously determined by the (lack of) participation in the past according to the long-run models of Chapter 8 , they are not expressed in lagged participation rates in (9.4), since there is direct empirical information on the norm beliefs.

Whereas the average consumption taste $\bar{\alpha}_{X t}$ has a positive participation effect, the average household time taste $\bar{\alpha}_{L \text {, }}$ has a negative participation effects for obvious reasons. The term $-\left|\bar{\alpha}_{L L}\right| \bar{T}_{1}$ represents a positive participation effect of the average total discretionary time $\bar{T}_{t}$. This results from more (less) satiation with discretionary household time when one does not participate $\left(L_{t}=T_{t}\right)$, as the total discretionary time $T_{1}$ is larger (smaller). The average total discretionary time $\bar{T}_{t}$ is equal to the total available time of 24 hours a day minus the average minimal necessary household time (see Sec. 2.3), and the latter minimal household time can be assumed to depend positively on the average number of children and negatively on the average age of the children, the availability of day care and home help, and the average efficiency of the household production. Since the average number of Dutch children has declined since the sixties and since the average household production efficiency has increased secularly, the average minimal necessary household time in the Netherlands may have declined, and hence the average total discretionary time $\bar{T}_{1}$ may have risen, implying a positive effect on the participation rate. ${ }^{9}$ However, it seems difficult to distinguish such effects from similar effects on the average household time taste $\bar{\alpha}_{L t}$.

The term $\left(\bar{\gamma}_{L L}+\bar{\gamma}_{L \bar{L}}\right) \bar{T}_{t-1}$ describes the negative participation effect of collective habit formation with respect to household time as far as determined by the average maximal discretionary household time $\bar{T}_{t-1}$ in the previous year. This negative participation effect counteracts the above positive participation effect of satiation with respect to household time and is larger (smaller) as $\bar{T}_{t-1}$ is larger (smaller). The next term, which is proportional to the

9 On the other hand, nowadays parents seem to spend more time on the upbringing of their children than in the past. This may be associated with a negative effect on $\bar{T}_{1}$, and hence on the participation rate. 
lagged participation rate $P_{t-1}$, describes the positive participation effect of behavioural preference interdependence (BPI) with respect to household time as far as determined by the corporate hours of other employed group members. Again, it implies a positive feedback of changes in the participation rate in the previous year on a determinant of the participation rate in the present year, and hence a long-run multiplier effect on the participation rate. This feedback is stronger as the average actual corporate hours of the employed group members $\bar{H}_{t-1}^{a e}$ are higher and as the unemployment rate $u_{t-1}$ is lower. Note that there is again no participation effect of individual habit formation (IHF) with respect to household time as far as determined by the corporate hours of the woman herself, since these corporate hours are zero before entry and only reinforce the entry decision after entry, so do not raise the participation rate. The next two terms model the positive participation effects of one's own belief and the average group belief in the household norm as a result of cognitive dissonance and reputation sanctions. These effects are larger as the average total discretionary time $\bar{T}_{t}$ is lower, since the extent of obedience to the household norm is then more sensitive to variations in the household time.

Although the participation equation $(9.4)$ is rather complex, its dynamic lag structure seems still too simple, since empirical results of Van de Stadt et al. (1985) and Clark and Summers (1982) strongly suggest that individual habits and (expected) social environments are adjusted to the corresponding individual and average behaviours in much more than one year. The individual habits and social environments can then be approximated as moving averages of the corresponding behaviours in preceding years $t-\tau$ with uniform exponentially declining weights $(1-\lambda) \lambda^{\tau-1}$, where the positive "memory coefficient" $\lambda$ is equal to 1 minus the positive adjustment speed $\delta$ (cf. Sec. 5.2). However, since the resulting expressions in the participation equation are terms in arguments of logarithms, we cannot simplify the participation equation by applying a Koyck transformation as in Clark and Summers (p. 834). Therefore, and since the modifications can be shown not to affect the long-run multipliers, the analysis in the next sections sticks to the unmodified participation equation (9.4).

Another complicating modification of the participation dynamics is implied by a certain degree of endogeneity of the average actual corporate hours of the employed group members $\bar{H}_{i-1}^{a \ell}$ which is suggested by the historical fact that since the end of the fifties employers have been offering an increasing number of part-time jobs in order to attract more married women to the labour market (Mol et al., 1988, p. 30). This means that employers have been taking the preferred corporate hours of married women into account in their offers of corporate hours, and implies a positive dependence of the average actual corporate hours $\bar{H}_{t-1}^{a c}$ on the average preferred corporate hours $\vec{H}_{t-1}^{c}$ of the employed group members. However. the latter corporate hours depend on the social environments (and the average norm beliefs). so on the average actual corporate hours $\bar{H}_{t-2}^{a}$ of all group members in the preceding year. and hence on the endogenous participation rate $P_{1-2}$ and the average preferred corporate hours of the employed group members $\bar{H}_{t-2}^{t}$ in that year, which again depend on the same variables in the preceding year $t-3$, etc.. These reinforcing feedbacks imply a complicated dynamics, which we consider as a second-order effect and neglect here. Thus, in the following we continue to assume exogeneity of the average actual corporate hours of the employed group members $\vec{H}_{t-1}^{a c}$.

Next. for a social group of women who do or do not keep their job when marrying or getting children, a logit participation equation similar to $(9.4)$ for a social group of women 
re-entering or not can be derived. This participation equation differs from (9.4) by additional terms which describe the negative effect on exits of IHF with respect to the higher (family) consumption and the lower household time as a result of having a job. This, however, is not elaborated here. Finally, in the case of re-entered women, such women will again leave the labour market when their short-run exit wages decline under their wage rates, since they become older, since their husbands reach a higher salary scale or since their children start to eam their own income. The resulting decline in the participation rate cannot be described by the participation equation (9.4), but can be described only by a participation equation for the subgroup of re-entered women which is similar to that for women who do or do not keep their job when marrying or getting children as mentioned above. However, for this case it can be argued that the density function of the exit wage-wage proportions in the subgroup of re-entered women may be very skew, so that an approximation of this density function by a logistic one seems dubious. Anyway, we then have a regime switch from a participation equation for entries to another participation equation for exits. This complication is related to the complex hysteresis structure of long-run labour supply schedules as represented by Figure 4.11 in Section 4.8 or Figure 7.1 in Section 7.2 , according to which labour supply paths depend on the sequence of rises and declines in the wage rate (relative to the reservation wages) in the past. Analogously to the discontinuous shifts of the long-run individual corporate hours between zero and maximal hours as a result of unstable IHF, we have here discrete shifts of an individual participation variable between the values zero and one (see Sec. 9.9). The complication also implies that the representation of the persistence effects of IHF on participation by Clark and Summers (1982) is inadequate (see Sec. 9.6).

\subsection{Aggregation and BPI between social groups}

The previous section has derived the logit participation equation (9.4) for one social group within a subpopulation of married women with grown-up or school-going children who do or do not re-enter the labour market. However, data for the norm belief and other variables in such a participation equation on a social group level do not seem available. Therefore, and in order to obtain insight in the determinants of the participation rate on the level of (birth) cohorts or an entire subpopulation, the participation equation (9.4) should be aggregated to such a level. The subpopulation which will be considered in this section, consists of all married women with school-going and no younger children who did not have a job before the youngest child reached the school-going age (and who do or do not re-enter the labour market).

A straightforward and approximative aggregation of the participation equation (9.4) is obtained by taking the averages of the left-hand and right-hand sides of $(9.4)$ over all social groups within a cohort of the subpopulation (or within the entire subpopulation) and by approximating the averages of logarithms, quotients and products by the logarithms, quotients and products of averages. This results in a participation equation of the same form as (9.4), where the constant factor $\kappa$ is replaced by its average $\bar{\kappa}$ over all social groups in the cohort, where $P_{t(-1)}$ and $u_{t-1}$ are the participation rate and unemployment rate of the cohort, and where the average variables and parameters denote averages over all individuals in the cohort. However, this specification of the aggregate participation equation contains a 
very severe aggregation error. This can be seen by deriving the participation equation for the cohort directly from the individual quotients $q_{i}$ of reservation wage and wage rate in the same way as the participation equation for one social group (9.3) in the previous two sections. We then obtain again a participation equation of the same form as (9.4) with the variables and parameters being defined on the cohor level, but now the constant factor $\tilde{\mathrm{K}}$ is inversely proportional to the standard deviation of the distribution of the individual quotients $q_{i}$ over the cohort. Since this standard deviation is likely to be much larger than the standard deviations for the separate social groups within the cohort, the constant factor $\tilde{\bar{K}}$ will be much smaller than the average constant factor $\bar{\kappa}$ over the social groups.

This result has very important implications for the values of the elasticities of the participation rate with respect to the exogenous variables on the cohort level versus the social group level. For example, the short-run wage elasticity of the participation rate can easily be shown to equal the constant factor $\kappa$ or $\bar{\kappa}$ times $1-P_{t}$. Consequently, for a given level of the participation rate $P_{l}$, this wage elasticity is much lower on the cohort level than on the social group level as a result of the aggregation. Similarly, other short as well as long-run elasticities as being positive functions of the constant factor will also be much lower. In Section 9.10 we will see that this properiy hinders the empirical identification of collective catastrophes in social groups.

On the other hand, Section 9.8 will show that the long-run elasticities of the participation rate on the cohort level are raised, when one adds the participation effects of behavioural preference interdependence (BPI) between social groups in the cohort to the participation effects of BPI within social groups. However, this rise will be relatively small, since the former BPI can be expected to be much smaller than the latter. In the context of our general BPI model as constructed in Section 5.2, adding BPI between social groups amounts to relaxing Assumption VI and extending the social reference group $R G_{i}$ of woman $i$ beyond $i$ 's social group. With respect to this extension at least two sets of simplifying assumptions can be made. First, as a generalization of Assumption VIII in Section 5.2, we may assume that the extended social reference group of each woman $i$ consists of all other women within i's cohort (of married women with school-going children) and that, alongside equivalent neighbour interactions between members of the same social group. there are weaker equivalent neighbour interactions between members of different social groups. This means that each woman $i$, in her perception of her social consumption and household time environment, assigns the same reference weight to every woman $j$ in other social groups. but that this reference weight is smaller than the uniform reference weight attached by $i$ to other members of the same social group. We can then decompose $i$ 's social environments in a social environment formed by the behaviour of all members of $i$ 's social group and a social environment formed by the behaviour of all members of other social groups. The former social environments can then be approximated as in the previous section, and if we assume that the number of women in the cohor is large compared to the number of women in i's social group, the latter social environments can be approximated as the average consumption $\bar{X}_{t-1}$ and the average household time $\bar{L}_{t-1}$ of all women in the cohort (cf. (5.8) as implied by Assumption IX in Section 5.2).

In the participation equation for the cohort these social environments formed by other social groups lead to additional terms $\bar{\gamma}_{X \bar{X}^{\circ}} \bar{X}_{t-1}$ for the consumption BPI and $\bar{\gamma}_{L \bar{L}^{\circ}} \bar{L}_{t-1}$ for the household time BPI in the arguments of the second and third logarithm, respectively. 
on the right-hand side (see (9.4)). Here the superscript $o$ stands for "other social groups" (see also Van de Stadt et al., 1985, Kapteyn and Wansbeek, 1985, Sec. 7.3, and Kapteyn and Woittiez, 1990). If we make the same substitutions for $\bar{X}_{t-1}$ and $\bar{L}_{t-1}$ as in the previous sections, we then obtain somewhat higher coefficients of $\bar{Y}_{O t-1}, \bar{w}_{t-1} \bar{H}_{t-1}^{a e}\left(1-u_{t-1}\right) P_{t-1}$, $\bar{T}_{t-1}$ and $\bar{H}_{t-1}^{a e}\left(1-u_{t-1}\right) P_{t-1}$ in the cohort variant of the participation equation (9.4). ${ }^{10}$

A second kind of extension of the social reference group $R G_{i}$ of woman $i$ beyond $i$ 's social group results when woman $i$ refers to the behaviour of women in some, but not all other social groups in her cohort or subpopulation. For example, Kapteyn et al. (1978) and Van Praag et al. (1979) (see also Kapteyn and Wansbeek, 1985, Sec. 7.2) find that in an evaluation of their income individuals refer to the incomes of other individuals whose level of education is the same or higher, but not lower. Similarly, in consumption demand models Pollak (1976a) and Schokkaert (1982b, Sec. V.B.IV) assume a hierarchical emulation pattern in which each individual's preferences are influenced by the consumption behaviour of members of higher(-status) groups. In his estimations of participation equations for Dutch female cohorts, Romme $(1987,1990)$ finds a significant impact of the participation rate of the preceding (on average) five year older cohort. Let us consider this impact in the context of our participation equation for a cohort of married women with school-going children, and let us assume for simplicity that it results from BPI between social groups in successive cohorts which have the same characteristics other than age (e.g., apart from marital status and having school-going children, the level of education). Moreover, assume equivalent neighbour interactions as above and approximate the social environments formed by these older social groups as the average consumption and the average household time in these groups in the previous year. This can easily be shown to lead to additional terms $\bar{\gamma}_{X \bar{X}^{-1}} \bar{X}_{t-1}^{k-1}$ for the consumption BPI and $\bar{\gamma}_{L \bar{L}^{-1}} \bar{L}_{t-1}^{k-1}$ for the household time BPI in the arguments of the second and third logarithm on the right-hand side of the participation equation for cohort $k$ (see (9.4)). Here the superscript $(k)-1$ refers to the preceding older cohort, and the averages are taken over this cohort. Making substitutions for $\bar{X}_{t-1}^{k-1}$ and $\bar{L}_{t-1}^{k-1}$ analogous to above, we then obtain additional terms in $\bar{Y}_{O t-1}^{k-1}, \bar{w}_{t-1}^{k-1} \bar{H}_{t-1}^{a k-1}\left(1-u_{t-1}^{k-1}\right) P_{t-1}^{k-1}, \bar{T}_{t-1}^{k-1}$ and $\bar{H}_{t-1}^{a k-1 e}\left(1-u_{t-1}^{k-1}\right) P_{t-1}^{k-1}$ in the variant of participation equation (9.4) for cohort $k$. Such terms can also be shown to result when we assume in addition equivalent neighbour interactions of BPI between social groups in successive cohorts which have different characteristics other than age (e.g., level of education).

Still another kind of extension of the social reference group $R G_{i}$ of woman $i$ beyond $i$ 's social group results when woman $i$ refers not only to the behaviour of other women, but also to that of men such as her husband and his and her male friends. However, in the labour supply model of Kapteyn and Woittiez (1990) this reference to the behaviour of men is estimated to be zero. On the other hand, woman i's normative reference groups for the household norm and financial independence norm are likely to consist not only of married

${ }^{10}$ Note that when these coefficients are estimated, the separate contributions of the BPI's within and between social groups are not identifiable. This lack of identifiability holds also for the mieroeconomic labour supply equations of Kapteyn and Woittiez (1990). 
women with school-going children to whom the norms apply, but also of persons like her husband, parents and friends to whom the norms do not apply. In this case the available SCP data for the norm beliefs (see Fig. 9.3 in Sec. 9.2) do not allow a distinction between a normative influence on woman $i$ of the average norm belief in her own cohort and that of the average norm belief outside her cohort. The SCP data provide only proxies for the overall average norm beliefs in the whole of Dutch (adult) population, so we are prompted to assume that these proxies can capture both kind of normative influences. The average norm belief variables $m_{F t}$ and $m_{L t}$ in the cohort variant of the participation equation (9.4) should then be redefined as the overall average norm beliefs in the Dutch population. Moreover, the average norm beliefs $\bar{n}_{F t}^{h}$ and $\bar{n}_{L t}^{h}$ in each cohort of married women with schoolgoing children, if all these women would not yet have re-entered the labour market, should be approximated by the overall average norm beliefs. This may not be a bad approximation. A disadvantage of the approximation is that it makes the contributions of the cognitive dissonance and reputation sanctions to the total effect of the nom beliefs unidentifiable.

\subsection{Social norm proxies and econometric evidence}

Our proxy for the overall average belief in the household norm is given by the percentage of the answer "objectionable" in Figure 9.3 on the question: "What do you think of a married woman with school-going children who has a job?". This percentage fell sharply from $84 \%$ in 1965 to $44 \%$ in 1970 and then further declined, up to some recoveries, to $20 \%$ in 1990 . According to our interpretation this implies that in the Netherlands the belief in the household norm eroded extremely fast in the roaring second half of the sixties and eroded at a more moderate pace in the seventies and eighties. The development of the social norrn proxy of Romme (see Sec. 9.2) is given by the complement of this time pattern. In regressions of a linear and a loglinear specification of a logit participation equation for Dutch female cohorts over the period 1971-1985, Romme finds consistently significant and stable coefficients of his social norm variable as well as of the participation rates of the same and older cohorts in the preceding year, and only weakly significant and less stable coefficients of the wage rate. Moreover, in the more plausible loglinear specification of the participation equation the coefficients of the social norm variable and the lagged participation rates are considerably higher than the coefficient of the wage rate. Furthermore, the significance of the social norm variable may become more pronounced when also the "cultural revolution" in the second half of the sixties could be included in the estimation. These results of Romme can be considered as a support for the empirical relevance of our theoretical model of the impacts of social norm change and collective habit formation on female labour supply. For when Romme would have used the household norm proxy instead of his complementary norm variable, the results mentioned would be the same in the case of his linear specification of the participation equation and presumably similar in the case of his loglinear specincation. In addition, together with some other empirical results of Romme, they raise serious doubts on the reliability of empirical purely neo-classical results like those of Hartog and Theeuwes (1985), who find strong and dominant own-wage effects in the development of Dutch female participation since 1947 (see footnote 3 and Sec. 9.8). 
On the basis of his estimation results Romme also makes projections of the aggregated female participation for 1987 and 1989. He distinguishes three routes: a minus-route where the social norm variable and unemployment rate change by $-2 \%$ and $2 \%$, respectively, a zero-route where these variables do not change, and a plus-route where they change by $2 \%$ and $-2 \%$, respectively. These routes turn out to imply projections which are considerably higher than conventional trend-extrapolations for the period 1985-1989 (see Romme, 1987). A later comparison of these forecasts with actual values reveals that the rise in female participation between 1987 and 1989 is predicted remarkably well by the loglinear specification of Romme's participation equation: while the predicted rise in participation lies in the route range between 140.000 and 164.000 , the actual rise in participation is 146.000 (CBS, 1992). ${ }^{11}$ Since our participation equation (9.4) resembles Romme's loglinear specification, this result too is encouraging for our model.

However, in comparison with our participation equation (9.4) the participation equations of Romme have three important drawbacks. First, unlike (9.4) Romme's equations have not systematically been derived from an underlying micro-economic labour supply model, but were specified ad hoc. These ad hoc specifications have the advantage of being simpler than (9.4) and therefore perhaps being more easy to estimate, but (9.4) and the implications of the loglinear specification for the long-run elasticities of the participation rate (see Sec. 9.9) suggest that both specifications contain severe specification errors. A second important drawback of Romme's participation equations is that the female cohorts are not distinguished by household composition such as being single or not and number and age of children. As a third related drawback, Romme includes neither such life-cycle variables nor variables for the level of education, the income of the husband, and the rationing of the number of job hours. The impacts of the rising level of education and the rising income of the husband are implicitly taken into account to some extent by the wage rate variable, but the probably important impact of the educational level other than via the wage rate is neglected (see Sec. 9.1). On the other hand, a participation equation like (9.4) which meets all these objections, has not yet been estimated and may pose thomy data and estimation problems.

A drawback of the participation equations of Romme which seems less severe, is the absence of a good proxy for an emerging "emancipation" norm of financial independence and/or self-fulfilment of non-single women. As an indication for this norm emergence in the Netherlands we could consider the development of the percentage of the answer "recommended" in Figure 9.3. More specifically, this percentage may serve as a proxy of the average beliefs in the financial independence norm $m_{F t}$ and $\bar{n}_{F l}^{h}$ in the cohort variant of the participation equation (9.4). While the percentage of "recommended" rose considerably from $2 \%$ in 1965 to $16 \%$ in 1970, it fluctuated between 1970 and 1981, and again rose considerably from $11 \%$ in 1981 to $32 \%$ in 1991 . Thus, in 1991 the percentage of the Dutch population which recommended labour force participation by married women with schoolgoing children, had become substantially higher than the percentage which objected to it. This rise in the proxy for the average belief in "emancipation" norms for non-single women may also be important in explaining the rise in the labour force participation of Dutch

${ }^{11}$ On the other hand, this remarkable prediction success may be a coincidence, since the so-called AKT data of the CBS, that were used in Romme's estimations and predictions, are based on another definition of participation than the so-called EBB data for the actual values. 
married women. However, the estimation results of Romme do not give indications on this, since Romme includes the percentage of "not objectionable" in his proxy of the general support for a norm in favour of female labour force participation.

A related important role in the participation increase may have been played by growing needs for financial independence and/or self-fulfilment and by collective habit formation with respect to a woman's own income and/or a woman's own corporate time. The former needs can be incorporated in the participation equation (9.4) as increases in the consumption taste parameter $\bar{\alpha}_{\chi t}$ and as decreases in the household time taste parameter $\bar{\alpha}_{L t}$, respectively. The latter collective habit formations can be shown to imply increments to the BPI coefficients of the own income term and the own corporate time term, respectively.

This brings us to a crucial feature of these lagged-participation-dependent terms, namely that they originate from BPI, but not from IHF. The source of this surprising result is that in a regime of (uniformly) declining proportions of the individual reservation wages and wage rates $q_{i}=w_{r i} / w_{i}$, and hence of an increasing proportion of (re-)entered married women, the IHF's only reinforce the entry decision after entry, so do not lead to an increase in the participation rate (see Sec. 9.4). Conversely, in a regime of (uniformly) rising proportions of the individual reservation wages and wage rates, and hence of an increasing proportion of women who have left the labour market, the IHF's only reinforce the exit decision after exit, so do not lower the participation rate. This essential property is not recognized by Clark and Summers, who incorporate IHF, but no BPI in their model. By an extremely loose aggregation procedure (p. 832) they "derive" an endogenous IHF term in their participation rate equation, which is subsequently expressed in lagged employment ratio's (p. 834) and hence implicitly in lagged participation rates (see Sec. 9.4). Thus, they make a severe aggregation error which leads to an incorrect interpretation of the persistence effects of IHF on participation. In our participation equation (9.4) such persistence effects of IHF are represented by the IHF parts of the terms in $\bar{Y}_{O 1-1}$ and $\bar{T}_{t-1}$, which describe the negative participation effects of a housewife being used to a low level of family consumption and a high level of household time as compared to the situation in which she would have a job. Alongside these persistence effects of IHF there are persistence effects of BPI in (9.4), which are represented by the BPI parts of the terms mentioned as well as by the negative participation effects of a low lagged participation rate.

Another feature of (9.4) which is absent in the model of Clark and Summers as well as in the participation equation of Romme, is the mediating influence of the average corporate hours of the employed group or cohort members, $\bar{H}_{i-1}^{a e}$, on the BPI effects of the lagged participation rate. As remarked at the end of Section 9.4, this is likely to reinforce the feedbacks of the lagged participation rate in a complicated way. On the other hand, this does not alter the following essential implications of these feedbacks. Whereas the feedbacks can cause persistence of a status quo of a relatively low or high participation rate, they can also, when the participation rate has nevertheless started to change due to exogenous causes, strongly amplify such changes. The latter property, which too seerns unrecognized by Clark and Summers, follows from the long-run dynamics of our model. This long-run dynamics is analysed in Section 9.8-9.10. However, the models for the long-run dynamics of social norm beliefs in Sections 8.1 and 8.5 suggest that then also such an endogenous dynamics of the average norm belief variables in the participation equation (9.4) should be taken into ac- 
count. Before we do so, we first discuss some historical evidence on the endogeneity of the dynamics of social norms in the next section.

\subsection{Endogenous dynamics of social norms}

Regarding social norms historical descriptions of the Duteh developments in female participation strongly suggest that the low female participation before 1960 originated from certain economic and social conditions and interests, and that it was legimitized ex post by social norms emphasizing the natural role of the woman as mother and housewife (see, e.g., Clason, 1981, and Molle et al., p. 26). ${ }^{12}$ Due to the late industrialization and the high birth rate in the Netheriands, there were sufficient men to work in the factories, so in contrast to other countries married women were not needed in the factories to suppress the male wages (Siegers, 1981, p. 557, and De Neubourg, 1988, pp. 156-161). This led to a strict labour division between paid work of men and unpaid work of women (including participation in the husband's business), which was raised to the status of social norms and institutionalized in several government regulations. In their turn, these social norms and institutions reinforced and consolidated the labour division, which confirmed the social norms and institutions, etc. (see Siegers, 1986, p. 87).

This consolidating process was further reinforced by the high unemployment in the thirties as well as by a social phenomenon which was typical for the Netherlands. This phenomenon was the emancipation movement of the Dutch Roman Catholics, which had a strong interest in catholic women bearing and raising a large number of children (see Van Heek, 1954, and De Neubourg, 1988, pp. 161-163). When a catholic woman married, she was supposed to start the reproduction immediately, and when she failed to do so, she and her husband were visited by a parish priest who urged them to do more their best. Only when in the fifties the Catholics achieved their emancipation, this social pressure loosened. Thus, the Catholic emancipation movement stood in the way of the "emancipation" of married women.

Another factor which contributed to the Netherlands having a particular historical background concerning female labour force participation, was the fact that in World War II the demand for female labour did not increase in contrast to other countries. Thus, when after World War II the demand for female workers increased, this demand was confronted with the very powerful social norm that married women should stay at home. This household norm had been built up and reinforced by a very long historical tradition in which neither employers nor married women, as groups, had acquired experience with and, as a result, got accustomed to labour force participation by married women. ${ }^{13}$ In spite of the increased demand for female labour, this led, in combination with the very low net eamings of married women, to a low participation of these women in the forties and fifties.

12 See Elster (1989) for some objections to such an explanation of the existence of social noms in terms of self or common interests. However. Elster's analysis has the drawback of being remarkably static and ahistorical.

${ }^{13}$ De Neubourg (1988. p. 155) even goes back to the seventeenth century in order to explain this historical tradition. 
Nevertheless, eventually the economic reality of a persistent surplus demand for labour began to undermine the household norm in the course of the fifties. Most political parties became more liberal on this point, the law requiring dismissal of female civil servants on marriage was abolished in 1955, and the business sector even tried to attract married women by offering part-time jobs. In combination with other factors as discussed in Section 9.1, this led to a strong rise in the participation of married women in the sixties. ${ }^{14}$ This rise was accompanied by a dramatic shift between 1965 and 1970 in the public opinion regarding married women with school-going children having a job (see the previous section). On the one hand, the implied strong erosion of the pertinent household norm is likely to have contributed substantially to the rise in participation. On the other hand, this erosion of the household norm may have been caused by, among other factors, the economic reality of a rising participation and hence a rising employment of married women with school-going children. Underlying mechanisms of the latter feedback may be formed by reduction of cognitive dissonance and social influencing of social norm beliefs as explained in Section 8.5. Together with the former causation it implies a mutually reinforcing process of changes in household nom beliefs and participation, which partially explains the booming character of both changes. Moreover, this process was further reinforced by other forms of collective habit formation.

However, this explanation can be shown to be insufficient to explain the fact that the erosion of the household norm between 1965 and 1970 was much stronger in absolute terms than the rise in participation of married women with school-going children in the entire sixties. On the other hand, the long-run model of Section 8.5 can be modified into a more complex model so as to give a good fit of the data, and this more complex model can be shown to imply dynamic effects similar to those of the simpler model of Section 8.5. Therefore, and since the more complex variant has not yet been fully elaborated, the analysis of the long-run dynamics of female participation in the next section assumes that the social norm beliefs develop according to the long-run model of Section 8.5, i.e. in a way analogous to the dynamics of collective habits.

Finally, at the end of Section 9.2 we also raised the questions of endogeneity of social attitudes and needs. Social attitudes can be considered as being moulded by social norms and collective habits, and hence as being endogenous in the long run. Section 9.11 will argue that needs are likely to be endogenous in the very long run and that this endogeneity may have been an important cause of the onset of the female participation increase in the sixties.

14 The statements of De Neubourg (1988) that the female participation rate in the Netherlands was very small in the sixties and the seventies and has been on the upgrade since 1980 (p. 153), and that the labour demand surplus in the sixties only marginally induced Dutch women to enter the labour force (footnote 8 on p. 159), are based on data for the total female participation rate in the Netherlands as compared to other industrialized countries (see Tab. 9.1 and Fig. 9.1). While being partially correct in this intemational context, the statements are misleading with regard to the development of the participation rate of Dutch married women over time. On the other hand, the rise in this participation rate in the sixties was insufficient to satisfy the increasing demand for labour. This was one of the factors inducing the massive recruitment of foreign workers in the course of the sixties and the first half of the seventies (Koot and Van der Wiel. 1982, Sec. 1.2, and De Neubourg, 1988, above footnote). 


\subsection{Long-run elasticities}

This section and the next two analyse the long-run dynamics of the participation rate of a social group or cohort of married women with school-going children according to the participation equation (9.2) in Section 9.3 or (9.4) in Section 9.4 and according to the long-run social norm model of Section 8.5. The latter model can be incorporated into the former equations by conceiving the individual and average group beliefs in the household norm and the financial independence norm as specific aspects of individual habit states and social environments with respect to household time and one's own income, respectively (see Secs. 8.4 and 8.5 ). This implies that, just as the individual habits and social environments, the social norm beliefs are assumed to be adjusted to the actual behaviour in one year, and hence to be proportional to the actual behaviour in the preceding year. The norm belief terms in the participation equation (9.4) or (9.2) then become a part of overall IHF and BPI terms with an average household time habit sensitivity $\bar{\gamma}_{L L}$ that includes the average cognitive dissonance effect of the household norm $\bar{\gamma}_{L} / \bar{T}^{2}$, an average susceptibility to the social household time environment $\bar{\gamma}_{L \bar{L}}$ that includes the average reputation effect of the household norm $\bar{\beta}_{L} \bar{\varepsilon}_{L} \bar{\rho}_{L} / \bar{T}^{2}$, and an average susceptibility to the social family consumption and own income environment $\bar{\gamma}_{X} \bar{X}$ that includes the average reputation effect of the financial independence norm $\bar{\beta}_{F} \bar{\varepsilon}_{F} \bar{\rho}_{F} / \bar{Y}_{I M}{ }^{2}$. Here the average total discretionary time $\bar{T}$ and the average individual minimum income $\bar{Y}_{I M}$ have been assumed independent of time, and there is no cognitive dissonance effect of one's own belief in the financial independence norm, since this belief has been assumed to be proportional to the zero own income before entry.

On the basis of the thus simplified participation equation (9.4), we can now give the following picture of the long-run dynamics of the participation rate of a social group or cohort of married women with school-going children. Consider a situation of approximately long-run equilibrium and suppose that an exogenous variable changes. For example, suppose that the average wage rate rises. Then also the participation rate of the social group (or cohort) rises. By several kinds of bandwagon effect (see Subsec. 5.1.2) this renders participation more attractive for the group members who have not yet entered, so lowers their entry wages. As a result, group members whose entry wages drop below the wage rates prevailing for them, also enter, implying a further rise of the participation rate. This induces again bandwagon effects, raising the participation rate, etc., until a new long-run equilibrium is reached.

The relative rise of the participation rate in long-run equilibrium as a result of a onepercent relative rise of the average wage rate is expressed by the long-run wage elasticity of the labour force participation rate $E_{\overline{w^{*}}}^{P^{*}}$. Here the long-run equilibrium $P^{*}$ is a function $P^{*}\left(\bar{w}_{t}, \bar{\alpha}_{X t}, \bar{Y}_{O l}, \bar{H}_{t}^{a e}, u_{t}, \bar{\alpha}_{L l}, \bar{H}_{t}^{a \ell}-\bar{H}_{t}^{e}\right)$. Similarly to relation $(3.10)$ in Section 3.4 , the long-run wage elasticity $E_{\overline{w^{*}}}^{P^{*}}$ can be related to the short-run wage elasticity of labour force participation $\left.E_{\bar{w}^{\prime}}^{P}\right|^{*}$ at the long-run equilibrium $P_{t}^{*}$ as

$$
E_{\bar{w}}^{P^{*}}=\frac{1}{1-P_{P_{t-1}^{*}}^{*}}\left[E_{\left.\bar{w}\right|^{*}}^{P}+E_{\left.\bar{w}_{t-1}\right|^{*}}^{P}\right]
$$




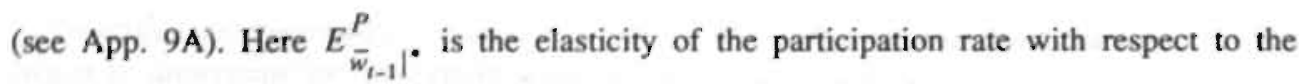
lagged wage rate in the pertinent term for consumption BPI. This elasticity appears in the formula for the long-run wage elasticity, since a change in the wage rate indaces, in the long run, a change in the lagged wage rate. More importantly, it induces, in the long run, also a change in the lagged participation rate, leading to a multiplier effect as indicated by the multiplier in front of the two elasticities on the right-hand side of (9.5) (see below).

The lagged-wage elasticity $E_{\left.\bar{w}_{1-1}\right|^{P}}$ in $(9.5)$ can be expressed in the short-run wage elasticity as $E_{\left.\bar{a}\right|^{*}}^{P} E_{\left.\bar{w}_{t-1}\right|^{*}}^{\bar{a}}=E_{\left.\bar{w}\right|^{*}}^{P} \cdot E_{\left.\bar{w}_{t-1}\right|^{*}}^{\bar{a}}$, where $E_{\left.\bar{w}_{t-1}\right|^{*}}^{\bar{a}}$, denotes the elasticity of $\bar{a}_{t}$ with respect to the lagged wage rate at the long-run equilibrium and can easily be shown to equal the share of the pertinent term for consumption BPI in $\bar{a}_{\ell}$. Hence, (9.5) can be rewritten as

$$
E_{\bar{w}}^{P}=\frac{1+E_{\left.\bar{w}_{t-1}\right|^{*}}^{\bar{a}}}{1-P_{P_{t-1}}^{*}} E_{\left.\bar{w}\right|^{*}}^{P}
$$

Thus, the long-run wage elasticity has become equal to a modified multiplier times the shortrun wage elasticity.

The derivative $P_{P_{1-1}^{*}}^{*}$ in the denominator of this multiplier is obtained by differentiating the right-hand side of the participation equation (9.2) in Section 9.3 to $P_{1-1}$ at the long-run equilibrium $P_{i}^{*}$ as

$$
P_{P_{t-1}}^{*}=f\left(\ln \bar{w}_{t}+\ln \bar{a}_{t}^{*}-\ln \bar{b}_{t}^{*}\right)\left(\frac{\bar{\gamma}_{X \bar{X}} \bar{w}_{t-1}}{\bar{a}_{t}^{*}}+\frac{\bar{\gamma}_{L \bar{L}}}{\bar{b}_{t}^{*}}\right) \bar{H}_{t-1}^{a c}\left(1-u_{t-1}\right)
$$

(see App. 9A). Here the function $f$ is the density function of $\ln (1+\varepsilon)$, where $\varepsilon$ is the relative deviation of the individual proportion of the short-run reservation wage and the wage rate, $q=w_{r} / w$, from its average. Furthermore, $\bar{a}_{i}^{*}$ and $\bar{b}_{i}^{*}$ are the values of $\bar{a}_{i}$ and $\bar{b}_{t}$ evaluated at $P_{i-1}=P_{i}^{*}$. The expression in $(9.7)$ has the interesting implieation that because the values of the usual density functions $f$ vary strongly with their argument, also the (modified) multiplier in (9.6) will vary strongly with that argument. Since the long-run participation rate is equal to the cortesponding distribution function by virtue of (9.2). and hence is monotonously increasing in the same argument. it then follows that the multiplier. and hence the long-run wage elasticity of the participation rate. will change considerably as the long-rin participation rate rises.

To be more precise, let us also consider what may then happen with the other timedependent factors in (9.7). In several OECD countries a rise in the female participation rate seems to have mostly been accompanied by a relatively strong rise in the average wage rate $\bar{w}_{t-1}$, a weaker (relative) decline in the employment rate $1-l_{t-1}$ and a weaker decline in the average corporate hours of employed women $\bar{H}_{t-1}^{a e}$ (see Killingsworth and Heckman, 1986. Sec. 2.1. for the U.S.A. and Great Britain). Moreover. the "parameter" $\bar{b}_{l}$ as representing the average marginal utility of household time when one does not participate $\left(L_{i}=T_{i}\right.$. see $(6.9$ b) in Subsec. 6.2 .1 ), is likely to have then declined relatively strongly and the develop- 
ment of the "parameter" $a_{t}$ as representing the average marginal utility of family consumption when one does not participate $\left(X_{i}=Y_{O i}\right.$, see $\left.(6.9 \mathrm{a})\right)$, seems ambiguous. The net result of these tendencies may have been a rise in the product of factors by which the density function value in (9.7) is multiplied, which rise could be expecied to be weak relative to the overall variation in this density function value. ${ }^{15}$

The participation equation (9.2) implies that the density function value in (9.7) times a small increase $d P_{t}^{*}$ in the long-run participation rate represents the number of group members who enter the labour market in long-run equilibrium as a result of that change. Assume that this density function value as a function of the long-run participation rate $P_{i}^{*}$ is unimodal and approximately symmetric. The maximum of this function is then reached for a long-run participation rate of about $50 \%$. Consider now the product of this function and the other factors in the expression for the derivative $P_{P_{t-1}^{*}}^{*}$ in (9.7). The supposed rise in the product of these factors as a function of the long-run participation rate has the effect of shifting the maximum point of the density function to a maximum point of $P_{P_{t-1}^{*}}$ at a higher long-run participation rate. Suppose that the maximum value of $P_{P_{h-1}^{*}}^{*}$ which is reached, is lower than one (see the next section for the opposite case). Then, as the long-run participation rate rises, one divided by the denominator in (9.6), i.e. the unmodified multiplier in (9.5), first increases from one to a maximum value for a long-run participation rate higher than (about) $50 \%$ and then decreases to one.

The elasticity $E_{\left.\bar{w}_{t-1}\right|^{-}}^{\bar{a}}$ in the numerator of the modified multiplier in (9.6) as representing the share of the term $\bar{\gamma}_{X \bar{X}} \bar{w}_{t-1} \bar{H}_{t-1}^{a *}$ in $\bar{a}_{t}^{*}$, where $\bar{H}_{t-1}^{a *}$ is the average corporate hours of all group members, can be argued to have typically risen in several OECD countries during the last three decades. The implied rise of this elasticity as a function of the long-run participation rate has the effect of shifting the maximum point of the unmodified multiplier to a maximum point of the modified multiplier at an even higher long-run participation rate. Empirical results of Romme (see the next section) suggest that the maximum value of the modified or unmodified multiplier may be in the order of tens or hundreds.

On the other hand, approximating the unimodal density function by a logistic one, leading to the participation equation (9.4), the short-run wage elasticity $E_{\bar{w}}^{P}$. at the long-run participation rate $P_{t}^{*}$ is given by $\kappa\left(1-P_{t}^{*}\right.$ ) (see App. 9.1), so decreases linearly with a rising long-run participation rate from the positive value $x$ to zero (see Fig. 9.4). As a result, we can expect that the long-run wage elasticity $E_{\bar{w}}^{p^{*}}$ will first decrease (somewhat) from $\kappa$, but next rise to a maximum value $E^{m}$ considerably higher than $\kappa$ and then decrease to zero. This profile is indicated in Figure 9.4, assuming a cautious maximum value 4 of the modified multiplier and supposing that the participation rate $P^{* 0}$ at which the maximum value

${ }^{15}$ A notable exception are the early eighties in especially the Netherlands, when the average wage rate declined, the employment rate declined strongly (Romme. 1986, p. 19), and the marginal utility of household time $\bar{b}_{t}$ may have risen due to a discouraged worker effect. However, the resulting decline in the product of factors by which the density function value in (9.7) is multiplied, may still be weak relative to the variation in this density funclion value, and is, as a first approximation, neglected in the following. 
$E^{m}$ of the long-run wage elasticity is reached, is not far from $50 \%{ }^{16}$ These assumptions imply that $E^{m}$ will be about equal to two times the maximum value of the short-run wage elasticity $\kappa$. Thus, the hump-shaped participation rate profile of the long-run wage elasticity is essentially different from the talus-shaped participation rate profile of the short-run wage elasticity.

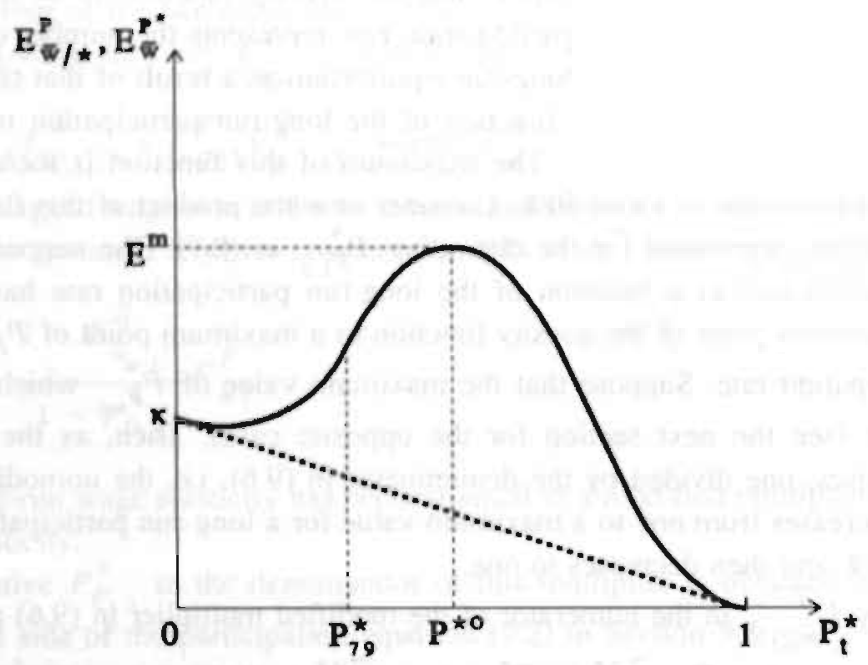

Figure 9.5 Talus-shaped participation rate profile of short-run wage elasticity $\left.E_{\overline{w^{*}}}^{P}\right|^{*}$ (dashed bold) $_{P^{*}}$ and hump-shaped participation rate profile of long-run wage elasticity $E_{\bar{w}}^{P^{*}}$ (solid) for Dutch married women.

Intuitively, the hump-shaped participation rate profile of the long-run wage elasticity can be explained as follows. When the average wage rate rises at a low or high value of the long-run participation rate, a relatively small number of group members in one of the tails of the unimodal and rather symmetric distribution of the entry wages will enter in the short run. By several kinds of bandwagon effect this will, in the long run, induce other group members in the distribution tail to enter also, but again the number of these group members will be relatively small. On the other hand, when the average wage rate rises at an intermediate value of the long-run participation rate. the group members who enter in the long run as a result of bandwagon effects, will be in the hump of the entry wage distribution, so their number will be relatively large. This leads to a hump shape of the multipliers and hence of the long-run wage elasticity as functions of the long-run participation rate, which shape is essentially different from the talus shape of the participation rate profile of the short-run wage elasticity.

A similar essential difference between the participation rate profiles of the long-run and short-run wage elasticities can be derived for a social group or cohort of other sub-

16 Note that multiplying the modified multiplier with the short-run elasticity has the effect of shifting the maximum point of the multiplier to a lower participation rate. 
populations of married women like married women with small children, who do or do not keep their job when getting children. Then, (approximately) aggregating the participationelasticity profiles over all cohorts in all subpopulations to the population of all married women can be expected also to yield a similar difference between the participation profiles of the long-run and short-run wage elasticities. This essential difference has adverse implications for the reliability of a backward simulation of the development of the participation rate of married women over time on the basis of cross-section estimates of a static labour supply function as carried out by Hartog and Theeuwes (1985, Sec. IV). For a 1979 nation-wide cross-section they estimated an own-wage elasticity of 1.99. The participation rate profile of such a static own-wage elasticity can be expected to have a talus-shape similar to that of the participation rate profile of our short-run wage elasticity (see also Hartog and Theeuwes, p. S248), Consequently, in Hartog and Theeuwes' backward simulation of the participation rates of married women from 1979 to 1947 , it is implicitly implied that the own-wage elasticity was higher than 1.99 in earlier years as the participation rate was lower. Therefore, it is not really surprising that they find a strongly dominating impact of the own wage on the development of the participation rate of married women between 1947 and 1979.

On the other hand, our model suggests a completely different picture. As the actual values of the participation rate can be expected to follow its long-run equilibrium values at a distance (see the end of Sec. 3.5), the long-run wage elasticity can be regarded as an adequate indicator of the total impact of the wage rate on the participation rate. Since the participation rate of married women in 1979 was $25 \%$ according to the data of Hartog and Theeuwes, the corresponding long-run participation rate $P_{79}^{*}$ will be higher than $25 \%$ and may be located in Figure 9.4 as indicated. Then the long-run wage elasticity would be lower than its 1979 estimate in earlier years, as the participation rate was lower.

Next, the question is whether an estimate of the long-run wage elasticity at 1979 would be higher or lower than the 1979 estimate 1.99 of the own-wage elasticity of Hartog and Theeuwes. Empirical results of Kapteyn, Woittiez and Ten Hacken (1989) suggest that the former estimate would be lower than the latter. For two Dutch cross-section samples in 1985 they find long-run wage elasticities of the average number of corporate hours of non-single women with and without children which are much lower than corresponding wage elasticities obtained from a standard model like that of Hartog and Theeuwes (see Kapteyn et al, p. 157). A similar result can be expected to hold with respect to the wage elasticities of participation for these samples. Since the participation rates of the non-single women in these samples were considerably below $50 \%$, the corresponding long-run participation rates at 1985 are likely to be left to the point $P^{* 0}$ in Figure 9.4, where the participation rate profile is assumed to reach its maximum. This would imply that the long-run wage elasticity of the participation rate of non-single women at 1979 would be lower than the same elasticity at 1985. Combined with the high probability that the standard wage elasticity of participation of non-single women at 1979 would be higher than the same elasticity at 1985, this leads to the expectation that the long-run wage elasticity at 1979 would be (considerably) Jower than the 1979 estimate 1.99 of the own-wage elasticity of Hartog and Theeuwes.

Thus, we arrive at the conclusion that, in a backward simulation of the participation rates of married women from 1979 to 1947 , the long-run wage elasticity could be expected to decline from a value (much) below 1.99 in 1979 to lower values in earlier years as the (long-run) participation rate was lower. This strongly suggests that the impact of the own 
wage on the development of the participation rate of married women between 1947 and 1979 has been greatly overestimated by Hartog and Theeuwes. On the other hand, the above analysis also suggests that low estimates of long-run wage elasticities for the eighties like those of Kapteyn et al. not need to hold for the nineties with their higher participation rates and hence presumably higher long-run wage elasticities (see also Kapteyn and Woittiez, 1987, Fig. 3). Therefore such estimates do not seem directly usable for projecting the participation and corporate hours effects of, for instance, a change in the fiscal regime in the nineties. ${ }^{17}$

Long-run wage elasticities vary strongly not only with time, but also with the level of aggregation. In Sections 9.4 and 9.5 we already saw that the constant $\boldsymbol{\kappa}$, and hence the short-run wage elasticity of participation $\kappa\left(1-P_{t}\right)$ (see Fig. 9.4), are inversely proportional to the standard deviation of the distribution of the individual proportions $q=w_{r} / w$ over the social group or cohort, and hence are lower at a higher aggregation level (and at given participation rate $P_{t}$ ). Thus, the participation rate profile of the short-run wage elasticity at the long-run participation rate $P_{i}^{*}$ has a more gradually declining talus shape as the aggregation level is higher. In addition, the density function $f$ and hence the modified multiplier in (9.6) will have a less pronounced participation profile as the aggregation level is higher and hence the individual variation stronger. This holds despite the higher susceptibilities to social influence $\bar{\gamma}_{X \bar{X}}$ and $\bar{\gamma}_{L L}$ in (9.7) as a result of BPI between social groups, since this BPI is relatively weak (see Sec. 9.5). Together the aggregation tendencies of the participation rate profiles of the short-run wage elasticity and the modified multiplier imply that the participation rate profile of the long-run wage elasticity will be (considerably) less pronounced as the aggregation level is higher. In particular, the maximum value $E^{m}$ of the long-run wage elasticity will be lower. The reason of this result is that, at a higher aggregation level and hence lower levels of the density function $f$ around its maximum, a one percent rise of the wage rate in this region induces a smaller percentage of the women to enter the labour market, and the resulting changes in the social environments of the staybehinds induce a relatively smaller percentage of them to enter also.

So far we have considered only wage elasticities of the participation rate. However, in Section 9.1 we saw that several other (more or less) exogenous variables like the number and age of children. the level of education (not via the wage rate), and the availability of part-time jobs are likely to have played an important role in the development of the participation rate of Dutch married women during the last century. With respect to these variables also short-run and long-run elasticities of the participation rate can be derived from the participation equations (9.2) and (9.4). Then it turns out that we obtain relations between long-run and short-run elasticities which are analogous to $(9.5)$. with the derivative $P_{P_{t-1}^{*}}^{*}$ again being given by the expression in (9.7), but in which the elasticity of the participation rate with respect to the lagged exogenous variable is mostly zero. Thus, in the latter case, in order to obtain the long-run elasticity the short-run elasticity should be multiplied by the unmodified multiplier in (9.5). Moreover, just as the long-run wage elasticity in (9.6), all long-nun elasticities can be expressed as a modified multiplier times the short-run wage

17 This does mot deny that for that purpose the full model structure of Kapteyn ef al. can be used (see their pertinent remark on p. 157). 
elasticity. The modified multiplier then has the same denominator as in (9.6), but the numerator is given by one "share elasticity" or the sum of two "share elasticities" of $\bar{a}$, or minus one "share elasticity" or minus the sum of two "share elasticities" of $\bar{b}$ with respect to the exogenous variable in year $t$ and, in the case of the sum, in year $t-1$. The pronotinced hump-shaped participation rate profile of such a multiplier implies that all long-run elasticities too will have a hump-shaped participation rate profile. The nexî section will consider the implications of such a profile for the development of the participation rate of married women over time.

\subsection{Participation rate dynamics and hysteresis}

This section analyses the long-run dynamics of the participation rate of a social group, cohort or (sub)population of married women, which results from hump-shaped participation rate profiles of the long-run elasticities of the participation rate with respect to the exogenous variables. We denote the exogenous variables that appear in $\bar{a}_{t}$ as $z_{1}^{a}, \ldots, z_{A}^{a}$ and the exogenous variables in $\bar{b}_{t}$ as $z_{1}^{b}, \ldots, z_{B}^{b}$. Using the expressions for the long-run elasticities obtained in the previous section, a relative change $\Delta \ln P_{t}^{*}:=\ln P_{t}^{*}-\ln P_{t-1}^{*}$ in the long-run participation rate $P_{1}^{*}$ can be expressed as

$$
\begin{aligned}
& \Delta \ln P_{t}^{*} \approx E_{\bar{w}}^{P^{*}} \Delta \ln \bar{w}_{l}+\sum_{l=1}^{A} E_{z_{l}^{a}}^{P^{*}} \Delta \ln z_{l l}^{a}+\sum_{l=1}^{B} E_{z_{l}^{b}}^{P^{*}} \Delta \ln z_{l l}^{b}= \\
& =\frac{E_{\bar{w} l^{*}}^{P}}{1-P_{P_{t-1}^{*}}^{*}}\left[\left(1+E_{\left.\bar{w}_{t-1}\right|^{*}}^{\bar{a}}\right) \Delta \ln \bar{w}_{l}+\sum_{l=1}^{A}\left(E_{z_{l l}^{a} l^{*}}^{\bar{a}}+E_{z_{l l-1}^{a} l^{*}}^{\bar{a}}\right) \Delta \ln z_{l l}^{a}-\sum_{l=1}^{B}\left(E_{z_{l i}^{b} l^{*}}^{\bar{b}}+E_{z_{l i-1}^{b} l^{*}}^{\bar{b}}\right) \Delta \ln z_{l l}^{b}\right] .
\end{aligned}
$$

Here the elasticities within the brackets are "share elasticities" of $\bar{a}_{1}$ and $\bar{b}$, with respect to the exogenous variables in years $t$ and $t-1$, where the "share elasticities" with respect to $t-1$ are mostly zero.

Since the last expression in (9.8) is rather complex, we make the simplifying assumption that the sum of the terms between the brackets is approximately proportional to the relative change $\Delta \ln \bar{w}_{1}$ in the average wage rate over time, so is equal to $\alpha \Delta \ln \bar{w}_{1}$, where $\alpha$ is a constant larger or smaller than one. The expression in (9.8) then becomes

$$
\Delta \ln P_{t}^{*}=\frac{\alpha E_{\left.\bar{w}\right|^{*}}^{P}}{1-P_{P_{t-1}^{*}}^{*}} \Delta \ln \bar{w}_{t} .
$$

The logarithm of the average wage rate may then serve as an indicator of the changes in all exogenous variables, so that the long-run participation rate $P_{i}^{*}$ can be considered as a function $P^{*}\left(\ln \bar{w}_{1}\right)$. Since the short-run wage elasticity $E_{\bar{w} \mid *}^{P}$ in $(9.9)$ can be logistically approximated as $\kappa\left(1-P_{l}^{*}\right)$, the graph of the function $P^{*}\left(\ln \bar{w}_{l}\right)$ would approximately have 
a logistic shape, if the derivative $P_{P_{t-1}^{*}}^{*}$ in (9.9) were zero for all values of $P_{t-1}^{*}=P_{t}^{*}$. This is the shape implied by static labour supply models like that of Hartog and Theeuwes (1985).

On the other hand, in our model the derivative $P_{P_{t-1}^{*}}^{*}$ in (9.9) is substantially larger than zero for intermediate values of $P_{t-1}^{*}=P_{i}^{*}$. Then the implied hump shape of the participation rate profile of the long-run "overall" wage elasticity (i.e. the factor by which $\Delta \ln \bar{w}_{t}$ in (9.9) is multiplied) has the effect of making the function $P^{*}\left(\ln \bar{w}_{t}\right)$ grow stronger than exponentially (constant growth rate or elasticity) for $P_{t}^{*}$ not too much lower than the maximum point of the profile (cf. $P^{* o}$ in Fig. 9.4), and decline stronger than logistically (linearly declining growth rate or elasticity) for $P_{i}^{*}$ sufficiently higher than the maximum point. This results in a sigmoid shape as roughly represented by the solid curve in Figure 9.5 which shape is considerably "sharper" than the logistic shape. It describes a (relatively) high degree of persistence at low as well as high levels of $P_{t}^{*}$ as a result of (relatively) low longrun elasticities, and strong changes at intermediate levels of $P_{t}^{*}$ as a result of relatively high long-run elasticities. We call such a curve a super-exponential-logistic curve (SEL curve). Its defining properties can be expected to be rather robust to relaxing the crude simplifying assumption made above.

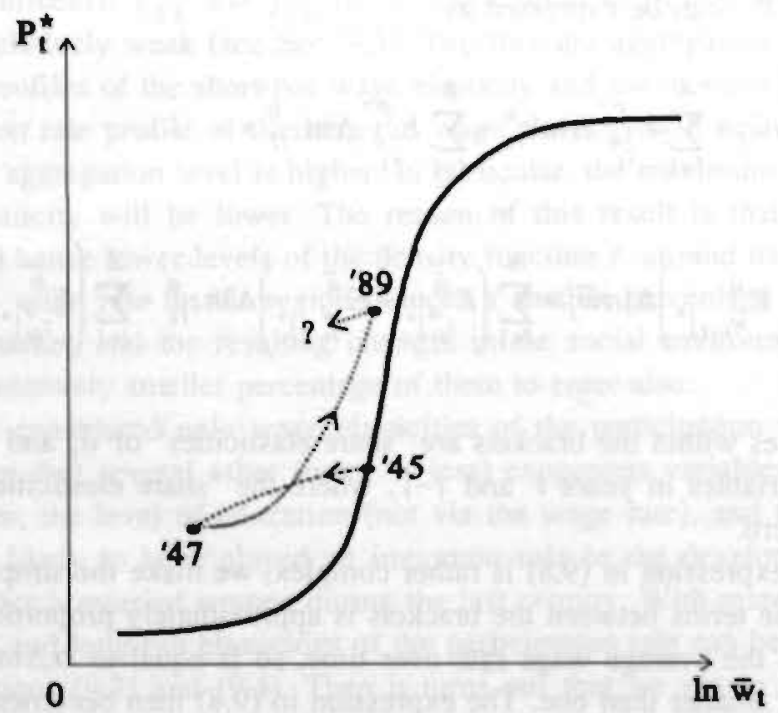

Figure 9.5 Super-exponential-logistic curve of $P_{i}^{*}$ as a function of $\ln \bar{w}_{q}$ (solid), and collective hysteresis loop of $P_{i}^{*}$ of American married women after World War II (dotted).

What are the implications of this SEL curve for the development of the participation rate of married women over time? Consider first a regime of a constant growth rate $\Delta \ln \bar{w}_{t}=\gamma$ of the average wage rate. By virtue of the simplifying assumption made above, this implies a constant, though generally different, weighted sum of the growth or decline rates of the other exogenous variables (including $\bar{w}_{t-1}$ ). If we substitute $\Delta \ln \bar{w}_{t}=\gamma$ in (9.9), it is easily 
seen that the long-run participation rate $P_{t}^{*}$ as a function of year $t$ will then develop according to a SEL curve. Since the shape of the development of the actual participation rate over time will be a "smoothed out" variant of the shape of the development of the long-run participation rate, the actual participation rate may then develop according to a SEL-like curve. Such a curve can account for the empirical pattern of a remarkably persistent low participation rate of Dutch married women before 1960 and a strongly growing participation rate of these women after 1960 (see Fig. 9.1). However, it does not seem to give a proper description of the less than exponential growth of this participation rate in the first half of the eighties. Possible causes may be the strong rise of the unemployment during that period, the fact that the average real wage rate of Dutch women then declined (Romme, 1986, p. 19), and the fact that the average number of children below five years did not decline any more in the first half of the eighties (Mol et al., 1988, Fig. 3.2). Such developments can be incorporated in the difference equations (9.8) or (9.9), but it then becomes an empirical question how the time profile of the participation rate is predicted to look like.

Still, on the explanation of the empirical pattern of a remarkably persistent low par. ticipation rate before 1960 and a strongly growing participation rate after 1960 we can make the following statements. Whereas conventional static labour supply models like that of Hartog and Theeuwes (1985) and Mol et al. (1988) try to explain this empirical pattern by unfavourable levels and small changes of exogenous variables before 1960 and favourable levels and large changes of such exogenous variables after 1960, our labour supply model of an endogenous dynamics of collective habit formation and social norms seerns to offer a more convincing explanation by adding the explanatory power of hump-shaped participation rate profiles of the long-run elasticities of exogenous variables. According to these profiles the persistence of the low participation rate of married women before 1960 is partially explained by low values of long-run elasticities of exogenous variables, and the booming character of the rise of the participation rate after 1960 is partially explained by higher values of such elasticities. Before 1960 the mutually reinforcing forces of collective habits and social norm beliefs on the one hand and of the actual low participation of married women on the other hand consolidated the status quo of a low participation rate and of corresponding collective habits and social norm beliefs (see Sec. 9.7). On the other hand, after 1960 a similar kind of mutually reinforcing forces strongly accelerated the changes in the participation rate of married women and in the corresponding collective habits and social norm beliefs. Of course, this theoretical explanation should be empirically tested by estimations of the participation equation (9.4) in Section 9.4, but it looks promising.

As noted in the previous section, the density function $f$, and hence the modified multiplier in (9.6), will have a less pronounced participation rate profile as the aggregation level is higher. This implies that the hump shape of the participation rate profile of the long-run elasticities, and hence the "sharp" sigmoid shape of the time profile of the participation rate, will be (considerably) less pronounced as the aggregation level is higher. This explains the more pronounced time profiles of the participation rates of married women in the age groups 
of 25-29, 30-39 and 40-49 years according to Figure 9.2 as compared to the time profile of the participation rate of all married women below 65 years in Figure 9.1. ${ }^{18}$

That the multipliers in (9.6), (9.8) and (9.9) may become very high, is suggested by the estimation results of Romme for the more plausible loglinear specification of his participation equation for female cohorts. The final variant of this specification can be shown to imply that the multiplier is equal to 500 (!) for zero long-run participation rate and declines as, in good approximation, $1 / P_{i}^{*}$ as the long-run participation rate rises. However, this participation rate profile of the multiplier as being essentially different from the humpshaped profile implied by (9.6) seems very implausible and suggests a severe specification error in Romme's loglinear participation equation. ${ }^{19}$ Still, at the lowest aggregation level of the social group the multiplier in (9.6) may become very high and even infinite at intermediate levels of the participation rate, when the BPI between the group members is strong and/or the individual variation of the reservation wage-wage proportions is weak. The case of an infinite multiplier will be analysed in the next section.

So far we have mainly analysed what happens when the average wage rate and the other exogenous variables change so as to encourage participation of married women. Suppose, however, that after a period of such encouraging changes the average wage rate or other exogenous variables stan to change in the opposite direction, and hence start to disencourage participation. This seems to have happened in the U.S.A. just after World War II (Clark and Summers, 1982, Sec. 2). Between 1940 and 1944, when large numbers of men were drawn into the Armed Forces increasing the job prospects and potential earnings of women, the participation rate of married women rose precipitously from $15.6 \%$ to $23.9 \%$. After the transitory developments of the war had passed, the participation rate was expected to fall back sharply to or below the long pre-war trend, but it declined only to $20.0 \%$ in 1947 and increased to $23.0 \%$ in 1950 . Clark and Summers ascribe this to persistence effects of, among other factors, individual habit formation (IHF).

In our model the persistence effect of IHF is described as a regime switch from a participation equation for entries without an IHF effect of corporate work to another participation equation for exits of (re-)entered married women with an IHF effect of corporate work (see the end of Sec. 9.4). The forner equation implies an upwards development of the long-run participation rate $P_{t}^{*}$ along a SEL curve like the solid curve in Figure 9.5. Let us assume that the lower half of this curve describes the rise of the long-run participation rate of American married women before and during World War II. The long-run participation rate at 1945 may then be located on the curve as indicated. The IHF effect of corporate work implies that after 1945 the long-run participation rate did not fall back along the curve. but developed along another curve as indicated by the dotted curve in Figure 9.5. This curve is a small (collective) hysteresis loop like those in Figure 4.11 in Section 4.8 , but it is less

${ }^{18}$ The less pronounced time profiles of the participation rates in the age groups of 50-59 and 6064 years as compared to the time profile for the whole population can be associated with the fact that the participation rates remained low, and hence were located in the relatively low left tails of the participation rate profiles of the long-nun elasticities.

${ }^{19}$ Even more severe specification ernors are made by Clark and Summers (1982), whose loglinear logarithmic participation equations imply constant short-run elasticities as well as constant multipliers. and hence constant long-run elasticities. 
pronounced, since the persistence of the participation of the (re-)entered married women is only a part of the development of the participation of the whole population of married women. The hysteresis loop indicates a second regime switch at (about) 1947, after which the participation rate again started to rise. (For reasons of exposition the distance between the 1945 and 1947 points is somewhat exaggerated in Figure 9.5.) After 1947 there was a persistence effect of IHF by those married women who had left the labour market between 1945 and 1947 and had got used to staying at home to a certain extent. However, as the average wage rate or other exogenous variables again became increasingly favourable for participation, such women re-entered the labour market and an increasing proportion of other married women (re-)entered or kept their job when marrying or getting children. The overall strong and steady increase of the participation rate of American married women between 1947 and 1990 can then be represented by an upward movement of the long-run participation rate along the dotted curve. As indicated in Figure 9.5, this growth path is predicted to approach the original pre-1945 growth path. However, in the course of 1990 the participation rate of American married women again started to decline (Breedeveld, 1991). Figure 9.5 describes this turning point by a regime switch at 1989 . The tuming point is likely to be due to cultural reactions, to which we will come back in Section 9.11.20

The hysteresis loop in Figure 9.5 has two interesting implications. First, it indicates that the persistence after the war of the strongly risen participation during the war led to a growth path which long remained at higher participation rates than the growth path which would have been followed, if there had not been a strong rise of the participation rate during the war and a subsequent decline. The latter situation applies to a country like the Netherlands, where the participation rate of married women remained low during the war. The resulting lack of experience with and accustomization to participation of married women can (partially) explain why also after the war the participation rate of Dutch married women remained lower than the participation rate of American married women and, more generally, than the corresponding participation rate in most other OECD countries. Besides this difference in participation experience during the war, also earlier differences are likely to have played an important role in the continuously lower participation rate in the Netherlands (see Sec. 9.7).

A second implication of the hysteresis loop in Figure 9.5 is that as the average wage rate and the other exogenous variables continue to change in a participation encouraging direction, the persistence effect fades away as indicated by the convergence of the dotted curve to the solid curve. This can partially explain why in the long run the paricipation rate of Dutch married women is likely to catch up with the corresponding participation rate in other OECD countries (see the strong rise in the paricipation rate of all Dutch women between 1985 and 1991 according to Table 9.1). Another explanatory factor for such a convergence might be BPI between countries.

${ }^{20}$ For these cultural reactions $\ln \bar{w}_{t}$ is likely to be not an adequate indicator, but for the sake of exposition Figure 9.5 pretends it is. 


\subsection{Collective catastrophes}

in the previous sections it has been assumed that the maximum value of the derivative $P_{P_{t-1}}^{*}$ in (9.7) is lower than one. This implies that as the long-run participation rate rises, the multiplier in formula (9.6) for the long-run wage elasticity of the participation rate remains finite. However, from (9.7) we can see that when the susceptibilities to social influence $\gamma_{X \bar{X}}$ and $\gamma_{L \bar{L}}$ are high and when the density function of the reservation wage-wage proportions $f$ is high-peaked by weak individual variation, the derivative $P_{P_{t-1}^{*}}^{*}$ can be larger than or equal to one. This may especially happen at the low aggregation level of a social group.

To analyse this possibility we consider the participation rate $P_{t}$ in year $t$ as a function $P\left(P_{t-1} ; z_{t}\right)$ of the participation rate $P_{t-1}$ in the preceding year for given values of the vector of all exogenous variables $z_{t}$. In general this function is given by the participation equation $(9.2)$ in Section 9.3 , where the norm belief terms in $\bar{a}_{t}$ and $\bar{b}_{t}$ have become a part of overall IHF and BPI terms (see the beginning of Sec. 9.8). This equation implies that $P_{i}$ as being equal to the distribution function value $F\left(\varepsilon_{t}\right)$ for $\varepsilon_{t}=\ln \bar{w}_{t}+\ln \bar{a}_{t}-\ln \bar{b}_{t}$ will usually have a sigmoid shape as a function of this argument, and hence probably as a function of $P_{t-1}$. For certain ranges of the exogenous variables $z_{t}$ the curve of the latter function $P\left(P_{t-1} ; z_{t}\right)$ may then look as in Figure 9.6. The intersections $A_{t}, B_{t}$ and $C_{t}$ of this curve with the equilibrium line $P_{t}=P_{t-1}$ indicate the loci of three long-run equilibria $P_{t}^{* A}$, $P_{t}^{* B}$ and $P_{t}^{* C}$ (cf. Fig. 5.4 in Subsec. 5.5.2). The slope of the curve at these long-run equilibria represents the derivative $P_{P_{t-1}^{*}}^{*}:=P_{P_{t-1}}\left(P_{t}^{*} ; z_{l}\right)$ referred to above. This slope is smaller than one for the equilibria $P_{t}^{* A}$ and $P_{t}^{* B}$ and larger than one for the equilibrium $P_{1}^{* C}$. By well-known arguments this can be shown to imply that the equilibria $P_{1}^{* A}$ and $P_{1}^{* B}$ are stable, whereas the equilibrium $P_{1}^{* C}$ is unstable (see the step-by-step adjustment path between $P_{t}^{* C}$ and $P_{t}^{* A}$ in Fig. 9.6 and cf. Secs. 3.2 and 5.3).

This configuration implies that the actual participation rate $P_{I}$ is converging to one of the two stable long-run participation rates $P_{1}^{* A}$ and $P_{1}^{* B}$. Hence, we are still in the situation of the derivative $P_{P_{t-1}^{*}}^{*}:=P_{P_{t-1}}\left(P_{t}^{*} ; z_{t}\right)$ being smaller than one, implying a finite multiplier, which has been extensively analysed in the previous section. However, in the configuration of Figure 9.5 this situation can become drastically different when the exogenous variables $z_{t}$ sufficiently change. Consider a social group of married women with school-going children that has a low participation rate near the long-run participation rate $P_{t}^{* A}$. This may describe the situation of many social groups of Dutch married women in the fifties and sixties. Suppose now that the exogenous variables $z_{l}$ start to change steadily over time so as to encourage participation. Examples from the Netherlands in the sixties and seventies are a declining birth rate, a rising level of education of married women, and a rising real wage rate of women. The effect of such changes on the curve of the participation function $P\left(P_{t-1} ; z_{t}\right)$ in Figure 9.6 can be an overall upward movement. As a result, the stable long-run equilibrium $A_{t}$ and the unstable long-run equilibrium $C_{t}$ move towards each other, and will, at certain values of the exogenous variables $z^{* 1}$, coincide into one unstable equilibrium and 


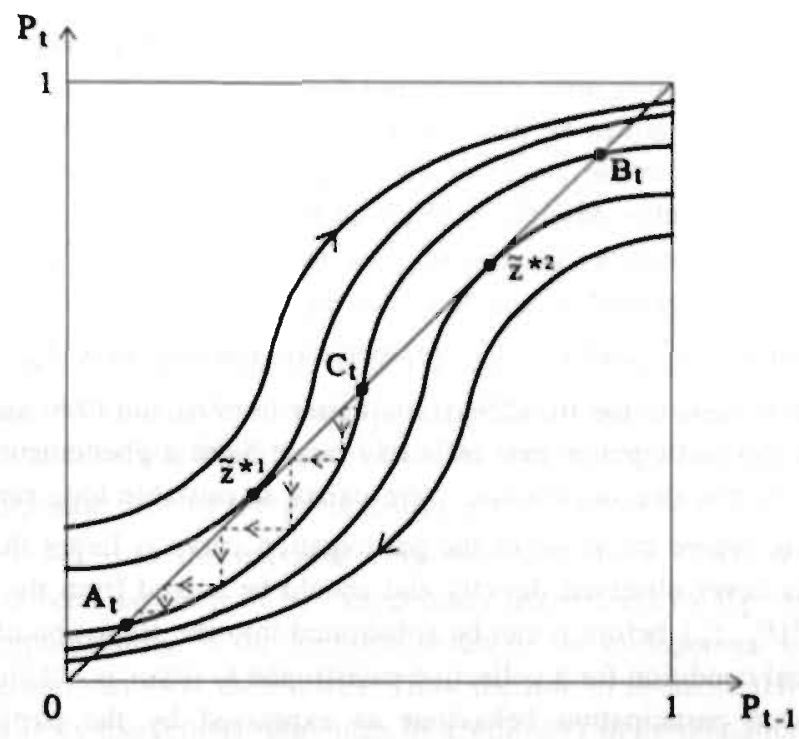

Figure 9.6 Participation functions $P\left(P_{t-1} ; z_{t}\right)$ with three long-run equilibria $A_{t}, B_{t}$ and $C_{t}$ for various values of the exogenous variables $z_{t}$. For $z_{l}$ beyond $z^{* 1}$ a collective entry catastrophe and for $z$, beyond $z^{* 2}$ a collective exit catastrophe occurs (as indicaled by the arrows).

subsequently, for $z_{t}$ beyond $z^{* 1}$, disappear. Then, only the stable equilibrium $B_{t}$ at a high participation rate is left, so the social group of married women will move to that $e$ quilibrium. This means that an infinitesimally small change in the exogenous variables $z$, beyond $z^{* 1}$ induces a large number of group members to follow each other in entering the labour market. Such a large shift in the participation rate as a consequence of an infinitesimally small change in the exogenous variables is a collective catastrophe (see Sec. 5.4 and Subsec. 5.5.3. and cf. Sec. 3.5).

Consider now a social group of married women with school-going children that has a high participation rate near the long-run participation rate $P_{t}^{* B}$. This may describe the situation of many social groups of American married women at the end of World War II (see the previous section). Suppose that the exogenous variables $z$, start to change over time so as to discourage participation to a considerable extent. This is likely to have happened in the U.S.A. just after the war as large numbers of men returned from the battle-field. Such changes can lead to an overall downward movement of the curve of the participation function $P\left(P_{t-1} ; z_{t}\right)$ in Figure 9.6. As a result, the stable long-run equilibrium $B_{t}$ and the unstable long-run equilibrium $C_{1}$ move towards each other, and may, at certain values of the exogenous variables $\tilde{z}^{* 2}$ and for some social groups, coincide into one unstable equilibrium and subsequently, for $z$, beyond $z^{* 2}$, disappear. Then, only the stable equilibrium $A_{\text {, }}$ at a low participation rate is left, so the social group of married women will move to that equilibrium. This means that an infinitesimally small change in the exogenous variables $z_{\text {, }}$ beyond $z^{* 2}$ induces a large number of group members to follow each other in leaving the 
labour market. Thus, we have here a collective exit catastrophe. However, in the abovementioned example of American women such a collective exit catastrophe may have occurred for some social groups only, considering the persistence of the overall participation rate. Moreover, it seems hard to be distinguished from a collective exit within a short time as a result of a large shift in the values of the exogenous variables within that time. Therefore, we will consider another possible example in the next section.

In both cases the collective catastrophe goes together with the participation curve in Figure 9.6 becoming tangential to the long-run equilibrium line at the relevant long-run participation rate for $z=z^{* 1}$ and $z=z^{* 2}$, respectively. The derivative $P_{P_{t-1}^{*}}^{*}:=P_{P_{t-1}}\left(P_{t}^{*}, z_{t}\right)$ is then equal to one, making the (modified) multiplier in (9.6) and (9.8) and hence the longrun elasticities of the participation rate infinitely large. Such a phenomenon can occur only when just before its possible occurrence, there exists an unstable long-run equilibrium like $P_{t}^{* C}$ in Figure 9.6, where the slope of the participation curve is larger than one. However, this equilibrium is never observed directly and should be solved from the highly non-linear equation $P_{t}^{*}=P\left(P_{t}^{*} ; z_{t}\right)$ before it can be substituted into the slope condition.

A more practical condition for a collective catastrophe to occur is obtained by considering the actual short-run participation behaviour as expressed by the participation equation $P_{t}=P\left(P_{t-1} ; z_{t}\right)$ and taking the first difference of this equation as

$$
\Delta P_{t}=\sum_{l=1}^{Z}\left(P_{z_{l}} \Delta z_{l t}+P_{z_{l t-1}} \Delta z_{l t-1}\right)+P_{P_{t-1}} \Delta P_{t-1}
$$

Here $Z$ is the total number of exogenous variables and $P_{z_{t t-1}}$ is zero for most of them. This difference equation describes an unstable explosive process if and only if the slope $P_{P_{t-1}}$ is larger than one. Such a process is a collective catastrophe, in which a small change in the participation rate $\Delta P_{t-1}$ as a result of small changes in exogenous variables amplifies itself, et cetera, into a large shift in the participation rate, until a domain of participation rates is reached where the slope $P_{P_{t-1}}$ is again smaller than one. ${ }^{21}$ The collective catastrophe condition that the slope $P_{P_{t-1}}$ should be larger than one in some range of the participation rates, is equivalent to the condition that the maximal slope $P_{P_{t-1}}$ over the whole range of the participation rates should be larger than one, i.e., analogously to (9.7),

$$
\max _{P_{t-1}}\left[\left(\frac{\gamma_{X} \bar{x}_{t-1}}{\bar{a}_{t}}+\frac{\gamma_{L \bar{L}}}{\bar{b}_{t}}\right) \bar{H}_{t-1}^{a c}\left(1-u_{t-1}\right) f\left(\ln \bar{w}_{t}+\ln \bar{a}_{t}-\ln \bar{b}_{t}\right)\right]>1 .
$$

It is interesting to compare this condition for collective catastrophes in participation with condition (7.4) in Section $\mathbf{7 . 2}$ for collective catastrophes in the average supply of corporate

${ }^{21}$ Such a collective catastrophe occurs also in the outbreak of an epidemic disease. The implied analogy is used in the epidemiological models of the diffusion of social nom beliefs and participation behaviour of De Neubourg $(1985,1988)$ and De Neubourg and Vendrik (1989). See, however. footnote 17 in Section 8.5 regarding the former references. 
hours in the case of BPI and unstable IHF with respect to household time only. We should then put $\bar{\gamma}_{X \bar{X}}=0$ in $(9.11)$, implying that the condition can be rewritten as

$$
\bar{\gamma}_{L \bar{L}}>\frac{1}{\max _{P_{t-1}}\left[\bar{H}_{t-1}^{a c}\left(1-u_{t-1}\right) \frac{f\left(\ln \bar{w}_{t}+\ln \bar{a}_{t}-\ln \bar{b}_{t}\right)}{\bar{b}_{t}}\right]}
$$

This condition differs from condition (7.4) in the following respects. First, the susceptibility to the social household time environment $\bar{\gamma}_{L} \bar{L}$ in (9.12) includes also the average reputation effect of the endogenous household norm (see the beginning of Sec. 9.8). Secondly, the average actual corporate time of the participating group members $\bar{H}_{t-1}^{a e}\left(1-u_{t-1}\right)$ in $(9.12)$ is usually much lower than the maximal corporate time $T$ in (7.4). Whereas the former corporate time has been assumed to be exogenously determined by the employers (see Sec. 9.4), the latter corporate time is the long-run result of the endogenous unstable household time IHF of the group members themselves. Thus, the role of unstable IHF in (7.4) has been taken over in (9.12) by exogenous rationings by employers in combination with the discrete participation variable. On the other hand, in reality the rationings by employers will be endogenous to a certain extent (see Sec. 9.4), so that the IHF partially regains its role and average habit sensitivities enter into the collective catastrophe conditions (as in Case IV in Table 6.1 in Subsec. 6.2.4). It seems, however, very hard to derive collective catastrophe condition for this more complex case.

A third difference between the conditions (9.12) and (7.4) is that the density function $f$ in (9.12) denotes the density function of $\tilde{\boldsymbol{\varepsilon}}=\ln (1+\varepsilon)$ with $\varepsilon$ being the relative deviation of the individual reservation wage-wage proportion, whereas $f$ in (7.4) stands for the density function of the individual household time taste $\alpha_{L}$. Condition (7.4) has been derived for the case of individual variation in the household time taste only, and in that particular case it is easily shown that $\bar{\varepsilon} \approx \varepsilon=\left(\alpha_{L}-\bar{\alpha}_{L}\right) / \bar{b}$. The denominator of the last expression explains the presence of an additional denominator $\bar{b}_{1}$ in (9.12) as compared to (7.4).

Although the condition (9.12) holds for collective entry as well as exit catastrophes, the participation curves in Figure 9.6 for given values of the exogenous variables $z$, have different positions for entry and exit behaviour. This is due to the additional terms in the participation equation for exits, describing the IHF effects of corporate work. These terms make the participation curves for exits higher than those for entries in Figure 9.6 and imply an even larger difference between the $z^{* 2}$ at which the collective exit catastrophe occurs and the $z^{* 1}$ at which the collective entry catastrophe occurs. This is illustrated by Figure 9.7. again assuming that $\ln \bar{w}_{1}$ is an adequate indicator for the changes in all exogenous variables. The right sigmoid curve is the entry curve, which describes how the long-run participation rate increases as $\ln \bar{w}_{l}$ rises and hence encourages entries (cf. Fig. 7.1 in Sec. 7.2). The solid part of this curve indicates stable long-run participation rates like $P_{t}^{* A}$ and $P_{t}^{* B}$ in Figure 9.6 and the dotted part represents unstable long-run participation rates like $P_{t}^{* C}$ in Figure 9.6. At $\ln \bar{w}^{* 1}$ a collective entry catastrophe occurs as indicated by the right dashed bold line. Similarly, the left sigmoid curve is the exit curve, which describes how the 
long-run participation decreases as $\ln \bar{w}_{t}$ declines and hence encourages exits. The solid and dotted parts of this curve again represent stable and unstable long-run participation rates, respectively. At $\ln \bar{w}^{\cdot 2}$ a collective exit catastrophe occurs as indicated by the left dashed bold line.

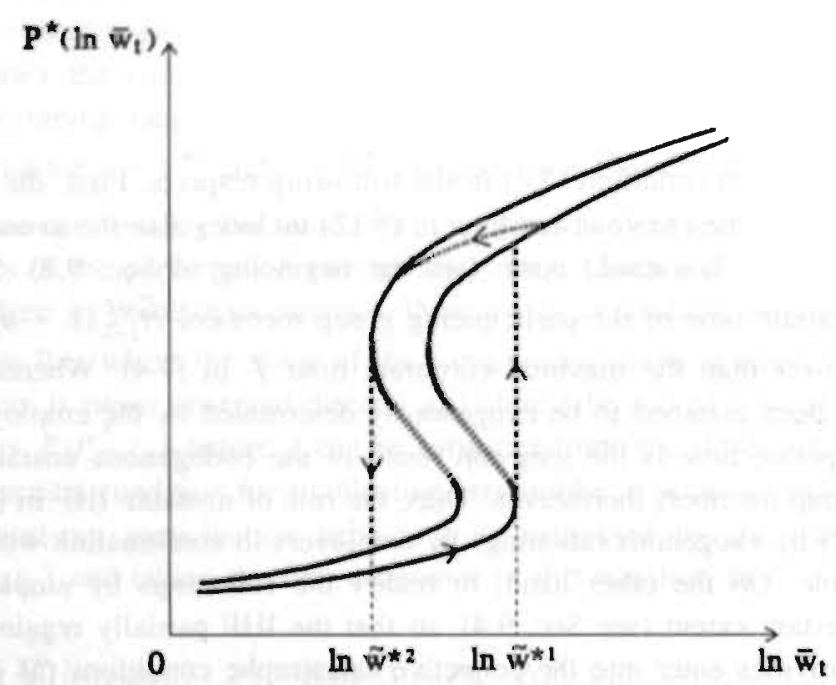

Figure 9.7 Long-run participation rate schedule for a social group of married women with a persistent hysteretic domain and two catastrophic domains.

This configuration implies (collective) hysteresis (just as Fig. 7.1 in Sec. 7.2, see Sec. 3.5). Consider, as a possible example, the case of American married women with schoolgoing children during and after World War II. During the war many social groups of these women may have been involved in a collective entry catastrophe as indicated by the entry curve in Figure 9.7. On the other hand, when just after the war the job opportunities, as indicated by $\ln \bar{w}_{1}$, got again worse, these social groups did not move back along the entry curve, but followed a persistent hysteresis path, as indicated by dots with an arrow in Figure 9.7, towards the exit curve and, when having more or less reached this exit curve, more or less moved along it afterwards. For some social groups the decline in $\operatorname{In} \bar{w}$, may have been so strong as to induce a collective exit catastrophe, but such social groups too continued to have a higher participation rate than they had at the same values of $\ln \bar{w}_{t}$ before the war, as a result of the hysteresis effect of IHF (in combination with the discrete participation variable). For the social groups who did not engage into a collective exit catastrophe, the difference in participation rate before and after the war was considerably higher, since for them the hysteresis effect was due not only to IHF as in the case of stable BPI in the previous section, but also to locally unstable BPI implying two levels of stable long-run participation rates at given $\ln \bar{w}_{t}$. When after about 1947 the job opportunities, as indicated by $\ln \bar{w}_{t}$, again improved, both kind of social groups are "predicted" to have followed a persistent hysteresis path towards the entry curve such as in Figure 9.5. More generally, the band between the entry and exit curve indicates a persistent domain where the long-run par- 
ticipation rate follows small hysteresis loops as in Figure 9.5. On the other hand, there are two domains outside this band where the long-run participation rate goes through collective catastrophes (see Sec. 7.2).

What happens with the long-run participation rate schedule in Figure 9.7, when it is aggregated to the level of a cohort or the whole subpopulation of married women with school-going children? In the collective catastrophe condition $(9.11)$ the density function $f$ will have a lower peak as the aggregation level is higher, but on the other hand the susceptibilities to the social consumption and household time environments $\bar{\gamma}_{X \bar{X}}$ and $\gamma_{L \bar{L}}$ will be higher by the additional BPI between social groups. Still, since the latter BPI is weak, the former effect of a lower peak of $f$ will dominate, and strongly diminish the probability of a collective catastrophe at the level of a cohort or the subpopulation. This implies that, whereas collective catastrophes may occur at the level of social groups, such collective catastrophes are likely to occur at entry wages $\hat{w}_{k}^{* 1}$ or exit wages $\bar{w}_{k}^{* 2}$ that differ among the social groups $k$. When the number $K$ of social groups within the cohort is very large, the relative contribution of each separate collective social group catastrophe to the development of the overall participation rate may be negligible (cf. Assumption IX in Sec. 5.2). Then, such collective catastrophes are fully "smoothed out" in the long-run participation rate schedule for the cohor or subpopulation (just as individual catastrophes are fully "smoothed out" in the long-run average labour supply schedule in Figure 4.11 in Section $4.8^{22}$ ). More specifically, if we assume that all members of the cohort or subpopulation are involved in collective social group catastrophes, the entry and exit curves can be derived to be SEL curves like the solid entry curve in Figure 9.5. On the basis of such aggregated curves collective catastrophes within social groups cannot be identified. More generally, the scarcity of disaggregated data on the level of social groups severely hinders the identification of collective catastrophes on that level. On the other hand, the collective hysteresis properties of Figure 9.7, as being the result of both IHF and BPI, survive the aggregation to a long-run participation rate schedule for a cohort or subpopulation of married women with schoolgoing children (as in Fig. 4.11 in Sec. 4.8). In addition, the small hysteresis loops in the band between the entry and exit curves are now caused not only by the IHF, but also by the unstable BPI within social groups (analogously to the unstable IHF within persons in Fig. 4.11).

To be able to compare the implications of such a schedule with those of Figure 9.5, we should further aggregate the former schedule to the level of a cohor or population of all married women. We should then also include social groups of women keeping their job or not when marrying or getting children. To such a social group an exit curve like that in Figure 9.7 can apply, but now such a curve should be given a different interpretation. It describes how, at a rise of $\ln \bar{w}_{1}$ beyond $\ln \bar{w}^{* 2}$, a much higher proportion of employed (married) women who get a child (or marry) in year 1 , keep their job, so that the participation rate of all married group members with small children converges to a much higher long-run value. Such a collective catastrophe has the complicating feature that only employed married women who get a child in year $t$ or later, will be involved in the collective

22 More generally, the effects of strong BPI within social groups and weak BPI between social groups are analogous to those of strong IHF within persons and weak BPI between persons, respectively. 
catastrophe, since other married group members with small children are likely to persist in either staying at home or keeping their job. This renders a demographic dynamics of flows in and out of the social group of married women with small children an essential element of the analysis and violates the assumption of a fixed social group which was made in the previous chapters of this book. Consequently, even with a constant density function of reservation wage-wage proportions as assumed in the present chapter, collective catastrophe conditions become more complex and stronger. Such conditions, however, are not elaborated here. On the other hand, the aggregation of the long-run participation rate schedules of all kinds of social groups of married women to the level of a cohort or population of all married women can be expected to yield SEL-like entry and exit curves that are more pronounced than the entry curve in Figure 9.5, as a result of strong or even unstable BPI within social groups and weak BPI between social groups. Moreover, the hysteresis loop of the participation rate of American married women in Figure 9.5 can now be explained not only by IHF, but also by unstable BPI within social groups.

Such results also hold when we take into account BPI of cohorts with respect to older cohorts. On the one hand, bandwagon effects (see Subsec. 5.1.2) in a social group with respect to a similar social group in an older cohort tend to delay a collective catastrophe, since an older cohort is usually less prone to change than a younger cohort (see also the next section). On the other hand, reverse bandwagon effects in a social group with respect to a similar social group in an older cohort may trigger a collective catastrophe. This interesting and important possibility will be discussed in the next section.

\subsection{Collective satiation}

At the end of Section 9.2 it was contended that the change in the long-run preferences of Dutch married women with respect to education, labour force participation and number of children after 1960 is likely to have been caused by certain "objective" opportunity factors as well as certain "subjective" preference factors. Of the latter factors social norms, collective habits and social attitudes were argued in Sections 9.7-9.10 to be endogenous in such a way that they either consolidate a status quo of a low or high participation rate or reinforce changes in the participation rate once these have set in. However, such endogenous processes of collective habit and norm belief formation and erosion do not explain why changes in the participation rate, and hence in the collective habits and norm beliefs, would set in. Such onsets of social changes may be explained by "objective" opportunity factors like birth control methods, education opportunities, reasonably paid part-time jobs and day care facilities becoming more broadly available for married women, but these factors too seem (partially) endogenous, since they tend to be promoted by the "emancipation" of married women. Moreover, the question may arise whether, in addition to more or less exogenous "objective" factors like the increase in the demand for female workers after World War II and the technical progress of birth control methods, certain "subjective" preference factors may also have played a role in the onset of "emancipatory" change in the Netherlands after 1960.

The answer to this question is affirmative; the onset of changes in the long-run preferences of Dutch married women with respect to education, labour force participation and 
number of children after 1960 may have partially been caused by a set of endogenous "subjective" preference factors, which we refer to as collective satiation (with respect to household time). This stands for a combination of individual long-run satiation, intergenerational long-run satiation, and reverse bandwagon effects with respect to the older generation and older (birth) cohorts. Individual long-run satiation with respect to household time has been described at the end of Section 3.5 as the phenomenon that for a housewife accumulation of satiation with her large amount of household time may eventually lower her entry wage under the prevailing market wage and induce her to enter the labour market. However, such an intrapersonal satiation effect may play a role only in the re-entries of women who have a long household time experience, i.e. for older women, whereas the participation changes are stronger for lower age groups.

In the onset of participation change in these lower age groups, intergenerational long-run satiation and reverse bandwagon effects may have been inportant. Intergenerational long-run satiation with respect to household time is the phenomenon that accumulation of satiation of a housewife with her large amount of household time may deter her daughter(s) to become also a housewife and induce her (them) to participate to the labour force. In addition, a daughter may find it attractive in itself to react against her mother and/or older sister(s) and to behave non-conformistic with respect to them by participating. This leads to reverse bandwagon effects in the generation of the daughters with respect to the generation of the mothers and/or in a younger cohort with respect to an older cohort (see Subsec. 5.1.2.a). The satiation as well as the reverse bandwagon effects can be incorporated into the reservation wage equation (9.1), and hence into the participation equations (9.2) and (9.4), by making the household time taste $\alpha_{L i}$ a negative function of state variables for the satiation and reverse bandwagon effects.

All these effects can start up changes in the participation rate and even trigger collective catastrophes within social groups. Then, however, the question arises why this may have happened in the Netherlands in the sixties and seventies, but not in earlier decades. Regarding the forties and fifties, World War II is likely to have played an important role. After the war there was a strong general feeling that the rebuilding of the country required a strict labour division between men rebuilding the country and women bearing and rearing children. In terms of the needs hierarchy of Maslow (1943, 1970), strong emphasis was laid on the satisfaction of needs of lower order like physical and social needs. ${ }^{23}$ This may have suppressed the working of long-run satiation with household time and may have motivated conformistic behaviour. On the other hand, when the rebuilding of the country was completed in the course of the fifties, the need for a strict labour division loosened, and long-run satiation with household time and non-conformistic tendencies began to get room to work. In terms of the gratification-activation principle of Maslow we could say that in the late fifties and sixties the gratification, or satiation of lower-order needs activated higher-order needs like individualistic needs for independence, non-conformism, power and self-fulfilment. ${ }^{24}$ This activation of higher-order needs may have been a strong force behind the onset of the

${ }^{23}$ This strong emphasis can be associated with the deprivation-domination principle of Maslow (see also Vendrik, 1988).

24 The gratification-activation principle is related to the distinction between necessary and luxury goods in economics. 
strong participation rise in the sixties and seventies. Moreover, it may have affected the social norm beliefs not only indirectly via the rise in participation, but also directly.

In other OECD countries similar social change processes may have taken place. For the U.S.A. Yankelovich (1981) finds in public opinion surveys a dramatic shift around 1970 from a self-sacrificing ethics to a self-fulfilment ethics. Surprisingly, however, this did not lead to a higher participation rate of American married women in 1980 relative to that in 1970 (Killingsworth and Heckman, 1986, Table 2.5; this may be due to the high unemployment rate in 1980). In 1990 the participation rate of American married women even declined, probably due to a cultural anti-movement in the form of groups like "Mothers home" and "Mothers first" (Breedeveld, 1991). This cultural anti-movement may be explained as the result of intergenerational and intrapersonal long-run satiation with corporate time of married women. More specifically, it may be explained in Maslovian terms as the result of an accumulating satiation of individualistic needs in combination with an accumulating deprivation of social needs and values with regard to raising and caring for children. One may then also think of an increasing awareness of the adverse long-term consequences of latchkey children. According to the deprivation-domination principle of Maslow such a situation is bound to lead to an increasing emphasis on social needs and values of raising and caring for children, and hence, in the American situation of limited day care, to a decline in the labour force participation of married women. For some social groups of married women the latter decline may take the form of a collective exit catastrophe or of a catastrophic fall in the proportion of group members who keep their job when getting children, or in the proportion of group members who re-enter the labour market when the children go to school. This, however, is merely a speculation.

In other OECD countries a similar cultural reaction to the "female participation movement" of the last decades can take place in this or the next decade(s). On the other hand, in a country like the Netherlands where the day care seems less inadequate than in the U.S.A., this cultural reaction may only curb the growth in the participation rate of married women (and curb the erosion of household nom beliefs and the growth in "emancipation" norm beliefs). The long-run development of the participation rate of Dutch married women since World War II into the coming decade may then be conceived in a stylized way as the result of a cultiural cycle superimposed on an upward trend in the "economically" demanded participation rate of married women (see Fig. 9.8). The latter participation rate is the participation rate of married women demanded by employers which is adjusted for the effects of collective habit formation and social norms on the demand side of the labour market. This theoretical concept of a latent "economic" labour demand needs further elaboration, but here I only introduce the idea and simply assume that this demanded participation rate increased exponentially since World War II except for a slump in the first half of the eighties. The cultural cycle is caused by the combination of collective habit (and norm belief) formation in a medium long run and collective satiation in a very long run (cf. the psychological long cycle in footnote 19 in Sec. 3.5). The collecrive habit formation can be associated with the Marxian conception that the economic situation in which a social class finds itself (e.g., participation or not) determines its social consciousness (including collective habits and norm beliefs). Therefore, and since collective satiation can be associated with the needs theory of Maslow. the cultural cycle may be referred to as the Marx-Maslow cycle (or, shorter, the M \& M cycle). 


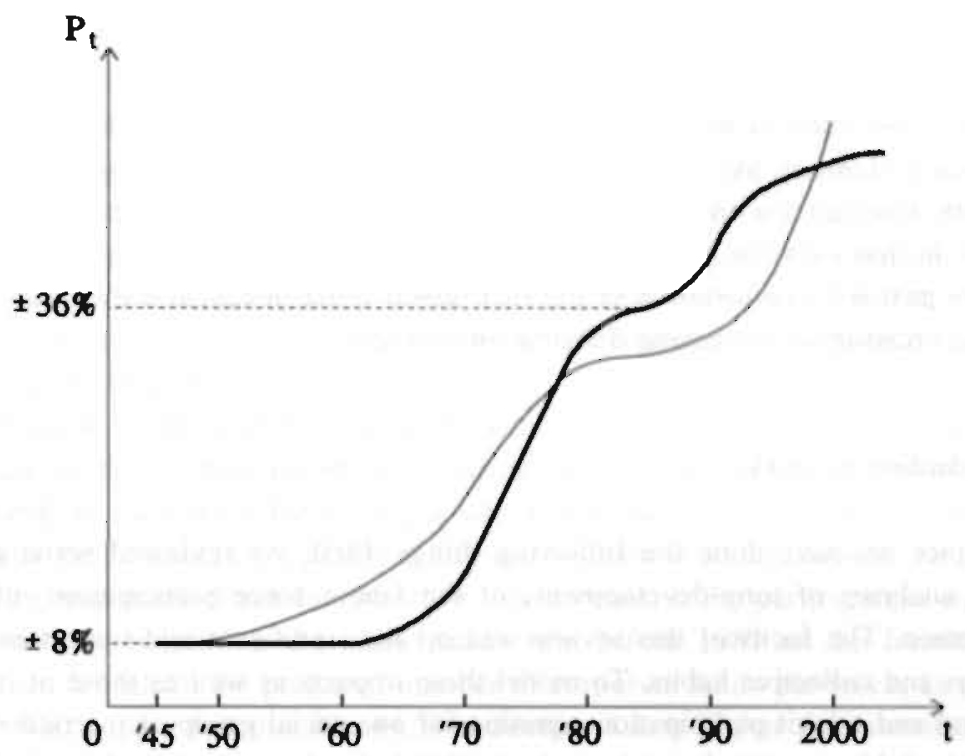

Figure 9.8 The long-run development of the participation rate of Dutch married women after World War II as a cultural cycle (bold) superimposed on an upward "economic" demand trend (thin).

Between 1945 and 1960 the Marx-Maslow cycle kept the participation rate at a stable low level below the demand trend as a result of the persistence effects of strong collective habits and household norm beliefs. This led to an increasing shortage of female labour supply, which in combination with collective satiation and other factors set the participation rate rising in the course of the sixties. ${ }^{25}$ This rise led to an erosion of collective household time habits and household norm beliefs and a growth of collective consumption and financial independence habits, which reinforced the participation increase, etc.. As a result of these reinforcing feedbacks the cultural cycle pushed the participation rate in the course of the seventies above the demand trend, causing a rise in the unemployment rate of married women. This rise curbed the growth in the participation rate in the first half of the eighties, but as the unemployment rate again declined in the second half of the eighties, the growth in the participation rate rallied. In the nineties collective satiation might again curb the growth in the participation rate, and hence again cause a shortage of female labour supply. This description is rather loose and should be refined.

Finally, we make two remarks. First, the more "progressive" social norm beliefs, collective habits and needs in more recent cohorts, which have been mentioned in Section 9.2, may partially be explained by intergenerational satiation and reverse bandwagon effects (in

${ }^{25}$ The increasing shortage of female labour supply can be deduced from the increasing recruilument of foreign workers between the mid-fifties and early seventies (see. Koot and Van der Wiel, 1982. Sec. 1.2, and De Neubourg. 1988, p. 159, footnote 8). 
addition to the endogenous factors of a higher level of education and a lower total number of children in more recent cohorts). Secondly, collective satiation may have played a role not only on the supply side of the labour market, but also on the demand side. The strong secular rise in the share of the service sector in total employment, which has been found to be a significant factor in the participation increase of married women by Mol et al. (1988), is sometimes associated with a working of the gratification-activation principle of Maslow (see above). In that view the subsequent growths of the agricultural sector and manufacturing sector in the past led to a satiation of physical lower-order needs, and this activated higherorder needs, creating an increasing demand for services.

\subsection{Concluding remarks}

In this chapter we have done the following things. First, we reviewed some econometric time-series analyses of long-developments of the labour force participation rate of Dutch married women. The focus of this review was on the significant and important impacts of social norms and collective habits. To model these impacts as well as those of other variables, a general and a logit participation equation for one social group of married women with school-going children were derived on the basis of the theoretical models of the previous chapters. These participation equations were aggregated to the level of a (birth) cohort or subpopulation of married women with school-going children, and the effects of BPI between social groups were added. The econometric evidence on the impacts of the norm belief and collective habit variables in these aggregated participation equations was discussed and the modelling of the persistence effects of IHF by Clark and Summers (1982) was criticized.

Next, we gave some historical evidence on the endogenous dynamics of social norms and collective habits in the participation of Dutch married women. Ensuing long-run elasticities of the participation rate with respect to the wage rate and other exogenous variables were shown to have a hump-shaped participation rate profile, which is essentially different from the participation rate profile of the corresponding short-run elasticities. On the basis of this theoretical result we criticized the empirical simulation finding of Hartog and Theeuwes (1985) of a strong and dominant impact of the own wage rate on the development of the participation rate of Dutch married women since World War II. As an improvement on the logistic shape of a simplified time profile of the participation rate implied by static labour supply models like that of Hartog and Theeuwes (1985), a super-exponential-logistic shape of this time profile was derived. According to the latter shape the remarkable persistence of the low participation rate of Dutch married women before 1960 is parrially explained by low values of long-run elasticities of exogenous variables, whereas the strong rise of the participation rate of these women after 1960 is partially explained by higher values of such elasticities. Moreover, the persistence of the participation rate of American married women just after World War II (Clark and Summers, 1982) can be explained as the result of hysteresis effects of IHF.

The possibility that the long-run elasticities of the participation rate of a social group become infinite as a result of unstable behavioural preference interdependence (BPI), was shown to imply collective entry and exit catastrophes. A condition for such catastrophes was derived and, just as in Section 7.2, we obtained a labour supply schedule with a persistent 
domain, where the long-run participation rate follows (small) hysteresis loops, and a catastrophic domain. Aggregating such schedules to the level of cohorts or (sub)populations was shown to preserve the hysteresis properties, but to "smooth out" the collective catastrophes. The hysteresis properties turned out to add unstable BPI within social groups to the explanation by IHF of the persistence of the participation rate of American married women just after World War II. Finally, we conceived the development of the participation rate of Dutch married women after World War II in a stylized way as the result of a cultural cycle superimposed on an upward labour demand trend. This cultural cycle was supposed to be caused by a combination of collective habit (and norm belief) formation in a medium long run and collective satiation in a very long run.

A complication in the Dutch data for female participation is that until 1987 the participation data of the Central Bureau of Statistics were based on an underlimit of twenty hours per week as a criterion for having a job. When this underlimit was lowered to zero hours as from 1987, suddenly 250.000 additional women working outside the home were observed! This important effect can be taken into account in the participation equations for married women by redefining the participation rate before 1987 as the fraction of those married women for which the preferred number of corporate hours per week, as given by an individual labour supply function like (4.5) in Section 4.1, is higher than twenty. This leads to additional terms and more complex IHF terms in the participation equation (9.4) in Section 9.4 , but the implied participation dynamics does not seem essentially different from the participation dynamics which has been analysed in this chapter.

\section{Appendix 9A Derivations of (9.5) and (9.7)}

Consider the long-run participation rate $P^{*}\left(\bar{w}_{1}, z_{1}\right)$ with $z$, denoting the vector of all exogenous variables (and time-dependent parameters) other than the average wage rate $w_{r}$. This participation rate is defined as the short-run participation rate $P\left(\bar{w}_{1}, \bar{w}_{t-1}, z_{t}, z_{t-1}, P_{t-1}\right)$ in long-run equilibrium for constant values of the exogenous variables, i.e. for $\bar{w}_{1-1}=\bar{w}_{1}$, $z_{t-1}=z_{1}$ and $P_{t-1}=P^{*}\left(\bar{w}_{1}, z_{1}\right)$. The long-run wage elasticity of the participation rate $E_{\bar{w}}^{P^{*}}$ can then be written as

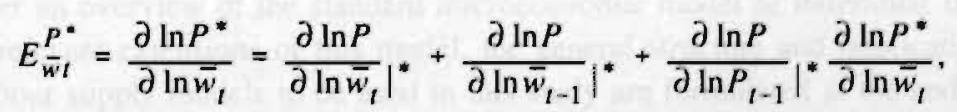

where " $\mid *$ "means that the "short-run derivatives" of $P\left(\bar{w}_{t}, \bar{w}_{t-1}, z_{t}, z_{t-1}, P_{t-1}\right)$ are evaluated at $\bar{w}_{t-1}=\bar{w}_{t}, z_{t-1}=z_{t}$ and $P_{t-1}=P^{*}\left(\bar{w}_{t}, z_{t}\right)$. Rearranging terms and dividing by $1-\partial \ln P /\left.\partial \ln P_{t-1}\right|^{*}=1-P_{P_{t-1}^{*}}^{*}$, we then obtain (9.5).

Differentiating the right-hand side of the participation equation (9.2) to $P_{1-1}$ at the longrun equilibrium $P_{i}^{*}$ yields 


$$
\begin{aligned}
P_{P_{t-1}}^{*} & =\left(\left.\frac{\partial F}{\partial \ln \bar{a}_{t} \mid} * \frac{\partial \ln \bar{a}_{t}}{\partial P_{t-1}}\right|^{*} * \frac{\partial F}{\partial \bar{b}_{t}} \cdot * \frac{\partial \ln \bar{b}_{t}}{\partial P_{t-1}} \mid *\right)= \\
& =f\left(\ln \bar{w}_{t}+\ln \bar{a}_{t}^{*}-\ln \bar{b}_{t}^{*}\right)\left(\frac{\frac{\partial \bar{a}_{t}}{\partial P_{t-1}} \mid}{\bar{a}_{t}^{*}}-\frac{\left.\frac{\partial \bar{b}_{t}}{\partial P_{t-1}}\right|^{*}}{\bar{b}_{t}^{*}}\right) .
\end{aligned}
$$

Then, dilterentiating the expressions for $\bar{a}_{1}$ and $\bar{b}$, given in $(94)$, we obtain $(9.7)$. 


\section{CHAPTER 10}

\section{Summary and Perspective}

In this final chapter we give a summary of the study in Section 10.1 and a short evaluation of its results in Section 10.2. Moreover, Section 10.3 puts forward some suggestions for further research.

\subsection{Summary}

This study applies three preference formation theories to labour supply, namely the theory of myopic individual habit formation and individual long-run satiation, the theory of behavioural preference interdependence, and the theory of social custom. These theories are incorporated into the microeconomic theory of labour supply and implications of this incorporation are derived. In doing so, the three preference formation theories are extended and integrated. Moreover, the focus is on the effects of non-linear forms of dynamics of the preference formations in combination with aggregation from the microeconomic to the macroeconomic behaviour. It is shown that in contrast to the existing models of stable individual habit formation (IHF) and stable behavioural preference interdependence (BPI) in labour supply, non-linear and locally unstable IHF and BPI can explain profound changes in labour supply preferences and behaviour such as when long-term unemployed become little motivated to get a new job or when former housewives develop a strong orientation towards paid work.

After an overview of the standard microeconomic model of individual labour supply and some relevant extensions of this model, the general structure and implications of the shortrun labour supply models to be used in this study are formulated at the end of Chapter 2 . In all following chapters these short-run models are complemented with specifications of the endogenous long-run dynamics of state variables in the context of the preference formation theories mentioned. The long-run labour supply effects of non-linear forms of these dynamics are analysed.

In particular, Chapter 3 and 4 analyse the implications of myopic individual habit formation (IHF). Chapter 3 argues that the existing models of stable IHF in labour supply cannot explain profound changes in preferences and behaviour as mentioned above. Therefore such changes are modelled as the outcomes of IHF which has an unstable long-run equilibrium between two stable long-run equilibria as in the addictive consumption model of 
Becker and Murphy. This leads to a polarization in behaviour and habit state: one of the two stable equilibria may describe the behaviour and habits of an "emancipated" woman with a job or a workaholic, whereas the other stable equilibrium may correspond to a housewife or a "voluntarily long-term unemployed" person. The time paths of exogenous variables like the wage rate or rationings in the past determine which of the two stable equilibria is actually approached. This is called individual hysteresis. At certain values of exogenous variables an unstable IHF process from one towards the other stable equilibrium may occur. Such a transition is referred to as an individual catastrophe in the sense of Thom (1975). Individual catastrophes in behaviour and habits may have occurred (and still occur) in the "emancipation" process of individual married women.

Chapter 3 analyses this non-linear dynamics of IHF in labour supply for IHF with respect to consumption, household lime and corporate time and for a general (additive) utility function. In particular, necessary and sufficient (in)stability conditions are derived and in the case of instability schedules of individual long-run labour supply as a function of the wage rate are obtained, displaying individual hysteresis and catastrophes. At the end of the chapter the unstable IHF model is complemented with individual long-run satiation, and cases of discontinuous short-run labour supply schedules are considered. Next, Chapter 4 derives necessary and sufficient conditions for individual hysteresis and catastrophes for an additive quadratic specification of the utility function. Moreover, this specification is used to trace the macroeconomic implications of the derived schedules of individual long-run labour supply by aggregating them to schedules of average long-run labour supply under various assumptions on the individual variation in utility function parameters, exogenous variables and habit states.

Chapter 5 analyses the non-linear dynamics of behavioural preference interdependence (BPI) with respect to average consumption, average household time and average corporate time for a general as well as an additive quadratic utility function. Analogously to the corresponding IHF cases, collective developments of a lack of corporate work motivation in subcultures of long-term unemployed persons and of a strong corporate work motivation in social groups of former housewives are modelled as the outcomes of (bandwagon) BPI which has an unstable long-run equilibrium between two stable long-run equilibria. In one of the latter stable equilibria a majority of the social group may not participate to the labour market. In the other stable equilibrium a majority of the same social group may have a job. Which of the two equilibria is actually approached at a certain moment depends on the past history of the social group. This is called collective hysteresis. Moreover, under certain conditions the social group may collectively enter or leave the labour market in an unstable BPI process from one towards the other equilibrium. This is referred to as a collective catastrophe. Such collective transitions within social groups may have played (and still play) a role in the strong rise of the labour force participation of married women in many OECD countries since World War II.

An attractive feature of the general and additive quadratic BPI models is that their structures are partly analogous to the structures of the corresponding IHF models of Chapters 3 and 4. This analogy is employed in deriving necessary and sufficient (in)stability conditions as well as schedules of average long-run labour supply as a function of the average wage rate, displaying collective hysteresis and catastrophes. On the other hand, the BPI models are shown to imply additional stabilizing effects of individual variation within 
the social group. For the additive quadratic BPI model necessary and sufficient conditions for collective catastrophes and hysteresis can then be derived. Finally, a comparison is made between the different effects on average labour supply of unstable BPI and unstable IHF.

Chapters 6 and 7 integrate the IHF models of Chapters 3 and 4 with the BPI models of Chapter 5 into models of collective habit formation (CHF). First, for two moderately general subcases of CHF with respect to household time only, simple necessary and sufficient (in)stability conditions are derived in Section 6.1. However, these conditions urn out not to be the relevant (in)stability conditions in the case of BPI and separately unstable IHF. For that case a modified relevant (in)stability condition is derived in Chapter 7. Accordingly, Section 6.2 and subsequent sections of Chapter 6 are restricted to the case of BPI and separately stable IHF, whereas Chapter 7 analyses the case of BPI and separately unstable IHF. In order to investigate the labour supply dynamics implied by the conditions in Section 6.1, Chapter 6 considers an additive quadratic specification of the CHF model (AQM). It is shown that, in the presence of exogenous individual variation, the IHF not only reinforces the destabilizing effect of BPI, but also counteracts it by amplifying the stabilizing individual variation. Again necessary and sufficient conditions for collective catastrophes and hysteresis are formulated, and BPI and separately stable IHF with respect to consumption and corporate time are added to the models.

The case of BPI and unstable IHF is analysed for the AQM specification in Chapter 7. Then collective catastrophes and hysteresis can arise as a result of a sort of "two-stage rocket". In the first stage the unstable IHF induces individual catastrophes of some group members from zero to maximal corporate hours or the reverse. The second stage fires when, due to a sufficiently strong BPI, these individual catastrophes trigger subsequent individual catastrophes of other group members to such an extent that collective catastrophes arise. Then, for instance, a rocketing number of group members starts to work a rocketing number of corporate hours. Considering unstable IHF and BPI with respect to household time only, two subcases of individual variation only in household time taste and IHF strength (Case A) and only in susceptibility to social influence (Case B) are distinguished. For both subcases (necessary and) sufficient conditions for collective catastrophes are derived as well as interesting long-run average labour supply schedules with two domains of catastrophic behaviour alongside a domain of persistent and hysteretic behaviour. Finally, unstable IHF and BPI with respect to consumption and corporate time are added to the models.

In Chapter 8 a variant of the theory of social custom (TSC) of Akerlof (1980) along the lines of Romer is applied to labour supply. Only the implications of social norms in female labour supply are elaborated, but the implications of social norms in male labour supply are similar. The application of the TSC to a so-called household norm is shown to be analogous in structure to a limiting case of Case B of the AQM of CHF in Chapter 7. This is with the proviso that the intergenerational process by which social norm beliefs change in the conception of Akerlof is replaced by an intrapersonal belief changing process. If we envision the concept of habit in a broader sense as general attitude or habitus and with the qualification just mentioned, the effects and dynamics of social norms in the TSC can then be interpreted as one of the possible "channels" through which unstable IHF and BPI may proceed. Moreover, it is argued that the destabilizing parameter restrictions on the AQM which correspond to the labour supply application of the TSC, are implausible. The chapter continues with a comparison with other applications of the TSC, which makes clear that the 
present application adds two pairs of essential elements to the overall picture, namely the pair of individual and collective catastrophes and the pair of individual and collective hysteresis. Finally, the female labour supply model is further extended by introducing the effects of a social norm of financial independence.

Chapter 9 investigates the empirical relevance of the theoretical models which have been set forth in the preceding chapters, by applying them to the developments of the labour force participation of Dutch and American married women since World War II. As an improvement on ad hoc participation equations of Romme (1987, 1990), general and logit participation equations for a social group, cohort and subpopulation of married women with school-going (or small) children are derived. These participation equations describe the impacts of CHF as well as social norm beliefs and have different specifications for entry and exit behaviour as a result of hysteretic persistence effects of IHF. The modeling of such effects by Clark and Summers (1982) is shown to be incorrect due to a severe aggregation error.

The endogenous dynamics of CHF and social norm beliefs tum out to imply a humpshaped participation rate profile of the long-run elasticities of the participation rate with respect to the wage rate and other exogenous variables. This is shown to imply serious doubts on the empirical simulation finding of Hartog and Theeuwes (1985) of a strong and dominant impact of the own wage rate on the development of the participation rate of Dutch married women since World War II. As an improvement on the logistic shape of a simplified time profile of the participation rate implied by static labour supply models like that of Hartog and Theeuwes, a so-called super-exponential-logistic shape of this time profile is derived. According to the latter shape the remarkable persistence of the low participation rate of Dutch married women before 1960 is partially explained by low values of long-run elasticities of exogenous variables, whereas the strong rise of the participation rate of these women after 1960 is partially explained by higher values of such elasticities. In close connection, the persistence of the participation rate of American married women just after World War II (Clark and Summers, 1982) is explained as the result of hysteresis effects of IHF. Such hysteresis effects are also shown to explain differences in as well as convergence of participation rates among countries.

The strong rises in the participation rate of Dutch married women after 1960 and of American married women after 1950 may have been caused by collective catastrophes within social groups. A necessary and sufficient condition for such a collective catastrophe in the participation rate is derived. Just as in Chapter 7, we obtain a labour supply schedule with two catastrophic domains alongside a persistent and hysteretic domain. Aggregating such schedules to the level of cohorts or (sub)populations is shown to preserve the hysteresis properties, but to "smooth out" the collective catastrophes. The hysteresis properties turn out to add unstable BPI within social groups to the explanation of the persistence of the participation rate of American married women just after World War II by IHF. Finally, the development of the participation rate of Dutch married women after World War II is conceived in a stylized way as the result of a cultural cycle superimposed on an upward labour demand trend. The cultural cycle is supposed to be caused by a combination of collective habit (and norm belief) formation in a medium long run and collective satiation in a very long run. Since the two mechanisms can be associated with conceptions of Marx and Maslow respectively, the cycle is referred to as the Marx-Maslow cycle. 


\subsection{Evaluation}

The approach which has been chosen in this study is theoretical and deductive. It can be regarded as an exploration of theoretical possibilities on the basis of non-linear dynamic extensions of the microeconomic theory of labour supply. In the view of the author, this exploration has been fruitful for the following reasons.

Especially Chapter 9 has made plausible that a number of the theoretical extensions analysed may have empirical relevance. In particular, these extensions offer an intuitively appealing explanation of the empirical pattern of a remarkably persistent low participation rate of Dutch married women before 1960 and a strongly growing participation rate of these women after 1960. Moreover, our explanatory model strongly suggests that the models underlying the few econometric studies on this long-run development (Hartog and Theeuwes, Mol et al., Romme) are severely misspecified. In addition, the modelling of hysteretic persistence effects of IHF on female participation by Clark and Summers is shown to be incorrect. Furthermore, Chapters 3 and 5 have argued that individual and collective developments of a lack of corporate work motivation of long-term unemployed persons cannot be explained by the existing stable IHF and BPI models (e.g., Kapteyn and Woittiez), but can be modelled as the outcomes of unstable IHF and BPI (including erosion of beliefs in work ethic norms).

However, this theoretical study is fruitful not only because it offers explanations of particular empirical phenomena. By modelling the impacts of IHF, BPI and social norms on labour supply within a general non-linear dynamic framework, the study leads to a thorough integration of the corresponding theories, in particular of the theory of social custom of Akerlof, into CHF theories. Moreover, by demonstrating that even extreme labour supply phenomena like profound preference changes can be covered by extensions of the microeconomic theory, the study shows the broad scope and strong flexibility of the oneutility maximizing model. Hopefully, this contributes to clarifying the deep controversy between neo-classical economists and their critics on the scope of microeconomic models.

More generally, the study aims to contribute to the integration of sociological and psychological insights into microeconomic models. Although the behavioural assumptions underlying the microeconomic models in this study may seem too crude to sociologists and psychologists, most of these assumptions may still be sufficiently accurate in a macro context (see Sec. 1.6). This issue should be further investigated by analysing the robustness of the models to alternative and possibly more plausible behavioural assumptions from sociology and psychology. However, I expect that this will not lead to essential differences in macro dynamics, but rather to refinements.

One of these refinements is suggested by Vendrik (1988), who generalizes the approach of Stigler and Becker (1977) to explaining preference formation in the context of labour supply and who uses it to derive a more plausible separability structure of utility functions than additivity (see Sec. 8.11). This generalization is organized in a general utility maximizing framework which is conceived as a hierarchy of means-ends relations and which consists of an "economic" and a psychological part. Such a hierarchy can be useful as an ordering and integrating framework for microeconomic and psychological (as well as sociological) motivation and satisfaction theories and demonstrates how psychological and 
sociological theories can be incorporated into a microeconomic framework without using a reductionistic economic terminology like that of the Chicago School.

This approach as well as that of the present study are similar in spirit to the work of economists like Akerlof, Frank and Granovetter and sociologists like Lindenberg (e.g., 1990) and Wippler (e.g., 1985, 1986). The great challenge is to integrate sociological and psychological insights into economic thinking while doing full justice to these insights. In the words of Baxter (1988, p. 260), "if economics is to become a social science in the fullest sense, it must build on the foundations of psychology and sociology, rather than rely on its own improvisations of these subjects. We might then hope for an economics with a truly human face." Moreover, a start can then be made towards reversing the overly strict division of labour in social science. The present study may be considered as a small step in that direction.

Notwithstanding this positive judgement of the study, it should be emphasized that the theoretical models developed remain to be tested empirically. This should be an important subjeet of further research, which may meet severe difficulties. Moreover, Akerlof's differential equation for the dynamics of social norms in female labour supply ought then to be modified into a more complex equation. This modification as well as other possible extensions and applications are discussed in the next section.

\subsection{Further research}

In the analysis of the long-run dynamics of female participation in Chapter 9 it has been assumed that the social norm beliefs develop according to the long-run model of Section 8.5 , i.e. in a way analogous to the dynamics of collective habits. However, as argued at the end of Section 9.7, this assumption is incorrect. Dutch survey data of the Social Cultural Planning Bureau $(1986,1992)$ and the Central Bureau of Statistics (see De Graaf, 1989) strongly suggest that, whereas collective habits are formed or wear off on the basis of the average participation behaviour in the past, changes in average social nom beliefs anticipate changes in the average participation behaviour. The SCP data imply an "own" dynamics of social norm beliefs, which led especially in the second half of the sixties to a very explosive swing in the social norm beliefs. On the other hand, this cultural dynamics does not seem fully autonomous, but seems to be affected by the participation behaviour of trendsetters. This again implies an endogenous dynamics of social norm beliefs, but now this dynamics is not described by Akerlof's differential equation ( 8.6 ) in Section 8.1, but by a more complex differential equation. Similarly to (8.6). this diffusion equation can be shown to imply collective and individual hysteresis and the possibility of collective catastrophes within social groups, the latter even with a higher probability.

Such an endogenous dynamics of social nom beliefs implies that the participation equations for cohorts of married women with school-going or small children should be estimated simultaneously with the diffusion equations for the social norm beliefs. However, such an estimation has not yet been carried out. If an implementation led to satisfactory estimates, it could be used to make simultaneous projections of future levels of participations and social norm beliefs of cohorts of married women. However, one should then take into account a demographic dynamics of flows in and out of subpopulations of married women as 
a result of life-cycle events like getting children or like children reaching the school-going age. Two important theoretical improvements on the participation projections of Romme as mentioned in Section 9.6 would then be that the developments of the social norm variables would be endogenously determined and that life-cycle events would be taken into account.

As another important theoretical improvement on Romme's study, our participation equations for cohorts of married women have been derived on the basis of an extension of the individual labour supply function (6.8) in Subsection 6.2.1. However, this individual labour supply function has implications not only for the participation of married women, but also for the average (preferred) number of corporate hours of these women. Ensuing kinds of dynamics of these average corporate hours have been analysed extensively in the Chapters 48. Similarly to the long-run elasticities of the participation rate. the long-run elasticities of the average corporate hours with respect to the wage rate and other exogenous variables can be expected to have a hump-shaped participation rate profile. However, in the context of subpopulations of married women, there are two additional complications that should be taken into account. First, Dutch data imply a certain degree of rationing of corporate hours in the sense that many women would like to work fewer corporate hours than they actually do (see, e.g., Allaart et al., 1989, 1991). Secondly, there is the complication of a demographic dynamics of flows in and out of subpopulations of married women as mentioned above. Both complications threaten to make a time-series analysis of the dynamics of average corporate hours of married women very difficult.

Both this analysis and the time-series analysis of the dynamics of female participation are based on an aggregation of a microeconomic labour supply function to, successively, the mesoeconomic level of social groups and the macroeconomic level of cohoris or subpopulations. These aggregations involve certain simplifying assumptions, which seem rather crude. For that and other reasons it seems worthwhile to complement the mesoeconomic and macroeconomic time series analyses with microeconomic cross-section and longitudinal analyses. Recently, an interesting microeconomic longitudinal study of the participation dynamics of an American cohort of mature married women has been conducted by Eckstein and Wolpin (1989). They present and estimate an intertemporal participation and fertility model in which, in addition to an effect of rational individual long-run satiation with respect to participation, the effect of corporate work experience on wages is explicitly taken into account. The latter effect, which has not been considered in the present study, is a result of the accumulation of human capital as an increasing function of corporate work experience and makes the wage rate into a state variable which is endogenous in the longer run. In estimations for the cohort of mature married women (between the ages of 39 and 59), this effect of experience on wages turns out to be significantly positive and to overwhelm the individual long-run satiation effect with respect to participation, so leading to persistence in the employment patterns of these women. Since Eckstein and Wolpin interpret the long-run satiation effect as a net effect of long-run satiation and individual habit persistence, their empirical results suggest that the mature American women studied were not kept at their job by individual (or collective) habit persistence as suggested by the theoretical participation model of Chapter 9, but rather by their increasing wages as a result of human capital accumulation and despite an increasing satiation stemming from their participation. This result seems unfavourable for our participation model, but the following objections car be: raised. 
Most importantly, Eckstein and Wolpin define and calculate work experience as a stock of the total number of years since age 18 in which the woman considered worked at least two weeks during the year. This implies a zero depreciation rate of the stock, which seems more appropriate for a modelling of satiation with participation in a very long run than for modelling individual habit persistence, that is likely to work in a shorter run. Individual habits with respect to participation seem more strongly influenced by work experience in recent years than in earlier years, implying a substantially positive depreciation rate. ${ }^{1}$ Thus, we can conclude that individual habit persistence is not properly modelled by Eckstein and Wolpin, whereas their finding of an individual long-run satiation effect in the very long run confirms our theoretical assumptions on such an effect at the end of Section 3.5 and in Section 9.11. On the other hand, the latter finding may be partially due to the neglect of a negative age effect of a diminishing physical condition on the marginal utility of participation. Moreover, the estimation results may change considerably when IHF and BPI with respect to participation as well as consumption are added to the model of Eckstein and Wolpin.

Nevertheless, their empirical results strongly suggest that the state dependence of the wage rate on work experience should be added to our participation model. Furthermore, in a longer run the wage rate is likely to be affected by market interactions of labour supply and demand. This implies a second form of state dependence of the wage rate on the average participation of other labour suppliers and works in the same direction as a reverse bandwagon effect. Research by Granovetter and Soong (1986) and Day (1986) on a related state dependence of prices in combination with BPI or IHF in consumption suggests that this state dependence of the wage rate and BPI or IHF in labour supply may be mutually destabilizing. However, empirical testing may then become even more difficult.

Another exogenous variable in the female labour supply models of this study which may be an endogenous state variable in a longer run, is the income of the husband $Y_{O}$. According to the individual utility-family budget constraint model of Leuthold (1968) the husband and wife will react to each other's labour supply decisions until a consistent and stable long-run equilibrium has been reached (see Subsec. 2.2.7). Ashworth and Ulph (1981) have extended this model with household time BPI between husband and wife (see also Kapteyn and Woittiez, 1990). As argued in Sections 7.8 and 8.11, these interactions between husband and wife may imply a fascinating dynamics of interacting collective habit and social norm formations and erosions in male and female labour supply. For example, a further increasing participation of Dutch married women in the coming decades may have a suppressing effect on the male supply of corporate hours and the corresponding collective habits and social noms. In combination with a further rising wage rate this might even trigger collective catastrophes within social groups in the now inelastic and persistent labour supply of men from five to, for instance, four days a week (in the backward-sloping part of their labour supply curve). In their tum, such declines in male labour supply will reinforce rises in female labour supply and corresponding changes in collective habits and social norms.

${ }^{1}$ Hotz et al. (1988, p. 351) estimate the rate of depreciation in the influence of past leisure on current iabour supply to range in value between 0.35 and 0.43 . 
On the other hand, the male supply of corporate hours may remain inelastic for quite some time because of the strong persistence effects of collective corporate time habits (including workaholism) and work ethic norms (see Secs. 8.2 and 8.11, and De Neubourg and Vendrik, 1992). This pattem of, on the one hand, strong persistence and, on the other hand, the possibility of strong and even catastrophic declines in male supply of corporate hours forms a kind of mirror image of the historical pattern of a persistently low participation of Dutch married women before 1960 and a strongly rising participation of these women after 1960. Accordingly, a modelling of the former pattern will resemble the participation model of Chapter 9.

Another interesting application of our theoretical models on male labour supply is related to the issue of hysteresis in long-term unemployment. The IHF and BPI models in Sections 3.2 and 5.3, respectively, explain how a temporary, but sufficiently long-lasting rationing of labour supply in the form of involuntary unemployment may lead to permanent "voluntary unemployment", i.e. non-participation, as a result of locally unstable IHF or BPI. Such processes involve a strong erosion of collective corporate time habits and work ethic norms and may occur in certain subcultures of the long-term unemployed (see also De Neubourg. 1991). They imply individual and collective hysteresis dependent on rationings by labour demand. Just like schedules of long-run individual and average labour supply displaying hysteresis as a function of the wage rate, schedules of long-run labour supply displaying hysteresis as a function of labour demand can be aggregated to the macro-level. Associating a lack of long-run labour supply with a kind of non-participation rate and a lack of labour demand with a kind of non-employment or labour slack ratio (see Sec. 9.4), we then obtain a macro-relation between these non-participation rate and non-employment ratio which is comparable with macro-relations between the natural unemployment rate and the actual unemployment rate, but which is genuinely hysteretic (see below). This macro-relation describes the case that, when the non-employment ratio rises from a relatively low level, the non-participation rate also (strongly) rises, whereas, on the other hand, when the nonemployment ratio again declines, the non-participation rate remains persistently at a relatively high level. This may be a good description of pertinent developments in many OECD countries in the seventies and eighties. In addition, our model predicts that, when as a result of the declining non-employment ratio the wage rate (as compared with the unemployment benefit) and/or other exogenous variables favouring paricipation rise and pass certain (high) levels. strong and even catastrophic declines in the non-participation rate may occur. Such a non-linear possibility does not seem to be recognized by the current linear models of hysteresis in unemployment and implies a more optimistic prospect than these models for the decades to come.

Finally, I would like to mention that the theoretical models which have been developed in this study in the context of labour supply. can also be applied in other fields. In the first place, they can easily be applied in microeconomic demand systems for various consumption categories by virtue of the integration of the microeconomic theories of consumption and labour supply (see Sec. 2.1). Furthermore, the theory of social custom of Akerlof has been applied to fair wage setting. trade union membership and collective strike action (see Sec. 8.7). In addition, collective habits and social norms seem to play important roles in the supply of work effort in organizations (think of shirking) and may form barriers to entry and exit in industrial as well as labour markets (sece. e.g. Van Witteloostuijn and Van Liẹ, 
1992). Hopefully, for such applications as well as others the present study can provide a firm theoretical basis or point of departure. 


\section{References}

Abbott, M. and O. Ashenfelter (1976), "Labour Supply, Commodity Demand and the Allocation of Time", Review of Economic Studies 43: 389-411.

Ajzen, I. and M. Fishbein (1977), "Attitude-Behavior Relations: A Theoretical Analysis and Review of Empirical Research", Psychological Bulletin 84: 888-918.

Akerlof, George A. (1980), "A Theory of Social Custom, of Which Unemployment May Be One Consequence", Quarterly Journal of Economics 94: 749-775.

Akerlof, George A. (1991), "Procrastination and Obedience", Richard T. Ely Lecture, AEA Papers and Proceedings 81, 2: 1-19.

Akerlof, George A. and W.T. Dickens (1982), "The Economic Consequences of Cognitive Dissonance", American Economic Review 72: 307-319.

Alessie, Rob and Arie Kapteyn (1991), "Habit Formation, Interdependent Preferences and Demographic Effects in the Almost Ideal Demand System", Economic Journal 101: 404-419.

Allaart, P.C., R. Kunnen and H.A. van Stiphout (1989), Trendrapport Arbeidsmarkt 1989, OSA-voorstudie V32, The Hague: SDU Grafische Bedrijven.

Allaart, P.C., R. Kunnen, W.C.M. Praat, H.A. van Stiphout en J.P. Vosse (1991), Trendrapport Aanbod van Arbeid 1991, OSA-rapport OSA12, The Hague: SDU Grafische Bedrijven.

Anderson, G.J. and R.W. Blundell (1982), "Estimation and Hypothesis Testing in Dynamic Singular Equation Systems", Econometrica 50, 6: 1559-1571.

Asch, S.E. (1951), Social Psychology, Englewoods Cliffs, N.J.: Prentice Hall.

Ashenfelter, Orley (1977), "Unemployment as Disequilibrium in a Model of Aggregate Labour Supply", Econometrica 48: 547-564.

Ashworth, J.S. and D.T. Ulph (1981), "Houschold Models", in: Brown, C.V. (ed.), Taxation and Labour Supply, London: Allen \& Unwin. pp. 117.133.

Atkinson. Anthony B. and Joseph E. Stiglitz (1980). Lectures on Public Economics, New York: McGraw-Hill.

Barzel, Yoram and Richard J. McDonald (1973), "Assets, Subsistence, and the Supply Curve of Labor". American Economic Review 63, 4: 621-633.

Baxter, J.L. (1988), Social and Psychological Foundations of Economic Analysis. London: Harvester-Wheatsheaf.

Becker, Gary S. (1965), "A Theory of the Allocation of Time", Economic Journal 75: 493-5!7.

Becker. Gary S. (1974). "A Theory of Social Interactions", Journal of Political Ecanomy 82, 6: $1063-1093$.

Becker, Gary S. (1976), The Economic Approach to Human Behavior, Chicago: University of Chicago Press. 
Becker, Gary S. (1981), A Treatise on the Family, Cambridge, MA: Harvard University Press.

Becker, Gary S. and Kevin M. Murphy (1988), "A Theory of Rational Addiction", Journal of Political Economy 96, 4: 675-700.

Benhabib, Jess and Richard H. Day (1981), "Rational Choice and Erratic Behaviour", Review of Economic Studies 48: 459-471.

Bentham, Jeremy (1789), Principles of Morals and Legislation, Oxford: Clarendon.

Blanchard, Olivier J. and Lawrence H. Summers (1987), "Hysteresis in Unemployment", European Economic Review 31: 288-295.

Booth, Alison L. (1985), "The Free Rider Problem and a Social Custom Model of Trade Union Membership", Quarterly Journal of Economics, 100: 253-261.

Bordley, Robert F. (1986), "Satiation and Habit Persistence (or the Dieter's Dilemma)", Journal of Economic Theory 38: 178-184.

Bourdieu, Pierre and Jean-Claude Passeron (1971), La Reproduction, Minuit.

Breedeveld, Marcella (1991), "Meer Moeders Thuis", Intermediair 33: 43.

Brown, M. and C.F. Chuang (1981), "Theoretical Constraints on a Household Bargaining Model", Working paper, Buffalo: State University of New York.

Bruyn-Hundt, M. and D. van der Linden (1989), De Invloed van Materiële Prikkels op het Arbeidsaanbod van Vrouwen, OSA-werkdocument W65, The Hague: SDU Drukkerij. Bürgenmeier, Beat (1991), "Time is Money or What? The Labour Market in a Socio-Economic Perspective", in: G. Antonides et al. (1991), The Consumption of Time and the Timing of Consumption: Toward a New Behavioral and Socio-Economics, Amsterdam: North-Holland.

CBS (1992), Enquête Beroepsbevolking 1991, Voorburg: CBS.

Chiappori, Pierre-André (1988), "Rational Household Labor Supply", Econometrica 56, 1: 63-89.

Clark, Kim B. and Lawrence H. Summers (1982), "Labour Force Participation: Timing and Persistence", Review of Economic Studies 49: 825-844.

Clason, C.E. (1981), "Vrouw en Arbeid" in: C.J. Weeda (ed.), Vrouw en Samenleving, Assen: Van Gorcum, pp. 39-54.

Cross, R. and A. Allen (1988), "On the History of Hysteresis", in: R. Cross (ed.), Unemployment, Hysteresis, and the Narural Rate Hypothesis, Oxford: Basil Blackwell, pp. 26. 38.

Day, Richard H. (1986), "A Note on Endogenous Preferences and Adaptive Economizing", in: Richard H. Day and Gunnar Eliasson (eds.). The Dynamics of Market Economies, Amsterdam: North-Holland, pp. 153-170,

Deaton, Angus and John Muel!bauer (1980), Economics and Consumer Behavior, Cambridge: Cambridge University Press.

De Graaf, A. (1989), "Moeder Hoeft Niet Meer Thuis te Blijven" ["Mothers No Longer Have to Stay Home"], Maandstatistiek van de Bevolking 89, 2: 12-14.

De Neubourg, Chris (1985), "The World According to Jantje van Leiden or the Culture of Unemployment: An Essay in Sociological Economics", Working Paper 85-003, Maastricht: Faculty of Economics, Limburg University, and published (1986) in Dutch in Tijdschrift voor Arbeidsvraagstukken 2, 1: 26-40. 
De Neubourg, Chris (1988), "Unemployment and Labour Slack: Theory and Evidence in Labour Market Accounting", Amsterdam: North-Holland.

De Neubourg, Chris (1991), "Job Libido and the Culture of Unemployment" , in: Richard Coughlin (ed.), Morality, Rationality and Efficiency: New Perspectives on Socioeconomics, Armonk, New York: M.E. Sharpe, pp. 61-78.

De Neubourg, Chris and Maarten C.M. Vendrik (1989), "Labour Supply Within a Complex Rationality Model", in: Tadideusz. Tyszka and Piotr Gasparski (eds.), Homo Oeconomicus: Presumptions \& Facts, Proc. 14th IAREP' annual colloquium, Warsaw: Dept. of Psychology, vol. 1, pp. 76-95, extended and forthcoming (1993) in: Journal of Economic Psychology.

Duesenberry, J.S. (1949), Income, Saving and the Theory of Consumer Behavior, Cambridge, MA.

Earl, Peter E., 1983. The Economic Imagination: Towards a Behavioural Analysis of Choice. Armonk, NY: M.E. Sharpe.

Eckstein, Zvi and Kenneth 1. Wolpin (1989), "Dynamic Labous Force Participation of Married Women and Endogenous Work Experience", Review of Economic Studies 56: 375-390.

Ehrenberg, Ronald G. and Roben S. Smith (1982), Modern Labor Economics: Theary amd Public Policy, Glenview, Illinois: Scott, Foresman and Co.

Elster, Jon (1989), "Social Norms and Economic Theory", Journal of Economic Perspectives 3, 4: 99-117.

Etzioni, Amitai (1988), The Moral Dimension: Toward a New Economics, New York: The. Free Press.

Festinger, L. (1954), "A Theory of Social Comparison", Human Relarions 7: 117-140.

Festinger, L. (1957), A Theory of Cognitive Dissonance, Evanston, IL: Row, Peterson and Co.

Frank, Robert H. (1984), "Interdependent Preferences and the Competitive Wage Structure", Rand Journal of Economics 15, 4: 510-520.

Frank, Robert H. (1985), Choosing the Right Pond, New York: Oxford University Press.

Frank, Robert H. (1989), "Frames of Reference and the Quality of Life", AEA Papers and Proceedings 79, 2: 80-85.

Franz, Wolfgang (1990), "Hysteresis in Economic Relationships: An Overview", Empirical Economics 15, 2: 109-125.

Furnham, Adrian (1990), The Protestant Work Ethic: The Psychology of Work-Related Beliefs and Behaviours, London: Routledge.

Gaerner, Wulf (1974), "A Dynamic Model of Interdependent Consumer Behavior", Zeitschrift für Nationalökonomie 34: 327-344.

Galbraith, John Kenneth (1958), The Affluent Society, Cambridge.

George, Donald (1981), "Equilibrium and Catastrophes in Economics", Scotrish Journal of Political Economy 28, 1: 43-61.

Georgescu-Roegen, N. (1971), The Entropy Law and the Economic Process, Cambridge, MA: Harvard University Press.

Goedhart, Theo and Ari Kapteyn (1978). "A Welfare Function of Income and Time", Statistical Studies 22, The Hague: Netherlands Central Bureau of Statistics, Staatsuitgeverij. 
Goodwin, P.B. (1977), "Habit and Hysteresis in Mode Choice", Urban Studies 14, 1: 95-98. Gossen, H.H. (1854, 1927), Entwicklung der Gesetze des Menschlichen Verkehrs und der daraus Fliessenden Regeln für Menschliches Handeln, Berlin: Prager.

Granovetter, Mark and Roland Soong (1986), "Threshold Models of Interpersonal Effects in Consumer Demand", Journal of Economic Behavior and Organization 7: 83-99.

Hall, Robert E. and John B. Taylor (1991), Macroeconomics, 3rd ed., New York: Norton \&. Co..

Hamermesh, Daniel S. (1974), "Enjoyable Work and Labor Supply: A Pedagogical Note", Unpublished manuscript, Dept. of Economics, Michigan State University.

Hanoch, G., and M. Honig (1978), "The Labor Supply Curve under Income Maintenance Programs", Journal of Public Economics 9: 1-16.

Hansen, Bent (1970), "Excess Demand, Unemployment, Vacancies, and Wages", Quarterly Journal of Economics 84: 1-23.

Hartog, Joop and Jules Theeuwes (1985), "The Emergence of the Working Wife in Holland", Journal of Labor Economics 3: S235-\$255.

Hausman, J.A. (1980), "The Effect of Wages, Taxes and Fixed Costs on Women's Labor Force Participation", Journal of Public Economics 14: 161-194.

Heiner, Ronald A. (1983), "The Origin of Predictable Behavior", American Economic Review 73, 4: 560-595.

Henderson, James M. and Richard E. Quandt (1958), Microeconomic Theory: A Mathematical Approach, New York: McGraw-Hill.

Hicks, J.R. (1936), Value and Capital, Oxford: Oxford University Press.

Hirsch, Fred (1976), Social Limits to Growth, Cambridge, MA: Harvard University Press.

Homan, Eitel, Aldi Hagenaars and Bernard Van Praag (1988), "The Allocation of Time and Goods: A Long-Run and Short-Run Analysis", Report 8806/A, Rotterdam: Econometric Institute, Erasmus University.

Hotz, V. Joseph, Finn E. Kydland, and Guilherme L. Sedlacek (1988), "Intertemporal Preferences and Labor Supply", Economerrica 56, 2: 335-360.

Houthakker, H.S. and Lester D. Taylor (1970). Consumer Demand in the United States: Analyses and Projections, 2nd ed., Cambridge, MA: Harvard University Press.

Hyman, H.H. (1942), "The Psychology of Status", Archives of Psychology 269: 5-38, 80-86. Iannacone, Laurence R. (1986), "Addiction and Satiation", Economics Letters 21: 95-99.

Iannacone, Laurence R. (1989), "Bandwagons and the Threat of Chaos, Interpersonal Effects. Revisited". Journal of Economic Behavior and Organization 11: 431-442.

Jahoda, Marie (1972), "Conformity and Independence: A Psychological Analysis", in: Joel Cohen (ed.), Behavioral Science Foundations of Consumer Behavior, pp. 339-554. New York: Free Press.

Johnson, T.R. and J.H. Pencavel (1984), "Dynamic Hours of Work Functions for Husbands, Wives, and Single Females". Econometrica 52, 2: 363-389.

Joshi, Heather and Elizabeth Overton (1984). "The Female Labour Force in Britain 19711991: Demographically Determined Components of Participation Rates over Ages 2059". Working paper, London: Center for Population Studies, University of London.

Kapteyn, Arie (1977), A Theory of Preference Formation, Doctoral thesis, Leiden, the Netherlands: University of Leiden.

Kapteyn, Arie (1985), "Utility and Economics", De Economist 133, 1: 1-20. 
Kapteyn, Arie, Sara van de Geer, Huib van de Stadt and Tom Wansbeek (1989), "Interdependent Preferences: An Econometric Analysis", CentER Discussion Paper no. 8954, Tilburg, the Netherlands: Center for Economic Research, Tilburg University. Kapteyn, Arie, Bernard M.S. van Praag and F.G. van Herwaarden (1978), "Individual Welfare Functions and Social Reference Spaces", Economics Letters 1: 173-178.

Kapteyn, Arie and Tom Wansbeek (1985), "The Individual Welfare Function: A Review", Journal of Economic Psychology 6: 333-363.

Kapteyn, Arie and Isolde Woittiez (1987, 1990), "Preference Interdependence and Habit Formation in Family Labor Supply", Research Memorandum FEW 262, Tilburg, the Netherlands: Dept. of Economics, Tilburg University, and in: Jean-Pierre Florens et al. (eds.), Microeconometrics: Surveys and Applications, Oxford: Basil Blackwell.

Kapteyn, Arie, Isolde Woittiez and Peter ten Hacken (1989), Household Labor Supply in the Netherlands in the Eighties and Nineties, OSA-werkdocument W61. The Hague: Staatsdrukkerij.

Kelman, H.C. (1961), "Processes of Opinion Change", Public Opinion Quarterly 25: 57-78. Killingsworth, Mark R. (1983), Labor Supply, Cambridge: Cambridge University Press.

Killingsworth, Mark R. and James J. Heckman (1986), "Female Labor: A Survey", in: Orley Ashenfelter and Richand Layard (eds.), Handbook of Labor Economics, Amsterdam: North-Holland, Vol. I, pp. 103-204.

Kok, Lucy and Chris de Neubourg (1986), Projecting Labour Supply: Theory, Methods and Research. An International Comparison, OSA-werkdocument W20, The Hague: Staatsdrukkerij.

Kooreman, Peter (1986), Essays on Microeconometric Analysis of Household Behavior, Doctoral thesis, Tilburg, the Netherlands: Tilburg University.

Kooreman, Peter (1988), "Household Labor Force Participation as a Cooperative Game: An Empirical Model", Research Memorandum FEW 323, Tilburg, the Netherlands: Dept. of Economics, Tilburg University.

Kooreman, Peter and Ari Kapteyn (1989), "On the Empirical Implementation of Some Game Theoretic Models of Household Labor Supply", Discussion Paper no. 8956, Tilburg, the Netherlands: Center for Economic Research, Tilburg University.

Koot, Willem and Arie van der Wiel (1982), Op Weg naar Werk Ver van Huis, Muiderberg: Coutinho.

Kotz, S. and N.L. Johnson (1985, eds.), Encyclopedia of Statistical Sciences, Vol. 5, New York: John Wiley \& Sons.

Krelle, W. (1968), Prüferenz- und Entscheidungstheorie, Tübingen: J.C.B. Mohr.

Leibenstein, Harvey (1950), "Bandwagon, Snob, and Veblen Effects in the Theory of Consumer Demand", Quarterly Journal of Economics, 64: 183-207, and in: Harvey Leibenstein (1970). Beyond Economic Man: A New Foundation for Microeconomics, Chapter 4, Cambridge, MA: Harvard University Press.

Leijonhufvud, Axel (1973). "Effective Demand Failures", Swedish Journal of Economics, 27-48.

Leontief, W. (1936), "Composite Commodities and the Problem of Index Numbers", Econometrica 15: 361-373.

Leuthold, J.H. (1968), "An Empirical Study of Formula Income Transfers and the Work Decision of the Poor, Journal of Human Resources 3: 312-323. 
Lindenberg, Siegwart (1990), "Homo Socio-Oeconomicus: The Emergence of a General Model of Man in the Social Sciences", Journal of Institutional and Theoretical Economics 146, 4: 727-748.

Lucas, Robert E.B. (1977), "Hedonic Wage Equations and Psychic Wages in the Returns to Schooling", American Economic Review 64: 549-558.

Lutz, Mark A. and Kenneth Lux (1988), Humanistic Economics: The New Challenge, New York: Bootstrap Press.

Maks, Hans (1981), Wetenschapsfilosofie en Economische Theorie, Lecture notes, Groningen, the Netherlands: Faculty of Economics, University of Groningen.

Manser, M. and M. Brown (1980), "Marriage and Household Decision-Making: A Bargaining Analysis", International Economic Review 21: 31-44.

Marshall, Alfred (1920), Principles of Economics, 8th ed., London: Macmillan.

Marx, Kari (1867), Das Kapital: Kritik der Politischen Ökonomie, 1st ed., Hamburg: Meissner.

Maslow, Abraham H. (1943), "A Theory of Human Motivation", Psychological Review 50: 370-396.

Maslow, Abraham H. (1970), Motivation and Personality, 2nd ed., New York: Harper and Row,

McCracken, Paul, et al. (1977), Towards Full Employment and Price Stability, Paris: OECD. Miller, Glenda (1988), "A Needs-Based Demand Theory", in: Piet Van den Abeele (ed.), Psychology in Micro \& Macro Economics, Proc. 13th annual IAREP colloquium, Vol. II, Leuven: Catholic University.

Moffitt, Robert (1983), "An Economic Model of Welfare Stigma", American Economic Review 73: 1023-1035.

Mol, P.W., J.C. van Ours and J.J.M. Theeuwes (1988), Honderd Jaar Gehuwde Vrouwen op de Arbeidsmarkt, OSA-werkdocument W 48, The Hague: Staatsdrukkerij.

Mroz, T.A. (1987), "The Sensitivity of an Empirical Model of Married Women's Hours of Work to Economic and Statistical Assumptions", Econometrica 55: 765-799.

Muellbauer, John (1988), "Habits, Rationality and Myopia in the Life Cycle Consumption Function", Annales dEconomie et de Statistique 9: 47-70.

Naylor, Robin (1989), "Strikes, Free Riders, and Social Customs", Quarterly Journal of Economics 104: 771-785.

Nelson, Philip (1970), "Information and Consumer Behavior". Journal of Political Economy 78: 311-329.

Newcomb. T.M. (1943), Personality and Social Change, New York: Dryden Press.

Ofshe, R. (1972), "Reference Conflict and Behavior", in: J. Berger, M. Zelditch and B. Anderson (eds.). Sociological Theories in Progress, Boston: Houghton Mifflin.

Park, C.W. and V.P. Lessig (1977). "Students and Housewives: Differences in Susceptibility to Reference Group Influence", Journal of Consumer Research 4: 102-109.

Parsons, D.O. (1977), "Health, Family Structure and Labor Supply", American Economic Review 67: 703-712.

Pashardes, Panos (1986), "Myopic and Forward Looking Behavior in a Dynamic Demand System", International Economic Review 27, 2: 387-397. 
Pencavel, John (1986), "Labor Supply of Men: A Survey", in: Orley Ashenfelter and Richard Layard (eds.), Handbook of Labor Economics, Amsterdam: North-Holland, Vol. I, Ch. 1.

Phlips, Louis (1974), Applied Consumption Analysis, Advanced Textbooks in Economics 5. Amsterdam: North-Holland.

Phlips, Louis (1978), "The Demand for Leisure and Money", Econometrica 46, 5: 10251043.

Pollak, Robert A. (1970), "Habit Formation and Dynamic Demand Functions", Jolirnal of Political Economy 78, 4: 745-763.

Pollak, Robert (1976a), "Interdependent Preferences", American Economic Review 66: 309-320.

Pollak, Robert (1976b), "Habit Formation and Long-Run Utility Functions", Journal of Economic Theory 13: 272-297.

Pollak, Robert A. (1977), "Price Dependent Preferences", American Economic Review 67: 64-75.

Pollak, Robert A. (1978), "Endogenous Tastes in Demand and Welfare Analysis", Proceedings of American Economic Association 68, 2: 374-377.

Renaud, P.S.A. and J.J. Siegers, "Income and Substitution Effects in Family Labour Supply", De Economist 3: 350-366.

Romer, David (1984), "The Theory of Social Custom: A Modification and Some Extensions", Quarterly Journal of Economics, Nov.: 717-727.

Romme, A. Georges L. (1986), "Endogenisering van het Arbeidsaanbod in het Kader van het CCSO-Jaarmodel", CCSO-Reeks no. 2, Maastricht: Faculty of Economics, Limburg University.

Romme, A. Georges L. (1987), "Het Macro-Economisch Arbeidsaanbod van Vrouwen: Een Verklaring voor de Periode 1971-85", Maandschrift Economie 51: 53-66.

Romme, A. Georges L. (1990), "Projecting Female Labor Supply: The Relevance of Social Norm Change", Journal of Economic Psychology 11: 85-99.

Runciman, W.G. (1966), Relative Deprivation and Social Justice, Henley: Routledge and Kegan Paul.

Samuelson, Paul A. (1956), "Social Indifference Curves", Quarterly Journal of Economics 70: 1-22.

Schelling, Thomas C. (1978), Micromotives and Macrobehavior, New York: Norton.

Schippers, Joop J. and Jacques J. Siegers (1991), "Labour Market Inequality between Men and Women: Allocational Differences versus Wage Differences", De Economist 139, 3: 401-427.

Schmalensee, Richard (1972), The Economics of Advertising, Amsterdam: North Holland.

Schokkaert, Erik (1982a), A General Framework for the Analysis of Preference Variation, European Economic Review 19: 159-179.

Schokkaert, Erik (1982b), Modelling Consumer Preference Formation, Doctoral thesis, Leuven: Catholic University.

Scitovsky, Tibor (1952), Welfare and Competition, London: Allen \& Unwin.

Scitovsky, Tibor (1977), The Joyless Economy: An Inquiry Into Human Satisfaction and Consumer Dissatisfaction, Oxford: Oxford University Press.

Sen, Amartya (1983), "Poor, Relatively Speaking", Oxford Economics Papers 35: 153-167. 
Sen, Amartya (1987), The Standard of Living, Cambridge: Cambridge University Press.

Sherif, M. (1953), "The Concept of Reference Groups in Human Relations", in: M. Sherif and M.O. Wilson (eds.), Group Relations at the Crossroads, New York: Harper.

Siebert, Jacques (1985), "Developments in the Economics of Human Capital", in D. Carline et al., Labour Economics.

Siegers, Jacques (1981), "Ongelijkheid tussen Mannen en Vrouwen op de Arbeidsmarkt", Sociaal Maandblad Arbeid, no. 7/8: 553-565.

Siegers, Jacques (1983), "An Economic-Demographic Ten Equation Model", De Economist 3: $400-443$.

Siegers, Jacques J. (1985), Arbeidsaanbod en Kindertal: een Micro-Economische Analyse, Doctoral thesis, Groningen, the Netherlands: University of Groningen.

Siegers, Jacques J. (1986), "Classificerend Beleid en Arbeid: Het Onderscheid naar Sekse", in: J.W. de Beus and J.A.A. van Doorn (eds.), De Geconstrueerde Samenleving, pp. 85-105, Amsterdam: Boom Meppel.

Smith, Adam (1759), The Theory of Moral Sentiments.

Social Cultural Planning Bureau (1986), Sociaal en Cultureel Rapport /Social and Cultural Report/, The Hague: Government Publishing Office.

Social Cultural Planning Bureau (1992), Sociaal en Cultureel Rapport /Social and Cultural Reportl, The Hague: Government Publishing Office.

Stigler, G.J., and G.S. Becker (1977), "De Gustibus Non Est Disputandum", American Economic Review 67, 2: 76-90.

Stone, Richard (1964), "British Economic Balances in 1970: A Trial Run on Rocket", in: P.E. Hart, G. Mills and J.K. Whitaker (eds.), Econometric Analysis for National Economic Planning, London: Butterworths.

Taylor, Lester D. (1974), "On the Dynamics of Dynamic Demand Models", Recherches Economiques de Louvain 40 : 21-31.

Taylor, Lester D. and Daniel Weiserbs (1972), "On the Estimation of Dynamic Demand Functions", Review of Economics and Statistics 54: 459-465.

Tazelaar, F. and R. Wippler (1981), "Die Theorie Mentaler Inkongruenzen und Ihre Anwendung in der Empirische Sozialforschung", Mimeo, Utrecht: University of Utrecht. Thierry, Hk., and A.M. Koopman-Iwema (1984), "Motivation and Satisfaction", in:

P.J.D. Drenth, Hk. Thierry, P.J. Willems and C.J. de Wolff (eds.), Handbook of Work and Organizational Psychology, pp. 270-313, John Wiley \& Sons.

Thom, René (1975), Structural Stability and Morphogenesis, New York: Benjamin.

Tomes, Nigel (1983), "Income Distribution, Happiness and Satisfaction: A Direct Test of the Interdependent Preferences Model". Working paper, Canada: Department of Economics, University of Western Ontario.

Tummers, Martijn P. and Isolde Woittiez (1988), "A Simultaneous Wage and Labour Supply Model with Hours Restrictions", Research Memorandum FEW 351, Tilburg, the Netherlands: Dept. of Economics, Tilburg University.

Van de Stadt. Huib (1981). "The Evaluation of Income and Leisure: An Analysis of Labour Supply Based on Individual Preferences". Report 81.13. Leiden, the Netherlands: Center for Research in Public Economics, University of Leiden. 
Van de Stadt, Huib, Arie Kapteyn and Sara A, van de Geer (1985), "The Relativity of Utility: Evidence from Panel Data", Review of Economics and Statistics 67, 2: 179 . 187.

Van Heek, F. (1954), Het Geboorteniveau van de Nederlandse Rooms-Katholieken, Publicaties van het Sociologisch Instituut der Rijksuniversiteit Leiden, III, Leiden: Stenfert Kroese.

Van Praag, Bernard M.S. (1968), Individual Welfare Functions and Consumer Behavior: A Theory of Rational Irrationality, Doctoral thesis, Amsterdam: North Holland.

Van Praag, Bernard M.S. (1971), "The Welfare Function of Income in Belgium: An Empirical Investigation", European Economic Review 2: 337-369.

Van Praag, Bernard M.S., Arie Kapteyn and F.G. van Herwaarden, "The Definition and Measurement of Social Reference Spaces", The Netherlands Journal of Sociology 15: 13-25.

Van Soest, Arthur, Isolde Woittiez, and Arie Kapteyn (1989), "Labour Supply, Income Taxes and Hours Restrictions", CentER Discussion Paper 8903, Tilburg, the Netherlands: Center for Economic Research, Tilburg University.

Van Witteloostuijn, Arjen and Arie van Lier (1992), "Organizational Inertia, Strategic Competition and Permanent Failure", Working paper, Maastricht: Faculty of Economics and Business Administration, University of Limburg.

Varian, Hal R. (1979), "Catastrophe theory and the Business Cycle", Economic Inquiry 17: 14-28.

Varian, Hal R. (1984), Microeconomic Analysis, 2nd ed., London: Norton and Co..

Varian, Hal R. (1987), Intermediate Microeconomics: A Modern Approach, London: Norton and $\mathrm{Co}$..

Veblen, Thorstein $(1899,1934)$, The Theory of the Leisure Class, Modern Library, New York: Random House.

Vendrik, Maarten C.M. (1979a), "A Classification of Phase Diagrams by means of "Elementary" Catastrophe Theory (ECT) I", Physica 99A: 103-144.

Vendrik, Maarten C.M. (1979b), "A Classification of Phase Diagrams by Means of "Elementary" Catastrophe Theory (ECT) II", Physica 99B: 513-544.

Vendrik, Maarten C.M. (1985), "Dynamic Models of Costs of Adjustment and Interrelated Factor Demands, with an Empirical Application to the Dutch Business Sector", Discussion Paper 8516/G, Rotterdam: Institute for Economic Research, Erasmus University.

Vendrik, Maarten C. M. (1986), "Adjustment and Anticipation: A Dynamic Model of Adjustment Costs and Interrelated Factor Demands with an Empirical Application to U.S. Manufacturing", Working Paper 86-008, Maastricht: Faculty of Economics and Business Administration, University of Limburg.

Vendrik, Maarten C. M. (1988), "Preference Drifts in Labour Supply: Towards an Integration of Economic and Psychological Motivation Theories", in: Piet van den Abeele (ed.), Psychology in Micro \& Macroeconomics, Proc. 13th IAREP annual colloquium, Vol. III, Leuven: Catholic University.

Weiserbs, Daniel (1974), "More about Dynamic Demand Systems", Recherches Economiques de Louvain 40: 33-43. 
Winder, Cornelius C.A. (1988), Modelling Intertemporal Consumer Behaviour: Theoretical Results and Empirical Evidence, Doctoral thesis, Amsterdam: Free University Press.

Winston, Gordon C. (1980), "Addiction and Backsliding: A Theory of Compulsive Consumption", Journal of Economic Behavior and Drganization, 1: 295-324.

Wippler, R. (1985), "Explanatory Sociology: The Development of a Theoretically Oriented Research Programme", The Netherlands Journal of Sociology 21: 63-74.

Wippler, R. and S. Lindenberg (1986), "Collective Phenomena and Rational Choice", in: J. Alexander et al. (eds.), Micro and Macro Levels in Sociological Theory, Berkeley: University of California Press.

Woittiez, Isolde and Arie Kapieyn (1987), "A Model of Job Choice, Labour Supply and Wages", Research Memorandum FEW 307, Tilburg, the Netherlands: Dept. of Economics, Tilburg University.

Wolfson, Dick (1988), Publieke Sector en Economische Orde, Groningen: Wolters Noordhoff.

Yankelovich, Daniel (1981), New Rules: Searching for Self-Fulfilment in a World Turned Upside Down.

Zabalza, A., C.A. Pissarides and M. Barton (1980), "Social Security and the Choice between Full-Time Work, Part-Time Work and Retirement", Journal of Public Economics 14: 245-276.

Zeeman, Christopher (1977), Catasirophe Theory, New York: Addison-Wesley. 


\section{Nederlandse Samenvatting Summary in Dutch}

Een opmerkelijk verschijnsel in de economische wetenschap is dat in veel van haar modellen nog steeds uitgegaan wordt van de veronderstelling dat de voorkeuren en behoeften van mensen niet veranderen in de loop der tijd. Dit ondanks het feit dat er steeds meer aanwijzingen van het tegendeel zijn. Een treffend voorbeeld op het gebied van arbeidsaanbod zijn de veranderingen in houding van emanciperende getrouwde vrouwen ten aanzien van het al of niet hebben van een baan. Sinds de jaren vijftig en zestig is de arbeidsmarktparticipatie van vrouwen in veel OESO-landen sterk toegenomen. Traditioneel hebben economen deze ontwikkelingen proberen te verklaren als het resultaat van veranderingen in financięle variabelen zoals het voor vrouwen geldende uurloon, het inkomen van de echtgenoot en het belastingstelsell, en van veranderingen in demografische en sociaal-economische variabelen zoals het aantal kinderen en het opleidingsnivo van vrouwen. Hoewel zulke variabelen voor een aanzienlijk deel de toename van de arbeidsmarktparticipatie van getrouwde vrouwen kunnen verklaren, lijken zij maar een deel van het verhaal. Zowel historische beschrijvingen als terloopse observatie wekken de sterke suggestie dat wijzigingen in gewoonten en sociale normen en sociale beïnvloedingsprocessen ook een belangrijke rol hebben gespeeld. Zulke wijzingen en processen hebben een rechtstreeks effekt op de voorkeuren van de betrokken vrouwen, zodat de aanname van onveranderlijke voorkeuren moet worden losgelaten.

Om veranderende voorkeuren in de arbeidsmarktparticipatie van getrouwde vrouwen te kunnen modelleren, is het echter nodig om psychologische en sociologische inzichten in de economische modellen te integreren (en dat is iets waar veel economen moeite mee hebben). Het belangrijkste doel van dit proefschrift is om aan deze integratie van inzichten een bijdrage te leveren. Hoewel het belang van veranderingen in voorkeuren al werd onderkend door klassieke economen, dateren pogingen om dit soort veranderingen op een systematische manier in de mainstream economische theorie te verwerken van na de Tweede Wereldoorlog. De twee belangrijkste ontwikkelde theorieèn van veranderende voorkeuren of m.a.w. voorkeursvorming zijn de theorie van individuele gewoontevorming en de theorie van onderlinge afhankelijkheid van voorkeuren. (Deze theorieën worden echter, blijkens de aanhef van deze samenvatting, in veel economische modellen niet gebruikt.) Individuele gewoontevorming is het verschijnsel dat een persoon gewend raakt aan een bepaald gedrag dat zij (of hij) vertoont, en dat dit haar voorkeur voor dat gedrag versterkt. Onderlinge afhankelijkheid van voorkeuren betekent dat de voorkeur van een persoon met betrekking tot een bepaald gedrag beïnvloed wordt door soortgelijk gedrag van voor haar belangrijke personen in haar sociale omgeving. Een andere, maar verwante theorie van voorkeursvorming is opgesteld door de Amerikaanse econoom George Akerlof. Zijn theorie van sociale gebruiken modelleert de effekten van het geloof in sociale normen op economisch gedrag en ook een versterkende terugkoppeling van dat gedrag op het geloof in sociale normen. 
Tenslotte is de tegenhanger van individuele gewoontevorming individuele verzadiging op lange termijn. Dit is het verschijnsel dat mensen op lange termijn genoeg krijgen van een bepaald gedrag, zodat hun voorkeur voor dat gedrag vermindert. Dit wordt doorgaans als een onderdeel van de theorie van individuele gewoontevorming beschouwd.

Alle drie genoemde theorieën lijken heel relevant voor de ontwikkeling van de arbeidsmarktparticipatie van vrouwen en, meer in het algemeen, voor het arbeidsaanbodgedrag van zowel vrouwen als mannen (denk aan arbeidsethosnormen). Aan de andere kant is hierover nog maar weinig onderzoek gedaan. Daarom worden de drie theorieën in dit boek toegepast op arbeidsaanbod door ze te incorporeren in de microeconomische theorie van het arbeidsaanbod en door de implicaties hiervan af te leiden. Hierbij worden de drie theorieën uitgebreid en onderling geïntegreerd. Bovendien ligt de nadruk op de effekten van een niet-lineaire dynamiek van voorkeursvorming (zie hieronder) en op het afleiden van het gemiddelde gedrag in een sociale groep van mensen uit het individuele gedrag. Een korte weergave van wat er in de verschillende hoofdstukken van dit boek aan de orde komt, ziet er als volgt uit.

\section{Individuele gewoontevorming}

De standaard micro-economische theorie van arbeidsaanbod modelleert het aantal uren dat men betaald wil werken ais het resuitaat van een afweging van inkomen (of consumptie) en vrije tijd. Uit empirische studies komt naar voren dat zowel aan een bepaald nivo van inkomen als aan een bepaalde hoeveelheid vrije tijd een sterke mate van gewenning plaatsvindt. Dit leidt tot een zichzelf versterkend proces van individuele gewoontevorming. B.v. stel: een huisvrouw neemt een kleine deeltijdbaan aan. Zij raakt dan geleidelijk gewend aan het hogere gezinsinkomen en aan de kleinere hoeveelheid tijd die ze overhoudt voor het huishouden, haar gezin en/of zichzelf (samengevat als "onbetaalde tijd"). Hierdoor kan ze gemotiveerd worden om een deeltijdbaan van meer uren aan te nemen. Olok hieraan zal ze gewend raken, en als gevolg hiervan kan ze een deeltijdbaan van nog meer uren ambiëren. Uiteindelijk zal zo'n zichzelf versterkend proces zich stabiliseren op een deeltijdbaan van een bepaald aantal uren. Dit aantal uren wordt een lange termijn evenwicht genoemd. In een extreem geval kan het lange termijn evenwicht liggen bij een hoog aantal uren per week en heeft de vrouw (of man) zich ontwikkeld tot een "workaholic". Hierbij dient wel opgemerkt te worden dat in dit model nog geen rekening wordt gehouden met het feit dat viouwen (en mannen) niet elk aantal uren betaald kunnen werken dat ze zouden willen. Zo'n zgn. rantsoenering kan echter in het model ingebracht worden, en de implicaties hiervan komen ook ter sprake in het boek.

Hoewel een dergelijk model van zgn. stabiele gewoontevorming veel kan verklaren, kan het niet verklaren waarom bepaalde langdurig werklozen, hoewel ze hun oude baan onvrijwillig verloren hebben, gedemotiveerd raken om een nieuwe baan asn te nemen. Bovendien geeft het wellicht een onvoldoende verklaring van emancipatieprocessen waarin voormalige huisvrouwen een sterke oriëntatie op betaald werk ontwikkelen. In dit boek worden zulke diepgaande veranderingen van voorkeuren en gedrag gemodelleerd als de uitkomsten van instabiele niet-lineaire gewoontevorming. Een model voor dergelijke gewoontevorming is ontwikkeld door de recente Nobelprijswinnaar Gary Becker in samenwerking met Kevin Murphy om het consumptiegedrag van verslaafden te verklaren. Er is dan sprake van een instabiel lange termijn evenwicht van het aantal betaalde arbeidsuren 
dat inligt tussen een stabiel lange termijn evenwicht voor een relatief hoog aantal uren en een ander stabiel lange termijn evenwicht voor een laag aantal uren of nul uur. Dit leidt tot een polarisatie in gedrag en gewoontes: aan de ene kant, als het aantal betaalde arbeidsuren aanvankelijk boven de instabiele evenwichtswaarde ligt, induceert de gewoontevorming een stijging van het aantal uren naar het stabiele evenwicht van een relatief hoog aantal uren en bijbehorende gewenning. Zo'n evenwicht kan het gedrag en de gewoontes van een "geëmancipeerde" vrouw met een baan of een "workaholic" (als het aantal werkuren hoog is) beschrijven. Aan de andere kant, als het aantal betaalde arbeidsuren aanvankelijk onder de instabiele evenwichtswaarde ligt (vgl. een waterscheiding), leidt de gewoontevorming tot een daling van het aantal uren naar het stabiele evenwicht van een laag aantal uren of nul uur en bijbehorende gewenning. Het evenwicht voor nul uur kan dan corresponderen met een huisvrouw of een "vrijwillig langdurig werkloze". Daar het aanvankelijke aantal uren afhangt van de ontwikkeling van exogene (= buiten het model bepaalde) variabelen zoals het uurloon of de vraag naar arbeid (b.v. werkloosheid) in het verleden, bepalen deze ontwikkelingen naar welk van de twee stabiele evenwichten het aantal uren toebeweegt. Deze eigenschap van het model wordt in het boek individuele hysterese genoemd. Voor bepaalde waarden van exogene variabelen kan het stabiele evenwicht waarnaar toebewogen wordt, instabiel worden en vervolgens verdwijnen. Als een soort sneeuwbaleffekt induceer dit een instabiel gewoontevormingsproces naar het andere stabiele evenwicht. Zo'n drastische overgang wordt een individuele katastrofe genoemd. Individuele katastrofes in gedrag en gewoontes kunnen zich hebben voorgedaan (en kunnen zich nog steeds voordoen) in het emancipatieproces van individuele getrouwde vrouwen.

In de hoofdstukken 3 en 4 van deze studie wordt deze niet-lineaire dynamiek van individuele gewoontevorming nader geanalyseerd. In het bijzonder worden grafieken van het individuele lange termijn aanbod van betaalde arbeidsuren als funktie van het uurloon geconstrueerd en worden voldoende en noodzakelijke voorwaarden voor het optreden van individuele hysterese en katastrofes afgeleid. Verder wordt het instabiele individuele gewoontevormingsmodel aangevuld met de effekten van individuele verzadiging op de zeer lange termijn. Tenslotte worden uit de grafieken van het individuele lange termijn arbeidsaanbod grafieken van het gemiddelde lange termijn arbeidsaanbod in een sociale groep afgeleid. Hierbij wordt rekening gehouden met verschillen tussen mensen in voorkeuren, gewoontes en externe omstandigheden.

\section{Onderlinge afhankelijkheid van voorkeuren}

Een belangrijk onderscheid bij onderlinge afhankelijkheid van voorkeuren is dat tussen zgn. bandwagoneffekten en omgekeerde bandwagoneffekten. Een bandwagoneffekt houdt in dat een bepaald gedrag van significante anderen iemands voorkeur met betrekking tot dat gedrag versterkt. Aan de andere kant is er sprake van een omgekeerd bandwagoneffekt, als een bepaald gedrag van significante anderen iemands voorkeur met betrekking tot dat gedrag afzwakt. De literatuur richt zich vooral op bandwagoneffekten, en de Nederlandse onderzoekers Arie Kapteyn en Isolde Woittiez vinden in een empirische studie met Nederlandse data significante bandwagoneffekten op het arbeidsaanbod van vrouwen en mannen. Net als individuele gewoontevorming leidt deze vorm van onderlinge afhankelijkheid van voorkeuren tot een zichzelf versterkend proces. Echter, nu speelt dit proces zich niet af op het nivo van afzonderlijke individuen, maar op dat van een sociale groep van 
individuen. B.v., stel dat in een sociale groep van huisvrouwen sommigen een baan aanvaarden. Dit kan andere leden van de sociale groep ertoe bewegen om ook een baan te nemen of om een baan van meer uren te zoeken. Dit proces van elkaar "opfokken" tot meer betaald werk zal uiteindelijk tot een lange termijn evenwicht leiden, waarbij b.v. een meerderheid van de groep banen van een bepaald (maar in het algemeen onderling verschillend) aantal uren heeft en een minderheid thuisblijft.

Ook in dit geval kan het zichzelf versterkende proces instabiel zijn rond een instabiel lange termijn evenwicht dat inligt tussen twee stabiele lange termijn evenwichten. Afhankelijk van de beginsituatie beweegt de sociale groep zich dan naar hetzij het ene, hetzij het andere stabiele evenwicht. In het ene evenwicht werkt b.v. een meerderheid van de sociale groep uitsluitend als onbetaalde huisvrouw en in het andere evenwicht heeft een meerderheid een betaalde baan. Naar welk evenwicht de sociale groep op weg is, hangt af van de voorgeschiedenis van de sociale groep. Deze afhankelijkheid wordt collectieve hysterese genoemd. Bovendien kan onder bepaalde omstandigheden, als een soort sneeuwbaleffekt, een instabiel bandwagonproces ontstaan van het ene naar het andere stabiele evenwicht. De sociale groep treedt dan en masse toe tot de arbeidsmarkt of treedt en masse uit. Dergelijke drastische overgangen binnen sociale groepen worden collectieve katastrofes genoemd en kunnen een rol gespeeld hebben (en nog spelen) in de sterke toename van de arbeidsmarkparticipatie van getrouwde vrouwen in veel OESO-landen sinds de jaren vijftig en zestig.

In hoofstuk 5 wordt deze niet-lineaire dynamiek van onderling afhankelijke voorkeuren in arbeidsaanbod nader geanalyseerd. Eerst wordt een classificatie gegeven van de verschillende soorten sociale interaktie die ten grondslag liggen aan de onderlinge afhankelijkheid van voorkeuren in arbeidsaanbod. Vervolgens worden deze sociale interakties samengevat in een algemeen model, dat een zelfde soort struktuur heeft als het individuele gewoontevormingsmodel. Deze analogie wordt gebruikt om grafieken af te leiden van het gemiddelde lange termijn arbeidsaanbod in een sociale groep als funktie van het gemiddelde uurloon. Deze grafieken zien er echter op wezenlijke punten anders uit dan de grafieken van het gemiddelde lange termijn arbeidsaanbod die volgen uit het individuele gewoontevormingsmodel (dat geldt op het nivo van afzonderlijke individuen). Dit komt door de verschillen in voorkeuren en omstandigheden tussen leden van de sociale groep. Deze heterogeniteit vormt een tegenkracht tegen de neiging tot confonmisme als gevolg van de onderlinge athankelijkheid van voorkeuren. Daarom leidt de heterogeniteit ook tot voldoende en noodzakelijke voorwaarden voor collectieve katastrofes en hysterese die sterker zijn dan in het geval van een homogene sociale groep.

\section{Collectieve gewoontevorming}

Om tot een vollediger modellering van bovengenoemde empirische verschijnselen te komen, worden de in de hoofdstukken 3 en 4 geanalyseerde modellen van individuele gewoontevorming en de in hoofdstuk 5 behandelde modelten van onderlinge afhankelijkheid van voorkeuren met elkaar geïntegreerd in de hoofdstukken 6 en 7. Dit leidt tot modellen van instabiele collectieve gewoontevorming. Deze modellen vallen uiteen in twee soorten. In hoofdstuk 6 worden modellen van onderling afhankelijke voorkeuren en stabiele individuele gewoontevoming onderzocht, terwijt in hoofdstuk 7 modellen van onderling afhankelijke voorkeuren en instabiele individuele gewoontevorming aan de orde komen. In het eerste 
geval kunnen de onderlinge afhankelijkheid van voorkeuren en de individuele gewoontevorming elkaar versterken tot een instabiel proces van collectieve gewoontevorming, maar werkt de individuele gewoontevorming ook remmend op de neiging tot conformisme, doordat ze verschillen in voorkeuren en gedrag tussen de leden van de sociale groep vergroot. In het geval van onderling afhankelijke voorkeuren en instabiele individuele gewoontevorming kunnen collectieve katastrofes en hysterese zich voordoen als het resultaat van een soort "tweetrapsraket". In de eerste trap induceert de instabiele individuele gewoontevorming individuele katastrofes van een aantal groepsleden van nul naar een maximaal aantal betaalde arbeidsuren of omgekeerd. De tweede trap ontbrandt, wanneer, ten gevolge van een voldoende sterke onderlinge afhankelijkheid van voorkeuren, de individuele katastrofes daaropvolgende individuele katastrofes van andere groepsleden "triggeren" in die mate dat een collectieve katastrofe ontstaat. Dan begint b.v, een explosief groeiend aantal groepsleden een explosief groeiend aantal betaalde arbeidsuren te werken. Deze arbeidsaanboddynamiek wordt geanalyseerd voor twee subgevallen, nl. Geval A van heterogeniteit van de groepsleden in persoonlijke voorkeur voor onbetaalde tijd en in de mate van geneigdheid tot individuele gewoontevorming en Geval B van heterogeniteit in de mate van ontvankelijkheid voor sociale beïnvloeding. Voor beide subgevallen worden voldoende (en noodzakelijke) voorwaarden voor collectieve katastrofes afgeleid en worden interessante grafieken van het gemiddelde lange termijn arbeidsaanbod geconstrueerd, die twee domeinen van katastrofaal gedrag aan weerskanten van een S-vormig domein van onveranderlijk hysteresegedrag vertonen (zie de figuur op de kaft van dit boek).

\section{Dynamiek van sociale normen}

Een theorie van voorkeursvorming die verwant is aan de hierboven besproken theorieën, is de door George Akerlof ontwikkelde theorie van sociale gebruiken. Deze theorie bestaat uit een model voor de korte termijn en een model voor de lange termijn. Het korte termijn model beschrijft de invloed van het geloof in sociale normen op economisch gedrag. Deze invloed is tweeërlei. In de eerste plaats zullen mensen, als zij geloven in de juistheid van een bepaalde sociale norm, zich overeenkomstig deze norm willen gedragen om niet met zichzelf in conflict te raken. Sociologen spreken dan over het effekt van een geinternalisecrde sociale norm. In de tweede plaats zullen mensen, als zij deel uitmaken van een sociale groep waarvan veel leden geloven in de sociale norm, zich willen conformeren aan deze norm om niet met de nek te worden aangekeken. M.a.w., de meeste mensen zijn gevoelig voor hun reputatie in een sociale groep waanoe zij behoren, en dit leidt tot wat sociologen sociale controle noemen. Daarnaast wordt in het lange termijn model van Akerlof aangenomen, dat naarmate meer groepsleden de sociale nomn in hun gedrag aan hun taars lappen (b.v. met het oog op economisch gewin). op langere termijn het geloof in de norm in de sociale groep wordt ondermijnd. Dat wil zeggen: het aantal groepsleden dat nog gelooft in de norm, neemt dan af. Aan de andere kant, naarmate meer groepsleden zich conformeren aan de norm, wordt op langere termijn het geloof in de norm in de sociale groep versterkt, d.w.z. het aantal groepsleden dat gelooft in de norm, neemt dan toe. Aldus beschrijft het lange termijn model van Akerlof processen van intemalisatie en "externalisatie" van een sociale norm, waardoor het geloof in die sociale norm verandert.

In hoofdstuk 8 wordt een variant van de theorie van sociale gebruiken toegepast op arbeidsaanbod. Eerst wordt een overzicht gegeven var sociale normen die een ro! lijken te 
spelen in het arbeidsaanbod van mannen en vrouwen. Alleen de implicaties van sociale normen in het arbeidsaanbod van vrouwen worden uitgewerkt, maar de implicaties van sociale normen in het arbeidsaanbod van mannen kunnen op een soortgelijke manier worden geanalyseerd (ze zijn wel op wezenlijke punten anders). In het bijzonder wordt de theorie van sociale gebruiken toegepast op een traditionele norm, die zegt dat getrouwde vrouwen met kinderen thuis behoren te blijven om voor de kinderen en het huishouden te zorgen. We noemen dit de huishoudnorm (of achter-het-aanrecht-norm). Het resulterende model blijkt een zelfde soort structuur te hebben als een grensgeval van het bovengenoemde Geval B van het collectieve gewoontevormingsmodel in hoofdstuk. 7. Dit betekent dat, als we het begrip "gewoonte" in bredere zin opvatten als "algemene houding" of "habitus", de effekten en dynamiek van sociale normen in de theorie van sociale gebruiken kunnen worden geïnterpreteerd als één van de mogelijke "kanalen" waarlangs instabiele collectieve gewoontevorming zich kan ontwikkelen. Verder maakt een vergelijking met andere toepassingen van de theorie van sociale gewoonten in de literatuur duidelijk, dat de toepassing in dit boek twee paar essentiēle elementen toevoegt aan het algemene beeld, nl. individuele en collectieve hysterese en individuele en collectieve katastrofes. Tenslotte wordt het arbeidsaanbodmodel voor getrouwde vrouwen uitgebreid met een emancipatoire sociale norm van economische zelfstandigheid, die de traditionele huishoudnorm steeds meer lijkt te verdringen en die zegt dat niet-alleenstaande vrouwen een baan behoren te hebben die hen financiëel onafhankelijk van hun partner maakt. Deze uitbreiding blijkt allerlei interessante effekten te hebben die ook worden geanalyseerd.

\section{Dynamiek van de arbeidsmarktparticipatie van vrouwen}

In hoofdstuk 9 wordt de empirische relevantie van de theoretische modellen die in de voorafgaande hoofdstukken zijn opgesteld, onderzocht door ze toe te passen op lange termijn ontwikkelingen van de arbeidsmarktparticipatie van Nederlandse en Amerikaanse getrouwde vrouwen. Terwijl de participatiegraad van Nederlandse getrouwde vrouwen opmerkelijk laag en stabiel was vanaf eind vorige eeuw tot begin jaren zestig, nam hij daarna sterk toe. De mogelijke rol van onderlinge afhankelijkheid van voorkeuren en sociale normen in laatstgenoemde toename is econometrisch onderzocht door de Maastrichtse onderzoeker Sjoerd Romme voor de periode 1971-1985. Hij vindt significante en aanzienlijke effekten van deze faktoren, maar zijn participatievergelijkingen zijn niet systematisch afgeleid van een achterliggend micro-economisch arbeidsaanbodmodel.

Om hierin verbetering te brengen, worden in hoofdstuk 9 participatievergelijkingen voor een sociale groep of deelpopulatie van getrouwde vrouwen met schoolgaande of kleine kinderen afgeleid uit de theoretische modellen van de voorafgaande hoofdstukken. Deze participatievergelijkingen hebben, als gevolg van hysterese-effekten van individuele gewoontevorming, verschillende specificaties voor toetreding tot de arbeidsmarkt en uittreding, en zij suggereren dat de participatievergelijkingen van Romme eñ andere onderzoekers emstige specificatiefouten bevatten. De sterk niet-lineaire dynamiek van collectieve gewoontevorming en sociale normen leidt ertoe dat de zgn. lange termijn elasticiteiten van de participatiegraad als funktie van het uurloon en andere exogene variabelen lage waarden hebben bij relatief lage waarden van de participatiegraad en veel hogere waarden bij hogere participatiegraden. Dit impliceent dat een verhoging van het uurloon met één procent in de jaren vijftig en daarvóór, toen de participatiegraad laag was, 
een veel kleiner procentueel effekt op de participatiegraad had dan in de jaren zeventig en tachtig met hun hogere participatiegraden. De achterliggende reden is dat de dynamiek van collectieve gewoontevorming en sociale normen in de jaren vijftig en daarvóór het traditionele rollenpatroon van man en vrouw bevestigde, terwijl zij in de jaren zeventig en tachtig de in de jaren zestig ingezette verandering van het rollenpatroon juist versterkte. Op een meer overtuigende wijze dan de gebruikelijke statische modellen, lijkt dit een verklaring te bieden van de opmerkelijk lage en stabiele participatiegraad van Nederlandse getrouwde vrouwen vóór het begin van de jaren zestig en de sterke toename van deze participatiegraad daama.

Laatstgenoemde sterke toename alsmede de eerder ingezette sterke stijging van de participatiegraad van getrouwde vrouwen in andere OESO-landen kan veroorzaakı zijn door collectieve katastrofes binnen sociale groepen. Een voldoende en noodzakelijke voorwaarde voor deze mogelijkheid wordt afgeleid en een zelfde soort arbeidsaanbodgrafiek als in hoofdstuk 7 wordt verkregen (zie de figuur op de kaft). Door de schaarste aan data op het nivo van sociale groepen is het echter erg moeilijk om collectieve katastrofes op dit nivo te identificeren. Verder wordt het feit dat de participatiegraad van getrouwde vrouwen in de Verenigde Staten niet sterk daalde, toen de Amerikaanse soldaten terugkeerden uit de Tweede Wereldoorlog, verklaard als het resultaat van hysterese-effekten van collectieve gewoontevorming. Tenslotte wordt de ontwikkeling van de participatiegraad van Nederlandse getrouwde vrouwen na de Tweede Wereldoorlog opgevat als het resultaat van een culturele golf rond een opwaarse trend in de door werkgevers uitgeoefende vraag naar arbeid van vrouwen. De culturele golf wordt veroorzakkt door een combinatie van collectieve gewoontevorming (inclusief sociale normen) op een middenlange termijn en collectieve verzadiging op een zeer lange termijn. Daar de twee mechanismen geassociëerd kunnen worden met concepties van respectievelijk Marx en Maslow, wordt de culturele golf betiteld als de Marx-Maslow-golf (of M \& M golf). 


\section{Curriculum Vitae}

Maarten Vendrik werd geboren op 10 juni 1950 te Utrecht. In 1968 sloot hij met goed gevolg het Gymnasium $\beta$ af aan het Stedelijk Gymnasium te Nijmegen. Van 1968 tot 1976 studeerde hij wis- en natuurkunde aan de Katholieke Universiteit Nijmegen, alwaar hij in 1976 cum laude afstudeerde in de theoretische natuurkunde. Daarna was hij een jaar werkzaam bij een pilot study over een milieubiologische inbreng in (economische) wereidmodellen aan het Instituut voor Theoretische Biologie van de Rijksuniversiteit Leiden, Vervolgens studeerde hij van 1977 tot 1986 algemene econometrie aan de Erasmusuniversiteit te Rotterdam en was hij van 1978 tot 1984 student-assistent bij onderzoek over energie, milieu en industrie in de vakgroep macro-economie van de Erasmusuniversiteit. Daarnaast publiceerde hij in de theoretische natuurkunde en volgde hij een reeks hoor- en werkcolleges in de sociale en economische filosofie en de sociologie. Na het behalen van zijn doctoraaldiploma in de algemene econometrie in 1986 (cum laude) trad hij in dienst van de vakgroep algemene economie van de Rijksuniversiteit Limburg te Maastricht. Aanvankelijk als wetenschappelijk assistent, later als toegevoegd onderzoeker en toegevoegd docent, verrichtte hij onderzoek over de rol van psychologische en sociologische factoren in een economische analyse van arbeidsaanbod. De resultaten hiervan werden onder meer vastgelegd in dit proefschrift. Sinds 1992 is hij universitair docent micro-economie in laatstgenoemde vakgroep. Bovendien heeft hij nu een post-doctoral research fellowship in deze vakgroep.

Maarten Vendrik (bom June 10,1950, at Utrecht, the Netherlands) studied physics at the Catholic University of Nijmegen from 1968 to 1976 and econometrics and philosophy at the Erasmus University of Rotterdam from 1977 to 1986. He worked as a research fellow on a pilot study in environmental biology at the University of Leiden (1976-1977) and as a research assistant in macroeconomics at the Erasmus University of Rotterdam (1978-1984). In addition, he has published in theoretical physics. Between 1986 and 1992 he was research fellow at the department of economics of the University of Limburg at Maastricht, and since 1992 he has been assistant professor of microeconomics at that department. Moreover, he now has a post-doctoral research fellowship at the department. 

A remarkable phenomenon in economic science is that the assumption of stable preferences is still a point of departure of many of its models, despite the fact that there is growing evidence to the contrary. One striking example of preference shifts in the field of labour supply is given by the changes in attitude of "emancipating" married women towards their participation in the labour market.

In order to construct explanatory models of such phenomena, this study applies three theories of preference formation to labour supply, namely the theory of habit formation and long-run satiation, the theory of preference interdependence, and the theory of social custom. These theories are incorporated into the microeconomic theory of labour supply, and implications of this incorporation are derived. In doing so, the three preference formation theories are extended and integrated. Moreover, the focus is on the effects of non-linear forms of dynamics of the preference formations in combination with aggregation from the microeconomic to the macroeconomic behaviour.

It is shown that in contrast to the existing models of stable habit formation and stable preference interdependence, non-linear and locally unstable habit formation and preference interdependence can explain profound changes in labour supply preferences and behaviour such as when long-term unemployed become little motivated to get a new job or when former housewives develop a strong orientation towards paid work. Furthermore, it is demonstrated that the effects and dynamics of social norms in the theory of social custom can be interpreted as one of the possible "channels" through which unstable habit formation and preference interdependence may proceed. Finally, the empirical relevance of the theoretical models developed is investigated by applying them to the developments of the labour force participation of Dutch and American married women since World War II. 